INEL-94/0059

SNF-REP-TR-001

\title{
Transportation Capabilities Study for DOE-Owned Spent Nuclear Fuel
}

\author{
G. L Clark \\ R. A. Johnson \\ R. W. Smith \\ D. G. Abbott ${ }^{a}$ \\ M. J. Tyacke"
}

Published October 1994

Packaging Technology, Inc.
Tacoma, Washington 98424

Prepared for Lockheed Idaho Technologles Company Under Subcontract No. C94-170778

and for the

U.S. Department of Energy

Assistant Secretary for Environmental Management Under DOE Idaho Operations Offlce

Contract DE-AC07-941D13223 
^ 


\section{DISCLAIMER}

This report was prepared as an account of work sponsored by an agency of the United States Government. Neither the United States Government nor any agency thereof, nor any of their employees, make any warranty, express or implied, or assumes any legal liability or responsibility for the accuracy, completeness, or usefuiness of any information, apparatus, product, or process disclosed, or represents that its use would not infringe privately owned rights. Reference herein to any specific commercial product, process, or service by trade name, trademark, manufacturer, or otherwise does not necessarily constitute or imply its endorsement, recommendation, or favoring by the United States Government or any agency thereof. The views and opinions of authors expressed herein do not necessarily state or reflect those of the United States Government or any agency thereof. 


\section{DISCLAIMER}

Portions of this document may be illegible in electronic image products. Images are produced from the best available original document. 


\begin{abstract}
This study evaluates current capabilities for transporting spent nuclear fuel owned by the U.S. Department of Energy. Currently licensed irradiated fuel shipping packages that have the potential for shipping the spent nuclear fuel are identified and then matched against the various spent nuclear fuel types. Also included are the results of a limited investigation into other certified packages and new packages currently under development. This study is intended to support top-level planning for the disposition of the Department of Energy's spent nuclear fuel inventory.
\end{abstract}




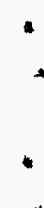




\section{ACKNOWLEDGMENTS}

A good deal of information contained in this report was obtained through direct communication with the various stakeholders (e.g., user sites, U.S. Nuclear Regulatory Commission and U.S. Department of Energy regulatory authorities, packaging developers or owners). Their contributions are too numerous to be listed in detail, but are gratefully acknowledged nonetheless. 
.

.

. 


\section{CONTENTS}

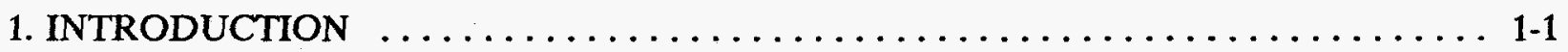

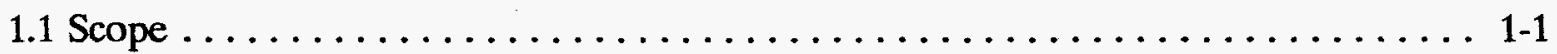

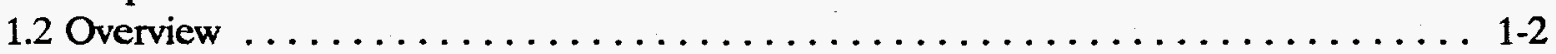

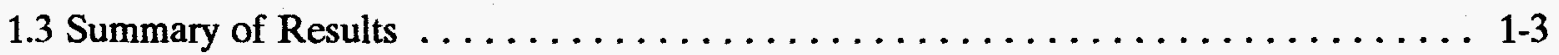

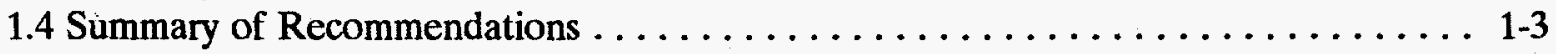

2. IDENTIFICATION OF DOE-OWNED SPENT NUCLEAR FUEL $\ldots \ldots \ldots \ldots \ldots \ldots 2-1$

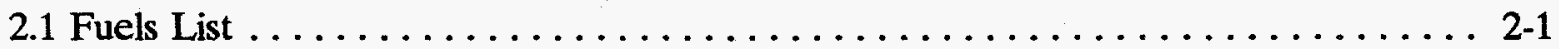

2.2 Discussion of Fuel Characteristics That Govern Transport $\ldots \ldots \ldots \ldots \ldots \ldots .2-1$

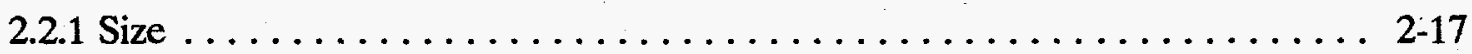

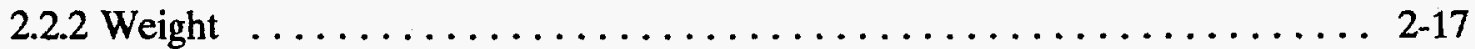

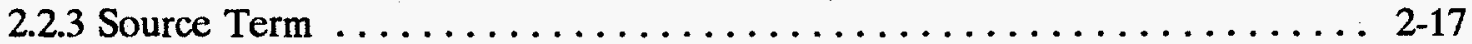

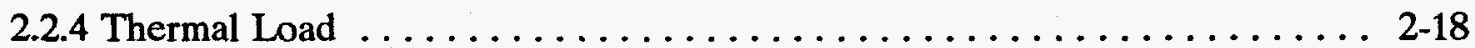

2.2 .5 Fragility $\ldots \ldots \ldots \ldots \ldots \ldots \ldots \ldots \ldots \ldots \ldots \ldots \ldots \ldots \ldots \ldots \ldots \ldots \ldots \ldots \ldots \ldots \ldots, 2-18$

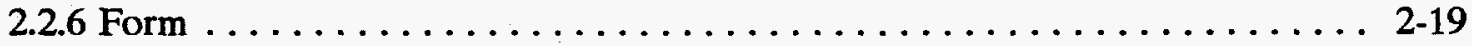

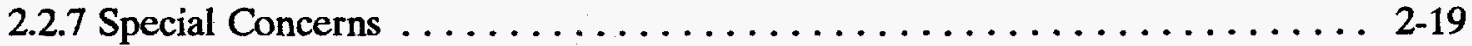

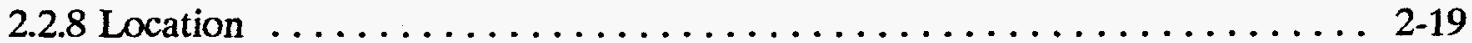

2.3 Discussion of Unknowns and Uncertainties Related to Fuels $\ldots \ldots \ldots \ldots \ldots$ 2-19

3. IDENTIFICATION OF CANDIDATE PACKAGINGS CURRENTLY IN EXISTENCE • 3-1

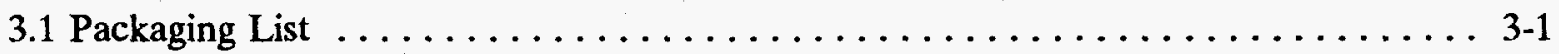

3.2 Discussion of Packaging-Imposed Payload Restrictions $\ldots \ldots \ldots \ldots \ldots \ldots \ldots$ 3-1

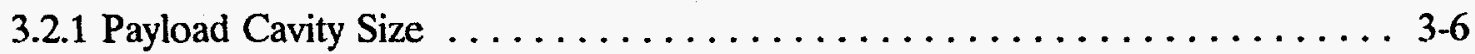

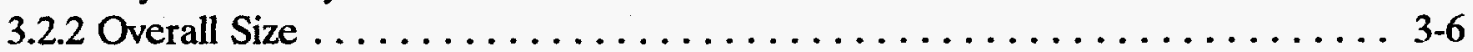

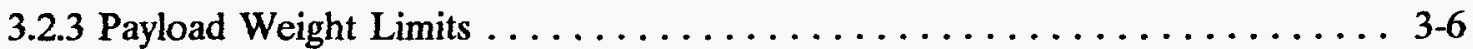

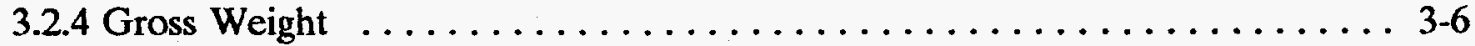

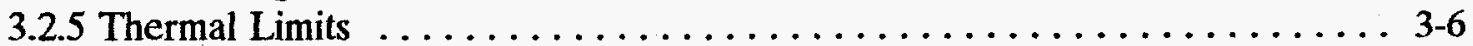

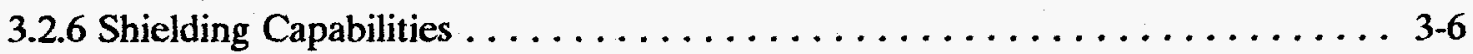

3.2.7 Containment Capabilities . . . . . . . . . . . . . .

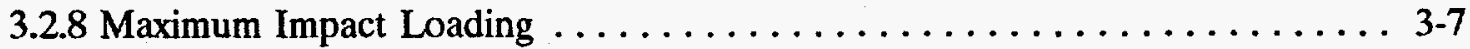

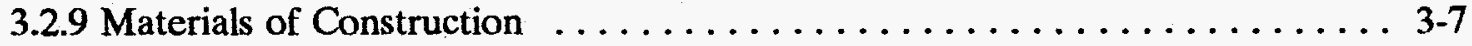

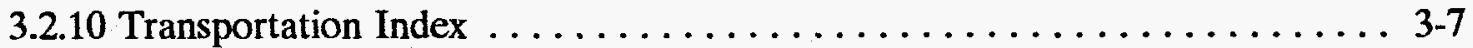

3.2.11 Operational Requirements $\ldots \ldots \ldots \ldots \ldots \ldots \ldots \ldots \ldots \ldots \ldots \ldots .3 .8$

3.2.12 Transport Mode.$\ldots \ldots \ldots \ldots \ldots \ldots \ldots \ldots \ldots \ldots \ldots \ldots \ldots \ldots \ldots$

3.3 Discussion of Unknowns and Uncertainties Related to Packagings $\ldots \ldots \ldots \ldots$ 3-8 
4.1 Capability That Currently Exists $\ldots \ldots \ldots \ldots \ldots \ldots \ldots \ldots \ldots \ldots \ldots \ldots \ldots \ldots$

4.2 Capability via Amended SARP and Certificate of Compliance (No Hardware

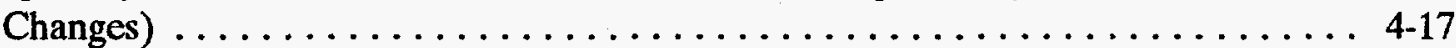

4.3 Capability via Revised SARP and Certificate of Compliance (No Hardware

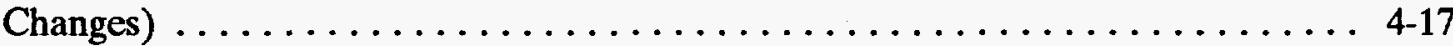

4.4 Capability via Revised SARP and Certificate of Compliance (Hardware

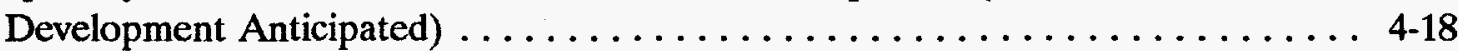

4.5 Capability via Packaging Design Change (New Certificate of Compliance) . . . . 4-18

4.6 No Apparent Capability (New Package Design Required) . . . . . . . . . . . . 4-18

4.7 Additional Study Needed to Establish Capability $\ldots \ldots \ldots \ldots \ldots \ldots \ldots \ldots \ldots .4 .18$

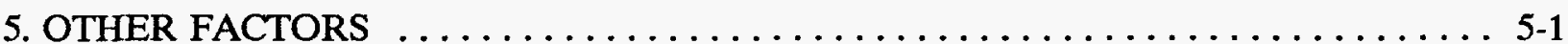

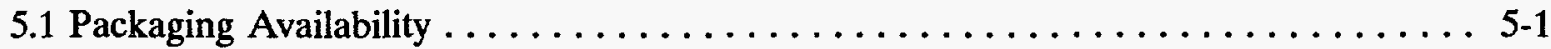

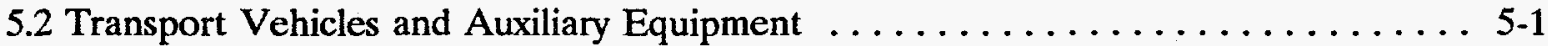

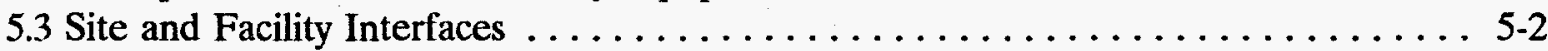

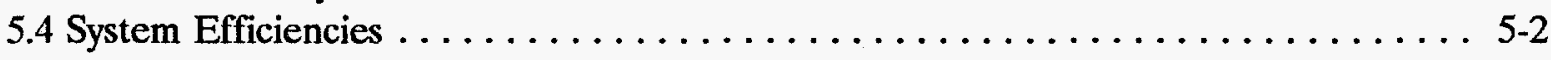

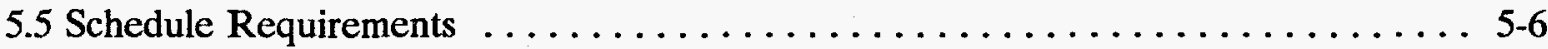

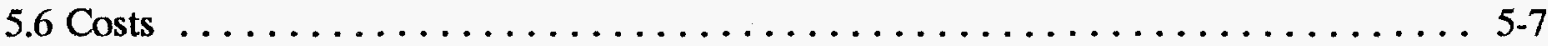

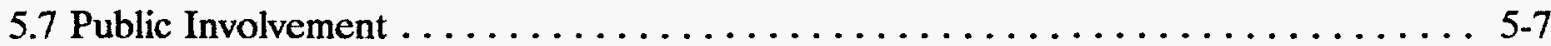

6. SUMMARY AND CONCLUSIONS $\ldots \ldots \ldots \ldots \ldots \ldots \ldots \ldots \ldots \ldots \ldots \ldots \ldots \ldots$

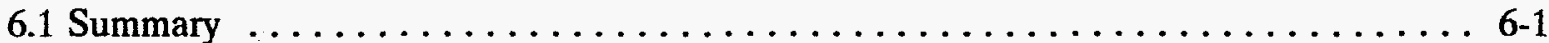

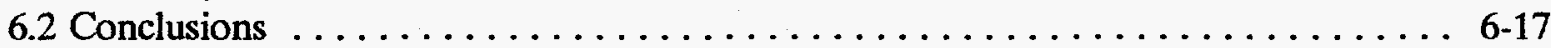

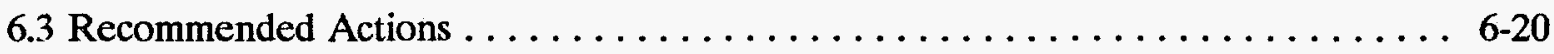

7. REFERENCES $\ldots \ldots \ldots \ldots \ldots \ldots \ldots \ldots \ldots \ldots \ldots \ldots \ldots \ldots \ldots \ldots \ldots \ldots \ldots \ldots$

Appendix A: Fuel Data Sheets and Design Drawings/Sketches . . . . . . . . . . . A-1

Appendix B: Packaging Data Sheets and Design Drawings/Sketches $\ldots \ldots \ldots \ldots \ldots \ldots$. . . . 


\section{FIGURE}

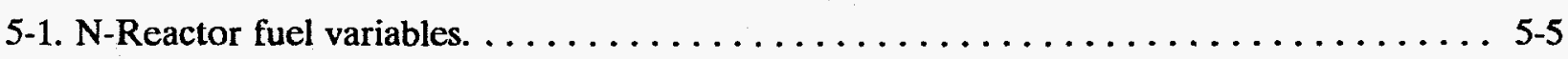

\section{TABLES}

2-1. Pressurized water reactor (PWR) DOE-owned SNF types $\ldots \ldots \ldots \ldots \ldots \ldots \ldots \ldots, 2-2$

2-2. Boiling water reactor (BWR) DOE-owned SNF types $\ldots \ldots \ldots \ldots \ldots \ldots \ldots \ldots \ldots \ldots \ldots \ldots \ldots$

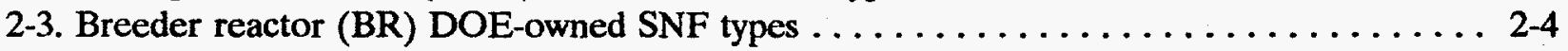

2-4. Gas-cooled reactor (GCR) DOE-owned SNF types $\ldots \ldots \ldots \ldots \ldots \ldots \ldots \ldots \ldots \ldots \ldots \ldots \ldots$

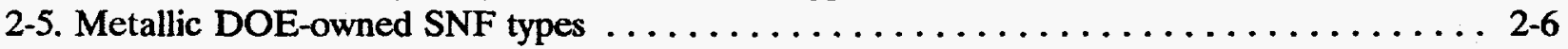

2-6. Graphite-moderated DOE-owned SNF types ..................... 2-10

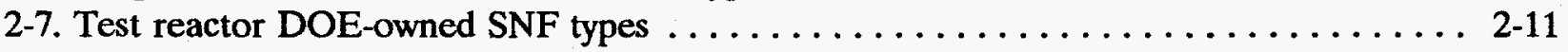

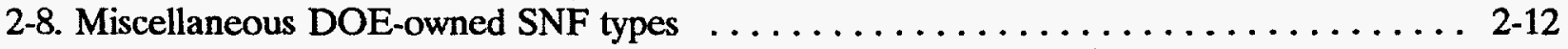

3-1. Transport package information $\ldots \ldots \ldots \ldots \ldots \ldots \ldots \ldots \ldots \ldots \ldots \ldots \ldots \ldots \ldots \ldots \ldots \ldots, 3-2$

4-1. Packaging compatibility with pressurized water reactor type SNF $\ldots \ldots \ldots \ldots \ldots \ldots$ 4-2

4-2. Packaging compatibility with boiling water reactor type SNF $\ldots \ldots \ldots \ldots \ldots \ldots \ldots .4$

4-3. Packaging compatibility with breeder reactor type SNF $\ldots \ldots \ldots \ldots \ldots \ldots \ldots$ 4-5

4-4. Packaging compatibility with gas-cooled reactor type SNF $\ldots \ldots \ldots \ldots \ldots \ldots \ldots$ 4-7

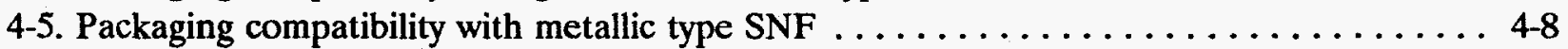

4-6. Packaging compatibility with graphite-moderated reactor type SNF $\ldots \ldots \ldots \ldots \ldots$ 4-15

$5-1$. Examples of single fuel types currently stored in multiple locations $\ldots \ldots \ldots \ldots \ldots .5-3$

6-1. Summary of potential transportation package and fuel type matchups. . . . . . . . 6 6-1

6-2. Fuel/package matchup summary matrix. $\ldots \ldots \ldots \ldots \ldots \ldots \ldots \ldots \ldots \ldots \ldots .6 \ldots \ldots$ 


\section{ACRONYMS}

$\begin{array}{ll}\text { AGN } & \text { Aerojet-General Nucleonics reactor } \\ \text { ANL-E } & \text { Argonne National Laboratory-East } \\ \text { ANL-W } & \text { Argonne National Laboratory-West } \\ \text { ARMF } & \text { Advanced Reactivity Measurement Facility } \\ \text { ATR } & \text { Advanced Test Reactor } \\ \text { ATSR } & \text { Argonne Thermal Source Reactor } \\ \text { B\&W } & \text { Babcock \& Wilcox } \\ \text { BNL } & \text { Brookhaven National Laboratory } \\ \text { BR } & \text { breeder reactor } \\ \text { BRP } & \text { Big Rock Point } \\ \text { BSR } & \text { Bulk Shielding Reactor } \\ \text { BWR } & \text { boiling water reactor } \\ \text { CFRMF } & \text { Coupled Fast Reactivity Measurement Facility } \\ \text { CPP } & \text { Chemical Processing Plant } \\ \text { DOE } & \text { U.S. Department of Energy } \\ \text { DOT } & \text { U.S. Department of Transportation } \\ \text { DU } & \text { depleted uranium } \\ \text { EBR } & \text { Experimental Breeder Reactor } \\ \text { EFPH } & \text { effective full-power hours } \\ \text { EH } & \text { Transportation and Packaging Safety Division } \\ \text { EPR } & \text { Experimental Power Reactor } \\ \text { ERR } & \text { Elk River Reactor } \\ \text { FFTF } & \text { Fast Flux Test Facility } \\ \text { FSV } & \text { Fort St. Vrain } \\ \text { FSVR } & \text { Fort St. Vrain Reactor } \\ \text { GCR } & \text { gas-cooled reactor } \\ \text { GCRE } & \text { Gas-Cooled Reactor Experiment } \\ \text { GE } & \text { General Electric } \\ \text { GETR } & \text { General Electric Test Reactor } \\ \text { HEU } & \text { high enriched uranium } \\ \text { HFBR } & \text { High-Flux Beam Reactor } \\ \text { HFEF } & \text { Hot Fuel Examination Facility } \\ \text { HFIR } & \text { High-Flux Isotope Reactor } \\ \text { HRE } & \text { Homogeneous Reactor Experiments } \\ \text { HWCTR } & \text { Heavy Water Component Test Reactor } \\ \text { ID } & \text { Idaho Operations Office } \\ \text { INEL } & \text { Idaho National Engineering Laboratory } \\ \text { JMTR } & \text { Japanese Material Test Reactor } \\ \text { LANL } & \text { Los Alamos National Laboratory } \\ \text { LEU } & \text { low enriched uranium } \\ \text { LM } & \text { liquid metal } \\ \text { LOCA } & \text { loss-of-coolant accident } \\ \text { LOFT } & \text { Loss-of-Fluid Test (reactor) } \\ \text { LW } & \text { light water } \\ & \\ & \end{array}$


LWR light water reactor

LWT legal weight truck

MIT Massachusetts Institute of Technology

MITHM metric initial ton of heavy metal

MNOP maximum normal operating pressure

MSRE Molten Salt Reactor Experiment

MTHM metric tons of heavy metal

MTR Materials Test Reactor

MURR Missouri University Research Reactor

NPR New Production Reactor

NR Naval Reactors Division

NRAD Neutron Radiography Facility

NRC U.S. Nuclear Regulatory Commission

ORNL Oak Ridge National Laboratory

ORR Oak Ridge Research Reactor

OSR Ohio State Reactor

PBF Power Burst Facility

PNL Pacific National Laboratory

PUREX Plutonium-Uranium Extraction (plant)

PWR pressurized water reactor

RBOF receiving basin for offsite fuel

REG R.E. Gina

RINC Rhode Island Nuclear Center

RSWF Radioactive Scrap and Waste Facility

SARP safety analysis report for packaging

SM Stationary Medium Power Plant

SNAP System for Nuclear Auxiliary Power

SNF spent nuclear fuel

SNL Sandia National Laboratories

SNM special nuclear material

SPERT Special Power Excursion Reactor Test

SPR Sandia Pulsed Reactor

SPSS Shield Plug Storage Station

SRS Savannah River Site

TAN Test Area North

TORY Experimental Propulsion Test Reactor

TREAT Transient Reactor Test Facility

TRIGA training, research, and isotope reactors (built by General Atomic)

TSR Tower Shielding Reactor

WAG Waste Area Group

ZPR Zero Power Reactor 


\section{Transportation Capabilities Study for DOE-Owned Spent Nuclear Fuel}

\section{INTRODUCTION}

This study assesses transportation capabilities for U.S. Department of Energy (DOE)-owned noncommercial spent nuclear fuel (SNF). The study was prepared to support top-level planning associated with management of DOE's SNF. The results of this study, when coupled with other recently completed and in-process studies of the various fuels themselves and their respective storage facilities (see References section), will provide guidelines for appropriate actions in dealing with DOE's SNF inventory.

\subsection{Scope}

Because of the limited time available for this study, the scope carefully focused on identifying currently licensed [U.S. Nuclear Regulatory Commission (NRC) or DOE] irradiated fuel shipping packages with the potential for shipping DOE-owned SNF. Also included are the results of a limited investigation into other International Atomic Energy Agency-certified packages and new packages currently under development.

Five primary goals for the study were established as follows:

1. Identify and discuss the issues potentially affecting transport of DOE-owned SNF.

2. Group and rank DOE-owned fuels in a way meaningful for transportation.

3. Identify currently licensed transport packages potentially suitable for conveying the fuels.

4. Determine, when possible, compatible fuel/package combinations.

5. Develop recommendations for followup actions.

It should be noted that the issues related to transportation of DOE's SNF, as set out in detail in this report, involve a large number of complex and often interrelated technical and political factors that must be resolved with a high degree of certainty before any realistic SNF transportation assessment can be initiated. Foremost among the uncertainties is a notable lack of adequate characterization of much of the SNF inventory. Many questions involving fuel form, radiological characteristics, and physical condition remain to be answered before licensing requirements for suitable transport packages can be determined.

Additionally, any package-related modifications that may be necessary to accommodate these fuels introduce a level of uncertainty that increases with the degree of modification required. Even with comprehensively characterized fuel, there is no guarantee that package analyses or designs can be modified sufficiently to accommodate the fuel. Furthermore, transportation 
regulations, and interpretations thereof, have been growing steadily more stringent over the years. Even if the proposed package-related modifications prove to be successful in theory, there is no assurance that they will be acceptable to the appropriate regulators. In the current regulatory and social climate, it should be expected that transporting the noncommercial SNF inventory will demand a very significant amount of time and resources.

\subsection{Overview}

Details of the DOE-owned SNF are presented in Section 2. This includes a complete listing of DOE-owned SNF. The SNF types are grouped in general categories, along with their most significant transportation-related characteristics. A detailed discussion of fuel characteristics that govern transport is also included in this section.

In many cases, insufficient fuel characteristics data exist at this time to allow a transportation assessment to be made for all DOE-owned SNF. It is estimated that the time frame required to fully characterize all of the SNF inventory will be on the order of five years. This estimate assumes that a relatively high priority in terms of resources will be given to this effort. As the various fuel types are characterized, transport packaging requirements can be developed sequentially, assuming resources are available for such a concurrent activity.

Transportation package characteristics are given in Section 3, and are summarized in Table 3-1. A number of key factors relating to transport packaging design and licensing are included and discussed.

Fuel/package compatibility relationships are discussed in Section 4. Current transport capability is discussed, as well as potential package modifications that might result in extended capability. With this background information in hand, fuel characteristics and cask design details were reviewed to appropriately categorize fuels and to match fuel categories with specific cask types. Considering such fuel-related items as geometry, decay heat and source term, and corresponding packaging characteristics (e.g., cavity size, heat transfer capability, and shielding characteristics), a fuel/package compatibility matrix was developed. This matrix is included as Tables 4-1 through 4-6, and is summarized in Table 6-2.

Additional factors, unrelated to specific fuel/package compatibility issues, are discussed in Section 5. Among these are facility interface modification or development requirements, funding procurement, and accommodation for public involvement.

Finally, the results of the study are summarized in Section 6 in a fuel/package compatibility matrix. Conclusions drawn from the study are included, as well as recommendations for further actions. Section 7 presents the references cited in this study. 


\subsection{Summary of Results}

Early on, the approach being developed indicated that very few direct matchups exist between fuels and currently licensed casks. Of 230 fuel types, only 36 types (16\%) qualified for transport in currently licensed casks (see Table 6-1). These matchups involve certain fuel types for which cask designs were specifically developed, and more general, commercial fuel types which can be accommodated by casks designed for such fuel. In all other cases, one or more of the following actions (ordered by increasing level of effort, cost, and risk), coupled with a corresponding NRC or DOE review and certification activity, will be required before shipping DOE-owned SNF:

1. Safety analysis report for packaging (SARP) and Certificate of Compliance amendment to add a new fuel definition (enveloped by the currently authorized payload limits).

2. SARP and Certificate of Compliance revision to accommodate a more demanding payload definition (one that is not enveloped by the currently authorized payload definition, and that extends the package capability or reduces currently documented safety margins).

3. SARP and Certificate of Compliance revision to accommodate a new fuel basket or canister design, and associated fabrication activity for the specific fuel to be transported.

4. SARP and Certificate of Compliance revision, and associated fabrication activity, for a modified packaging design to accommodate a new, more demanding fuel definition.

5. Entirely new package design, license and fabrication activity.

The timeline associated with completing the above changes, including certification, can be as little as three months (in the case of a simple payload amendment) to as much as three or more years (for a new package or major SARP and hardware revision). These time estimates do not include the time that may be required for adequate fuel characterization. The actual overall time frame will be strongly dependent on the priority associated with a particular shipping campaign (within DOE or NRC) and on the demonstration approaches employed, as well as the conservatism embodied in the design or demonstration.

\subsection{Summary of Recommendations}

Recommendations for further actions are summarized as follows:

- A fairly large amount of DOE-owned SNF can potentially be transported in existing licensed packages. However, these packages will, in most cases, likely require at least some modification before DOE-owned SNF can be transported in them. New transportation systems will have to be developed for the remainder of the SNF inventory. 
- A significant fuel characterization effort remains to be accomplished before required package modifications or new package development can be initiated. This characterization effort may result in additional fuel types that can be transported in existing packages.

- An overall materials management and disposition program should be initiated to coordinate fuel characterization and transportation planning among all of the pertinent DOE sites.

- Prioritization of fuel shipments based on fuel or storage facility conditions should be incorporated in any overall transportation plan. Additionally, the transportation plan should consider overall system efficiencies, which will essentially entail a tradeoff between a lower level of transportation optimization with a corresponding greater number of shipments required, or greater initial optimization effort resulting in fewer, more efficient shipments. 


\section{IDENTIFICATION OF DOE-OWNED SPENT NUCLEAR FUEL}

This section identifies all DOE-owned spent nuclear fuel types and the characteristics pertinent to the selection of suitable transport packages in which to ship them. This includes a complete listing of DOE-owned SNF as derived from Reference 1 and Footnote a. Additional fuel information was obtained from References 2, 3, 4, and Footnote b. The SNF types are grouped in general categories in Tables 2-1 through 2-8, along with their most significant transportation-related characteristics. The discussion of fuels is mainly generic in that specific recommendations for package/fuel transport combinations are not developed, but the fuel-related factors involved in making such determinations are set out in detail. Specific package/fuel matchups are discussed in Section 4.

\subsection{Fuels List}

All noncommercial DOE-owned SNF types are listed in Tables 2-1 through 2-8, along with their current storage locations, total mass in metric tons of heavy metal (MTHM), enrichment (\% ${ }^{235} \mathrm{U}$ ), decay heat (Kw) and burnup in megawatt days per metric initial ton of heavy metal (MWd/MITHM). The information for this section was derived from References 1 through 4 and Footnotes a and b. Tables 2-7 (test reactor SNF) and 2-8 (miscellaneous SNF) list fuel types for which inadequate information currently exists for purposes of transportation assessments. Additionally, some of the fuels in the other categories (Tables 2-1 through 2-6) are likewise inadequately characterized.

\subsection{Discussion of Fuel Characteristics That Govern Transport}

A number of factors are involved in matching fuel types with potential transport packages. For a given fuel type, all of these factors must be evaluated to ensure that they fall within the acceptable limits imposed by each candidate package. The parameters defining these limits for transport packages are set out in Section 3.

Additionally, fuel and package characteristics may be interdependent in terms of determining suitability of fuel types as potential payloads (see, for instance, Section 2.2.1). All fuel-related characteristics, as they pertain to transportation packaging, are listed and discussed below.

a. "Spent Nuclear Fuel Inventory Data (OPE-EIS-94.273)," attached to a letter from Tom Wichmann (DOE Idaho Operations Office) to addressees, May 11, 1994.

b. Private communications between Packaging Technology personnel and knowledgeable spent nuclear fuel authorities. 
Table 2-1. Pressurized water reactor (PWR) DOE-owned SNF types.

\begin{tabular}{|c|c|c|c|c|c|c|}
\hline $\begin{array}{l}\text { Source } \\
\text { (form) }\end{array}$ & Type & Location & MTHM & $\begin{array}{l}\text { Enrichment } \\
\text { (\% U-235) }\end{array}$ & $\begin{array}{l}\text { Decay heat } \\
(\mathrm{kW})\end{array}$ & $\begin{array}{c}\text { Burnup } \\
\text { (MWd/MITHM) }\end{array}$ \\
\hline Shippingport C-2 (assemblies) & $\mathrm{UO}_{2}$ & Hanford T-Plant & 16.2 & 0.714 & $0.104 /$ assbly & Unknown \\
\hline Point Beach-1 (assemblies) & $\mathrm{UO}_{2}$ & Hanford Bldg 324, 5,7 & 1.16 & 0.88 & $0.6 /$ assembly & Unknown \\
\hline Calvert Cliffs (rods) & $\mathrm{UO}_{2}$ & Hanford Bldg $324,5,7$ & 0.664 & 0.65 & Unknown & Unknown \\
\hline H. B. Robinson (rods) & $\mathrm{UO}_{2}$ & Hanford Bldg $324,5,7$ & 0.03 & 0.7 & Unknown & Unknown \\
\hline H. B. Robinson (rods) & $\mathrm{UO}_{2}$ & INEL TAN-607 & 0.258 & 1.85 & Unknown & Unknown \\
\hline H. B. Robinson (canister) & $\mathrm{UO}_{2}+\mathrm{PuO}_{2}$ & SRS RBOF & 0.0005 & Unknown & Unknown & $30,000 \max$ \\
\hline Shippingport C1-S4 (subassembly) & $\mathrm{UO}_{2}$ & INEL CPP-666 & 0.08 & 80.4 & $<0.33 /$ assbly & $38-53 \%$ \\
\hline Shippingport C2-S1 (clusters) & $\mathrm{UO}_{2}$ & INEL CPP-666 & 0.219 & 69.1 & $<0.33 /$ assbly & $38-53 \%$ \\
\hline Shippingport C2-S2 (clusters) & $\mathrm{UO}_{2}$ & INEL CPP-666 & 0.3 & 80.8 & $<0.33 /$ assbly & $38-53 \%$ \\
\hline Shippingport (canister) & $\mathrm{UO}_{2}+\mathrm{PuO}_{2}$ & SRS RBOF & 0.0162 & 0.14 & Unknown & 18,000 \\
\hline Conn Yankee (assembly) & $\mathrm{UO}_{2}$ & INEL TAN-607 & 0.377 & $1.4(1.85) ?$ & Unknown & 32,151 \\
\hline LOFT (can, assembly) & $\mathrm{UO}_{2}$ & INEL TAN-607 & 2.178 & $4.3 \max$ & Unknown & 1,178 avg \\
\hline Surrey (assembly, canister) & $\mathrm{UO}_{2}$ & INEL TAN-790 & 30.47 & 1.85 & Unknown & 32,521 \\
\hline Turkey Point B17 & $\mathrm{UO}_{2}$ & INEL TAN-790 & 0.412 & 1.85 & Unknown & 25,662 \\
\hline Turkey Point EMAD (assembly, can) & $\mathrm{UO}_{2}$ & INEL TAN-790 & 7.49 & 1.85 & Unknown & 27,525 \\
\hline Battelle Memorial Institute (can) & $\mathrm{UO}_{2}$ & INEL CPP-603 & 0.0013 & 93.2 & Unknown & Unknown \\
\hline R. E. Gina (assembly) & $\mathrm{UO}_{2}$ & West Valley FRS & 15.06 & 3.473 & $0.132 /$ assbly & Unknown \\
\hline TMI-2 (canisters) & $\mathrm{UO}_{2}$ & INEL TAN-607 & 81.595 & 2.98 & $<0.1 /$ can & 3,165 \\
\hline Arkansas ANO-1 (canisters) & $\mathrm{UO}_{2} ?$ & B\&W Lynchburg & 0.0119 & 0.4 & Unknown & Unknown \\
\hline Oconee (canisters) & $\mathrm{UO}_{2}$ ? & B\&W Lynchburg & 0.001 & 1.3 & Unknown & Unknown \\
\hline
\end{tabular}


Table 2-2. Boiling water reactor (BWR) DOE-owned SNF types.

\begin{tabular}{|c|c|c|c|c|c|c|}
\hline $\begin{array}{l}\text { Source } \\
\text { (form) }\end{array}$ & Type & Location & MTHM & $\begin{array}{l}\text { Enrichment } \\
\text { (\% U-235) }\end{array}$ & $\begin{array}{l}\text { Decay heat } \\
(\mathrm{kW})\end{array}$ & $\begin{array}{c}\text { Burnup } \\
\text { (MWd/MITHM) }\end{array}$ \\
\hline EBWR (assembly) & $\begin{array}{l}\mathrm{UO}_{2} \mathrm{Zr} \\
\mathrm{UO}_{2}+\mathrm{PuO}_{2} \\
\end{array}$ & SRS RBOF & 2.21 & $\begin{array}{l}\text { LEU/Natural/ } \\
\text { Depleted }\end{array}$ & Unknown & 1,600 \\
\hline Dresden (assemblies) & $\mathrm{UO}_{2}$ & INEL TAN-607 & 0.166 & 1.59 & Unknown & Unknown \\
\hline Peach Bottom 1 (elements) & $\mathrm{UO}_{2}$ & INEL CPP-603 & 0.046 & $75.7(93.15) ?$ & Unknown & Unknown \\
\hline Peach Bottom 2 (partial assembly) & $\mathrm{UO}_{2}$ & INEL TAN-607 & 0.356 & $1.4(2.19) ?$ & Unknown & 11,440 \\
\hline Peach Bottom (rods in canisters) & $\mathrm{UO}_{2}$ & INEL TRA-603 & 0.010 & 1.4 & Unknown & Unknown \\
\hline Pathfinder (rod) & $\mathrm{UO}_{2}$ & INEL CPP-603 & 0.053 & 93 & Unknown & $0.97 \%$ \\
\hline SPSS - SPERT (can) & $\mathrm{UO}_{2}$ & INEL CPP-603 & 0.001 & 93 & $0.4 \mathrm{~W}$ & Unknown \\
\hline Borax V (assembly) & $\mathrm{UO}_{2}$ & INEL CPP-603 & 0.021 & 93 & Unknown & Unknown \\
\hline Cooper (rods) & $\mathrm{UO}_{2}$ & Hanford Bldg $324,5,7$ & 0.365 & $4 \max ?$ & Unknown & Unknown \\
\hline Vallecitos (can) & $\mathrm{UO}_{2}$ & SRS RBOF & 0.001 & 93 & Unknown & $8 \%$ \\
\hline Vallecitos (can) & $\mathrm{UO}_{2}$ & INEL CPP-603 & 0.012 & $21.1(93) ?$ & Unknown & $8 \%$ \\
\hline
\end{tabular}


Table 2-3. Breeder reactor (BR) DOE-owned SNF types.

\begin{tabular}{|c|c|c|c|c|c|c|}
\hline $\begin{array}{l}\text { Source } \\
(\text { form })^{\mathrm{a}}\end{array}$ & Type & Location & MTHM & $\begin{array}{l}\text { Enrichment } \\
\text { (\% U-235) }\end{array}$ & $\begin{array}{c}\text { Decay heat } \\
(\mathrm{kW})\end{array}$ & $\begin{array}{c}\text { Burnup } \\
\text { (MWd/MITHM) }\end{array}$ \\
\hline FFTF - LM (DA) & $\mathrm{UO}_{2}+\mathrm{PuO}_{2}$ & Hanford FFTF & 9.0 & 0.71 & $<2.0$ & Unknown \\
\hline FFTF - LM (TFA) & $\begin{array}{l}\mathrm{UO}_{2}+\mathrm{PuO}_{2}+ \\
\mathrm{ThO}, \mathrm{UN}_{3}\end{array}$ & Hanford FFTF & 2.43 & 0.71 & Very Low & Unknown \\
\hline Shippingport - LW (pellets) & $\mathrm{UO}_{2}$ & INEL CPP-749 & 42.93 & 0.98 U-233 & Unknown & $29,048 \mathrm{EFPH}$ \\
\hline Shippingport - LW (canister) & $\mathrm{UO}_{2}+\mathrm{ThO}_{2}$ & ANL-E Bldg 212 & $0.045 \mathrm{E}-3$ & Unknown & Unknown & $53,000 \max$ \\
\hline Fermi Cores $1 \& 2$ - LM (can) & UMo & INEL CPP-666 & 3.913 & 25.7 & $<0.021 /$ can & $2 \%$ \\
\hline Fermi I Blanket - LM (assembly) & U-238, Mo & INEL CPP-749 & 34.172 & 0.36 & Unknown & $0.35 \%$ \\
\hline EBR-II - LM (casks) & U metal & Hanford Bldg 325 & 0.08 & 18 & Unknown & Unknown \\
\hline EBR-II - LM (canister) & U metal & INEL CPP-666 & 0.8 & 67 & $<0.5$ & $25-30,000$ \\
\hline EBR-II - LM (scrap) & $U$ metal & INEL CPP-603 & 1.173 & 60 & Unknown & N/A \\
\hline EBR-II ANL-6 - LM (can) & U metal & INEL CPP-603 & 0.002 & 60 & Unknown & N/A \\
\hline EBR - ? (pieces) & Unknown & Hanford $200 \mathrm{~W}$ & Unknown & Unknown & Unknown & Unknown \\
\hline EBR-II - LM (subassembly) & $\mathrm{U}-10 \% \mathrm{Zr}$ & ANL-W EBR-II & 17.81 & 69778 & $<1.0 ; 0.004$ & Unknown \\
\hline EBR-II - LM (subassembly, element) & $\mathrm{U} \cdot 10 \% \mathrm{Zr}$ & ANL-W HFEF & 11.89 & $69 / 78$ & $<1.0 ; 0.004$ & Unknown \\
\hline EBR-II - LM (subassembly) & Metal & ANL-W RSWF & $11: 33$ & $69 / 78$ & $<0.001$ & Unknown \\
\hline EBR-II - LM (canister) & $\mathrm{UO}_{2}+\mathrm{PuO}_{2}$ & SRS RBOF & 0.0006 & Unknown & Unknown & 62 \\
\hline EBR-II - LM (canister) & $\mathrm{UO}_{2}+\mathrm{PuO}_{2}$ & SRS RBOF & 0.0027 & Unknown & Unknown & $34,000 \max$ \\
\hline EBR-II - LM targets (canister) & U declad & SRS RBOF & 16.9791 & Unknown & Unknown & Unknown \\
\hline Saxton (canister) & $\mathrm{UO}_{2}+\mathrm{PuO}_{2}$ & SRS RBOF & 0.2974 & Unknown & Unknown & 1,000 \\
\hline Saxton (canister) & $\mathrm{UO}_{2}$ & SRS RBOF & 0.0893 & Unknown & Unknown & 1,600 \\
\hline Dresden - LW (sleeves, canisters) & $\mathrm{UO}_{2}+\mathrm{ThO}_{2}$ & SRS RBOF & 2.542 & HEU/LEU ? & Unknown & Unknown \\
\hline ERR (bundle) & $\mathrm{UO}_{2}+\mathrm{ThO}_{2}$ & SRS RBOF & 5.043 & Unknown/83? & Unknown & $50,000 \max$ \\
\hline ML-1 (assemblies) & $\begin{array}{l}\mathrm{UO}_{2}+\mathrm{PuO}_{2}+ \\
\mathrm{BeO}\end{array}$ & SRS RBOF & 0.0586 & Unknown & Unknown & Unknown \\
\hline
\end{tabular}

a. $L W=$ Light Water; $L M=$ Liquid Metal. 
Table 2-4. Gas-cooled reactor (GCR) DOE-owned SNF types.

\begin{tabular}{|c|c|c|c|c|c|c|}
\hline $\begin{array}{l}\text { Source } \\
\text { (form) }\end{array}$ & Type & Location & MTHM & $\begin{array}{l}\text { Enrichment } \\
\text { (\% U-235) }\end{array}$ & $\begin{array}{c}\text { Decay heat } \\
(\mathrm{kW})\end{array}$ & $\begin{array}{c}\text { Burnup } \\
\text { (MWd/MITHM) }\end{array}$ \\
\hline Peach Bottom 1 - HT (elements) & $\mathrm{UC}$ & INEL CPP-749 & 1.627 & $75.7(93.15) ?$ & Unknown & Unknown \\
\hline Peach Bottom 2 - HT (elements) & UC & INEL CPP-603 GSF & 1.295 & $53.3(93.15) ?$ & Unknown & Unknown \\
\hline FSVR - HT (assembly) & UC & INEL CPP-603 GSF & 8.62 & 93.5 & Unknown & $6-26,000$ \\
\hline FSVR (assembly) & UC & FSVR & 16 & 54 & Unknown & Unknown \\
\hline TORY-IIC (can) & $\mathrm{UO}_{2}$ & INEL CPP-603 GSF & 0.059 & 93.2 & Unknown & $1.5 \%$ \\
\hline TORY-IIA (can) & $\mathrm{UO}_{2}$ & INEL CPP-603 & 0.049 & 93 & Unknown & $0.002 \%$ \\
\hline GCRE Can (can) & $\mathrm{UO}_{2}$ & INEL CPP-603 & 0.001 & 93 & Unknown & Unknown \\
\hline GCRE (canisters) & $\begin{array}{l}\mathrm{UO}_{2} \text { or } \\
\mathrm{UO}_{2}+\mathrm{BeO}\end{array}$ & SRS RBOF & 0.061 & Unknown & Unknown & Unknown \\
\hline
\end{tabular}


Table 2-5. Metallic DOE-owned SNF types.

\begin{tabular}{|c|c|c|c|c|c|c|}
\hline $\begin{array}{l}\text { Source } \\
\text { (form) }\end{array}$ & Type & Location & MTHM & $\begin{array}{l}\text { Enrichment } \\
\text { (\% U-235) }\end{array}$ & $\begin{array}{l}\text { Decay heat } \\
(\mathbf{k W})\end{array}$ & $\begin{array}{c}\text { Burnup } \\
\text { (MWd/MITHM) }\end{array}$ \\
\hline TRIGA Al (rod) & $\mathrm{UZrHx}$ & INEL CPP-603 & 0.104 & 20 & $<.001 \mathrm{~W} / \mathrm{rod}$ & Varies \\
\hline TRIGA FLIP (rod) & UZrHx & INEL CPP-603 & 0.001 & 69.7 & N/A & $5.7-14.8 \%$ \\
\hline TRIGA FLIP and Std (element) & $\begin{array}{l}\text { UErZrH, } \\
\text { UZrH std }\end{array}$ & ANL-W NRAD & 0.010 & $\begin{array}{l}\text { FLIP } 70 \\
\text { std } 20\end{array}$ & Negligible & Unknown \\
\hline TRIGA SST (element) & $\mathrm{UZrHx}$ & INEL CPP-603 & 0.050 & 20 & $\begin{array}{c}<.001 \\
\text { W/element }\end{array}$ & Varies \\
\hline TRIGA SST (assembly) & $\mathrm{UZrHx}$ & Hanford Bldg 308 & 0.019 & 20 & Low & Unknown \\
\hline TRIGA (assembly) & $\mathrm{UZrHx}$ & Hanford $200 \mathrm{~W}$ & 0.017 & 20 & Unknown & Unknown \\
\hline TRIGA BER-II (element) & $\mathrm{UZrHx}$ & INEL CPP-603 GSF & 0.009 & 44 & Unknown & $0.13 \%$ \\
\hline TRIGA Areotest (element) & $\mathrm{UZrHx}$ ? & San Remon & 0.015 & 19.9 & Unknown & Unknown \\
\hline AFRRI-TRIGA (element) & $\mathrm{UZrHx}$ ? & Armed Forces & 0.019 & 19.9 & Unknown & Unknown \\
\hline GA-TRIGA (element) & $\mathrm{UZrHx}$ ? & GA San Diego & 0.045 & 19.9 & Unknown & Unknown \\
\hline U.S. Geo-TRIGA (element) & UZrHx? & Denver & 0.02 & 19.9 & Unknown & Unknown \\
\hline TRIGA (element) & $\mathrm{UZrHx} ?$ & McLellam & 0.015 & 19.9 & Unknown & Unknown \\
\hline DOW-TRIGA (element) & $\mathrm{UZrHx}$ ? & Midland & 0.014 & 19.9 & Unknown & Unknown \\
\hline TRIGA (assembly?) & $\mathrm{UZrHx}$ & University of Arizona & 0.081 & 19.8 & Unknown & Unknown \\
\hline TRIGA (assembly?) & $\mathrm{UZrHx}$ & University of Wisconsin & 0.039 & $70 / 19.8$ & Unknown & Unknown \\
\hline TRIGA (assembly?) & UZrHx & U. of Illinois (2 reactors) & 0.038 & 19.8 & Unknown & Unknown \\
\hline TRIGA FLIP (assembly?) & $\mathrm{UZrHx}$ & Washington State & 0.037 & $70 / 20$ & Unknown & Unknown \\
\hline TRIGA (assembly?) & $\mathrm{UZrHx}$ & Pennsylvania State & 0.036 & 20 & Unknown & Unknown \\
\hline TRIGA (assembly?) & $\mathrm{UZrHx}$ & University of Texas & 0.029 & 19.8 & Unknown & Unknown \\
\hline
\end{tabular}


Table 2-5. (continued).

\begin{tabular}{|c|c|c|c|c|c|c|}
\hline $\begin{array}{l}\text { Source } \\
\text { (form) }\end{array}$ & Type & Location & MTHM & $\begin{array}{l}\text { Enrichment } \\
\text { (\% U-235) }\end{array}$ & $\begin{array}{c}\text { Decay heat } \\
(\mathrm{kW})\end{array}$ & $\begin{array}{c}\text { Burnup } \\
\text { (MWd/MITHM) }\end{array}$ \\
\hline TRIGA FLIP (assembly?) & $\mathrm{UZrHx}$ & Texas A\&M University & 0.027 & $70 / 19.8$ & Unknown & Unknown \\
\hline TRIGA (assembly?) & $\mathrm{UZrHx}$ & University of Utah & 0.026 & 19.8 & Unknown & Unknown \\
\hline TRIGA (assembly?) & $\mathrm{UZrHx}$ & Kansas State & 0.025 & 20 & Unknown & Unknown \\
\hline TRIGA (assembly?) & $\mathrm{UZrHx}$ & Cornell Univ. (2 reactors) & 0.021 & 20 & Unknown & Unknown \\
\hline TRIGA (assembly?) & $\mathrm{UZrHx}$ & U. of California (Irvine) & 0.021 & 19.8 & Unknown & Unknown \\
\hline TRIGA (assembly?) & UZrHx & University of Maryland & 0.017 & 19.8 & Unknown & Unknown \\
\hline TRIGA FLIP (assembly?) & $\mathrm{UZrHx}$ & Oregon State & 0.017 & 85 & Unknown & Unknown \\
\hline TRIGA (assembly?) & $\mathrm{UZrHx}$ & Reed University & 0.013 & 20 & Unknown & Unknown \\
\hline SNAP (can) & $\mathrm{UZrHx}$ & INEL CPP-603 & 0.02 & 93 & Unknown & $<1 \%$ \\
\hline MURR (assembly) & UAlx & INEL CPP-603 & 0.022 & 93.4 & Unknown & Unknown \\
\hline MURR (bundle) & UAl & SRS RBOF & 0.0486 & Unknown & Unknown & 120 days \\
\hline MURR (assembly?) & UAl ? & U. of Missouri (Columbia) & 0.047 & 93.15 & Unknown & Unknown \\
\hline MURR (assembly?) & UAl ? & U. of Missouri (Rolla) & 0.018 & 93.15 & Unknown & Unknown \\
\hline HFBR (element) & UAlx \& $\mathrm{U}_{3} \mathrm{O}_{8}$ & INEL CPP-603 & 0.058 & 93 & Unknown & $40.5 \%$ \\
\hline HFBR (fuel handling unit) & $\mathrm{U}_{3} \mathrm{O}_{8}$ & BNL HFBR Canal & 0.248 & 93.17 & $<4.0 / \mathrm{FHU}$ & Unknown \\
\hline ORR (element) & UAlx & INEL CPP-603 & 0.003 & 93 & Unknown & Unknown \\
\hline ATR (assemblies) & UAlx & INEL CPP-603 & 0.09 & 93 & $\begin{array}{c}<634 \\
\text { W/assbly }\end{array}$ & $41 \%$ \\
\hline ATR recycle (element) & UAlx? & INEL TRA-670 & 0.586 & 86 & Unknown & Unknown \\
\hline ATR spent (assembly) & UAlx? & INEL TRA-670 & 0.095 & 82.6 & Unknown & Unknown \\
\hline
\end{tabular}


Table 2-5. (continued).

\begin{tabular}{|c|c|c|c|c|c|c|}
\hline $\begin{array}{l}\text { Source } \\
\text { (form) }\end{array}$ & Type & Location & MTHM & $\begin{array}{l}\text { Enrichment } \\
\text { (\% U-235) }\end{array}$ & $\begin{array}{l}\text { Decay heat } \\
(\mathrm{kW})\end{array}$ & $\begin{array}{c}\text { Burnup } \\
\text { (MWd/MITHM) }\end{array}$ \\
\hline ATR core (elements) & UAlx ? & INEL ATR Facility & 0.046 & 83 & Unknown & Unknown \\
\hline ATR (assembly) & UAlx & INEL CPP-666 & 0.649 & 93 & $634 \mathrm{~W} /$ assbly & $41 \%$ \\
\hline HFIR (assembly) & $\mathrm{U}_{3} \mathrm{O}_{8} \mathrm{Al}$ & ORNL HFIR Pool & 0.404 & 93 & $<0.25$ & Unknown \\
\hline RINC (bundles) & UAlx \& UAl & SRS RBOF & 0.0037 & Unknown & Unknown & $<4,000$ \\
\hline Nereide (bundle) & USiAl & SRS RBOF & 0.0354 & Unknown & Unknown & 600 \\
\hline ANLJ (bundle) & UAl & SRS RBOF & 0.0028 & Unknown & Unknown & $55 \%$ \\
\hline ATSR (bundle) & $\mathrm{UAl}$ & SRS RBOF & 0.0032 & Unknown & Unknown & $<20 \%$ \\
\hline JMTR (bundles) & UA1 & SRS RBOF & 0.0167 & Unknown & Unknown & $<100,000$ \\
\hline RHF (cylinder) & UAl & SRS RBOF & 0.0251 & Unknown & Unknown & Unknown \\
\hline S. Forest (bundle) & UAl & SRS RBOF & 0.0284 & Unknown & Unknown & 1,900 \\
\hline Atomics International - LM (pin) & $\mathrm{UZrHx}$ & INEL CPP-603 & 0.008 & 93 & Unknown & Unknown \\
\hline ARMF (plates) & UAl & INEL CPP-666 & 0.0002 & 92.3 & Unknown & Negligible \\
\hline ARMF/CFRMF (element) & UAlx & INEL TRA-660 & 0.0118 & 100 & Very Low & Very Low \\
\hline ARMF/CFRMF (unknown) & UAlx & INEL TRA-660 & 0.0016 & 100 & Very Low & Very Low \\
\hline ARMF/CFRMF (insert) & UAlx & INEL TRA-660 & 0.0008 & 100 & Very Low & Very Low \\
\hline Taiwan RR (canister) & UAl & SRS RBOF & 20.4845 & Unknown & Unknown & 4 \\
\hline SPEC (can) & U metal & SRS RBOF & 0.002 & Unknown & Unknown & Unknown \\
\hline $\begin{array}{l}\text { Tower Shielding Reactor } \\
\text { (assembly) }\end{array}$ & UAl & ORNL TSR Vessel & 0.009 & 93.15 & Unknown & Unknown \\
\hline BNL MRR (element) & UAl & BNL MRR & 0.0058 & $93 \max$ & Unknown & Unknown \\
\hline
\end{tabular}


Table 2-5. (continued).

\begin{tabular}{|c|c|c|c|c|c|c|}
\hline $\begin{array}{l}\text { Source } \\
\text { (form) }\end{array}$ & Type & Location & MTHM & $\begin{array}{l}\text { Enrichment } \\
\text { (\% U-235) }\end{array}$ & $\begin{array}{c}\text { Decay heat } \\
(\mathrm{kW})\end{array}$ & $\begin{array}{c}\text { Burnup } \\
\text { (MWd/MITHM) }\end{array}$ \\
\hline Univ of Wash (bundle) & UAlx & INEL CPP-666 & 0.004 & 93 & Unknown & $0.44 \%$ \\
\hline Univ of Virginia (bundle) & UAl & SRS RBOF & 0.00193 & Unknown & Unknown & $63 \mathrm{MWd} /$ assmbly \\
\hline Univ of Michigan (bundles) & UAl & SRS RBOF & 0.0338 & Unknown & Unknown & 633 days \\
\hline MTR Type (assembly?) & UAl ? & University of Virginia & 0.025 & 93 & Unknown & Unknown \\
\hline MTR Type (assembly?) & UAl ? & University of Michigan & 0.093 & 20 & Unknown & Unknown \\
\hline MTR Type (assembly?) & Unknown & Georgia Tech & 0.029 & 93 & Unknown & Unknown \\
\hline MTR Type (assembly?) & Unknown & Michigan Inst. of Tech. & 0.026 & 93 & Unknown & Unknown \\
\hline MTR Type (assembly?) & Unknown & U. of Mass. (Lowell) & 0.023 & 93 & Unknown & Unknown \\
\hline MTR Type (assembly?) & Unknown & Iowa State & 0.02 & 93 & Unknown & Unknown \\
\hline MTR Type (assembly?) & Unknown & Worster Polytech. Inst. & 0.018 & 20 & Unknown & Unknown \\
\hline MTR Type (assembly?) & Unknown & Purdue University & 0.014 & 93 & Unknown & Unknown \\
\hline MTR Type (assembly?) & Unknown & Rhode Is Nuclear Sci Cen & 0.03 & 93 & Unknown & Unknown \\
\hline Argonaut (assembly?) & Unknown & University of Florida & 0.024 & 93 & Unknown & Unknown \\
\hline OSR (assembly?) & Unknown & Ohio State & 0.02 & 20 & Unknown & Unknown \\
\hline
\end{tabular}


Table 2-6. Graphite-moderated DOE-owned SNF types.

\begin{tabular}{|l|l|l|l|l|l|l|}
\hline \multicolumn{1}{|c|}{$\begin{array}{c}\text { Source } \\
\text { (form) }\end{array}$} & \multicolumn{1}{|c|}{ Type } & \multicolumn{1}{|c|}{ Location } & MTHM & $\begin{array}{c}\text { Enrichment } \\
(\% \text { U-235) }\end{array}$ & $\begin{array}{c}\text { Decay heat } \\
(\mathrm{kW})\end{array}$ & $\begin{array}{c}\text { Burnup } \\
(\mathrm{MWd} / \mathrm{MITHM})\end{array}$ \\
\hline SPR (element) & $\mathrm{U}$ metallic & Hanford 105-KW Basin & $2.9 \mathrm{U} ?$ & $1.25 \mathrm{max}$ & $0.5 \mathrm{~W}$ & Unknown \\
\hline SPR (element) & U metallic & Hanford PUREX & $2.9 \mathrm{U} ?$ & $1.25 \max$ & $0.5 \mathrm{~W}$ & Unknown \\
\hline SPR (element) & U metallic & Hanford 105-KE Basin & $0.4 \mathrm{U} ?$ & $1.25 \max$ & $0.5 \mathrm{~W}$ & Unknown \\
\hline N Reactor (element) & U metallic & Hanford 105-KW/KE & 2,106 & $1.25 \max$ & 3.5 W/elemnt & Unknown \\
\hline N Reactor (element) & $\mathrm{U}$ metallic & Hanford PUREX & $0.3 \mathrm{U} ?$ & $1.25 \max$ & 3.5 W/elemnt & Unknown \\
\hline Big Rock Point (assembly) & $\mathrm{UO}_{2}$ & West Valley FRS & 11.5 & $5.6 \max$ & $0.06 /$ assmbly & Unknown \\
\hline TREAT (assembly) & $\mathrm{UO}_{2}$ in graphite & ANL-W TREAT & 0.010 & 93 & Negligible & Unknown \\
\hline
\end{tabular}


Table 2-7. Test reactor DOE-owned SNF types.

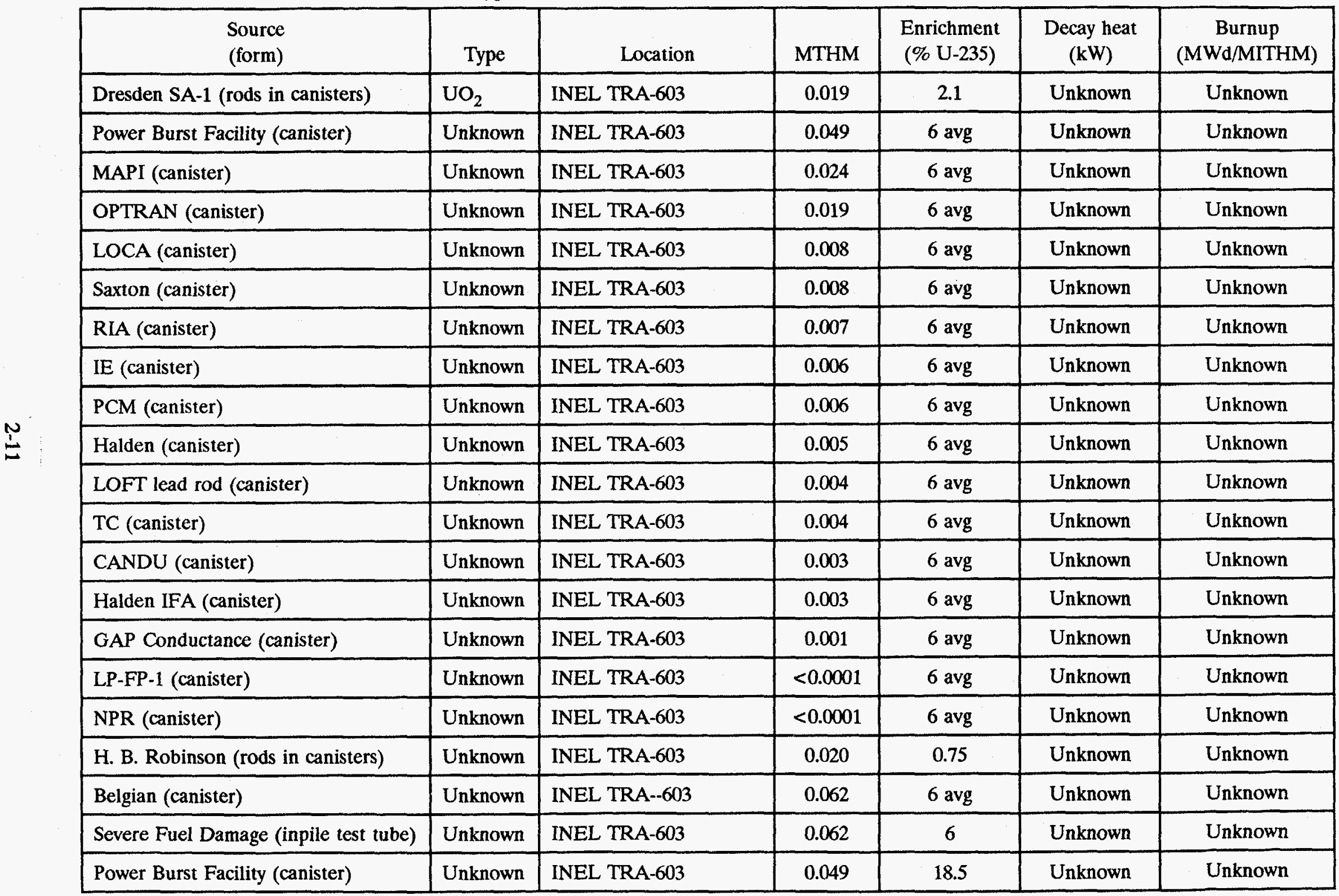


Table 2-8. Miscellaneous DOE-owned SNF types.

\begin{tabular}{|c|c|c|c|c|c|c|}
\hline $\begin{array}{l}\text { Source } \\
\text { (form) }\end{array}$ & Type & Location & MTHM & $\begin{array}{l}\text { Enrichment } \\
(\% \text { U-235) }\end{array}$ & $\begin{array}{c}\text { Decay heat } \\
(\mathrm{kW})\end{array}$ & $\begin{array}{c}\text { Burnup } \\
\text { (MWd/MITHM) }\end{array}$ \\
\hline Target (unknown) & Unknown & SRS F-Canyon & 22.634 & Unknown & Unknown & Unknown \\
\hline Driver (unknown) & Unknown & SRS H-Canyon & 0.068 & Unknown & Unknown & Unknown \\
\hline Driver (unknown) & Unknown & SRS K-Basin & 3.256 & Unknown & Unknown & Unknown \\
\hline Non-U target (unknown) & Unknown & SRS KLP-Basin & 0.059 & Unknown & Unknown & Unknown \\
\hline Driver/target (unknown) & Unknown & SRS L-Basin & 118.11 & Unknown & Unknown & Unknown \\
\hline Driver (unknown) & Unknown & SRS P-Basin & 1.408 & Unknown & Unknown & Unknown \\
\hline B\&W scrap (canister) & $\mathrm{UO}_{2}+\mathrm{PuO}_{2}$ & SRS RBOF & 0.00008 & Unknown & Unknown & 54 \\
\hline CANDU (canister) & $\mathrm{UO}_{2}$ & SRS RBOF & 0.05 & Unknown & Unknown & 6,500 \\
\hline Carolina-Virginia TR & $\mathrm{UO}_{2}$ & SRS RBOF & 0.0675 & Unknown & Unknown & $10,000 \max$ \\
\hline EPR-1 (canister) & $\mathrm{PuO}_{2}$ & SRS RBOF & 0.00002 & Unknown & Unknown & Unknown \\
\hline HTRE (canister) & $\mathrm{UO}_{2}+\mathrm{BeO}$ & SRS RBOF & 0.004 & Unknown & Unknown & Unknown \\
\hline HWCTR (tubes, canisters) & $\mathrm{U}$ or $\mathrm{UO}_{2}$ & SRS RBOF & 1.8267 & LEU & Unknown & Unknown \\
\hline HWCTR (assembly, canisters) & $\mathrm{UZr}$ & SRS RBOF & 0.0387 & $\mathrm{HEU}$ & Unknown & Unknown \\
\hline HWCTR (assembly) & UTh & SRS RBOF & 0.0791 & Unknown & Unknown & Unknown \\
\hline MIT (bundle) & $\mathrm{UO}_{2}+\mathrm{PuO}_{2}$ & SRS RBOF & 0.0159 & Unknown & Unknown & Unknown \\
\hline ORNL-SIW-1 (canister) & $\mathrm{U}$ & SRS RBOF & 0.00018 & Unknown & Unknown & Low \\
\hline ORNL Mixed Oxide (canister) & $\mathrm{UO}_{2}+\mathrm{PuO}_{2}$ & SRS RBOF & Unknown & Unknown & Unknown & 18,000 \\
\hline ORRa (bundle) & $\mathrm{U}_{3} \mathrm{Si}_{2} \mathrm{Al}$ & SRS RBOF & 0.090 & 16 & Unknown & 15,600 \\
\hline ORRb (bundle) & $\mathrm{U}_{3} \mathrm{Si}_{2} \mathrm{Al}$ & SRS RBOF & 0.0055 & 11 & Unknown & 15,600 \\
\hline ORRc (bundle) & $\mathrm{U}_{3} \mathrm{O}_{8} \mathrm{Al}$ & SRS RBOF & 0.2051 & $\mathrm{HEU}$ & Unknown & 250 days \\
\hline Saxton UO (canister) & $\mathrm{UO}_{2} ?$ & SRS RBOF & 0.089 & 7.7 & Unknown & Unknown \\
\hline S. Forest (canister) & $\mathrm{UO}_{2}$ & SRS RBOF & 0.09429 & HEU & Unknown & $<16$ days \\
\hline
\end{tabular}


Table 2-8. (continued).

\begin{tabular}{|c|c|c|c|c|c|c|}
\hline $\begin{array}{l}\text { Source } \\
\text { (form) }\end{array}$ & Type & Location & MTHM & $\begin{array}{l}\text { Enrichment } \\
\text { (\% U-235) }\end{array}$ & $\begin{array}{l}\text { Decay heat } \\
(\mathrm{kW})\end{array}$ & $\begin{array}{c}\text { Burnup } \\
\text { (MWd/MITHM) }\end{array}$ \\
\hline SPERT-3 (canister) & $\mathrm{UO}_{2}$ & SRS RBOF & 0.0097 & Unknown & Unknown & Unknown' \\
\hline SPERT (assembly?) & $\mathrm{UO}_{2}$ & Rensselaer University & 0.388 & 5 & Unknown & Unknown \\
\hline $\begin{array}{l}\text { Sodium Reactor Experiment } \\
\text { (unknown) }\end{array}$ & $\mathrm{UO}_{2}+\mathrm{ThO}_{2}$ & SRS RBOF & 2.1274 & $\mathrm{HEU}$ & Unknown & 10,000 \\
\hline $\begin{array}{l}\text { Sodium Reactor Experiment } \\
\text { (canister) }\end{array}$ & UC & SRS RBOF & 0.0443 & Unknown & Unknown & 10,000 \\
\hline SRS-P (tubes) & $\begin{array}{l}\mathrm{U}_{3} \mathrm{O}_{8} \mathrm{Al} \\
\mathrm{UAl}\end{array}$ & SRS RBOF & 0.0061 & Unknown & Unknown & Unknown \\
\hline SRS (unknown) & Unknown & SRS RBOF & 153.625 & Unknown & Unknown & Unknown \\
\hline SRS target samples (assembly) & $\mathbf{U}$ & SRS RBOF & 0.369 & Unknown & Unknown & Unknown \\
\hline SRS target material (assembly) & $\mathrm{U}$ base & SRS RBOF & Class. & Unknown & Unknown & Unknown \\
\hline LWR samples (canister) & $\mathrm{UO}_{2}+\mathrm{PuO}_{2}$ & SRS RBOF & 0.0127 & Unknown & Unknown & Unknown \\
\hline ZPPR (plates and rods) & $\begin{array}{l}\mathrm{PuAl} \text {, } \\
\mathrm{PuMo} \\
\mathrm{PuO}_{2}, \mathrm{U} \\
\text { metal, } \mathrm{U}\end{array}$ & ANL-W ZPPR & 9.47 & 93 & Unknown & Unknown \\
\hline ZPTR (assembly?) & $\mathrm{UO}_{2}$ & Cornell University & 1.7 & 2 & Unknown & Unknown \\
\hline ZPR (assembly?) & $\mathrm{UO}_{2}$ ? & Manhattan University & 0.019 & 20 & Unknown & Unknown \\
\hline LFRSB (assembly) & $\mathrm{UO}_{2}$ & INEL TAN-607 & 0.312 & N/A & Unknown & Unknown \\
\hline PULSTAR Buffalo (pin) & $\mathrm{UO}_{2}$ & INEL CPP-603 & 0.251 & 6 & $<7 \mathrm{~W} /$ pin & 12,000 max \\
\hline PULSTAR Buffalo? (pins) & $\mathrm{UO}_{2}$ & $\begin{array}{l}\text { State Univ of NY } \\
\text { (Buffalo) }\end{array}$ & 0.493 & 6 & $<7 \mathrm{~W} /$ pin ? & $12,000 \max ?$ \\
\hline PULSTAR (unknown) & $\mathrm{UO}_{2}$ & North Carolina State & 0.445 & 4 & Unknown & Unknown \\
\hline SM-1A Army (assembly) & $\mathrm{UO}_{2}$ & INEL CPP-603 & 0.066 & 93 & Unknown & 5,124 \\
\hline
\end{tabular}


Table 2-8. (continued).

\begin{tabular}{|c|c|c|c|c|c|c|}
\hline $\begin{array}{l}\text { Source } \\
\text { (form) }\end{array}$ & Type & Location & MTHM & $\begin{array}{l}\text { Enrichment } \\
\text { (\% U-235) }\end{array}$ & $\begin{array}{l}\text { Decay heat } \\
(\mathrm{kW})\end{array}$ & $\begin{array}{c}\text { Burnup } \\
\text { (MWd/MITHM) }\end{array}$ \\
\hline PBF Driver Core (rod) & UZr/calcia & INEL PBF-620 & 0.56 & 18.5 & Unknown & 1,849 \\
\hline APP [AGE-2] (can) & $\mathrm{UO}_{2}$ & INEL CPP-603 & 0.00002 & 94 & Unknown & $30 \%$ \\
\hline GETR Filters (basket) & $\mathrm{UO}_{2}, \mathrm{U}_{3} \mathrm{O}_{8}$ & INEL CPP-603 & 0.004 & 93 & Unknown & $5 \%$ \\
\hline Core Filter (unknown) & Unknown & INEL TRA-660 & 0.217 & Depleted & Unknown & Unknown \\
\hline LWR Commercial Fuel (pieces) & $\mathrm{UO}_{2}$ & Hanford 327 Bldg & 0.007 & $4 \max$ & $\begin{array}{l}\text { Unknown, } \\
\text { low }\end{array}$ & Unknown \\
\hline DOE, Commercial Fuel (pieces) & Various & Hanford 327 Bldg & 0.010 & $4 \max$ & $\begin{array}{l}\text { Unknown, } \\
\text { low }\end{array}$ & Unknown \\
\hline LWR Commercial Fuel (pieces) & $\mathrm{UO}_{2}$ & Hanford 325 Bldg & 0.010 & $4 \max$ & $\begin{array}{l}\text { Unknown, } \\
\text { low }\end{array}$ & Unknown \\
\hline LWR Commercial Fuel (assembly) & $\mathrm{UO}_{2}$ & Hanford 324 Bldg & 2.24 & $4 \max$ & $0.6 /$ assmbly & Unknown \\
\hline LWR Commercial Fuel (pieces) & $\mathrm{UO}_{2}$ & Hanford $200 \mathrm{~W}$ & 0.007 & 4 & $0.6 /$ assmbly & Unknown \\
\hline DOE, Commercial Fuel (pieces) & Various & Hanford $200 \mathrm{~W}$ & 0.223 & Varies & Varies & Unknown \\
\hline Bulk Shielding Reactor (element) & $\mathrm{U}_{3} \mathrm{O}_{8}$ & ORNL BSR POOI & Unknown & 93.15 & Unknown & Unknown \\
\hline ORRR Fuel (element) & $\mathrm{U}_{3} \mathrm{O}_{8}$ & ORNL BSR Pool & Unknown & 19.8 & Unknown & Unknown \\
\hline MSRE Fuel (storage tank) & $\mathrm{UF}_{4}$ & ORNL MSRE Vessel & 0.037 & 18.37 & 0.2 & Unknown \\
\hline JASPER Fuel (pins) & Unknown & ORNL TSR Vessel & 0.868 & 4.81 & Unknown & Unknown \\
\hline Con Edison (can) & $\mathrm{U}_{3} \mathrm{O}_{8}$ & $\begin{array}{l}\text { ORNL Bldg } 3019 \\
\text { Drywell }\end{array}$ & $1.215 ?$ & $93\left(7^{233} U\right)$ & $0.1 \mathrm{~W} / \mathrm{can}$ & Unknown \\
\hline Savannah River Oxide (can) & $\mathrm{U}_{3} \mathrm{O}_{8}, \mathrm{UF}_{4}$ & $\begin{array}{l}\text { ORNL Bldg } 3019 \\
\text { Drywell }\end{array}$ & 0.070 & $100{ }^{233} \mathrm{U}$ & $0.1 \mathrm{~W} / \mathrm{can}$ & Unknown \\
\hline Hanford \& Misc. Oxide (can) & $\mathrm{U}_{3} \mathrm{O}_{8}$ & $\begin{array}{l}\text { ORNL Bldg } 3019 \\
\text { Drywell }\end{array}$ & 0.023 & $100^{233} \mathrm{U}$ & $0.1 \mathrm{~W} / \mathrm{can}$ & Unknown \\
\hline
\end{tabular}


Table 2-8. (continued).

\begin{tabular}{|c|c|c|c|c|c|c|}
\hline $\begin{array}{l}\text { Source } \\
\text { (form) }\end{array}$ & Type & Location & MTHM & $\begin{array}{l}\text { Enrichment } \\
\text { (\% U-235) }\end{array}$ & $\begin{array}{l}\text { Decay heat } \\
(\mathrm{kW})\end{array}$ & $\begin{array}{c}\text { Burnup } \\
\text { (MWd/MITHM) }\end{array}$ \\
\hline Misc. Irrad/Unirrad Fuels (misc.) & $\begin{array}{l}\mathrm{UO}_{2}, \mathrm{UCO}, \\
\mathrm{ThO}_{2}\end{array}$ & ORNL Bldg 3525 & Unknown & $\begin{array}{l}\text { LEU to } \\
\text { HEU }\end{array}$ & Unknown & Unknown \\
\hline LWR Commercial (rod section) & $\mathrm{UO}_{2}$ & $\begin{array}{l}\text { ORNL Bldg } 4501 \\
\text { Drywell }\end{array}$ & Unknown & $7.6 \max$ & $\begin{array}{l}\text { Too low to } \\
\text { measure }\end{array}$ & Unknown \\
\hline Peach Bottom Specimens (elements) & $\mathrm{UO}_{2}+\mathrm{ThO}_{2}$ & $\begin{array}{l}\text { ORNL Dry Storage } \\
7929\end{array}$ & Unknown & Unknown & Unknown & Unknown \\
\hline Homogeneous Fuel (aqueous) & $\mathrm{U}$ in $\mathrm{H}_{2} \mathrm{SO}_{4}$ & $\begin{array}{l}\text { ORNL HRE Wells, } \\
\text { WAG7 }\end{array}$ & Unknown & 86 & Unknown & Unknown \\
\hline AGN (assembly?) & $\mathrm{UO}_{2}$-poly & Idaho State & 0.011 & 20 & Unknown & Unknown \\
\hline AGN (assembly?) & $\mathrm{UO}_{2}$-poly & $\begin{array}{l}\text { University of New } \\
\text { Mexico }\end{array}$ & 0.004 & 20 & Unknown & Unknown \\
\hline HPRR Fuel (plates, rods, etc.) & UMo & ORNL Y-12, 9720-5 & Unknown & 93.14 & Negligible & Unknown \\
\hline SNAP 10A (core assembly in NaK) & $\mathrm{UZr}$ & ORNL Y-12, 9720-5 & Unknown & 93.29 & Negligible & Unknown \\
\hline DOE Demonstration (core assembly) & Unknown & ORNL Y-12, 9720-5 & Unknown & $93 \max$ & Unknown & Unknown \\
\hline MTR Type (element) & $\mathrm{U}_{3} \mathrm{O}_{8}$ & LANL Omega West & 0.0064 & 93.5 & $4 \mathrm{~W} /$ element & Unknown \\
\hline MTR Type (element) & $\mathrm{U}_{3} \mathrm{O}_{8}$ & LANL CMR & Unknown & 93.5 & $4 \mathrm{~W} /$ element & Unknown \\
\hline EBR-II, FFTF, TREAT (canister) & $\mathrm{U}+\mathrm{Pu}$ & ANL-E Bldg 212 & 0.005 & Unknown & Unknown & $180,000 \max$ \\
\hline EBR-II, TREAT, MTR (canister) & $\begin{array}{l}\text { U metal, } \\
\text { alloys }\end{array}$ & ANL-E Bldg 212 & 0.004 & Unknown & Unknown & $100,000 \max$ \\
\hline EBR-II, TREAT (canister) & $\mathrm{UO}_{2}+\mathrm{PuO}_{2}$ & ANL-E Bldg 212 & 0.018 & Unknown & Unknown & $130,000 \max$ \\
\hline $\begin{array}{l}\text { Dresden II, H. B Robinson, BR-3, } \\
\text { TMI-2 (canister) }\end{array}$ & $\mathrm{UO}_{2}$ & ANL-E Bldg 212 & 0.020 & Unknown & Unknown & $40,000 \max$ \\
\hline ORR Experiments (canister) & $\mathrm{U}_{3} \mathrm{Si}$ & ANL-E Bldg 212 & 0.001 & Unknown & Unknown & $80,000 \max$ \\
\hline SPR-III (elements) & Unknown & SNL SPR & 0.22 & 93 (est) & Unknown & Unknown \\
\hline
\end{tabular}


Table 2-8. (continued).

\begin{tabular}{|c|c|c|c|c|c|c|}
\hline $\begin{array}{l}\text { Source } \\
\text { (form) }\end{array}$ & Type & Location & MTHM & $\begin{array}{l}\text { Enrichment } \\
\text { (\% U-235) }\end{array}$ & $\begin{array}{l}\text { Decay heat } \\
(\mathrm{kW})\end{array}$ & $\begin{array}{c}\text { Burnup } \\
\text { (MWd/MITHM) }\end{array}$ \\
\hline SPR-II (elements) & Unknown & SNL SPR & 0.09 (est) & 93 (est) & Unknown & Unknown \\
\hline ACRR (unknown) & Unknown & SNL ACRR & 0.03 (est) & 35 (est) & Unknown & Unknown \\
\hline ACRR (experiments) & Unknown & SNL ACRR & 0.001 & Unknown & Unknown & Unknown \\
\hline SPR-II \& III (unknown) & Unknown & SNL SPR & 0.03 (est) & Unknown & Unknown & Unknown \\
\hline PNL Mixed (unknown) & Unknown & SNL Mansano & 0.025 & Unknown & Unknown & Unknown \\
\hline PNL Mixed (unknown) & Unknown & SNL HCF & 0.009 & Unknown & Unknown & Unknown \\
\hline CX (elements) & Unknown & SNL & 0.02 (est) & 93 (est) & Unknown & Unknown \\
\hline FREC (elements) & Unknown & SNL & 0.01 (est) & 20 (est) & Unknown & Unknown \\
\hline SPR-II (unknown) & Unknown & SNL SNM Storage & 0.01 (est) & 93 (est) & Unknown & Unknown \\
\hline Experiments (unknown) & Unknown & SNL ACRR & 0.001 & Unknown & Unknown & Unknown \\
\hline GENTR (elements) & $\mathrm{UO}_{2} ?$ & $\begin{array}{l}\text { GE Vallecitos } \\
\text { (Pleasanton) }\end{array}$ & 0.008 & 93 & Unknown & Unknown \\
\hline NIS(T?) (elements) & Unknown & Gaithersburg & 0.019 & 93 & Unknown & Unknown \\
\hline Naval (classified) & Unknown & INEL CPP-603 & 0.947 & classified & classified & classified \\
\hline Naval (classified) & Unknown & INEL CPP-666 & 4.816 & classified & classified & classified \\
\hline Naval (classified) & Unknown & NRF & 2.5 & classified & classified & classified \\
\hline
\end{tabular}

Note: Question marks (?) indicate that data could not be directly determined from the references, but that reasonable deductions were made based on similar fuel types or other known factors. 


\subsubsection{Size}

Obviously, the size of a particular fuel type must be smaller than the payload cavity of the particular candidate transport package under consideration. In those cases where more than one fuel assembly of a given type will fit within the payload cavity, an optimum loading configuration may have to be determined. Additionally, questions pertaining to package cavity size may also arise from a consideration of the fragility of the fuel. Fuel fragility will determine the robustness (and hence, size) of any support structure that will be necessary to safely transport the fuel while maintaining a noncritical configuration under hypothetical accident condition loading. Robustness of the support structure will in turn be a function of package maximum impact loading (orientation-dependent), which will dictate structural strength requirements. By necessity, the resulting net size (fuel plus support structure) must be smaller than the package payload cavity. Additionally, the support structure may be required to incorporate neutron poisoning, potentially resulting in additional structural size and mass and corresponding reduction in payload capability.

\subsubsection{Weight}

Maximum payload weight limitations of the package must not be exceeded. Considerations regarding the potential need for payload support structure or poisoning are the same as those described in Section 2.2.1.

Note that package payload weight limitations may not necessarily be restricted to currently licensed values. Package designs could potentially be upgraded for increased carrying capacity, as detailed in Section 4.3 below.

\subsubsection{Source Term}

This fuel characteristic has implications in two major subcategories: radiation level and radionuclide distribution, and criticality.

2.2.3.1 Radiation Level and Radionuclide Distribution. This factor will determine if the fuel type can be adequately shielded by a particular transport package. That is, maximum dose rates exterior to the package must be maintained according to the regulatory requirements of 10 CFR \$71.47, "Normal Conditions of Transport" and 10 CFR \$71.51(a)(2), "Hypothetical Accident Conditions," of Reference 5. Obviously, many factors are involved in determining radiation levels. Chief among these are

- Initial fissile material content ( $\mathrm{kg} / \mathrm{assembly)}$

- Initial enrichment (without U-235 equivalent)

- Burnup (megawatt days per metric ton of uranium-MWD/MTU)

- $\quad$ Cooling time (years)

- Potential presence of activation products. 
These factors must be determined for each fuel type in order to develop even a first-level approximation of fuel and package compatibility. A potential complication may arise due to the fact that the Nuclear Regulatory Commission Transportation Committee (the licensing authority) has, in the past, expressed great reluctance in acknowledging anything but the potentially worstcase burnup assumptions for any spent fuel being transported in NRC-licensed packages.

Other factors may also be of importance. For instance, the isotopic distribution of the payload may be a significant factor. Shielding designed for a uniformly distributed source may not be adequate for a source of the same activity level that is concentrated in a relatively small volume within the payload. Even for a nominally "homogeneous" distribution, axial burnup variations may be significant.

2.2.3.2 Criticality. For any given fuel type, fissile content will govern the classification of packaging required, under 10 CFR \$71.55, "General Requirements," and 10 CFR \$71.59, "Standards for Arrays of Fissile Material Packages." This will also be a key parameter in determining the requirements for payload support in the form of a fuel basket that can withstand regulatory load applications and maintain the fuel in a noncritical configuration. If neutron poisoning is required to be incorporated into the fuel basket, size and weight implications will be involved, as detailed in Sections 2.2.1 and 2.2.2.

This could be a particularly troublesome issue for fuel types that cannot be well characterized because of a lack of sufficient historical data, or for fuels that may be in a deteriorated state. The computer codes used to perform criticality analyses require precise inputs of fuel characteristics. If such data are not available or well defined, conservative bounding analyses would have to be performed. This could potentially result in overly severe limitations on payload capacities and a resulting reduction in transportation efficiency.

\subsubsection{Thermal Load}

Total heat output of the payload cannot exceed the specified thermal limitations of the package. Again, heat source distribution may be a consideration, with concerns similar to those set out in Section 2.2.3.1. Also, with additional effort (refer to Section 4.3), upward adjustments to a package's thermal limits could potentially be made.

Additionally, even if currently specified packaging thermal limits are maintained, excessive thermal loading may detrimentally affect fuel cladding or basket/support structure integrity.

\subsubsection{Fragility}

Fragility will be a factor that must be considered in conjunction with size and weight (Sections 2.2.1 and 2.2.2). Robust fuel may need little in the way of support structure, with its accompanying size and weight penalty. Highly fragile fuel may need a fairly massive support structure, at the expense of total fuel payload capacity. Badly deteriorated fuel may require repackaging. The governing consideration will be support of the payload in a noncritical configuration after the hypothetical accident condition loading sequence. 


\subsubsection{Form}

Damaged fuel (i.e., breached or deteriorated cladding) that falls under the restrictions imposed by $10 \mathrm{CFR} \$ 71.63$ (i.e., quantities of plutonium greater than $20 \mathrm{Ci}$ per shipment) will probably be required to be transported in double-containment, leaktight packages. The significance of this requirement should be strongly emphasized. Currently, only one of the candidate SNF transport packages (the 125-B) features a double containment design. Even this package, with its highly specialized inner containment vessel configuration, is not expected to be generally useful for payload types other than that for which it was specifically designed. Accordingly, it is anticipated that, if double containment is necessary, all of the candidate packages will have to be requalified for custom-designed, separate inner containment vessels. The implications of this requirement are quite profound. From a licensing aspect, qualifying an existing package with a newly designed level of containment could be tantamount to licensing an entirely new packaging design.

\subsubsection{Special Concerns}

- Chemical compatibility (payload reactions with package cavity materials, seal materials, etc.)

- Chemical stability (pyrophoric form, etc.)

- Radiolytic gas generation [effect on package maximum normal operating pressure (MNOP), etc.]

- Water content (dry transport is mandatory in currently NRC or DOE-licensed packages).

\subsubsection{Location}

The fuel storage facility must be accessible by the transport package under consideration, unless multiple transfers are undertaken. This factor is discussed in greater detail in Section 5.3.

\subsection{Discussion of Unknowns and Uncertainties Related to Fuels}

Note that some fuel types may involve multiple variations of the above parameters. For instance, a single fuel type may exhibit various degrees of integrity, from very robust to very fragile, as well as demonstrate a wide range of radiological and thermal activity. In addition, the fuel may be stored at several facilities.

If the worst-case fuel condition is used for purposes of selecting a transport package for a particular fuel type, it is possible that a highly inefficient transportation campaign would result. That is, the worst-case fuel of the selected fuel type may compose only a small portion of the total inventory of that particular fuel type. The bulk of the fuel type might possibly be transported more efficiently (more fuel per trip) in a different type of package, or several types of packages. 
Consequently, it is conceivable that, in some instances, more than one type of package may be required to efficiently transport a single type of fuel.

Additionally, priority may be an important concern in the process of determining the suitability of transport packages. Some fuels are badly deteriorated or stored in poorly controlled conditions (Reference 4). These fuels would likely require the most immediate attention in assessing their transportability.

Finally, while detailed checks were not undertaken to confirm the fuels data in the listed references, a number of inconsistencies between data sets were discovered in the course of the study. In those cases, the worst-case quantity or other physical characteristic was used to estimate total transportation requirements. 


\section{IDENTIFICATION OF CANDIDATE PACKAGINGS CURRENTLY IN EXISTENCE}

This section identifies all current NRC or DOE-licensed irradiated fuel transport packages and the characteristics pertinent to the selection of suitable spent fuel types to ship in them. The discussion of transport packages is mainly generic, in that specific recommendations for package/fuel transport combinations are not developed, but the package-related factors involved in making such determinations are set out in detail. Packaging information was derived from References 6, 7, 8, 9, and Footnote d. Section 4 discusses specific package/fuel matchups.

For this transportation assessment, it is assumed that no "out-of-commerce" shipments will be allowed. That is, all shipments will be in accordance with current federal regulations [for NRC or U.S. Department of Transportation (DOT) rules] or DOE Order 5480.3 (Reference 10, for DOE rules) governing transportation of radioactive material in the public domain. The applicable DOT rules are set out in 49 CFR 173 (Reference 11), Sections 416, "Authorized Type B Packages," and 417, "Authorized Packaging-Fissile Materials." These regulations cover shipments of radioactive materials, but (except in the case of overseas shipments) they generally either defer to the control of the NRC regulations (10 CFR 71, Reference 5) or involve packages that either are too small, are obsolete and no longer available, or are designed for very limited, specific payload types (e.g., $\mathrm{UF}_{6}$ containers).

\subsection{Packaging List}

All current NRC or DOE-licensed irradiated fuel packages are listed in Table 3-1, along with the design characteristics that could limit and otherwise determine which types of spent fuels can potentially be transported in them. The information shown in Table 3-1 was derived from a variety of sources, listed as References 6 through 9.

Design details, including top-level engineering drawings and current Certificates of Compliance, can be found in Appendix B of this report.

\subsection{Discussion of Packaging-Imposed Payload Restrictions}

Because of the stringency of the governing regulations for the transportation of radioactive materials, as set out in 10 CFR 71, specific limits are delineated for all significant design and operational factors for each licensed package. These limits are summarized in the specific Certificates of Compliance, and are listed and discussed in detail below.

In many cases, there may be little compatibility between certified package parameters and the corresponding SNF parameters set out in Section 2.2. Since use of the packagings outside of their specific operating parameters is not legally permitted, it is anticipated that, at a minimum, amendments or revisions to the relevant SARPs and Certificates of Compliance will be required for most of the DOE's SNF.

d. Private communications between Packaging Technology and owners and users of the listed packagings. 
Table 3-1. Transport package information. ${ }^{\text {a }}$

\begin{tabular}{|c|c|c|c|c|c|c|c|c|c|c|c|c|c|c|}
\hline $\begin{array}{c}\text { Package } \\
\text { name }\end{array}$ & $\begin{array}{c}\text { Certificate } \\
\text { of } \\
\text { compliance }\end{array}$ & $\begin{array}{l}\text { Certificate } \\
\text { expiration }\end{array}$ & $\begin{array}{c}\text { Gross } \\
\text { weight } \\
\text { (pounds) }\end{array}$ & $\begin{array}{c}\text { External } \\
\text { dimensions } \\
\text { (inches) }^{b}\end{array}$ & $\begin{array}{c}\text { Transport } \\
\text { mode }\end{array}$ & $\begin{array}{l}\text { Payload } \\
\text { weight } \\
\text { (pounds) }\end{array}$ & $\begin{array}{l}\text { Internal } \\
\text { dimensions } \\
\text { (inches) }^{b}\end{array}$ & Shielding config. ${ }^{c}$ & Content(s) ${ }^{d}$ & $\begin{array}{l}\text { Maximum } \\
\text { heat load }\end{array}$ & $\begin{array}{l}\text { Maximum } \\
\text { impact } \\
\text { load (g's) / } \\
\text { orientation }\end{array}$ & Owner $^{f}$ & Availability & Number \\
\hline GE T-2 & $\begin{array}{l}\text { USA/5607/ } \\
\mathrm{B}(\mathrm{)}) \\
(\mathrm{DOE})\end{array}$ & $5 / 31 / 98$ & 18,400 & $\begin{array}{l}36.0 \phi x \\
133 \text { long }\end{array}$ & $\begin{array}{c}\text { Legal } \\
\text { weight } \\
\text { truck }\end{array}$ & 160 & $\begin{array}{c}6.06 \phi x \\
99.69 \text { long }\end{array}$ & $\begin{array}{c}\text { Double stl wall w/ } 4.2^{\prime \prime}- \\
8^{\prime \prime} \mathrm{Pb}(\mathrm{s})\end{array}$ & $\begin{array}{c}3 \mathrm{w}_{\mathrm{o}}{ }^{235} \mathrm{U} \text { fuel } \\
\text { pins } \\
57 \mathrm{Kci} \\
18 \mathrm{Mwd} / \mathrm{kg}\end{array}$ & $\begin{array}{c}290 \mathrm{w} \\
\text { (+other) }\end{array}$ & N/A & DOE-EH & N/A & 2 \\
\hline GE-100 & $\begin{array}{c}\text { USA/5926/ } \\
\text { B( )F }\end{array}$ & $2 / 28 / 98$ & 4,800 & $\begin{array}{l}20.25 \text { \$x } \\
26.88 \text { long }\end{array}$ & $\begin{array}{c}\text { Legal } \\
\text { weight } \\
\text { truck }\end{array}$ & N/A & $\begin{array}{l}7.625 \phi x \\
10.00 \text { long }\end{array}$ & $\begin{array}{c}\text { Double stl wall w/ } \\
5.88^{\prime \prime} \mathrm{Pb}(\mathrm{s})\end{array}$ & $\begin{array}{c}500 \mathrm{~g} \\
235 \mathrm{U} \\
(\mathrm{TI}=5.6)\end{array}$ & $400 w$ & N/A & $\begin{array}{l}\text { General } \\
\text { Electric }\end{array}$ & $\begin{array}{c}\text { Approx. } \\
50 \%\end{array}$ & 3 \\
\hline BMI-1 & $\begin{array}{c}\text { USA } 5957 / \\
\mathrm{B}(\mathrm{)})\end{array}$ & $5 / 31 / 95$ & 23,660 & $\begin{array}{l}33.37 \text { \$ } x \\
73.37 \text { high }\end{array}$ & $\begin{array}{l}\text { Legal } \\
\text { weight } \\
\text { truck }\end{array}$ & 1,800 & $\begin{array}{l}15.50 \text { 中 X } \\
54.00 \text { high }\end{array}$ & $\begin{array}{l}\text { Double stl wall }\left(0.75^{\prime \prime}\right. \\
\text { total) w/ } 8.0^{\prime \prime} \mathrm{Pb}(\mathrm{s}) \\
7.75^{\prime \prime} \mathrm{Pb}(\mathrm{t})\end{array}$ & $\begin{array}{c}93.5 \mathrm{w} / \mathrm{o} / \\
510 \mathrm{~g} 235_{\mathrm{U}} \\
25.6 \mathrm{w}_{/ \mathrm{o}} 235_{\mathrm{U}} \text { for } \\
\text { Fermi }\end{array}$ & N/A & $\begin{array}{c}\text { 375/end } \\
500 / \text { side } \\
128 / \text { corner }\end{array}$ & DOE-EH & $\begin{array}{c}\text { Limited } \\
\text { availability }\end{array}$ & 1 \\
\hline FSV-1 & $\begin{array}{c}\text { USA/6346/ } \\
\mathrm{B}(\mathrm{)}) \mathrm{F}\end{array}$ & $4 / 30 / 96$ & 47,600 & $\begin{array}{c}31.0 \text { \& } \mathrm{x} \\
208.0 \text { long }\end{array}$ & $\begin{array}{l}\text { Legal } \\
\text { weight } \\
\text { truck }\end{array}$ & 4,430 & $\begin{array}{c}17.7 \text { \& } x \\
187.6 \text { long }\end{array}$ & $\begin{array}{c}\text { Double stl wall w/ 6.4" } \\
\text { DU (max) (s) } \\
3.75^{\prime \prime} \text { stl }+2.25^{n} \mathrm{DU} \\
(\mathrm{t})\end{array}$ & $\begin{array}{c}93.5 \mathrm{w} / \mathrm{o} \mathrm{max}{ }^{235} \mathrm{U} \\
\text { (+other) }\end{array}$ & $4.1 \mathrm{Kw}$ & SARP? & $\begin{array}{l}\text { Public } \\
\text { Service of } \\
\text { Colorado }\end{array}$ & $\begin{array}{l}\text { Readily } \\
\text { avail. }\end{array}$ & 2 \\
\hline NLI-1/2 & $\begin{array}{c}\text { USA/9010/ } \\
\text { B( )F }\end{array}$ & $3 / 31 / 96$ & 49,250 & $\begin{array}{c}75.0 \text { \& } \mathrm{x} \\
178.0 \mathrm{long}\end{array}$ & $\begin{array}{c}\text { Legal } \\
\text { weight } \\
\text { truck }\end{array}$ & 3,000 & $\begin{array}{c}12.63 \phi x \\
237.00 \text { long }\end{array}$ & $\begin{array}{l}\text { 2.75" DU } \\
5.0^{\prime \prime} \mathrm{H}_{2} \mathrm{O} / \\
\text { glycol } \\
\text { 2.13" Pb (s) } \\
5.0^{\prime \prime} \mathrm{H}_{2} \mathrm{O}(\mathrm{t} ?)\end{array}$ & $\begin{array}{c}3.7{ }^{\mathrm{W}} / \mathrm{omax} \\
235 \mathrm{U}+\mathrm{Pu} / \\
40000 \mathrm{Mwd} \text { for } \\
\text { PWR } \\
2.65 \mathrm{w} / \mathrm{o} / 34000 \\
\text { Mwd for BWR }\end{array}$ & $750 \mathrm{w}$ & $\begin{array}{c}\text { 39.4/end } \\
\text { 38.6/side } \\
\text { 37.5/corner }\end{array}$ & $\begin{array}{l}\text { Nuclear } \\
\text { Assurance } \\
\text { Corp. }\end{array}$ & $\begin{array}{c}\text { Possibly full } \\
\text { time }\end{array}$ & 5 \\
\hline $\begin{array}{l}\text { NLI. } \\
6502\end{array}$ & $\begin{array}{c}\text { USA/9103/ } \\
\text { B( )F }\end{array}$ & $10 / 31 / 93$ & 45,300 & $\begin{array}{c}33.5 \phi x \\
130.0 \text { long }\end{array}$ & $\begin{array}{c}\text { Legal } \\
\text { weight } \\
\text { truck }\end{array}$ & N/A & $\begin{array}{l}11.5 \square x \\
120.0 \text { long }\end{array}$ & $\begin{array}{c}\text { Triple stl wall (2" } \\
\text { total) w/ } 3^{n} \mathrm{~Pb} \& 5.72^{\prime \prime} \\
(\max ) \mathrm{DU}+\mathrm{Pb}(\mathrm{s}) \\
?^{\prime \prime} \text { stl w/ 6.63" } \mathrm{Pb}(\mathrm{t} \& \mathrm{~b})\end{array}$ & $\begin{array}{c}550 \mathrm{~g} \text { max }{ }^{235} \mathrm{U} \\
45 \% \text { burnup }\end{array}$ & $4.1 \mathrm{Kw}$ & N/A & $\begin{array}{l}\text { Nuclear } \\
\text { Assurance } \\
\text { Corp. }\end{array}$ & N/A & N/A \\
\hline $\mathrm{T}-3$ & $\begin{array}{l}\text { USA/9132/ } \\
\text { B(M)F }\end{array}$ & $3 / 31 / 96$ & 38,200 & $\begin{array}{c}52.00 \phi x \\
213.20 \text { long }\end{array}$ & $\begin{array}{l}\text { Legal } \\
\text { weight } \\
\text { truck }\end{array}$ & 700 & $\begin{array}{c}7.98 \phi x \\
147.0 \text { long }\end{array}$ & $\begin{array}{c}\text { Double stl wall (1.3" } \\
\text { total) w/ 8.0" } \mathrm{Pb}(\mathrm{s}) \\
11.83^{\prime \prime} \text { stl (b) } \\
11.625^{\prime \prime} \text { stl (t) }\end{array}$ & $\begin{array}{c}311^{\mathrm{w}} / \mathrm{o} \mathrm{PuO} \\
69^{\mathrm{w}} / \mathrm{o} \mathrm{U}_{2}\end{array}$ & $1.4 \mathrm{Kw}$ & $\begin{array}{c}\text { 87.8/end } \\
94.5 / \text { side } \\
50.2 / \text { corner }\end{array}$ & DOE -EH & $\begin{array}{c}\text { Immed. } \\
\text { (1 custom } \\
\text { trailer only) }\end{array}$ & 3 \\
\hline NAC-1 & $\begin{array}{c}\text { USA/9183/ } \\
\text { B( )F }\end{array}$ & $9 / 30 / 94$ & 49,000 & $\begin{array}{c}50.0 \text { \& } \mathrm{x} \\
214.0 \text { long }\end{array}$ & $\begin{array}{c}\text { Legal } \\
\text { weight } \\
\text { truck }\end{array}$ & 3,700 & $\begin{array}{c}13.5 \phi x \\
178.0 \text { long }\end{array}$ & $\begin{array}{l}\text { Double stl wall (1.56" } \\
\text { total) w/ } 5^{\prime \prime}(\mathrm{min}) \mathrm{Pb} \\
(\mathrm{s}) \\
2^{\prime \prime} \mathrm{stl}(\mathrm{b}) \\
7.5^{\prime \prime} \mathrm{stl}(\mathrm{l})\end{array}$ & $\begin{array}{c}1600 \mathrm{Mwd} / \mathrm{MTU} \\
\text { metallic } \mathrm{U}\end{array}$ & $750 w$ & $\begin{array}{c}\text { 76.6/end } \\
96 / \text { side } \\
45.5 / \text { corner }\end{array}$ & $\begin{array}{l}\text { Nuclear } \\
\text { Assurance } \\
\text { Corp. }\end{array}$ & $\begin{array}{c}\text { Possibly full } \\
\text { time }\end{array}$ & 6 \\
\hline
\end{tabular}


Table 3-1. (continued).

\begin{tabular}{|c|c|c|c|c|c|c|c|c|c|c|c|c|c|c|}
\hline $\begin{array}{c}\text { Package } \\
\text { name }\end{array}$ & $\mid \begin{array}{c}\text { Certificate } \\
\text { of } \\
\text { compliance }\end{array}$ & $\begin{array}{l}\text { Certificate } \\
\text { expiration }\end{array}$ & $\begin{array}{c}\text { Gross } \\
\text { weight } \\
\text { (pounds) }\end{array}$ & $\mid \begin{array}{c}\text { External } \\
\text { dimensions } \\
\text { (inches) }^{\mathrm{b}}\end{array}$ & $\begin{array}{c}\text { Transport } \\
\text { mode }\end{array}$ & $\begin{array}{c}\text { Payload } \\
\text { weight } \\
\text { (pounds) }\end{array}$ & $\begin{array}{c}\text { Internal } \\
\text { dimensions } \\
\text { (inches) }^{\mathrm{b}}\end{array}$ & Shielding config. ${ }^{c}$ & Content(s) ${ }^{d}$ & \begin{tabular}{|l|} 
Maximum \\
heat load
\end{tabular} & $\begin{array}{c}\text { Maximum } \\
\text { impact } \\
\text { load (g's) / } \\
\text { orientation }\end{array}$ & Owner $f$ & Availability & Number \\
\hline $\begin{array}{l}\text { CNS } \\
1-13 G\end{array}$ & $\begin{array}{l}\text { USA9216/ } \\
\text { B( }) F\end{array}$ & $12 / 31 / 97$ & 25,500 & $\begin{array}{c}68.00 \phi x \\
81.80 \text { high } \\
38.5 \phi x \\
67.19 \text { high } \\
(w / 0 \text { I/L) }\end{array}$ & $\begin{array}{l}\text { Legal } \\
\text { weight } \\
\text { truck }\end{array}$ & $\begin{array}{l}\text { "Not } \\
\text { restricted" }\end{array}$ & $\begin{array}{l}26.5 \phi x \\
54.00 \text { high }\end{array}$ & $\begin{array}{c}\text { Dbl stl wall (1.0n total) } \\
\mathrm{w} / 5.0^{\prime \prime} \mathrm{Pb}(\mathrm{s})\end{array}$ & $\begin{array}{c}\mathrm{PuO}_{2} \text { and } \mathrm{UO}_{2} \text { in } \\
\mathrm{Zr} \text { or SST }\end{array}$ & $\left|\begin{array}{c}600 \mathrm{w} \\
(+ \text { other })\end{array}\right|$ & SARP? & $\begin{array}{l}\text { Chem- } \\
\text { Nuclear } \\
\text { Systems }\end{array}$ & $\begin{array}{l}\text { Available } \\
\text { after } \\
\text { Barnwell } \\
\text { closes }\end{array}$ & 1 \\
\hline $\begin{array}{c}\text { NRBK- } \\
41\end{array}$ & $\begin{array}{l}\text { USA/9221/ } \\
\text { B( ) F }\end{array}$ & $1 / 31 / 96$ & 9,000 & $\begin{array}{c}27.16 \phi x \\
40.00 \text { long } \\
\text { (high?) }\end{array}$ & $\begin{array}{l}\text { Legal } \\
\text { weight } \\
\text { truck }\end{array}$ & N/A & $\begin{array}{c}5.00 \phi x \\
16.00 \text { long } \\
\text { (high?) }\end{array}$ & $\begin{array}{l}\text { Double stl shell }\left(0.5^{\prime \prime}\right. \\
\text { total) w/ } 10.00^{\prime \prime} \mathrm{Pb}(\mathrm{s})\end{array}$ & $350 \mathrm{~g}$ equiv. ${ }^{235} \mathrm{U}$ & $\mid \begin{array}{c}264 \mathrm{w} \\
\text { (exclusive } \\
\text { use) }\end{array}$ & N/A & DOE-NR & N/A & N/A \\
\hline $\begin{array}{l}\text { NAC- } \\
\text { LWT }\end{array}$ & $\begin{array}{l}\text { USA/9225/ } \\
\text { B(U)F }\end{array}$ & $12 / 31 / 94$ & 51,200 & \begin{tabular}{|c|}
$65.0 \phi x$ \\
232.0 long \\
$44.0 \phi x$ \\
200.0 long \\
$(w / 01 / L)$
\end{tabular} & $\begin{array}{l}\text { Legal } \\
\text { weight } \\
\text { truck }\end{array}$ & 4,000 & $\begin{array}{c}13.4 \phi \mathrm{x} \\
178.0 \text { long }\end{array}$ & $\begin{array}{c}1.95^{\prime \prime} \text { stl }+5.75^{\prime \prime} \mathrm{Pb}+ \\
5^{\prime \prime} \mathrm{H}_{2} \mathrm{O} / \text { glycol(s) } \\
3.5^{\prime \prime} \mathrm{stl}+3^{\prime \prime} \mathrm{Pb}(\mathrm{b}) \\
11.3^{\prime \prime} \mathrm{stl}(\mathrm{t})\end{array}$ & \begin{tabular}{|}
$1 \mathrm{PWR}$ or $2 \mathrm{BWR}$ \\
or $15 \mathrm{rods}$ \\
$3.7 \mathrm{w} / \mathrm{o} /$ \\
$4.0 \mathrm{w} / \mathrm{o}$ \\
$35000 /$ \\
$30000 \mathrm{MWd} / \mathrm{MTU}$
\end{tabular} & $\begin{array}{c}2.5 \mathrm{~kW} \\
\text { (tother) }\end{array}$ & SARP? & $\begin{array}{c}\text { Nuclear } \\
\text { Assurance } \\
\text { Corp. }\end{array}$ & $\begin{array}{c}\text { Possibly full } \\
\text { time }\end{array}$ & 4 \\
\hline $\begin{array}{c}\text { GE } \\
\text { Model } \\
2000\end{array}$ & $\begin{array}{l}\text { USA/9228/ } \\
\mathrm{B}(\mathrm{U}) \mathrm{F}\end{array}$ & $10 / 31 / 94$ & 33,550 & $\begin{array}{c}72.0 \phi x \\
131.5 \text { high } \\
38.5 \phi x \\
71.0 \text { high } \\
(w / 01 / L)\end{array}$ & $\begin{array}{l}\text { Legal } \\
\text { weight } \\
\text { truck }\end{array}$ & 5,450 & $\begin{array}{l}26.5 \notin x \\
54.0 \text { high }\end{array}$ & $\begin{array}{c}\text { Double stl wall (2" } \\
\text { total) w/ } 4^{\prime \prime} \mathrm{Pb}(\mathrm{s}) \\
6 \text { "stl (b) } \\
\text { ?" stl + ?" } \mathrm{Pb}(\mathrm{t})\end{array}$ & $\begin{array}{c}1.75 \mathrm{~kg} 5 \mathrm{w} / \mathrm{o} \text { or } \\
500 \mathrm{~g} 235 \mathrm{U} \text { equiv. } \\
\text { mass } 45000 \\
\text { Mwd/MTU }\end{array}$ & $600 \mathrm{w}$ & SARP? & $\begin{array}{l}\text { General } \\
\text { Electric }\end{array}$ & $\begin{array}{l}\text { Approx. } \\
50 \%\end{array}$ & $\begin{array}{c}2 \\
\text { (plus 1 } \\
\text { currently } \\
\text { in fab) }\end{array}$ \\
\hline TN-FSV & $\begin{array}{l}\text { USA/9253/ } \\
\mathrm{B}(\mathrm{U}) \mathrm{F}\end{array}$ & $5 / 31 / 99$ & 47,000 & $\begin{array}{c}78.0 \phi \mathrm{x} \\
247.0 \text { long } \\
31.0 \phi \mathrm{x} \\
\text { ? long } \\
\text { (w/o I/L) }\end{array}$ & $\begin{array}{c}\text { Legal } \\
\text { weight } \\
\text { truck }\end{array}$ & 5,000 & $\begin{array}{l}18.0 \phi x \\
199.0 \text { long }\end{array}$ & $\begin{array}{c}\text { Double stl wall }\left(2.62^{\prime \prime}\right. \\
\text { total) w/ 3.44" Pb (s) } \\
5.5^{\prime \prime} \mathrm{stl}(\mathrm{b}) \\
2.5^{\prime \prime} \mathrm{stl} \text { w/ ?" DU (t) }\end{array}$ & $\begin{array}{c}6 \text { irradiated HTGR } \\
\text { fuel elements } \\
\left(8.4 \mathrm{~kg} \text { total } 235_{\mathrm{U}}\right. \\
-93.5 \mathrm{w} / \mathrm{o}+66 \mathrm{~kg} \\
\mathrm{Th})\end{array}$ & $\begin{array}{l}360 \mathrm{w} \\
\text { (total) }\end{array}$ & SARP? & $\begin{array}{c}\text { Public } \\
\text { Service Co. } \\
\text { of Colorado }\end{array}$ & $\begin{array}{c}\text { Depends on } \\
\text { availability } \\
\text { of storage } \\
\text { for FSV } \\
\text { fuel at } \\
\text { INEL }\end{array}$ & 2 \\
\hline $\begin{array}{l}\text { TN-8 } \\
\text { TN-8L }\end{array}$ & $\begin{array}{l}\text { USAYO15/ } \\
\text { B( ) }\end{array}$ & $5 / 31 / 96$ & 79,380 & $\begin{array}{l}67.64 \phi x \\
217.2 \text { long }\end{array}$ & $\begin{array}{l}\text { Over- } \\
\text { weight } \\
\text { truck }\end{array}$ & 4,850 & $\begin{array}{c}3 \times 9.06 \square \times \\
168.5 \text { long }\end{array}$ & $\begin{array}{c}1.023^{\prime \prime} \mathrm{stl}+5.315^{\prime \prime} \mathrm{Pb} \\
+5.905^{\prime \prime} \text { resin }\end{array}$ & $\begin{array}{c}4 \mathrm{w}_{/ \mathrm{o}}^{235} \mathrm{U}, 38500 \\
\mathrm{Mwd} / \mathrm{MTU}\end{array}$ & $\begin{array}{l}35.5 \mathrm{Kw} \\
\text { (+other) }\end{array} \mid$ & $\begin{array}{l}155 / \text { end } \\
155 / \text { side } \\
80 / \text { corner }\end{array}$ & Transnuclear & $\begin{array}{c}1 \text { immed. } \\
1 \text { soon } \\
\text { (after } \\
\text { Barnwell } \\
\text { campaign) }\end{array}$ & 2 \\
\hline TN-9 & $\begin{array}{c}\text { USA/9016/ } \\
B() F\end{array}$ & $5 / 31 / 96$ & 79,380 & $\left|\begin{array}{c}67.64 \phi x \\
226.61 \text { high }\end{array}\right|$ & $\begin{array}{l}\text { Over- } \\
\text { weight } \\
\text { truck }\end{array}$ & $4,652.6$ & $\begin{array}{l}7 \times 5.91 \mathrm{Dx} \\
177.95 \mathrm{long}\end{array}$ & {$\left[\begin{array}{c}1.023^{\prime \prime} \mathrm{stl}+5.039^{\mathrm{n}} \mathrm{Pb} \\
+5.905^{\prime \prime} \text { resin }(\mathrm{s})\end{array}\right.$} & $\begin{array}{c}2.655^{\mathrm{w}} / \mathrm{o}^{235} \mathrm{U} \\
36500 \mathrm{Mwd} / \mathrm{MTU}\end{array}$ & $\left|\begin{array}{c}24.4 \mathrm{Kw} \\
\text { total } 3.54 \\
\mathrm{Kw} / \mathrm{ass} ' \mathrm{y}\end{array}\right|$ & $\begin{array}{c}155 / \text { end } \\
155 / \text { side } \\
80 / \text { corner }\end{array}$ & Transnuclear & $\begin{array}{l}\text { Immed. } \\
\text { (trailers } \\
\text { need } \\
\text { refurbish) }\end{array}$ & 2 \\
\hline
\end{tabular}


Table 3-1. (continued).

\begin{tabular}{|c|c|c|c|c|c|c|c|c|c|c|c|c|c|c|}
\hline $\begin{array}{c}\text { Package } \\
\text { name }\end{array}$ & $\begin{array}{l}\text { Certificate } \\
\text { of } \\
\text { compliance }\end{array}$ & $\begin{array}{l}\text { Certificate } \\
\text { expiration }\end{array}$ & $\begin{array}{c}\text { Gross } \\
\text { weight } \\
\text { (pounds) }\end{array}$ & $\begin{array}{c}\text { External } \\
\text { dimensions } \\
\text { (inches) }^{b}\end{array}$ & $\begin{array}{c}\text { Transport } \\
\text { mode }\end{array}$ & $\begin{array}{l}\text { Payload } \\
\text { weight } \\
\text { (pounds) }\end{array}$ & $\begin{array}{c}\text { Internal } \\
\text { dimensions } \\
\text { (inches) }^{b}\end{array}$ & Shielding config. ${ }^{c}$ & Content(s) ${ }^{\mathrm{d}}$ & $\begin{array}{l}\text { Maximum } \\
\text { heat load }\end{array}$ & $\begin{array}{l}\text { Maximum } \\
\text { impact } \\
\text { load (g's)/ } \\
\text { orientation }\end{array}$ & Owner ${ }^{f}$ & Availability & Number \\
\hline M-130 & $\begin{array}{c}\text { USA/6003/ } \\
\text { B( )F }\end{array}$ & $12 / 31 / 97$ & 228,000 & $\begin{array}{l}84.00 \phi x \\
158.0 \text { high }\end{array}$ & Rail & N/A & $\begin{array}{c}55.0 \text { od } x \\
29.0 \text { id } x \\
130.0 \text { high }\end{array}$ & $\begin{array}{l}\text { Double stl wall (2" } \\
\text { total) } \mathrm{w} / 10.0^{\prime \prime} \mathrm{Pb}(\mathrm{s})\end{array}$ & fuel elements & $9815.5 \mathrm{w}$ & $\mathbf{N} / \mathbf{A}$ & DOE-NR & $\begin{array}{c}\text { Not } \\
\text { available }\end{array}$ & 27 \\
\hline IF-300 & $\begin{array}{c}\text { USA/9001/ } \\
\mathrm{B}(\mathrm{)}) \mathrm{F}\end{array}$ & $5 / 31 / 95$ & $\begin{array}{c}185,000 \\
\text { w/ skid } \\
140,000 \\
\text { cask only }\end{array}$ & $\begin{array}{l}64.0 \phi x \\
210.0 \text { long }\end{array}$ & Rail & 21,000 & $\begin{array}{c}37.5 \phi x \\
180.25 \text { long }\end{array}$ & $\begin{array}{c}\text { Double stl wall }\left(2.13^{\prime \prime}\right. \\
\text { total) w/ } 4^{\prime \prime} \mathrm{DU}+4.5^{\prime \prime} \\
\mathrm{H}_{2} \mathrm{O}(\mathrm{s}) \\
?^{\prime \prime} \mathrm{stl}+3^{\prime \prime} \mathrm{DU}(\mathrm{t} \& \mathrm{~b})\end{array}$ & $\begin{array}{c}4^{\mathrm{w} / \mathrm{o}}{ }^{235} \mathrm{U}, 35000 \\
\mathrm{Mwd} / \mathrm{MTU}\end{array}$ & $11.72 \mathrm{Kw}$ & $\begin{array}{l}\text { 234/end } \\
\text { 109/side } \\
69 / \text { corner }\end{array}$ & $\begin{array}{c}\text { CP\&L } \\
-- \\
\text { Vectra } \\
\text { Technologies }\end{array}$ & $\begin{array}{c}2 \text { not } \\
\text { available } \\
-- \\
2 \text { readily } \\
\text { available }\end{array}$ & $\begin{array}{c}2 \mathrm{CP} \& \mathrm{~L} \\
2 \mathrm{Vectra}\end{array}$ \\
\hline $\begin{array}{l}\text { NLI- } \\
10 / 24\end{array}$ & $\begin{array}{c}\text { USA/9023/ } \\
\mathrm{B}(\mathrm{)}) \mathrm{F}\end{array}$ & $3 / 31 / 97$ & 194,000 & $\begin{array}{l}96.00 \text { ф } \mathrm{x} \\
204.5 \text { long }\end{array}$ & Rail & 34,100 & $\begin{array}{c}45.00 \phi x \\
179.50 \text { long } \\
\end{array}$ & $\begin{array}{c}\text { Triple stl wall }\left(3.5^{\prime \prime} \text { stl }\right) \\
\text { w/ } 6.0^{\prime \prime} \mathrm{Pb}+9.0^{\prime \prime} \mathrm{H}_{2} \mathrm{O} \\
(\mathrm{s}) \\
\text { ?" DU }+?^{\prime \prime} \mathrm{stl}+ \\
\text { polymer (t\&b) }\end{array}$ & $\begin{array}{c}3.5^{\mathrm{w}} / \mathrm{o}{ }^{235} \mathrm{U} \\
35500 \mathrm{Mwd} / \mathrm{MTU} \\
\text { for } \mathrm{PWR} \\
2.8^{\mathrm{w}} / \mathrm{o}{ }^{235} \mathrm{U} \\
29700 \mathrm{Mwd} / \mathrm{MTU} \\
\text { for } \mathrm{BWR}\end{array}$ & $70 \mathrm{Kw}$ & $\begin{array}{l}\text { 29.4/end } \\
80.8 / \text { side } \\
31 / \text { corner }\end{array}$ & $\begin{array}{l}\text { Nuclear } \\
\text { Assurance } \\
\text { Corp. }\end{array}$ & $\begin{array}{c}\text { Possibly full } \\
\text { time }\end{array}$ & 2 \\
\hline $125-B$ & $\begin{array}{c}\text { USA/9200/ } \\
\mathrm{B}(\mathrm{M}) \mathrm{F}\end{array}$ & $5 / 31 / 96$ & 181,500 & $\begin{array}{l}120.0 \phi x \\
279.5 \text { long } \\
65.5 \phi x \\
207.5 \text { long } \\
(w / 0 \mathrm{~L} / \mathrm{L})\end{array}$ & Rail & $\begin{array}{c}57,580 \mathrm{w} / \\
\text { Outer vessel } \\
\text { only } \\
20,580 \mathrm{w} / \\
\text { Inner vessel } \\
\text { also (for } \\
\text { double } \\
\text { containment) }\end{array}$ & $\begin{array}{c}51.25 \phi x \\
192.5 \text { long } \\
\text { (outer vessel) } \\
7 \times 14.5 \phi x \\
183.25 \text { long } \\
\text { (inner vessel; } \\
\text { for double } \\
\text { containment) }\end{array}$ & \begin{tabular}{|c} 
Double stl wall (3" \\
total) w/ 3.88" Pb (s) \\
(Outer Vessel) + \\
1" stl (s) (Inner \\
Vessel) \\
$7.5^{\prime \prime}$ stl (t) \\
$7.5^{\prime \prime}$ stl (b) (Outer \\
Vessel) + \\
$5^{\prime \prime}$ stl (t) \\
$4^{\prime \prime}$ stl (b) (Inner \\
Vessel)
\end{tabular} & $\begin{array}{c}2.98 \mathrm{w}_{/ \mathrm{o}}^{235} \mathrm{U} \\
3165 \mathrm{Mwd} / \mathrm{MTU}, \\
6 \text { year cooling }\end{array}$ & $\left|\begin{array}{c}100 \mathrm{w} \text { per } \\
\text { canister } \\
(700 \mathrm{w} \\
\text { total })\end{array}\right|$ & $\begin{array}{c}\text { 51.6/end } \\
67.8 / \text { side } \\
\text { 34.7/corner }\end{array}$ & $\mid \begin{array}{c}\text { DOE-1D } \\
- \\
\text { Vectra } \\
\text { Technologies }\end{array}$ & Immediate & $\begin{array}{l}2 \mathrm{DOE} \\
1 \text { vectra }\end{array}$ \\
\hline $\begin{array}{l}\text { TN- } \\
\text { BRP }\end{array}$ & $\begin{array}{l}\text { USA/9202/ } \\
\mathrm{B}(\mathrm{U}) \mathrm{F}\end{array}$ & $6 / 30 / 94$ & 215,000 & $\begin{array}{c}131.0 \phi x \\
244.5 \text { long } \\
83.25 \text { \$ } x \\
196.5 \text { long } \\
(w / o ~ I / L)\end{array}$ & Rail & 41,250 & $\begin{array}{c}64.0 \phi x \\
171.0 \text { long }\end{array}$ & $\begin{array}{c}9.62 " \text { stl (s) } 9.75^{\prime \prime} \text { stl } \\
\text { (b\&t) }\end{array}$ & $\begin{array}{c}44 \mathrm{BWR} \text { ass ys } \\
5.6 \mathrm{w} / \mathrm{o}{ }^{235} \mathrm{U} \\
25000 \mathrm{Mwd} / \mathrm{MTU}, \\
14 \text { year cooling }\end{array}$ & $\begin{array}{c}3.1 \mathrm{Kw} \\
\text { total } 103 \\
\text { w/ass'y }\end{array}$ & SARP? & DOE-ID & $\begin{array}{c}\text { None if W. } \\
\text { Valley } \\
\text { campaign } \\
\text { begins (low } \\
\text { probability) } \\
\text { also limited } \\
\text { on time of } \\
\text { year \& } \\
\text { payload }\end{array}$ & 1 \\
\hline
\end{tabular}


Table 3-1. (continued).

\begin{tabular}{|c|c|c|c|c|c|c|c|c|c|c|c|c|c|c|}
\hline $\begin{array}{c}\text { Package } \\
\text { name }\end{array}$ & $\begin{array}{l}\text { Certificate } \\
\text { of } \\
\text { compliance }\end{array}$ & $\begin{array}{l}\text { Certificate } \\
\text { expiration }\end{array}$ & $\begin{array}{c}\text { Gross } \\
\text { weight } \\
\text { (pounds) }\end{array}$ & $\begin{array}{c}\text { External } \\
\text { dimensions } \\
\text { (inches) }^{b}\end{array}$ & $\begin{array}{c}\text { Transport } \\
\text { mode }\end{array}$ & $\begin{array}{l}\text { Payload } \\
\text { weight } \\
\text { (pounds) }\end{array}$ & $\begin{array}{l}\text { Internal } \\
\text { dimensions } \\
\text { (inches) }^{\mathrm{b}}\end{array}$ & Shielding config. $c$ & Content(s) ${ }^{d}$ & $\begin{array}{l}\text { Maximum } \\
\text { heat load }\end{array}$ & $\begin{array}{c}\text { Maximum } \\
\text { impact } \\
\text { load }\left(g^{\prime} s\right) / \\
\text { orientation }\end{array}$ & Owner $f$ & Availability & Number \\
\hline $\begin{array}{l}\text { TN. } \\
\text { REG }\end{array}$ & $\begin{array}{l}\text { USA/9206/ } \\
\text { B(U)F }\end{array}$ & $5 / 31 / 95$ & 225,000 & $\begin{array}{l}131.0 \phi x \\
234.0 \text { long }\end{array}$ & Rail & 50,500 & $\begin{array}{c}71.75 \phi x \\
163.25 \text { long }\end{array}$ & $\begin{array}{l}9.25 " \text { stl (s) } \\
8.5 " \text { stl (t) }\end{array}$ & $\begin{array}{c}20 \mathrm{PWR}^{2} \text { ass }{ }^{\prime} \mathrm{ys} \\
3 . \mathrm{w}^{\mathrm{w}} / \mathrm{o} 235 \mathrm{U}, \\
15000 \mathrm{Mwd} / \mathrm{MTU}\end{array}$ & $\left|\begin{array}{c}2.7 \mathrm{Kw} \\
\text { (+other) }\end{array}\right|$ & SARP? & DOE-ID & $\begin{array}{c}\text { Same as } \\
\text { TN-BRP } \\
\text { above }\end{array}$ & 1 \\
\hline$M-160$ & $\begin{array}{l}\text { USA/9781/ } \\
\text { B( )F }\end{array}$ & $7 / 31 / 97$ & 237,000 & $\begin{array}{l}79.00 \phi x \\
199.0 \text { high }\end{array}$ & Rail & N/A & $\begin{array}{l}55.0 \text { od } x \\
21.0 \text { id } x \\
160.0 \text { high }\end{array}$ & $\begin{array}{c}\text { Double (?) stl wall } \\
\text { (2.5" total) w/ } 9.44^{\prime \prime} \mathrm{Pb} \\
\text { (s) } \\
15^{\prime \prime} \text { stl (t) } \\
7^{\prime \prime} \text { stl (b) }\end{array}$ & Fuel elements & $6240.9 \mathrm{w}$ & N/A & DOE-NR & $\begin{array}{c}\text { Not } \\
\text { available }\end{array}$ & 1 \\
\hline M-140 & $\begin{array}{l}\text { USA/9793/ } \\
\mathrm{B}(\mathrm{U}) \mathrm{F}\end{array}$ & $10 / 31 / 96$ & 375,000 & $\begin{array}{l}126.0 \phi x \\
194.0 \text { high }\end{array}$ & Rail & N/A & $\begin{array}{c}70.0 \text { \$x } \\
146.0 \mathrm{high}\end{array}$ & $\begin{array}{l}14^{\prime \prime} \text { stl (s) } \\
12^{\prime \prime} \text { stl (b) } \\
13^{\prime \prime} \text { stl (t) }\end{array}$ & Fuel elements & $\left|\begin{array}{l}18.25 \mathrm{Kw} \\
\text { (+other) }\end{array}\right|$ & N/A & DOE-NR & $\begin{array}{c}\text { Not } \\
\text { available }\end{array}$ & $\begin{array}{c}24 \\
\text { currently }\end{array}$ \\
\hline
\end{tabular}

in

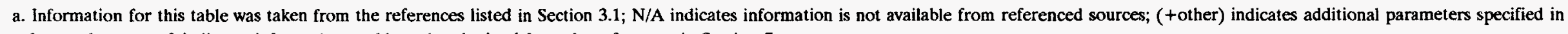
referenced sources; ? indicates information could not be obtained from the references in Section 7.

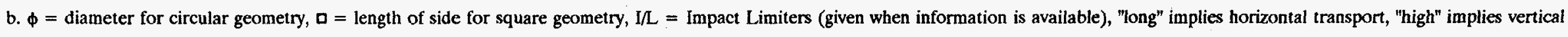
transport.

c. stl $=$ steel, $\mathrm{Pb}=$ lead, $\mathrm{DU}=$ depleted uranium, $\mathrm{H}_{2} \mathrm{O}=$ water, $(\mathrm{s})=$ sides, $(\mathrm{t})=$ top, $(\mathrm{b})=$ bottom.

d. $\mathrm{TI}=$ Transportation Index.

e. SARP? indicates missing information may be available from the safety analysis report for packaging (SARP).

f. $\mathrm{EH}=$ Transportation and Packaging Safety Division, ID = Idaho Operations Office, NR = Naval Reactors Division. 


\subsubsection{Payload Cavity Size}

The packaging payload cavity size will be a primary factor in determining what fuel types and how many of each may be transported. Size considerations relating to fuel-specific internal structures (baskets or canisters) must be accounted for, in addition to the spent fuel itself.

\subsubsection{Overall Size}

Facility interface considerations will govern maximum package size that can be accommodated without facility modifications. This factor is discussed in greater detail in Section 5.3.

\subsubsection{Payload Weight Limits}

Payload weight cannot exceed specified package limits. Weight capacity can potentially be increased by detailed analysis and Certificate of Compliance revision, as discussed in Section 5.3.

\subsubsection{Gross Weight}

Facility interface considerations (e.g., crane capacity, may limit package access for particular fuel types). Refer to Section 5.3 for details.

\subsubsection{Thermal Limits}

Maximum permissible payload thermal loading is usually specified as an operational limit on a transport package. The implications of these limits are discussed in Sections 3.2.6 through 3.2.9.

\subsubsection{Shielding Capabilities}

Radiological activity will be governed by regulatory dose rate limits of 10 CFR $\$ 71.47$, "Normal Conditions of Transport," and 10 CFR \$71.51(a)(2), "Hypothetical Accident Conditions." In addition, thermal limits must be maintained to preclude biological shielding deterioration (e.g., lead melt, neutron shielding degeneration).

\subsubsection{Containment Capabilities}

Fuels with breached cladding and plutonium content in excess of $20 \mathrm{Ci}$ per shipment will probably have to be transported in double-containment, leak-tight packages, in accordance with 10 CFR $\$ 71.63$ (note that intact fuel meets the double containment exemption). As discussed in Section 2.2.6, the implications of these restrictions are very serious. Qualifying an existing package for a new inner containment vessel could be an undertaking similar in magnitude to qualifying an entirely new package. Furthermore, the leaktight restriction could introduce even more complications. Most of the candidate transport packages do not currently feature qualified leaktight closure configurations on even the single level of containment they incorporate. Accordingly, these packages would either have to be requalified for maximum leakage rates well below currently licensed levels, or would have to be retrofitted with leaktight closure designs. Of special importance is the requirement to demonstrate that the leaktight closure remains leaktight 
after the hypothetical accident condition impact and fire tests. In addition, excessive thermal loading, particularly that which may develop from the accident fire test, may adversely affect intact fuel cladding, seal material integrity, or package maximum normal operating pressure, all potentially leading to additional containment concerns.

\subsubsection{Maximum Impact Loading}

Impact loading at various orientations arising from the hypothetical accident condition $30-\mathrm{ft}$ free drop requirement will drive requirements for payload protection (i.e., baskets or other structural support). Typically, a nonyielding support structure is desired. Thermal loading must not result in excessive loss of strength of these structures. In addition, elevated temperatures of impact limiting materials may affect their mechanical properties and, hence, associated impact loadings. Structurally fragile SNF assemblies will require special consideration, and criticality evaluations hinge directly on post-impact payload configurations.

Variations in package weight also directly affect impact acceleration magnitudes; reduced weight typically increases g-loading, increased weight typically reduces g-loads. Shielding deformation in the hypothetical accident condition $30-\mathrm{ft}$ free drop may be increased under greater impact loading, leading to compromise of package shielding capabilities. Finally, the load path from the packaging to the payload may be an important factor. For instance, the inner containment vessel of the 125-B package spans the inner surface of the outer vessel, resulting in reaction loads being applied at either end of both vessels. Any new basket developed for this package would have to either span the loads to the ends of the basket or employ a suitably reduced payload weight.

\subsubsection{Materials of Construction}

Package materials, particularly payload cavity and seal materials, must be chemically compatible with proposed payloads, as well as with associated thermal loadings.

\subsubsection{Transportation Index}

10 CFR \$71.59, "Standards for Arrays of Fissile Material Packages," governs the shipping configuration for irradiated fuel transport packages. Such packages are normally shipped via an exclusive use vehicle, though multiple package shipments might be feasible with certain payload restrictions (reduced fissile content or other form of inherent criticality control) that would reduce the transportation index to the required level.

Fissile-rated packages (i.e., packages rated for transportation of fissile contents greater than $15 \mathrm{~g}$ total) must undergo strenuous criticality evaluations to ensure that nuclear criticality safety is maintained during transportation. These criticality analyses require detailed information concerning the payload characteristics. In many instances, adequate SNF characteristics are not currently available to make such determinations. Additionally, maintenance of criticality safe payload configurations following the hypothetical accident condition tests will almost surely require positive geometrical configuration control (i.e., internal support structures). Design and qualification of such structures for the often unusual, nonstandard SNF geometries is expected to 
be a significant undertaking. The level of effort involved in qualifying an existing package for a new basket structure and payload type could be on par with that required to qualify an entirely new package.

\subsubsection{Operational Requirements}

Special tools, specially trained personnel, remote handling equipment, etc., may be required at the facility or facilities where the fuel is stored. Refer to Section 5.2 for details.

\subsubsection{Transport Mode}

Facilities containing proposed fuel types must be accessible by the particular transport package. A rail-mounted package will be of little use at a facility with road access only. Additionally, augmentation of a package's payload weight capacity, according to Section 3.2.3, may affect the transport limitations of the package (e.g., 80,000-lb maximum gross weight for legal weight truck transport). Refer to Section 5.3 for details.

\subsection{Discussion of Unknowns and Uncertainties Related to Packagings}

There are a number of uncertainties relating to packaging capabilities for any given fuel type. Fuel parameters set out in Section 2.2 are all interrelated insofar as all factors for a given fuel type must be fully bounded by the documented and certified capabilities of any package under consideration for purposes of transporting the fuel type. The final determination and categorization of such a wide range of parameters for the large number of fuel types in the DOE inventory with the degree of accuracy required to satisfy regulatory requirements will be a daunting task.

Economics will be a significant factor in grouping fuel types for transportation. At one extreme, an attempt could be made to reduce the overall number of package modifications required by qualifying a relatively small number of packages. This would result in the necessity of qualifying each candidate package for a relatively large group of fuel types. Such an effort would, in general, tend to result in limitations on capacity for each of the fuel types in any transport group. The final outcome could be an unacceptably large number of shipments required for each fuel type and, hence, a level of transportation inefficiency that would offset the initial developmental savings. Conversely, small groups of fuel types will lead to a need for a greater number of individual package amendments or revisions. This opposite extreme could also potentially impose unnecessarily severe cost and schedule hardships.

Additionally, while some currently licensed packages in their present form may not be suitable for shipment of any significant quantities of DOE-owned SNF, it is possible that modifications could be made to increase their payload capacity. However, a new level of uncertainty is thereby introduced. Categorizing SNF based on the postulated performance of a modified package would be an even greater undertaking than attempts to do so for well-defined, 
known package capabilities. This is particularly true since several levels of performance enhancement, each increasingly more demanding (and expensive), are potentially possible. Refer to Sections 4.2 through 4.5 for details of possible modifications of existing packages for purposes of upgrading their payload capabilities. 


\section{DISCUSSION OF COMPATIBILITY BETWEEN FUELS AND PACKAGINGS}

The regulations governing the transportation of radioactive material are very restrictive. The packaging Certificates of Compliance are normally quite explicit as to the definition of the material that is qualified for transportation in a particular package. Because of these factors, it is likely that, apart from the fuel types referred to in Section 4.1 below, transport of DOE-owned SNF will not be able to proceed without at least some additional effort. This effort could include adjustments to existing packages (either design or SARP related), or development of entirely new packages. These adjustments or replacements are categorized in Sections 4.2 through 4.6 below by the level of effort, as well as risk and cost, expected to be required. Fuels/packaging compatibilities were developed with the aid of Footnote d and Reference 12.

DOE-owned spent nuclear fuel will have to be comprehensively characterized by the parameters set out in Section 2 before transportation can take place. Once this is accomplished, candidate packages whose design characteristics (as detailed in Section 3) indicate a potential matchup can be further investigated. Depending on the desired carrying capability, packaging adjustments will be made, as detailed below. If transportation economics indicate that a large quantity or variety of fuels is to be transported in a single package, extensive package modifications or package development will probably have to be considered. More modest transportation goals will likely require less extensive packaging changes.

Using some the most important considerations set out in Sections 2 and 3, a fuels/packages compatibility matrix has been developed, and is included as Tables 4-1 through 4-6. The listed matchups have been based on size, thermal and shielding compatibility between each package, and the various types of SNF. For many of the SNF types, insufficient data exist to make a realistic determination of transportation requirements. These fuel types include all those listed in Tables 2 7 and 2-8, as well as some of the fuels listed in the other tables of Section 2. For the remainder of the fuels, the estimated fuel/package matchups generally fall into two categories:

1. Those fuels whose characteristics are sufficiently well defined so as to provide reasonable confidence in the selection of transportation packages for their conveyance. This category includes fuels for which transportation packages have been specifically designed (e.g., the Fort St. Vrain fuel, which both the FSV-1 and TN-FSV packages are licensed to transport).

2. Those fuels whose characteristics definitions were insufficient as listed in the reference documents, but which could be reasonably deduced by comparison to similar fuels with known transportation capabilities.

d. "Department of Energy-Owned Spent Nuclear Fuel/Certified Transportation Cask Compatibility Matrix; Forwarding of Preliminary Results," attached to a letter from R. A. Roesener (EG\&G Idaho) to D. G. Abbott, RAR-07-94, April 29, 1994. 
Table 4-1. Packaging compatibility with pressurized water reactor type SNF.

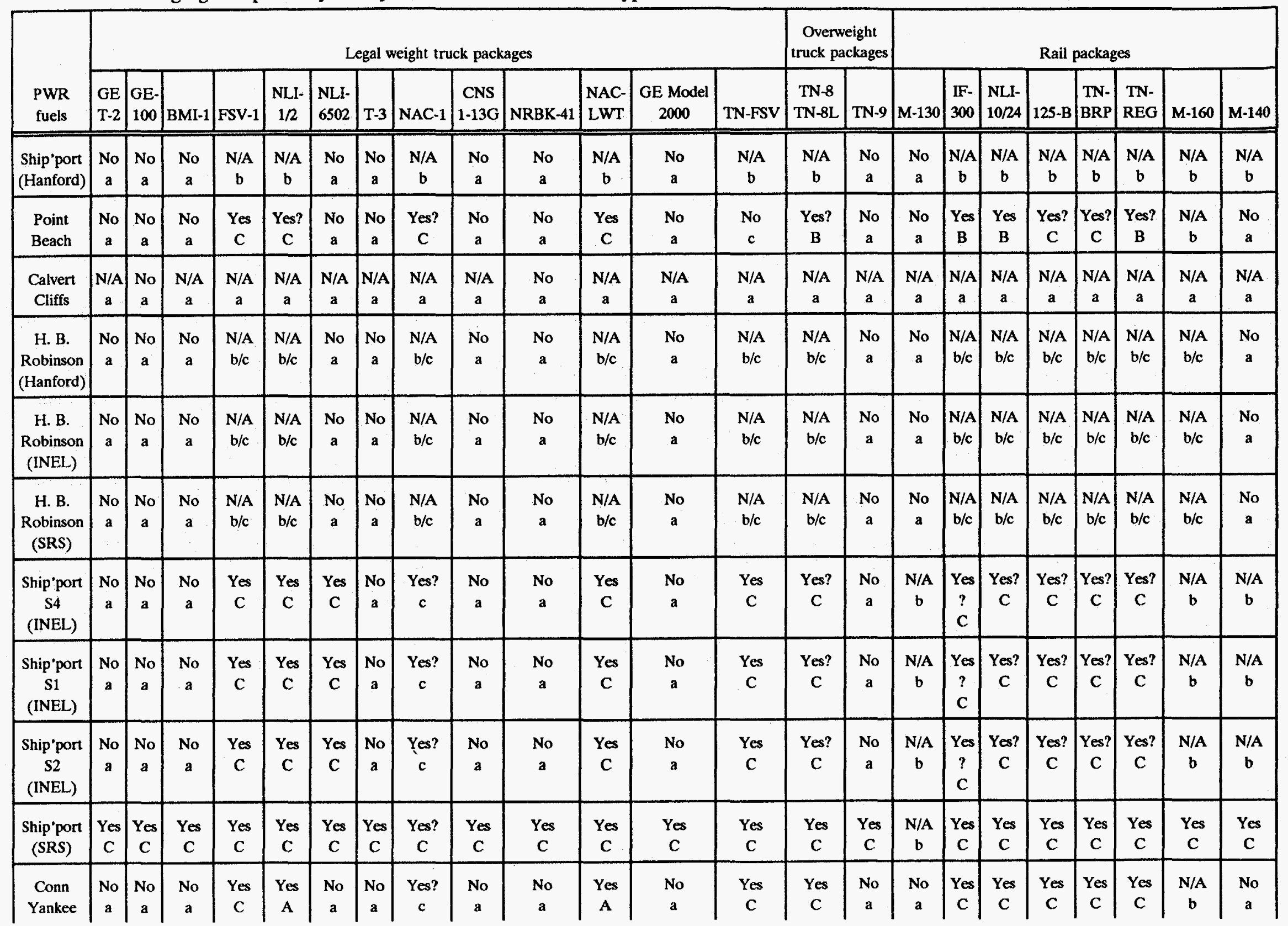


Table 4-1. (continued).

\begin{tabular}{|c|c|c|c|c|c|c|c|c|c|c|c|c|c|c|c|c|c|c|c|c|c|c|c|}
\hline \multirow[b]{2}{*}{$\begin{array}{l}\text { PWR } \\
\text { fuels }\end{array}$} & \multicolumn{13}{|c|}{ Legal weight truck packages } & \multicolumn{2}{|c|}{$\begin{array}{c}\text { Overweight } \\
\text { truck packages }\end{array}$} & \multicolumn{8}{|c|}{ Rail packages } \\
\hline & $\begin{array}{c}G E \\
T-2\end{array} \mid$ & $\begin{array}{l}\text { GE- } \\
100\end{array}$ & BMI-1 & FSV-1 & $\begin{array}{c}\text { NLI- } \\
1 / 2\end{array}$ & $\begin{array}{l}\text { NLI- } \\
6502\end{array}$ & $\mathrm{~T}-3$ & NAC-1 & $\begin{array}{c}\text { CNS } \\
1-13 G\end{array}$ & NRBK-41 & $\begin{array}{l}\text { NAC- } \\
\text { LWT }\end{array}$ & $\begin{array}{c}\text { GE Model } \\
2000\end{array}$ & TN-FSV & \begin{tabular}{|c|} 
TN-8 \\
TN-8L
\end{tabular} & TN-9 & $M-130$ & $\mid \begin{array}{l}\text { IF- } \\
300\end{array}$ & $\begin{array}{c}\text { NLI- } \\
10 / 24\end{array}$ & 125-B & $\begin{array}{l}\text { TN- } \\
\text { BRP }\end{array}$ & $\begin{array}{l}\text { TN- } \\
\text { REG }\end{array}$ & M-160 & M-140 \\
\hline LOFT & $\begin{array}{c}\text { Yes } \\
\text { C }\end{array}$ & $\begin{array}{c}\text { No } \\
a\end{array}$ & $\begin{array}{c}\text { No } \\
\text { a }\end{array}$ & $\begin{array}{l}\text { Yes } \\
\text { C }\end{array}$ & $\begin{array}{c}\text { Yes } \\
\text { C }\end{array}$ & $\begin{array}{c}\text { Yes } \\
\text { C }\end{array}$ & $\begin{array}{c}\text { No } \\
a\end{array}$ & $\begin{array}{l}\text { Yes } \\
\text { C }\end{array}$ & $\begin{array}{c}\text { No } \\
\text { a }\end{array}$ & $\begin{array}{c}\text { No } \\
\text { a }\end{array}$ & $\begin{array}{l}\text { Yes } \\
\text { C }\end{array}$ & $\begin{array}{c}\text { No } \\
\mathbf{a}\end{array}$ & $\begin{array}{l}\text { Yes } \\
\text { C }\end{array}$ & $\begin{array}{l}\text { Yes } \\
\text { C }\end{array}$ & $\begin{array}{c}\text { No } \\
\mathrm{a}\end{array}$ & $\begin{array}{l}\text { Yes } \\
\text { C }\end{array}$ & $\begin{array}{c}\text { Yes } \\
\text { C }\end{array}$ & $\begin{array}{l}\text { Yes } \\
\text { C }\end{array}$ & $\begin{array}{l}\text { Yes } \\
\text { C }\end{array}$ & $\begin{array}{c}\text { Yes } \\
\text { C }\end{array}$ & $\begin{array}{l}\text { Yes } \\
\text { C }\end{array}$ & $\begin{array}{c}N / A \\
b\end{array}$ & $\begin{array}{c}\text { N/A } \\
\text { b }\end{array}$ \\
\hline Surrey & $\begin{array}{c}\text { No } \\
a\end{array}$ & $\begin{array}{c}\text { No } \\
a\end{array}$ & $\begin{array}{c}\text { No } \\
a\end{array}$ & $\begin{array}{l}\text { Yes } \\
\text { C }\end{array}$ & $\begin{array}{l}\text { Yes } \\
\text { B }\end{array}$ & $\begin{array}{c}\text { No } \\
a \text {. }\end{array}$ & $\begin{array}{c}\text { No } \\
a\end{array}$ & $\begin{array}{l}\text { Yes } \\
\text { C }\end{array}$ & $\begin{array}{c}\text { No } \\
a\end{array}$ & $\begin{array}{c}\text { No } \\
a\end{array}$ & $\begin{array}{l}\text { Yes } \\
\text { A }\end{array}$ & $\begin{array}{c}\text { No } \\
a\end{array}$ & $\begin{array}{l}\text { Yes } \\
\text { C }\end{array}$ & $\begin{array}{l}\text { Yes } \\
\text { A }\end{array}$ & $\begin{array}{c}\text { No } \\
a\end{array}$ & $\begin{array}{c}\text { No } \\
a\end{array}$ & $\begin{array}{c}\text { Yes } \\
\text { A }\end{array}$ & $\begin{array}{l}\text { Yes } \\
\text { A }\end{array}$ & $\begin{array}{c}\text { Yes? } \\
\text { C }\end{array}$ & $\begin{array}{c}\text { Yes } \\
\text { C }\end{array}$ & $\begin{array}{l}\text { Yes } \\
\text { C }\end{array}$ & $\begin{array}{c}\text { N/A } \\
b\end{array}$ & $\begin{array}{c}\text { No } \\
a\end{array}$ \\
\hline $\begin{array}{c}\text { Turkey } \\
\text { Point B17 }\end{array}$ & $\begin{array}{c}\text { No } \\
\text { a }\end{array}$ & $\begin{array}{c}\text { No } \\
\text { a }\end{array}$ & $\begin{array}{c}\text { No } \\
\text { a }\end{array}$ & $\begin{array}{l}\text { Yes } \\
\text { C }\end{array}$ & $\begin{array}{c}\text { Yes } \\
\text { A }\end{array}$ & $\begin{array}{c}\text { No } \\
\text { a }\end{array}$ & $\begin{array}{c}\text { No } \\
a\end{array}$ & $\begin{array}{l}\text { Yes } \\
\text { C }\end{array}$ & $\begin{array}{c}\text { No } \\
\text { a }\end{array}$ & $\begin{array}{c}\text { No } \\
\text { a }\end{array}$ & $\begin{array}{l}\text { Yes } \\
\text { A }\end{array}$ & $\begin{array}{c}\text { No } \\
\text { a }\end{array}$ & $\begin{array}{l}\text { Yes } \\
\text { C }\end{array}$ & $\begin{array}{l}\text { Yes } \\
\text { A }\end{array}$ & $\begin{array}{c}\text { No } \\
\text { a }\end{array}$ & $\begin{array}{c}\text { No } \\
\mathrm{a}\end{array}$ & $\begin{array}{c}\text { Yes } \\
\text { A }\end{array}$ & $\begin{array}{c}\text { Yes } \\
\text { A }\end{array}$ & $\begin{array}{c}\text { Yes? } \\
\text { C }\end{array}$ & $\begin{array}{c}\text { Yes } \\
\text { C }\end{array}$ & $\begin{array}{l}\text { Yes } \\
\text { C }\end{array}$ & $\begin{array}{c}\mathrm{N} / \mathrm{A} \\
\mathrm{b}\end{array}$ & $\begin{array}{c}\text { No } \\
\text { a }\end{array}$ \\
\hline $\begin{array}{l}\text { Turkey } \\
\text { Point } \\
\text { EMAD }\end{array}$ & $\begin{array}{c}\text { No } \\
\text { a }\end{array}$ & $\begin{array}{c}\text { No } \\
\text { a }\end{array}$ & $\begin{array}{c}\text { No } \\
\text { a }\end{array}$ & $\begin{array}{c}\text { Yes } \\
\text { C }\end{array}$ & $\begin{array}{c}\text { Yes } \\
\text { A }\end{array}$ & $\begin{array}{c}\text { No } \\
\text { a }\end{array}$ & $\begin{array}{c}\text { No } \\
\text { a }\end{array}$ & $\begin{array}{c}\text { Yes } \\
\text { C }\end{array}$ & $\begin{array}{c}\text { No } \\
\text { a }\end{array}$ & $\begin{array}{c}\text { No } \\
\text { a }\end{array}$ & $\begin{array}{c}\text { Yes } \\
\text { A }\end{array}$ & $\begin{array}{c}\text { No } \\
\text { a }\end{array}$ & $\begin{array}{l}\text { Yes } \\
\text { C }\end{array}$ & $\begin{array}{c}\text { Yes } \\
\text { A }\end{array}$ & $\begin{array}{c}\text { No } \\
\text { a }\end{array}$ & $\begin{array}{c}\text { No } \\
\text { a }\end{array}$ & $\begin{array}{c}\text { Yes } \\
\text { A }\end{array}$ & $\begin{array}{c}\text { Yes } \\
\text { A }\end{array}$ & $\begin{array}{c}\text { Yes? } \\
\text { C }\end{array}$ & $\begin{array}{c}\text { Yes } \\
\text { C }\end{array}$ & $\begin{array}{l}\text { Yes } \\
\text { C }\end{array}$ & $\begin{array}{c}N / A \\
b\end{array}$ & $\begin{array}{c}\text { No } \\
\text { a }\end{array}$ \\
\hline BMI & $\left|\begin{array}{c}N / A \\
b / c\end{array}\right|$ & $\begin{array}{c}\text { No } \\
\mathrm{a}\end{array}$ & $\begin{array}{l}\text { Yes } \\
\text { A }\end{array}$ & $\begin{array}{l}\text { N/A } \\
b / c\end{array}$ & $\begin{array}{l}N / A \\
b / c\end{array}$ & $\begin{array}{l}\text { N/A } \\
b / c\end{array}$ & $\mid \begin{array}{l}N / A \\
b / c\end{array}$ & $\begin{array}{l}\mathrm{N} / \mathrm{A} \\
\mathrm{b} / \mathrm{c}\end{array}$ & $\begin{array}{l}\text { N/A } \\
\text { b/c }\end{array}$ & $\begin{array}{c}\text { No } \\
\text { a }\end{array}$ & $\begin{array}{l}\mathrm{N} / \mathrm{A} \\
\mathrm{b} / \mathrm{c}\end{array}$ & $\begin{array}{l}\mathrm{N} / \mathrm{A} \\
\mathrm{b} / \mathrm{c}\end{array}$ & $\begin{array}{l}\mathrm{N} / \mathrm{A} \\
\mathrm{b} / \mathrm{c}\end{array}$ & $\begin{array}{l}\mathrm{N} / \mathrm{A} \\
\mathrm{b} / \mathrm{c}\end{array}$ & $\begin{array}{c}\text { No } \\
a\end{array}$ & $\begin{array}{l}\mathrm{N} / \mathrm{A} \\
\mathrm{b} / \mathrm{c}\end{array}$ & $\left|\begin{array}{c}N / A \\
b / c\end{array}\right|$ & $\begin{array}{l}\text { N/A } \\
\mathbf{b} / \mathrm{c}\end{array}$ & $\begin{array}{l}\mathrm{N} / \mathrm{A} \\
\mathrm{b} / \mathrm{c}\end{array}$ & $\begin{array}{c}N / A \\
b / c\end{array}$ & $\begin{array}{l}\mathrm{N} / \mathrm{A} \\
\mathrm{b} / \mathrm{c}\end{array}$ & $\begin{array}{l}\mathrm{N} / \mathrm{A} \\
\mathrm{b} / \mathrm{c}\end{array}$ & $\begin{array}{l}N / A \\
b / c\end{array}$ \\
\hline $\begin{array}{l}\text { R. E. } \\
\text { Gina }\end{array}$ & $\begin{array}{c}\text { No } \\
\text { a }\end{array}$ & $\begin{array}{c}\text { No } \\
\text { a }\end{array}$ & $\begin{array}{c}\text { N/A } \\
c\end{array}$ & $\begin{array}{l}\text { Yes } \\
\text { C }\end{array}$ & $\begin{array}{c}\text { Yes } \\
\text { A }\end{array}$ & $\begin{array}{l}N / A \\
b / c\end{array}$ & $\mid \begin{array}{c}\text { Yes } \\
\text { C }\end{array}$ & $\begin{array}{l}\text { Yes } \\
\text { C }\end{array}$ & $\begin{array}{l}\text { Yes } \\
\text { C }\end{array}$ & $\begin{array}{c}\text { No } \\
\text { a }\end{array}$ & $\begin{array}{l}\text { Yes } \\
\text { C }\end{array}$ & $\begin{array}{l}\text { Yes } \\
\text { C }\end{array}$ & $\begin{array}{c}\text { Yes } \\
\text { C }\end{array}$ & $\begin{array}{c}\text { Yes } \\
\text { C }\end{array}$ & $\begin{array}{c}\text { No } \\
\text { a }\end{array}$ & $\begin{array}{c}\text { N/A } \\
b\end{array}$ & $\begin{array}{c}\text { Yes } \\
\text { A }\end{array}$ & $\begin{array}{c}\text { Yes } \\
\text { A }\end{array}$ & $\begin{array}{c}\text { Yes } \\
\text { C }\end{array}$ & $\begin{array}{c}\text { Yes } \\
\text { C }\end{array}$ & $\begin{array}{c}\text { Yes } \\
\text { A }\end{array}$ & $\begin{array}{l}N / A \\
b / c\end{array}$ & $\begin{array}{l}N / A \\
b / c\end{array}$ \\
\hline TMI-2 & $\begin{array}{c}\text { No } \\
\mathrm{a}\end{array}$ & $\begin{array}{c}\text { No } \\
a\end{array}$ & $\begin{array}{c}\text { No } \\
\mathrm{a}\end{array}$ & $\begin{array}{c}\text { No } \\
\mathrm{a}\end{array}$ & $\begin{array}{c}\text { No } \\
\text { a }\end{array}$ & $\begin{array}{c}\text { No } \\
\text { a }\end{array}$ & $\begin{array}{c}\text { No } \\
a\end{array} \mid$ & $\begin{array}{c}\text { No } \\
\mathrm{a}\end{array}$ & $\begin{array}{c}\text { No } \\
\text { a }\end{array}$ & $\begin{array}{c}\text { No } \\
\mathrm{a}\end{array}$ & $\begin{array}{c}\text { No } \\
\text { a }\end{array}$ & $\begin{array}{c}\text { No } \\
\text { a }\end{array}$ & $\begin{array}{c}\text { No } \\
\mathbf{a}\end{array}$ & $\begin{array}{c}\text { No } \\
\text { a }\end{array}$ & $\begin{array}{c}\text { No } \\
\text { a }\end{array}$ & $\begin{array}{c}\text { N/A } \\
b\end{array}$ & \begin{tabular}{|c} 
Yes \\
B
\end{tabular} & $\begin{array}{c}\text { Yes } \\
\text { B }\end{array}$ & $\begin{array}{c}\text { Yes } \\
\text { A }\end{array}$ & $\begin{array}{c}\text { Yes } \\
\text { C }\end{array}$ & $\begin{array}{c}\text { Yes } \\
\text { B }\end{array}$ & $\begin{array}{c}\mathrm{N} / \mathrm{A} \\
\mathrm{b}\end{array}$ & $\begin{array}{c}\text { No } \\
\mathrm{a}\end{array}$ \\
\hline $\begin{array}{c}\text { Arkansas } \\
\text { ANO-1 }\end{array}$ & $\begin{array}{c}\mathrm{N} / \mathrm{A} \\
\mathrm{a}\end{array} \mid$ & $\begin{array}{c}\mathrm{N} / \mathrm{A} \\
\mathrm{a}\end{array}$ & $\begin{array}{c}\text { N/A } \\
a\end{array}$ & $\begin{array}{c}\text { N/A } \\
\text { a }\end{array}$ & $\begin{array}{c}\text { N/A } \\
\text { a }\end{array}$ & $\begin{array}{c}N / A \\
a\end{array}$ & $\begin{array}{c}\mathrm{N} / \mathrm{A} \\
\mathrm{a}\end{array}$ & $\begin{array}{c}\text { N/A } \\
\mathrm{a}\end{array}$ & $\begin{array}{c}\text { N/A } \\
\text { a }\end{array}$ & $\begin{array}{c}\mathrm{N} / \mathrm{A} \\
\mathbf{a}\end{array}$ & $\begin{array}{c}\mathrm{N} / \mathrm{A} \\
\mathbf{a}\end{array}$ & $\begin{array}{c}\text { N/A } \\
a\end{array}$ & $\begin{array}{c}\mathrm{N} / \mathrm{A} \\
\mathbf{a}\end{array}$ & $\begin{array}{c}\text { N/A } \\
a\end{array}$ & $\begin{array}{c}\text { N/A } \\
a\end{array}$ & $\begin{array}{c}\text { N/A } \\
\mathbf{a}\end{array}$ & $\begin{array}{c}\mathrm{N} / \mathrm{A} \\
\mathrm{a}\end{array} \mid$ & $\begin{array}{c}\text { N/A } \\
a\end{array}$ & $\begin{array}{c}\text { N/A } \\
a\end{array}$ & $\begin{array}{c}\mathrm{N} / \mathrm{A} \\
\mathrm{a}\end{array}$ & $\begin{array}{c}\text { N/A } \\
a\end{array}$ & $\begin{array}{c}\mathrm{N} / \mathrm{A} \\
\mathrm{a}\end{array}$ & $\begin{array}{c}\text { N/A } \\
a\end{array}$ \\
\hline Oconee & $\begin{array}{c}\mathrm{N} / \mathrm{A} \\
\mathrm{a}\end{array}$ & $\begin{array}{c}\mathrm{N} / \mathrm{A} \\
\mathrm{a}\end{array}$ & $\begin{array}{c}\text { N/A } \\
a\end{array}$ & $\begin{array}{c}\text { N/A } \\
\text { a }\end{array}$ & $\begin{array}{c}\text { N/A } \\
\text { a }\end{array}$ & $\begin{array}{c}N / A \\
a\end{array}$ & $\begin{array}{c}N / A \\
a\end{array}$ & $\begin{array}{c}\text { N/A } \\
\text { a }\end{array}$ & $\begin{array}{c}\text { N/A } \\
\text { a }\end{array}$ & $\begin{array}{c}\text { N/A } \\
\text { a }\end{array}$ & $\begin{array}{c}N / A \\
a\end{array}$ & $\begin{array}{c}N / A \\
a\end{array}$ & $\begin{array}{c}\text { N/A } \\
a\end{array}$ & $\underset{a}{\text { N/A }}$ & $\begin{array}{c}\text { N/A } \\
a\end{array}$ & $\begin{array}{c}\text { N/A } \\
\text { a }\end{array}$ & $\left|\begin{array}{c}\mathrm{N} / \mathrm{A} \\
\mathrm{a}\end{array}\right|$ & $\begin{array}{c}\text { N/A } \\
a\end{array}$ & $\begin{array}{c}\text { N/A } \\
a\end{array}$ & $\begin{array}{c}\text { N/A } \\
a\end{array}$ & $\begin{array}{c}\text { N/A } \\
a\end{array}$ & $\begin{array}{c}\text { N/A } \\
a\end{array}$ & $\begin{array}{c}\mathrm{N} / \mathrm{A} \\
\mathrm{a}\end{array}$ \\
\hline
\end{tabular}


Table 4-2. Packaging compatibility with boiling water reactor type SNF.

\begin{tabular}{|c|c|c|c|c|c|c|c|c|c|c|c|c|c|c|c|c|c|c|c|c|c|c|c|}
\hline \multirow[b]{2}{*}{ BWR Fuels } & \multicolumn{13}{|c|}{ Legal weight truck packages } & \multicolumn{2}{|c|}{$\begin{array}{c}\text { Overweight } \\
\text { truck packages }\end{array}$} & \multicolumn{8}{|c|}{ Rail packages } \\
\hline & $\begin{array}{l}\mathrm{GE} \\
\mathrm{T}-2\end{array}$ & $\begin{array}{l}\text { GE- } \\
100\end{array}$ & BMI-1 & FSV-1 & $\begin{array}{c}\text { NLI- } \\
1 / 2\end{array}$ & $\mid \begin{array}{l}\text { NLI- } \\
6502\end{array}$ & $\mathrm{~T}-3$ & NAC-1 & $\begin{array}{c}\text { CNS } \\
1-13 G\end{array}$ & NRBK-41 & $\begin{array}{l}\text { NAC- } \\
\text { LWT }\end{array}$ & $\begin{array}{c}\text { GE Model } \\
2000\end{array}$ & $\begin{array}{l}\text { TN- } \\
\text { FSV }\end{array}$ & $\begin{array}{l}\text { TN-8 } \\
\text { TN-8L }\end{array}$ & TN-9 & M-130 & $\begin{array}{l}\text { IF- } \\
300\end{array}$ & $\begin{array}{l}\text { NLI- } \\
10 / 24\end{array}$ & $125 \cdot B$ & $\begin{array}{l}\mathrm{TN}- \\
\mathrm{BRP}\end{array}$ & $\begin{array}{l}\text { TN- } \\
\text { REG }\end{array}$ & M-160 & M-140 \\
\hline EBWR & $\begin{array}{c}\text { Yes } \\
\text { C }\end{array}$ & $\begin{array}{c}\text { No } \\
\text { a }\end{array}$ & $\begin{array}{c}\text { No } \\
\text { a }\end{array}$ & $\begin{array}{c}\text { Yes } \\
\text { C }\end{array}$ & $\begin{array}{c}\text { Yes } \\
\text { C }\end{array}$ & $\begin{array}{c}\text { Yes? } \\
\mathrm{C}\end{array}$ & $\begin{array}{c}\text { Yes } \\
\text { C }\end{array}$ & $\begin{array}{c}\text { Yes } \\
\text { B }\end{array}$ & $\begin{array}{c}\text { No } \\
\text { a }\end{array}$ & $\begin{array}{c}\text { No } \\
\text { a }\end{array}$ & $\begin{array}{c}\text { Yes } \\
\text { C }\end{array}$ & $\begin{array}{l}\text { Yes } \\
\text { C }\end{array}$ & $\begin{array}{c}\text { Yes } \\
\mathrm{C}\end{array}$ & $\begin{array}{c}\text { Yes } \\
\text { C }\end{array}$ & $\begin{array}{c}\text { Yes } \\
\mathrm{C}\end{array}$ & $\begin{array}{c}\text { N/A } \\
\mathbf{b}\end{array}$ & $\begin{array}{c}\text { Yes } \\
\text { C }\end{array}$ & $\begin{array}{c}\text { Yes } \\
\text { C }\end{array}$ & $\begin{array}{c}\text { Yes } \\
\text { C }\end{array}$ & $\begin{array}{c}\text { Yes } \\
\text { C }\end{array}$ & $\begin{array}{c}\text { Yes } \\
\text { C }\end{array}$ & $\begin{array}{c}\text { N/A } \\
\text { b }\end{array}$ & $\begin{array}{c}\text { N/A } \\
\mathbf{b}\end{array}$ \\
\hline $\begin{array}{l}\text { Dresden } \\
\text { (Ass'ys) }\end{array}$ & No & $\begin{array}{c}\text { No } \\
\text { a }\end{array}$ & $\begin{array}{c}\text { No } \\
\text { a }\end{array}$ & $\begin{array}{c}\text { N/A } \\
\text { b }\end{array}$ & $\begin{array}{c}\text { N/A } \\
b\end{array}$ & $\begin{array}{c}\text { No } \\
\text { a }\end{array}$ & $\begin{array}{c}\text { No } \\
\text { a }\end{array}$ & $\begin{array}{c}\text { N/A } \\
b\end{array}$ & $\begin{array}{c}\text { No } \\
\text { a }\end{array}$ & $\begin{array}{c}\text { No } \\
\text { a }\end{array}$ & $\begin{array}{c}\text { N/A } \\
b\end{array}$ & $\begin{array}{c}\text { No } \\
\text { a }\end{array}$ & $\begin{array}{c}\text { N/A } \\
b\end{array}$ & $\begin{array}{c}\text { No } \\
\text { a }\end{array}$ & $\begin{array}{c}\text { N/A } \\
\text { b }\end{array}$ & $\begin{array}{c}\text { No } \\
\text { a }\end{array}$ & $\begin{array}{c}\text { N/A } \\
\text { b }\end{array}$ & $\begin{array}{c}\text { N/A } \\
\text { b }\end{array}$ & $\begin{array}{c}\text { N/A } \\
\text { b }\end{array}$ & $\begin{array}{c}\text { No } \\
\text { a }\end{array}$ & $\begin{array}{c}\text { No } \\
\text { a }\end{array}$ & $\begin{array}{c}\text { No } \\
\text { a }\end{array}$ & $\begin{array}{c}\text { No } \\
\text { a }\end{array}$ \\
\hline $\begin{array}{c}\text { Peach } \\
\text { Bottom } 1 \\
\text { (elements) }\end{array}$ & $\begin{array}{c}\mathbf{N} / \mathbf{A} \\
\mathbf{a}\end{array}$ & $\begin{array}{c}\text { N/A } \\
\text { a }\end{array}$ & $\begin{array}{c}\text { N/A } \\
\text { a }\end{array}$ & $\begin{array}{c}\text { N/A } \\
\text { a }\end{array}$ & $\begin{array}{c}\text { N/A } \\
a\end{array}$ & $\begin{array}{c}\mathrm{N} / \mathrm{A} \\
\mathrm{a}\end{array}$ & $\begin{array}{c}\text { N/A } \\
\mathbf{a}\end{array}$ & $\begin{array}{c}\text { N/A } \\
\mathbf{a}\end{array}$ & $\begin{array}{c}\text { N/A } \\
\text { a }\end{array}$ & $\begin{array}{c}\text { N/A } \\
\mathbf{a}\end{array}$ & $\begin{array}{c}\text { N/A } \\
\mathbf{a}\end{array}$ & $\begin{array}{c}\text { N/A } \\
\text { a }\end{array}$ & $\begin{array}{c}\text { N/A } \\
a\end{array}$ & $\begin{array}{c}\text { N/A } \\
a\end{array}$ & $\begin{array}{c}\text { N/A } \\
\text { a }\end{array}$ & $\begin{array}{c}\text { N/A } \\
\mathbf{a}\end{array}$ & $\begin{array}{c}\text { N/A } \\
\mathbf{a}\end{array}$ & $\begin{array}{c}\text { N/A } \\
\mathbf{a}\end{array}$ & $\begin{array}{c}\text { N/A } \\
\mathbf{a}\end{array}$ & $\begin{array}{c}\text { N/A } \\
a\end{array}$ & $\begin{array}{c}\text { N/A } \\
\mathbf{a}\end{array}$ & $\begin{array}{c}\text { N/A } \\
\text { a }\end{array}$ & $\begin{array}{c}\text { N/A } \\
a\end{array}$ \\
\hline $\begin{array}{c}\text { Peach } \\
\text { Bottom } 2 \\
\text { (partial } \\
\text { ass'ys) }\end{array}$ & $\begin{array}{c}\text { No } \\
\text { a }\end{array}$ & $\begin{array}{c}\text { No } \\
\text { a }\end{array}$ & $\begin{array}{c}\text { No } \\
\text { a }\end{array}$ & $\begin{array}{c}\text { Yes } \\
\text { C }\end{array}$ & $\begin{array}{c}\text { Yes } \\
\text { A }\end{array}$ & $\begin{array}{c}\text { No } \\
\text { a }\end{array}$ & $\begin{array}{c}\text { No } \\
\text { a }\end{array}$ & $\begin{array}{l}\text { Yes } \\
\text { C }\end{array}$ & $\begin{array}{c}\text { No } \\
\text { a }\end{array}$ & $\begin{array}{c}\text { No } \\
\text { a }\end{array}$ & $\begin{array}{c}\text { Yes } \\
\text { B }\end{array}$ & $\begin{array}{c}\text { No } \\
\text { a }\end{array}$ & $\begin{array}{c}\text { Yes } \\
\text { C }\end{array}$ & $\begin{array}{c}\text { No } \\
\text { a }\end{array}$ & $\begin{array}{c}\text { Yes } \\
\text { B }\end{array}$ & $\begin{array}{c}\text { No } \\
\text { a }\end{array}$ & $\begin{array}{c}\text { Yes } \\
\text { B }\end{array}$ & $\begin{array}{c}\text { Yes } \\
\text { B }\end{array}$ & $\begin{array}{c}\text { Yes } \\
\text { C }\end{array}$ & $\begin{array}{c}\text { No } \\
\text { a }\end{array}$ & $\begin{array}{c}\text { No } \\
\text { a }\end{array}$ & $\begin{array}{c}\text { No } \\
\text { a }\end{array}$ & $\begin{array}{c}\text { No } \\
\text { a }\end{array}$ \\
\hline $\begin{array}{c}\text { Peach } \\
\text { Bottom } \\
\text { (rods in } \\
\text { canisters) }\end{array}$ & $\begin{array}{c}\text { No } \\
\text { a }\end{array}$ & $\begin{array}{c}\text { No } \\
\text { a }\end{array}$ & $\begin{array}{c}\text { No } \\
\text { a }\end{array}$ & $\begin{array}{c}\text { No } \\
\text { a }\end{array}$ & $\begin{array}{c}\text { No } \\
\text { a }\end{array}$ & $\begin{array}{c}\text { No } \\
\text { a }\end{array}$ & $\begin{array}{c}\text { No } \\
\text { a }\end{array}$ & $\begin{array}{c}\text { No } \\
\text { a }\end{array}$ & $\begin{array}{c}\text { No } \\
\text { a }\end{array}$ & $\begin{array}{c}\text { No } \\
\text { a }\end{array}$ & $\begin{array}{c}\text { No } \\
\text { a }\end{array}$ & $\begin{array}{c}\text { No } \\
\text { a }\end{array}$ & $\begin{array}{c}\text { No } \\
\text { a }\end{array}$ & $\begin{array}{c}\text { No } \\
\text { a }\end{array}$ & $\begin{array}{c}\text { No } \\
\text { a }\end{array}$ & $\begin{array}{c}\text { No } \\
\text { a }\end{array}$ & $\begin{array}{c}\text { No } \\
\text { a }\end{array}$ & $\begin{array}{c}\text { No } \\
\text { a }\end{array}$ & $\begin{array}{c}\text { No } \\
\text { a }\end{array}$ & $\begin{array}{c}\text { No } \\
\text { a }\end{array}$ & $\begin{array}{c}\text { No } \\
\text { a }\end{array}$ & $\begin{array}{c}\text { No } \\
\text { a }\end{array}$ & $\begin{array}{c}\text { No } \\
\text { a }\end{array}$ \\
\hline Pathfinder & $\begin{array}{c}\text { N/A } \\
b\end{array}$ & $\begin{array}{c}\text { No } \\
\text { a }\end{array}$ & $\begin{array}{c}\text { No } \\
\text { a }\end{array}$ & $\begin{array}{c}\text { Yes } \\
\mathrm{c}\end{array}$ & $\begin{array}{c}\text { Yes } \\
\text { C }\end{array}$ & $\begin{array}{c}\text { Yes } \\
\text { C }\end{array}$ & $\begin{array}{c}\text { Yes } \\
\text { C }\end{array}$ & $\begin{array}{c}\text { Yes } \\
\text { C }\end{array}$ & $\begin{array}{c}\text { No } \\
\text { a }\end{array}$ & $\begin{array}{c}\text { No } \\
\text { a }\end{array}$ & $\begin{array}{c}\text { Yes } \\
\text { C }\end{array}$ & $\begin{array}{c}\text { No } \\
\text { a }\end{array}$ & $\begin{array}{c}\text { Yes } \\
\text { C }\end{array}$ & $\begin{array}{c}\text { Yes } \\
\text { C }\end{array}$ & $\begin{array}{c}\text { Yes } \\
\text { C }\end{array}$ & $\begin{array}{c}\text { N/A } \\
b\end{array}$ & $\begin{array}{c}\text { Yes } \\
\text { C }\end{array}$ & $\begin{array}{c}\text { Yes } \\
\text { C }\end{array}$ & $\begin{array}{c}\text { Yes } \\
\text { C }\end{array}$ & $\begin{array}{c}\text { Yes } \\
\text { C }\end{array}$ & $\begin{array}{c}\text { Yes } \\
\text { C }\end{array}$ & $\begin{array}{c}\text { N/A } \\
b\end{array}$ & $\begin{array}{c}\text { N/A } \\
\text { b }\end{array}$ \\
\hline $\begin{array}{c}\text { SPSS- } \\
\text { SPERT }\end{array}$ & $\begin{array}{c}\text { Yes } \\
\text { C }\end{array}$ & $\begin{array}{c}\text { No } \\
\text { a }\end{array}$ & $\begin{array}{c}\text { Yes } \\
\text { A }\end{array}$ & $\begin{array}{c}\text { Yes } \\
\text { B }\end{array}$ & $\begin{array}{c}\text { Yes } \\
\text { C }\end{array}$ & $\begin{array}{c}\text { Yes } \\
\text { C }\end{array}$ & $\begin{array}{c}\text { Yes } \\
\text { C }\end{array}$ & $\begin{array}{c}\text { Yes } \\
\text { C }\end{array}$ & $\begin{array}{c}\text { Yes } \\
\text { C }\end{array}$ & $\begin{array}{c}\text { No } \\
\text { a }\end{array}$ & $\begin{array}{c}\text { Yes } \\
\text { C }\end{array}$ & $\begin{array}{c}\text { Yes } \\
\text { C }\end{array}$ & $\begin{array}{c}\text { Yes } \\
\text { B }\end{array}$ & $\begin{array}{c}\text { Yes } \\
\text { C }\end{array}$ & $\begin{array}{c}\text { Yes } \\
\text { C }\end{array}$ & $\begin{array}{c}\text { N/A } \\
\text { b }\end{array}$ & $\begin{array}{c}\text { Yes } \\
\text { C }\end{array}$ & $\begin{array}{c}\text { Yes } \\
\text { C }\end{array}$ & $\begin{array}{c}\text { Yes } \\
\text { C }\end{array}$ & $\begin{array}{c}\text { Yes } \\
\text { C }\end{array}$ & $\begin{array}{c}\text { Yes } \\
\text { C }\end{array}$ & $\begin{array}{c}\text { N/A } \\
b\end{array}$ & $\begin{array}{c}\text { N/A } \\
\text { b }\end{array}$ \\
\hline BORAX V & $\begin{array}{c}N / A \\
b\end{array}$ & $\begin{array}{c}\text { N/A } \\
b\end{array}$ & $\begin{array}{c}\text { N/A } \\
\text { b }\end{array}$ & $\begin{array}{c}\text { N/A } \\
b\end{array}$ & $\begin{array}{c}\text { N/A } \\
b\end{array}$ & $\begin{array}{c}\text { N/A } \\
b\end{array}$ & $\begin{array}{c}\text { N/A } \\
b\end{array}$ & $\begin{array}{c}\text { N/A } \\
\text { b }\end{array}$ & $\begin{array}{c}N / A \\
b\end{array}$ & $\begin{array}{c}\text { N/A } \\
b\end{array}$ & $\begin{array}{c}\text { N/A } \\
\mathbf{b}\end{array}$ & $\begin{array}{c}\text { N/A } \\
\mathbf{b}\end{array}$ & $\begin{array}{c}\text { N/A } \\
\text { b }\end{array}$ & $\begin{array}{c}\text { N/A } \\
b\end{array}$ & $\begin{array}{c}\text { N/A } \\
b\end{array}$ & $\begin{array}{c}\text { N/A } \\
b\end{array}$ & $\begin{array}{c}\text { N/A } \\
\mathbf{b}\end{array}$ & $\begin{array}{c}\text { N/A } \\
b\end{array}$ & $\begin{array}{c}\text { N/A } \\
\mathbf{b}\end{array}$ & $\begin{array}{c}\text { N/A } \\
\text { b }\end{array}$ & $\begin{array}{c}\text { N/A } \\
b\end{array}$ & $\begin{array}{c}\text { N/A } \\
b\end{array}$ & $\begin{array}{c}\text { N/A } \\
b\end{array}$ \\
\hline Cooper & $\begin{array}{c}\text { N/A } \\
b\end{array}$ & $\begin{array}{c}\text { N/A } \\
b\end{array}$ & $\begin{array}{c}\text { N/A } \\
\text { b }\end{array}$ & $\begin{array}{c}\text { N/A } \\
\text { b }\end{array}$ & $\begin{array}{c}\text { N/A } \\
b\end{array}$ & $\begin{array}{c}\text { N/A } \\
b\end{array}$ & $\begin{array}{c}\text { N/A } \\
b\end{array}$ & $\begin{array}{c}\text { N/A } \\
b\end{array}$ & $\begin{array}{c}\text { N/A } \\
\text { b }\end{array}$ & $\begin{array}{c}\text { N/A } \\
\text { b }\end{array}$ & $\begin{array}{c}\mathbf{N} / \mathbf{A} \\
\mathbf{b}\end{array}$ & $\begin{array}{c}\text { N/A } \\
\text { b }\end{array}$ & $\begin{array}{c}\text { N/A } \\
b\end{array}$ & $\begin{array}{c}\text { N/A } \\
\text { b }\end{array}$ & $\begin{array}{c}\text { N/A } \\
b\end{array}$ & $\begin{array}{c}\text { N/A } \\
b\end{array}$ & $\begin{array}{c}\mathbf{N} / \mathbf{A} \\
\mathbf{b}\end{array}$ & $\begin{array}{c}\mathbf{N} / \mathbf{A} \\
\mathbf{b}\end{array}$ & $\begin{array}{c}\text { N/A } \\
\mathbf{b}\end{array}$ & $\begin{array}{c}\text { N/A } \\
\text { b }\end{array}$ & $\begin{array}{c}\text { N/A } \\
b\end{array}$ & $\begin{array}{c}\text { N/A } \\
b\end{array}$ & $\underset{b}{N / A}$ \\
\hline $\begin{array}{c}\text { Vallecitos } \\
\text { (SRS) }\end{array}$ & $\begin{array}{c}\text { No } \\
\text { a }\end{array}$ & $\begin{array}{c}\text { No } \\
\text { a }\end{array}$ & $\begin{array}{c}\text { Yes } \\
\text { B }\end{array}$ & $\begin{array}{c}\text { Yes } \\
\text { C }\end{array}$ & $\begin{array}{c}\text { Yes } \\
\text { C }\end{array}$ & $\begin{array}{c}\text { Yes } \\
\text { C }\end{array}$ & $\begin{array}{c}\text { Yes } \\
\text { C }\end{array}$ & $\begin{array}{c}\text { Yes } \\
\text { C }\end{array}$ & $\begin{array}{c}\text { N/A } \\
\text { b }\end{array}$ & $\begin{array}{c}\text { No } \\
\text { a }\end{array}$ & $\begin{array}{c}\text { Yes } \\
\text { C }\end{array}$ & $\begin{array}{c}\text { Yes } \\
\text { C }\end{array}$ & $\begin{array}{c}\text { Yes } \\
\mathrm{C}\end{array}$ & $\begin{array}{c}\text { Yes } \\
\text { C }\end{array}$ & $\begin{array}{c}\text { No } \\
\text { a }\end{array}$ & $\begin{array}{c}\text { N/A } \\
\text { b }\end{array}$ & $\begin{array}{c}\text { Yes } \\
\text { C }\end{array}$ & $\begin{array}{c}\text { Yes } \\
\text { C }\end{array}$ & $\begin{array}{c}\text { Yes } \\
\text { C }\end{array}$ & $\begin{array}{c}\text { Yes } \\
\text { C }\end{array}$ & $\begin{array}{c}\text { Yes } \\
\text { C }\end{array}$ & $\begin{array}{c}\text { N/A } \\
\text { b }\end{array}$ & $\begin{array}{c}\text { N/A } \\
\text { b }\end{array}$ \\
\hline $\begin{array}{l}\text { Vallecitos } \\
\text { (INEL) }\end{array}$ & $\begin{array}{c}\text { No } \\
\text { a }\end{array}$ & $\begin{array}{c}\text { No } \\
\text { a }\end{array}$ & $\begin{array}{c}\text { Yes } \\
\text { B }\end{array}$ & $\begin{array}{c}\text { Yes } \\
\text { C }\end{array}$ & $\begin{array}{c}\text { Yes } \\
\mathrm{C}\end{array}$ & $\begin{array}{c}\text { Yes } \\
\text { C }\end{array}$ & $\begin{array}{c}\text { Yes } \\
\text { C }\end{array}$ & $\begin{array}{c}\text { Yes } \\
\text { C }\end{array}$ & $\begin{array}{c}\text { N/A } \\
b\end{array}$ & $\begin{array}{c}\text { No } \\
\text { a }\end{array}$ & $\begin{array}{c}\text { Yes } \\
\text { C }\end{array}$ & $\begin{array}{c}\text { Yes } \\
\text { C }\end{array}$ & $\begin{array}{c}\text { Yes } \\
\text { C }\end{array}$ & $\begin{array}{c}\text { Yes } \\
\text { C }\end{array}$ & $\begin{array}{c}\text { No } \\
\text { a }\end{array}$ & $\begin{array}{c}\mathbf{N} / \mathbf{A} \\
\mathbf{b}\end{array}$ & $\begin{array}{c}\text { Yes } \\
\text { C }\end{array}$ & $\begin{array}{c}\text { Yes } \\
\text { C }\end{array}$ & $\begin{array}{c}\text { Yes } \\
\text { C }\end{array}$ & $\begin{array}{c}\text { Yes } \\
\text { C }\end{array}$ & $\begin{array}{c}\text { Yes } \\
\text { C }\end{array}$ & $\begin{array}{c}\text { N/A } \\
\text { b }\end{array}$ & $\begin{array}{c}\text { N/A } \\
\text { b }\end{array}$ \\
\hline
\end{tabular}


Table 4-3. Packaging compatibility with breeder reactor type SNF.

\begin{tabular}{|c|c|c|c|c|c|c|c|c|c|c|c|c|c|c|c|c|c|c|c|c|c|c|c|}
\hline \multirow[b]{2}{*}{$\begin{array}{c}\text { Breeder } \\
\text { reactor fuels }\end{array}$} & \multicolumn{13}{|c|}{ Legal weight truck packages } & \multicolumn{2}{|c|}{$\begin{array}{c}\text { Overweight } \\
\text { truck packages }\end{array}$} & \multicolumn{8}{|c|}{ Rail packages } \\
\hline & $\begin{array}{l}\text { GE } \\
\text { T-2 }\end{array}$ & $\begin{array}{l}\text { GE- } \\
100\end{array}$ & BMI-1 & FSV-1 & $\begin{array}{c}\text { NL.I- } \\
1 / 2\end{array}$ & $\begin{array}{l}\text { NLI- } \\
6502\end{array}$ & $\mathrm{~T}-3$ & NAC-1 & $\begin{array}{c}\mathrm{CNS} \\
1-13 \mathrm{G}\end{array}$ & NRBK-41 & $\begin{array}{l}\text { NAC- } \\
\text { LWT }\end{array}$ & $\begin{array}{c}\text { GE Model } \\
2000\end{array}$ & $\begin{array}{l}\text { TN- } \\
\text { FSV }\end{array}$ & $\begin{array}{l}\text { TN-8 } \\
\text { TN-8L }\end{array}$ & TN-9 & M-130 & $\begin{array}{l}\text { IF- } \\
300\end{array}$ & $\begin{array}{l}\text { NLI- } \\
10 / 24\end{array}$ & $125-\mathrm{B}$ & $\begin{array}{l}\text { TN- } \\
\text { BRP }\end{array}$ & $\begin{array}{c}\text { TN- } \\
\text { REG }\end{array}$ & $M-160$ & $M-140$ \\
\hline $\begin{array}{l}\text { FFTF-LM } \\
\text { (DA) }\end{array}$ & $\begin{array}{c}\text { No } \\
\text { a }\end{array}$ & $\begin{array}{c}\text { No } \\
\text { a }\end{array}$ & $\begin{array}{c}\text { No } \\
\text { a }\end{array}$ & $\begin{array}{c}\text { Yes } \\
\text { C }\end{array}$ & $\begin{array}{c}\text { No } \\
\text { b }\end{array}$ & No & $\begin{array}{c}\text { Yes } \\
\text { B }\end{array}$ & $\begin{array}{c}\text { No } \\
b\end{array}$ & $\begin{array}{c}\text { No } \\
\text { a }\end{array}$ & $\begin{array}{l}\text { No } \\
\text { a }\end{array}$ & $\begin{array}{c}\text { No } \\
\text { b }\end{array}$ & $\begin{array}{c}\text { No } \\
\text { a }\end{array}$ & $\begin{array}{c}\text { No } \\
\text { b }\end{array}$ & $\begin{array}{c}\text { No } \\
\text { b }\end{array}$ & $\begin{array}{l}\text { No } \\
b\end{array}$ & $\begin{array}{c}\text { No } \\
\text { a }\end{array}$ & $\begin{array}{c}\text { Yes } \\
\text { C }\end{array}$ & $\begin{array}{c}\text { No } \\
b\end{array}$ & $\begin{array}{c}\text { No } \\
\text { b }\end{array}$ & $\begin{array}{c}\text { No } \\
b\end{array}$ & $\begin{array}{c}\text { No } \\
\text { b }\end{array}$ & $\begin{array}{c}\text { N/A } \\
b\end{array}$ & $\begin{array}{c}N / A \\
b\end{array}$ \\
\hline $\begin{array}{c}\text { FFTF-LM } \\
\text { (TFA) }\end{array}$ & $\begin{array}{c}\text { No } \\
\text { a }\end{array}$ & $\begin{array}{c}\text { No } \\
\text { a }\end{array}$ & $\begin{array}{c}\text { No } \\
\text { a }\end{array}$ & $\begin{array}{c}\text { Yes } \\
\text { C }\end{array}$ & $\begin{array}{c}\text { No } \\
b\end{array}$ & $\begin{array}{c}\text { No } \\
\text { a }\end{array}$ & $\begin{array}{c}\text { Yes } \\
\text { A }\end{array}$ & $\begin{array}{c}\text { No } \\
\text { b }\end{array}$ & $\begin{array}{c}\text { No } \\
\text { a }\end{array}$ & $\begin{array}{c}\text { No } \\
\text { a }\end{array}$ & $\begin{array}{c}\text { No } \\
b\end{array}$ & $\begin{array}{c}\text { No } \\
\text { a }\end{array}$ & $\begin{array}{c}\text { No } \\
b\end{array}$ & $\begin{array}{l}\text { No } \\
b\end{array}$ & $\begin{array}{c}\text { No } \\
b\end{array}$ & $\begin{array}{c}\text { No } \\
\text { a }\end{array}$ & $\begin{array}{c}\text { Yes } \\
\text { C }\end{array}$ & $\begin{array}{c}\text { No } \\
\mathbf{b}\end{array}$ & $\begin{array}{c}\text { No } \\
b\end{array}$ & $\begin{array}{c}\text { No } \\
b\end{array}$ & $\begin{array}{c}\text { No } \\
\text { b }\end{array}$ & $\begin{array}{c}\text { N/A } \\
\mathbf{b}\end{array}$ & $\begin{array}{c}\text { N/A } \\
\text { b }\end{array}$ \\
\hline $\begin{array}{l}\text { Ship'port- } \\
\text { LW (INEL) }\end{array}$ & $\begin{array}{c}\mathrm{N} / \mathrm{A} \\
\mathrm{a}\end{array}$ & $\begin{array}{c}\text { N/A } \\
a\end{array}$ & $\begin{array}{c}\text { N/A } \\
a\end{array}$ & $\begin{array}{c}\text { N/A } \\
a\end{array}$ & $\begin{array}{c}\text { N/A } \\
\mathbf{a}\end{array}$ & $\begin{array}{c}\mathrm{N} / \mathrm{A} \\
\mathrm{a}\end{array}$ & $\begin{array}{c}\text { N/A } \\
\mathbf{a}\end{array}$ & $\begin{array}{c}\mathrm{N} / \mathrm{A} \\
\mathrm{a}\end{array}$ & $\begin{array}{c}\text { N/A } \\
\mathbf{a}\end{array}$ & $\begin{array}{c}\text { N/A } \\
a\end{array}$ & $\begin{array}{c}\text { N/A } \\
\mathbf{a}\end{array}$ & $\begin{array}{c}\text { N/A } \\
a\end{array}$ & $\begin{array}{c}\text { N/A } \\
\mathbf{a}\end{array}$ & $\begin{array}{c}\mathbf{N} / \mathbf{A} \\
\mathrm{a}\end{array}$ & $\begin{array}{c}\text { N/A } \\
a\end{array}$ & $\begin{array}{c}\mathrm{N} / \mathrm{A} \\
\mathbf{a}\end{array}$ & $\begin{array}{c}\text { N/A } \\
\mathbf{a}\end{array}$ & $\begin{array}{c}\text { N/A } \\
a\end{array}$ & $\begin{array}{c}\text { N/A } \\
\mathbf{a}\end{array}$ & $\begin{array}{c}\text { N/A } \\
\text { a }\end{array}$ & $\begin{array}{c}\text { N/A } \\
a\end{array}$ & $\begin{array}{c}\mathrm{N} / \mathrm{A} \\
\mathrm{a}\end{array}$ & $\begin{array}{c}\mathrm{N} / \mathrm{A} \\
\mathrm{a}\end{array}$ \\
\hline $\begin{array}{l}\text { Ship'port- } \\
\text { LW (ANL-E) }\end{array}$ & $\begin{array}{c}\text { N/A } \\
a\end{array}$ & $\begin{array}{c}\text { N/A } \\
a\end{array}$ & $\begin{array}{c}\text { N/A } \\
a\end{array}$ & $\begin{array}{c}\text { N/A } \\
a\end{array}$ & $\begin{array}{c}\text { N/A } \\
\mathbf{a}\end{array}$ & $\begin{array}{c}\text { N/A } \\
a\end{array}$ & $\begin{array}{c}\mathrm{N} / \mathrm{A} \\
\mathrm{a}\end{array}$ & $\begin{array}{c}\text { N/A } \\
a\end{array}$ & $\begin{array}{c}\text { N/A } \\
\mathbf{a}\end{array}$ & $\begin{array}{c}\text { N/A } \\
\mathbf{a}\end{array}$ & $\begin{array}{c}\text { N/A } \\
\text { a }\end{array}$ & $\begin{array}{c}\text { N/A } \\
\mathbf{a}\end{array}$ & $\begin{array}{c}\text { N/A } \\
\mathbf{a}\end{array}$ & $\begin{array}{c}\text { N/A } \\
\mathbf{a}\end{array}$ & $\begin{array}{c}\text { N/A } \\
\text { a }\end{array}$ & $\begin{array}{c}\text { N/A } \\
\mathbf{a}\end{array}$ & $\begin{array}{c}\text { N/A } \\
a\end{array}$ & $\begin{array}{c}\text { N/A } \\
\text { a }\end{array}$ & $\begin{array}{c}\mathbf{N} / \mathbf{A} \\
\mathbf{a}\end{array}$ & $\begin{array}{c}\text { N/A } \\
a\end{array}$ & $\begin{array}{c}\text { N/A } \\
a\end{array}$ & $\begin{array}{c}\text { N/A } \\
\text { a }\end{array}$ & $\begin{array}{c}\text { N/A } \\
\mathbf{a}\end{array}$ \\
\hline $\begin{array}{c}\text { Fermi Core } 1 \\
\& 2-\mathrm{LM}\end{array}$ & $\begin{array}{c}\text { Yes } \\
\text { C }\end{array}$ & $\begin{array}{c}\text { No } \\
\text { a }\end{array}$ & $\begin{array}{c}\text { Yes } \\
\text { A }\end{array}$ & $\begin{array}{c}\text { Yes } \\
\text { C }\end{array}$ & $\begin{array}{c}\text { Yes } \\
\text { C }\end{array}$ & $\begin{array}{c}\text { Yes } \\
\text { C }\end{array}$ & $\begin{array}{c}\text { Yes } \\
\text { C }\end{array}$ & $\begin{array}{l}\text { Yes } \\
\text { C }\end{array}$ & $\begin{array}{l}\text { Yes } \\
\text { C }\end{array}$ & $\begin{array}{c}\text { No } \\
\text { a }\end{array}$ & $\begin{array}{c}\text { Yes } \\
\text { C }\end{array}$ & $\begin{array}{c}\text { Yes } \\
\text { C }\end{array}$ & $\begin{array}{c}\text { Yes } \\
\text { C }\end{array}$ & $\begin{array}{c}\text { Yes } \\
\text { C }\end{array}$ & $\begin{array}{c}\text { Yes } \\
\text { C }\end{array}$ & $\begin{array}{c}\text { N/A } \\
b\end{array}$ & $\begin{array}{c}\text { Yes } \\
\text { C }\end{array}$ & $\begin{array}{c}\text { Yes } \\
\text { C }\end{array}$ & $\begin{array}{c}\text { Yes } \\
\text { C }\end{array}$ & $\begin{array}{c}\text { Yes } \\
\text { C }\end{array}$ & $\begin{array}{c}\text { Yes } \\
\text { C }\end{array}$ & $\begin{array}{c}\mathrm{N} / \mathrm{A} \\
\mathrm{b}\end{array}$ & $\begin{array}{c}\text { N/A } \\
\text { b }\end{array}$ \\
\hline $\begin{array}{c}\text { Fermi } 1 \\
\text { Blanket-LM }\end{array}$ & $\begin{array}{c}\text { Yes } \\
\text { B }\end{array}$ & $\begin{array}{c}\text { No } \\
\text { a }\end{array}$ & $\begin{array}{c}\text { Yes } \\
\text { B }\end{array}$ & $\begin{array}{c}\text { Yes } \\
\text { C }\end{array}$ & $\begin{array}{c}\text { Yes } \\
\text { C }\end{array}$ & $\begin{array}{c}\text { Yes } \\
\mathrm{C}\end{array}$ & $\begin{array}{c}\text { Yes } \\
\text { C }\end{array}$ & $\begin{array}{c}\text { Yes } \\
\text { C }\end{array}$ & $\begin{array}{l}\text { Yes } \\
\text { C }\end{array}$ & $\begin{array}{c}\text { No } \\
\text { a }\end{array}$ & $\begin{array}{l}\text { Yes } \\
\text { C }\end{array}$ & $\begin{array}{c}\text { Yes } \\
\text { C }\end{array}$ & $\begin{array}{c}\text { Yes } \\
\text { C }\end{array}$ & $\begin{array}{l}\text { Yes } \\
\text { C }\end{array}$ & $\begin{array}{c}\text { Yes } \\
\text { C }\end{array}$ & $\begin{array}{c}\mathbf{N} / \mathbf{A} \\
\mathbf{b}\end{array}$ & $\begin{array}{c}\text { Yes } \\
\text { C }\end{array}$ & $\begin{array}{l}\text { Yes } \\
\text { C }\end{array}$ & $\begin{array}{c}\text { Yes } \\
\text { C }\end{array}$ & $\begin{array}{c}\text { Yes } \\
\text { C }\end{array}$ & $\begin{array}{c}\text { Yes } \\
\text { C }\end{array}$ & $\begin{array}{c}\text { N/A } \\
b\end{array}$ & $\begin{array}{c}N / A \\
b\end{array}$ \\
\hline $\begin{array}{l}\text { EBR II-LM } \\
\text { (Hanford - } \\
\text { casks) }\end{array}$ & $\begin{array}{c}\text { N/A } \\
a\end{array}$ & $\begin{array}{c}\text { N/A } \\
a\end{array}$ & $\begin{array}{c}\text { N/A } \\
a\end{array}$ & $\begin{array}{c}\text { N/A } \\
a\end{array}$ & $\begin{array}{c}\mathrm{N} / \mathrm{A} \\
\mathrm{a}\end{array}$ & $\begin{array}{c}\mathrm{N} / \mathrm{A} \\
\mathrm{a}\end{array}$ & $\begin{array}{c}\text { N/A } \\
a\end{array}$ & $\begin{array}{c}\mathrm{N} / \mathrm{A} \\
\mathbf{a}\end{array}$ & $\begin{array}{c}\text { N/A } \\
a\end{array}$ & $\begin{array}{c}\text { N/A } \\
\mathbf{a}\end{array}$ & $\begin{array}{c}\text { N/A } \\
a\end{array}$ & $\begin{array}{c}\mathbf{N} / \mathbf{A} \\
\mathbf{a}\end{array}$ & $\begin{array}{c}\mathrm{N} / \mathrm{A} \\
\mathbf{a}\end{array}$ & $\begin{array}{c}\text { N/A } \\
a\end{array}$ & $\begin{array}{c}\text { N/A } \\
a\end{array}$ & $\begin{array}{c}\text { N/A } \\
\mathbf{a}\end{array}$ & $\begin{array}{c}\text { N/A } \\
\text { a }\end{array}$ & $\begin{array}{c}\text { N/A } \\
a\end{array}$ & $\begin{array}{c}\text { N/A } \\
\text { a }\end{array}$ & $\begin{array}{c}\text { N/A } \\
\text { a }\end{array}$ & $\begin{array}{c}\mathrm{N} / \mathrm{A} \\
\mathrm{a}\end{array}$ & $\begin{array}{c}\mathrm{N} / \mathrm{A} \\
\mathbf{a}\end{array}$ & $\begin{array}{c}\mathrm{N} / \mathrm{A} \\
\mathbf{a}\end{array}$ \\
\hline $\begin{array}{l}\text { EBR II-LM } \\
\text { (INEL } \\
\text { canister) }\end{array}$ & $\begin{array}{c}\text { No } \\
\text { c }\end{array}$ & $\begin{array}{c}\text { No } \\
\text { a }\end{array}$ & $\begin{array}{l}\text { Yes } \\
\text { B }\end{array}$ & $\begin{array}{c}\text { Yes } \\
\text { B }\end{array}$ & $\begin{array}{c}\text { Yes } \\
\text { C }\end{array}$ & $\begin{array}{c}\text { Yes } \\
\text { C }\end{array}$ & $\begin{array}{c}\text { Yes } \\
\text { C }\end{array}$ & $\begin{array}{c}\text { Yes } \\
\text { C }\end{array}$ & $\begin{array}{l}\text { Yes } \\
\text { C }\end{array}$ & $\begin{array}{c}\text { No } \\
\text { a }\end{array}$ & $\begin{array}{c}\text { Yes } \\
\text { C }\end{array}$ & $\begin{array}{c}\text { Yes } \\
\text { C }\end{array}$ & $\begin{array}{c}\text { Yes } \\
\text { C }\end{array}$ & $\begin{array}{l}\text { Yes } \\
\text { C }\end{array}$ & $\begin{array}{c}\text { Yes } \\
\text { C }\end{array}$ & $\begin{array}{c}\text { N/A } \\
\text { b }\end{array}$ & $\begin{array}{c}\text { Yes } \\
\text { C }\end{array}$ & $\begin{array}{c}\text { Yes } \\
\text { C }\end{array}$ & $\begin{array}{c}\text { Yes } \\
\text { C }\end{array}$ & $\begin{array}{c}\text { Yes } \\
\text { c }\end{array}$ & $\begin{array}{c}\text { Yes } \\
\text { C }\end{array}$ & $\begin{array}{c}\text { N/A } \\
\text { b }\end{array}$ & $\begin{array}{c}\text { N/A } \\
b\end{array}$ \\
\hline $\begin{array}{l}\text { EBR II-LM } \\
\text { (INEL pin) }\end{array}$ & $\begin{array}{c}\text { Yes } \\
\text { C }\end{array}$ & $\begin{array}{c}\text { No } \\
\text { a }\end{array}$ & $\begin{array}{c}\text { Yes } \\
\text { B }\end{array}$ & $\begin{array}{c}\text { Yes } \\
\text { B }\end{array}$ & $\begin{array}{c}\text { Yes } \\
\text { C }\end{array}$ & $\begin{array}{c}\text { Yes } \\
\text { C }\end{array}$ & $\begin{array}{c}\text { Yes } \\
\text { C }\end{array}$ & $\begin{array}{c}\text { Yes } \\
\text { C }\end{array}$ & $\begin{array}{c}\text { Yes } \\
\text { C }\end{array}$ & $\begin{array}{c}\text { No } \\
\text { a }\end{array}$ & $\begin{array}{l}\text { Yes } \\
\text { C }\end{array}$ & $\begin{array}{c}\text { Yes } \\
\text { C }\end{array}$ & $\begin{array}{c}\text { Yes } \\
\text { B }\end{array}$ & $\begin{array}{c}\text { Yes } \\
\text { C }\end{array}$ & $\begin{array}{c}\text { Yes } \\
\text { C }\end{array}$ & $\begin{array}{c}\text { N/A } \\
\mathbf{b}\end{array}$ & $\begin{array}{c}\text { Yes } \\
\text { C }\end{array}$ & $\begin{array}{c}\text { Yes } \\
\text { C }\end{array}$ & $\begin{array}{c}\text { Yes } \\
\text { C }\end{array}$ & $\begin{array}{c}\text { Yes } \\
\text { C }\end{array}$ & $\begin{array}{c}\text { Yes } \\
\text { C }\end{array}$ & $\begin{array}{c}\text { N/A } \\
\text { b }\end{array}$ & $\begin{array}{c}\text { N/A } \\
\mathbf{b}\end{array}$ \\
\hline $\begin{array}{c}\text { EBR-II } \\
\text { (ANL-6) }\end{array}$ & $\begin{array}{c}\text { Yes } \\
\text { C }\end{array}$ & $\begin{array}{c}\text { No } \\
\text { a }\end{array}$ & $\begin{array}{c}\text { Yes } \\
\text { B }\end{array}$ & $\begin{array}{c}\text { Yes } \\
\text { B }\end{array}$ & $\begin{array}{c}\text { Yes } \\
\text { C }\end{array}$ & $\begin{array}{c}\text { Yes } \\
\text { C }\end{array}$ & $\begin{array}{c}\text { Yes } \\
\text { C }\end{array}$ & $\begin{array}{c}\text { Yes } \\
\text { B }\end{array}$ & $\begin{array}{c}\text { Yes } \\
\text { C }\end{array}$ & $\begin{array}{c}\text { No } \\
\text { a }\end{array}$ & $\begin{array}{c}\text { Yes } \\
\text { C }\end{array}$ & $\begin{array}{c}\text { Yes } \\
\text { C }\end{array}$ & $\begin{array}{c}\text { Yes } \\
\text { B }\end{array}$ & $\begin{array}{l}\text { Yes } \\
\text { C }\end{array}$ & $\begin{array}{c}\text { Yes } \\
\text { C }\end{array}$ & $\begin{array}{c}\text { N/A } \\
\mathbf{b}\end{array}$ & $\begin{array}{c}\text { Yes } \\
\text { C }\end{array}$ & $\begin{array}{c}\text { Yes } \\
\text { C }\end{array}$ & $\begin{array}{c}\text { Yes } \\
\text { C }\end{array}$ & $\begin{array}{c}\text { Yes } \\
\text { C }\end{array}$ & $\begin{array}{c}\text { Yes } \\
\text { C }\end{array}$ & $\begin{array}{c}\text { N/A } \\
b\end{array}$ & $\begin{array}{c}\text { N/A } \\
\text { b }\end{array}$ \\
\hline $\begin{array}{c}\text { EBR } \\
\text { (Hanford - } \\
\text { pieces) }\end{array}$ & $\begin{array}{c}\mathrm{N} / \mathrm{A} \\
\mathrm{a}\end{array}$ & $\begin{array}{c}\text { N/A } \\
a\end{array}$ & $\begin{array}{c}\text { N/A } \\
a\end{array}$ & $\begin{array}{c}\text { N/A } \\
\mathbf{a}\end{array}$ & $\begin{array}{c}\text { N/A } \\
a\end{array}$ & $\begin{array}{c}\text { N/A } \\
a\end{array}$ & $\begin{array}{c}\text { N/A } \\
a\end{array}$ & $\begin{array}{c}\mathrm{N} / \mathrm{A} \\
\mathbf{a}\end{array}$ & $\begin{array}{c}\text { N/A } \\
\mathbf{a}\end{array}$ & $\begin{array}{c}\text { No } \\
\text { a }\end{array}$ & $\begin{array}{c}\text { N/A } \\
a\end{array}$ & $\begin{array}{c}\text { N/A } \\
a\end{array}$ & $\begin{array}{c}\text { N/A } \\
a\end{array}$ & $\begin{array}{c}\text { N/A } \\
a\end{array}$ & $\begin{array}{c}\text { N/A } \\
a\end{array}$ & $\begin{array}{c}\text { N/A } \\
a\end{array}$ & $\begin{array}{c}\mathbf{N} / \mathbf{a} \\
\mathbf{a}\end{array}$ & $\begin{array}{c}\text { N/A } \\
\mathbf{a}\end{array}$ & $\begin{array}{c}\mathrm{N} / \mathrm{A} \\
\mathrm{a}\end{array}$ & $\begin{array}{c}\text { N/A } \\
a\end{array}$ & $\begin{array}{c}\text { N/A } \\
\text { a }\end{array}$ & $\begin{array}{c}\text { N/A } \\
a\end{array}$ & $\begin{array}{c}\text { N/a } \\
\mathbf{a}\end{array}$ \\
\hline $\begin{array}{c}\text { EBR II-LM } \\
\text { (ANL-W - } \\
\text { EBR-II) }\end{array}$ & $\begin{array}{c}\mathrm{N} / \mathrm{A} \\
\mathrm{a}\end{array}$ & $\begin{array}{c}\text { No } \\
\text { a }\end{array}$ & $\begin{array}{c}\text { N/A } \\
\mathbf{a}\end{array}$ & $\begin{array}{c}\text { N/A } \\
a\end{array}$ & $\begin{array}{c}\mathrm{N} / \mathrm{A} \\
\mathrm{a}\end{array}$ & $\begin{array}{c}\text { N/A } \\
a\end{array}$ & $\begin{array}{c}\text { N/A } \\
a\end{array}$ & $\begin{array}{c}\text { N/A } \\
\mathbf{a}\end{array}$ & $\begin{array}{c}\text { N/A } \\
\mathbf{a}\end{array}$ & $\begin{array}{c}\text { N/A } \\
a\end{array}$ & $\begin{array}{c}\text { N/A } \\
\mathbf{a}\end{array}$ & $\begin{array}{c}\text { N/A } \\
a\end{array}$ & $\begin{array}{c}\text { N/A } \\
\mathbf{a}\end{array}$ & $\begin{array}{c}\text { N/A } \\
\mathbf{a}\end{array}$ & $\begin{array}{c}\text { N/A } \\
a\end{array}$ & $\begin{array}{c}\text { N/A } \\
\mathbf{a}\end{array}$ & $\begin{array}{c}\mathbf{N} / \mathbf{a} \\
\mathbf{a}\end{array}$ & $\begin{array}{c}\text { N/A } \\
\mathbf{a}\end{array}$ & $\begin{array}{c}\text { N/A } \\
\mathbf{a}\end{array}$ & $\begin{array}{c}\text { N/A } \\
\text { a }\end{array}$ & $\begin{array}{c}\mathbf{N} / \mathbf{A} \\
\mathrm{a}\end{array}$ & $\begin{array}{c}\text { N/A } \\
\text { a }\end{array}$ & $\begin{array}{c}\mathbf{N} / \mathbf{a} \\
\mathbf{a}\end{array}$ \\
\hline
\end{tabular}


Table 4-3. (continued).

\begin{tabular}{|c|c|c|c|c|c|c|c|c|c|c|c|c|c|c|c|c|c|c|c|c|c|c|c|}
\hline \multirow[b]{2}{*}{$\begin{array}{l}\text { Breeder } \\
\text { reactor fuels } \mathrm{s}^{\mathrm{a}}\end{array}$} & \multicolumn{13}{|c|}{ Legal weight truck packages } & \multicolumn{2}{|c|}{$\begin{array}{c}\text { Overweight } \\
\text { truck packages }\end{array}$} & \multicolumn{8}{|c|}{ Rail packages } \\
\hline & $\begin{array}{l}\mathrm{GE} \\
\mathrm{T}-2\end{array}$ & $\begin{array}{l}\text { GE- } \\
100\end{array}$ & BMI-1 & FSV-1 & $\begin{array}{c}\mathrm{NLI}- \\
1 / 2\end{array}$ & $\left|\begin{array}{l}\mathrm{NLI}- \\
6502\end{array}\right|$ & $\mathrm{T}-3$ & NAC-1 & $\begin{array}{c}\text { CNS } \\
1-13 G\end{array}$ & NRBK-41 & $\begin{array}{l}\text { NAC- } \\
\text { LWT }\end{array}$ & $\begin{array}{c}\text { GE Model } \\
2000\end{array}$ & $\begin{array}{l}\text { TN- } \\
\text { FSV }\end{array}$ & $\begin{array}{l}\text { TN-8 } \\
\text { TN-8L }\end{array}$ & $\mid$ IN-9| & $\mathrm{M}-130$ & $\begin{array}{l}\text { IF- } \\
300\end{array}$ & $\begin{array}{l}\text { NLI- } \\
10 / 24\end{array}$ & $125-B$ & $\begin{array}{l}\text { TN- } \\
\text { BRP }\end{array}$ & $\begin{array}{c}\text { TN- } \\
\text { REG }\end{array}$ & M-160 & M-140 \\
\hline $\begin{array}{c}\text { EBR II-LM } \\
\text { (ANL-W - } \\
\text { HFEF) }\end{array}$ & $\begin{array}{c}\text { No } \\
a\end{array}$ & $\begin{array}{c}\text { No } \\
\text { a }\end{array}$ & $\begin{array}{c}\text { No } \\
\text { a }\end{array}$ & $\begin{array}{l}\text { Yes } \\
\text { B }\end{array}$ & $\begin{array}{l}\text { Yes } \\
\text { C }\end{array}$ & $\begin{array}{l}\text { Yes } \\
\text { C }\end{array}$ & $\begin{array}{c}\text { No } \\
\mathrm{a}\end{array}$ & $\begin{array}{l}\text { Yes } \\
\text { C }\end{array}$ & $\begin{array}{c}\text { No } \\
\mathbf{a}\end{array}$ & $\begin{array}{c}\text { No } \\
\text { a }\end{array}$ & $\begin{array}{l}\text { Yes } \\
\text { C }\end{array}$ & $\begin{array}{c}\text { No } \\
\text { a }\end{array}$ & $\begin{array}{l}\text { No } \\
\text { c }\end{array}$ & $\begin{array}{l}\text { Yes } \\
\text { C }\end{array}$ & $\begin{array}{l}\text { Yes } \\
\text { C }\end{array}$ & $\begin{array}{c}\text { N/A } \\
\text { b }\end{array}$ & $\begin{array}{c}\text { Yes } \\
\text { c }\end{array}$ & $\begin{array}{c}\text { Yes } \\
\text { C }\end{array}$ & $\begin{array}{l}\text { No } \\
\text { c }\end{array}$ & $\begin{array}{l}\text { Yes } \\
\text { C }\end{array}$ & $\begin{array}{c}\text { Yes } \\
\text { C }\end{array}$ & $\begin{array}{c}\text { N/A } \\
b\end{array}$ & $\begin{array}{c}\text { N/A } \\
\text { b }\end{array}$ \\
\hline $\begin{array}{c}\text { EBR II-LM } \\
\text { (ANL-W - } \\
\text { RSWF) }\end{array}$ & $\begin{array}{c}\text { No } \\
\text { a }\end{array}$ & $\begin{array}{c}\text { No } \\
\text { a }\end{array}$ & $\begin{array}{c}\text { No } \\
\mathbf{a}\end{array}$ & $\begin{array}{c}\text { No } \\
\mathbf{a}\end{array}$ & $\begin{array}{c}\text { No } \\
a\end{array}$ & $\begin{array}{c}\text { No } \\
\text { a }\end{array}$ & $\begin{array}{c}\text { No } \\
\text { a }\end{array}$ & $\begin{array}{c}\text { No } \\
\text { a }\end{array}$ & $\begin{array}{c}\text { No } \\
\text { a }\end{array}$ & $\begin{array}{c}\text { No } \\
\text { a }\end{array}$ & $\begin{array}{c}\text { No } \\
\text { a }\end{array}$ & $\begin{array}{c}\text { No } \\
\text { a }\end{array}$ & $\begin{array}{c}\text { No } \\
\text { a }\end{array}$ & $\begin{array}{c}\text { No } \\
\text { a }\end{array}$ & $\begin{array}{c}\text { No } \\
\text { a }\end{array}$ & $\begin{array}{c}\text { No } \\
\text { a }\end{array}$ & $\begin{array}{c}\text { Yes } \\
\text { C }\end{array}$ & $\begin{array}{c}\text { Yes } \\
\text { C }\end{array}$ & $\begin{array}{c}\text { Yes } \\
\text { C }\end{array}$ & $\begin{array}{c}\text { Yes } \\
\text { C }\end{array}$ & $\begin{array}{c}\text { Yes } \\
\text { C }\end{array}$ & $\underset{b}{\text { N/A }}$ & $\begin{array}{c}\text { N/A } \\
b\end{array}$ \\
\hline $\begin{array}{l}\text { EBR-II } \\
\text { (SRS) }\end{array}$ & $\left|\begin{array}{c}\mathrm{N} / \mathrm{A} \\
\mathrm{b}\end{array}\right|$ & $\begin{array}{c}\text { No } \\
\text { a }\end{array}$ & $\begin{array}{c}\text { N/A } \\
b\end{array}$ & $\begin{array}{c}\text { N/A } \\
b\end{array}$ & $\begin{array}{c}\text { N/A } \\
b\end{array}$ & $\begin{array}{c}N / A \\
b\end{array} \mid$ & $\begin{array}{c}\text { N/A } \\
b\end{array}$ & $\begin{array}{c}\text { N/A } \\
b\end{array}$ & $\begin{array}{c}\text { N/A } \\
b\end{array}$ & $\begin{array}{c}\text { No } \\
\text { a }\end{array}$ & $\begin{array}{c}\text { N/A } \\
b\end{array}$ & $\begin{array}{c}\text { N/A } \\
b\end{array}$ & $\begin{array}{c}N / A \\
b\end{array}$ & $\begin{array}{c}\text { N/A } \\
b\end{array}$ & $\begin{array}{c}\mathrm{N} / \mathrm{A} \\
\mathrm{b}\end{array}$ & $\begin{array}{c}\text { N/A } \\
b\end{array}$ & $\begin{array}{c}\text { N/A } \\
b\end{array}$ & $\begin{array}{c}N / A \\
b\end{array}$ & $\begin{array}{c}\text { N/A } \\
b\end{array}$ & $\begin{array}{c}\text { N/A } \\
0\end{array}$ & $\left|\begin{array}{c}\mathrm{N} / \mathrm{A} \\
\mathrm{b}\end{array}\right|$ & $\begin{array}{c}\text { N/A } \\
b\end{array}$ & $\begin{array}{c}\mathrm{N} / \mathrm{A} \\
\mathrm{b}\end{array}$ \\
\hline $\begin{array}{c}\text { EBR-II } \\
\text { (SRS) }\end{array}$ & $\begin{array}{c}\mathrm{N} / \mathrm{A} \\
\mathrm{b}\end{array}$ & $\begin{array}{c}\text { No } \\
\mathrm{a}\end{array}$ & $\begin{array}{c}\text { N/A } \\
b\end{array}$ & $\begin{array}{c}\text { N/A } \\
b\end{array}$ & $\begin{array}{c}\text { N/A } \\
b\end{array}$ & $\begin{array}{c}\text { N/A } \\
b\end{array} \mid$ & $\begin{array}{c}\text { N/A } \\
b\end{array}$ & $\begin{array}{c}\text { N/A } \\
\text { b }\end{array}$ & $\underset{b}{N / A}$ & $\begin{array}{c}\text { No } \\
\text { a }\end{array}$ & $\begin{array}{c}\text { N/A } \\
b\end{array}$ & $\begin{array}{c}\text { N/A } \\
b\end{array}$ & $\begin{array}{c}N / A \\
b\end{array}$ & $\begin{array}{c}\text { N/A } \\
b\end{array}$ & $\begin{array}{c}N / A \\
b\end{array}$ & $\begin{array}{c}\mathrm{N} / \mathrm{A} \\
\mathrm{b}\end{array}$ & $\begin{array}{c}\text { N/A } \\
b\end{array}$ & $\begin{array}{c}\text { N/A } \\
\text { b }\end{array}$ & $\begin{array}{c}\text { N/A } \\
b\end{array}$ & $\begin{array}{c}\text { N/A } \\
b\end{array}$ & $\begin{array}{c}\mathrm{N} / \mathrm{A} \\
\mathrm{b}\end{array}$ & $\begin{array}{c}\text { N/A } \\
\text { b }\end{array}$ & $\begin{array}{c}\text { N/A } \\
b\end{array}$ \\
\hline $\begin{array}{l}\text { EBR-II } \\
\text { Targets }\end{array}$ & $\begin{array}{c}\mathrm{N} / \mathrm{A} \\
\mathrm{a}\end{array}$ & $\begin{array}{c}\mathrm{N} / \mathrm{A} \\
\mathrm{a}\end{array}$ & $\begin{array}{c}\mathrm{N} / \mathrm{A} \\
\mathrm{a}\end{array}$ & $\begin{array}{c}\text { N/A } \\
\text { a }\end{array}$ & $\begin{array}{c}\text { N/A } \\
a\end{array}$ & $\begin{array}{c}\mathrm{N} / \mathrm{A} \\
\mathrm{a}\end{array}$ & $\begin{array}{c}\mathrm{N} / \mathrm{A} \\
\mathrm{a}\end{array}$ & $\begin{array}{c}\text { N/A } \\
\mathbf{a}\end{array}$ & $\begin{array}{c}N / a \\
a\end{array}$ & $\stackrel{N / A}{a}$ & $\begin{array}{c}\text { N/A } \\
\text { a }\end{array}$ & $\underset{\mathrm{a}}{\mathrm{N} / \mathrm{A}}$ & $\begin{array}{c}\mathrm{N} / \mathrm{A} \\
\mathrm{a}\end{array}$ & $\begin{array}{c}\text { N/A } \\
\text { a }\end{array}$ & $\begin{array}{c}\mathrm{N} / \mathrm{A} \\
\mathrm{a}\end{array}$ & $\begin{array}{c}\text { N/A } \\
a\end{array}$ & $\begin{array}{c}\mathrm{N} / \mathbf{a} \\
\mathbf{a}\end{array}$ & $\begin{array}{c}\mathrm{N} / \mathrm{A} \\
\mathrm{a}\end{array}$ & $\begin{array}{c}\mathrm{N} / \mathrm{A} \\
\mathrm{a}\end{array}$ & $\begin{array}{c}\text { N/A } \\
a\end{array}$ & $\begin{array}{c}\mathrm{N} / \mathrm{A} \\
\mathrm{a}\end{array}$ & $\begin{array}{c}\text { N/A } \\
\text { a }\end{array}$ & $\begin{array}{c}N / a \\
a\end{array}$ \\
\hline $\begin{array}{c}\text { Saxton (Mixed } \\
\text { oxide) }\end{array}$ & $\begin{array}{c}\mathrm{N} / \mathrm{A} \\
\mathrm{b}\end{array}$ & $\begin{array}{c}\text { No } \\
\mathrm{a}\end{array}$ & $\begin{array}{c}\text { N/A } \\
b\end{array}$ & $\begin{array}{c}\text { N/A } \\
\text { b }\end{array}$ & $\begin{array}{c}\text { N/A } \\
b\end{array}$ & $\begin{array}{c}\text { N/A } \\
b\end{array}$ & $\begin{array}{c}\text { N/A } \\
b\end{array}$ & $\begin{array}{c}\text { N/A } \\
b\end{array}$ & $\begin{array}{c}\text { N/A } \\
b\end{array}$ & $\begin{array}{c}\text { No } \\
\text { a }\end{array}$ & $\begin{array}{c}\text { N/A } \\
b\end{array}$ & $\begin{array}{c}N / A \\
b\end{array}$ & $\begin{array}{c}\text { N/A } \\
b\end{array}$ & $\begin{array}{c}\text { N/A } \\
\text { b }\end{array}$ & $\begin{array}{c}\text { N/A } \\
b\end{array}$ & $\begin{array}{c}\text { N/A } \\
\text { b }\end{array}$ & $\begin{array}{c}\text { N/A } \\
b\end{array}$ & $\begin{array}{c}\text { N/A } \\
b\end{array}$ & $\begin{array}{c}\text { N/A } \\
b\end{array}$ & $\begin{array}{c}\text { N/A } \\
b\end{array}$ & $\begin{array}{c}\text { N/A } \\
b\end{array}$ & $\begin{array}{c}\text { N/A } \\
b\end{array}$ & $\begin{array}{c}\text { N/A } \\
b\end{array}$ \\
\hline Saxton $\left(\mathrm{UO}_{2}\right)$ & $\begin{array}{c}\mathrm{N} / \mathrm{A} \\
\mathrm{a}\end{array}$ & $\begin{array}{c}\mathrm{N} / \mathrm{A} \\
\mathrm{a}\end{array}$ & $\begin{array}{c}\mathrm{N} / \mathrm{A} \\
\mathrm{a}\end{array}$ & $\begin{array}{c}\text { N/A } \\
\text { a }\end{array}$ & $\begin{array}{c}\text { N/A } \\
a\end{array}$ & $\left|\begin{array}{c}\mathrm{N} / \mathrm{A} \\
\mathrm{a}\end{array}\right|$ & $\begin{array}{c}\mathrm{N} / \mathrm{A} \\
\mathrm{a}\end{array}$ & $\begin{array}{c}\text { N/A } \\
\text { a }\end{array}$ & $\begin{array}{c}N / a \\
a\end{array}$ & $\begin{array}{c}\text { N/A } \\
a\end{array}$ & $\begin{array}{c}\text { N/A } \\
a\end{array}$ & $\begin{array}{c}N / A \\
a\end{array}$ & $\begin{array}{c}\text { N/A } \\
a\end{array}$ & $\begin{array}{c}\mathrm{N} / \mathrm{A} \\
\mathrm{a}\end{array}$ & $\begin{array}{c}\mathrm{N} / \mathrm{A} \\
\mathrm{a}\end{array}$ & $\begin{array}{c}\text { N/A } \\
\text { a }\end{array}$ & $\begin{array}{c}\mathrm{N} / \mathrm{A} \\
\mathrm{a}\end{array}$ & $\begin{array}{c}\text { N/A } \\
\text { a }\end{array}$ & $\begin{array}{c}\mathrm{N} / \mathrm{A} \\
\mathrm{a}\end{array}$ & $\begin{array}{c}\text { N/A } \\
a\end{array}$ & $\begin{array}{c}\mathrm{N} / \mathrm{A} \\
\mathrm{a}\end{array}$ & $\begin{array}{c}\mathrm{N} / \mathrm{A} \\
\mathrm{a}\end{array}$ & $\begin{array}{c}N / a \\
a\end{array}$ \\
\hline Dresden & $\begin{array}{c}N / A \\
b / c\end{array}$ & $\begin{array}{c}N / A \\
b / c\end{array}$ & $\begin{array}{l}\mathrm{N} / \mathrm{A} \\
\mathrm{b} / \mathrm{c}\end{array}$ & $\begin{array}{l}\mathrm{N} / \mathrm{A} \\
\mathrm{b} / \mathrm{c}\end{array}$ & $\begin{array}{l}\mathrm{N} / \mathrm{A} \\
\mathrm{b} / \mathrm{c}\end{array}$ & $\begin{array}{c}N / A \\
b / c\end{array}$ & $\begin{array}{l}N / A \\
b / c\end{array}$ & $\begin{array}{l}\mathrm{N} / \mathrm{A} \\
\mathrm{b} / \mathrm{c}\end{array}$ & $\begin{array}{l}\text { N/A } \\
b / c\end{array}$ & $\begin{array}{l}\text { N/A } \\
b / c\end{array}$ & $\begin{array}{l}N / A \\
b / c\end{array}$ & $\begin{array}{l}\mathrm{N} / \mathrm{A} \\
\mathrm{b} / \mathrm{c}\end{array}$ & $\begin{array}{l}N / A \\
b / c\end{array}$ & $\begin{array}{l}N / A \\
b / c\end{array}$ & $\begin{array}{l}N / A \\
b / c\end{array}$ & $\begin{array}{l}N / A \\
b / c\end{array}$ & $\begin{array}{l}N / A \\
b / c\end{array}$ & $\begin{array}{l}N / A \\
b / c\end{array}$ & $\begin{array}{l}N / A \\
b / c\end{array}$ & $\begin{array}{l}N / A \\
b / c\end{array}$ & $\begin{array}{c}N / A \\
b / c\end{array}$ & $\begin{array}{l}N / A \\
b / c\end{array}$ & $\begin{array}{l}\mathrm{N} / \mathrm{A} \\
\mathrm{b} / \mathrm{c}\end{array}$ \\
\hline ERR & $\begin{array}{c}N / A \\
b\end{array}$ & $\begin{array}{c}\text { No } \\
\text { a }\end{array}$ & $\begin{array}{c}\text { No } \\
\mathbf{a}\end{array}$ & $\begin{array}{c}\text { N/A } \\
b\end{array}$ & $\begin{array}{c}\text { N/A } \\
b\end{array}$ & $\begin{array}{c}N / A \\
b\end{array}$ & $\begin{array}{c}N / A \\
b\end{array}$ & $\begin{array}{c}N / A \\
b\end{array}$ & $\begin{array}{c}\text { No } \\
\text { a }\end{array}$ & $\begin{array}{c}\text { No } \\
\text { a }\end{array}$ & $\stackrel{N / A}{b}$ & $\begin{array}{c}\text { No } \\
a\end{array}$ & $\begin{array}{c}\mathrm{N} / \mathrm{A} \\
\mathrm{b}\end{array}$ & $\begin{array}{c}\text { N/A } \\
b\end{array}$ & $\begin{array}{c}N / A \\
b\end{array}$ & $\begin{array}{c}\text { N/A } \\
b\end{array}$ & $\begin{array}{c}\text { N/A } \\
b\end{array}$ & $\begin{array}{c}N / A \\
b\end{array}$ & $\begin{array}{c}\text { N/A } \\
b\end{array}$ & $\begin{array}{c}\mathrm{N} / \mathrm{A} \\
\mathrm{b}\end{array}$ & $\begin{array}{c}\text { N/A } \\
b\end{array}$ & $\begin{array}{c}\mathrm{N} / \mathrm{A} \\
\mathrm{b}\end{array}$ & $\underset{b}{N / A}$ \\
\hline ML-1 & $\begin{array}{c}\mathrm{N} / \mathrm{A} \\
\mathrm{a}\end{array}$ & $\begin{array}{c}\text { No } \\
\mathbf{a}\end{array}$ & $\begin{array}{c}\text { N/A } \\
\mathbf{a}\end{array}$ & $\begin{array}{c}\text { N/A } \\
\text { a }\end{array}$ & $\begin{array}{c}\text { N/A } \\
a\end{array}$ & $\begin{array}{c}\text { N/A } \\
a\end{array} \mid$ & $\begin{array}{c}\mathrm{N} / \mathrm{A} \\
\mathrm{a}\end{array}$ & $\begin{array}{c}\text { N/A } \\
\mathbf{a}\end{array}$ & $\begin{array}{c}N / a \\
a\end{array}$ & $\begin{array}{c}\text { N/A } \\
\mathbf{a}\end{array}$ & $\begin{array}{c}\text { N/A } \\
a\end{array}$ & $\begin{array}{c}\text { N/A } \\
\text { a }\end{array}$ & $\begin{array}{c}\mathrm{N} / \mathrm{A} \\
\mathbf{a}\end{array}$ & $\begin{array}{c}\text { N/A } \\
\text { a }\end{array}$ & $\begin{array}{c}\text { N/A } \\
a\end{array}$ & $\begin{array}{c}\text { N/A } \\
\text { a }\end{array}$ & $\begin{array}{c}N / a \\
a\end{array}$ & $\begin{array}{c}\text { N/A } \\
\text { a }\end{array}$ & $\begin{array}{c}\text { N/A } \\
a\end{array}$ & $\begin{array}{c}\text { N/A } \\
a\end{array}$ & $\begin{array}{c}\mathrm{N} / \mathrm{A} \\
\mathrm{a}\end{array}$ & $\begin{array}{c}\text { N/A } \\
\text { a }\end{array}$ & $\begin{array}{c}\mathrm{N} / \mathrm{a} \\
\mathrm{a}\end{array}$ \\
\hline
\end{tabular}


Table 4-4. Packaging compatibility with gas-cooled reactor type SNF.

\begin{tabular}{|c|c|c|c|c|c|c|c|c|c|c|c|c|c|c|c|c|c|c|c|c|c|c|c|}
\hline \multirow{2}{*}{$\begin{array}{l}\text { Gas-cooled } \\
\text { reactor } \\
\text { fuels }\end{array}$} & \multicolumn{13}{|c|}{ Legal weight truck packages } & \multicolumn{2}{|c|}{$\begin{array}{c}\text { Overweight } \\
\text { truck packages }\end{array}$} & \multicolumn{8}{|c|}{ Rail packages } \\
\hline & $\begin{array}{l}\text { GE } \\
\mathrm{T}-2\end{array}$ & $\begin{array}{l}\text { GE- } \\
100\end{array}$ & BMI-1 & FSV-1 & $\begin{array}{l}\text { NLI- } \\
1 / 2\end{array}$ & $\begin{array}{l}\text { NLI- } \\
6502\end{array}$ & $\mathrm{~T}-\mathbf{3}$ & NAC-1 & $\begin{array}{c}\text { CNS } \\
1-13 G\end{array}$ & NRBK-41 & $\begin{array}{l}\text { NAC- } \\
\text { LWT }\end{array}$ & $\begin{array}{c}\text { GE Model } \\
2000\end{array}$ & $\begin{array}{l}\text { TN. } \\
\text { FSV }\end{array}$ & $\begin{array}{l}\text { TN-8 } \\
\text { TN-8L }\end{array}$ & TN-9 & $\mathbf{M}-130$ & IF-300 & $\begin{array}{l}\text { NLI- } \\
10 / 24\end{array}$ & 125-B & $\begin{array}{l}\text { TN- } \\
\text { BRP }\end{array}$ & $\begin{array}{c}\text { TN- } \\
\text { REG }\end{array}$ & $M-160$ & M-140 \\
\hline $\begin{array}{c}\text { Peach } \\
\text { Bottom } 1\end{array}$ & $\begin{array}{c}\text { No } \\
\text { a }\end{array}$ & $\begin{array}{c}\text { No } \\
\text { a }\end{array}$ & $\begin{array}{c}\text { No } \\
\text { a }\end{array}$ & $\begin{array}{c}\text { N/A } \\
b\end{array}$ & $\begin{array}{c}\text { N/A } \\
\text { b }\end{array}$ & $\begin{array}{c}\text { No } \\
\text { a }\end{array}$ & $\begin{array}{c}\text { N/A } \\
b\end{array}$ & $\begin{array}{c}\text { N/A } \\
b\end{array}$ & $\begin{array}{c}\text { No } \\
\text { a }\end{array}$ & $\begin{array}{c}\text { No } \\
\text { a }\end{array}$ & $\begin{array}{c}\text { N/A } \\
\text { b }\end{array}$ & $\begin{array}{c}\text { No } \\
\text { a }\end{array}$ & $\begin{array}{c}\text { N/A } \\
b\end{array}$ & $\begin{array}{c}\text { N/A } \\
b\end{array}$ & $\begin{array}{c}\text { N/A } \\
b\end{array}$ & $\begin{array}{c}\text { No } \\
\text { a }\end{array}$ & $\begin{array}{c}\text { N/A } \\
b\end{array}$ & $\underset{b}{\text { N/A }}$ & $\begin{array}{c}\text { N/A } \\
b\end{array}$ & $\begin{array}{c}\text { N/A } \\
b\end{array}$ & $\begin{array}{c}\text { N/A } \\
b\end{array}$ & $\begin{array}{c}\text { N/A } \\
b\end{array}$ & $\begin{array}{c}\text { N/A } \\
b\end{array}$ \\
\hline $\begin{array}{c}\text { Peach } \\
\text { Bottom } 2\end{array}$ & $\begin{array}{c}\text { No } \\
\mathbf{a}\end{array}$ & $\begin{array}{c}\text { No } \\
\text { a }\end{array}$ & $\begin{array}{l}\text { No } \\
\text { a }\end{array}$ & $\begin{array}{c}\text { N/A } \\
b\end{array}$ & $\begin{array}{c}\text { N/A } \\
b\end{array}$ & $\begin{array}{c}\text { No } \\
\text { a }\end{array}$ & $\begin{array}{c}\text { N/A } \\
b\end{array}$ & $\begin{array}{c}\text { N/A } \\
b\end{array}$ & $\begin{array}{c}\text { No } \\
\text { a }\end{array}$ & $\begin{array}{l}\text { No } \\
\text { a }\end{array}$ & $\begin{array}{c}\text { N/A } \\
b\end{array}$ & $\begin{array}{l}\text { No } \\
\text { a }\end{array}$ & $\begin{array}{c}\text { N/A } \\
b\end{array}$ & $\begin{array}{c}\text { N/A } \\
\text { b }\end{array}$ & $\begin{array}{c}\text { N/A } \\
b\end{array}$ & $\begin{array}{c}\text { No } \\
\text { a }\end{array}$ & $\begin{array}{c}\text { N/A } \\
\text { b }\end{array}$ & $\begin{array}{c}\text { N/A } \\
b\end{array}$ & $\begin{array}{c}\text { N/A } \\
b\end{array}$ & $\begin{array}{c}\text { N/A } \\
b\end{array}$ & $\begin{array}{c}\text { N/A } \\
\mathbf{b}\end{array}$ & $\begin{array}{c}\text { N/A } \\
\mathbf{b}\end{array}$ & $\begin{array}{c}\text { N/A } \\
\text { b }\end{array}$ \\
\hline FSVR-HT & $\begin{array}{c}\text { No } \\
\text { a }\end{array}$ & $\begin{array}{c}\text { No } \\
\text { a }\end{array}$ & $\begin{array}{c}\text { No } \\
\text { a }\end{array}$ & $\begin{array}{c}\text { Yes } \\
\text { A }\end{array}$ & $\begin{array}{c}\text { Yes } \\
\text { A }\end{array}$ & $\begin{array}{c}\text { No } \\
\text { a }\end{array}$ & $\begin{array}{c}\text { No } \\
\text { a }\end{array}$ & $\begin{array}{c}\text { No } \\
\text { a }\end{array}$ & $\begin{array}{c}\text { N/A } \\
b\end{array}$ & $\begin{array}{c}\text { No } \\
\text { a }\end{array}$ & $\begin{array}{c}\text { No } \\
\text { a }\end{array}$ & $\begin{array}{c}\text { N/A } \\
b\end{array}$ & $\begin{array}{c}\text { Yes } \\
\text { A }\end{array}$ & $\begin{array}{c}\text { No } \\
\text { a }\end{array}$ & $\begin{array}{c}\text { No } \\
\text { a }\end{array}$ & $\begin{array}{c}\text { No } \\
\text { a }\end{array}$ & $\begin{array}{c}\text { N/A } \\
b\end{array}$ & $\begin{array}{c}\text { N/A } \\
b\end{array}$ & $\begin{array}{c}\text { N/A } \\
\text { b }\end{array}$ & $\begin{array}{c}\text { N/A } \\
\mathbf{b}\end{array}$ & $\begin{array}{c}\text { N/A } \\
\text { b }\end{array}$ & $\begin{array}{c}\text { N/A } \\
b\end{array}$ & $\begin{array}{c}\text { N/A } \\
b\end{array}$ \\
\hline FSVR & $\begin{array}{c}\text { No } \\
\text { a }\end{array}$ & $\begin{array}{c}\text { No } \\
\text { a }\end{array}$ & $\begin{array}{c}\text { No } \\
a\end{array}$ & $\begin{array}{c}\text { Yes } \\
\text { A }\end{array}$ & $\begin{array}{c}\text { Yes } \\
\text { A }\end{array}$ & $\begin{array}{c}\text { No } \\
\text { a }\end{array}$ & $\begin{array}{c}\text { No } \\
\text { a }\end{array}$ & $\begin{array}{c}\text { No } \\
\mathbf{a}\end{array}$ & $\begin{array}{c}\text { N/A } \\
b\end{array}$ & $\begin{array}{c}\text { No } \\
a\end{array}$ & $\begin{array}{c}\text { No } \\
\text { a }\end{array}$ & $\begin{array}{c}\text { N/A } \\
b\end{array}$ & $\begin{array}{c}\text { Yes } \\
\text { A }\end{array}$ & $\begin{array}{c}\text { No } \\
\text { a }\end{array}$ & $\begin{array}{c}\text { No } \\
a\end{array}$ & $\begin{array}{c}\text { No } \\
\text { a }\end{array}$ & $\begin{array}{c}\text { N/A } \\
b\end{array}$ & $\begin{array}{c}\text { N/A } \\
b\end{array}$ & $\begin{array}{c}\text { N/A } \\
b\end{array}$ & $\begin{array}{c}\text { N/A } \\
b\end{array}$ & $\begin{array}{c}\mathbf{N} / \mathbf{A} \\
b\end{array}$ & $\begin{array}{c}\text { N/A } \\
b\end{array}$ & $\begin{array}{c}\text { N/A } \\
b\end{array}$ \\
\hline TORY-IIC & $\begin{array}{c}\text { Yes } \\
\text { C }\end{array}$ & $\begin{array}{c}\text { No } \\
\text { a }\end{array}$ & $\begin{array}{c}\text { No } \\
\text { a }\end{array}$ & $\begin{array}{c}\text { Yes } \\
\text { C }\end{array}$ & $\begin{array}{c}\text { Yes } \\
\text { C }\end{array}$ & $\begin{array}{c}\text { Yes } \\
\text { C }\end{array}$ & $\begin{array}{c}\text { Yes } \\
\text { C }\end{array}$ & $\begin{array}{c}\text { Yes } \\
\text { C }\end{array}$ & $\begin{array}{c}\text { No } \\
\text { a }\end{array}$ & $\begin{array}{c}\text { No } \\
\text { a }\end{array}$ & $\begin{array}{c}\text { Yes } \\
\text { C }\end{array}$ & $\begin{array}{l}\text { No } \\
\text { a }\end{array}$ & $\begin{array}{c}\text { Yes } \\
\text { C }\end{array}$ & $\begin{array}{c}\text { Yes } \\
\text { C }\end{array}$ & $\begin{array}{c}\text { Yes } \\
\text { C }\end{array}$ & $\begin{array}{c}\text { N/A } \\
\text { b }\end{array}$ & $\begin{array}{c}\text { Yes } \\
\text { C }\end{array}$ & $\begin{array}{c}\text { Yes } \\
\text { C }\end{array}$ & $\begin{array}{c}\text { Yes } \\
\text { C }\end{array}$ & $\begin{array}{c}\text { Yes } \\
\text { C }\end{array}$ & $\begin{array}{c}\text { Yes } \\
\text { C }\end{array}$ & $\begin{array}{c}\text { N/A } \\
b\end{array}$ & $\begin{array}{c}\text { N/A } \\
\text { b }\end{array}$ \\
\hline TORY-IIA & $\begin{array}{c}\text { Yes } \\
\text { C }\end{array}$ & $\begin{array}{c}\text { No } \\
\text { a }\end{array}$ & $\begin{array}{c}\text { Yes } \\
\text { C }\end{array}$ & $\begin{array}{c}\text { Yes } \\
\text { C }\end{array}$ & $\begin{array}{c}\text { Yes } \\
\text { C }\end{array}$ & $\begin{array}{c}\text { Yes } \\
\text { C }\end{array}$ & $\begin{array}{c}\text { Yes } \\
\text { C }\end{array}$ & $\begin{array}{l}\text { Yes } \\
\text { C }\end{array}$ & $\begin{array}{c}\text { Yes } \\
\text { C }\end{array}$ & $\begin{array}{c}\text { No } \\
\text { a }\end{array}$ & $\begin{array}{c}\text { Yes } \\
\text { C }\end{array}$ & $\begin{array}{c}\text { Yes } \\
\text { C }\end{array}$ & $\begin{array}{c}\text { Yes } \\
\text { C }\end{array}$ & $\begin{array}{c}\text { Yes } \\
\text { C }\end{array}$ & $\begin{array}{c}\text { Yes } \\
\text { C }\end{array}$ & $\begin{array}{c}\text { N/A } \\
b\end{array}$ & $\begin{array}{c}\text { Yes } \\
\text { C }\end{array}$ & $\begin{array}{c}\text { Yes } \\
\text { C }\end{array}$ & $\begin{array}{c}\text { Yes } \\
\text { C }\end{array}$ & $\begin{array}{c}\text { Yes } \\
\text { C }\end{array}$ & $\begin{array}{c}\text { Yes } \\
\text { C }\end{array}$ & $\begin{array}{c}\text { N/A } \\
b\end{array}$ & $\begin{array}{c}\text { N/A } \\
b\end{array}$ \\
\hline $\begin{array}{l}\text { GCRE } \\
\text { (INEL) }\end{array}$ & $\begin{array}{c}\text { N/A } \\
b\end{array}$ & $\begin{array}{c}\text { No } \\
\text { a }\end{array}$ & $\begin{array}{c}N / A \\
b\end{array}$ & $\begin{array}{c}\text { N/A } \\
b\end{array}$ & $\begin{array}{c}\text { N/A } \\
b\end{array}$ & $\begin{array}{c}\text { N/A } \\
b\end{array}$ & $\begin{array}{c}\text { N/A } \\
b\end{array}$ & $\begin{array}{c}\text { N/A } \\
\text { b }\end{array}$ & $\begin{array}{c}\text { N/A } \\
b\end{array}$ & $\begin{array}{c}\text { No } \\
\text { a }\end{array}$ & $\begin{array}{c}\text { N/A } \\
b\end{array}$ & $\begin{array}{c}\text { N/A } \\
b\end{array}$ & $\begin{array}{c}\text { N/A } \\
b\end{array}$ & $\begin{array}{c}\text { N/A } \\
\mathrm{b}\end{array}$ & $\begin{array}{c}\text { N/A } \\
b\end{array}$ & $\begin{array}{c}\text { N/A } \\
b\end{array}$ & $\begin{array}{c}\text { N/A } \\
b\end{array}$ & $\begin{array}{c}\text { N/A } \\
b\end{array}$ & $\begin{array}{c}\text { N/A } \\
b\end{array}$ & $\begin{array}{c}\text { N/A } \\
\text { b }\end{array}$ & $\begin{array}{c}\text { N/A } \\
\text { b }\end{array}$ & $\begin{array}{c}\text { N/A } \\
b\end{array}$ & $\begin{array}{c}\text { N/A } \\
\text { b }\end{array}$ \\
\hline $\begin{array}{l}\text { GCRE } \\
\text { (SRS) }\end{array}$ & $\begin{array}{c}\mathrm{N} / \mathrm{A} \\
\mathrm{b}\end{array}$ & $\begin{array}{c}\text { No } \\
\text { a }\end{array}$ & $\begin{array}{c}\text { N/A } \\
b\end{array}$ & $\begin{array}{c}\text { N/A } \\
b\end{array}$ & $\begin{array}{c}\text { N/A } \\
b\end{array}$ & $\begin{array}{c}\text { N/A } \\
b\end{array}$ & $\begin{array}{c}\text { N/A } \\
b\end{array}$ & $\begin{array}{c}\text { N/A } \\
b\end{array}$ & $\begin{array}{c}\text { N/A } \\
b\end{array}$ & $\begin{array}{c}\text { No } \\
\text { a }\end{array}$ & $\begin{array}{c}\text { N/A } \\
b\end{array}$ & $\begin{array}{c}\text { N/A } \\
b\end{array}$ & $\begin{array}{c}N / A \\
b\end{array}$ & $\begin{array}{c}N / A \\
b\end{array}$ & $\begin{array}{c}\text { N/A } \\
b\end{array}$ & $\begin{array}{c}\text { N/A } \\
b\end{array}$ & $\begin{array}{c}\text { N/A } \\
b\end{array}$ & $\begin{array}{c}N / A \\
b\end{array}$ & $\begin{array}{c}\text { N/A } \\
b\end{array}$ & $\begin{array}{c}\text { N/A } \\
b\end{array}$ & $\begin{array}{c}\text { N/A } \\
b\end{array}$ & $\begin{array}{c}N / A \\
b\end{array}$ & $\begin{array}{c}\text { N/A } \\
b\end{array}$ \\
\hline
\end{tabular}


Table 4-5. Packaging compatibility with metallic type SNF.

\begin{tabular}{|c|c|c|c|c|c|c|c|c|c|c|c|c|c|c|c|c|c|c|c|c|c|c|c|}
\hline \multirow[b]{2}{*}{$\begin{array}{l}\text { Metallic } \\
\text { fuels }\end{array}$} & \multicolumn{13}{|c|}{ Legal weight truck packages } & \multicolumn{2}{|c|}{$\begin{array}{c}\text { Overweight } \\
\text { truck packages }\end{array}$} & \multicolumn{8}{|c|}{ Rail packages } \\
\hline & $\begin{array}{l}\mathrm{GE} \\
\mathrm{T}-2\end{array}$ & $\begin{array}{l}\text { GE- } \\
100\end{array}$ & BMI-1 & FSV-1 & $\begin{array}{l}\text { NLI- } \\
1 / 2\end{array}$ & $\begin{array}{l}\text { NLI- } \\
6502\end{array}$ & T-3 & NAC-1 & $\begin{array}{c}\text { CNS } \\
1-13 G\end{array}$ & NRBK-41 & $\begin{array}{l}\text { NAC- } \\
\text { LWT }\end{array}$ & $\begin{array}{c}\text { GE Model } \\
2000\end{array}$ & $\begin{array}{l}\text { TN- } \\
\text { FSV }\end{array}$ & $\begin{array}{l}\text { TN-8 } \\
\text { TN-8L }\end{array}$ & $|\mathrm{TN}-9|$ & $\mathrm{M}-130$ & $\begin{array}{l}\text { IF- } \\
300\end{array}$ & $\begin{array}{l}\text { NLI- } \\
10 / 24\end{array}$ & $125-\mathrm{B}$ & $\left|\begin{array}{c}\mathrm{TN}- \\
\mathrm{BRP}\end{array}\right|$ & $\begin{array}{l}\text { TN- } \\
\text { REG }\end{array}$ & M-160 & M-140 \\
\hline $\begin{array}{c}\text { TRIGA AL } \\
\text { (INEL) }\end{array}$ & $\begin{array}{c}\text { Yes } \\
\text { C }\end{array}$ & $\begin{array}{c}\text { No } \\
\text { a }\end{array}$ & $\begin{array}{c}\text { Yes } \\
\text { A }\end{array}$ & $\begin{array}{c}\text { Yes } \\
\text { C }\end{array}$ & $\begin{array}{l}\text { Yes } \\
\text { C }\end{array}$ & $\begin{array}{c}\text { Yes } \\
\text { C }\end{array}$ & $\begin{array}{l}\text { Yes } \\
\text { C }\end{array}$ & $\begin{array}{c}\text { Yes } \\
\text { C }\end{array}$ & $\begin{array}{c}\text { Yes } \\
\text { C }\end{array}$ & $\begin{array}{c}\text { No } \\
\text { a }\end{array}$ & $\begin{array}{c}\text { Yes } \\
\text { C }\end{array}$ & $\begin{array}{c}\text { Yes } \\
\text { C }\end{array}$ & $\begin{array}{c}\text { Yes } \\
\text { C }\end{array}$ & $\begin{array}{c}\text { Yes } \\
\text { C }\end{array}$ & $\begin{array}{c}\text { Yes } \\
\text { C }\end{array}$ & $\begin{array}{l}\text { Yes } \\
\text { C }\end{array}$ & $\begin{array}{c}\text { Yes } \\
\text { C }\end{array}$ & $\begin{array}{c}\text { Yes } \\
\text { C }\end{array}$ & $\begin{array}{l}\text { Yes } \\
\text { C }\end{array}$ & $\begin{array}{c}\text { Yes } \\
\text { C }\end{array}$ & $\begin{array}{c}\text { Yes } \\
\text { C }\end{array}$ & $\begin{array}{l}\text { Yes } \\
\text { C }\end{array}$ & $\begin{array}{l}\text { Yes } \\
\text { C }\end{array}$ \\
\hline $\begin{array}{c}\text { TRIGA } \\
\text { FLIP } \\
\text { (INEL) }\end{array}$ & $\begin{array}{l}\text { Yes } \\
\text { C }\end{array}$ & $\begin{array}{c}\text { No } \\
\text { a }\end{array}$ & $\begin{array}{c}\text { Yes } \\
\text { A }\end{array}$ & $\begin{array}{c}\text { Yes } \\
\text { C }\end{array}$ & $\begin{array}{l}\text { Yes } \\
\text { C }\end{array}$ & $\begin{array}{c}\text { Yes } \\
\text { C }\end{array}$ & $\begin{array}{c}\text { Yes } \\
\text { C }\end{array}$ & $\begin{array}{l}\text { Yes } \\
\text { C }\end{array}$ & $\begin{array}{l}\text { Yes } \\
\text { C }\end{array}$ & $\begin{array}{l}\text { No } \\
\text { a }\end{array}$ & $\begin{array}{c}\text { Yes } \\
\text { C }\end{array}$ & $\begin{array}{c}\text { Yes } \\
\text { C }\end{array}$ & $\begin{array}{c}\text { Yes } \\
\text { C }\end{array}$ & $\begin{array}{l}\text { Yes } \\
\text { C }\end{array}$ & $\begin{array}{c}\text { Yes } \\
\text { C }\end{array}$ & $\begin{array}{l}\text { Yes } \\
\text { C }\end{array}$ & $\begin{array}{c}\text { Yes } \\
\text { C }\end{array}$ & $\begin{array}{c}\text { Yes } \\
\text { C }\end{array}$ & $\begin{array}{l}\text { Yes } \\
\text { C }\end{array}$ & $\begin{array}{l}\text { Yes } \\
\text { C }\end{array}$ & $\begin{array}{l}\text { Yes } \\
\text { C }\end{array}$ & $\begin{array}{c}\text { Yes } \\
\text { C }\end{array}$ & $\begin{array}{l}\text { Yes } \\
\text { C }\end{array}$ \\
\hline $\begin{array}{c}\text { TRIGA } \\
\text { FLIP } \\
\text { (ANL-W) }\end{array}$ & $\begin{array}{l}\text { Yes } \\
\text { C }\end{array}$ & $\begin{array}{c}\text { No } \\
\text { a }\end{array}$ & $\begin{array}{l}\text { Yes } \\
\mathrm{A}\end{array}$ & $\begin{array}{c}\text { Yes } \\
\text { C }\end{array}$ & $\begin{array}{l}\text { Yes } \\
\text { C }\end{array}$ & $\begin{array}{l}\text { Yes } \\
\text { C }\end{array}$ & $\begin{array}{c}\text { Yes } \\
\text { C }\end{array}$ & $\begin{array}{c}\text { Yes } \\
\text { C }\end{array}$ & $\begin{array}{l}\text { Yes } \\
\text { C }\end{array}$ & $\begin{array}{c}\text { No } \\
\text { a }\end{array}$ & $\begin{array}{c}\text { Yes } \\
\text { C }\end{array}$ & $\begin{array}{l}\text { Yes } \\
\text { C }\end{array}$ & $\begin{array}{l}\text { Yes } \\
\text { C }\end{array}$ & $\begin{array}{c}\text { Yes } \\
\text { C }\end{array}$ & $\begin{array}{c}\text { Yes } \\
\text { C }\end{array}$ & $\begin{array}{c}\text { Yes } \\
\text { C }\end{array}$ & $\begin{array}{c}\text { Yes } \\
\text { C }\end{array}$ & $\begin{array}{c}\text { Yes } \\
\text { C }\end{array}$ & $\begin{array}{l}\text { Yes } \\
\text { C }\end{array}$ & $\begin{array}{l}\text { Yes } \\
\text { C }\end{array}$ & $\begin{array}{c}\text { Yes } \\
\text { C }\end{array}$ & $\begin{array}{l}\text { Yes } \\
\text { C }\end{array}$ & $\begin{array}{c}\text { Yes } \\
\text { C }\end{array}$ \\
\hline $\begin{array}{c}\text { TRIGA SST } \\
\text { (INEL) }\end{array}$ & $\begin{array}{c}\text { Yes } \\
\text { C }\end{array}$ & $\begin{array}{c}\text { No } \\
\mathrm{a}\end{array}$ & $\begin{array}{c}\text { Yes } \\
\text { A }\end{array}$ & $\begin{array}{l}\text { Yes } \\
\text { C }\end{array}$ & $\begin{array}{l}\text { Yes } \\
\text { C }\end{array}$ & $\begin{array}{l}\text { Yes } \\
\text { C }\end{array}$ & $\begin{array}{c}\text { Yes } \\
\text { C }\end{array}$ & $\begin{array}{l}\text { Yes } \\
\text { C }\end{array}$ & $\begin{array}{c}\text { Yes } \\
\text { C }\end{array}$ & $\begin{array}{c}\text { No } \\
\text { a }\end{array}$ & $\begin{array}{l}\text { Yes } \\
\text { C }\end{array}$ & $\begin{array}{l}\text { Yes } \\
\text { C }\end{array}$ & $\begin{array}{l}\text { Yes } \\
\mathrm{C}\end{array}$ & $\begin{array}{l}\text { Yes } \\
\text { C }\end{array}$ & $\begin{array}{c}\text { Yes } \\
\text { C }\end{array}$ & $\begin{array}{l}\text { Yes } \\
\text { C }\end{array}$ & $\begin{array}{c}\text { Yes } \\
\text { C }\end{array}$ & $\begin{array}{l}\text { Yes } \\
\text { C }\end{array}$ & $\begin{array}{l}\text { Yes } \\
\text { C }\end{array}$ & $\begin{array}{c}\text { Yes } \\
\text { C }\end{array}$ & $\begin{array}{l}\text { Yes } \\
\text { C }\end{array}$ & $\begin{array}{c}\text { Yes } \\
\text { C }\end{array}$ & $\begin{array}{c}\text { Yes } \\
\text { C }\end{array}$ \\
\hline $\begin{array}{c}\text { TRIGA SST } \\
\text { (Hanford) }\end{array}$ & Yes & $\begin{array}{c}\text { No } \\
\text { a }\end{array}$ & $\begin{array}{c}\text { Yes } \\
\text { A }\end{array}$ & $\begin{array}{c}\text { Yes } \\
\text { C }\end{array}$ & $\begin{array}{l}\text { Yes } \\
\text { C }\end{array}$ & $\begin{array}{l}\text { Yes } \\
\text { C }\end{array}$ & $\begin{array}{c}\text { Yes } \\
\text { C }\end{array}$ & $\begin{array}{c}\text { Yes } \\
\text { C }\end{array}$ & $\begin{array}{c}\text { Yes } \\
\text { C }\end{array}$ & $\begin{array}{c}\text { No } \\
\text { a }\end{array}$ & $\begin{array}{l}\text { Yes } \\
\text { C }\end{array}$ & $\begin{array}{l}\text { Yes } \\
\text { C }\end{array}$ & $\begin{array}{l}\text { Yes } \\
\text { C }\end{array}$ & $\begin{array}{l}\text { Yes } \\
\text { C }\end{array}$ & $\begin{array}{c}\text { Yes } \\
\text { C }\end{array}$ & $\begin{array}{l}\text { Yes } \\
\text { C }\end{array}$ & $\begin{array}{c}\text { Yes } \\
\text { C }\end{array}$ & $\begin{array}{l}\text { Yes } \\
\text { C }\end{array}$ & $\begin{array}{l}\text { Yes } \\
\text { C }\end{array}$ & $\begin{array}{l}\text { Yes } \\
\text { C }\end{array}$ & $\begin{array}{c}\text { Yes } \\
\text { C }\end{array}$ & $\begin{array}{c}\text { Yes } \\
\text { C }\end{array}$ & $\begin{array}{c}\text { Yes } \\
\text { C }\end{array}$ \\
\hline $\begin{array}{l}\text { TRIGA } \\
\text { Ass'y } \\
\text { (Hanford) }\end{array}$ & $\begin{array}{c}\text { Yes } \\
\text { C }\end{array}$ & $\begin{array}{c}\text { No } \\
\text { a }\end{array}$ & $\begin{array}{c}\text { Yes } \\
\text { A }\end{array}$ & $\begin{array}{l}\text { Yes } \\
\text { C }\end{array}$ & $\begin{array}{l}\text { Yes } \\
\text { C }\end{array}$ & $\begin{array}{l}\text { Yes } \\
\text { C }\end{array}$ & $\begin{array}{c}\text { Yes } \\
\text { C }\end{array}$ & $\begin{array}{l}\text { Yes } \\
\text { C }\end{array}$ & $\begin{array}{l}\text { Yes } \\
\text { C }\end{array}$ & $\begin{array}{c}\text { No } \\
\text { a }\end{array}$ & $\begin{array}{l}\text { Yes } \\
\text { C }\end{array}$ & $\begin{array}{c}\text { Yes } \\
C\end{array}$ & $\begin{array}{l}\text { Yes } \\
\text { C }\end{array}$ & $\begin{array}{c}\text { Yes } \\
\text { c }\end{array}$ & $\begin{array}{c}\text { Yes } \\
\text { C }\end{array}$ & $\begin{array}{l}\text { Yes } \\
\text { C }\end{array}$ & $\begin{array}{c}\text { Yes } \\
\text { C }\end{array}$ & $\begin{array}{l}\text { Yes } \\
\text { C }\end{array}$ & $\begin{array}{l}\text { Yes } \\
\text { C }\end{array}$ & $\begin{array}{l}\text { Yes } \\
\text { C }\end{array}$ & $\begin{array}{l}\text { Yes } \\
\text { C }\end{array}$ & $\begin{array}{c}\text { Yes } \\
\text { C }\end{array}$ & $\begin{array}{l}\text { Yes } \\
\text { C }\end{array}$ \\
\hline $\begin{array}{l}\text { TRIGA } \\
\text { BER-II }\end{array}$ & $\begin{array}{c}\text { Yes } \\
\text { C }\end{array}$ & $\begin{array}{c}\text { No } \\
a\end{array}$ & $\begin{array}{c}\text { Yes } \\
\text { B }\end{array}$ & $\begin{array}{c}\text { Yes } \\
\text { C }\end{array}$ & $\begin{array}{l}\text { Yes } \\
\text { C }\end{array}$ & $\begin{array}{l}\text { Yes } \\
\text { C }\end{array}$ & $\begin{array}{c}\text { Yes } \\
\text { C }\end{array}$ & $\begin{array}{l}\text { Yes } \\
\text { C }\end{array}$ & $\begin{array}{l}\text { Yes } \\
\text { C }\end{array}$ & $\begin{array}{c}\text { No } \\
\text { a }\end{array}$ & $\begin{array}{l}\text { Yes } \\
\text { C }\end{array}$ & $\begin{array}{l}\text { Yes } \\
\text { C }\end{array}$ & $\begin{array}{l}\text { Yes } \\
\text { C }\end{array}$ & $\begin{array}{l}\text { Yes } \\
\text { C }\end{array}$ & $\begin{array}{l}\text { Yes } \\
\text { C }\end{array}$ & $\begin{array}{l}\text { Yes } \\
\text { C }\end{array}$ & $\left|\begin{array}{c}\text { Yes } \\
\text { C }\end{array}\right|$ & $\begin{array}{l}\text { Yes } \\
\text { C }\end{array}$ & $\begin{array}{l}\text { Yes } \\
\text { C }\end{array}$ & $\begin{array}{l}\text { Yes } \\
\text { C }\end{array}$ & $\begin{array}{c}\text { Yes } \\
\text { C }\end{array}$ & $\begin{array}{l}\text { Yes } \\
\text { C }\end{array}$ & $\begin{array}{c}\text { Yes } \\
\text { C }\end{array}$ \\
\hline $\begin{array}{c}\text { Areotest } \\
\text { TRIGA } \\
\text { (San } \\
\text { Ramon) }\end{array}$ & $\begin{array}{c}\text { Yes } \\
\text { C }\end{array}$ & $\begin{array}{c}\text { No } \\
\text { a }\end{array}$ & $\begin{array}{c}\text { Yes } \\
\text { B }\end{array}$ & $\begin{array}{l}\text { Yes } \\
\text { C }\end{array}$ & $\begin{array}{c}\text { Yes } \\
\text { C }\end{array}$ & $\begin{array}{l}\text { Yes } \\
\text { C }\end{array}$ & $\begin{array}{c}\text { Yes } \\
\text { C }\end{array}$ & $\begin{array}{c}\text { Yes } \\
\text { C }\end{array}$ & $\begin{array}{c}\text { Yes } \\
\text { C }\end{array}$ & $\begin{array}{c}\text { No } \\
\text { a }\end{array}$ & $\begin{array}{l}\text { Yes } \\
\text { C }\end{array}$ & $\begin{array}{l}\text { Yes } \\
\text { C }\end{array}$ & $\begin{array}{l}\text { Yes } \\
\text { C }\end{array}$ & $\begin{array}{l}\text { Yes } \\
\text { C }\end{array}$ & $\begin{array}{l}\text { Yes } \\
\text { C }\end{array}$ & $\begin{array}{l}\text { Yes } \\
\text { C }\end{array}$ & $\mid \begin{array}{c}\text { Yes } \\
\text { C }\end{array}$ & $\begin{array}{c}\text { Yes } \\
\text { C }\end{array}$ & $\begin{array}{l}\text { Yes } \\
\text { C }\end{array}$ & $\begin{array}{c}\text { Yes } \\
\text { C }\end{array}$ & \begin{tabular}{|c|} 
Yes \\
C
\end{tabular} & $\begin{array}{c}\text { Yes } \\
\text { C }\end{array}$ & $\begin{array}{c}\text { Yes } \\
\text { C }\end{array}$ \\
\hline $\begin{array}{l}\text { AFRRI- } \\
\text { TRIGA }\end{array}$ & $\begin{array}{l}\text { Yes } \\
\text { C }\end{array}$ & $\begin{array}{c}\text { No } \\
a\end{array}$ & $\begin{array}{c}\text { Yes } \\
\text { B }\end{array}$ & $\begin{array}{l}\text { Yes } \\
\text { C }\end{array}$ & $\begin{array}{l}\text { Yes } \\
\text { C }\end{array}$ & $\begin{array}{l}\text { Yes } \\
\text { C }\end{array}$ & $\begin{array}{c}\text { Yes } \\
\mathrm{C}\end{array}$ & $\begin{array}{l}\text { Yes } \\
\text { C }\end{array}$ & $\begin{array}{l}\text { Yes } \\
\text { C }\end{array}$ & $\begin{array}{c}\text { No } \\
\mathrm{a}\end{array}$ & $\begin{array}{l}\text { Yes } \\
\text { C }\end{array}$ & $\begin{array}{l}\text { Yes } \\
\text { C }\end{array}$ & $\begin{array}{l}\text { Yes } \\
\text { C }\end{array}$ & $\begin{array}{l}\text { Yes } \\
\text { C }\end{array}$ & $\begin{array}{c}\text { Yes } \\
\text { C }\end{array}$ & $\begin{array}{c}\text { Yes } \\
\text { C }\end{array}$ & $\begin{array}{c}\text { Yes } \\
\text { C }\end{array}$ & $\begin{array}{l}\text { Yes } \\
\text { C }\end{array}$ & $\begin{array}{l}\text { Yes } \\
\text { C }\end{array}$ & $\begin{array}{c}\text { Yes } \\
\text { C }\end{array}$ & $\begin{array}{c}\text { Yes } \\
\mathrm{C}\end{array}$ & $\begin{array}{l}\text { Yes } \\
\text { C }\end{array}$ & $\begin{array}{l}\text { Yes } \\
\text { C }\end{array}$ \\
\hline GA-TRIGA & $\begin{array}{c}\text { Yes } \\
\text { C }\end{array}$ & $\begin{array}{c}\text { No } \\
a\end{array}$ & $\begin{array}{l}\text { Yes } \\
\text { B }\end{array}$ & $\begin{array}{l}\text { Yes } \\
\text { C }\end{array}$ & $\begin{array}{l}\text { Yes } \\
\text { C }\end{array}$ & $\begin{array}{c}\text { Yes } \\
\text { C }\end{array}$ & $\begin{array}{c}\text { Yes } \\
\text { C }\end{array}$ & $\begin{array}{l}\text { Yes } \\
\text { C }\end{array}$ & $\begin{array}{l}\text { Yes } \\
\text { C }\end{array}$ & $\begin{array}{c}\text { No } \\
\text { a }\end{array}$ & $\begin{array}{c}\text { Yes } \\
\text { C }\end{array}$ & $\begin{array}{l}\text { Yes } \\
\text { C }\end{array}$ & $\begin{array}{l}\text { Yes } \\
\text { C }\end{array}$ & $\begin{array}{c}\text { Yes } \\
\text { C }\end{array}$ & $\begin{array}{c}\text { Yes } \\
\text { C }\end{array}$ & $\begin{array}{c}\text { Yes } \\
\text { C }\end{array}$ & Yes & $\begin{array}{c}\text { Yes } \\
\text { C }\end{array}$ & $\begin{array}{c}\text { Yes } \\
\text { C }\end{array}$ & $\begin{array}{c}\text { Yes } \\
\text { C }\end{array}$ & $\begin{array}{c}\text { Yes } \\
\mathrm{C}\end{array}$ & $\begin{array}{c}\text { Yes } \\
\text { C }\end{array}$ & $\begin{array}{c}\text { Yes } \\
\text { C }\end{array}$ \\
\hline $\begin{array}{l}\text { USGS- } \\
\text { TRIGA }\end{array}$ & $\begin{array}{c}\text { Yes } \\
\text { C }\end{array}$ & $\begin{array}{c}\text { No } \\
\mathrm{a}\end{array}$ & $\begin{array}{l}\text { Yes } \\
\text { B }\end{array}$ & $\begin{array}{l}\text { Yes } \\
\text { C }\end{array}$ & $\begin{array}{l}\text { Yes } \\
\text { C }\end{array}$ & $\begin{array}{l}\text { Yes } \\
\text { C }\end{array}$ & $\begin{array}{c}\text { Yes } \\
\text { C }\end{array}$ & $\begin{array}{l}\text { Yes } \\
\text { C }\end{array}$ & $\begin{array}{l}\text { Yes } \\
\text { C }\end{array}$ & $\begin{array}{c}\text { No } \\
\mathrm{a}\end{array}$ & $\begin{array}{l}\text { Yes } \\
\text { C }\end{array}$ & $\begin{array}{c}\text { Yes } \\
\text { C }\end{array}$ & $\begin{array}{l}\text { Yes } \\
\text { C }\end{array}$ & $\begin{array}{l}\text { Yes } \\
\text { C }\end{array}$ & $\begin{array}{l}\text { Yes } \\
\text { C }\end{array}$ & $\begin{array}{c}\text { Yes } \\
\text { C }\end{array}$ & $\begin{array}{c}\text { Yes } \\
\text { C }\end{array}$ & $\begin{array}{l}\text { Yes } \\
\text { C }\end{array}$ & $\begin{array}{c}\text { Yes } \\
\text { C }\end{array}$ & $\begin{array}{c}\text { Yes } \\
\text { C }\end{array}$ & \begin{tabular}{|c|} 
Yes \\
C
\end{tabular} & $\begin{array}{l}\text { Yes } \\
\text { C }\end{array}$ & $\begin{array}{l}\text { Yes } \\
\text { C }\end{array}$ \\
\hline $\begin{array}{c}\text { TRIGA } \\
\text { (McLellam) }\end{array}$ & $\begin{array}{c}\text { Yes } \\
\text { C }\end{array}$ & $\begin{array}{c}\text { No } \\
\text { a }\end{array}$ & $\begin{array}{c}\text { Yes } \\
\text { B }\end{array}$ & $\begin{array}{c}\text { Yes } \\
\text { C }\end{array}$ & $\begin{array}{c}\text { Yes } \\
\text { C }\end{array}$ & $\begin{array}{c}\text { Yes } \\
\text { C }\end{array}$ & $\begin{array}{c}\text { Yes } \\
\text { C }\end{array}$ & $\begin{array}{c}\text { Yes } \\
\text { C }\end{array}$ & $\begin{array}{l}\text { Yes } \\
\text { C }\end{array}$ & $\begin{array}{c}\text { No } \\
\mathrm{a}\end{array}$ & $\begin{array}{l}\text { Yes } \\
\text { C }\end{array}$ & $\begin{array}{c}\text { Yes } \\
\text { C }\end{array}$ & $\begin{array}{c}\text { Yes } \\
\text { C }\end{array}$ & $\begin{array}{c}\text { Yes } \\
\text { C }\end{array}$ & $\begin{array}{c}\text { Yes } \\
\text { C }\end{array}$ & $\begin{array}{c}\text { Yes } \\
\text { C }\end{array}$ & $\mid \begin{array}{c}\text { Yes } \\
\text { C }\end{array}$ & $\begin{array}{c}\text { Yes } \\
\text { C }\end{array}$ & $\begin{array}{l}\text { Yes } \\
\text { C }\end{array}$ & $\begin{array}{c}\text { Yes } \\
\text { C }\end{array}$ & $\begin{array}{c}\text { Yes } \\
\text { C }\end{array}$ & $\begin{array}{c}\text { Yes } \\
\text { C }\end{array}$ & $\begin{array}{c}\text { Yes } \\
\text { C }\end{array}$ \\
\hline
\end{tabular}




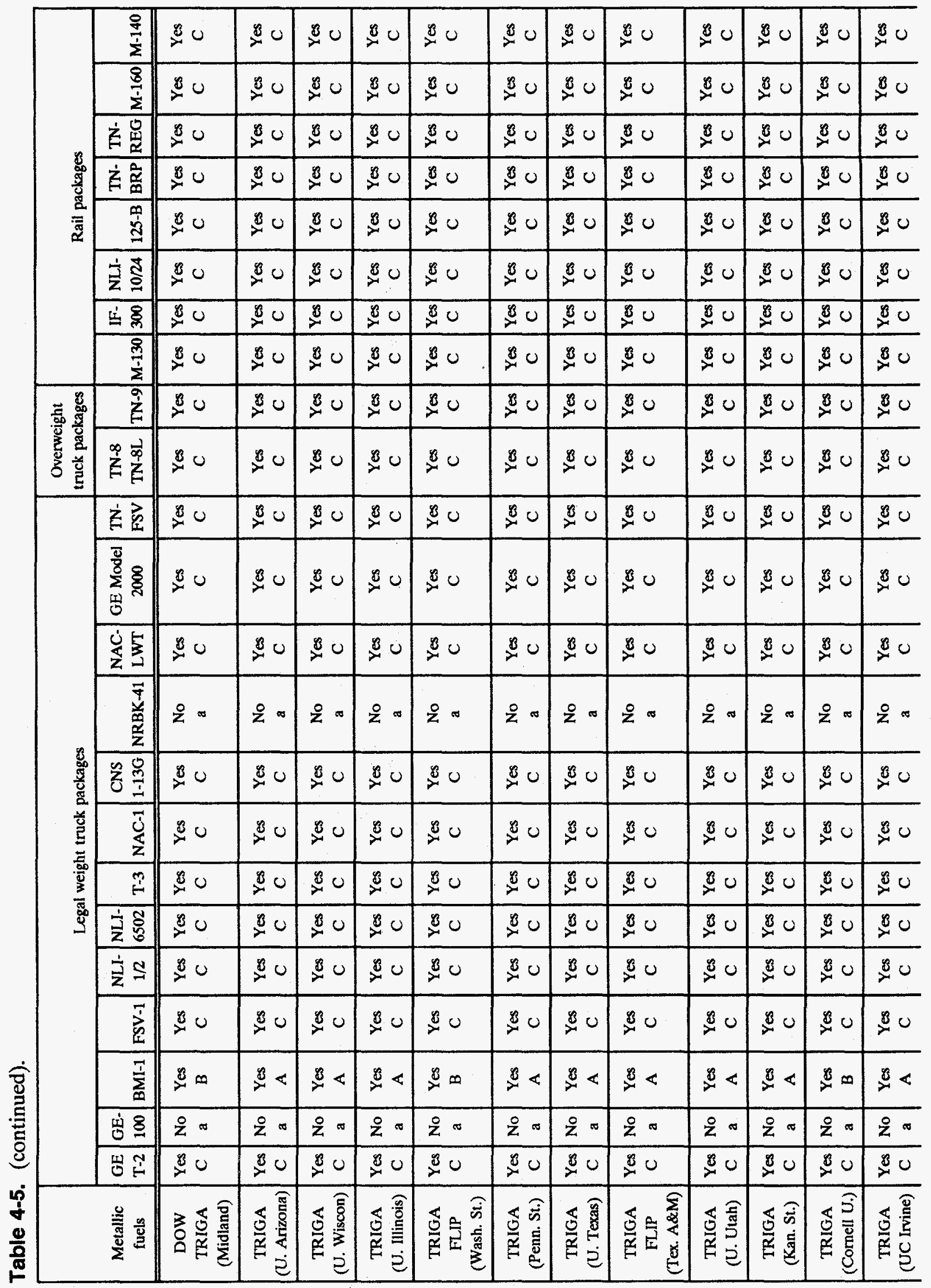


Table 4-5. (continued).

\begin{tabular}{|c|c|c|c|c|c|c|c|c|c|c|c|c|c|c|c|c|c|c|c|c|c|c|c|}
\hline \multirow[b]{2}{*}{$\begin{array}{c}\text { Metallic } \\
\text { fuels }\end{array}$} & \multicolumn{13}{|c|}{ Legal weight truck packages } & \multicolumn{2}{|c|}{$\begin{array}{c}\text { Overweight } \\
\text { truck packages }\end{array}$} & \multicolumn{8}{|c|}{ Rail packages } \\
\hline & $\begin{array}{l}\mathrm{GE} \\
\mathrm{T}-2\end{array}$ & $\begin{array}{l}\mathrm{GE} \\
100 \\
\end{array}$ & BMI-1 & FSV-1 & $\begin{array}{l}\mathrm{NLI}- \\
1 / 2\end{array}$ & $\begin{array}{l}\mathrm{NLI}- \\
6502\end{array}$ & $\mathrm{~T}-\mathbf{3}$ & NAC-1 & \begin{tabular}{|c|}
$\mathrm{CNS}$ \\
$1-13 \mathrm{G}$
\end{tabular} & NRBK-41 & $\begin{array}{l}\text { NAC- } \\
\text { LWT }\end{array}$ & $\begin{array}{c}\text { GE Model } \\
2000\end{array}$ & $\begin{array}{l}\text { TN- } \\
\text { FSV }\end{array}$ & $\begin{array}{l}\text { TN-8 } \\
\text { TN-8L }\end{array}$ & TN-9 & M-130 & $\begin{array}{l}\text { IF- } \\
300\end{array}$ & $\begin{array}{l}\text { NLI- } \\
10 / 24\end{array}$ & $125-\mathrm{B}$ & $\begin{array}{l}\text { TN- } \\
\text { BRP }\end{array}$ & $\begin{array}{c}\text { TN- } \\
\text { REG }\end{array}$ & M-160 & M-14 \\
\hline $\begin{array}{c}\text { TRIGA } \\
\text { (U. Mrylnd) }\end{array}$ & $\begin{array}{c}\text { Yes } \\
\text { C }\end{array}$ & $\begin{array}{c}\text { No } \\
\text { a }\end{array}$ & $\begin{array}{c}\text { Yes } \\
\text { A }\end{array}$ & $\begin{array}{l}\text { Yes } \\
\text { C }\end{array}$ & $\begin{array}{l}\text { Yes } \\
\text { C }\end{array}$ & $\begin{array}{l}\text { Yes } \\
\text { C }\end{array}$ & $\begin{array}{l}\text { Yes } \\
\text { C }\end{array}$ & $\begin{array}{c}\text { Yes } \\
\text { C }\end{array}$ & $\begin{array}{l}\text { Yes } \\
\text { C }\end{array}$ & $\begin{array}{c}\text { No } \\
\mathrm{a}\end{array}$ & $\begin{array}{l}\text { Yes } \\
\text { C }\end{array}$ & $\begin{array}{c}\text { Yes } \\
\text { C }\end{array}$ & $\begin{array}{l}\text { Yes } \\
\text { C }\end{array}$ & $\begin{array}{c}\text { Yes } \\
\text { C }\end{array}$ & $\begin{array}{c}\text { Yes } \\
\text { C }\end{array}$ & $\begin{array}{l}\text { Yes } \\
\text { C }\end{array}$ & $\begin{array}{c}\text { Yes } \\
\text { C }\end{array}$ & $\begin{array}{c}\text { Yes } \\
\text { C }\end{array}$ & $\begin{array}{c}\text { Yes } \\
\text { C }\end{array}$ & $\begin{array}{l}\text { Yes } \\
\text { C }\end{array}$ & \begin{tabular}{|c|} 
Yes \\
C
\end{tabular} & $\begin{array}{c}\text { Yes } \\
\text { C }\end{array}$ & $\begin{array}{c}\text { Yes } \\
\text { C }\end{array}$ \\
\hline $\begin{array}{c}\text { TRIGA } \\
\text { FLIP } \\
\text { (Oregon St) }\end{array}$ & $\begin{array}{c}\text { Yes } \\
\mathrm{C}\end{array}$ & $\begin{array}{c}\text { No } \\
\text { a }\end{array}$ & $\begin{array}{c}\text { Yes } \\
\text { A }\end{array}$ & $\begin{array}{l}\text { Yes } \\
\text { c }\end{array}$ & $\begin{array}{l}\text { Yes } \\
\text { C }\end{array}$ & $\begin{array}{l}\text { Yes } \\
\text { C }\end{array}$ & $\begin{array}{l}\text { Yes } \\
\text { C }\end{array}$ & $\begin{array}{l}\text { Yes } \\
\text { c }\end{array}$ & $\begin{array}{l}\text { Yes } \\
\text { C }\end{array}$ & $\begin{array}{l}\text { No } \\
\text { a }\end{array}$ & $\begin{array}{c}\text { Yes } \\
\text { C }\end{array}$ & $\begin{array}{c}\text { Yes } \\
\text { C }\end{array}$ & $\begin{array}{c}\text { Yes } \\
\text { C }\end{array}$ & $\begin{array}{c}\text { Yes } \\
\text { C }\end{array}$ & $\begin{array}{c}\text { Yes } \\
\text { C }\end{array}$ & $\begin{array}{l}\text { Yes } \\
\text { C }\end{array}$ & $\mid \begin{array}{c}\text { Yes } \\
\text { C }\end{array}$ & $\begin{array}{c}\text { Yes } \\
\text { C }\end{array}$ & $\begin{array}{c}\text { Yes } \\
\text { C }\end{array}$ & $\begin{array}{c}\text { Yes } \\
\text { C }\end{array}$ & $\begin{array}{c}\text { Yes } \\
\text { C }\end{array}$ & $\begin{array}{l}\text { Yes } \\
\text { C }\end{array}$ & $\begin{array}{l}\text { Yes } \\
\text { C }\end{array}$ \\
\hline $\begin{array}{c}\text { TRIGA } \\
\text { (Reed U.) }\end{array}$ & $\begin{array}{c}\text { Yes } \\
\text { C }\end{array}$ & $\begin{array}{c}\text { No } \\
\text { a }\end{array}$ & $\begin{array}{c}\text { Yes } \\
\text { A }\end{array}$ & $\begin{array}{l}\text { Yes } \\
\text { C }\end{array}$ & $\begin{array}{l}\text { Yes } \\
\text { C }\end{array}$ & $\begin{array}{c}\text { Yes } \\
\mathrm{C}\end{array}$ & $\begin{array}{l}\text { Yes } \\
\text { C }\end{array}$ & $\begin{array}{l}\text { Yes } \\
\text { c }\end{array}$ & $\begin{array}{c}\text { Yes } \\
\text { c }\end{array}$ & $\begin{array}{c}\text { No } \\
\text { a }\end{array}$ & $\begin{array}{l}\text { Yes } \\
\text { c }\end{array}$ & $\begin{array}{l}\text { Yes } \\
\text { C }\end{array}$ & $\begin{array}{l}\text { Yes } \\
\text { C }\end{array}$ & $\begin{array}{c}\text { Yes } \\
\text { C }\end{array}$ & $\begin{array}{c}\text { Yes } \\
\text { C }\end{array}$ & $\begin{array}{l}\text { Yes } \\
\text { C }\end{array}$ & $\begin{array}{c}\text { Yes } \\
\text { C }\end{array}$ & $\begin{array}{c}\text { Yes } \\
\text { C }\end{array}$ & $\begin{array}{l}\text { Yes } \\
\text { C }\end{array}$ & $\begin{array}{l}\text { Yes } \\
\text { C }\end{array}$ & $\begin{array}{l}\text { Yes } \\
\text { C }\end{array}$ & $\begin{array}{l}\text { Yes } \\
\text { C }\end{array}$ & $\begin{array}{c}\text { Yes } \\
\text { C }\end{array}$ \\
\hline SNAP & $\begin{array}{c}\text { Yes } \\
\text { C }\end{array}$ & $\begin{array}{c}\text { No } \\
\text { a }\end{array}$ & $\begin{array}{c}\text { Yes } \\
\text { C }\end{array}$ & $\begin{array}{l}\text { Yes } \\
\text { C }\end{array}$ & $\begin{array}{l}\text { Yes } \\
\text { C }\end{array}$ & $\begin{array}{l}\text { Yes } \\
\text { C }\end{array}$ & $\begin{array}{l}\text { Yes } \\
\text { C }\end{array}$ & $\begin{array}{c}\text { Yes } \\
\text { C }\end{array}$ & $\begin{array}{l}\text { Yes } \\
\text { C }\end{array}$ & $\begin{array}{c}\text { No } \\
\text { a }\end{array}$ & $\begin{array}{l}\text { Yes } \\
\text { C }\end{array}$ & $\begin{array}{l}\text { Yes } \\
\text { C }\end{array}$ & $\begin{array}{l}\text { Yes } \\
\text { C }\end{array}$ & $\begin{array}{l}\text { Yes } \\
\text { C }\end{array}$ & $\begin{array}{c}\text { Yes } \\
\text { C }\end{array}$ & $\begin{array}{c}N / A \\
b\end{array}$ & $\begin{array}{c}\text { Yes } \\
\text { C }\end{array}$ & $\begin{array}{c}\text { Yes } \\
\text { C }\end{array}$ & $\begin{array}{l}\text { Yes } \\
\text { C }\end{array}$ & $\begin{array}{c}\text { Yes } \\
\text { C }\end{array}$ & $\begin{array}{c}\text { Yes } \\
\text { C }\end{array}$ & $\begin{array}{c}\text { N/A } \\
b\end{array}$ & $\begin{array}{c}\text { N/A } \\
b\end{array}$ \\
\hline $\begin{array}{l}\text { MURR } \\
\text { (INEL) }\end{array}$ & $\begin{array}{c}\text { No } \\
a\end{array}$ & $\begin{array}{c}\text { No } \\
\text { a }\end{array}$ & $\begin{array}{c}N / A \\
b\end{array}$ & $\begin{array}{c}\text { N/A } \\
b\end{array}$ & $\begin{array}{c}\mathrm{N} / \mathrm{A} \\
\mathrm{b}\end{array}$ & $\begin{array}{c}\text { No } \\
\mathrm{a}\end{array}$ & $\begin{array}{c}\text { No } \\
\text { a }\end{array}$ & $\begin{array}{c}\text { N/A } \\
\text { b }\end{array}$ & $\begin{array}{c}\text { N/A } \\
b\end{array}$ & $\begin{array}{c}\text { No } \\
\text { a }\end{array}$ & $\begin{array}{c}\text { N/A } \\
b\end{array}$ & $\begin{array}{c}\text { N/A } \\
b\end{array}$ & $\begin{array}{c}\text { No } \\
\text { a }\end{array}$ & $\begin{array}{c}\mathrm{N} / \mathrm{A} \\
b\end{array}$ & $\begin{array}{c}\text { N/A } \\
b\end{array}$ & $\begin{array}{c}\text { N/A } \\
b\end{array}$ & $\begin{array}{l}\mathrm{N} / \\
\mathrm{A}\end{array}$ & $\begin{array}{c}\text { N/A } \\
b\end{array}$ & $\begin{array}{c}\mathrm{N} / \mathrm{A} \\
\mathrm{b}\end{array}$ & $\begin{array}{c}\mathrm{N} / \mathrm{A} \\
\mathrm{b}\end{array}$ & $\begin{array}{c}\mathrm{N} / \mathrm{A} \\
\mathrm{b}\end{array}$ & $\begin{array}{c}\text { N/A } \\
b\end{array}$ & $\begin{array}{c}\text { N/A } \\
b\end{array}$ \\
\hline $\begin{array}{c}\text { MURR } \\
\text { (SRS) }\end{array}$ & $\begin{array}{c}\text { No } \\
\text { a }\end{array}$ & $\begin{array}{c}\text { No } \\
\text { a }\end{array}$ & $\begin{array}{c}\text { No } \\
\text { a }\end{array}$ & $\begin{array}{c}\text { N/A } \\
b\end{array}$ & $\begin{array}{c}\mathrm{N} / \mathrm{A} \\
\mathrm{b}\end{array}$ & $\begin{array}{c}\text { No } \\
\text { a }\end{array}$ & $\begin{array}{c}\text { No } \\
\text { a }\end{array}$ & $\begin{array}{c}\text { N/A } \\
b\end{array}$ & $\begin{array}{c}\text { No } \\
\text { a }\end{array}$ & $\begin{array}{c}\text { No } \\
a\end{array}$ & $\begin{array}{c}\text { N/A } \\
b\end{array}$ & $\begin{array}{c}\text { No } \\
\text { a }\end{array}$ & $\begin{array}{c}\text { N/A } \\
b\end{array}$ & $\begin{array}{c}\mathrm{N} / \mathrm{A} \\
b\end{array}$ & $\begin{array}{c}\text { N/A } \\
b\end{array}$ & $\begin{array}{c}\text { No } \\
\text { a }\end{array}$ & $\begin{array}{l}\mathbf{N} / \\
\mathrm{A}\end{array}$ & $\begin{array}{c}\text { N/A } \\
b\end{array}$ & $\begin{array}{c}\text { N/A } \\
b\end{array}$ & $\begin{array}{c}\mathrm{N} / \mathrm{A} \\
\mathrm{b}\end{array}$ & $\begin{array}{c}\text { No } \\
\text { a }\end{array}$ & $\begin{array}{c}\text { No } \\
\text { a }\end{array}$ & $\begin{array}{c}\text { No } \\
a\end{array}$ \\
\hline $\begin{array}{c}\text { MURR } \\
\text { (U. Missouri } \\
\text { Columbia) }\end{array}$ & $\begin{array}{c}\mathrm{N} / \mathrm{A} \\
\mathrm{b}\end{array}$ & $\begin{array}{c}\text { No } \\
\text { a }\end{array}$ & $\begin{array}{c}\mathrm{N} / \mathrm{A} \\
\mathrm{b}\end{array}$ & $\begin{array}{c}\text { N/A } \\
b\end{array}$ & $\begin{array}{c}\mathrm{N} / \mathrm{A} \\
\mathrm{b}\end{array}$ & $\begin{array}{c}\mathrm{N} / \mathrm{A} \\
\mathrm{b}\end{array}$ & $\begin{array}{c}\text { N/A } \\
b\end{array}$ & $\begin{array}{c}\text { N/A } \\
b\end{array}$ & $\begin{array}{c}\mathrm{N} / \mathrm{A} \\
b\end{array}$ & $\begin{array}{c}\text { No } \\
\text { a }\end{array}$ & $\begin{array}{c}\text { N/A } \\
b\end{array}$ & $\begin{array}{c}\text { N/A } \\
b\end{array}$ & $\begin{array}{c}\mathrm{N} / \mathrm{A} \\
\mathrm{b}\end{array}$ & $\begin{array}{c}\text { N/A } \\
a\end{array}$ & $\begin{array}{c}\text { N/A } \\
a\end{array}$ & $\begin{array}{c}N / A \\
b\end{array}$ & $\begin{array}{l}\mathbf{N} / \\
\mathbf{A} \\
\mathrm{b}\end{array}$ & $\begin{array}{c}\mathrm{N} / \mathrm{A} \\
\mathrm{b}\end{array}$ & $\begin{array}{c}\mathrm{N} / \mathrm{A} \\
\mathrm{b}\end{array}$ & $\begin{array}{c}\mathrm{N} / \mathrm{A} \\
\mathrm{b}\end{array}$ & $\begin{array}{c}\text { N/A } \\
\text { b }\end{array}$ & $\begin{array}{c}\text { N/A } \\
\text { b }\end{array}$ & $\begin{array}{c}\text { N/A } \\
\text { b }\end{array}$ \\
\hline $\begin{array}{l}\text { MURR } \\
\text { (U. Missouri } \\
\text { Rolla) }\end{array}$ & $\begin{array}{c}\mathrm{N} / \mathrm{A} \\
\mathrm{b}\end{array}$ & $\begin{array}{c}\text { No } \\
a\end{array}$ & $\begin{array}{c}\mathrm{N} / \mathrm{A} \\
\mathrm{b}\end{array}$ & $\begin{array}{c}\text { N/A } \\
b\end{array}$ & $\begin{array}{c}\text { N/A } \\
b\end{array}$ & $\begin{array}{c}\text { N/A } \\
b\end{array}$ & $\begin{array}{c}\text { N/A } \\
\text { b }\end{array}$ & $\begin{array}{c}\mathrm{N} / \mathrm{A} \\
\mathrm{b}\end{array}$ & $\begin{array}{c}\text { N/A } \\
b\end{array}$ & $\begin{array}{c}\text { No } \\
\text { a }\end{array}$ & $\begin{array}{c}\text { N/A } \\
b\end{array}$ & $\begin{array}{c}\text { N/A } \\
\text { b }\end{array}$ & $\begin{array}{c}\mathrm{N} / \mathrm{A} \\
\mathrm{b}\end{array}$ & $\begin{array}{c}\text { N/A } \\
\text { b }\end{array}$ & $\begin{array}{c}\text { N/A } \\
\text { b }\end{array}$ & $\begin{array}{c}\text { N/A } \\
\text { b }\end{array}$ & $\begin{array}{l}\text { N/ } \\
\text { A } \\
\text { b }\end{array}$ & $\begin{array}{c}\text { N/A } \\
b\end{array}$ & $\begin{array}{c}\text { N/A } \\
b\end{array}$ & $\begin{array}{c}\mathrm{N} / \mathrm{A} \\
\mathrm{b}\end{array}$ & $\begin{array}{c}\text { N/A } \\
\text { b }\end{array}$ & $\begin{array}{c}\text { N/A } \\
\text { b }\end{array}$ & $\begin{array}{c}\mathbf{N} / \mathbf{A} \\
\mathbf{b}\end{array}$ \\
\hline $\begin{array}{l}\text { HFBR } \\
\text { (INEL) }\end{array}$ & $\begin{array}{c}N / A \\
b / c\end{array}$ & $\begin{array}{c}\text { No } \\
\text { a }\end{array}$ & $\begin{array}{c}N / A \\
b / c\end{array}$ & $\begin{array}{l}\text { N/A } \\
b / c\end{array}$ & $\begin{array}{l}N / A \\
b / c\end{array}$ & $\begin{array}{c}N / A \\
b / c\end{array}$ & $\begin{array}{c}N / A \\
b / c\end{array}$ & $\begin{array}{c}\text { N/A } \\
b / c\end{array}$ & $\begin{array}{l}\mathrm{N} / \mathrm{A} \\
\mathrm{b} / \mathrm{c}\end{array}$ & $\begin{array}{c}\text { No } \\
\text { a }\end{array}$ & $\begin{array}{l}\mathbf{N} / \mathrm{A} \\
\mathbf{b} / \mathfrak{c}\end{array}$ & $\begin{array}{l}N / A \\
b / c\end{array}$ & $\begin{array}{c}\mathbf{N} / \mathrm{A} \\
\mathrm{b} / \mathrm{c}\end{array}$ & $\begin{array}{c}\text { N/A } \\
b / c\end{array}$ & $\begin{array}{c}\text { N/A } \\
b / c\end{array}$ & $\begin{array}{c}N / A \\
b / c\end{array}$ & $\begin{array}{l}N / \\
A \\
b / c\end{array}$ & $\begin{array}{c}\mathrm{N} / \mathrm{A} \\
\mathrm{b} / \mathrm{c}\end{array}$ & $\begin{array}{c}\mathrm{N} / \mathrm{A} \\
\mathrm{b} / \mathrm{c}\end{array}$ & $\begin{array}{l}\mathrm{N} / \mathrm{A} \\
\mathrm{b} / \mathrm{c}\end{array}$ & $\begin{array}{l}\mathrm{N} / \mathrm{A} \\
\mathrm{b} / \mathrm{c}\end{array}$ & $\begin{array}{c}\mathrm{N} / \mathrm{A} \\
\mathrm{b} / \mathrm{c}\end{array}$ & $\begin{array}{l}\text { N/A } \\
b / c\end{array}$ \\
\hline $\begin{array}{l}\text { HFBR } \\
\text { (BNL) }\end{array}$ & $\begin{array}{c}\text { No } \\
\text { a }\end{array}$ & $\begin{array}{c}\text { No } \\
\text { a }\end{array}$ & $\begin{array}{c}\text { N/A } \\
b\end{array}$ & $\begin{array}{c}\text { N/A } \\
\text { b }\end{array}$ & $\begin{array}{l}\text { No } \\
c\end{array}$ & $\begin{array}{c}\text { N/A } \\
b\end{array}$ & $\begin{array}{c}\text { No } \\
\text { c }\end{array}$ & $\begin{array}{c}\text { No } \\
\text { c }\end{array}$ & $\begin{array}{l}\text { No } \\
\text { c }\end{array}$ & $\begin{array}{c}\text { No } \\
\text { a }\end{array}$ & $\begin{array}{c}\text { No } \\
\text { c }\end{array}$ & $\begin{array}{c}\text { No } \\
\text { c }\end{array}$ & $\begin{array}{c}\text { No } \\
\mathfrak{c}\end{array}$ & $\begin{array}{c}\text { N/A } \\
\text { b }\end{array}$ & $\begin{array}{c}\text { N/A } \\
\text { b }\end{array}$ & $\begin{array}{c}\text { N/A } \\
\text { b }\end{array}$ & $\begin{array}{l}\text { N/ } \\
\text { A } \\
\text { b }\end{array}$ & $\begin{array}{c}\mathbf{N} / \mathbf{A} \\
\mathrm{b}\end{array}$ & $\begin{array}{c}\text { No } \\
\text { c }\end{array}$ & $\begin{array}{c}\mathrm{N} / \mathrm{A} \\
\mathrm{b}\end{array}$ & $\begin{array}{c}\text { No } \\
\text { c }\end{array}$ & $\begin{array}{c}\text { N/A } \\
\text { b }\end{array}$ & $\begin{array}{c}\mathbf{N} / \mathbf{A} \\
\mathbf{b}\end{array}$ \\
\hline
\end{tabular}


Table 4-5. (continued).

\begin{tabular}{|c|c|c|c|c|c|c|c|c|c|c|c|c|c|c|c|c|c|c|c|c|c|c|c|}
\hline \multirow[b]{2}{*}{$\begin{array}{c}\text { Metallic } \\
\text { fuels }\end{array}$} & \multicolumn{13}{|c|}{ Legal weight truck packages } & \multicolumn{2}{|c|}{$\begin{array}{c}\text { Overweight } \\
\text { truck packages }\end{array}$} & \multicolumn{8}{|c|}{ Rail packages } \\
\hline & $\begin{array}{l}\mathrm{GE} \\
\mathrm{T}-2\end{array}$ & $\begin{array}{l}\text { GE- } \\
100\end{array}$ & BMI-1 & FSV-1 & $\begin{array}{c}\mathrm{NLI}- \\
1 / 2\end{array}$ & $\begin{array}{l}\mathrm{NLI}- \\
6502\end{array}$ & $\mathrm{~T}-\mathbf{3}$ & NAC-1 & $\begin{array}{c}\text { CNS } \\
1-13 G\end{array}$ & NRBK-41 & $\begin{array}{l}\text { NAC- } \\
\text { LWT }\end{array}$ & $\begin{array}{c}\text { GE Model } \\
2000\end{array}$ & $\begin{array}{l}\text { TN. } \\
\text { FSV }\end{array}$ & $\begin{array}{l}\text { TN-8 } \\
\text { TN-8L }\end{array}$ & TN-9 & M-130 & \begin{tabular}{|l|}
$\mathrm{IF}-$ \\
300
\end{tabular} & $\begin{array}{l}\text { NLI- } \\
10 / 24\end{array}$ & $125-\mathrm{B}$ & \begin{tabular}{|l|} 
TN- \\
BRP
\end{tabular} & $\begin{array}{c}\text { TN- } \\
\text { REG }\end{array}$ & M-160 & M-140 \\
\hline ORR & $\begin{array}{c}\mathrm{N} / \mathrm{A} \\
\mathbf{b}\end{array}$ & $\begin{array}{c}\text { No } \\
\text { a }\end{array}$ & $\begin{array}{c}\text { N/A } \\
b\end{array}$ & $\begin{array}{c}\text { N/A } \\
b\end{array}$ & $\begin{array}{c}N / A \\
b\end{array}$ & $\begin{array}{c}\mathbf{N} / \mathrm{A} \\
\mathbf{b}\end{array}$ & $\begin{array}{c}\text { N/A } \\
b\end{array}$ & $\begin{array}{c}\text { N/A } \\
b\end{array}$ & $\begin{array}{c}\text { N/A } \\
b\end{array}$ & $\begin{array}{c}\text { No } \\
\text { a }\end{array}$ & $\underset{b}{N / A}$ & $\begin{array}{c}\text { N/A } \\
b\end{array}$ & $\begin{array}{c}\text { N/A } \\
b\end{array}$ & $\begin{array}{c}\text { N/A } \\
b\end{array}$ & $\begin{array}{c}\text { N/A } \\
b\end{array}$ & $\begin{array}{c}\text { N/A } \\
b\end{array}$ & $\begin{array}{l}\mathbf{N} / \\
\mathbf{A}\end{array}$ & $\begin{array}{c}\text { N/A } \\
b\end{array}$ & $\begin{array}{c}\text { N/A } \\
b\end{array}$ & $\begin{array}{c}N / A \\
b\end{array}$ & $\begin{array}{c}N / A \\
b\end{array}$ & $\begin{array}{c}\text { N/A } \\
b\end{array}$ & $\begin{array}{c}N / A \\
b\end{array}$ \\
\hline $\begin{array}{l}\text { ATR Ass'ys } \\
\text { (INEL } \\
\text { CPP-603) }\end{array}$ & $\begin{array}{c}\text { No } \\
\text { c }\end{array}$ & $\begin{array}{c}\text { No } \\
\text { a }\end{array}$ & $\begin{array}{c}\text { N/A } \\
c\end{array}$ & $\begin{array}{c}\text { Yes } \\
\mathrm{C}\end{array}$ & $\begin{array}{c}\mathrm{N} / \mathrm{A} \\
\mathrm{b}\end{array}$ & $\begin{array}{c}\text { Yes } \\
\mathrm{C}\end{array}$ & $\begin{array}{c}\text { Yes } \\
\text { C }\end{array}$ & $\begin{array}{c}\text { Yes? } \\
\text { C }\end{array}$ & $\begin{array}{l}\text { No } \\
\text { c }\end{array}$ & $\begin{array}{l}\text { No } \\
\text { a }\end{array}$ & $\begin{array}{c}\text { Yes } \\
\text { C }\end{array}$ & $\begin{array}{l}\text { No } \\
\text { c }\end{array}$ & $\begin{array}{c}\text { Yes } \\
\text { C }\end{array}$ & $\begin{array}{c}\text { Yes } \\
\text { C }\end{array}$ & $\begin{array}{c}\text { Yes } \\
\text { C }\end{array}$ & $\begin{array}{c}N / A \\
b\end{array}$ & $\begin{array}{c}\text { Yes } \\
\text { C }\end{array}$ & $\begin{array}{c}\text { Yes } \\
\text { C }\end{array}$ & $\begin{array}{c}\text { Yes } \\
\text { C }\end{array}$ & $\begin{array}{c}\text { Yes } \\
\text { C }\end{array}$ & $\begin{array}{c}\text { Yes } \\
\text { C }\end{array}$ & $\underset{b}{N / A}$ & $\begin{array}{c}N / A \\
b\end{array}$ \\
\hline $\begin{array}{c}\text { ATR } \\
\text { Recycle }\end{array}$ & $\begin{array}{c}\text { N/A } \\
b\end{array}$ & $\begin{array}{c}\text { No } \\
\text { a }\end{array}$ & $\begin{array}{c}\text { N/A } \\
b\end{array}$ & $\begin{array}{c}\text { N/A } \\
b\end{array}$ & $\begin{array}{c}N / A \\
b\end{array}$ & $\begin{array}{c}\text { N/A } \\
b\end{array}$ & $\begin{array}{c}N / A \\
b\end{array}$ & $\begin{array}{c}N / A \\
b\end{array}$ & $\begin{array}{c}N / A \\
b\end{array}$ & $\begin{array}{c}\text { No } \\
\text { a }\end{array}$ & $\begin{array}{c}\text { N/A } \\
b\end{array}$ & $\begin{array}{c}\text { N/A } \\
b\end{array}$ & $\begin{array}{c}\text { N/A } \\
b\end{array}$ & $\begin{array}{c}\text { N/A } \\
b\end{array}$ & $\begin{array}{c}\text { N/A } \\
b\end{array}$ & $\begin{array}{c}N / A \\
b\end{array}$ & $\begin{array}{l}\text { N/ } \\
\text { A }\end{array}$ & $\begin{array}{c}\text { N/A } \\
b\end{array}$ & $\begin{array}{c}\text { N/A } \\
\text { b }\end{array}$ & $\begin{array}{c}\text { N/A } \\
b\end{array}$ & $\begin{array}{c}\text { N/A } \\
b\end{array}$ & $\begin{array}{c}\text { N/A } \\
b\end{array}$ & $\begin{array}{c}\text { N/A } \\
b\end{array}$ \\
\hline ATR Spent & $\begin{array}{c}\text { N/A } \\
b\end{array}$ & $\begin{array}{c}\text { No } \\
\text { a }\end{array}$ & $\begin{array}{c}\text { N/A } \\
b\end{array}$ & $\begin{array}{c}\text { N/A } \\
b\end{array}$ & $\begin{array}{c}\text { N/A } \\
b\end{array}$ & $\begin{array}{c}\text { N/A } \\
b\end{array}$ & $\begin{array}{c}N / A \\
b\end{array}$ & $\begin{array}{c}N / A \\
b\end{array}$ & $\begin{array}{c}\text { N/A } \\
b\end{array}$ & $\begin{array}{c}\text { No } \\
\text { a }\end{array}$ & $\begin{array}{c}N / A \\
b\end{array}$ & $\begin{array}{c}\text { N/A } \\
b\end{array}$ & $\begin{array}{c}\text { N/A } \\
b\end{array}$ & $\begin{array}{c}\text { N/A } \\
\text { b }\end{array}$ & $\begin{array}{c}\text { N/A } \\
b\end{array}$ & $\begin{array}{c}N / A \\
b\end{array}$ & $\begin{array}{l}\text { N/ } \\
\text { A }\end{array}$ & $\begin{array}{c}\text { N/A } \\
b\end{array}$ & $\begin{array}{c}\text { N/A } \\
b\end{array}$ & $\begin{array}{c}\text { N/A } \\
b\end{array}$ & $\begin{array}{c}\mathrm{N} / \mathrm{A} \\
\mathrm{b}\end{array}$ & $\begin{array}{c}\mathbf{N} / \mathbf{A} \\
\mathbf{b}\end{array}$ & $\begin{array}{c}\text { N/A } \\
b\end{array}$ \\
\hline ATR Core & $\begin{array}{c}\text { N/A } \\
b\end{array}$ & $\begin{array}{c}\text { No } \\
\text { a }\end{array}$ & $\begin{array}{c}\text { N/A } \\
b\end{array}$ & $\begin{array}{c}\text { N/A } \\
b\end{array}$ & $\begin{array}{c}\mathrm{N} / \mathrm{A} \\
\mathrm{b}\end{array}$ & $\begin{array}{c}\text { N/A } \\
b\end{array}$ & $\begin{array}{c}\text { N/A } \\
b\end{array}$ & $\begin{array}{c}\text { N/A } \\
b\end{array}$ & $\begin{array}{c}\text { N/A } \\
\text { b }\end{array}$ & $\begin{array}{c}\text { No } \\
\text { a }\end{array}$ & $\begin{array}{c}\text { N/A } \\
b\end{array}$ & $\begin{array}{c}\text { N/A } \\
\text { b }\end{array}$ & $\begin{array}{c}\text { N/A } \\
b\end{array}$ & $\begin{array}{c}\text { N/A } \\
b\end{array}$ & $\begin{array}{c}\text { N/A } \\
\text { b }\end{array}$ & $\begin{array}{c}\mathbf{N} / \mathbf{A} \\
\mathbf{b}\end{array}$ & $\begin{array}{l}\mathbf{N} / \\
\mathrm{A}\end{array}$ & $\begin{array}{c}\text { N/A } \\
b\end{array}$ & $\begin{array}{c}\mathbf{N} / \mathbf{A} \\
\mathbf{b}\end{array}$ & $\begin{array}{c}\text { N/A } \\
b\end{array}$ & $\begin{array}{c}\mathbf{N} / \mathbf{A} \\
\mathrm{b}\end{array}$ & $\begin{array}{c}N / A \\
b\end{array}$ & $\begin{array}{c}N / A \\
b\end{array}$ \\
\hline $\begin{array}{l}\text { ATR Ass'ys } \\
\text { (INEL } \\
\text { CPP-666) }\end{array}$ & $\begin{array}{c}\text { No } \\
\text { c }\end{array}$ & $\begin{array}{c}\text { No } \\
\text { a }\end{array}$ & $\begin{array}{c}\text { N/A } \\
c\end{array}$ & $\begin{array}{c}\text { Yes } \\
\text { C }\end{array}$ & $\begin{array}{c}N / A \\
b\end{array}$ & $\begin{array}{c}\text { Yes } \\
\text { C }\end{array}$ & $\begin{array}{c}\text { Yes } \\
\text { C }\end{array}$ & $\begin{array}{c}\text { Yes? } \\
\text { C }\end{array}$ & $\begin{array}{l}\text { No } \\
\text { c }\end{array}$ & $\begin{array}{c}\text { No } \\
\text { a }\end{array}$ & $\begin{array}{c}\text { Yes } \\
\text { C }\end{array}$ & $\begin{array}{c}\text { No } \\
\text { c }\end{array}$ & $\begin{array}{c}\text { Yes } \\
\text { C }\end{array}$ & $\begin{array}{c}\text { Yes } \\
\text { C }\end{array}$ & $\begin{array}{c}\text { Yes } \\
\text { C }\end{array}$ & $\begin{array}{c}\text { N/A } \\
b\end{array}$ & $\begin{array}{c}\text { Yes } \\
\text { C }\end{array}$ & $\begin{array}{c}\text { Yes } \\
\text { C }\end{array}$ & $\begin{array}{c}\text { Yes } \\
\text { C }\end{array}$ & $\begin{array}{c}\text { Yes } \\
\text { C }\end{array}$ & $\begin{array}{c}\text { Yes } \\
\text { C }\end{array}$ & $\begin{array}{c}\text { N/a } \\
b\end{array}$ & $\begin{array}{c}\text { N/A } \\
b\end{array}$ \\
\hline HFIR & $\begin{array}{c}\text { No } \\
\text { a }\end{array}$ & $\begin{array}{c}\text { No } \\
\mathrm{a}\end{array}$ & $\begin{array}{c}\text { No } \\
\text { a }\end{array}$ & $\begin{array}{c}\text { No } \\
\text { a }\end{array}$ & $\begin{array}{c}\text { No } \\
\text { a }\end{array}$ & $\begin{array}{c}\text { No } \\
\text { a }\end{array}$ & $\begin{array}{c}\text { No } \\
\text { a }\end{array}$ & $\begin{array}{c}\text { No } \\
\text { a }\end{array}$ & $\begin{array}{c}\text { Yes } \\
\text { C }\end{array}$ & $\begin{array}{c}\text { No } \\
\text { a }\end{array}$ & $\begin{array}{c}\text { No } \\
\mathbf{a}\end{array}$ & $\begin{array}{c}\text { Yes } \\
\text { A }\end{array}$ & $\begin{array}{c}\text { No } \\
\text { a }\end{array}$ & $\begin{array}{c}\text { No } \\
\text { a }\end{array}$ & $\begin{array}{c}\text { No } \\
\text { a }\end{array}$ & $\begin{array}{c}\text { No } \\
\text { a }\end{array}$ & $\begin{array}{c}\text { Yes } \\
\mathrm{C}\end{array}$ & $\begin{array}{c}\text { Yes } \\
\text { C }\end{array}$ & $\begin{array}{c}\text { Yes } \\
\text { C }\end{array}$ & $\begin{array}{c}\text { Yes } \\
\text { C }\end{array}$ & $\begin{array}{c}\text { Yes } \\
\text { C }\end{array}$ & $\begin{array}{c}\text { Yes } \\
\text { C }\end{array}$ & $\begin{array}{c}\text { Yes } \\
\text { C }\end{array}$ \\
\hline RINC & $\begin{array}{c}\text { N/A } \\
\mathbf{a}\end{array}$ & $\begin{array}{c}\text { No } \\
\text { a }\end{array}$ & $\begin{array}{c}\text { N/A } \\
a\end{array}$ & $\begin{array}{c}\text { N/A } \\
a\end{array}$ & $\begin{array}{c}\text { N/A } \\
a\end{array}$ & $\begin{array}{c}\text { N/A } \\
a\end{array}$ & $\begin{array}{c}\text { N/A } \\
a\end{array}$ & $\begin{array}{c}\text { N/A } \\
a\end{array}$ & $\begin{array}{c}\text { N/A } \\
a\end{array}$ & $\begin{array}{c}\text { No } \\
\text { a }\end{array}$ & $\begin{array}{c}\text { N/A } \\
a\end{array}$ & $\begin{array}{c}\mathbf{N} / \mathbf{A} \\
\mathbf{a}\end{array}$ & $\begin{array}{c}N / A \\
a\end{array}$ & $\begin{array}{c}\text { N/A } \\
a\end{array}$ & $\begin{array}{c}\text { N/A } \\
a\end{array}$ & $\begin{array}{c}\text { N/A } \\
a\end{array}$ & $\begin{array}{l}\text { N/ } \\
\text { A }\end{array}$ & $\begin{array}{c}\text { N/A } \\
a\end{array}$ & $\begin{array}{c}\text { N/A } \\
\mathbf{a}\end{array}$ & $\begin{array}{c}\text { N/A } \\
a\end{array}$ & $\begin{array}{c}\text { N/A } \\
a\end{array}$ & $\begin{array}{c}\text { N/A } \\
a\end{array}$ & $\begin{array}{c}\text { N/A } \\
\mathbf{a}\end{array}$ \\
\hline Nereide & $\begin{array}{c}\text { N/A } \\
b\end{array}$ & $\begin{array}{c}\text { No } \\
\text { a }\end{array}$ & $\begin{array}{c}\text { N/A } \\
\text { b }\end{array}$ & $\begin{array}{c}N / A \\
b\end{array}$ & $\begin{array}{c}\text { N/A } \\
b\end{array}$ & $\begin{array}{c}\text { N/A } \\
b\end{array}$ & $\begin{array}{c}N / A \\
b\end{array}$ & $\begin{array}{c}\text { N/A } \\
b\end{array}$ & $\begin{array}{c}\text { N/A } \\
\text { b }\end{array}$ & $\begin{array}{c}\text { No } \\
\text { a }\end{array}$ & $\begin{array}{c}\text { N/A } \\
b\end{array}$ & $\begin{array}{c}\text { N/A } \\
\text { b }\end{array}$ & $\begin{array}{c}\text { N/A } \\
\mathbf{b}\end{array}$ & $\begin{array}{c}\text { N/A } \\
b\end{array}$ & $\begin{array}{c}\text { N/A } \\
b\end{array}$ & $\begin{array}{c}\text { N/A } \\
b\end{array}$ & $\begin{array}{l}\mathbf{N} / \\
\mathrm{A}\end{array}$ & $\begin{array}{c}\text { N/A } \\
b\end{array}$ & $\begin{array}{c}\text { N/A } \\
b\end{array}$ & $\begin{array}{c}N / A \\
b\end{array}$ & $\begin{array}{c}\mathrm{N} / \mathrm{A} \\
\mathrm{b}\end{array}$ & $\begin{array}{c}\text { N/A } \\
\mathbf{b}\end{array}$ & $\begin{array}{c}\text { N/A } \\
b\end{array}$ \\
\hline ANLJ & $\begin{array}{c}\text { No } \\
\mathrm{a}\end{array}$ & $\begin{array}{c}\text { No } \\
\text { a }\end{array}$ & $\begin{array}{c}\text { No } \\
\text { a }\end{array}$ & $\begin{array}{c}\text { N/A } \\
b\end{array}$ & $\begin{array}{c}\text { N/A } \\
b\end{array}$ & $\begin{array}{c}\text { No } \\
\text { a }\end{array}$ & $\begin{array}{c}\text { No } \\
\text { a }\end{array}$ & $\begin{array}{c}\text { N/A } \\
b\end{array}$ & $\begin{array}{c}\text { No } \\
\mathrm{a}\end{array}$ & $\begin{array}{c}\text { No } \\
\text { a }\end{array}$ & $\begin{array}{c}\text { N/A } \\
b\end{array}$ & $\begin{array}{c}\text { No } \\
\mathrm{a}\end{array}$ & $\begin{array}{c}\mathbf{N} / \mathbf{A} \\
\mathbf{b}\end{array}$ & $\begin{array}{c}\text { N/A } \\
\text { b }\end{array}$ & $\begin{array}{c}\text { N/A } \\
\mathbf{b}\end{array}$ & $\begin{array}{c}\text { No } \\
\text { a }\end{array}$ & $\begin{array}{l}\mathrm{N} / \\
\mathrm{A}\end{array}$ & $\begin{array}{c}\text { N/A } \\
\text { b }\end{array}$ & $\begin{array}{c}\text { N/A } \\
\mathbf{b}\end{array}$ & $\begin{array}{c}\text { N/A } \\
b\end{array}$ & $\begin{array}{c}\text { No } \\
\text { a }\end{array}$ & $\begin{array}{c}\text { No } \\
\text { a }\end{array}$ & $\begin{array}{c}\text { No } \\
\text { a }\end{array}$ \\
\hline
\end{tabular}


Table 4-5. (continued).

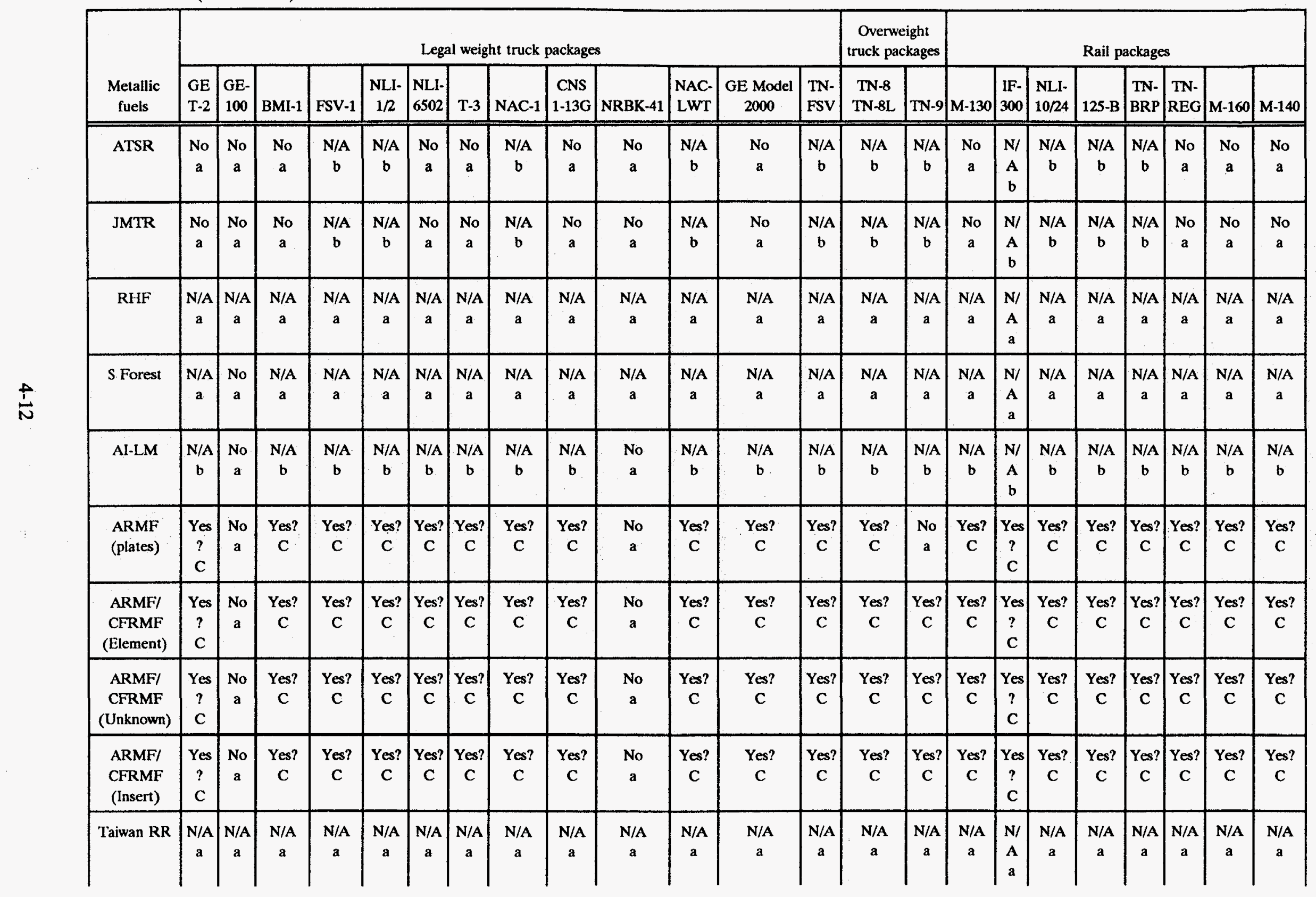


Table 4-5. (continued).

\begin{tabular}{|c|c|c|c|c|c|c|c|c|c|c|c|c|c|c|c|c|c|c|c|c|c|c|c|}
\hline \multirow[b]{2}{*}{$\begin{array}{c}\text { Metallic } \\
\text { fuels }\end{array}$} & \multicolumn{13}{|c|}{ Legal weight truck packages } & \multicolumn{2}{|c|}{$\begin{array}{l}\text { Overweight } \\
\text { truck packages }\end{array}$} & \multicolumn{8}{|c|}{ Rail packages } \\
\hline & $\left|\begin{array}{c}\mathrm{GE} \\
\mathrm{T}-2\end{array}\right|$ & $\begin{array}{l}\text { GE- } \\
100\end{array}$ & BMI-1 & FSV-1 & $\begin{array}{l}\text { NLI- } \\
1 / 2\end{array}$ & $\left|\begin{array}{l}\mathrm{NLI}- \\
6502\end{array}\right|$ & T-3 & NAC-1 & $\begin{array}{c}\text { CNS } \\
1-13 G\end{array}$ & NRBK-41 & $\begin{array}{l}\text { NAC- } \\
\text { LWT }\end{array}$ & $\begin{array}{c}\text { GE Model } \\
2000\end{array}$ & $\begin{array}{l}\text { TN- } \\
\text { FSV }\end{array}$ & $\begin{array}{c}\text { TN-8 } \\
\text { TN-8L }\end{array}$ & TN-9 & M-130 & $\begin{array}{l}\text { IF- } \\
300\end{array}$ & $\begin{array}{l}\text { NLI- } \\
10 / 24\end{array}$ & 125-B & $\begin{array}{l}\text { TN- } \\
\text { BRP }\end{array}$ & $\begin{array}{c}\text { TN- } \\
\text { REG }\end{array}$ & M-160 & M-140 \\
\hline SPEC & $\begin{array}{c}\mathrm{N} / \mathrm{A} \\
\mathrm{a}\end{array}$ & $\begin{array}{c}\mathrm{N} / \mathrm{A} \\
\mathrm{a}\end{array}$ & $\begin{array}{c}\text { N/A } \\
a\end{array}$ & $\begin{array}{c}\text { N/A } \\
a\end{array}$ & $\begin{array}{c}\mathrm{N} / \mathrm{A} \\
\mathbf{a}\end{array}$ & $\begin{array}{c}\text { N/A } \\
\text { a }\end{array}$ & $\begin{array}{c}\text { N/A } \\
a\end{array}$ & $\begin{array}{c}\text { N/A } \\
\mathbf{a}\end{array}$ & $\begin{array}{c}\text { N/A } \\
a\end{array}$ & $\begin{array}{c}\text { N/A } \\
a\end{array}$ & $\begin{array}{c}\text { N/A } \\
\text { a }\end{array}$ & $\begin{array}{c}\mathrm{N} / \mathrm{A} \\
\mathrm{a}\end{array}$ & $\begin{array}{c}\text { N/A } \\
\text { a }\end{array}$ & $\begin{array}{c}\text { N/A } \\
\mathbf{a}\end{array}$ & $\begin{array}{c}\mathrm{N} / \mathrm{A} \\
\mathrm{a}\end{array}$ & $\begin{array}{c}\text { N/A } \\
\text { a }\end{array}$ & $\begin{array}{l}\text { N/ } \\
\text { A }\end{array}$ & $\begin{array}{c}\mathrm{N} / \mathrm{A} \\
\mathrm{a}\end{array}$ & $\begin{array}{c}\text { N/A } \\
a\end{array}$ & $\begin{array}{c}\mathrm{N} / \mathrm{A} \\
\mathrm{a}\end{array}$ & $\begin{array}{c}\mathrm{N} / \mathrm{A} \\
\mathrm{a}\end{array}$ & $\begin{array}{c}\text { N/A } \\
\text { a }\end{array}$ & $\begin{array}{c}\mathrm{N} / \mathrm{A} \\
\mathrm{a}\end{array}$ \\
\hline TSR & $\begin{array}{c}\mathrm{N} / \mathrm{A} \\
\mathrm{a}\end{array}$ & $\begin{array}{c}\text { No } \\
\text { a }\end{array}$ & $\begin{array}{c}\text { No } \\
\text { a }\end{array}$ & $\begin{array}{c}\text { No } \\
\text { a }\end{array}$ & $\begin{array}{c}\text { No } \\
\text { a }\end{array}$ & $\begin{array}{c}\text { No } \\
\text { a }\end{array}$ & $\begin{array}{c}\text { No } \\
\text { a }\end{array}$ & $\begin{array}{c}\text { No } \\
\text { a }\end{array}$ & $\begin{array}{c}\text { No } \\
\text { a }\end{array}$ & $\begin{array}{c}\text { No } \\
\text { a }\end{array}$ & $\begin{array}{c}\text { No } \\
\text { a }\end{array}$ & $\begin{array}{c}\text { No } \\
\text { a }\end{array}$ & $\begin{array}{c}\text { No } \\
\text { a }\end{array}$ & $\begin{array}{c}\text { No } \\
\text { a }\end{array}$ & $\begin{array}{c}\text { No } \\
\text { a }\end{array}$ & $\begin{array}{c}\text { No } \\
\text { a }\end{array}$ & $\begin{array}{l}\mathbf{N} / \\
\mathbf{A}\end{array}$ & $\begin{array}{c}\text { N/A } \\
b\end{array}$ & $\begin{array}{c}\text { N/A } \\
b\end{array}$ & $\begin{array}{c}\text { N/A } \\
b\end{array}$ & $\begin{array}{c}\mathrm{N} / \mathrm{A} \\
\mathrm{b}\end{array}$ & $\begin{array}{c}\text { N/A } \\
\text { b }\end{array}$ & $\begin{array}{c}\mathrm{N} / \mathrm{A} \\
\mathrm{b}\end{array}$ \\
\hline BNL MRR & $\begin{array}{c}\mathrm{N} / \mathrm{A} \\
\mathrm{b}\end{array}$ & $\begin{array}{c}\text { No } \\
\text { a }\end{array}$ & $\begin{array}{c}\text { N/A } \\
b\end{array}$ & $\begin{array}{c}\text { N/A } \\
b\end{array}$ & $\begin{array}{c}\text { N/A } \\
b\end{array}$ & $\left|\begin{array}{c}\mathrm{N} / \mathrm{A} \\
\mathrm{b}\end{array}\right|$ & $\begin{array}{c}\text { N/A } \\
b\end{array}$ & $\begin{array}{c}\mathrm{N} / \mathrm{A} \\
\mathrm{b}\end{array}$ & $\begin{array}{c}\text { N/A } \\
b\end{array}$ & $\begin{array}{c}\text { No } \\
\text { a }\end{array}$ & $\begin{array}{c}\text { N/A } \\
b\end{array}$ & $\begin{array}{c}\text { N/A } \\
b\end{array}$ & $\begin{array}{c}\text { N/A } \\
b\end{array}$ & $\begin{array}{c}\text { N/A } \\
b\end{array}$ & $\begin{array}{c}\text { N/A } \\
b\end{array}$ & $\begin{array}{c}\text { N/A } \\
b\end{array}$ & $\begin{array}{l}\mathbf{N} / \\
\mathbf{A}\end{array}$ & $\begin{array}{c}\text { N/A } \\
\text { b }\end{array}$ & $\begin{array}{c}\text { N/A } \\
b\end{array}$ & $\begin{array}{c}\text { N/A } \\
b\end{array}$ & $\left|\begin{array}{c}\mathrm{N} / \mathrm{A} \\
\mathrm{b}\end{array}\right|$ & $\begin{array}{c}\text { N/A } \\
b\end{array}$ & $\begin{array}{c}\mathrm{N} / \mathrm{A} \\
\mathrm{b}\end{array}$ \\
\hline U. Wash. & $\begin{array}{c}\text { Yes } \\
\text { C }\end{array}$ & $\begin{array}{c}\text { No } \\
\text { a }\end{array}$ & $\begin{array}{c}\text { Yes } \\
\text { B }\end{array}$ & $\begin{array}{c}\text { Yes } \\
\text { C }\end{array}$ & $\begin{array}{c}\text { Yes } \\
\text { C }\end{array}$ & $\begin{array}{c}\text { Yes } \\
\text { C }\end{array}$ & $\begin{array}{l}\text { Yes } \\
\text { C }\end{array}$ & $\begin{array}{l}\text { Yes } \\
\text { C }\end{array}$ & $\begin{array}{c}\text { Yes } \\
\text { C }\end{array}$ & $\begin{array}{c}\text { No } \\
\text { a }\end{array}$ & $\begin{array}{c}\text { Yes } \\
\text { C }\end{array}$ & $\begin{array}{c}\text { Yes } \\
\text { C }\end{array}$ & $\begin{array}{c}\text { Yes } \\
\text { C }\end{array}$ & $\begin{array}{l}\text { Yes } \\
\text { C }\end{array}$ & $\begin{array}{l}\text { Yes } \\
\text { C }\end{array}$ & $\begin{array}{l}\text { Yes } \\
\text { C }\end{array}$ & $\begin{array}{c}\text { Yes } \\
\text { C }\end{array}$ & $\begin{array}{l}\text { Yes } \\
\text { C }\end{array}$ & $\begin{array}{l}\text { Yes } \\
\text { C }\end{array}$ & $\begin{array}{c}\text { Yes } \\
\text { C }\end{array}$ & $\begin{array}{c}\text { Yes } \\
\mathrm{C}\end{array}$ & $\begin{array}{c}\text { Yes } \\
\text { C }\end{array}$ & $\begin{array}{c}\text { Yes } \\
\text { C }\end{array}$ \\
\hline U. Virginia & $\begin{array}{c}\mathrm{N} / \mathrm{A} \\
\mathrm{a}\end{array}$ & $\begin{array}{c}\mathrm{N} / \mathrm{A} \\
\mathrm{a}\end{array}$ & $\begin{array}{c}\mathrm{N} / \mathrm{A} \\
\mathrm{a}\end{array}$ & $\begin{array}{c}\text { N/A } \\
\text { a }\end{array}$ & $\begin{array}{c}\mathrm{N} / \mathrm{A} \\
\mathrm{a}\end{array}$ & $\begin{array}{c}\mathrm{N} / \mathrm{A} \\
\mathrm{a}\end{array} \mid$ & $\begin{array}{c}\text { N/A } \\
a\end{array}$ & $\begin{array}{c}\text { N/A } \\
a\end{array}$ & $\begin{array}{c}\text { N/A } \\
\text { a }\end{array}$ & $\begin{array}{c}\mathrm{N} / \mathrm{A} \\
\mathrm{a}\end{array}$ & $\begin{array}{c}\text { N/A } \\
\mathbf{a}\end{array}$ & $\begin{array}{c}\text { N/A } \\
\mathbf{a}\end{array}$ & $\begin{array}{c}\mathrm{N} / \mathrm{A} \\
\mathrm{a}\end{array}$ & $\begin{array}{c}\text { N/A } \\
\text { a }\end{array}$ & $\begin{array}{c}\text { N/A } \\
a\end{array}$ & $\begin{array}{c}\text { N/A } \\
\text { a }\end{array}$ & $\begin{array}{l}\text { N/ } \\
\text { A }\end{array}$ & $\begin{array}{c}\text { N/A } \\
\text { a }\end{array}$ & $\begin{array}{c}\mathrm{N} / \mathrm{A} \\
\mathrm{a}\end{array}$ & $\begin{array}{c}\text { N/A } \\
a\end{array}$ & $\begin{array}{c}\mathrm{N} / \mathrm{A} \\
\mathrm{a}\end{array}$ & $\begin{array}{c}\mathrm{N} / \mathrm{A} \\
\mathrm{a}\end{array}$ & $\begin{array}{c}\text { N/A } \\
\mathbf{a}\end{array}$ \\
\hline U. Michigan & $\begin{array}{c}\mathrm{N} / \mathrm{A} \\
\mathrm{a}\end{array}$ & $\begin{array}{c}\mathrm{N} / \mathrm{A} \\
\mathrm{a}\end{array}$ & $\begin{array}{c}\mathrm{N} / \mathrm{A} \\
\mathrm{a}\end{array}$ & $\begin{array}{c}\text { N/A } \\
\text { a }\end{array}$ & $\begin{array}{c}\mathrm{N} / \mathrm{A} \\
\mathrm{a}\end{array}$ & $\begin{array}{c}\mathrm{N} / \mathrm{A} \\
\mathrm{a}\end{array} \mid$ & $\begin{array}{c}\text { N/A } \\
a\end{array}$ & $\begin{array}{c}\text { N/A } \\
a\end{array}$ & $\begin{array}{c}\text { N/A } \\
a\end{array}$ & $\begin{array}{c}\mathrm{N} / \mathrm{A} \\
\mathrm{a}\end{array}$ & $\begin{array}{c}\mathrm{N} / \mathrm{A} \\
\mathrm{a}\end{array}$ & $\begin{array}{c}\mathrm{N} / \mathrm{A} \\
\mathrm{a}\end{array}$ & $\begin{array}{c}\mathrm{N} / \mathrm{A} \\
\mathrm{a}\end{array}$ & $\begin{array}{c}\mathrm{N} / \mathrm{A} \\
\mathbf{a}\end{array}$ & $\begin{array}{c}\text { N/A } \\
a\end{array}$ & $\begin{array}{c}\mathrm{N} / \mathrm{A} \\
\mathrm{a}\end{array}$ & N/ & $\begin{array}{c}\mathrm{N} / \mathrm{A} \\
\mathrm{a}\end{array}$ & $\begin{array}{c}\text { N/A } \\
a\end{array}$ & $\begin{array}{c}\mathrm{N} / \mathrm{A} \\
\mathrm{a}\end{array}$ & $\left|\begin{array}{c}\mathrm{N} / \mathrm{A} \\
\mathrm{a}\end{array}\right|$ & $\begin{array}{c}\text { N/A } \\
a\end{array}$ & $\begin{array}{c}\mathrm{N} / \mathrm{A} \\
\mathbf{a}\end{array}$ \\
\hline $\begin{array}{c}\text { MTR Type } \\
\text { (U. Virginia) }\end{array}$ & $\begin{array}{c}\mathrm{N} / \mathrm{A} \\
\mathrm{b}\end{array}$ & $\begin{array}{c}\text { No } \\
\mathrm{a}\end{array}$ & $\begin{array}{c}\text { Yes } \\
\text { A }\end{array}$ & $\begin{array}{c}\text { N/A } \\
\text { b }\end{array}$ & $\begin{array}{c}\mathrm{N} / \mathrm{A} \\
\mathrm{b}\end{array}$ & $\left|\begin{array}{c}\mathrm{N} / \mathrm{A} \\
\mathrm{b}\end{array}\right|$ & $\begin{array}{c}\text { N/A } \\
b\end{array}$ & $\begin{array}{c}\mathrm{N} / \mathrm{A} \\
\mathrm{b}\end{array}$ & $\begin{array}{c}\mathrm{N} / \mathrm{A} \\
\mathrm{b}\end{array}$ & $\begin{array}{c}\text { No } \\
\text { a }\end{array}$ & $\underset{b}{N / A}$ & $\begin{array}{c}\text { N/A } \\
b\end{array}$ & $\begin{array}{c}\text { N/A } \\
b\end{array}$ & $\begin{array}{c}\text { N/A } \\
b\end{array}$ & $\begin{array}{c}\text { N/A } \\
b\end{array}$ & $\begin{array}{c}\text { N/A } \\
b\end{array}$ & $\begin{array}{l}\text { N/ } \\
\text { A }\end{array}$ & $\begin{array}{c}\text { N/A } \\
b\end{array}$ & $\begin{array}{c}\text { N/A } \\
b\end{array}$ & $\begin{array}{c}N / A \\
b\end{array}$ & $\begin{array}{c}\mathrm{N} / \mathrm{A} \\
\mathrm{b}\end{array}$ & $\begin{array}{c}\text { N/A } \\
b\end{array}$ & $\begin{array}{c}\text { N/A } \\
b\end{array}$ \\
\hline $\begin{array}{l}\text { MTR Type } \\
\text { (U. Mich.) }\end{array}$ & $\begin{array}{c}\mathrm{N} / \mathrm{A} \\
\mathrm{b}\end{array}$ & $\begin{array}{c}\text { No } \\
\mathrm{a}\end{array}$ & $\begin{array}{l}\text { Yes } \\
\text { A }\end{array}$ & $\begin{array}{c}\text { N/A } \\
\text { b }\end{array}$ & $\begin{array}{c}\text { N/A } \\
b\end{array}$ & $\left|\begin{array}{c}\mathrm{N} / \mathrm{A} \\
\mathrm{b}\end{array}\right|$ & $\begin{array}{c}\text { N/A } \\
b\end{array}$ & $\begin{array}{c}\text { N/A } \\
\text { b }\end{array}$ & $\begin{array}{c}\text { N/A } \\
\text { b }\end{array}$ & $\begin{array}{c}\text { No } \\
\mathbf{a}\end{array}$ & $\begin{array}{c}\text { N/A } \\
b\end{array}$ & $\begin{array}{c}\text { N/A } \\
\text { b }\end{array}$ & $\begin{array}{c}\text { N/A } \\
\text { b }\end{array}$ & $\begin{array}{c}\text { N/A } \\
\text { b }\end{array}$ & $\begin{array}{c}\text { N/A } \\
\text { b }\end{array}$ & $\begin{array}{c}\text { N/A } \\
\text { b }\end{array}$ & $\begin{array}{l}\text { N/ } \\
\text { A }\end{array}$ & $\begin{array}{c}\text { N/A } \\
b\end{array}$ & $\stackrel{\text { N/A }}{b}$ & $\begin{array}{c}\text { N/A } \\
b\end{array}$ & $\begin{array}{c}\mathrm{N} / \mathrm{A} \\
\mathrm{b}\end{array}$ & $\begin{array}{c}\text { N/A } \\
\text { b }\end{array}$ & $\begin{array}{c}\text { N/A } \\
\text { b }\end{array}$ \\
\hline $\begin{array}{l}\text { MTR Type } \\
\text { (Georgia } \\
\text { Tech) }\end{array}$ & $\begin{array}{c}\mathbf{N} / \mathrm{A} \\
\mathbf{b}\end{array}$ & $\begin{array}{c}\text { No } \\
\mathrm{a}\end{array}$ & $\begin{array}{c}\text { Yes } \\
\text { A }\end{array}$ & $\begin{array}{c}\mathrm{N} / \mathrm{A} \\
\mathrm{b}\end{array}$ & $\begin{array}{c}\text { N/A } \\
b\end{array}$ & $\left|\begin{array}{c}\mathrm{N} / \mathrm{A} \\
\mathrm{b}\end{array}\right|$ & $\begin{array}{c}\text { N/A } \\
\text { b }\end{array}$ & $\begin{array}{c}\text { N/A } \\
b\end{array}$ & $\begin{array}{c}\text { N/A } \\
\text { b }\end{array}$ & $\begin{array}{c}\text { No } \\
\text { a }\end{array}$ & $\begin{array}{c}\text { N/A } \\
b\end{array}$ & $\begin{array}{c}\text { N/A } \\
\text { b }\end{array}$ & $\begin{array}{c}\text { N/A } \\
\text { b }\end{array}$ & $\begin{array}{c}\text { N/A } \\
b\end{array}$ & $\begin{array}{c}\text { N/A } \\
\text { b }\end{array}$ & $\begin{array}{c}\text { N/A } \\
\text { b }\end{array}$ & $\begin{array}{l}\text { N/ } \\
\text { A } \\
\text { b }\end{array}$ & $\begin{array}{c}\text { N/A } \\
\text { b }\end{array}$ & $\begin{array}{c}\text { N/A } \\
b\end{array}$ & $\begin{array}{c}\text { N/A } \\
b\end{array}$ & $\begin{array}{c}\mathrm{N} / \mathrm{A} \\
\mathrm{b}\end{array}$ & $\begin{array}{c}\text { N/A } \\
\text { b }\end{array}$ & $\begin{array}{c}\text { N/A } \\
b\end{array}$ \\
\hline $\begin{array}{l}\text { MTR-Type } \\
\text { (MIT) }\end{array}$ & $\begin{array}{c}\mathbf{N} / \mathrm{A} \\
\mathrm{b}\end{array}$ & $\begin{array}{c}\text { No } \\
\text { a }\end{array}$ & $\begin{array}{c}\text { N/A } \\
b\end{array}$ & $\begin{array}{c}\text { N/A } \\
b\end{array}$ & $\begin{array}{c}\mathrm{N} / \mathrm{A} \\
\mathrm{b}\end{array}$ & $\left|\begin{array}{c}\mathrm{N} / \mathrm{A} \\
\mathrm{b}\end{array}\right|$ & $\begin{array}{c}\text { N/A } \\
\text { b }\end{array}$ & $\begin{array}{c}\text { N/A } \\
b\end{array}$ & $\begin{array}{c}\text { N/A } \\
b\end{array}$ & $\begin{array}{c}\text { No } \\
\text { a }\end{array}$ & $\begin{array}{c}\mathbf{N} / \mathbf{A} \\
\mathbf{b}\end{array}$ & $\begin{array}{c}\text { N/A } \\
\text { b }\end{array}$ & $\begin{array}{c}\text { N/A } \\
b\end{array}$ & $\begin{array}{c}\text { N/A } \\
\text { b }\end{array}$ & $\begin{array}{c}\text { N/A } \\
b\end{array}$ & $\begin{array}{c}\text { N/A } \\
b\end{array}$ & $\begin{array}{l}\text { N/ } \\
\text { A }\end{array}$ & $\begin{array}{c}\text { N/A } \\
\text { b }\end{array}$ & $\begin{array}{c}\text { N/A } \\
b\end{array}$ & $\begin{array}{c}\text { N/A } \\
b\end{array}$ & $\begin{array}{c}\mathrm{N} / \mathrm{A} \\
\mathrm{b}\end{array}$ & $\begin{array}{c}\text { N/A } \\
b\end{array}$ & $\begin{array}{c}\text { N/A } \\
\text { b }\end{array}$ \\
\hline
\end{tabular}


Table 4-5. (continued).

\begin{tabular}{|c|c|c|c|c|c|c|c|c|c|c|c|c|c|c|c|c|c|c|c|c|c|c|c|}
\hline \multirow[b]{2}{*}{$\begin{array}{l}\text { Metallic } \\
\text { fuels }\end{array}$} & \multicolumn{13}{|c|}{ Legal weight truck packages } & \multicolumn{2}{|c|}{$\begin{array}{c}\text { Overweight } \\
\text { truck packages }\end{array}$} & \multicolumn{8}{|c|}{ Rail packages } \\
\hline & $\begin{array}{l}\mathrm{GE} \\
\mathrm{T} \cdot 2\end{array}$ & $\begin{array}{l}\text { GE- } \\
100\end{array}$ & BMI-1 & FSV-1 & $\begin{array}{l}\text { NLI- } \\
1 / 2\end{array}$ & $\mid \begin{array}{l}\text { NLI- } \\
6502\end{array}$ & T-3 & NAC-1 & \begin{tabular}{|c|} 
CNS \\
$1-13 \mathrm{G}$
\end{tabular} & NRBK-41 & $\begin{array}{l}\text { NAC } \\
\text { LWT }\end{array}$ & $\begin{array}{c}\text { GE Model } \\
2000\end{array}$ & $\begin{array}{l}\text { TN- } \\
\text { FSV }\end{array}$ & $\begin{array}{l}\text { TN-8 } \\
\text { TN-8L }\end{array}$ & $\mathrm{TN}-9$ & $\mathrm{M}-130$ & $\begin{array}{l}\text { IF- } \\
300\end{array}$ & $\begin{array}{l}\text { NLI- } \\
10 / 24\end{array}$ & $125-\mathrm{B}$ & $\begin{array}{l}\mathrm{TN}- \\
\mathrm{BRP}\end{array}$ & $\begin{array}{l}\text { TN. } \\
\text { REG }\end{array}$ & M-160 & M-140 \\
\hline $\begin{array}{l}\text { MTR Type } \\
\text { (U. Lowell) }\end{array}$ & $\begin{array}{c}\mathrm{N} / \mathrm{A} \\
\mathrm{b}\end{array}$ & $\begin{array}{c}\text { No } \\
\text { a }\end{array}$ & $\begin{array}{c}\mathrm{N} / \mathrm{A} \\
\mathrm{b}\end{array}$ & $\begin{array}{c}\text { N/A } \\
\text { b }\end{array}$ & $\underset{b}{N / A}$ & $\begin{array}{c}\text { N/A } \\
b\end{array}$ & $\begin{array}{c}\mathrm{N} / \mathrm{A} \\
\mathrm{b}\end{array}$ & $\begin{array}{c}\mathrm{N} / \mathrm{A} \\
\mathrm{b}\end{array}$ & $\begin{array}{c}\text { N/A } \\
b\end{array}$ & $\begin{array}{c}\text { No } \\
\text { a }\end{array}$ & $\begin{array}{c}\text { N/A } \\
\text { b }\end{array}$ & $\begin{array}{c}\mathrm{N} / \mathrm{A} \\
\mathrm{b}\end{array}$ & $\begin{array}{c}N / A \\
b\end{array}$ & $\begin{array}{c}\text { N/A } \\
b\end{array}$ & $\stackrel{\mathrm{N} / \mathrm{A}}{\mathrm{b}}$ & $\begin{array}{c}\text { N/A } \\
b\end{array}$ & $\begin{array}{l}\text { N/ } \\
\text { A } \\
\text { b }\end{array}$ & $\begin{array}{c}\text { N/A } \\
b\end{array}$ & $\begin{array}{c}\mathrm{N} / \mathrm{A} \\
\mathrm{b}\end{array}$ & $\begin{array}{c}\mathrm{N} / \mathrm{A} \\
\mathrm{b}\end{array}$ & $\begin{array}{c}\text { N/A } \\
b\end{array}$ & $\begin{array}{c}\text { N/A } \\
b\end{array}$ & $\begin{array}{c}\mathrm{N} / \mathrm{A} \\
\mathrm{b}\end{array}$ \\
\hline $\begin{array}{l}\text { MTR Type } \\
\text { (lowa St.) }\end{array}$ & $\begin{array}{c}\text { No } \\
\mathrm{a}\end{array}$ & $\begin{array}{c}\text { No } \\
a\end{array}$ & $\begin{array}{c}\mathrm{N} / \mathrm{A} \\
\mathrm{b}\end{array}$ & $\begin{array}{c}\text { N/A } \\
\text { b }\end{array}$ & $\begin{array}{c}\mathrm{N} / \mathrm{A} \\
\mathrm{b}\end{array}$ & $\begin{array}{c}\text { N/A } \\
\text { b }\end{array}$ & $\begin{array}{c}\text { N/A } \\
b\end{array}$ & $\begin{array}{c}\text { N/A } \\
b\end{array}$ & $\begin{array}{c}\text { N/A } \\
b\end{array}$ & $\begin{array}{c}\text { No } \\
\text { a }\end{array}$ & $\begin{array}{c}\text { N/A } \\
b\end{array}$ & $\begin{array}{c}\text { N/A } \\
\mathrm{b}\end{array}$ & $\begin{array}{c}\text { N/A } \\
b\end{array}$ & $\begin{array}{c}\text { N/A } \\
b\end{array}$ & $\begin{array}{c}\mathrm{N} / \mathrm{A} \\
\mathrm{b}\end{array}$ & $\begin{array}{c}\text { N/A } \\
b\end{array}$ & $\begin{array}{l}\text { N/ } \\
\text { A }\end{array}$ & $\begin{array}{c}\text { N/A } \\
b\end{array}$ & $\begin{array}{c}\mathrm{N} / \mathrm{A} \\
\mathrm{b}\end{array}$ & $\begin{array}{c}\text { N/A } \\
b\end{array}$ & $\begin{array}{c}\text { N/A } \\
b\end{array}$ & $\begin{array}{c}\text { N/A } \\
b\end{array}$ & $\begin{array}{c}\text { N/A } \\
\mathrm{b}\end{array}$ \\
\hline $\begin{array}{l}\text { MTR Type } \\
\text { (Worster } \\
\text { Tech. Inst.) }\end{array}$ & $\begin{array}{c}N / A \\
b\end{array}$ & $\begin{array}{c}\text { No } \\
a\end{array}$ & $\begin{array}{c}\mathrm{N} / \mathrm{A} \\
\mathrm{b}\end{array}$ & $\begin{array}{c}\mathrm{N} / \mathrm{A} \\
\mathrm{b}\end{array}$ & $\begin{array}{c}\mathrm{N} / \mathrm{A} \\
\mathrm{b}\end{array}$ & $\begin{array}{c}N / A \\
b\end{array}$ & $\begin{array}{c}\text { N/A } \\
b\end{array}$ & $\begin{array}{c}\text { N/A } \\
b\end{array}$ & $\begin{array}{c}N / A \\
b\end{array}$ & $\begin{array}{c}\text { No } \\
\text { a }\end{array}$ & $\begin{array}{c}\text { N/A } \\
b\end{array}$ & $\begin{array}{c}\text { N/A } \\
b\end{array}$ & $\begin{array}{c}\text { N/A } \\
b\end{array}$ & $\begin{array}{c}\text { N/A } \\
b\end{array}$ & $\begin{array}{c}\mathrm{N} / \mathrm{A} \\
\mathrm{b}\end{array}$ & $\begin{array}{c}\text { N/A } \\
b\end{array}$ & $\begin{array}{l}\text { N/ } \\
\text { A } \\
\text { b }\end{array}$ & $\begin{array}{c}\mathrm{N} / \mathrm{A} \\
\mathrm{b}\end{array}$ & $\begin{array}{c}\mathrm{N} / \mathrm{A} \\
\mathrm{b}\end{array}$ & $\begin{array}{c}\mathrm{N} / \mathrm{A} \\
\mathrm{b}\end{array}$ & $\begin{array}{c}N / A \\
b\end{array}$ & $\begin{array}{c}\mathrm{N} / \mathrm{A} \\
\mathrm{b}\end{array}$ & $\begin{array}{c}N / A \\
b\end{array}$ \\
\hline $\begin{array}{l}\text { MTR Type } \\
\text { (Purdue U.) }\end{array}$ & $\begin{array}{c}N / A \\
b\end{array} \mid$ & $\begin{array}{c}\text { No } \\
\text { a }\end{array}$ & $\begin{array}{c}\mathrm{N} / \mathrm{A} \\
\mathrm{b}\end{array}$ & $\begin{array}{c}\text { N/A } \\
\text { b }\end{array}$ & $\begin{array}{c}\text { N/A } \\
b\end{array}$ & $\begin{array}{c}\mathrm{N} / \mathrm{A} \\
\mathrm{b}\end{array}$ & $\begin{array}{c}\mathrm{N} / \mathrm{A} \\
\mathrm{b}\end{array}$ & $\begin{array}{c}\text { N/A } \\
b\end{array}$ & $\begin{array}{c}\text { N/A } \\
b\end{array}$ & $\begin{array}{c}\text { No } \\
\mathbf{a}\end{array}$ & $\begin{array}{c}\text { N/A } \\
\text { b }\end{array}$ & $\begin{array}{c}N / A \\
b\end{array}$ & $\begin{array}{c}\text { N/A } \\
b\end{array}$ & $\begin{array}{c}\mathrm{N} / \mathrm{A} \\
\mathrm{b}\end{array}$ & $\begin{array}{c}\mathrm{N} / \mathrm{A} \\
\mathrm{b}\end{array}$ & $\begin{array}{c}\text { N/A } \\
b\end{array}$ & $\begin{array}{l}\text { N/ } \\
\text { A } \\
\text { b }\end{array}$ & $\begin{array}{c}\text { N/A } \\
b\end{array}$ & $\begin{array}{c}\mathrm{N} / \mathrm{A} \\
\mathrm{b}\end{array}$ & $\begin{array}{c}\mathrm{N} / \mathrm{A} \\
\mathrm{b}\end{array}$ & $\begin{array}{c}N / A \\
b\end{array}$ & $\begin{array}{c}\text { N/A } \\
b\end{array}$ & $\begin{array}{c}\mathrm{N} / \mathrm{A} \\
\mathrm{b}\end{array}$ \\
\hline $\begin{array}{l}\text { MTR Type } \\
\text { (RINSC) }\end{array}$ & $\begin{array}{c}\mathrm{N} / \mathrm{A} \\
\mathrm{a}\end{array}$ & $\begin{array}{c}\mathrm{N} / \mathrm{A} \\
\mathrm{a}\end{array}$ & $\begin{array}{c}\text { N/A } \\
a\end{array}$ & $\begin{array}{c}\text { N/A } \\
\mathbf{a}\end{array}$ & $\begin{array}{l}\text { N/A } \\
a\end{array}$ & $\begin{array}{c}\mathrm{N} / \mathrm{A} \\
\mathrm{a}\end{array}$ & $\begin{array}{c}\text { N/A } \\
a\end{array}$ & $\begin{array}{c}\text { N/A } \\
\mathbf{a}\end{array}$ & $\begin{array}{c}\text { N/A } \\
a\end{array}$ & $\begin{array}{c}\text { No } \\
\text { a }\end{array}$ & $\begin{array}{c}\text { N/A } \\
a\end{array}$ & $\begin{array}{c}\mathrm{N} / \mathrm{A} \\
\mathrm{a}\end{array}$ & $\begin{array}{c}\mathrm{N} / \mathrm{A} \\
\mathrm{a}\end{array}$ & $\begin{array}{c}\text { N/A } \\
a\end{array}$ & $\begin{array}{c}\mathrm{N} / \mathrm{A} \\
\mathrm{a}\end{array}$ & $\begin{array}{c}\text { N/A } \\
\mathbf{a}\end{array}$ & $\begin{array}{l}\mathbf{N} / \\
\mathrm{A}\end{array}$ & $\begin{array}{c}\mathrm{N} / \mathrm{A} \\
\mathrm{a}\end{array}$ & $\begin{array}{c}\mathrm{N} / \mathrm{A} \\
\mathrm{a}\end{array}$ & $\begin{array}{c}\mathbf{N} / \mathbf{A} \\
\mathrm{a}\end{array}$ & $\begin{array}{c}\mathrm{N} / \mathrm{A} \\
\mathrm{a}\end{array}$ & $\begin{array}{l}\mathrm{N} / \mathrm{A} \\
\mathrm{a}\end{array}$ & $\begin{array}{c}\mathrm{N} / \mathrm{A} \\
\mathrm{a}\end{array}$ \\
\hline $\begin{array}{c}\text { Argonaut } \\
\text { (U. Florida) }\end{array}$ & $\begin{array}{c}\mathrm{N} / \mathrm{A} \\
\mathrm{b}\end{array}$ & $\begin{array}{c}\text { No } \\
\text { a }\end{array}$ & $\begin{array}{c}\text { N/A } \\
b\end{array}$ & $\begin{array}{c}\text { N/A } \\
\text { b }\end{array}$ & $\begin{array}{c}\text { N/A } \\
b\end{array}$ & $\begin{array}{c}\text { N/A } \\
b\end{array}$ & $\begin{array}{c}\text { N/A } \\
b\end{array}$ & $\begin{array}{c}\text { N/A } \\
b\end{array}$ & $\begin{array}{c}\text { N/A } \\
\text { b }\end{array}$ & $\begin{array}{c}\text { No } \\
\text { a }\end{array}$ & $\begin{array}{c}\text { N/A } \\
b\end{array}$ & $\begin{array}{c}\text { N/A } \\
\text { b }\end{array}$ & $\begin{array}{c}\text { N/A } \\
\text { b }\end{array}$ & $\begin{array}{c}\text { N/A } \\
b\end{array}$ & $\begin{array}{c}N / A \\
b\end{array}$ & $\begin{array}{c}\text { N/A } \\
b\end{array}$ & $\begin{array}{l}\text { N/ } \\
\text { A }\end{array}$ & $\begin{array}{c}\text { N/A } \\
b\end{array}$ & $\begin{array}{c}\mathrm{N} / \mathrm{A} \\
\mathrm{b}\end{array}$ & $\begin{array}{c}\mathrm{N} / \mathrm{A} \\
\mathrm{b}\end{array}$ & $\begin{array}{c}\text { N/A } \\
b\end{array}$ & $\begin{array}{c}\text { N/A } \\
b\end{array}$ & $\begin{array}{c}\text { N/A } \\
\text { b }\end{array}$ \\
\hline $\begin{array}{c}\text { OSR } \\
\text { (Ohio St.) }\end{array}$ & $\begin{array}{c}\text { N/A } \\
b\end{array}$ & $\begin{array}{c}\text { No } \\
\text { a }\end{array}$ & $\begin{array}{c}\mathrm{N} / \mathrm{A} \\
\mathrm{b}\end{array}$ & $\begin{array}{c}\text { N/A } \\
\text { b }\end{array}$ & $\begin{array}{c}\text { N/A } \\
\text { b }\end{array}$ & $\begin{array}{c}\mathrm{N} / \mathrm{A} \\
\mathrm{b}\end{array}$ & $\begin{array}{c}\text { N/A } \\
b\end{array}$ & $\begin{array}{c}\text { N/A } \\
\text { b }\end{array}$ & $\begin{array}{c}\text { N/A } \\
\text { b }\end{array}$ & $\begin{array}{c}\text { No } \\
\text { a }\end{array}$ & $\begin{array}{c}\text { N/A } \\
\text { b }\end{array}$ & $\begin{array}{c}\text { N/A } \\
b\end{array}$ & $\begin{array}{c}\text { N/A } \\
b\end{array}$ & $\begin{array}{c}\text { N/A } \\
\text { b }\end{array}$ & $\begin{array}{c}N / A \\
b\end{array}$ & $\begin{array}{c}\text { N/A } \\
b\end{array}$ & $\begin{array}{c}\text { N/ } \\
\text { A } \\
\text { b }\end{array}$ & $\begin{array}{c}\text { N/A } \\
\mathrm{b}\end{array}$ & $\begin{array}{c}\text { N/A } \\
b\end{array}$ & $\begin{array}{c}\mathrm{N} / \mathrm{A} \\
\mathrm{b}\end{array}$ & $\begin{array}{c}\text { N/A } \\
b\end{array}$ & $\begin{array}{c}\text { N/A } \\
b\end{array}$ & $\begin{array}{c}\text { N/A } \\
\text { b }\end{array}$ \\
\hline
\end{tabular}




\begin{tabular}{|c|c|c|c|c|c|c|c|c|c|}
\hline \multirow{8}{*}{ 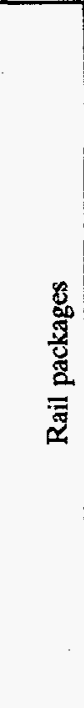 } & $\frac{9}{\frac{1}{2}}$ & 20 & $B O$ & $\frac{B}{2} 0$ & 20 & 20 & $\underset{\gamma}{\mathscr{C}} 0$ & $\sum_{2}^{0} 0$ & \multirow{8}{*}{ 它 } \\
\hline & $\frac{8}{5}$ & $\sum_{\nu}^{8}$ & $\sum_{2} 0$ & ఫ્ર 0 & 20 & ن & $\sum_{20} 0$ & $\sum^{\infty} 0$ & \\
\hline & 忘岕 & $\sum_{2}^{8}$ & $\sum_{2}^{8} 0$ & 0 & $\otimes 0$ & 20 & $\stackrel{0}{*} 0$ & $\underset{Z}{B} 0$ & \\
\hline & 悹空 & 20 & $\sum_{2}^{\infty} 0$ & $y^{3} u$ & $D_{0}$ & $\stackrel{8}{8}$ & ${ }_{2}^{8}<$ & $\sum_{2} 0$ & \\
\hline & 品 & 20 & 80 & $\frac{3}{2} 0$ & 00 & $\gg 0$ & $\frac{1}{2} 0$ & 20 & \\
\hline & $\dot{\vec{z}} \mathrm{~S}$ & $\sum_{0} 0$ & 20 & $\frac{1}{2} 0$ & 20 & 20 & 20 & $\geqslant 0$ & \\
\hline & 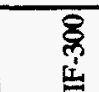 & 20 & ن & 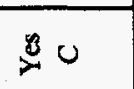 & $\sum_{>}^{8} 0$ & ن & $\sum^{2} 0$ & ن & \\
\hline & 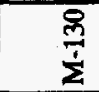 & $\leqslant$ & $\frac{1}{z}$ & $\leqslant$ & $\leqslant 0$ & $\leqslant$ & $\leqslant 0$ & $\leqslant$ & \\
\hline \multirow{2}{*}{ 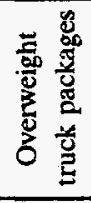 } & 完 & $y_{2} u$ & $\sum 0$ & 20 & $\sum_{x}$ & 20 & zo $=$ & $\underbrace{8}_{2} 0$ & \multirow{16}{*}{ 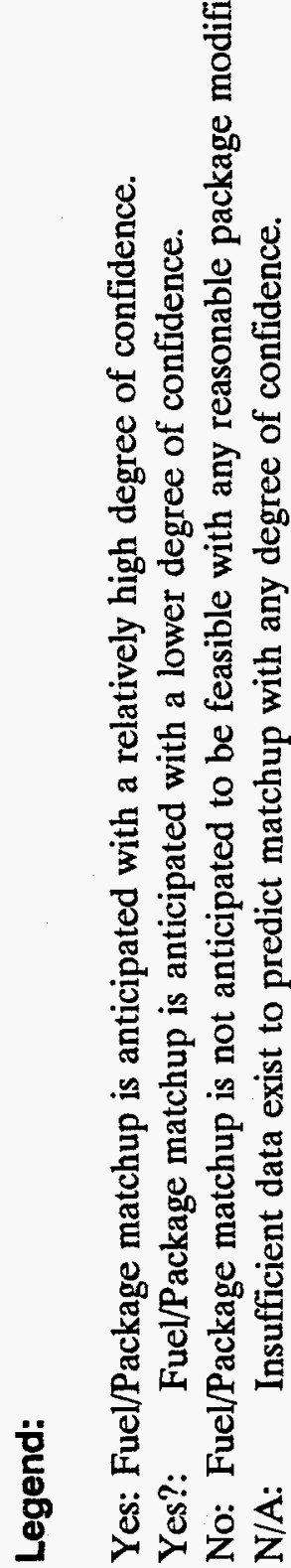 } \\
\hline & 串京 & $\sum_{2}^{2} 0$ & $\stackrel{0}{2} 0$ & $\frac{B}{2} 0$ & $x_{\nu}^{B} 0$ & $\sum_{\nu}^{\infty} 0$ & $\underset{0}{2} 0$ & 0 & \\
\hline & 它密 & $x_{0} 0$ & $\sum 0$ & $\sum_{2}^{8} 0$ & 20 & 20 & 20 & 20 & \\
\hline & 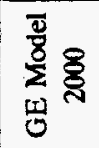 & $\sum_{2} 0$ & U & $D_{2} 0$ & 20 & $\frac{80}{20}$ & $\stackrel{z}{z}$ & $\stackrel{2}{z}=$ & \\
\hline & 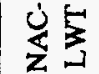 & ע & $\$ 0$ & o & 20 & 20 & $\searrow 0$ & 20 & \\
\hline & $\begin{array}{l}\vec{y} \\
\vec{z} \\
\vec{z} \\
\frac{z}{z}\end{array}$ & 20 & 20 & 20 & 之 $\sigma$ & z $\sigma$ & 之 & $\stackrel{\circ}{z} \sigma$ & \\
\hline & $\overbrace{3}^{0}$ & D & 赵 & $\underbrace{}_{0}$ & 20 & 20 & z $\pi$ & $z=$ & \\
\hline & 总 & $\sum_{\nu}^{\mathscr{B}} \infty$ & $\underbrace{D}$ & $\stackrel{D}{\infty} \infty$ & $\underset{\gamma}{\mathscr{C}} \infty$ & $\stackrel{\mathscr{Z}}{\not} \infty$ & D & $\sum_{\nu}^{B} U$ & \\
\hline & 3 & 20 & 20 & $\underbrace{80}_{0}$ & $\sum_{2}^{8} 0$ & $\sum_{\gamma}^{8}$ & $\dot{z} \approx$ & $\sum_{2}^{\infty} 0$ & \\
\hline & $\dot{\vec{Z}}$ & $i_{>} 0$ & 80 & $\infty 0$ & 20 & $\sum_{\gamma} 0$ & $\$ 0$ & $\$ 0$ & \\
\hline & $\frac{\dot{B}}{z}$ & 80 & 20 & 20 & 20 & 20 & $\approx 0$ & 20 & \\
\hline & $\overrightarrow{\overrightarrow{0}}$ & $\gtrsim 0$ & $\underbrace{2} u$ & $\sum 0$ & $\underbrace{}_{x}$ & $\sum 0$ & $\sum_{0}$ & $\sum^{\infty} 0$ & \\
\hline & $\overrightarrow{\dot{\vec{m}}}$ & $\stackrel{0}{*}$ & $\gtrsim 0$ & $\underset{\nu}{*}$ & $\sum_{\nu}$ & $\overbrace{\nearrow}^{\mathscr{B}}$ & $\dot{z}=$ & $z=$ & \\
\hline & 빙 응 & $\sum_{2} 0$ & $\sum_{0}^{\infty} 0$ & 20 & $\stackrel{\circ}{z}=$ & $\dot{z}=$ & $\stackrel{\circ}{z}=$ & $\stackrel{2}{z} \approx$ & \\
\hline & 罗 & $\$ 0$ & 20 & 20 & $\sum 0$ & $\underbrace{}_{0} 0$ & 之o & z $\sigma$ & \\
\hline & 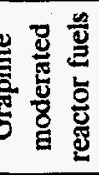 & 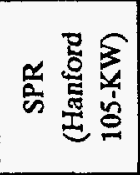 & 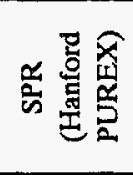 & 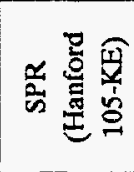 & 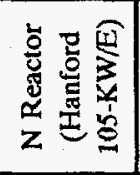 & 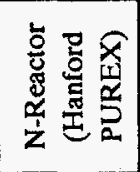 & 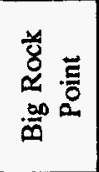 & 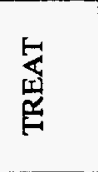 & \\
\hline
\end{tabular}


Table 4-6. (continued).

\section{Legend (continued):}

a. Matchup is not feasible due to dimensional discrepancies (No), or matchup cannot be evaluated because available fuel dimensional data is insufficient to make a determination with any degree of confidence (N/A).

b. Matchup is not feasible due to shielding requirements (No), or matchup cannot be evaluated because available fuel radiological data is insufficient to make a determination with any degree of confidence (N/A).

c. Matchup is not feasible due to thermal limitations (No), or matchup cannot be evaluated because available fuel thermal data is insufficient to make a determination with any degree of confidence (N/A).

d. Matchup is not feasible due to payload weight restrictions (No), or matchup cannot be evaluated because available fuel weight data is insufficient to make a determination with any degree of confidence (N/A).

A: No package modifications expected to be required (see Section 4.1)

B: Package Certificate of Compliance amendment probably required (see Section 4.2)

C: Package SARP and Certificate of Compliance revisions probably required (see Section 4.3) 


\subsection{Capability That Currently Exists}

The specific cask/fuel matchups which currently permit transport are as follows:

- FFTF fuel and certain other irradiated materials are authorized for shipment in the T-3 package

- Three-Mile Island damaged fuel is authorized for shipment in the 125-B package

- Naval reactor fuel is authorized for shipment in the M-130, M-140, and M-160 packages

- Fort St. Vrain spent fuel is authorized for shipment in the FSV-1 and TN-FSV packages

- $\quad$ R.E. Gina (REG) spent fuel is authorized for shipment in the TN-REG package

- $\quad$ Big Rock Point (BRP) spent fuel is authorized for shipment in the TN-BRP package

- GE Model 2000 has been specifically modified to transport HFIR fuel

- BMI-1 has been authorized to carry several research reactor fuel types

- Commercial-type fuels that are adequately characterized can be shipped in casks specifically developed for commercial fuel.

\subsection{Capability via Amended SARP and Certificate of Compliance (No Hardware Changes)}

This action will be the least effort expected in order to qualify a package for a payload for which it is not specifically licensed. If a fuel type or types can be determined which are fully bounded by a package's current payload definition, as determined by all the parameters listed in Section 2.2, then an amendment to the certificate of compliance should be obtainable. Based on recent experience of licensing the T-3 package for alternative payloads, this effort could be completed in anywhere from 3 to 12 months.

\subsection{Capability via Revised SARP and Certificate of Compliance (No Hardware Changes)}

For fuel types whose governing parameters fall outside of the payload capabilities of a currently licensed package, it is possible that the safety analysis report for that package could be revisited with an eye to revising calculations based on extended payload demands (heavier payload, greater heat load, higher operating pressure, etc.). If the resulting margins of safety remain positive, it is conceivable that a revised Certificate of Compliance might be obtained for the package, qualifying it for the desired fuel type(s). However, smaller margins almost automatically dictate a significantly greater level of analysis refinement and regulatory interaction. 
It is also possible that some currently licensed packages not specifically designed to transport irradiated fuel could be requalified for that purpose. It is unlikely that any revisions such as detailed above could be accomplished in less than a year's time.

\subsection{Capability via Revised SARP and Certificate of Compliance (Hardware Development Anticipated)}

This type of modification is not directly indicated in Tables 4-1 through 4-6, because it is expected be the minimum level of effort required for nearly all of the fuel types. With the exception of those listed in Section 4.1, very few of the SNF types have geometries compatible with existing transport packages. Almost all will likely require some type of support before they can be transported in packages that were generally designed for different payload geometries. This support could take the form of either custom-designed baskets (possibly incorporating neutron poisoning material for criticality control), or possibly repackaging (canistering). The required design development and fabrication of the associated hardware, along with the related licensing activities, would probably not be accomplished in less than 18 months.

\subsection{Capability via Packaging Design Change (New Certificate of Compliance)}

It is possible that, with fairly modest design changes (new impact limiters, different types of seals, additional closure bolts, etc.), an existing package could be qualified for a new certificate of compliance for increased payload capability. It is anticipated that such an approach could not be successfully accomplished in less than two years' time.

\subsection{No Apparent Capability (New Package Design Required)}

For fuel types (or possibly, certain subsets of fuel types) that cannot be accommodated by the above approaches, a new package design or designs would have to be undertaken. Based on recent experience, development of an entirely new package through the design, licensing, and fabrication phases is unlikely to be accomplished in a time frame of less than three years. If packages currently undergoing development could be used, this time frame could be shortened, depending on the stage of development of the particular package.

\subsection{Additional Study Needed to Establish Capability}

The many types of fuels, and wide variety of conditions within some fuel types, will require that careful and detailed evaluations be performed in order to characterize the fuel adequately for determination of a suitable packaging matchup (or development) for transportation of that fuel. This study can only provide a rough order of magnitude of the effort that will be required for transportation of a portion of the noncommercial spent nuclear fuel inventory.

As a final note, it should be emphasized that the above estimates on time required for package licensing are based largely on experience with the Nuclear Regulatory Commission's Transportation Branch. The built-in assumption is that the licensing contractor will be well 
qualified and experienced, with a good, long-term working relationship with the NRC regulators, who themselves have had many years of experience in transportation system design review. These factors will assure a timely and efficient review process. Since the relative youth of the current DOE regulatory system generally precludes these conditions, it can be expected that, on the whole, obtaining a DOE Certificate of Compliance will probably require a longer time frame. 


\section{OTHER FACTORS}

In addition to all of the fuels and packaging parameters listed in the above sections, numerous other factors will affect the transport feasibility of the SNF inventory.

\subsection{Packaging Availability}

Some of the currently licensed packages may be involved in shipping campaigns in the near or intermediate future, which will reduce or eliminate their availability for purposes of transporting DOE-owned SNF. Approximate anticipated availability for the irradiated fuel packages is summarized in Table 3-1. Exact figures are difficult to determine in many cases, since the shipping campaigns depend on a variety of other factors, not the least of which is availability of storage sites to which the designated payloads are to be shipped. An example would be the TN-REG and TN-BRP packages, which are earmarked for transport of West Valley fuels to intermediate storage facilities yet to be developed. In some cases, the planned shipping campaigns involve the study fuels, such as the TN-FSV package is scheduled to transport Fort St. Vrain fuel, and the M-series packages (M-130, M-140 and M-160) will be utilized to ship naval reactor fuels. Unless program priorities change, these packages would not be available for other duty. Other packages are readily available, and their owners are eager to have them utilized as soon as possible. Whether the immediately available packages are those which would be of greatest utility for transporting DOE-owned SNF remains to be determined.

Another factor to be considered is that existing packages are limited in number, sometimes one of a kind. Due to recent regulatory revisions, additional fabrication of most of these packages (those fabricated prior to August 31, 1986) cannot be undertaken. By December 31, 1995, additional fabrication of the remainder of the currently licensed package designs will also likely be prohibited unless they are demonstrated to be in compliance with revised (more stringent) transportation rules.

\subsection{Transport Vehicles and Auxiliary Equipment}

Some of the currently licensed packages require specially designed, dedicated transport vehicles (e.g., the 125-B requires an articulated railcar). Some have specialty transporters, but are capable of being transported on other vehicles, suitably modified (e.g., the T-3, which has three packages but only one dedicated specialty trailer). The trailers for the TN-9 packages will require refurbishment before being put back into service. Unless periodic maintenance is performed on these specialty transporters, it is likely that, over time, they will deteriorate and eventually become unusable without some amount of rework.

Additionally, specialty auxiliary equipment will almost certainly be required for each package for loading and unloading operations. The 125-B was originally furnished with remote handling equipment suitable for its design payload. This equipment may or may not still exist in a serviceable condition. Handling equipment for this and other packages will likely have to be developed specifically for the off-design fuels they may be required to transport. While the development of such equipment does not require the extensive effort required for licensed 
transport packages, it will still require a not-insignificant expenditure of time and resources, and must be included in any SNF transportation management plan.

In the case of leak-tight sealing requirements, which is likely to be necessary, at least for damaged and deteriorated fuels, specialty leak-detection equipment will be required. For the very small leak rate defined as "leaktight" by Reference $13\left(1 \times 10^{-7} \mathrm{scc} / \mathrm{sec}\right.$, air maximum), a rather complex leak-check procedure utilizing precision equipment, such as a helium mass spectrometer, will be necessary. Training of the personnel who will undertake this activity will be required. Other loading activities will also require special training (dewatering or drying, inert gas backfill, etc.). Allowance must be made for developing procedures and training personnel for these activities for all affected facilities.

\subsection{Site and Facility Interfaces}

As mentioned earlier, an important consideration is facility accessibility by the particular transport package required to service that facility. A summary of facility interface parameters is given in Table 5-1. The main interface considerations are as follows:

- Transport mode (road, rail, ship)

- Loading mode (wet or dry)

- Gross package weight (should not exceed facility crane capacity)

- Dimensional restrictions (access door dimensions, ceiling height, etc., as applicable)

- Operation restrictions (electrical equipment important to safety).

These restrictions could potentially limit access by the package (or packages) that might be required to remove spent fuel from the particular facility where it is located. In that event, multiple transfers or facility modifications might be required. Further complication is introduced by the fact that some of the fuel types are stored in more than one location. See Table 5-1 for a partial listing of these fuels.

\subsection{System Efficiencies}

As previously set out in Section 2.3 , it is conceivable that the most efficient shipping campaign for even a single fuel type might require more than one transport package, or multiple authorized payload definitions for a given package. This is because any single fuel type might involve a large variety of significant transport-related factors.

As an example, the $\mathrm{N}$-Reactor fuel at the Hanford site spans a wide range of key parameters. As shown in Figure 5-1, this fuel type has two major configurations, Mark IV and Mark IA. Between these two configurations, there are a total of seven different sizes, each size with two primary burnup levels, and each of these with a potentially large span of time out of 
Table 5-1. Examples of single fuel types currently stored in multiple locations.

\begin{tabular}{|c|c|c|c|}
\hline Fuel Type & Location (Facility) & MTHM & Total MTHM \\
\hline \multirow[t]{3}{*}{ N Reactor } & Hanford (KE-Basin) & 1145 & - \\
\hline & Hanford (KW-Basin) & 961 & - \\
\hline & Hanford (PUREX) & 0.3 & - \\
\hline N Reactor Total & & & $2,106.30$ \\
\hline \multirow[t]{3}{*}{ Target } & SRS (L-Basin) & 115.498 & - \\
\hline & SRS (F-Canyon) & 22.634 & - \\
\hline & SRS (RBOF) & 0.37 & - \\
\hline Target Total & & & 138.50 \\
\hline \multirow[t]{5}{*}{ EBR-II } & ANL-W (EBR-II) & 25 & - \\
\hline & ANL-W (HFEF) & 11.89 & - \\
\hline & INEL (CPP-603) & 1.2 & - \\
\hline & INEL (CPP-666) & 0.799 & - \\
\hline & Hanford (Bldg-325) & 0.08 & - \\
\hline EBR-II Total & & & 38.97 \\
\hline \multirow[t]{2}{*}{ FSVR } & PSC (FSVR) & 16 & - \\
\hline & INEL (CPP-IFSF) & 8.6 & - \\
\hline FSVR Total & & & 24.60 \\
\hline \multirow[t]{3}{*}{ Shippingport PWR } & Hanford (T-Plant) & 16.2 & - \\
\hline & NRF (ECF) & 1.6 & - \\
\hline & INEL (CPP-666) & 0.599 & 一 \\
\hline Ship'pt PWR Total & & & 18.40 \\
\hline
\end{tabular}


Table 5-1. (continued).

\begin{tabular}{|c|c|c|c|}
\hline Fuel Type & Location (Facility) & MTHM & Total MTHM \\
\hline \multirow[t]{3}{*}{ Naval } & INEL (CPP-666) & 4.816 & - \\
\hline & NRF (ECF) & 2.5 & - \\
\hline & INEL (CPP-603) & 0.952 & - \\
\hline Naval Total & & & 8.27 \\
\hline \multirow[t]{3}{*}{ SPR } & Hanford (PUREX) & 2.9 & - \\
\hline & Hanford (KE-Basin) & 0.4 & - \\
\hline & Hanford (KW-Basin) & 0.1 & 一 \\
\hline SPR Total & & & 3.40 \\
\hline \multirow[t]{5}{*}{ Peachbottom } & INEL (CPP-749) & 1.654 & - \\
\hline & INEL (CPP-603) & 1.346 & - \\
\hline & INEL (TAN-607) & 0.356 & - \\
\hline & INEL (TRA-603) & 0.010 & - \\
\hline & ORNL (Bldg-7829) & $\begin{array}{l}\text { unknown } \\
\text { (probably } \\
<.005 \text { ) }\end{array}$ & - \\
\hline Peachbottom Total & & & 3.37 \\
\hline \multicolumn{4}{|l|}{ HFBR } \\
\hline & BNL (HFBR) & 0.25 & - \\
\hline & INEL (CPP-666) & 0.0584 & - \\
\hline & INEL (CPP-603) & 0.005 & - \\
\hline HFBR Total & & & 0.31 \\
\hline
\end{tabular}




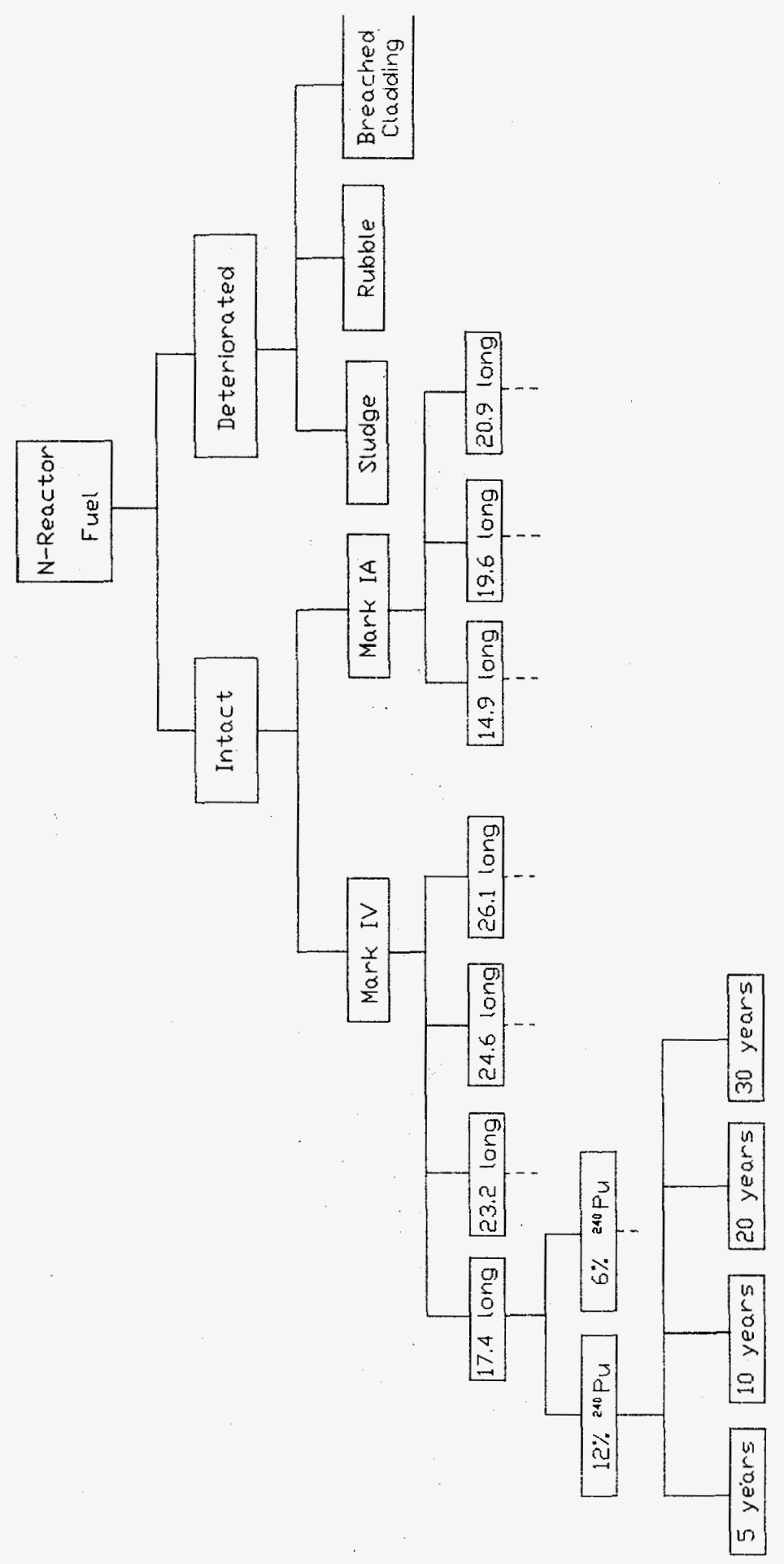

5-1. N-Reactor fuel variables. 
reactor. Additionally, some of this fuel is deteriorated, involving every condition from breached cladding down to disintegration into sludge. It is highly unlikely that a single transport package could be efficiently utilized to transport all of this fuel.

If the worst-condition fuel were used as the baseline for package selection, the selected transport package would probably be greatly overdesigned for the remainder of the fuel. The resulting limited transport capability would necessarily involve more shipments than would be required for a package more optimally matched with the non-worst-case fuel.

Even in those instances where all of a given fuel type can be transported in a single package, transportation efficiency might be unacceptably low. An example would be the T-3 package, which is capable of carrying only one Fast Flux Test Facility (FFTF) fuel assembly per shipment.

As discussed in Section 3.3, if several fuel types can be categorized and administratively controlled to the satisfaction of the regulatory authority, multiple payload definitions or amendments for a given package could be developed, significantly improving overall system efficiency. However, it must be emphasized that this goal will not be easily obtainable. In general, the greater the variety of payload types, the larger will be the envelope of characteristics (thermal, radiological, etc.) imposed on a candidate packaging. It should be realized that the process of obtaining a Certificate of Compliance for a package design that includes only a single payload type, or a small, very well-defined subset of payload types, is itself a very laborious undertaking. Attempting to broaden the payload definition will likely only compound the effort required to license the candidate package.

\subsection{Schedule Requirements}

There are a great number of factors affecting schedule. The single biggest schedule driver will likely be privatization of shipping campaigns within the DOE system. Priorities will depend strongly on fuel or storage facility conditions, as well as politics, and certain shipping campaigns may be found to require immediate action.

Once governing schedule constraints are identified and priorities established, detailed implementation strategies and schedules can then be developed. If schedule constraints are minimal, more efficient shipping campaigns can be achieved. Tight schedules will tend to minimize implementation options (due to funding availability problems, time frames which dictate simple licensing amendments, etc.), and inherently lead to less efficient shipments.

It must be kept in mind that, although preparations for an efficient shipping campaign may require more up-front time and cost, the savings realized once shipments begin may be found to easily offset these impacts. The extent of necessary package modifications, or possibly development efforts, would determine when a shipping campaign for a particular fuel could commence. Multiple transfers, or facility modifications, where there is an incompatible package/facility interface might be another consideration affecting a shipping campaign. Package availability would be a concern, as well as time required for transfer equipment and procedure development, and personnel training. 
In any case, except for those few cases where fuel/package compatibility already exists, a minimum of six months should be allowed for preparation of a shipping campaign. The subsequent length of the campaign will depend on quantity of fuel and efficiency of shipments. If program priorities are low or design/SARP revisions are significant, a three or more year preparation time may be required.

\subsection{Costs}

All of the factors mentioned in the above section will have a direct impact on total transport costs, as well. It is expected that the following factors will play the largest role in overall transportation costs:

- Lease or purchase costs for transport existing packages

(The IF-300 lease fees are currently on the order of $\$ 3000-\$ 4000$ per day)

- $\quad$ SARP revision costs

(Recent T-3 amendments have cost from $\$ 20,000$ to $\$ 50,000$, exclusive of any necessary design and review costs; NRC fees are currently $\$ 134$ per hour for SARP review)

- New package development and fabrication costs (including SARP preparation) (A current package development project is just entering its licensing stage (i.e., drop testing and Rev 0 SARP submittal) after more than three and a half years since contract award; projected total development costs are anticipated to be in excess of $\$ 15$ million)

- Facility upgrade or development is expected to be a major cost driver.

\subsection{Public Involvement}

A major factor in the development of SNF Transportation Plans is the very large degree of public involvement which can be expected. The single shipping campaign involving the Three-Mile Island damaged fuel transport attracted an enormous amount of attention and sparked an unprecedented public relations campaign on the part of the DOE (see References 14 and 15 for details). An undertaking involving up to 230 different types of SNF from a large number of different locations over an extensive variety of routes will provoke a proportionally greater response. Preparing and coordinating transportation plans with the numerous state and local officials, congressional delegations, local emergency planning groups, interest groups, etc., will require a significant expenditure of resources on the part of DOE before the first SNF shipment is even initiated. 


\section{SUMMARY AND CONCLUSIONS}

This section includes a summary of the fuel/package compatibility results, draws conclusions based on the results, and then suggests recommended actions.

\subsection{Summary}

The overall results are condensed and summarized in Table 6-1; see Table 6-2 for a more detailed summary. Table 6-1 shows that, of the approximately 230 different fuel types in the DOE-owned SNF inventory, $66 \%$ either do not have a packaging matchup, or insufficient fuel characteristics data were available to make a transportation assessment. Of the remaining $34 \%$ of the fuel types, $16 \%$ appear to have at least one compatible package as is. Another $12 \%$ could potentially have at least one compatible package if minor Certificate of Compliance changes are made and a new basket assembly is designed and qualified, as necessary. The final $6 \%$ could potentially have at least one compatible package if extensive changes are made to the safety analysis report for packaging and the Certificate of Compliance, and a new basket assembly is designed and qualified, as necessary.

A great deal of engineering judgment has been used in formulating Table 6-1 and 6-2, and it is accordingly provided as a top-level approximation of SNF transportation capabilities only. For much of the SNF inventory, fuel characterization is currently inadequate to make even a firstorder approximation of transportation capabilities, and these fuels are not included in Table 6-2. The matchups have been estimated using primarily the data from Reference 1, which is incomplete and, in some cases, has been found to be erroneous. Consequently, some of the postulated matchups may not agree with the opinions of those with a more detailed or accurate knowledge of specific fuel types.

Table 6-1. Summary of potential transportation package and fuel type matchups.

\begin{tabular}{|c|c|c|}
\hline Transportation package acceptability category & $\begin{array}{l}\text { Number of fuel } \\
\text { types }\end{array}$ & $\begin{array}{l}\text { Percentage of total } \\
\text { fuel types }\end{array}$ \\
\hline $\begin{array}{l}\text { Package/fuel matchup acceptable as is (no Certificate of } \\
\text { Compliance or SARP changes required) }\end{array}$ & 36 & $16 \%$ \\
\hline $\begin{array}{l}\text { Package/fuel matchup requires Certificate of Compliance } \\
\text { changes and probably design and qualification of new } \\
\text { basket }\end{array}$ & 28 & $12 \%$ \\
\hline $\begin{array}{l}\text { Package/fuel matchup requires extensive SARP and } \\
\text { Certificate of Compliance changes and probably design } \\
\text { and qualification of new basket }\end{array}$ & 13 & $6 \%$ \\
\hline $\begin{array}{l}\text { No package/fuel matchup, either no matchup found or } \\
\text { insufficient fuel type data available for determination }\end{array}$ & 153 & $66 \%$ \\
\hline Totals & 230 & $100 \%$ \\
\hline
\end{tabular}


Table 6-2 summarizes the results of the study in a fuel/package compatibility matrix. The reader is strongly urged to carefully review both this section as well as the legend and notes included at the end of the table before attempting to use Table 6-2. The table provides estimated matchups between various DOE-owned SNF types and currently licensed transportation packages that are, or that might be, capable of conveying them. The table includes only those fuel types for which sufficient data exist to estimate transportation requirements. It is condensed from Tables 41 through 4-6, which are in turn derived from Tables 2-1 through 2-8. It is these latter tables that contain the full set of DOE-owned SNF. It must be emphasized that this study involves a toplevel assessment of potential transportation capabilities. No attempt has been made to optimize transportation. Hence, many of the postulated fuel and package matchups, while theoretically feasible, are highly impractical (e.g., extensive modifications required to allow a 125-B rail cask to transport one or two light-water reactor fuel assemblies).

The postulated matchups of Table 6-2 are divided into two categories: Those for which sufficient fuel information exists to afford a fairly high level of confidence in transportation requirements (marked simply "yes"), and those for which insufficient direct data exist, but which can be reasonably deduced from similar fuels of known characteristics or transport requirements (marked "yes?"). Where lack of compatibility between a specific package and fuel is determined, the corresponding cell in the matrix is marked "no." When insufficient fuel data exist to make a determination, the corresponding matrix cell is marked with "N/A" (not available). For the latter two categories, the reason for the incompatibility (or lack of ability to make a determination) is indicated with a lower case letter: "a" indicates dimensional incompatibility (or lack of fuel dimensional data), " $b$ " indicates shielding incompatibility (or lack of fuel radiological data), "c" indicates thermal incompatibility (or lack of fuel thermal data), and "d" indicates weight incompatibility (or lack of fuel weight data). Refer to the legend for details.

In addition to estimated compatibility, Table 6-2 also provides an appraisal of the additional packaging-related work, if any, that may be required in order to transport a particular fuel in the package. These modifications range from "none" through two progressively greater degrees of effort, as discussed in detail in Sections 4.1 through 4.3. These classifications are indicated by uppercase letters, with " $\mathrm{A}$ " signifying no additional effort needed, through " $\mathrm{C}$ ", where the most extensive effort is expected to be required. Refer to the legend for details. It should be strongly emphasized that the highest level of effort must generally be viewed as a major undertaking, and entails a significant amount of risk. Furthermore, it is anticipated that most projected matchups, other than level " $A$ " matchups, will require the design and qualification (licensing) of some sort of internal payload support structure (basket). This essentially implies a complete relicensing effort for most " $\mathrm{B}$ " as well as " $\mathrm{C}$ " matchups (refer to Note 1 for details).

Finally, the table also provides an estimate of the amount of a particular fuel type that each qualified cask can transport, as well as an approximate total number of shipments that the cask will be required to make in order to transport all of that particular fuel. These two estimates are given in the last line of each appropriate cell, separated by a slash mark. For instance, 5/15 would indicate that a total of 5 fuel units can be transported at one time, with a total of 15 shipments expected to be required to transport all of that particular fuel. Specific fuel units are listed for each fuel type in Tables 2-1 through 2-6. It should be realized that these quantities are estimates at best, and do not make detailed allowances for additional structure that may be required to support or poison a given fuel during transport. As such, they should be considered at least 
somewhat optimistic. If fuel data are not sufficient to estimate package capacity, the lower part of the matrix cell will contain "N/A" (not available), along with the appropriate lowercase letter signifying the specific data required but not available. If payload capacity can be estimated, but no data on total fuel quantities exist, the estimated number of shipments is signified by an "e." Refer to the legend for details. 
Table 6-2. Fuel/package matchup summary matrix.

\begin{tabular}{|c|c|c|c|c|c|c|c|c|c|c|c|c|c|c|c|c|c|c|}
\hline \multirow[b]{2}{*}{ DOE SNF } & \multicolumn{11}{|c|}{ Legal weight truck packages } & \multicolumn{2}{|c|}{$\begin{array}{c}\text { Overweight } \\
\text { truck packages }\end{array}$} & \multicolumn{5}{|c|}{ Rail packages } \\
\hline & $\begin{array}{l}\mathrm{GE} \\
\mathrm{T}-2\end{array}$ & BMI-1 & FSV-1 & NLI-1/2 & NLI-6502 & T-3 & NAC-1 & $\begin{array}{l}\text { CNS 1- } \\
13 G\end{array}$ & $\begin{array}{l}\text { NAC- } \\
\text { LWT }\end{array}$ & $\begin{array}{c}\text { GE Model } \\
2000\end{array}$ & $\begin{array}{l}\text { TN- } \\
\text { FSV }\end{array}$ & $\begin{array}{c}\text { TN-8 } \\
\text { TN-8L }\end{array}$ & TN-9 & IF-300 & $\begin{array}{l}\text { NLI- } \\
10 / 24\end{array}$ & $125-\mathrm{B}$ & $\begin{array}{l}\text { TN- } \\
\text { BRP }\end{array}$ & $\begin{array}{l}\text { TN- } \\
\text { REG }\end{array}$ \\
\hline \multicolumn{19}{|l|}{ PWR } \\
\hline $\begin{array}{l}\text { Point Beach } \\
\text { (N/A) }\end{array}$ & $\begin{array}{c}\text { No } \\
\text { a }\end{array}$ & $\begin{array}{c}\text { No } \\
\text { a }\end{array}$ & $\begin{array}{c}\text { Yes } \\
\text { C } \\
1 / ?(e)\end{array}$ & $\begin{array}{c}\text { Yes? } \\
\mathrm{C} \\
1 / ?(\mathrm{e})\end{array}$ & $\begin{array}{c}\text { No } \\
\text { a }\end{array}$ & $\begin{array}{c}\text { No } \\
\text { a }\end{array}$ & $\begin{array}{c}\text { Yes? } \\
\text { C } \\
1 / ?(\mathrm{e})\end{array}$ & $\begin{array}{c}\text { No } \\
\text { a }\end{array}$ & $\begin{array}{c}\text { Yes } \\
C \\
1 / 2(e)\end{array}$ & $\begin{array}{c}\text { No } \\
\text { a }\end{array}$ & $\begin{array}{l}\text { No } \\
\text { c }\end{array}$ & $\begin{array}{c}\text { Yes? } \\
\text { B } \\
3 / ?(e)\end{array}$ & $\begin{array}{c}\text { No } \\
\text { a }\end{array}$ & $\begin{array}{c}\text { Yes } \\
\text { B } \\
7 / ?(e)\end{array}$ & $\begin{array}{c}\text { Yes } \\
\text { B } \\
10 / ?(e)\end{array}$ & $\begin{array}{c}\text { Yes? } \\
\mathrm{C} \\
1 / ?(\mathrm{e})\end{array}$ & $\begin{array}{c}\text { Yes? } \\
C \\
6 / ?(e)\end{array}$ & $\begin{array}{c}\text { Yes? } \\
B \\
5 / ?(e)\end{array}$ \\
\hline $\begin{array}{c}\text { Shippingport } \\
\text { (INEI) } \\
\text { (43) }\end{array}$ & $\begin{array}{c}\text { No } \\
\text { a }\end{array}$ & $\begin{array}{c}\text { No } \\
\text { a }\end{array}$ & $\begin{array}{c}\text { Yes } \\
\text { C } \\
1 / 43\end{array}$ & $\begin{array}{c}\text { Yes } \\
\text { C } \\
1 / 43\end{array}$ & $\begin{array}{c}\text { Yes } \\
\text { C } \\
1 ?(d) / 43\end{array}$ & $\begin{array}{c}\text { No } \\
\text { a }\end{array}$ & $\begin{array}{c}\text { Yes? } \\
\text { C } \\
1 / 43\end{array}$ & $\begin{array}{c}\text { No } \\
\text { a }\end{array}$ & $\begin{array}{c}\text { Yes } \\
\text { C } \\
1 / 43\end{array}$ & $\begin{array}{c}\text { No } \\
\text { a }\end{array}$ & $\begin{array}{c}\text { Yes } \\
C \\
1 / 43\end{array}$ & $\begin{array}{c}\text { Yes? } \\
\text { C } \\
3 / 15\end{array}$ & $\begin{array}{c}\text { No } \\
\text { a }\end{array}$ & $\begin{array}{c}\text { Yes? } \\
\text { C } \\
7 / 7\end{array}$ & $\begin{array}{c}\text { Yes? } \\
\text { C } \\
10 / 5\end{array}$ & $\begin{array}{c}\text { Yes? } \\
\text { C } \\
2 / 22\end{array}$ & $\begin{array}{c}\text { Yes? } \\
\text { C } \\
11 / 4\end{array}$ & $\begin{array}{c}\text { Yes? } \\
\mathrm{C} \\
10 / 5\end{array}$ \\
\hline $\begin{array}{c}\text { Shippingport } \\
\text { (SRS) } \\
\text { (1) }\end{array}$ & $\begin{array}{l}\text { Yes } \\
\mathrm{C} \\
\text { N/A } \\
\text { c/d }\end{array}$ & $\begin{array}{c}\text { Yes } \\
\text { C } \\
\text { N/A } \\
\text { c/d }\end{array}$ & $\begin{array}{c}\text { Yes } \\
\mathrm{C} \\
\mathrm{N} / \mathrm{A} \\
\mathrm{c} / \mathrm{d}\end{array}$ & $\begin{array}{l}\text { Yes } \\
C \\
\text { N/A } \\
\text { c/d }\end{array}$ & $\begin{array}{c}\text { Yes } \\
\mathrm{C} \\
\mathrm{N} / \mathrm{A} \\
\mathrm{c} / \mathrm{d}\end{array}$ & $\begin{array}{l}\text { Yes } \\
\text { C } \\
\text { N/A } \\
\text { c/d }\end{array}$ & $\begin{array}{l}\text { Yes? } \\
\text { C } \\
\text { N/A } \\
\text { c/d }\end{array}$ & $\begin{array}{c}\text { Yes } \\
\text { C } \\
\text { N/A } \\
\text { c/d }\end{array}$ & $\begin{array}{c}\text { Yes } \\
\mathrm{C} \\
\mathrm{N} / \mathrm{A} \\
\mathrm{c} / \mathrm{d}\end{array}$ & $\begin{array}{l}\text { Yes } \\
\text { C } \\
\text { N/A } \\
\text { c/d }\end{array}$ & $\begin{array}{l}\text { Yes } \\
C \\
N / A \\
c / d\end{array}$ & $\begin{array}{l}\text { Yes } \\
\text { C } \\
\text { N/A } \\
c / d\end{array}$ & $\begin{array}{c}\text { Yes } \\
C \\
\text { N/A } \\
c / d\end{array}$ & $\begin{array}{c}\text { Yes } \\
\text { C } \\
\text { N/A } \\
\text { c/d }\end{array}$ & $\begin{array}{c}\text { Yes } \\
\text { C } \\
\text { N/A } \\
c / d\end{array}$ & $\begin{array}{c}\text { Yes } \\
\text { C } \\
\text { N/A } \\
\text { c/d }\end{array}$ & $\begin{array}{c}\text { Yes } \\
\text { C } \\
\text { N/A } \\
c / d\end{array}$ & $\begin{array}{c}\text { Yes } \\
\mathrm{C} \\
\mathrm{N} / \mathrm{A} \\
\mathrm{c} / \mathrm{d}\end{array}$ \\
\hline $\begin{array}{c}\text { Conn Yankee } \\
\text { (1) }\end{array}$ & $\begin{array}{c}\text { No } \\
\text { a }\end{array}$ & $\begin{array}{c}\text { No } \\
\text { a }\end{array}$ & $\begin{array}{c}\text { Yes } \\
\text { C } \\
1 ?(c) / 1\end{array}$ & $\begin{array}{c}\text { Ycs } \\
\text { A } \\
1 ?(c) / 1\end{array}$ & $\begin{array}{c}\text { No } \\
\text { a }\end{array}$ & $\begin{array}{l}\text { No } \\
\text { a }\end{array}$ & $\begin{array}{c}\text { Yes? } \\
\text { C } \\
1 ?(c) / 1\end{array}$ & $\begin{array}{c}\text { No } \\
\text { a }\end{array}$ & $\begin{array}{c}\text { Yes } \\
\text { A } \\
1 ?(\mathrm{c}) / 1\end{array}$ & $\begin{array}{c}\text { No } \\
\text { a }\end{array}$ & $\begin{array}{c}\text { Yes } \\
\mathrm{C} \\
1 ?(\mathrm{c}) / 1\end{array}$ & $\begin{array}{c}\text { Yes } \\
\mathrm{C} \\
1 ?(\mathrm{c}) / 1\end{array}$ & $\begin{array}{c}\text { No } \\
\text { a }\end{array}$ & $\begin{array}{c}\text { Yes } \\
\text { C } \\
1 ?(\mathrm{c}) / 1\end{array}$ & $\begin{array}{c}\text { Yes } \\
\mathrm{C} \\
1 ?(\mathrm{c}) / 1\end{array}$ & $\begin{array}{c}\text { Yes } \\
\mathrm{C} \\
1 ?(\mathrm{c}) / 1\end{array}$ & $\begin{array}{c}\text { Yes } \\
\mathrm{C} \\
1 ?(\mathrm{c}) / 1\end{array}$ & $\begin{array}{c}\text { Yes } \\
\mathrm{C} \\
1 ?(\mathrm{c}) / 1\end{array}$ \\
\hline $\begin{array}{l}\text { LOFT } \\
(19)\end{array}$ & $\begin{array}{c}\text { Yes } \\
C \\
\text { N/A } \\
c\end{array}$ & $\begin{array}{c}\text { No } \\
\text { a }\end{array}$ & $\begin{array}{c}\text { Yes } \\
\text { C } \\
\text { N/A } \\
c\end{array}$ & $\begin{array}{c}\text { Yes } \\
\text { C } \\
\text { N/A } \\
c\end{array}$ & $\begin{array}{c}\text { Yes } \\
C \\
\text { N/A } \\
c\end{array}$ & $\begin{array}{c}\text { No } \\
\text { a }\end{array}$ & $\begin{array}{c}\text { Yes } \\
C \\
\text { N/A } \\
c\end{array}$ & $\begin{array}{c}\text { No } \\
\text { a }\end{array}$ & $\begin{array}{l}\text { Yes } \\
\text { C } \\
\text { N/A } \\
\text { c }\end{array}$ & $\begin{array}{c}\text { No } \\
\text { a }\end{array}$ & $\begin{array}{c}\text { Yes } \\
C \\
\text { N/A } \\
c\end{array}$ & $\begin{array}{c}\text { Yes } \\
C \\
\text { N/A } \\
c\end{array}$ & $\begin{array}{c}\text { No } \\
\text { a }\end{array}$ & $\begin{array}{c}\text { Yes } \\
C \\
\text { N/A } \\
c\end{array}$ & $\begin{array}{c}\text { Yes } \\
C \\
\text { N/A } \\
c\end{array}$ & $\begin{array}{c}\text { Yes } \\
C \\
\text { N/A } \\
c\end{array}$ & $\begin{array}{c}\text { Yes } \\
C \\
N / A \\
c\end{array}$ & $\begin{array}{c}\text { Yes } \\
C \\
\text { N/A } \\
c\end{array}$ \\
\hline $\begin{array}{l}\text { Surrcy } \\
(69)\end{array}$ & $\begin{array}{c}\text { No } \\
\text { a }\end{array}$ & $\begin{array}{c}\text { No } \\
\text { a }\end{array}$ & $\begin{array}{c}\text { Yes } \\
\text { C } \\
1 ?(c) / 69\end{array}$ & $\begin{array}{c}\text { Yes } \\
\text { B } \\
1 ?(c) / 69\end{array}$ & $\begin{array}{c}\text { No } \\
\text { a }\end{array}$ & $\begin{array}{c}\text { No } \\
\text { a }\end{array}$ & $\begin{array}{c}\text { Yes } \\
\text { C } \\
1 ?(c) / 69\end{array}$ & $\begin{array}{c}\text { No } \\
\text { a }\end{array}$ & $\begin{array}{c}\text { Yes } \\
\text { A } \\
17(c) / 69\end{array}$ & $\begin{array}{c}\text { No } \\
\text { a }\end{array}$ & $\begin{array}{c}\text { Yes } \\
\text { C } \\
1 ?(\mathrm{c}) / \\
69\end{array}$ & $\begin{array}{c}\text { Yes } \\
\text { A } \\
3 ?(c) / \\
32\end{array}$ & $\begin{array}{c}\text { No } \\
\text { a }\end{array}$ & $\begin{array}{c}\text { Yes } \\
\text { A } \\
7 ?(c) / 10\end{array}$ & $\begin{array}{c}\text { Yes } \\
A \\
10 ?(c) / \\
7\end{array}$ & $\begin{array}{c}\text { Yes? } \\
\text { C } \\
\text { N/A } \\
c\end{array}$ & $\begin{array}{c}\text { Yes } \\
C \\
\text { N/A } \\
c\end{array}$ & $\begin{array}{c}\text { Yes } \\
C \\
\text { N/A } \\
c\end{array}$ \\
\hline $\begin{array}{c}\text { Turtey Point B17 } \\
\text { (17) }\end{array}$ & $\begin{array}{c}\text { No } \\
\text { a }\end{array}$ & $\begin{array}{c}\text { No } \\
\text { a }\end{array}$ & $\begin{array}{c}\text { Yes } \\
\text { C } \\
1 ?(c) / 17\end{array}$ & $\begin{array}{c}\text { Yes } \\
\text { A } \\
1 ?(\mathrm{c}) / 17\end{array}$ & $\begin{array}{c}\text { No } \\
\text { a }\end{array}$ & $\begin{array}{c}\text { No } \\
\text { a }\end{array}$ & $\begin{array}{c}\text { Yes } \\
\mathrm{C} \\
1 ?(\mathrm{c}) / 17\end{array}$ & $\begin{array}{c}\text { No } \\
\text { a }\end{array}$ & $\begin{array}{c}\text { Yes } \\
\text { A } \\
1 ?(\mathrm{c}) / 17\end{array}$ & $\begin{array}{c}\text { No } \\
\text { a }\end{array}$ & $\begin{array}{c}\text { Yes } \\
\text { C } \\
1 ?(c) \backslash \\
17\end{array}$ & $\begin{array}{c}\text { Yes } \\
\text { A } \\
3 ?(c) / 6\end{array}$ & $\begin{array}{c}\text { No } \\
\text { a }\end{array}$ & $\begin{array}{c}\text { Yes } \\
\text { A } \\
7 ?(\mathrm{c}) / 3\end{array}$ & $\begin{array}{c}\text { Yes } \\
\text { A } \\
10 ?(c) / \\
2\end{array}$ & $\begin{array}{l}\text { Yes? } \\
C \\
\text { N/A } \\
c\end{array}$ & $\begin{array}{l}\text { Yes } \\
C \\
\text { N/A } \\
c\end{array}$ & $\begin{array}{c}\text { Yes } \\
C \\
\text { N/A } \\
c\end{array}$ \\
\hline $\begin{array}{c}\text { Turtey Point } \\
\text { EMAD } \\
(1)\end{array}$ & $\begin{array}{c}\text { No } \\
\text { a }\end{array}$ & $\begin{array}{c}\text { No } \\
\text { a }\end{array}$ & $\begin{array}{c}\text { Yes } \\
\mathrm{C} \\
1 ?(\mathrm{c}) / 1\end{array}$ & $\begin{array}{c}\text { Yes } \\
\text { A } \\
1 ?(\mathrm{c}) / 1\end{array}$ & $\begin{array}{c}\text { No } \\
\text { a }\end{array}$ & $\begin{array}{c}\text { No } \\
\text { a }\end{array}$ & $\begin{array}{c}\text { Yes } \\
\text { C } \\
1 ?(\mathrm{c}) / 1\end{array}$ & $\begin{array}{c}\text { No } \\
\text { a }\end{array}$ & $\begin{array}{c}\text { Yes } \\
\mathbf{A} \\
1 ?(\mathrm{c}) / 1\end{array}$ & $\begin{array}{c}\text { No } \\
\text { a }\end{array}$ & $\begin{array}{c}\text { Yes } \\
\mathrm{C} \\
1 ?(\mathrm{c}) / 1 \\
\mathrm{C}\end{array}$ & $\begin{array}{c}\text { Yes } \\
\text { A } \\
3 ?(\mathrm{c}) / 1\end{array}$ & $\begin{array}{c}\text { No } \\
\text { a }\end{array}$ & $\begin{array}{c}\text { Yes } \\
\text { A } \\
7 ?(\mathrm{c}) / 1\end{array}$ & $\begin{array}{c}\text { Yes } \\
\mathbf{A} \\
10 ?(\mathrm{c}) / \\
1\end{array}$ & $\begin{array}{l}\text { Yes? } \\
C \\
\text { N/A } \\
c\end{array}$ & $\begin{array}{c}\text { Yes } \\
C \\
\text { N/A } \\
c\end{array}$ & $\begin{array}{c}\text { Yes } \\
C \\
\text { N/A } \\
c\end{array}$ \\
\hline
\end{tabular}




\begin{tabular}{|c|c|c|c|c|c|c|c|c|c|c|}
\hline & 事兽 & $\frac{\pi}{2}$ & 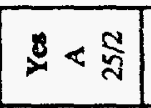 & $\stackrel{s \rightarrow \infty}{8}$ & & $\otimes \cup \frac{\leq}{2} \Xi$ & 20 & $\searrow 0 \leqslant 0$ & $80 \stackrel{5}{0}$ & 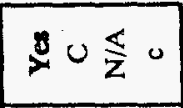 \\
\hline & 妾 & $\frac{1}{2}=0$ & 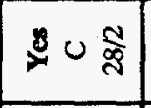 & 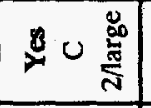 & & $\otimes 0 \leqslant \frac{\pi}{2}$ & $\stackrel{0}{z} \%$ & $80 \frac{5}{2} 0$ & $80 \stackrel{5}{0}$ & $\therefore 0 \frac{5}{2} 0$ \\
\hline & $\stackrel{0}{3}$ & $\frac{5}{2}$ & 005 & $\frac{8}{2}<\frac{f}{1}$ & & $\forall \cup \frac{5}{z} g$ & $g \cup \frac{\pi}{z} \circ$ & $\nexists 0 \frac{x}{2} 0$ & $80 \stackrel{5}{0}$ & $\otimes \cup \frac{5}{2} 0$ \\
\hline & $\dot{\vec{\Delta}}$ & $\frac{50}{2}$ & $8<\frac{\delta}{8}$ & 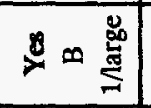 & & $\$ 0 \frac{5}{2}$ & sid & $80 \leq 0$ & $200 \stackrel{0}{8}$ & $\otimes 0 \leq \frac{5}{2}$ \\
\hline & $\begin{array}{l}8 \\
\end{array}$ & $\frac{\pi}{2}$ & $x<\xi$ & 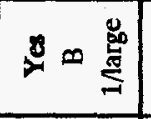 & & $\frac{5}{z}$ & 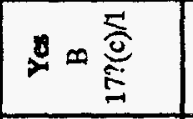 & $\forall 0 \frac{5}{2} 0$ & 00 窝 & $\otimes 0 \frac{\pi}{2} 0$ \\
\hline \multirow{2}{*}{ 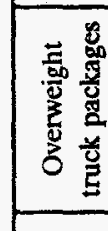 } & $\stackrel{i}{\dot{z}}$ & 这 & $\stackrel{\circ}{2} \approx$ & 呈。 & & $\nexists \cup \frac{5}{2}:$ & 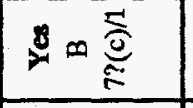 & $\Delta \cup \frac{5}{2} 0$ & 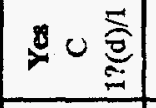 & 宅 \\
\hline & 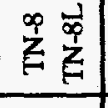 & $\frac{5}{2}$ & 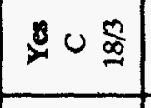 & 요 & & $\triangle \cup \frac{5}{2}=$ & ¿̊。 & 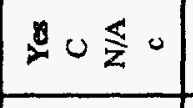 & $80 \stackrel{0}{0}$ & $\otimes \cup \frac{\pi}{z} 0$ \\
\hline \multirow{11}{*}{ 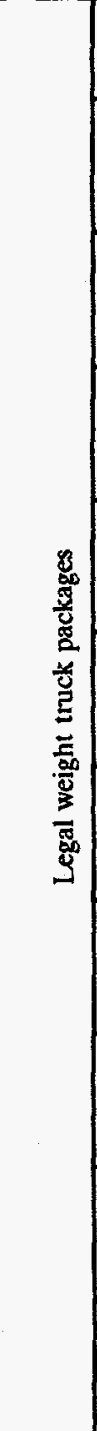 } & $\dot{z}$ & $\frac{1}{z}:$ & 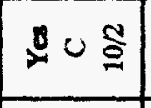 & z \% & & $\forall \cup \frac{s}{3}$ & $200 \frac{\bar{c}}{\tilde{c}}$ & $80 \frac{5}{2}$ & $\therefore \rightarrow$ & 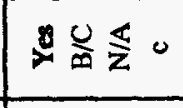 \\
\hline & 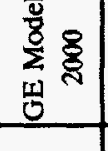 & $\frac{5}{2}$ & 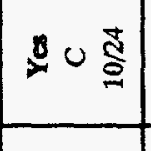 & 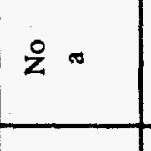 & & $\forall \cup \frac{5}{2}$ & $\ddot{z}$ & z: & $80 \stackrel{5}{0}$ & $\because \cup \frac{\Delta}{2}$ \\
\hline & 妾管 & $\frac{5}{2}$ & $80 \frac{\pi}{2}$ & z̊ & & $\stackrel{\circ}{z} \sigma$ & 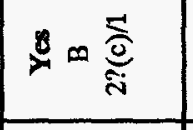 & $90 \frac{\pi}{z} 0$ & 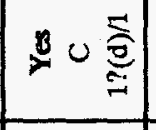 & $\otimes 0 \frac{\pi}{2} 0$ \\
\hline & 吾品 & $\frac{4}{z} \frac{0}{8}$ & 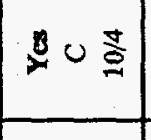 & 竞。 & & $g=\leq s$ & $\stackrel{2}{z}=$ & $\stackrel{\circ}{2}$ & 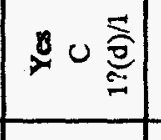 & $\frac{\pi}{z}=$ \\
\hline & $\begin{array}{l}\overrightarrow{\tilde{v}} \\
\underline{z} \\
\end{array}$ & $\frac{5}{2}$ & 805 & z & & 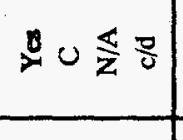 & $800 \frac{5}{x}$ & $g \cup \frac{\pi}{z} 0$ & $\therefore 0$ 竘 & $\otimes \cup \frac{\pi}{z} 0$ \\
\hline & 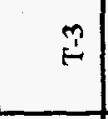 & $\leqslant \frac{0}{2}$ & 닐 & 台 & & $\frac{8}{2} \cup \leq \frac{g}{0}$ & $\stackrel{0}{2} \approx$ & $D \cup \frac{\pi}{z} 0$ & $80 \stackrel{0}{0}$ & $\frac{8}{2} \cup \frac{\pi}{z} 0$ \\
\hline & $\begin{array}{l} \\
\frac{5}{2} \\
\\
\end{array}$ & $\frac{5}{2}$ & $\frac{S}{2}$ & 요 & & $\mathscr{B} \cup \leq \frac{8}{2}$ & 完 & $8 \cup \frac{\pi}{z} 0$ & 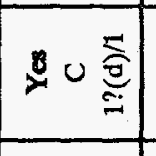 & 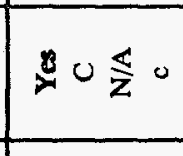 \\
\hline & $\begin{array}{l}\frac{5}{3} \\
\frac{1}{2} \\
\end{array}$ & $\frac{5}{2}$ & $g<x$ & $\stackrel{\circ}{z}$ & & $80 \leq \frac{g}{0}$ & $20<\frac{5}{\tilde{N}}$ & $\frac{8}{x} \cup \frac{\pi}{2} 0$ & 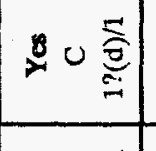 & $\gg \cup \frac{S}{z} 0$ \\
\hline & 窝 & $\frac{\leq 0}{z}$ & 00 & $\stackrel{\circ}{2}$ & & $\stackrel{\circ}{2} \sigma$ & \%० & $80 \frac{\pi}{z} 0$ & 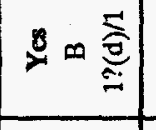 & 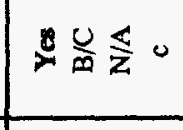 \\
\hline & $\overline{\overline{\mathbf{m}}}$ & $\ddot{0}<<\frac{5}{\tilde{m}}$ & $\frac{\pi}{z} 0$ & $\stackrel{0}{2}$ & & $\stackrel{2}{2}$ & $\stackrel{2}{z}=$ & $\stackrel{\circ}{2}$. & 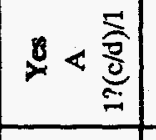 & $\ddot{x} \infty \frac{\mathbb{Z}}{z} 0$ \\
\hline & 圆 : & $\frac{5}{z} \frac{0}{b}$ & $\frac{\mathbb{S}}{z} \pi$ & $\stackrel{\circ}{z}=$ & & $8 \cup \leq \frac{5}{2}$ & 宅。 & $\frac{x}{z}=$ & $₫ 0 \stackrel{\frac{\pi}{0}}{=}$ & zㅇ. \\
\hline & $\begin{array}{l}\text { 崇 } \\
\text { 荘 } \\
0\end{array}$ & $\bar{\sum}$ & 喽 & 䇺需 & 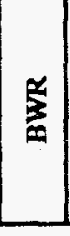 & 着度 & 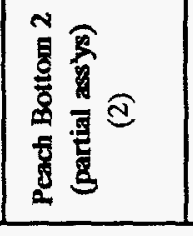 & 童列 & 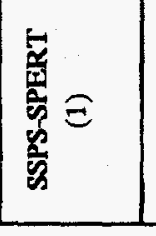 & 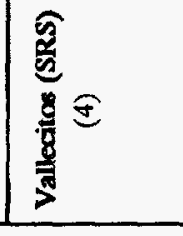 \\
\hline
\end{tabular}


Table 6-2. (continued).

\begin{tabular}{|c|c|c|c|c|c|c|c|c|c|c|c|c|c|c|c|c|c|c|}
\hline \multirow[b]{2}{*}{ DOE SNF } & \multicolumn{11}{|c|}{ Legal weight truck packages } & \multicolumn{2}{|c|}{$\begin{array}{l}\text { Overweight } \\
\text { truck packages }\end{array}$} & \multicolumn{5}{|c|}{ Rail packages } \\
\hline & $\begin{array}{l}\mathrm{GE} \\
\mathrm{T}-2\end{array}$ & BMI-1 & FSV.1 & NLI-1/2 & NLI-6502 & $\mathrm{T}-3$ & NAC-1 & $\begin{array}{c}\text { CNS 1- } \\
13 G\end{array}$ & $\begin{array}{l}\text { NAC- } \\
\text { LWT }\end{array}$ & $\begin{array}{c}\text { GE Model } \\
2000\end{array}$ & $\begin{array}{l}\text { TN- } \\
\text { FSV }\end{array}$ & $\begin{array}{l}\text { TN-8 } \\
\text { TN-8L }\end{array}$ & TN-9 & IF-300 & $\begin{array}{l}\text { NLI- } \\
10 / 24\end{array}$ & $125-\mathrm{B}$ & $\begin{array}{l}\text { TN- } \\
\text { BRP }\end{array}$ & $\begin{array}{l}\text { TN- } \\
\text { REG }\end{array}$ \\
\hline $\begin{array}{c}\text { Vallecitos (INEL) } \\
\text { (4) }\end{array}$ & $\begin{array}{c}\text { No } \\
\text { a }\end{array}$ & $\begin{array}{c}\text { Yes } \\
B \\
\text { N/A } \\
\text { c }\end{array}$ & $\begin{array}{c}\text { Yes } \\
\text { B/C } \\
\text { N/A } \\
\text { c }\end{array}$ & $\begin{array}{c}\text { Yes } \\
\text { C } \\
\text { N/A } \\
c\end{array}$ & $\begin{array}{c}\text { Yes } \\
\text { C } \\
\text { N/A } \\
c\end{array}$ & $\begin{array}{c}\text { Ye } \\
C \\
\text { N/A } \\
c\end{array}$ & $\begin{array}{c}\text { Yes } \\
C \\
\text { N/A } \\
c\end{array}$ & $\begin{array}{c}\mathrm{N} / \mathrm{A} \\
\mathrm{b}\end{array}$ & $\begin{array}{c}\text { Yes } \\
\text { C } \\
\text { N/A } \\
c\end{array}$ & $\begin{array}{c}\text { Yes } \\
\text { C } \\
\text { N/A } \\
\text { c }\end{array}$ & $\begin{array}{c}\text { Yes } \\
C \\
\text { N/A } \\
c\end{array}$ & $\begin{array}{c}\text { Yes } \\
\text { C } \\
\text { N/A } \\
\text { c }\end{array}$ & $\begin{array}{c}\text { No } \\
\text { a }\end{array}$ & $\begin{array}{c}\text { Yes } \\
\text { C } \\
\text { N/A } \\
c\end{array}$ & $\begin{array}{c}\text { Yes } \\
\text { C } \\
\text { N/A } \\
\text { c }\end{array}$ & $\begin{array}{c}\text { Yes } \\
\text { C } \\
\text { N/A } \\
c\end{array}$ & $\begin{array}{c}\text { Ycs } \\
\mathrm{C} \\
\mathrm{N} / \mathrm{A} \\
\mathrm{c}\end{array}$ & $\begin{array}{c}\text { Yes } \\
\text { C } \\
\text { N/A } \\
\text { c }\end{array}$ \\
\hline \multicolumn{19}{|l|}{ BR } \\
\hline $\begin{array}{c}\text { FFIF-DA } \\
(310)\end{array}$ & $\begin{array}{c}\text { No } \\
\text { a }\end{array}$ & $\begin{array}{c}\text { No } \\
\text { a }\end{array}$ & $\begin{array}{c}\text { Yes } \\
\text { C } \\
\text { 2/arge }\end{array}$ & $\begin{array}{c}\text { No } \\
b\end{array}$ & $\begin{array}{c}\text { No } \\
\text { a }\end{array}$ & $\begin{array}{c}\text { Yes } \\
\text { B } \\
\text { 1/large }\end{array}$ & $\begin{array}{l}\text { No } \\
\text { b }\end{array}$ & $\begin{array}{c}\text { No } \\
\text { a }\end{array}$ & $\begin{array}{c}\text { No } \\
b\end{array}$ & $\begin{array}{c}\text { No } \\
\text { a }\end{array}$ & $\begin{array}{c}\text { No } \\
b\end{array}$ & $\begin{array}{c}\text { No } \\
b\end{array}$ & $\begin{array}{c}\mathrm{No} \\
\mathrm{b}\end{array}$ & $\begin{array}{c}\text { Yes } \\
\text { C } \\
6 / 52\end{array}$ & $\begin{array}{c}\text { No } \\
\text { b }\end{array}$ & $\begin{array}{c}\text { No } \\
\text { b }\end{array}$ & $\begin{array}{c}\text { No } \\
b\end{array}$ & $\begin{array}{c}\text { No } \\
\text { b }\end{array}$ \\
\hline $\begin{array}{c}\text { FFTF-TFA } \\
\text { (8) }\end{array}$ & $\begin{array}{c}\text { No } \\
\text { a }\end{array}$ & $\begin{array}{c}\text { No } \\
\text { a }\end{array}$ & $\begin{array}{l}\text { Yes } \\
\text { C } \\
5 / 2\end{array}$ & $\begin{array}{c}\text { No } \\
b\end{array}$ & $\begin{array}{c}\text { No } \\
\text { a }\end{array}$ & $\begin{array}{c}\text { Yes } \\
\text { A } \\
1 / 8\end{array}$ & $\begin{array}{c}\text { No } \\
b\end{array}$ & $\begin{array}{c}\text { No } \\
\text { a }\end{array}$ & $\begin{array}{l}\text { No } \\
b\end{array}$ & $\begin{array}{c}\text { No } \\
\text { a }\end{array}$ & $\begin{array}{c}\text { No } \\
b\end{array}$ & $\begin{array}{c}\text { No } \\
\text { b }\end{array}$ & $\begin{array}{c}\text { No } \\
b\end{array}$ & $\begin{array}{c}\text { Yes } \\
\text { C } \\
13 / 1\end{array}$ & $\begin{array}{c}\text { No } \\
b\end{array}$ & $\begin{array}{c}\text { No } \\
b\end{array}$ & $\begin{array}{c}\text { No } \\
b\end{array}$ & $\begin{array}{c}\text { No } \\
\text { b }\end{array}$ \\
\hline $\begin{array}{c}\text { Fermi Core } 1822 \\
(214)\end{array}$ & $\begin{array}{c}\text { Yes } \\
\text { C } \\
16 / 2\end{array}$ & $\begin{array}{c}\text { Yes } \\
\text { A } \\
\text { 1/arge }\end{array}$ & $\begin{array}{c}\text { Yes } \\
\text { C } \\
60 / 4\end{array}$ & $\begin{array}{l}\text { Yes } \\
\text { C } \\
35 \pi\end{array}$ & $\begin{array}{c}\text { Yes } \\
\text { C } \\
\text { 9?(d)/24 }\end{array}$ & $\begin{array}{c}\text { Yes } \\
\text { C } \\
6 / 36\end{array}$ & $\begin{array}{c}\text { Yes } \\
\text { C } \\
24 / 9\end{array}$ & $\begin{array}{c}\text { Yes } \\
C \\
24 / 9\end{array}$ & $\begin{array}{c}\text { Yes } \\
\text { C } \\
24 / 9\end{array}$ & $\begin{array}{c}\text { Yes } \\
\text { C } \\
24 / 9\end{array}$ & $\begin{array}{c}\text { Yes } \\
C \\
20 / 11\end{array}$ & $\begin{array}{c}\text { Yes } \\
\text { C } \\
27 / 8\end{array}$ & $\begin{array}{c}\text { Yes } \\
\text { C } \\
28 / 8\end{array}$ & $\begin{array}{c}\text { Yes } \\
\text { C } \\
208 / 2\end{array}$ & $\begin{array}{c}\text { Yes } \\
C \\
304 / 1\end{array}$ & $\begin{array}{c}\text { Yes } \\
\text { C } \\
40 / 6\end{array}$ & $\begin{array}{c}\text { Yes } \\
\text { C } \\
177 / 2\end{array}$ & $\begin{array}{c}\text { Yes } \\
\text { C } \\
100 / 3\end{array}$ \\
\hline $\begin{array}{c}\text { Fermi } 1 \text { Blanket } \\
\quad(510)\end{array}$ & $\begin{array}{c}\text { Yes } \\
\text { B } \\
\text { N/A } \\
\text { c }\end{array}$ & $\begin{array}{c}\text { Yes } \\
\text { B } \\
\text { N/A } \\
\text { c }\end{array}$ & $\begin{array}{c}\text { Yes } \\
\text { C } \\
\text { N/A } \\
c\end{array}$ & $\begin{array}{c}\text { Yes } \\
\text { C } \\
\text { N/A } \\
\text { c }\end{array}$ & $\begin{array}{l}\text { Yes } \\
\text { C } \\
\text { N/A } \\
\text { c }\end{array}$ & $\begin{array}{c}\text { Yes } \\
\text { C } \\
\text { N/A } \\
\text { c }\end{array}$ & $\begin{array}{l}\text { Yes } \\
\text { C } \\
\text { N/A } \\
\text { c }\end{array}$ & $\begin{array}{c}\text { Yes } \\
C \\
N / A \\
c\end{array}$ & $\begin{array}{c}\text { Yes } \\
\text { C } \\
\text { N/A } \\
c\end{array}$ & $\begin{array}{c}\text { Yes } \\
\text { C } \\
\text { N/A } \\
c\end{array}$ & $\begin{array}{c}\text { Yes } \\
\text { C } \\
\text { N/A } \\
c\end{array}$ & $\begin{array}{c}\text { Yes } \\
\text { C } \\
\text { N/A } \\
\text { c }\end{array}$ & $\begin{array}{c}\text { Yes } \\
\text { C. } \\
\text { N/A } \\
\text { c }\end{array}$ & $\begin{array}{c}\text { Yes } \\
\text { C } \\
\text { N/A } \\
\text { c }\end{array}$ & $\begin{array}{c}\text { Yes } \\
\text { C } \\
\text { N/A } \\
c\end{array}$ & $\begin{array}{c}\text { Yos } \\
\text { C } \\
\text { N/A } \\
\text { c }\end{array}$ & $\begin{array}{c}\text { Yes } \\
\mathrm{C} \\
\mathrm{N} / \mathrm{A} \\
\mathrm{c}\end{array}$ & $\begin{array}{c}\text { Yes } \\
\text { C } \\
\text { N/A } \\
\text { c }\end{array}$ \\
\hline $\begin{array}{c}\text { EBR-II (INEL } \\
\text { canister) } \\
(1472)\end{array}$ & $\begin{array}{c}\text { No } \\
\text { c }\end{array}$ & $\begin{array}{c}\text { Yes } \\
\text { B } \\
\text { N/A } \\
\text { c }\end{array}$ & $\begin{array}{c}\text { Yes } \\
\text { B } \\
\text { 4/arge }\end{array}$ & $\begin{array}{c}\text { Yes } \\
\text { C } \\
\text { 2/large }\end{array}$ & $\begin{array}{c}\text { Yes } \\
\text { C } \\
4 ?(d) / \\
\text { large }\end{array}$ & $\begin{array}{c}\text { Yes } \\
\text { C } \\
\text { 2harge }\end{array}$ & $\begin{array}{c}\text { Yes } \\
\text { C } \\
\text { 1/large }\end{array}$ & $\begin{array}{c}\text { Yes } \\
\text { C } \\
\text { 1//arge }\end{array}$ & $\begin{array}{c}\text { Yes } \\
\text { C } \\
\text { 5/large }\end{array}$ & $\begin{array}{c}\text { Yca } \\
\text { C } \\
\text { 1/large }\end{array}$ & $\begin{array}{c}\text { Yes } \\
\mathrm{C} \\
\text { 1/large }\end{array}$ & $\begin{array}{c}\text { Yes } \\
C \\
75 / 20\end{array}$ & $\begin{array}{c}\text { Yes } \\
\mathrm{C} \\
50 / 30\end{array}$ & $\begin{array}{c}\text { Yes } \\
\text { C } \\
25 / 62\end{array}$ & $\begin{array}{c}\text { Ye } \\
C \\
168 / 9\end{array}$ & $\begin{array}{c}\text { Yes } \\
\mathrm{C} \\
\text { 1/harge }\end{array}$ & $\begin{array}{c}\text { Yes } \\
\text { C } \\
\text { 7/harge }\end{array}$ & $\begin{array}{c}\text { Yes } \\
\text { C } \\
\text { 6/arge }\end{array}$ \\
\hline $\begin{array}{c}\text { EBR-II } \\
\text { (INEL pin) } \\
\text { (2165) }\end{array}$ & $\begin{array}{c}\text { No } \\
\text { c }\end{array}$ & $\begin{array}{c}\text { Yes } \\
\text { B } \\
\text { N/A } \\
\text { c }\end{array}$ & $\begin{array}{c}\text { Yes } \\
\text { B } \\
\text { 8/arge }\end{array}$ & $\begin{array}{c}\text { Yes } \\
\text { C } \\
\text { 2/large }\end{array}$ & $\begin{array}{c}\text { Yes } \\
\text { C } \\
8 ?(\mathbf{c}) / \\
\text { large }\end{array}$ & $\begin{array}{c}\text { Yes } \\
\text { C } \\
\text { 3/arge }\end{array}$ & $\begin{array}{c}\text { Yes } \\
\text { C } \\
\text { 2/large }\end{array}$ & $\begin{array}{c}\text { Yes } \\
\text { C } \\
\text { 2/arge }\end{array}$ & $\begin{array}{c}\text { Yes } \\
\text { C } \\
\text { 5/arge }\end{array}$ & $\begin{array}{c}\text { Yes } \\
\text { C } \\
\text { 2/large }\end{array}$ & \begin{tabular}{|c} 
Yes \\
B \\
1/arge
\end{tabular} & $\begin{array}{c}\text { Yes } \\
C \\
66 / 33\end{array}$ & $\begin{array}{c}\text { Yes } \\
\text { C } \\
50 / 44\end{array}$ & $\begin{array}{c}\text { Yes } \\
\text { C } \\
28 / 78\end{array}$ & \begin{tabular}{|c|} 
Yes \\
C \\
$168 / 13$
\end{tabular} & \begin{tabular}{|c|} 
Yes \\
C \\
1/Aarge
\end{tabular} & $\begin{array}{c}\text { Yes } \\
\text { C } \\
\text { 7/harge }\end{array}$ & $\begin{array}{c}\text { Yes } \\
\text { C } \\
\text { 6/large }\end{array}$ \\
\hline $\begin{array}{c}\text { EBR-II } \\
\text { (ANL-6) } \\
\text { (4) }\end{array}$ & $\begin{array}{c}\text { Yes } \\
\text { C } \\
\text { N/A } \\
\text { c }\end{array}$ & $\begin{array}{c}\text { Yes } \\
B \\
\text { N/A } \\
\text { c }\end{array}$ & $\begin{array}{c}\text { Yes } \\
\text { B } \\
\text { N/A } \\
\text { c }\end{array}$ & $\begin{array}{c}\text { Yes } \\
\text { C } \\
\text { N/A } \\
\text { c }\end{array}$ & $\begin{array}{c}\text { Ycs } \\
C \\
\text { N/A } \\
c\end{array}$ & $\begin{array}{c}\text { Yos } \\
C \\
\text { N/A } \\
c\end{array}$ & $\begin{array}{c}\text { Yes } \\
\text { B } \\
\text { N/A } \\
\text { c }\end{array}$ & $\begin{array}{c}\text { Yes } \\
C \\
\text { N/A } \\
c\end{array}$ & $\begin{array}{c}\text { Yos } \\
C \\
\text { N/A } \\
c\end{array}$ & $\begin{array}{c}\text { Yes } \\
\text { C } \\
\text { N/A } \\
\text { c }\end{array}$ & $\begin{array}{c}\text { Yes } \\
B \\
\text { N/A } \\
\text { c }\end{array}$ & $\begin{array}{c}\text { Yes } \\
\text { C } \\
\text { N/A } \\
\text { c }\end{array}$ & $\begin{array}{c}\text { Yes } \\
\text { C } \\
\text { N/A } \\
\text { c }\end{array}$ & $\begin{array}{c}\text { Yes } \\
C \\
\text { N/A } \\
c\end{array}$ & $\begin{array}{c}\text { Yes } \\
C \\
\text { N/A } \\
\text { c }\end{array}$ & $\begin{array}{c}\text { Yes } \\
\text { C } \\
\text { N/A } \\
c\end{array}$ & $\begin{array}{c}\text { Yes } \\
C \\
\text { N/A } \\
c\end{array}$ & $\begin{array}{c}\text { Yes } \\
C \\
\text { N/A } \\
c\end{array}$ \\
\hline
\end{tabular}


Table 6-2. (continued).

\begin{tabular}{|c|c|c|c|c|c|c|c|c|c|c|c|c|c|c|c|c|c|c|}
\hline \multirow[b]{2}{*}{ DOE SNF } & \multicolumn{11}{|c|}{ Legal weight truck packages } & \multicolumn{2}{|c|}{$\begin{array}{c}\text { Overweight } \\
\text { truck packages }\end{array}$} & \multicolumn{5}{|c|}{ Rail packages } \\
\hline & $\begin{array}{l}\mathrm{GE} \\
\mathrm{T}-2\end{array}$ & BMI-1 & FSV-1 & NLI-1/2 & NLI-6502 & $T-3$ & NAC-1 & $\begin{array}{l}\text { CNS 1- } \\
13 G\end{array}$ & $\begin{array}{l}\text { NAC- } \\
\text { LWT }\end{array}$ & $\mid \begin{array}{c}\text { GE Model } \\
2000\end{array}$ & $\begin{array}{l}\text { TN- } \\
\text { FSV }\end{array}$ & $\begin{array}{l}\text { TN-8 } \\
\text { TN-8L }\end{array}$ & TN-9 & IF-300 & $\begin{array}{l}\text { NLI- } \\
10 / 24\end{array}$ & $125-\mathrm{B}$ & $\begin{array}{l}\text { TN- } \\
\text { BRP }\end{array}$ & $\begin{array}{l}\text { TN- } \\
\text { REG }\end{array}$ \\
\hline $\begin{array}{c}\text { EBR-II } \\
\text { (ANL-W/HFEA) } \\
(1790)\end{array}$ & $\begin{array}{c}\text { No } \\
\text { a }\end{array}$ & $\begin{array}{c}\text { No } \\
\text { a }\end{array}$ & $\begin{array}{c}\text { Yes } \\
\text { B } \\
\text { 4/arge }\end{array}$ & $\begin{array}{c}\text { Yes } \\
\text { C } \\
\text { 1/large }\end{array}$ & $\begin{array}{c}\text { Yes } \\
\mathrm{C} \\
1 ?(\mathrm{~d}) / \\
\text { large }\end{array}$ & $\begin{array}{c}\text { No } \\
\text { a }\end{array}$ & $\begin{array}{c}\text { Yes } \\
\text { C } \\
\text { 2/large }\end{array}$ & $\begin{array}{c}\text { No } \\
\text { a }\end{array}$ & $\begin{array}{c}\text { Yes } \\
\text { C }\end{array}$ & $\begin{array}{c}\text { No } \\
\text { a }\end{array}$ & $\begin{array}{c}\text { No } \\
\text { c }\end{array}$ & $\begin{array}{c}\text { Yes } \\
\text { C } \\
\text { 3/arge }\end{array}$ & $\mid \begin{array}{c}\text { Yes } \\
\text { C } \\
\text { 7narge }\end{array}$ & $\begin{array}{c}\text { Yes } \\
C \\
14 / \text { arge }\end{array}$ & $\mid \begin{array}{c}\text { Yes } \\
C \\
26 / 35\end{array}$ & $\begin{array}{c}\text { No } \\
\text { c }\end{array}$ & $\mid \begin{array}{c}\text { Yes } \\
\mathrm{C} \\
\text { 3/arge }\end{array}$ & $\begin{array}{c}\text { Yes } \\
\text { C } \\
\text { 3/arge }\end{array}$ \\
\hline $\begin{array}{c}\text { EBR-II } \\
\text { (ANL-W/RSWF) } \\
(15000)\end{array}$ & $\begin{array}{c}\text { Yes } \\
C \\
\text { N/A } \\
c\end{array}$ & $\begin{array}{c}\text { No } \\
\text { a }\end{array}$ & $\begin{array}{l}\text { No } \\
\text { a }\end{array}$ & $\begin{array}{l}\text { No } \\
\text { a }\end{array}$ & $\begin{array}{c}\text { No } \\
\text { a }\end{array}$ & $\begin{array}{c}\text { No } \\
\text { a }\end{array}$ & $\begin{array}{c}\text { No } \\
\text { a }\end{array}$ & $\begin{array}{c}\text { No } \\
\text { a }\end{array}$ & $\begin{array}{c}\text { No } \\
\text { a }\end{array}$ & $\begin{array}{c}\text { No } \\
\text { a }\end{array}$ & $\begin{array}{c}\text { No } \\
\text { a }\end{array}$ & $\begin{array}{l}\text { No } \\
\mathbf{a}\end{array}$ & $\begin{array}{l}\text { No } \\
\text { a }\end{array}$ & $\begin{array}{c}\text { Yes } \\
\text { C } \\
\text { 42/arge }\end{array}$ & $\begin{array}{c}\text { Yes } \\
\mathrm{C} \\
60 \text { /arge }\end{array}$ & $\mid \begin{array}{c}\text { Yes } \\
\text { C } \\
40 \text { harge }\end{array}$ & $\begin{array}{c}\text { Yes } \\
\text { C } \\
116 / \\
\text { large }\end{array}$ & $\begin{array}{c}\text { Yes } \\
\text { C } \\
135 n \\
\text { large }\end{array}$ \\
\hline \multicolumn{19}{|l|}{ GCR } \\
\hline $\begin{array}{c}\text { FSVR-HT } \\
(744)\end{array}$ & $\begin{array}{c}\text { No } \\
\text { a }\end{array}$ & $\begin{array}{c}\text { No } \\
\mathbf{a}\end{array}$ & $\begin{array}{c}\text { Yes } \\
\text { A } \\
6 ?(c) / \\
\text { large }\end{array}$ & $\begin{array}{c}\text { No } \\
\text { a }\end{array}$ & $\begin{array}{c}\text { No } \\
\text { a }\end{array}$ & $\begin{array}{c}\text { No } \\
\text { a }\end{array}$ & $\begin{array}{l}\text { No } \\
\text { a }\end{array}$ & $\begin{array}{c}\mathrm{N} / \mathrm{A} \\
\mathrm{b}\end{array}$ & $\begin{array}{c}\text { No } \\
\text { a }\end{array}$ & $\begin{array}{c}\mathrm{N} / \mathrm{A} \\
\mathrm{b}\end{array}$ & $\begin{array}{c}\text { Yes } \\
\mathrm{A} \\
6 ?(\mathrm{c}) / \\
\text { large }\end{array}$ & $\begin{array}{c}\text { No } \\
\text { a }\end{array}$ & $\begin{array}{c}\text { No } \\
\text { a }\end{array}$ & $\begin{array}{c}\text { N/A } \\
b\end{array}$ & $\begin{array}{c}\text { N/A } \\
\text { b }\end{array}$ & $\begin{array}{c}\text { N/A } \\
b\end{array}$ & $\begin{array}{c}\text { N/A } \\
b\end{array}$ & $\begin{array}{c}\mathrm{N} / \mathrm{A} \\
\mathrm{b}\end{array}$ \\
\hline $\begin{array}{l}\text { FSVR } \\
(1500)\end{array}$ & $\begin{array}{c}\text { No } \\
\text { a }\end{array}$ & $\begin{array}{c}\text { No } \\
\text { a }\end{array}$ & $\begin{array}{c}\text { Yes } \\
\text { A } \\
6 ?(\mathrm{c}) / \\
\text { large }\end{array}$ & $\begin{array}{c}\text { No } \\
\text { a }\end{array}$ & $\begin{array}{c}\text { No } \\
\text { a }\end{array}$ & $\begin{array}{c}\text { No } \\
\text { a }\end{array}$ & $\begin{array}{c}\text { No } \\
\text { a }\end{array}$ & $\begin{array}{c}\mathrm{N} / \mathrm{A} \\
\mathrm{b}\end{array}$ & $\begin{array}{c}\text { No } \\
\text { a }\end{array}$ & $\begin{array}{c}\text { N/A } \\
b\end{array}$ & $\begin{array}{c}\text { Yes } \\
\text { A } \\
6 ?(\mathrm{c}) / \\
\text { large }\end{array}$ & $\begin{array}{c}\text { No } \\
\text { a }\end{array}$ & $\begin{array}{c}\text { No } \\
\text { a }\end{array}$ & $\begin{array}{c}\text { N/A } \\
b\end{array}$ & $\begin{array}{c}\text { N/A } \\
\text { b }\end{array}$ & $\begin{array}{c}\text { N/A } \\
b\end{array}$ & $\begin{array}{c}\text { N/A } \\
b\end{array}$ & $\begin{array}{c}\text { N/A } \\
b\end{array}$ \\
\hline $\begin{array}{c}\text { TORY-IIC } \\
(655)\end{array}$ & $\begin{array}{c}\text { Yes } \\
C \\
\text { N/A } \\
c\end{array}$ & $\begin{array}{l}\text { No } \\
\text { a }\end{array}$ & $\begin{array}{c}\text { Ycs } \\
C \\
\text { N/A } \\
c\end{array}$ & $\begin{array}{c}\text { Yes } \\
\text { C } \\
\text { N/A } \\
\text { c }\end{array}$ & $\begin{array}{c}\text { Yes } \\
C \\
\text { N/A } \\
c\end{array}$ & $\begin{array}{c}\text { Yes } \\
C \\
\text { N/A } \\
c\end{array}$ & $\begin{array}{c}\text { Yes } \\
C \\
\text { N/A } \\
c\end{array}$ & $\begin{array}{c}\text { No } \\
\text { a }\end{array}$ & $\begin{array}{c}\text { Yes } \\
C \\
\text { N/A } \\
c\end{array}$ & $\begin{array}{c}\text { No } \\
\text { a }\end{array}$ & $\begin{array}{c}\text { Yes } \\
C \\
\text { N/A } \\
c\end{array}$ & $\begin{array}{c}\text { Yes } \\
C \\
\text { N/A } \\
c\end{array}$ & $\begin{array}{c}\text { Yes } \\
C \\
\text { N/A } \\
c\end{array}$ & $\begin{array}{c}\text { Yes } \\
C \\
\text { N/A } \\
c\end{array}$ & $\begin{array}{c}\text { Yes } \\
C \\
N / A \\
c\end{array}$ & $\begin{array}{c}\text { Yes } \\
C \\
\text { N/A } \\
c\end{array}$ & $\begin{array}{c}\text { Yes } \\
C \\
\text { N/A } \\
\text { c }\end{array}$ & $\begin{array}{c}\text { Yes } \\
C \\
\text { N/A } \\
c\end{array}$ \\
\hline $\begin{array}{c}\text { TORY-IIA } \\
(146)\end{array}$ & $\begin{array}{c}\text { Yes } \\
C \\
\text { N/A } \\
c\end{array}$ & $\begin{array}{c}\text { Yes } \\
C \\
N / A \\
c\end{array}$ & $\begin{array}{c}\text { Yes } \\
C \\
N / A \\
c\end{array}$ & $\begin{array}{c}\text { Yes } \\
C \\
\text { N/A } \\
\text { c }\end{array}$ & $\begin{array}{c}\text { Yes } \\
C \\
\text { N/A } \\
c\end{array}$ & $\begin{array}{c}\text { Yes } \\
C \\
N / A \\
c\end{array}$ & $\begin{array}{c}\text { Yes } \\
\text { C } \\
\text { N/A } \\
c\end{array}$ & $\begin{array}{c}\text { Yes } \\
C \\
N / A \\
c\end{array}$ & $\begin{array}{c}\text { Yes } \\
C \\
\text { N/A } \\
c\end{array}$ & $\begin{array}{c}\text { Yes } \\
C \\
\text { N/A } \\
c\end{array}$ & $\begin{array}{c}\text { Ye } \\
C \\
\text { N/A } \\
c\end{array}$ & $\begin{array}{c}\text { Yes } \\
C \\
\text { N/A } \\
c\end{array}$ & $\begin{array}{c}\text { Yes } \\
C \\
\text { N/A } \\
c\end{array}$ & $\begin{array}{c}\text { Yes } \\
C \\
\text { N/A } \\
c\end{array}$ & $\begin{array}{c}\text { Yes } \\
\text { C } \\
\text { N/A } \\
c\end{array}$ & $\begin{array}{c}\text { Yes } \\
\text { C } \\
\text { N/A } \\
c\end{array}$ & $\begin{array}{c}\text { Yes } \\
\text { C } \\
\text { N/A } \\
\text { c }\end{array}$ & $\begin{array}{c}\text { Yes } \\
\text { C } \\
\text { N/A } \\
c\end{array}$ \\
\hline \multicolumn{19}{|l|}{ Metallic } \\
\hline $\begin{array}{l}\text { TRIGA-AL } \\
\text { (INEL) } \\
(578)\end{array}$ & $\begin{array}{c}\text { Yes } \\
\text { C } \\
30 / 20\end{array}$ & $\begin{array}{c}\text { Yes } \\
\text { A } \\
38 ?(\mathrm{c}) / \\
16\end{array}$ & $\begin{array}{c}\text { Yes } \\
C \\
240 / 3\end{array}$ & $\begin{array}{c}\text { Yes } \\
\text { C } \\
170 / 4\end{array}$ & $\begin{array}{c}\text { Yes } \\
\text { C } \\
98 ?(d) / 6\end{array}$ & $\begin{array}{c}\text { Yes } \\
C \\
50 / 12\end{array}$ & $\begin{array}{c}\text { Yes } \\
C \\
150 / 4\end{array}$ & $\begin{array}{c}\text { Yes } \\
C \\
120 / 5\end{array}$ & $\begin{array}{c}\text { Yes } \\
\text { C } \\
150 / 4\end{array}$ & $\begin{array}{c}\text { Yes } \\
C \\
120 / 5\end{array}$ & $\begin{array}{c}\text { Yes } \\
C \\
240 / 3\end{array}$ & $\begin{array}{c}\text { Yes } \\
\text { C } \\
225 / 3\end{array}$ & $\begin{array}{c}\text { Yes } \\
C \\
280 / 3\end{array}$ & $\begin{array}{c}\text { Yes } \\
C \\
900 / 1\end{array}$ & $\begin{array}{c}\text { Yes } \\
C \\
1600 / 1\end{array}$ & $\mid \begin{array}{c}Y c a \\
C \\
1428 / 1\end{array}$ & $\mid \begin{array}{c}\text { Yes } \\
\text { C } \\
3700 / 1\end{array}$ & $\begin{array}{c}\text { Yes } \\
\text { C } \\
3800 / 1\end{array}$ \\
\hline
\end{tabular}


Table 6-2. (continued).

\begin{tabular}{|c|c|c|c|c|c|c|c|c|c|c|c|c|c|c|c|c|c|c|}
\hline \multirow[b]{2}{*}{ DOE SNF } & \multicolumn{11}{|c|}{ Legal weight truck packages } & \multicolumn{2}{|c|}{$\begin{array}{c}\text { Overweight } \\
\text { truck packages }\end{array}$} & \multicolumn{5}{|c|}{ Rail packages } \\
\hline & $\begin{array}{l}\mathrm{GE} \\
\mathrm{T}-2\end{array}$ & BMI-1 & FSV-1 & NLI-1/2 & NLI-6502 & $T \cdot 3$ & NAC-1 & $\begin{array}{c}\text { CNS } 1- \\
13 G\end{array}$ & $\begin{array}{l}\text { NAC- } \\
\text { LWT }\end{array}$ & $\left|\begin{array}{c}\text { GE Model } \\
2000\end{array}\right|$ & \begin{tabular}{|l} 
TN. \\
FSV
\end{tabular} & $\begin{array}{l}\text { TN-8 } \\
\text { TN-8L }\end{array}$ & TN-9 & IF-300 & $\begin{array}{l}\text { NLI- } \\
10 / 24\end{array}$ & $125-B$ & $\begin{array}{l}\text { TN- } \\
\text { BRP }\end{array}$ & $\begin{array}{l}\text { TN- } \\
\text { REG }\end{array}$ \\
\hline $\begin{array}{l}\text { TRIGA FLIP } \\
\text { (INEL) } \\
\text { (7) }\end{array}$ & $\begin{array}{c}\text { Yes } \\
\text { C } \\
72(c) / 1\end{array}$ & $\begin{array}{c}\text { Yes } \\
\text { A } \\
19 ?(\mathrm{c}) / 1\end{array}$ & $\begin{array}{c}\text { Yes } \\
\text { C } \\
120 ?(\mathrm{c}) / 1\end{array}$ & $\begin{array}{c}\text { Yes } \\
\text { C } \\
85 ?(\mathrm{c}) / 1\end{array}$ & $\left|\begin{array}{c}\text { Yes } \\
\mathrm{C} \\
49 ?(\mathrm{c} / \mathrm{d}) / 1\end{array}\right|$ & $\begin{array}{c}\text { Yes } \\
\mathbf{C} \\
25 ?(\mathrm{c}) / \\
1\end{array}$ & $\begin{array}{c}\text { Yes } \\
\text { C } \\
75 ?(\mathrm{c}) / 1\end{array}$ & $\begin{array}{c}\text { Yes } \\
\mathrm{C} \\
60 ?(\mathrm{c}) / 1\end{array}$ & $\begin{array}{c}\text { Yes } \\
\text { C } \\
75 ?(\mathrm{c}) / 1\end{array}$ & $\begin{array}{c}\text { Yes } \\
C \\
607(c) / 1\end{array}$ & $\begin{array}{c}\text { Yes } \\
\text { C } \\
120 ? \\
\text { (c) } / 1\end{array}$ & $\begin{array}{c}\text { Yes } \\
\text { C } \\
105 ? \\
\text { (c) } / 1\end{array}$ & $\begin{array}{c}\text { Yes } \\
\text { C } \\
140 ?(\mathrm{c}) \\
/ 1\end{array}$ & $\begin{array}{c}\mathrm{Yes} \\
\mathrm{C} \\
450 ?(\mathrm{c}) / 1\end{array}$ & $\mid \begin{array}{c}\text { Yes } \\
\mathrm{C} \\
800 ?(\mathrm{c}) \\
11\end{array}$ & $\begin{array}{c}\text { Yes } \\
\mathrm{C} \\
714 ?(\mathrm{c}) \\
/ 1\end{array}$ & \begin{tabular}{|c|} 
Ye \\
C \\
$1850 ?$ \\
$(c) / 1$
\end{tabular} & $\begin{array}{c}\text { Yes } \\
C \\
1900 ? \\
(c) / 1\end{array}$ \\
\hline $\begin{array}{l}\text { TRIGA FLIP } \\
\text { (ANL-W) } \\
(116)\end{array}$ & $\begin{array}{c}\text { Yes } \\
C \\
30 ?(d) / 9\end{array}$ & $\begin{array}{c}\text { Yes } \\
\text { A } \\
19 ?(c / d) / 7\end{array}$ & $\begin{array}{c}\text { Yes } \\
C \\
120 ?(d) / 1\end{array}$ & $\begin{array}{c}\text { Yes } \\
C \\
49 ?(d) / 3\end{array}$ & $\begin{array}{c}\text { Yes } \\
C \\
\text { N/A } \\
d\end{array}$ & $\begin{array}{c}\text { Yes } \\
\text { C } \\
25 ?(d) / 5\end{array}$ & $\begin{array}{c}\text { Yes } \\
\text { C } \\
75 ?(\mathrm{~d}) / 2\end{array}$ & $\begin{array}{c}Y e s \\
C \\
60 ?(d) / 2\end{array}$ & $\begin{array}{c}\text { Yes } \\
\mathrm{C} \\
75 ?(\mathrm{~d}) / 2\end{array}$ & $\begin{array}{c}Y e s \\
C \\
60 ?(d) / 2\end{array}$ & $\begin{array}{c}\text { Yes } \\
\text { C } \\
120 ? \\
\text { (d) } / 1\end{array}$ & $\begin{array}{c}\text { Yes } \\
\text { C } \\
105 ? \\
\text { (d) } / 2\end{array}$ & $\begin{array}{c}\text { Yes } \\
\text { C } \\
140 ?(d) \\
/ 1\end{array}$ & $\begin{array}{c}\text { Yes } \\
\text { C } \\
450 ?(d) / \\
1\end{array}$ & $\begin{array}{c}\text { Yes } \\
\text { C } \\
800 ? \\
\text { (d) } / 1\end{array}$ & $\begin{array}{c}\text { Yes } \\
\text { C } \\
714 ? \\
\text { (d)/1 }\end{array}$ & $\begin{array}{c}\text { Yes } \\
\text { C } \\
1850 ? \\
\text { (d) } / 1\end{array}$ & $\begin{array}{c}\text { Yes } \\
\text { C } \\
1900 ? \\
\text { (d) } / 1\end{array}$ \\
\hline $\begin{array}{c}\text { TRIGA SST } \\
\text { (INEL) } \\
\text { (263) }\end{array}$ & $\begin{array}{c}\text { Yes } \\
\mathrm{C} \\
30 ?(d) / 4\end{array}$ & $\begin{array}{c}\text { Ye } \\
\text { A } \\
38 ?(\mathrm{c} / \mathrm{d}) /\end{array}$ & $\begin{array}{c}\text { Yes } \\
\text { C } \\
240 ?(d) / 2\end{array}$ & $\begin{array}{c}\text { Yes } \\
C \\
98 ?(d) / 3\end{array}$ & $\begin{array}{c}\text { Yes } \\
\text { C } \\
\text { N/A } \\
d\end{array}$ & $\begin{array}{c}\text { Yes } \\
C \\
50 ?(d) / 6\end{array}$ & $\begin{array}{c}Y e s \\
C \\
150 ?(d) / 2\end{array}$ & $\begin{array}{c}\text { Yes } \\
\text { C } \\
120 ?(d) / 3\end{array}$ & $\begin{array}{c}\text { Yes } \\
C \\
150 ?(d) / 2\end{array}$ & $\mid \begin{array}{c}\text { Yes } \\
C \\
120 ?(d) / 3\end{array}$ & $\begin{array}{c}\text { Yes } \\
\text { C } \\
240 ? \\
\text { (d) } / 2\end{array}$ & $\begin{array}{c}\text { Yes } \\
\text { C } \\
225 ? \\
\text { (d) } / 2\end{array}$ & $\begin{array}{c}\text { Ycs } \\
C \\
280 ?(d) \\
/ 1\end{array}$ & $\begin{array}{c}\text { Yes } \\
C \\
900 ?(d) / \\
1\end{array}$ & \begin{tabular}{|c|} 
Yes \\
$C$ \\
$1600 ?$ \\
(d)/1
\end{tabular} & \begin{tabular}{|c|} 
Ycs \\
$\mathrm{C}$ \\
$1428 ?$ \\
$(d) / 1$
\end{tabular} & $\begin{array}{c}\text { Yes } \\
C \\
3700 ? \\
\text { (d) } / 1\end{array}$ & \begin{tabular}{|c|} 
Yes \\
C \\
$3800 ?$ \\
(d) $/ 1$
\end{tabular} \\
\hline $\begin{array}{c}\text { TRIGA SST } \\
\text { (Hanford) } \\
\text { (101) }\end{array}$ & $\begin{array}{c}\text { Yes } \\
C \\
30 ?(d) / 4\end{array}$ & $\begin{array}{c}\text { Yes } \\
\text { A } \\
38 ?(\mathrm{c} / \mathrm{d}) / 3\end{array}$ & $\begin{array}{c}\text { Yes } \\
C \\
240 ?(d) / 1\end{array}$ & $\begin{array}{c}\text { Ycs } \\
C \\
170 ?(d) / 1\end{array}$ & $\begin{array}{c}\text { Yes } \\
\text { C } \\
\text { N/A } \\
\text { d }\end{array}$ & $\begin{array}{c}\text { Yes } \\
C \\
50 ?(d) / 3\end{array}$ & $\begin{array}{c}\text { Yes } \\
C \\
150 ?(d) / 1\end{array}$ & $\begin{array}{c}\text { Yes } \\
C \\
120 ?(d) / 1\end{array}$ & $\begin{array}{c}\text { Yes } \\
C \\
150 ?(d) / 1\end{array}$ & $\begin{array}{c}\text { Yes } \\
\mathrm{C} \\
120 ?(\mathrm{~d}) / 1\end{array}$ & $\begin{array}{c}\text { Yes } \\
\text { C } \\
240 ? \\
\text { (d) } / 1\end{array}$ & $\begin{array}{c}\text { Yes } \\
\text { C } \\
225 ? \\
\text { (d) } / 1\end{array}$ & $\begin{array}{c}\text { Yes } \\
\text { C } \\
280 ?(d) \\
/ 1\end{array}$ & $\begin{array}{c}\text { Yes } \\
\mathrm{C} \\
900 ?(\mathrm{~d}) / \\
1\end{array}$ & \begin{tabular}{|c|} 
Ycs \\
$C$ \\
$1600 ?$ \\
(d) $/ 1$
\end{tabular} & \begin{tabular}{|c|} 
Yes \\
$C$ \\
$1428 ?$ \\
(d)/1
\end{tabular} & $\begin{array}{c}Y e s \\
C \\
3700 ? \\
\text { (d) } / 1\end{array}$ & $\begin{array}{c}\text { Yes } \\
\text { C } \\
3800 ? \\
\text { (d)/1 }\end{array}$ \\
\hline $\begin{array}{l}\text { TRIGA Ass'y } \\
\text { (Hanford) } \\
\text { (N/A) }\end{array}$ & $\begin{array}{c}\text { Yes } \\
C \\
\text { N/A } \\
c\end{array}$ & $\begin{array}{c}\text { Ye } \\
\text { A } \\
38 ?(\mathrm{c} / \mathrm{d}) / ? \\
\text { (e) }\end{array}$ & $\begin{array}{c}\text { Yes } \\
\text { C } \\
240 ?(\mathrm{c} / \\
\text { d)/?(e) }\end{array}$ & $\begin{array}{c}\text { Yes } \\
C \\
170 ?(c / \\
\text { d)/?(e) }\end{array}$ & $\begin{array}{c}\text { Yes } \\
C \\
\text { N/A } \\
d\end{array}$ & $\begin{array}{c}\text { Yes } \\
\text { C } \\
50 ?(\mathrm{c} / \\
\text { d) } /(\mathrm{e})\end{array}$ & $\begin{array}{c}\text { Yes } \\
\text { C } \\
150 ?(\mathrm{c} / \\
\text { d)??(e) }\end{array}$ & $\begin{array}{c}\text { Yes } \\
\text { C } \\
\text { 120?(d) } \\
\text { d)/?(e) }\end{array}$ & $\begin{array}{c}\text { Yes } \\
\text { C } \\
150 ?(c / \\
\text { d)/?(e) }\end{array}$ & $\begin{array}{c}\text { Yes } \\
\mathrm{C} \\
120 ?(\mathrm{c} / \\
\mathrm{d}) ?(\mathrm{e})\end{array}$ & $\begin{array}{c}\text { Yes } \\
\text { C } \\
240 ?(\mathrm{c} / \\
\text { d) ??(e) }\end{array}$ & $\begin{array}{c}\text { Yes } \\
\text { C } \\
225 ?(\mathrm{c} / \\
\text { d)?(e) }\end{array}$ & $\begin{array}{c}\text { Yes } \\
\text { C } \\
280 ?(\mathrm{c} \\
\text { d) } / ?(\mathrm{e})\end{array}$ & $\begin{array}{c}\text { Yes } \\
\text { C } \\
900 ?(\mathrm{c} / \\
\text { d) } /(\mathrm{e})\end{array}$ & $\begin{array}{c}\text { Yes } \\
\text { C } \\
1600 ?(c \\
I \\
\text { d) } / \text { (e) }\end{array}$ & $\begin{array}{c}\text { Yes } \\
C \\
1428 ?(c \\
/ \\
\text { d) } ? \text { ?(e) }\end{array}$ & $\begin{array}{c}\text { Yes } \\
\mathrm{C} \\
3700 ?(\mathrm{c} \\
/ \\
\text { d) } / \text { (e) }\end{array}$ & $\begin{array}{c}\text { Yes } \\
\text { C } \\
3800 ?(\mathrm{c} \\
/ \\
\text { d) } / \text { (e) }\end{array}$ \\
\hline $\begin{array}{l}\text { TRIGA BER-II } \\
\text { (21) }\end{array}$ & $\begin{array}{c}\text { Yes } \\
C \\
\text { N/A } \\
c / d\end{array}$ & $\begin{array}{c}\text { Yes } \\
B \\
\text { N/A } \\
c / d\end{array}$ & $\begin{array}{c}\text { Yes } \\
C \\
\text { N/A } \\
c / d\end{array}$ & $\begin{array}{c}\text { Yes } \\
C \\
\text { N/A } \\
\text { c/d }\end{array}$ & $\begin{array}{c}\text { Yes } \\
C \\
\text { N/A } \\
\text { c/d }\end{array}$ & $\begin{array}{c}\text { Yes } \\
C \\
\text { N/A } \\
c / d\end{array}$ & $\begin{array}{c}\text { Yes } \\
C \\
\text { N/A } \\
c / d\end{array}$ & $\begin{array}{c}\text { Yes } \\
C \\
\text { N/A } \\
\text { c/d }\end{array}$ & $\begin{array}{c}\text { Yes } \\
C \\
N / A \\
c / d\end{array}$ & $\begin{array}{c}\text { Yes } \\
C \\
\text { N/A } \\
\text { c/d }\end{array}$ & $\begin{array}{c}\text { Yes } \\
C \\
\text { N/A } \\
\text { c/d }\end{array}$ & $\begin{array}{c}\text { Yes } \\
C \\
\text { N/A } \\
\text { c/d }\end{array}$ & $\begin{array}{c}\text { Yes } \\
C \\
\text { N/A } \\
\text { c/d }\end{array}$ & $\begin{array}{c}\text { Yes } \\
C \\
\text { N/A } \\
\text { c/d }\end{array}$ & $\begin{array}{c}\text { Yes } \\
C \\
\text { N/A } \\
\text { c/d }\end{array}$ & $\begin{array}{c}\text { Yes } \\
C \\
\text { N/A } \\
c / d\end{array}$ & $\begin{array}{c}\text { Yes } \\
C \\
\text { N/A } \\
\text { c/d }\end{array}$ & $\begin{array}{c}\text { Yes } \\
C \\
\text { N/A } \\
c / d\end{array}$ \\
\hline $\begin{array}{l}\text { Aerolest TRIGA } \\
\text { (San Ramon) } \\
\text { (93) }\end{array}$ & $\begin{array}{c}\text { Yes } \\
C \\
\text { N/A } \\
c / d\end{array}$ & $\begin{array}{c}\text { Yes } \\
B \\
\text { N/A } \\
c / d\end{array}$ & $\begin{array}{c}\text { Yes } \\
C \\
\text { N/A } \\
c / d\end{array}$ & $\begin{array}{c}\text { Yes } \\
C \\
\text { N/A } \\
\text { c/d }\end{array}$ & $\begin{array}{c}\text { Yes } \\
C \\
\text { N/A } \\
\text { c/d }\end{array}$ & $\begin{array}{c}\text { Yes } \\
C \\
\text { N/A } \\
c / d\end{array}$ & $\begin{array}{c}\text { Yes } \\
\text { C } \\
\text { N/A } \\
\text { c/d }\end{array}$ & $\begin{array}{c}\text { Yes } \\
C \\
\text { N/A } \\
\text { c/d }\end{array}$ & $\begin{array}{c}\text { Yes } \\
C \\
\text { N/A } \\
\text { c/d }\end{array}$ & $\begin{array}{c}\text { Yes } \\
\text { C } \\
\text { N/A } \\
\text { c/d }\end{array}$ & $\begin{array}{c}\text { Yes } \\
C \\
\text { N/A } \\
\text { c/d }\end{array}$ & $\begin{array}{c}\text { Yos } \\
\text { C } \\
\text { N/A } \\
\text { c/d }\end{array}$ & $\begin{array}{c}\text { Yes } \\
C \\
\text { N/A } \\
\text { c/d }\end{array}$ & $\begin{array}{c}\text { Yes } \\
\text { C } \\
\text { N/A } \\
\text { c/d }\end{array}$ & $\begin{array}{c}\text { Yes } \\
\text { C } \\
\text { N/A } \\
\text { c/d }\end{array}$ & $\begin{array}{c}\text { Yes } \\
\mathrm{C} \\
\mathrm{N} / \mathrm{A} \\
\mathrm{c} / \mathrm{d}\end{array}$ & $\begin{array}{c}\text { Yes } \\
\text { C } \\
\text { N/A } \\
\text { c/d }\end{array}$ & $\begin{array}{c}\text { Yes } \\
\text { C } \\
\text { N/A } \\
\text { c/d }\end{array}$ \\
\hline
\end{tabular}




\begin{tabular}{|c|c|c|c|c|c|c|c|c|}
\hline \multirow{5}{*}{ 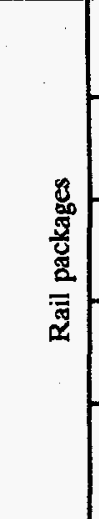 } & 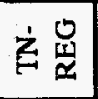 & $80 \leqslant 8$ & $\frac{y}{\gamma} \cup \frac{5}{2}$ & $\frac{0}{8} \cup \frac{\pi}{z}$ & $\nabla 0 \frac{\pi}{z}$ & 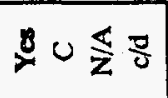 & $80 \stackrel{8}{8}$ & 융 \\
\hline & $\dot{\xi}$ & $\geqslant 0 \leqslant \frac{2}{2}$ & $\frac{8}{2} \cup \frac{5}{z}$ & $\gamma \cup \frac{1}{z} D$ & $80 \frac{z}{z}$ & $0 \leq \frac{5}{z} D$ & 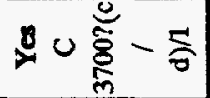 & 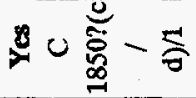 \\
\hline & $\stackrel{\infty}{\dot{\Delta}}$ & 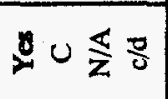 & $80 \frac{3}{z}$ & $\delta 0 \leqslant z$ & $20 \leqslant 8$ & $\varnothing \cup \leqslant \frac{2}{2}$ & • & ¿ \\
\hline & $\dot{\vec{z}}$ & $\searrow \cup \leqslant \frac{\pi}{2}$ & $\geqslant 0 \leqslant \frac{3}{2}$ & $\varnothing \cup \leqslant z$ & $8 \cup \leq \frac{0}{z}$ & 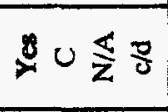 & 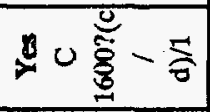 & 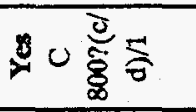 \\
\hline & 务 & $x \cup \frac{\pi}{2}$ & 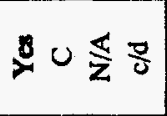 & 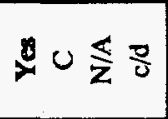 & $00 \frac{8}{2}$ & 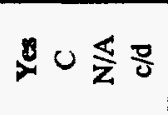 & ¿ & 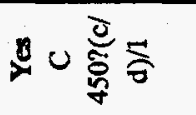 \\
\hline \multirow{2}{*}{ 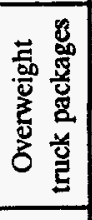 } & ż & $\searrow 0 \leqslant \frac{\pi}{z}$ & $\$ 0 \frac{\pi}{z} \frac{D}{0}$ & $d 0 \frac{\pi}{z}$ & $00 \leqslant \frac{0}{2}$ & 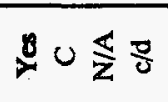 & 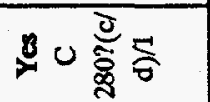 & Du \\
\hline & 竞审 & $0<\frac{3}{2}$ & $80 \leqslant \frac{1}{z}$ & $0 \quad \frac{\pi}{z}$ & $20 \frac{8}{z}$ & $80 \leq \frac{8}{2}$ & Ð & 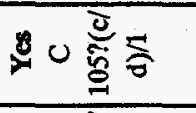 \\
\hline \multirow{11}{*}{ 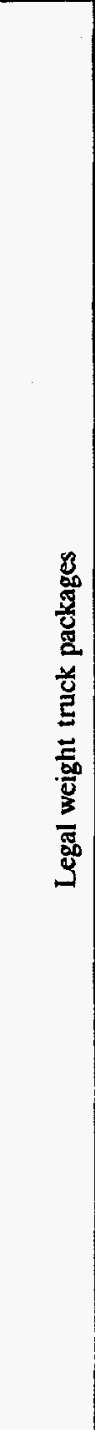 } & 它密 & 눈 & $00 \frac{3}{2}$ & 인 & $00 \frac{5}{2}$ & $20 \frac{8}{2}$ & 잉 & ๑ \\
\hline & 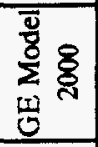 & $y u \frac{z}{2}$ & $00 \frac{\pi}{2}$ & $\searrow \cup \frac{\pi}{z} g$ & $\oslash 0 \leqslant \frac{2}{z}$ & D्र $\cup \frac{z}{z}$ & ن & $\frac{\pi}{\frac{8}{8}}$ \\
\hline & 这占 & $D \cup \frac{2}{z}$ & $D 0 \leqslant s$ & 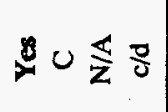 & $D_{x} \cup \frac{z}{z}$ & 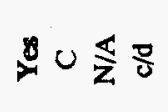 & 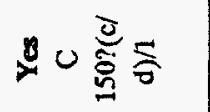 & 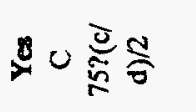 \\
\hline & 它总 & $\sum \cup \frac{\pi}{z}$ & $80 \leqslant 8$ & $D \cup \frac{\pi}{2}$ & $D \cup \leqslant \frac{\pi}{2}$ & $D \cup \frac{\pi}{z} g$ & 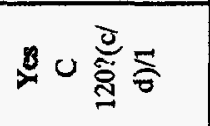 & 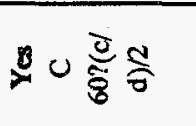 \\
\hline & $\overrightarrow{\dot{z}}$ & $y 0 \leqslant 3$ & $80 \leqslant \frac{2}{2}$ & 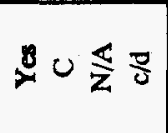 & $y 0 \frac{\pi}{2}$ & \& & 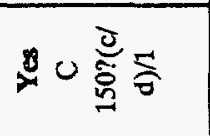 & 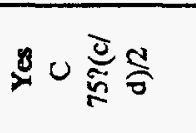 \\
\hline & $\stackrel{9}{H}$ & $\succsim 0 \leqslant \frac{2}{z}$ & $D \cup \leqslant \frac{\pi}{z}$ & $y<\frac{2}{2}$ & $00 \leqslant \frac{2}{z}$ & D $0 \frac{5}{z}$ & 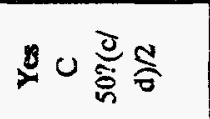 & 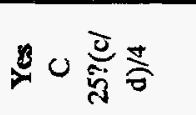 \\
\hline & 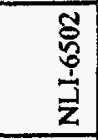 & 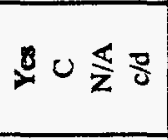 & $\searrow 0 \leqslant \frac{2}{z}$ & $\varnothing \cup \leqslant 3$ & $\searrow 0 \frac{\pi}{z}$ & 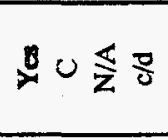 & 00 & $\mathscr{*} \cup \frac{1}{z}=$ \\
\hline & $\underset{\mathbf{z}}{\frac{S}{2}}$ & $80 \frac{3}{z}$ & $0<\frac{5}{z}$ & $\frac{8}{\gamma} \cup \frac{\pi}{2} \frac{z}{2}$ & $80 \frac{5}{z}$ & $y \cup \frac{\pi}{z}$ & 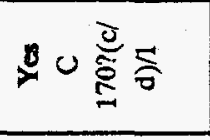 & DU \\
\hline & $\overrightarrow{\dot{3}}$ & 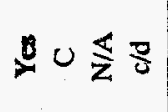 & 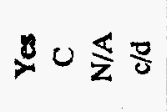 & $\frac{8}{2} \cup \frac{8}{2}$ & $\pm 0 \leqslant 8$ & $d \cup \frac{2}{z}$ & • & 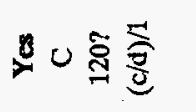 \\
\hline & $\overrightarrow{\dot{\overrightarrow{\mid}}}$ & $D \infty \frac{D}{\partial}$ & $y \infty \frac{8}{z}$ & $\searrow \infty \frac{8}{z}$ & 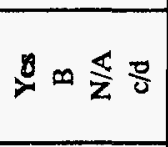 & 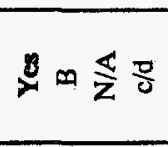 & 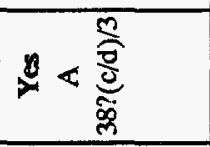 & $\frac{n}{2}$ \\
\hline & 멍연 & $\searrow 0 \leqslant 8$ & $y<\leqslant \frac{2}{z}$ & 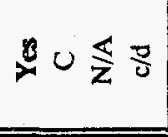 & $y<\frac{3}{z}$ & $0<\frac{5}{z}$ & 낭 & 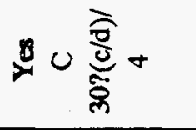 \\
\hline & 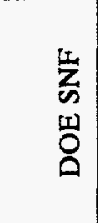 & 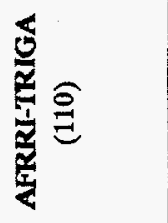 & 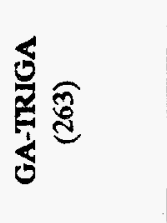 & 㝴 & 㝴息 & 起量 & 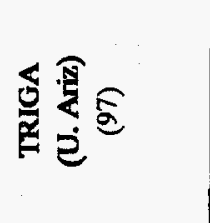 & 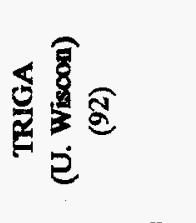 \\
\hline
\end{tabular}


Table 6-2. (continued).

\begin{tabular}{|c|c|c|c|c|c|c|c|c|c|c|c|c|c|c|c|c|c|c|}
\hline \multirow[b]{2}{*}{ DOE SNF } & \multicolumn{11}{|c|}{ Legal weight truck packages } & \multicolumn{2}{|c|}{$\begin{array}{c}\text { Overweight } \\
\text { truck packages }\end{array}$} & \multicolumn{5}{|c|}{ Rail packages } \\
\hline & $\begin{array}{l}\mathrm{GE} \\
\mathrm{T}-2\end{array}$ & BMI-1 & FSV-1 & NLI-1/2 & $\mid$ NLI-6502 $\mid$ & $\mathrm{T}-3$ & NAC-1 & $\begin{array}{l}\text { CNS 1- } \\
13 G\end{array}$ & $\begin{array}{l}\text { NAC- } \\
\text { LWT }\end{array}$ & $\mid \begin{array}{c}\text { GE Model } \\
2000\end{array}$ & $\begin{array}{l}\text { TN- } \\
\text { FSV }\end{array}$ & $\begin{array}{l}\text { TN-8 } \\
\text { TN-8L }\end{array}$ & TN-9 & IF-300 & $\begin{array}{l}\text { NLI- } \\
10 / 24\end{array}$ & 125-B & $\begin{array}{l}\text { TN- } \\
\text { BRP }\end{array}$ & $\begin{array}{l}\text { TN- } \\
\text { REG }\end{array}$ \\
\hline $\begin{array}{c}\text { TRIGA } \\
\text { (U. Illinois) } \\
\text { (193) }\end{array}$ & $\begin{array}{c}\text { Yes } \\
C \\
30 ?(\mathrm{c}) / \\
7\end{array}$ & $\mid \begin{array}{c}\text { Yes } \\
\mathrm{B} \\
38 ?(\mathrm{c} / \mathrm{d}) / 6\end{array}$ & $\begin{array}{c}\text { Yes } \\
\text { C } \\
240 ?(\mathrm{c} / \\
\text { d) } / 1\end{array}$ & $\begin{array}{c}\text { Yes } \\
\text { C } \\
170 ?(\mathrm{c} / \\
\text { d) } / 21\end{array}$ & $\begin{array}{c}\text { Yos } \\
\text { C } \\
\text { N/A } \\
d\end{array}$ & $\begin{array}{c}\text { Yes } \\
\text { C } \\
50 ?(c / \\
\text { d) } / 4\end{array}$ & $\begin{array}{c}\text { Yes } \\
\text { C } \\
150 ?(\mathrm{c} / \\
\text { d) } / 2\end{array}$ & $\begin{array}{c}\text { Yes } \\
\text { C } \\
120 ?(\mathrm{c} / \\
\text { d) } / 2\end{array}$ & $\begin{array}{c}\text { Yes } \\
\text { C } \\
150 ?(\mathrm{c} / \\
\text { d) } / 2\end{array}$ & $\begin{array}{c}\text { Yes } \\
\text { C } \\
120 ?(\mathrm{c} / \\
\text { d) } / 2\end{array}$ & $\begin{array}{c}\text { Yes } \\
\mathrm{C} \\
240 ?(\mathrm{cl} \\
\mathrm{d}) / 2\end{array}$ & $\begin{array}{c}\text { Yes } \\
\mathrm{C} \\
225 ?(\mathrm{cl} \\
\mathrm{d}) / 1\end{array}$ & $\begin{array}{c}\text { Yes } \\
\text { C } \\
280 ?(c / \\
\text { d) } / 1\end{array}$ & $\begin{array}{c}\text { Yes } \\
\text { C } \\
900 ?(c / \\
\text { d) } / 1\end{array}$ & $\begin{array}{c}\text { Yes } \\
C \\
1600 ?(c \\
I \\
\text { d) } / 1\end{array}$ & $\mid \begin{array}{c}Y e s \\
C \\
1428 ?(c \\
I \\
\text { d) } / 1\end{array}$ & $\begin{array}{c}\text { Yes } \\
\mathrm{C} \\
37000 ?(\mathrm{c} \\
1 \\
\text { d) } / 1\end{array}$ & $\begin{array}{c}\text { Yes } \\
\mathrm{C} \\
3800 ?(\mathrm{c} \\
/ \\
\text { d) } / 1\end{array}$ \\
\hline $\begin{array}{l}\text { TRIGA FL.IP } \\
\text { (Wash St) } \\
\text { (25) }\end{array}$ & $\begin{array}{c}\text { Yes } \\
\text { C } \\
\text { N/A } \\
\text { d }\end{array}$ & $\begin{array}{c}\text { Yes } \\
\text { B } \\
\text { N/A } \\
\text { d }\end{array}$ & $\begin{array}{c}\text { Yes } \\
C \\
\text { N/A } \\
d\end{array}$ & $\begin{array}{c}\text { Yes } \\
C \\
\text { N/A } \\
d\end{array}$ & $\begin{array}{c}\text { Yes } \\
C \\
\text { N/A } \\
d\end{array}$ & $\begin{array}{c}\text { Yes } \\
C \\
\text { N/A } \\
d\end{array}$ & $\begin{array}{c}\text { Yes } \\
\text { C } \\
\text { N/A } \\
d\end{array}$ & $\begin{array}{c}\text { Yes } \\
\text { C } \\
\text { N/A } \\
\text { d }\end{array}$ & $\begin{array}{c}\text { Yes } \\
\text { C } \\
\text { N/A } \\
d\end{array}$ & $\begin{array}{c}\text { Yes } \\
C \\
\text { N/A } \\
d\end{array}$ & $\begin{array}{c}\text { Yes } \\
\text { C } \\
\text { N/A } \\
d\end{array}$ & $\begin{array}{c}\text { Yes } \\
\text { C } \\
\text { N/A } \\
\text { d }\end{array}$ & $\begin{array}{c}\text { Yes } \\
C \\
N / A \\
d\end{array}$ & $\begin{array}{c}\text { Yes } \\
C \\
\text { N/A } \\
d\end{array}$ & $\begin{array}{c}\text { Yes } \\
C \\
\text { N/A } \\
d\end{array}$ & $\begin{array}{c}\text { Yes } \\
C \\
\text { N/A } \\
d\end{array}$ & $\begin{array}{c}\text { Yes } \\
\text { C } \\
\text { N/A } \\
d\end{array}$ & $\begin{array}{c}\text { Yes } \\
\text { C } \\
\text { N/A } \\
\text { d }\end{array}$ \\
\hline $\begin{array}{c}\text { TRIGA } \\
\text { (Penn St) } \\
(175)\end{array}$ & $\begin{array}{c}\text { Yes } \\
\mathrm{C} \\
30 ?(\mathrm{c} / \mathrm{d}) / \\
6\end{array}$ & $\left|\begin{array}{c}\text { Yes } \\
\mathrm{A} \\
38 ?(\mathrm{c} / \mathrm{d}) / 5\end{array}\right|$ & $\begin{array}{c}\text { Yes } \\
\text { C } \\
240 ?(\mathrm{c} / \\
\text { d) } / 1\end{array}$ & $\begin{array}{c}\text { Yes } \\
\text { C } \\
170 ?(\mathrm{c} / \\
\text { d) } / 2\end{array}$ & $\begin{array}{c}\text { Yes } \\
\text { C } \\
\text { N/A } \\
d\end{array}$ & $\begin{array}{c}\text { Yes } \\
C \\
50 ?(\mathrm{c} / \\
\text { d) } / 4\end{array}$ & $\begin{array}{c}\text { Yes } \\
\text { C } \\
150 ?(\mathrm{c} / \\
\text { d) } / 2\end{array}$ & $\begin{array}{c}\text { Yes } \\
\text { C } \\
120 ?(\mathrm{c} / \\
\text { d) } / 2\end{array}$ & $\begin{array}{c}\text { Yes } \\
\text { C } \\
150 ?(\mathrm{c} / \\
\text { d) } / 2\end{array}$ & $\begin{array}{c}\text { Yes } \\
\text { C } \\
120 ?(\mathrm{c} / \\
\text { d) } / 2\end{array}$ & $\begin{array}{c}\text { Yes } \\
\mathrm{C} \\
240 ?(\mathrm{cl} \\
\text { d) } 2\end{array}$ & $\begin{array}{c}\text { Yes } \\
\mathrm{C} \\
225 ?(\mathrm{~cd} \\
\mathrm{d}) / 1\end{array}$ & $\mid \begin{array}{c}\text { Yes } \\
C \\
280 ?(c / \\
\text { d) } / 1\end{array}$ & $\begin{array}{c}\text { Yes } \\
\text { C } \\
900 ?(c / \\
\text { d) } / 1\end{array}$ & $\begin{array}{c}\text { Yes } \\
C \\
1600 ?(c \\
I \\
\text { d) } / 1\end{array}$ & $\mid \begin{array}{c}\text { Yes } \\
\mathrm{C} \\
1428 ?(\mathrm{c} \\
/ \\
\mathrm{d}) / 1\end{array}$ & \begin{tabular}{|c|} 
Yes \\
$\mathrm{C}$ \\
$3700 ?(\mathrm{c}$ \\
1 \\
$\mathrm{~d}) / 1$
\end{tabular} & $\begin{array}{c}\text { Yes } \\
\text { C } \\
3800 ?(c \\
/ \\
\text { d) } / 1\end{array}$ \\
\hline $\begin{array}{c}\text { TRIGA } \\
\text { (U. Towas) } \\
\text { (126) }\end{array}$ & $\begin{array}{c}\text { Yes } \\
C \\
30 ?(c / d) / \\
5\end{array}$ & $\mid \begin{array}{c}Y e \\
\mathrm{~A} \\
38 ?(\mathrm{c} / \mathrm{d}) / 4\end{array}$ & $\begin{array}{c}\text { Yes } \\
C \\
240 ?(\mathrm{c} / \\
\text { d) } / 1\end{array}$ & $\begin{array}{c}\text { Yes } \\
\text { C } \\
170 ?(\mathrm{c} / \\
\text { d) } / 1\end{array}$ & $\begin{array}{c}\text { Yes } \\
\text { C } \\
\text { N/A } \\
\text { d }\end{array}$ & $\begin{array}{c}\text { Ycs } \\
C \\
50 ?(\mathrm{c} / \\
\text { d) } / 3\end{array}$ & $\begin{array}{c}\text { Yes } \\
\mathrm{C} \\
150 ?(\mathrm{c} / \\
\text { d) } / 1\end{array}$ & $\begin{array}{c}\text { Yes } \\
\text { C } \\
120 ?(\text { c/ } \\
\text { d) } / 2\end{array}$ & $\begin{array}{c}\text { Yes } \\
\text { C } \\
150 ?(c / \\
\text { d) } / 1\end{array}$ & $\begin{array}{c}\text { Yes } \\
\text { C } \\
120 ?(\mathrm{c} / \\
\text { d) } 2\end{array}$ & $\begin{array}{c}\text { Yes } \\
\mathrm{C} \\
240 ?(\mathrm{c} / \\
\mathrm{d}) / 2\end{array}$ & $\begin{array}{c}\text { Yes } \\
\mathrm{C} \\
225 ?(\mathrm{c} / \\
\mathrm{d}) / 1\end{array}$ & $\begin{array}{c}\text { Yes } \\
C \\
280 ?(\mathrm{cl} \\
\text { d) } / 1\end{array}$ & $\begin{array}{c}\text { Yes } \\
\mathrm{C} \\
900 ?(\mathrm{c} / \\
\text { d) } / 1\end{array}$ & $\begin{array}{c}\text { Yes } \\
C \\
1600 ?(c \\
I \\
\text { d) } / 1\end{array}$ & $\mid \begin{array}{c}Y c s \\
C \\
1428 ?(c \\
/ \\
\text { d) } / 1\end{array}$ & $\begin{array}{c}\text { Yes } \\
\text { C } \\
3700 ?(c \\
I \\
\text { d) } / 1\end{array}$ & $\begin{array}{c}\text { Yes } \\
C \\
3800 ?(c \\
/ \\
\text { d) } / 1\end{array}$ \\
\hline $\begin{array}{c}\text { TRIGA FLIP } \\
\text { (Tex. A\&MM) } \\
(186)\end{array}$ & $\begin{array}{c}\text { Yes } \\
C \\
30 ?(c / d) / \\
7\end{array}$ & $\begin{array}{c}\text { Yes } \\
\text { A } \\
19 ?(c / d) / 1 \\
0\end{array}$ & $\begin{array}{c}\text { Yes } \\
\text { C } \\
120 ? \\
(c / d) / 2\end{array}$ & $\begin{array}{c}\text { Yes } \\
\mathrm{C} \\
85 ?(\mathrm{c} / \mathrm{d}) / 3\end{array}$ & $\begin{array}{c}\text { Ycs } \\
\text { C } \\
\text { N/A } \\
d\end{array}$ & $\begin{array}{c}Y c s \\
C \\
25 ?(c / \\
\text { d) } / 8\end{array}$ & $\begin{array}{c}\text { Yes } \\
\text { C } \\
75 ?(c / \\
\text { d) } / 3\end{array}$ & $\begin{array}{c}\text { Yes } \\
\text { C } \\
60 ?(c / \\
d) / 4\end{array}$ & $\begin{array}{c}\text { Yes } \\
\text { C } \\
75 ?(\mathrm{c} / \\
\text { d) } / 3\end{array}$ & $\begin{array}{c}\text { Yes } \\
\text { C } \\
60 ?(c / \\
\text { d) } / 4\end{array}$ & $\begin{array}{c}\text { Yes } \\
C \\
120 ?(\mathrm{c} / \\
\text { d) } 2\end{array}$ & $\begin{array}{c}\text { Yes } \\
C \\
105 ?(c / \\
d) / 2\end{array}$ & $\begin{array}{c}\text { Yes } \\
C \\
140 ?(\mathrm{c} / \\
\text { d) } / 2\end{array}$ & $\begin{array}{c}\text { Yes } \\
\text { C } \\
450 ?(\mathrm{c} / \\
\text { d) } / 1\end{array}$ & $\begin{array}{c}\text { Yes } \\
\text { C } \\
800 ?(\mathrm{c} / \\
\text { d) } / 1\end{array}$ & $\mid \begin{array}{c}\text { Yes } \\
\mathrm{C} \\
714 ?(\mathrm{c} / \\
\mathrm{d}) / 1\end{array}$ & $\begin{array}{c}\text { Yes } \\
\mathrm{C} \\
1850 ?(\mathrm{c} \\
1 \\
\mathrm{~d}) / 1\end{array}$ & $\begin{array}{c}\text { Yes } \\
C \\
1900 ?(c \\
/ \\
\text { d) } / 1\end{array}$ \\
\hline $\begin{array}{c}\text { TRIGA } \\
\text { (U. Utah) } \\
(100)\end{array}$ & $\begin{array}{c}\text { Yes } \\
\text { C } \\
30 ?(\mathrm{c} / \mathrm{d}) / \\
4\end{array}$ & $\mid \begin{array}{c}\text { Yes } \\
\mathrm{A} \\
38 ?(\mathrm{c} / \mathrm{d}) / 3\end{array}$ & $\begin{array}{c}\text { Yes } \\
\text { C } \\
240 ?(\mathrm{c} / \\
\text { d) } / 1\end{array}$ & $\begin{array}{c}\text { Yes } \\
\text { C } \\
170 ?(c / \\
\text { d) } / 1\end{array}$ & $\begin{array}{c}\text { Yes } \\
\text { C } \\
\text { N/A } \\
\text { d }\end{array}$ & $\begin{array}{c}Y c \\
C \\
50 ?(c / \\
\text { d) } / 2\end{array}$ & $\begin{array}{c}\text { Yes } \\
\text { C } \\
150 ?(\mathrm{c} / \\
\text { d) } / 1\end{array}$ & $\begin{array}{c}\text { Yes } \\
\text { C } \\
120 ?(\mathrm{c} / \\
\text { d) } / 1\end{array}$ & $\begin{array}{c}\text { Yes } \\
\text { C } \\
150 ?(\mathrm{c} / \\
\text { d) } / 1\end{array}$ & $\begin{array}{c}\text { Yes } \\
\text { C } \\
120 ?(\mathrm{c} / \\
\text { d)/1 }\end{array}$ & $\begin{array}{c}\text { Yes } \\
\text { C } \\
240 ?(\mathrm{c} / \\
\text { d)/1 }\end{array}$ & $\begin{array}{c}\text { Yc } \\
\mathrm{C} \\
225 ?(\mathrm{c} / \\
\mathrm{d}) / 1\end{array}$ & $\begin{array}{c}\text { Yes } \\
C \\
280 ?(d / 1 \\
\text { d) } / 1\end{array}$ & $\begin{array}{c}\text { Yes } \\
\text { C } \\
900 ?(c / \\
\text { d) } / 1\end{array}$ & $\begin{array}{c}\text { Yc } \\
C \\
1600 ?(c \\
/ \\
\text { d) } / 1\end{array}$ & $\mid \begin{array}{c}Y c \\
C \\
1428 ?(c \\
/ \\
\text { d) } / 1\end{array}$ & $\begin{array}{c}\text { Yes } \\
\mathrm{C} \\
3700 ?(\mathrm{c} \\
1 \\
\text { d) } / 1\end{array}$ & $\begin{array}{c}\text { Yes } \\
\mathrm{C} \\
3800 ?(\mathrm{c} \\
/ \\
\text { d) } / 1\end{array}$ \\
\hline
\end{tabular}


Table 6-2. (continued).

\begin{tabular}{|c|c|c|c|c|c|c|c|c|c|c|c|c|c|c|c|c|c|c|}
\hline \multirow[b]{2}{*}{ DOE SNF } & \multicolumn{11}{|c|}{ Legal weight truck packages } & \multicolumn{2}{|c|}{$\begin{array}{l}\text { Overweight } \\
\text { truck packages }\end{array}$} & \multicolumn{5}{|c|}{ Rail packages } \\
\hline & $\begin{array}{l}\mathrm{GE} \\
\mathrm{T}-2\end{array}$ & BMI-1 & FSV-1 & NLI-1/2 & NLI-6502 & $\mathrm{T}-\mathbf{3}$ & NAC-1 & $\begin{array}{c}\text { CNS 1- } \\
13 G\end{array}$ & $\begin{array}{l}\text { NAC- } \\
\text { LWT }\end{array}$ & $\begin{array}{c}\text { GE Model } \\
2000\end{array}$ & $\begin{array}{l}\text { TN- } \\
\text { FSV }\end{array}$ & $\begin{array}{l}\text { TN-8 } \\
\text { TN-8L }\end{array}$ & TN-9 & IF-300 & $\begin{array}{l}\text { NLI- } \\
10 / 24\end{array}$ & 125-B & $\begin{array}{l}\text { TN- } \\
\text { BRP }\end{array}$ & $\begin{array}{l}\text { TN- } \\
\text { REG }\end{array}$ \\
\hline $\begin{array}{c}\text { TRIGA } \\
\text { (Kan. St) } \\
(105)\end{array}$ & $\begin{array}{c}\text { Yes } \\
C \\
30 ?(c / d) / \\
4\end{array}$ & $\mid \begin{array}{c}Y e s \\
A \\
38 ?(c / d) / 3\end{array}$ & $\begin{array}{c}\text { Yes } \\
\text { C } \\
240 ?(\mathrm{~d} / \\
\text { d) } / 1\end{array}$ & $\begin{array}{c}\text { Yes } \\
\text { C } \\
170 ?(\mathrm{c} / \\
\text { d) } / 1\end{array}$ & $\begin{array}{c}\text { Yes } \\
C \\
\text { N/A } \\
\text { d }\end{array}$ & $\begin{array}{c}\text { Yes } \\
C \\
50 ?(c / \\
\text { d) } / 3\end{array}$ & $\begin{array}{c}\text { Yes } \\
\text { C } \\
150 ?(\mathrm{c} / \\
\text { d) } / 1\end{array}$ & $\begin{array}{c}\text { Yes } \\
\text { C } \\
120 ?(\mathrm{c} / \\
\text { d) } / 1\end{array}$ & $\begin{array}{c}\text { Yes } \\
\text { C } \\
150 ?(\mathrm{c} / \\
\text { d) } / 1\end{array}$ & $\begin{array}{c}\text { Yes } \\
C \\
120 ?(\mathrm{c} / \\
\text { d) } / 1\end{array}$ & $\begin{array}{c}\text { Yes } \\
\text { C } \\
240 ?(c / \\
\text { d) } / 1\end{array}$ & $\begin{array}{c}\text { Yes } \\
C \\
225 ?(\mathrm{c} / \\
\text { d) } / 1\end{array}$ & $\begin{array}{c}\text { Yes } \\
\text { C } \\
280 ?(\mathrm{c} / \\
\text { d) } / 1\end{array}$ & $\begin{array}{c}\text { Yes } \\
\text { C } \\
900 ?(\mathrm{c} / \\
\text { d) } / 1\end{array}$ & $\left|\begin{array}{c}Y e s \\
C \\
1600 ?(c \\
I \\
d) / 1\end{array}\right|$ & $\begin{array}{c}\text { Yes } \\
\text { C } \\
1428 ?(\mathrm{c} \\
/ \\
\text { d) } / 1\end{array}$ & $\begin{array}{c}\text { Yes } \\
\mathrm{C} \\
3700 ?(\mathrm{c} \\
/ \\
\text { d) } / 1\end{array}$ & $\begin{array}{c}Y c \\
C \\
3800 ?(0 \\
1 \\
\text { d) } / 1\end{array}$ \\
\hline $\begin{array}{c}\text { TRIGA } \\
\text { (Corneli) } \\
(100)\end{array}$ & $\begin{array}{c}\text { Yes } \\
\text { C } \\
\text { N/A } \\
\text { c/d }\end{array}$ & $\begin{array}{c}\text { Yes } \\
B \\
\text { N/A } \\
\text { c/d }\end{array}$ & $\begin{array}{c}\text { Yes } \\
C \\
\text { N/A } \\
c / d\end{array}$ & $\begin{array}{c}\text { Yes } \\
\text { C } \\
\text { N/A } \\
c / d\end{array}$ & $\begin{array}{c}\text { Yes } \\
C \\
N / A \\
c / d\end{array}$ & $\begin{array}{c}\text { Yes } \\
C \\
\text { N/A } \\
\text { c/d }\end{array}$ & $\begin{array}{c}\text { Yes } \\
\text { C } \\
\text { N/A } \\
\text { c/d }\end{array}$ & $\begin{array}{c}\text { Yes } \\
C \\
N / A \\
c / d\end{array}$ & $\begin{array}{c}\text { Yes } \\
C \\
\text { N/A } \\
c / d\end{array}$ & $\begin{array}{c}\text { Yes } \\
C \\
\text { N/A } \\
\text { c/d }\end{array}$ & $\begin{array}{c}\text { Yes } \\
C \\
\text { N/A } \\
c / d\end{array}$ & $\begin{array}{c}\text { Yes } \\
C \\
\text { N/A } \\
c / d\end{array}$ & $\begin{array}{c}\text { Yes } \\
\text { C } \\
\text { N/A } \\
\text { c/d }\end{array}$ & $\begin{array}{c}\text { Yes } \\
C \\
\text { N/A } \\
\text { c/d }\end{array}$ & $\begin{array}{c}\text { Yes } \\
C \\
\text { N/A } \\
\text { c/d }\end{array}$ & $\begin{array}{c}\text { Yes } \\
C \\
\text { N/A } \\
c / d\end{array}$ & $\begin{array}{c}\text { Yos } \\
C \\
\text { N/A } \\
c / d\end{array}$ & $\begin{array}{c}\text { Yes } \\
C \\
\text { N/A } \\
c / d \\
\end{array}$ \\
\hline $\begin{array}{c}\text { TRIGA } \\
\text { (UC Irvine) } \\
\text { (113) }\end{array}$ & $\begin{array}{c}\text { Yes } \\
\mathrm{C} \\
30 ?(\mathrm{c} / \mathrm{d}) / \\
4\end{array}$ & $\left|\begin{array}{c}\text { Yes } \\
\mathrm{A} \\
38 ?(\mathrm{c} / \mathrm{d}) / 3\end{array}\right|$ & $\begin{array}{c}\text { Yes } \\
\text { C } \\
240 ?(\mathrm{c} / \\
\text { d) } / 1\end{array}$ & $\begin{array}{c}\text { Yes } \\
\mathrm{C} \\
170 ?(\mathrm{c} / \\
\mathrm{d}) / 1\end{array}$ & $\begin{array}{c}\text { Yes } \\
C \\
\text { N/A } \\
d\end{array}$ & $\begin{array}{c}\text { Yes } \\
\text { C } \\
50 ?(\mathrm{c} / \\
\text { d) } / 3\end{array}$ & $\begin{array}{c}\text { Yes } \\
\text { C } \\
150 ?(\mathrm{c} / \\
\text { d) } / 1\end{array}$ & $\begin{array}{c}\text { Yes } \\
\text { C } \\
120 ?(\mathrm{c} / \\
\text { d) } /\end{array}$ & $\begin{array}{c}\text { Yes } \\
C \\
150 ?(\mathrm{c} / \\
\text { d) } / 1\end{array}$ & $\begin{array}{c}\text { Yes } \\
\text { C } \\
120 ?(\mathrm{c} / \\
\text { d) } / 1\end{array}$ & $\begin{array}{c}\text { Yes } \\
C \\
240 ?(d \\
\text { d) } / 1\end{array}$ & $\begin{array}{c}\text { Yes } \\
\mathrm{C} \\
225 ?(\mathrm{c} / \\
\text { d) } / 1\end{array}$ & $\begin{array}{c}\text { Yes } \\
C \\
280 ?(\mathrm{c} / \\
\text { d) } / 1\end{array}$ & $\begin{array}{c}\text { Yes } \\
\text { C } \\
900 ?(\mathrm{c} / \\
\text { d) } / 1\end{array}$ & $\begin{array}{c}\text { Yes } \\
c \\
1600 ?(c \\
\prime \\
\text { d) } / 1\end{array}$ & $\begin{array}{c}\text { Yes } \\
C \\
1428 ?(c \\
/ \\
\text { d) } / 1\end{array}$ & $\begin{array}{c}\text { Yes } \\
\mathrm{C} \\
3700 ?(\mathrm{c} \\
/ \\
\text { d) } / 1\end{array}$ & $\begin{array}{c}\text { Yes } \\
C \\
3800 ?(\mathrm{c} \\
/ \\
\text { d) } / 1\end{array}$ \\
\hline $\begin{array}{c}\text { TRIGA } \\
\text { (U. Mrylnd) } \\
\text { (93) }\end{array}$ & $\begin{array}{c}\text { Yes } \\
\text { C } \\
30 ?(\mathrm{c} / \mathrm{d}) / \\
4\end{array}$ & $\left|\begin{array}{c}Y e s \\
A \\
38 ?(c / d) / 3\end{array}\right|$ & $\begin{array}{c}\text { Yes } \\
\text { C } \\
240 ?(\mathrm{~d} / \\
\text { d) } / 1\end{array}$ & $\begin{array}{c}\text { Yes } \\
\text { C } \\
170 ?(\mathrm{c} / \\
\text { d) } / 1\end{array}$ & $\begin{array}{c}\text { Yes } \\
\text { C } \\
\text { N/A } \\
d\end{array}$ & $\begin{array}{c}\text { Yes } \\
\text { C } \\
50 ?(\mathrm{c} / \\
\text { d) } / 2\end{array}$ & $\begin{array}{c}\text { Yes } \\
C \\
150 ?(\mathrm{c} / \\
\text { d) } / 1\end{array}$ & $\begin{array}{c}\text { Yes } \\
\text { C } \\
120 ?(\mathrm{c} / \\
\text { d) } / 1\end{array}$ & $\begin{array}{c}\text { Yes } \\
\text { C } \\
150 ?(\mathrm{c} / \\
\text { d) } / 1\end{array}$ & $\begin{array}{c}\text { Yes } \\
\text { C } \\
120 ?(\mathrm{c} / \\
\text { d) } / 1\end{array}$ & $\begin{array}{c}\text { Yes } \\
C \\
240 ?(d / \\
\text { d) } / 1\end{array}$ & $\begin{array}{c}\text { Yes } \\
\mathrm{C} \\
225 ?(\mathrm{c} / \\
\mathrm{d}) / 1\end{array}$ & $\begin{array}{c}\text { Yes } \\
C \\
280 ?(c / \\
d) / 1\end{array}$ & $\begin{array}{c}\text { Yes } \\
\text { C } \\
900 ?(\mathrm{cl} \\
\text { d) } / 1\end{array}$ & $\mid \begin{array}{c}Y c s \\
C \\
1600 ?(c \\
I \\
d) / 1\end{array}$ & $\begin{array}{c}Y e s \\
C \\
1428 ?(\mathrm{c} \\
/ \\
\text { d) } /\end{array}$ & $\begin{array}{c}\text { Yes } \\
C \\
3700 ? \text { ?c } \\
/ \\
\text { d) } / 1 \\
\end{array}$ & $\begin{array}{c}\text { Yes } \\
\text { C } \\
3800 ? \text { ?c } \\
1 \\
\text { d) } / 1\end{array}$ \\
\hline $\begin{array}{l}\text { TRIGA FLIP } \\
\text { (Ore. St.) } \\
\text { (85) }\end{array}$ & $\begin{array}{c}\text { Yes } \\
C \\
30 ?(c / d) / \\
3\end{array}$ & $\begin{array}{c}\text { Yes } \\
\mathrm{A} \\
19 ?(\mathrm{c} / \mathrm{d}) / 5\end{array}$ & $\begin{array}{c}\text { Yes } \\
\text { C } \\
120 ? \\
\text { (c/d)/1 }\end{array}$ & $\begin{array}{c}\text { Yes } \\
\mathrm{C} \\
85 ?(\mathrm{c} / \mathrm{d}) / 1\end{array}$ & $\begin{array}{c}\text { Yes } \\
\text { C } \\
\text { N/A } \\
d\end{array}$ & $\begin{array}{c}\text { Yes } \\
\text { C } \\
25 ?(\mathrm{c} / \\
\text { d) } / 4\end{array}$ & $\begin{array}{c}\text { Yes } \\
C \\
75 ?(c / \\
\text { d) } / 2\end{array}$ & $\begin{array}{c}\text { Yes } \\
\mathrm{C} \\
60 ?(\mathrm{c} / \\
\text { d) } 2\end{array}$ & $\begin{array}{c}\text { Yes } \\
C \\
75 ?(c / \\
\text { d) } / 2\end{array}$ & $\begin{array}{c}\text { Yes } \\
\text { C } \\
60 ?(c / \\
\text { d) } / 2\end{array}$ & $\begin{array}{c}\text { Yes } \\
\text { C } \\
120 ?(\mathrm{cl} \\
\text { d) } / 1\end{array}$ & $\begin{array}{c}\text { Yes } \\
C \\
105 ?(c / \\
\text { d)/1 }\end{array}$ & $\begin{array}{c}\text { Yes } \\
C \\
140 ?(c / \\
\text { d) } / 1\end{array}$ & $\begin{array}{c}\text { Yes } \\
\text { C } \\
450 ?(\mathrm{c} / \\
\text { d) } / 1\end{array}$ & $\mid \begin{array}{c}\text { Yes } \\
\mathrm{C} \\
800 ?(\mathrm{c} / \\
\mathrm{d}) / 1\end{array}$ & $\begin{array}{c}\text { Yes } \\
\text { C } \\
714 ?(\mathrm{~d} / \\
\mathrm{d}) / 1\end{array}$ & $\begin{array}{c}\text { Yes } \\
C \\
1850 ? \text { ?c } \\
/ \\
\text { d) } / 1 \\
\end{array}$ & \begin{tabular}{|c|} 
Yes \\
$C$ \\
$1900 ?(0)$ \\
1 \\
d) $/ 1$ \\
\end{tabular} \\
\hline $\begin{array}{c}\text { TRIGA } \\
\text { (Reod U.) } \\
(60)\end{array}$ & $\begin{array}{c}\text { Yes } \\
\text { C } \\
30 ?(\mathrm{c} / \mathrm{d}) / \\
2\end{array}$ & $\mid \begin{array}{c}Y c s \\
\mathrm{~A} \\
38 ?(\mathrm{c} / \mathrm{d}) / 2\end{array}$ & $\begin{array}{c}\text { Yes } \\
\text { C } \\
240 ?(d / \\
\text { d) } / 1\end{array}$ & $\begin{array}{c}\text { Yes } \\
\text { C } \\
170 ?(\mathrm{c} / \\
\text { d) } / 1\end{array}$ & $\begin{array}{c}\text { Yes } \\
\text { C } \\
\text { N/A } \\
d\end{array}$ & $\begin{array}{c}Y e s \\
C \\
50 ?(c / \\
\text { d) } / 2\end{array}$ & $\begin{array}{c}\text { Yes } \\
\text { C } \\
150 ?(\mathrm{c} / \\
\text { d) } / 1\end{array}$ & $\begin{array}{c}\text { Yes } \\
\text { C } \\
120 ?(\mathrm{c} / \\
\text { d) } / 1\end{array}$ & $\begin{array}{c}\text { Yes } \\
\text { C } \\
150 ?(\mathrm{c} / \\
\text { d) } / 1\end{array}$ & $\begin{array}{c}\text { Yes } \\
\text { C } \\
120 ?(\mathrm{c} / \\
\text { d) } / 2\end{array}$ & $\begin{array}{c}\text { Yes } \\
\text { C } \\
240 ?(\mathrm{c} / \\
\text { d) } / 1\end{array}$ & $\begin{array}{c}\text { Yes } \\
C \\
225 ?(\mathrm{c} / \\
\text { d) } / 1\end{array}$ & $\begin{array}{c}\text { Yes } \\
\text { C } \\
280 ?(c / \\
\text { d) } / 1\end{array}$ & $\begin{array}{c}\text { Yes } \\
\mathrm{C} \\
900 ?(\mathrm{c} / \\
\mathrm{d}) / 1\end{array}$ & $\begin{array}{c}\text { Yes } \\
C \\
1600 ?(c \\
/ \\
\text { d) } / 1\end{array}$ & $\begin{array}{c}\text { Yes } \\
\text { C } \\
1428 ?(\mathrm{c} \\
/ \\
\text { d) } / 1\end{array}$ & \begin{tabular}{|c|} 
Yes \\
C \\
$3700 ?(\mathrm{c}$ \\
$/$ \\
d) $/ 1$
\end{tabular} & $\begin{array}{c}Y e \\
C \\
3800 ?(0 \\
1 \\
d) / 1\end{array}$ \\
\hline
\end{tabular}


Table 6-2. (continued).

\begin{tabular}{|c|c|c|c|c|c|c|c|c|c|c|c|c|c|c|c|c|c|c|}
\hline \multirow[b]{2}{*}{ DOE SNF } & \multicolumn{11}{|c|}{ Legal weight truck packages } & \multicolumn{2}{|c|}{$\begin{array}{c}\text { Overweight } \\
\text { truck packages }\end{array}$} & \multicolumn{5}{|c|}{ Rail packages } \\
\hline & $\begin{array}{l}\mathrm{GE} \\
\mathrm{T}-2\end{array}$ & BMI-1 & FSV-1 & $\mathrm{NLI} \cdot 1 / 2$ & NLI-6502 & $\mathrm{T}-3$ & NAC-1 & $\begin{array}{c}\text { CNS 1- } \\
13 \mathrm{G}\end{array}$ & $\begin{array}{l}\text { NAC- } \\
\text { LWT }\end{array}$ & $\begin{array}{c}\text { GE Model } \\
2000\end{array}$ & $\begin{array}{l}\text { TN- } \\
\text { FSV }\end{array}$ & $\begin{array}{l}\text { TN-8 } \\
\text { TN-8L }\end{array}$ & TN-9 & IF-300 & $\begin{array}{l}\text { NLI- } \\
10 / 24\end{array}$ & $125-\mathrm{B}$ & $\begin{array}{l}\text { TN- } \\
\text { BRP }\end{array}$ & $\begin{array}{l}\text { TN- } \\
\text { REG }\end{array}$ \\
\hline $\begin{array}{c}\text { SNAP } \\
(19)\end{array}$ & $\begin{array}{c}\text { Yes } \\
\text { C } \\
\text { N/A } \\
\text { c }\end{array}$ & $\begin{array}{c}\text { Yes } \\
\text { C } \\
\text { N/A } \\
c\end{array}$ & $\begin{array}{c}\text { Yes } \\
\text { C } \\
\text { N/A } \\
\text { c }\end{array}$ & $\begin{array}{c}\text { Yes } \\
C \\
N / A \\
c\end{array}$ & $\begin{array}{c}\text { Yes } \\
C \\
N / A \\
c / d\end{array}$ & $\begin{array}{c}\text { Ye } \\
C \\
\text { N/A } \\
c\end{array}$ & $\begin{array}{c}\text { Yes } \\
\text { C } \\
\text { N/A } \\
\text { c }\end{array}$ & $\begin{array}{c}\text { Yes } \\
C \\
\text { N/A } \\
c\end{array}$ & $\begin{array}{c}\text { Yes } \\
C \\
\text { N/A } \\
\text { c }\end{array}$ & $\begin{array}{c}\text { Yes } \\
\text { C } \\
\text { N/A } \\
\text { c }\end{array}$ & $\begin{array}{c}\text { Yes } \\
C \\
\text { N/A } \\
c\end{array}$ & $\begin{array}{c}\text { Yes } \\
\text { C } \\
\text { N/A } \\
\text { c } \\
\end{array}$ & $\begin{array}{c}\text { Yes } \\
\text { C } \\
\text { N/A } \\
\text { c } \\
\end{array}$ & $\begin{array}{c}\text { Yes } \\
\text { C } \\
\text { N/A } \\
c\end{array}$ & $\begin{array}{c}\text { Yes } \\
\text { C } \\
\text { N/A } \\
\text { c } \\
\end{array}$ & $\begin{array}{c}\text { Yes } \\
\text { C } \\
\text { N/A } \\
\text { c } \\
\end{array}$ & $\begin{array}{c}\text { Yes } \\
\text { C } \\
\text { N/A } \\
\text { c } \\
\end{array}$ & $\begin{array}{c}\text { Yes } \\
C \\
\text { N/A } \\
\text { c }\end{array}$ \\
\hline $\begin{array}{c}\text { ATR Ass'y } \\
\text { (INEL } \\
\text { CPP-603) } \\
(109)\end{array}$ & $\begin{array}{c}\text { No } \\
\text { c }\end{array}$ & $\begin{array}{c}\mathrm{N} / \mathrm{A} \\
\mathrm{c}\end{array}$ & $\begin{array}{c}\text { Yes } \\
\text { C } \\
6 / 19\end{array}$ & $\begin{array}{c}\text { N/A } \\
b\end{array}$ & $\begin{array}{c}\text { Yes } \\
\text { C } \\
4 ?(d) / 28\end{array}$ & $\begin{array}{c}\text { Yes } \\
\text { C } \\
2 / 55\end{array}$ & $\begin{array}{c}\text { Yes } \\
\text { C } \\
\text { 1/Aarge }\end{array}$ & $\begin{array}{l}\text { No } \\
\text { c }\end{array}$ & $\begin{array}{l}\text { Yes } \\
\text { C } \\
4 / 28\end{array}$ & $\begin{array}{l}\text { No } \\
\text { c }\end{array}$ & $\begin{array}{l}\text { No } \\
\text { c }\end{array}$ & $\begin{array}{l}\text { Yes } \\
\text { C } \\
18 \pi\end{array}$ & $\begin{array}{c}\text { Yes } \\
\text { C } \\
21 / 6\end{array}$ & $\begin{array}{c}\text { Yes } \\
\text { C } \\
22 / 5\end{array}$ & $\begin{array}{l}\text { Yes } \\
\text { C } \\
88 / 2\end{array}$ & $\begin{array}{c}\text { Yes } \\
\text { C } \\
\text { 1/narge }\end{array}$ & $\begin{array}{c}\text { Yes } \\
\text { C } \\
5 / 22\end{array}$ & $\begin{array}{c}\text { Yes } \\
\text { C } \\
5 / 22\end{array}$ \\
\hline $\begin{array}{c}\text { ATR ASs'y } \\
\text { (INEU/ } \\
\text { CPP-666) } \\
(785)\end{array}$ & $\begin{array}{l}\text { No } \\
\text { c }\end{array}$ & $\begin{array}{c}\mathrm{N} / \mathrm{A} \\
\mathrm{c}\end{array}$ & $\begin{array}{c}\text { Yes } \\
\text { C } \\
6 / 13\end{array}$ & $\begin{array}{c}\text { Yes } \\
\text { C } \\
\text { 1/harge }\end{array}$ & $\begin{array}{c}\text { Yos } \\
\text { C } \\
4 ?(d) / \\
\text { large }\end{array}$ & $\begin{array}{c}\text { Yea } \\
\text { C } \\
\text { 2/arge }\end{array}$ & $\begin{array}{c}\text { Yes? } \\
\text { C } \\
\text { 1/narge }\end{array}$ & $\begin{array}{l}\text { No } \\
\text { c }\end{array}$ & $\begin{array}{c}\text { Yes } \\
\text { C } \\
\text { 4/arge }\end{array}$ & $\begin{array}{l}\text { No } \\
\text { c }\end{array}$ & $\mid \begin{array}{c}\text { Yes } \\
\mathrm{C} \\
\text { G/arge }\end{array}$ & $\begin{array}{c}\text { Yex } \\
\text { C } \\
18 / 44\end{array}$ & $\begin{array}{c}\text { Yes } \\
\text { C } \\
21 / 38\end{array}$ & $\begin{array}{c}\text { Yes } \\
\text { C } \\
22 / 36\end{array}$ & $\begin{array}{c}\text { Yes } \\
\text { C } \\
88 / 9\end{array}$ & \begin{tabular}{|c|} 
Yes \\
C \\
1/large
\end{tabular} & $\begin{array}{c}\text { Yes } \\
\text { C } \\
s / 22\end{array}$ & $\begin{array}{c}\text { Yes } \\
\text { C } \\
5 / 22\end{array}$ \\
\hline $\begin{array}{c}\text { HFIR } \\
(43)\end{array}$ & $\begin{array}{c}\text { No } \\
\text { a }\end{array}$ & $\begin{array}{c}\text { No } \\
\text { a }\end{array}$ & $\begin{array}{c}\text { No } \\
\text { a }\end{array}$ & $\begin{array}{c}\text { No } \\
\text { a }\end{array}$ & $\begin{array}{c}\text { No } \\
\text { a }\end{array}$ & $\begin{array}{c}\text { No } \\
\text { a }\end{array}$ & $\begin{array}{c}\text { No } \\
\text { a }\end{array}$ & $\begin{array}{c}\text { Yes } \\
\text { C } \\
1 / 43\end{array}$ & $\begin{array}{c}\text { No } \\
\text { a }\end{array}$ & $\begin{array}{c}\text { Yes } \\
\text { A } \\
1 / 43\end{array}$ & $\begin{array}{c}\text { No } \\
\text { a }\end{array}$ & $\begin{array}{c}\text { No } \\
\text { a }\end{array}$ & $\begin{array}{c}\text { No } \\
\text { a }\end{array}$ & $\begin{array}{c}\text { Yes } \\
\text { C } \\
10 / 5\end{array}$ & $\begin{array}{c}\text { Yes } \\
\text { C } \\
10 / 5\end{array}$ & $\begin{array}{c}\text { Yes } \\
\text { C } \\
3 / 15\end{array}$ & $\begin{array}{c}\text { Yes } \\
\text { C } \\
14 / 4\end{array}$ & $\begin{array}{c}\text { Yes } \\
\text { C } \\
13 / 4\end{array}$ \\
\hline $\begin{array}{l}\text { ARMF (Plates) } \\
\quad(15)\end{array}$ & $\begin{array}{c}\text { Yes? } \\
C \\
>15 ?(\mathrm{c}) / 1\end{array}$ & $\left|\begin{array}{c}\text { Yes? } \\
C \\
>15 ?(c) / 1\end{array}\right|$ & $\begin{array}{c}\text { Yes? } \\
\mathrm{C} \\
>15 ?(\mathrm{c}) / 1\end{array}$ & $\begin{array}{c}\text { Yes? } \\
C \\
>15 ?(\mathbf{c}) / 1\end{array}$ & $\begin{array}{c}\text { Yes? } \\
C \\
>15 ? \\
(\mathrm{c} / \mathrm{d}) / 1\end{array}$ & $\begin{array}{l}\text { Yca? } \\
\text { C } \\
>15 ? \\
(c) / 1 \\
\end{array}$ & $\begin{array}{l}\text { Yes? } \\
\text { C } \\
>15 ? \\
\text { (c) } / 1 \\
\end{array}$ & $\begin{array}{l}\text { Yes? } \\
\text { C } \\
>15 ? \\
\text { (c) } / 1\end{array}$ & $\begin{array}{c}\text { Yes? } \\
\text { C } \\
>15 ? \\
\text { (c) } / 1\end{array}$ & $\left|\begin{array}{c}\text { Yes? } \\
\mathrm{C} \\
>15 ?(\mathrm{c}) / 1\end{array}\right|$ & $\begin{array}{c}\text { Yes? } \\
C \\
>15 ? \\
(c) / 1 \\
\end{array}$ & $\begin{array}{c}\text { Yes? } \\
\text { C } \\
>15 ? \\
\text { (c) } / 1 \\
\end{array}$ & $\begin{array}{c}\text { No } \\
\text { a }\end{array}$ & $\begin{array}{c}\text { Yeat } \\
\mathrm{C} \\
>15 ?(\mathrm{c}) / \\
1\end{array}$ & $\begin{array}{c}\text { Yea? } \\
\mathrm{C} \\
>15 ?(\mathrm{c} \\
\mathrm{x} / 1 \\
\end{array}$ & $\begin{array}{c}\text { Yes? } \\
\mathrm{C} \\
>15 ?(\mathrm{c} \\
\mathrm{y} / 1 \\
\end{array}$ & $\begin{array}{c}\text { Yes? } \\
\mathrm{C} \\
>15 ?(\mathrm{c} \\
\mathrm{y} / 1 \\
\end{array}$ & $\begin{array}{c}\text { Yes? } \\
\mathrm{C} \\
>15 ?(\mathrm{c} \\
\mathrm{T} / 1 \\
\end{array}$ \\
\hline $\begin{array}{c}\text { ARMF/ } \\
\text { CRFMF } \\
\text { (Ekement) } \\
(58)\end{array}$ & $\begin{array}{c}\text { Yes? } \\
\text { C } \\
\text { N/A } \\
\text { d }\end{array}$ & $\begin{array}{c}\text { Yes? } \\
\mathrm{C} \\
\mathrm{N} / \mathrm{A} \\
\mathrm{c} / \mathrm{d}\end{array}$ & $\begin{array}{c}\text { Yes? } \\
\text { C } \\
\text { N/A } \\
\text { d } \\
\end{array}$ & $\begin{array}{c}\text { Ycs? } \\
\text { C } \\
\text { N/A } \\
d\end{array}$ & $\begin{array}{c}\text { Yes? } \\
\text { C } \\
\text { N/A } \\
d\end{array}$ & $\begin{array}{c}\text { Yes? } \\
\text { C } \\
\text { N/A } \\
d\end{array}$ & $\begin{array}{c}\text { Yes? } \\
\text { C } \\
\text { N/A } \\
d\end{array}$ & $\begin{array}{c}\text { Yes? } \\
\text { C } \\
\text { N/A } \\
d\end{array}$ & $\begin{array}{c}\text { Yes? } \\
\text { C } \\
\text { N/A } \\
d\end{array}$ & $\begin{array}{c}\text { Yes? } \\
\text { C } \\
\text { N/A } \\
\text { d }\end{array}$ & $\begin{array}{c}\text { Yes? } \\
\text { C } \\
\text { N/A } \\
\text { d } \\
\end{array}$ & $\begin{array}{c}\text { Yes? } \\
\text { C } \\
\text { N/A } \\
\text { d }\end{array}$ & $\begin{array}{c}\text { Yes? } \\
\text { C } \\
\text { N/A } \\
d\end{array}$ & $\begin{array}{c}\text { Yes? } \\
\text { C } \\
\text { N/A } \\
\text { d }\end{array}$ & $\begin{array}{c}\text { Yes? } \\
\text { C } \\
\text { N/A } \\
d\end{array}$ & $\begin{array}{c}\text { Yes? } \\
\text { C } \\
\text { N/A } \\
d\end{array}$ & $\begin{array}{c}\text { Yes? } \\
\text { C } \\
\text { N/A } \\
\text { d }\end{array}$ & $\begin{array}{c}\text { Yes? } \\
\text { C } \\
\text { N/A } \\
\text { d }\end{array}$ \\
\hline $\begin{array}{c}\text { ARMF/CRFMF } \\
\text { (Unknown) } \\
(8)\end{array}$ & $\begin{array}{c}\text { Yes? } \\
\text { C } \\
\text { N/A } \\
\text { d }\end{array}$ & $\begin{array}{c}\text { Yes? } \\
C \\
\text { N/A } \\
c / d\end{array}$ & $\begin{array}{c}\text { Yes? } \\
\text { C } \\
\text { N/A } \\
\text { d }\end{array}$ & $\begin{array}{c}\text { Yes? } \\
\text { C } \\
\text { N/A } \\
\text { d }\end{array}$ & $\begin{array}{c}\text { Yes? } \\
\text { C } \\
\text { N/A } \\
\text { d }\end{array}$ & $\begin{array}{c}\text { Yex? } \\
\text { C } \\
\text { N/A } \\
\text { d } \\
\end{array}$ & $\begin{array}{c}\text { Yes? } \\
\text { C } \\
\text { N/A } \\
\text { d }\end{array}$ & $\begin{array}{c}\text { Yes? } \\
\text { C } \\
\text { N/A } \\
\text { d }\end{array}$ & $\begin{array}{c}\text { Yes? } \\
\text { C } \\
\text { N/A } \\
\text { d } \\
\end{array}$ & $\begin{array}{c}\text { Yes? } \\
\text { C } \\
\text { N/A } \\
\text { d }\end{array}$ & $\begin{array}{c}\text { Yes? } \\
\text { C } \\
\text { N/A } \\
\text { d } \\
\end{array}$ & $\begin{array}{c}\text { Yes? } \\
\text { C } \\
\text { N/A } \\
\text { d } \\
\end{array}$ & $\begin{array}{c}\text { Yes? } \\
\text { C } \\
\text { N/A } \\
\text { d }\end{array}$ & $\begin{array}{c}\text { Yes? } \\
\text { C } \\
\text { N/A } \\
\text { d }\end{array}$ & $\begin{array}{c}\text { Yes? } \\
\text { C } \\
\text { N/A } \\
\text { d }\end{array}$ & $\begin{array}{c}\text { Yes? } \\
\text { C } \\
\text { N/A } \\
d\end{array}$ & $\begin{array}{c}\text { Yes? } \\
\text { C } \\
\text { N/A } \\
\text { d }\end{array}$ & $\begin{array}{c}\text { Yes? } \\
\text { C } \\
\text { N/A } \\
\text { d }\end{array}$ \\
\hline $\begin{array}{l}\text { ARMF/CRFMF } \\
\text { (Insert) } \\
(4)\end{array}$ & $\begin{array}{c}\text { Yes? } \\
\text { C } \\
\text { N/A } \\
\text { d }\end{array}$ & $\begin{array}{c}\text { Yes? } \\
\text { C } \\
\text { N/A } \\
\text { c/d }\end{array}$ & $\begin{array}{c}\text { Yes? } \\
\text { C } \\
\text { N/A } \\
\text { d }\end{array}$ & $\begin{array}{c}\text { Yes? } \\
\text { C } \\
\text { N/A } \\
\text { d }\end{array}$ & $\begin{array}{c}\text { Yes? } \\
\text { C } \\
\text { N/A } \\
\text { d }\end{array}$ & $\begin{array}{c}\text { Yes? } \\
\text { C } \\
\text { N/A } \\
\text { d }\end{array}$ & $\begin{array}{c}\text { Yes? } \\
\text { C } \\
\text { N/A } \\
d\end{array}$ & $\begin{array}{c}\text { Yes? } \\
\text { C } \\
\text { N/A } \\
\text { d }\end{array}$ & $\begin{array}{c}\text { Yes? } \\
\text { C } \\
\text { N/A } \\
\text { d }\end{array}$ & $\begin{array}{c}\text { Yes? } \\
\text { C } \\
\text { N/A } \\
\text { d }\end{array}$ & $\begin{array}{c}\text { Yas? } \\
\text { C } \\
\text { N/A } \\
\text { d }\end{array}$ & $\begin{array}{c}\text { Yes? } \\
\text { C } \\
\text { N/A } \\
\text { d }\end{array}$ & $\begin{array}{c}\text { Yes? } \\
\text { C } \\
\text { N/A } \\
\text { d }\end{array}$ & $\begin{array}{c}\text { Yes? } \\
\text { C } \\
\text { N/A } \\
\text { d }\end{array}$ & $\begin{array}{c}\text { Yes? } \\
\text { C } \\
\text { N/A } \\
\text { d }\end{array}$ & $\begin{array}{c}\text { Yes? } \\
\text { C } \\
\text { N/A } \\
\text { d }\end{array}$ & $\begin{array}{c}\text { Yes? } \\
\text { C } \\
\text { N/A } \\
\text { d }\end{array}$ & $\begin{array}{c}\text { Yes? } \\
\text { C } \\
\text { N/A } \\
\text { d }\end{array}$ \\
\hline
\end{tabular}




\begin{tabular}{|c|c|c|c|c|c|c|c|c|c|}
\hline \multirow{5}{*}{ 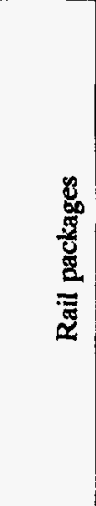 } & 望品 & શ્ર & $\frac{1}{z} 0$ & 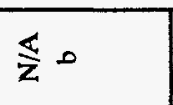 & $\frac{1}{z} 0$ & & ڤ్ర & క & ○० హ్ \\
\hline & 忘管 & $\searrow 0 \overline{8}$ & $\underline{z}$ & $\frac{\widehat{s}}{z} 0$ & $\frac{\pi}{z} 0$ & & 낭 & ఎ & 인 \\
\hline & 安 & 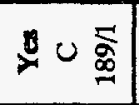 & $\frac{\varsigma}{z} 0$ & $\underline{z}$ & $\frac{\widehat{s}}{\mathrm{z}}$ & & 串 & ○一 & 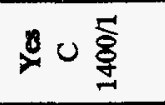 \\
\hline & 芸至 & ○一 蛋 & $\widehat{s} 0$ & 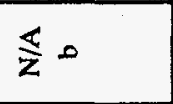 & 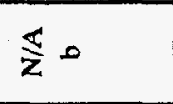 & & ๖્ર & ৩ & 닗 \\
\hline & $\begin{array}{l}\text { \&्లે } \\
\text { 至 }\end{array}$ & 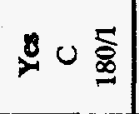 & $\frac{s}{z} 0$ & $\frac{S}{z} 0$ & $\frac{S}{z} 0$ & & 》0 & \ن & ๑ \\
\hline \multirow{2}{*}{ 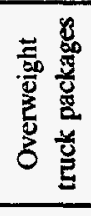 } & i & ㄴำ & $\frac{1}{z} 0$ & $\frac{s}{z} 0$ & $\overleftarrow{z} 0$ & & 시 & 싱 & ט \\
\hline & 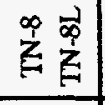 & $\searrow 0$ 雨 & $\frac{S}{z} 0$ & $\frac{1}{z} 0$ & $\frac{\pi}{z} 0$ & & 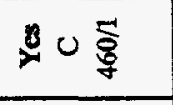 & 只 & 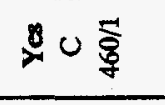 \\
\hline \multirow{11}{*}{ 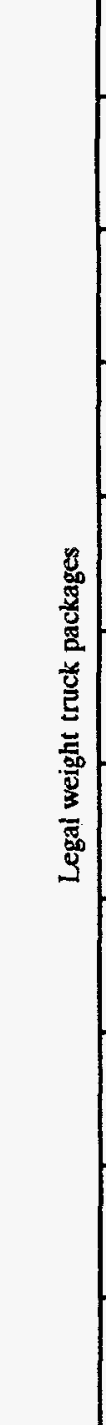 } & 忘勇 & ఇ & $\leqslant 0$ & $\frac{\delta}{z}$ & $\frac{\$}{z}$ & & Q & 일 & ఇ \\
\hline & 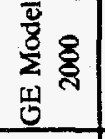 & $\otimes \cup$ & $\widehat{\Sigma} 0$ & $\widehat{s}$ & $\frac{\pi}{z} 0$ & & 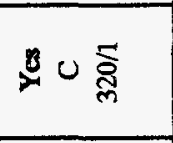 & 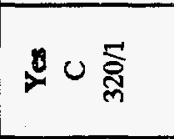 & 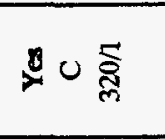 \\
\hline & 安忽 & 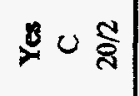 & $\leqslant 0$ & $\frac{5}{z} 0$ & $\frac{\pi}{2}$ & & 805 & $\stackrel{B}{\circ} \stackrel{n}{\varrho}$ & $20 \leqq$ \\
\hline & $\dot{\vec{c}} 0$ & $\forall 05$ & $\frac{5}{z}$ & $\frac{s}{z} 0$ & $\frac{1}{z}$ & & ৩ & ○० & ๑ \\
\hline & $\begin{array}{l}\bar{u} \\
\dot{z}\end{array}$ & ถั & $\frac{S}{z}$ & $\underline{\Sigma}$ & $\frac{\pi}{z} 0$ & & $8 \infty$ & $\stackrel{\theta}{x} \infty \stackrel{\circ}{g}$ & 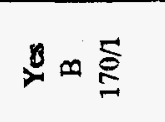 \\
\hline & $\stackrel{m}{H}$ & $20 \div$ & $\frac{5}{z} 0$ & $\frac{5}{z} 0$ & $\frac{5}{z}=$ & & 닝 & 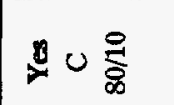 & $\nexists 0$ \\
\hline & 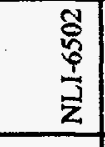 & 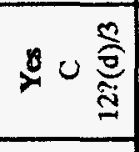 & $\widehat{z}$ & $\frac{\pi}{z}$ & $\frac{\pi}{z} 0$ & & 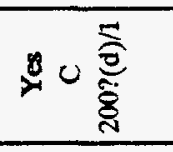 & 刃्र & 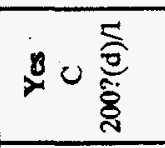 \\
\hline & $\underset{\mathrm{a}}{\mathrm{a}}$ & 80 ำ & $\frac{\varsigma}{z} 0$ & $\frac{5}{z} 0$ & $\frac{\pi}{z} 0$ & & $80 \underset{7}{5}$ & $80 \underset{్}{\sigma}$ & $80 \underset{7}{5}$ \\
\hline & 窟 & 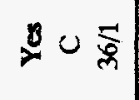 & $\frac{5}{z}=$ & $\frac{5}{z} 0$ & $\frac{3}{z}$ & & ¿ & ๖u & 구윯 \\
\hline & $\overline{\bar{\Sigma}}$ & 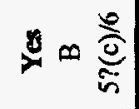 & $g<\leqslant \frac{5}{\sigma}$ & $g<\leq \frac{5}{2}$ & $g<\frac{5}{z} g$ & & $\mathscr{B} 0 \frac{5}{z} 0$ & $\frac{x}{x} \cup \frac{5}{z} 0$ & $80 \leq$ \\
\hline & 罟 & 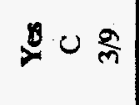 & $\frac{5}{z}=$ & $\widehat{\Sigma}$ & $\frac{\widehat{s}}{z}$ & & 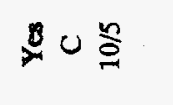 & $\stackrel{0}{x} 0 \stackrel{\circ}{0}$ & 舟U \\
\hline & 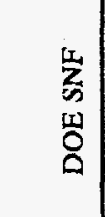 & $\begin{array}{l}\text { 戛 } \\
3 \\
\dot{3}\end{array}$ & 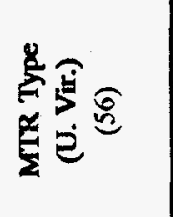 & 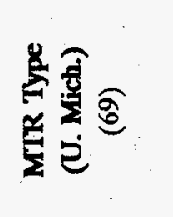 & 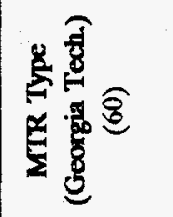 & 送 & 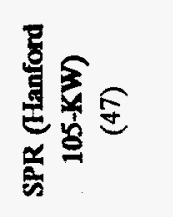 & 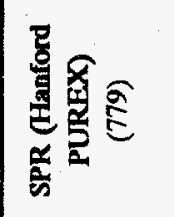 & 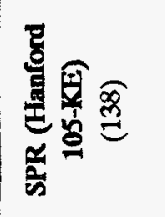 \\
\hline
\end{tabular}


Table 6-2. (continued).

\begin{tabular}{|c|c|c|c|c|c|c|c|c|c|c|c|c|c|c|c|c|c|c|}
\hline \multirow[b]{2}{*}{ DOE SNF } & \multicolumn{11}{|c|}{ Legal weight truck packages } & \multicolumn{2}{|c|}{$\begin{array}{c}\text { Overweight } \\
\text { truck packages }\end{array}$} & \multicolumn{5}{|c|}{ Rail packages } \\
\hline & $\begin{array}{l}\mathrm{GE} \\
\mathrm{T}-2\end{array}$ & BMI-1 & FSV-1 & NLI-1/2 & NLI-6502 & $\mathrm{T}-3$ & NAC-1 & $\begin{array}{c}\text { CNS 1- } \\
13 G\end{array}$ & $\begin{array}{l}\text { NAC- } \\
\text { LWT }\end{array}$ & $\begin{array}{c}\text { GE Model } \\
2000\end{array}$ & $\begin{array}{l}\text { TN- } \\
\text { FSV }\end{array}$ & $\begin{array}{r}\text { TN-8 } \\
\text { TN-8L }\end{array}$ & TN-9 & IF-300 & $\begin{array}{l}\text { NLI- } \\
10 / 24\end{array}$ & 125-B & $\begin{array}{l}\text { TN- } \\
\text { BRP }\end{array}$ & $\begin{array}{l}\text { TN- } \\
\text { REG }\end{array}$ \\
\hline $\begin{array}{c}\text { N-Reactor } \\
\text { (Hanford } \\
\text { 105-KW) } \\
(52959)\end{array}$ & $\begin{array}{c}\text { Yes } \\
\mathrm{C} \\
\text { 9/arge }\end{array}$ & $\begin{array}{c}\text { Yes } \\
\mathrm{C} \\
\mathrm{N} / \mathrm{A} \\
\mathrm{C}\end{array}$ & $\begin{array}{c}\text { Yes } \\
\text { C } \\
\text { 70/large }\end{array}$ & $\begin{array}{c}\text { Yes } \\
\text { C } \\
\text { 32/large }\end{array}$ & $\begin{array}{c}\text { Yes } \\
\text { C } \\
22 ?(d) / \\
\text { large }\end{array}$ & $\begin{array}{c}\text { Yes } \\
\text { C } \\
\text { 18/arge }\end{array}$ & $\begin{array}{c}\text { Yes } \\
\text { B } \\
60 / \text { arge }\end{array}$ & $\begin{array}{c}\text { Yes } \\
\text { C } \\
\text { 85/large }\end{array}$ & $\begin{array}{c}\text { Yes } \\
\text { C } \\
\text { 60/large }\end{array}$ & $\begin{array}{c}\text { Yes } \\
\text { C } \\
\text { 85/large }\end{array}$ & $\begin{array}{c}\text { Yes } \\
\mathrm{C} \\
70 / \text { large }\end{array}$ & $\begin{array}{c}\text { Yes } \\
\text { C } \\
108 / \\
\text { large }\end{array}$ & $\begin{array}{c}\text { Yes } \\
\mathrm{C} \\
84 / \\
\text { large }\end{array}$ & $\begin{array}{c}\text { Yes } \\
\text { C } \\
528 / 101\end{array}$ & $\begin{array}{c}\text { Yes } \\
\mathrm{C} \\
708 / 75\end{array}$ & $\begin{array}{c}\text { Yes } \\
C \\
147 / \\
\text { large }\end{array}$ & $\begin{array}{c}\text { Yes } \\
C \\
1620 / 33\end{array}$ & $\begin{array}{c}\text { Yes } \\
\text { C } \\
2140 / 25\end{array}$ \\
\hline $\begin{array}{c}\text { N-Reactor } \\
\text { (Hanford 105-KE) } \\
(50683)\end{array}$ & $\begin{array}{c}\text { Yes } \\
\text { C } \\
\text { 9/large }\end{array}$ & $\begin{array}{c}\text { Yes } \\
\mathrm{C} \\
\mathrm{N} / \mathrm{A} \\
\mathrm{c}\end{array}$ & $\begin{array}{c}\text { Ya } \\
C \\
\text { 70/large }\end{array}$ & $\begin{array}{c}\text { Yes } \\
\text { C } \\
\text { 32/large }\end{array}$ & $\begin{array}{c}\text { Yes } \\
\text { C } \\
22 ?(d) / \\
\text { large }\end{array}$ & $\begin{array}{c}\text { Yes } \\
\mathrm{C} \\
\text { 18/large }\end{array}$ & $\begin{array}{c}\text { Yes } \\
\text { B } \\
\text { 60/large }\end{array}$ & $\begin{array}{c}\text { Yes } \\
\text { C } \\
\text { 85/large }\end{array}$ & $\begin{array}{c}\text { Yes } \\
C \\
\text { 60/large }\end{array}$ & $\begin{array}{c}\text { Yes } \\
C \\
85 / \text { large }\end{array}$ & $\begin{array}{c}\text { Yes } \\
\text { C } \\
70 \text { /arge }\end{array}$ & $\begin{array}{c}\text { Yes } \\
\text { C } \\
108 / \\
\text { large }\end{array}$ & $\begin{array}{c}\text { Yes } \\
C \\
84 / \\
\text { large }\end{array}$ & $\begin{array}{c}\text { Yes } \\
\text { C } \\
528 / 96\end{array}$ & $\begin{array}{c}\text { Yes } \\
C \\
708 / 72\end{array}$ & $\begin{array}{c}\text { Yes } \\
C \\
147 / \\
\text { large }\end{array}$ & $\begin{array}{c}\text { Yes } \\
\mathrm{C} \\
1620 / 32\end{array}$ & $\begin{array}{c}\text { Yes } \\
\mathrm{C} \\
2140 / 24\end{array}$ \\
\hline $\begin{array}{l}\text { N Reactor } \\
\text { (Hanford } \\
\text { PUREX) } \\
(19)\end{array}$ & $\begin{array}{c}\text { Yes } \\
C \\
9 / 3\end{array}$ & $\begin{array}{c}\text { Yes } \\
C \\
N / A \\
c\end{array}$ & $\begin{array}{c}\text { Yes } \\
C \\
70 / 1\end{array}$ & $\begin{array}{c}\text { Yes } \\
\text { C } \\
32 / 1\end{array}$ & $\begin{array}{c}\text { Yes } \\
C \\
22 ?(d) / 1\end{array}$ & $\begin{array}{c}\text { Yes } \\
\text { C } \\
18 / 2\end{array}$ & $\begin{array}{c}\text { Yes } \\
\text { B } \\
60 / 1\end{array}$ & $\begin{array}{c}\text { Yes } \\
C \\
85 / 1\end{array}$ & $\begin{array}{c}\text { Yes } \\
C \\
60 / 1\end{array}$ & $\begin{array}{c}\text { Yes } \\
\text { C } \\
85 / 1\end{array}$ & $\begin{array}{c}\text { Yes } \\
\text { C } \\
70 / 1\end{array}$ & $\begin{array}{c}\text { Yes } \\
C \\
108 / 1\end{array}$ & $\begin{array}{c}\text { Yes } \\
\text { C } \\
84 / 1\end{array}$ & $\begin{array}{c}\text { Yes } \\
\text { C } \\
528 / 1\end{array}$ & $\begin{array}{c}\text { Yes } \\
\text { C } \\
708 / 1\end{array}$ & $\begin{array}{c}\text { Yes } \\
\text { C } \\
147 / 1\end{array}$ & $\begin{array}{c}\text { Yes } \\
C \\
1620 / 1\end{array}$ & $\begin{array}{c}\text { Yes } \\
\text { C } \\
2140 / 1\end{array}$ \\
\hline $\begin{array}{l}\text { Big Rock Point } \\
\text { (85) }\end{array}$ & $\begin{array}{c}\text { No } \\
\text { a }\end{array}$ & $\begin{array}{l}\text { No } \\
\text { a }\end{array}$ & $\begin{array}{c}\text { Yes } \\
C \\
2 / 43\end{array}$ & $\begin{array}{c}\text { Yes } \\
\text { C } \\
2 / 43\end{array}$ & $\begin{array}{c}\text { Yes } \\
C \\
1 ?(d) / 85\end{array}$ & $\begin{array}{c}\text { No } \\
\text { a }\end{array}$ & $\begin{array}{c}\text { Yes } \\
\text { C } \\
2 / 43\end{array}$ & $\begin{array}{c}\text { No } \\
\text { a }\end{array}$ & $\begin{array}{c}\text { Yes } \\
\text { C } \\
2 / 43\end{array}$ & $\begin{array}{c}\text { No } \\
\text { a }\end{array}$ & $\begin{array}{c}\text { Yes } \\
\text { C } \\
2 / 43\end{array}$ & $\begin{array}{c}\text { Yes } \\
\text { C } \\
6 / 15\end{array}$ & $\begin{array}{l}\text { No } \\
\text { a }\end{array}$ & $\begin{array}{c}\text { Yes } \\
\text { C } \\
21 / 5\end{array}$ & $\begin{array}{c}\text { Yes } \\
\text { C } \\
28 / 4\end{array}$ & $\begin{array}{c}\text { Yes } \\
\text { C } \\
14 / 6\end{array}$ & $\begin{array}{c}\text { Yes } \\
\text { A } \\
62 / 2\end{array}$ & $\begin{array}{c}\text { Yes } \\
\text { C } \\
54 / 2\end{array}$ \\
\hline $\begin{array}{c}\text { TREAT } \\
(390)\end{array}$ & $\begin{array}{l}\text { No } \\
\text { a }\end{array}$ & $\begin{array}{c}\text { No } \\
\text { a }\end{array}$ & $\begin{array}{c}\text { Yes } \\
\text { C } \\
\text { N/A } \\
\text { d }\end{array}$ & $\begin{array}{c}\text { Yes } \\
\text { C } \\
\text { N/A } \\
\text { d }\end{array}$ & $\begin{array}{c}\text { Yes } \\
C \\
\text { N/A } \\
d\end{array}$ & $\begin{array}{c}\text { Yes } \\
\text { C } \\
\text { N/A } \\
d\end{array}$ & $\begin{array}{c}\text { Yes } \\
C \\
\text { N/A } \\
d\end{array}$ & $\begin{array}{c}\text { No } \\
\text { a }\end{array}$ & $\begin{array}{c}\text { Yes } \\
\text { C } \\
\text { N/A } \\
d\end{array}$ & $\begin{array}{c}\text { No } \\
\text { a }\end{array}$ & $\begin{array}{c}\text { Yes } \\
\text { C } \\
\text { N/A } \\
\text { d }\end{array}$ & $\begin{array}{c}\text { Yes } \\
C \\
\text { N/A } \\
d\end{array}$ & $\begin{array}{c}\text { Yes } \\
C \\
\text { N/A } \\
d\end{array}$ & $\begin{array}{c}\text { Yes } \\
C \\
\text { N/A } \\
d\end{array}$ & $\begin{array}{c}\text { Yes } \\
C \\
\text { N/A } \\
d\end{array}$ & $\begin{array}{c}\text { Yes } \\
\text { C } \\
\text { N/A } \\
\text { d }\end{array}$ & $\begin{array}{c}\text { Yes } \\
C \\
\text { N/A } \\
d\end{array}$ & $\begin{array}{c}\text { Yex } \\
\text { C } \\
\text { N/A } \\
d\end{array}$ \\
\hline
\end{tabular}




\section{Legend:}

Yes: Fuel/package matchup is anticipated with a relatively high degree of confidence.

Yes?: Fuel/package matchup is anticipated with a lower degree of confidence.

No: Fuel/package matchup is not anticipated to be feasible with any reasonable package modifications.

N/A: Insufficient data exist to predict matchup with any degree of confidence.

a: Matchup is not feasible because of dimensional discrepancies (No), or matchup cannot be evaluated because available fuel dimensional data are insufficient to make a determination with any degree of confidence (N/A).

b: Matchup is not feasible because of shielding requirements (No), or matchup cannot be evaluated because available fuel radiological data are insufficient to make a determination with any degree of confidence (N/A).

c: Matchup is not feasible because of thermal limitations (No), or matchup cannot be evaluated because available fuel thermal data are insufficient to make a determination with any degree of confidence (N/A).

d: Matchup is not feasible because of payload weight restrictions (No), or matchup cannot be evaluated because available fuel weight data are insufficient to make a determination with any degree of confidence (N/A).

e: Payload quantities cannot be estimated because total fuel quantity data are not available.

A: No package modifications are expected to be required (see Section 4.1)

B: Package Certificate of Compliance amendment probably required (see Section 4.2)

C: $\quad$ Package SARP and Certificate of Compliance revisions probably required (see Section 4.3)

Number of fuel units per payload and total number of shipments for the particular fuel inventory are given for each potential package/fuel matchup below the "Yes" or "Yes?" as $\mathrm{x} / \mathrm{y}$, where "x" represents units per payload and " $\mathrm{y}$ " represents total number of shipments. When the total number of shipments exceeds 100 , "y" is simply given as "large." Where sufficient information exists to postulate a package/fuel matchup, but not enough to estimate specific payload capacities, an "N/A" is given instead. If sufficient information is not directly available to determine payload capacities, but adequate indirect means exist (comparison to other, similar fuels, etc.), the payload estimate is followed by a question mark (?) and a lowercase letter indicating the area of uncertainty (see letters "a" through " $\mathrm{e}$ " above). Total fuel units for each fuel type are given in parentheses below the fuel type name (column 1), where specific units are stated in Tables 2-1 through 3-6. If total number of units of a particular fuel is not known, "N/A" is given in parentheses below the fuel type name. 
Notes:

1. Only coarse allowance has been made in payload capacity estimates for payload support structure, such as baskets (see Section 4.4). In general, except for direct (level "A") fuel/package matchups, it can be expected that new internal support structures of some kind will have to be designed and qualified for almost all payloads. The significance of this assumption cannot be overemphasized. It essentially entails a complete relicensing effort for the affected packages. For packages that might be capable of transporting more than one fuel type, this will involve either a more complicated qualification effort (licensing for multiple payloads), or relicensing the cask for each individual payload type.

2. Only coarse allowance has been made for criticality control in payload capacity estimates. Consequently, reductions in payload capacity, either through the introduction of neutron poisoning material, or reduction in the quantity of fuel transported per shipment, may occur after final criticality analyses for the SNF have been performed.

3. No estimates have been made for payload capacities using revised package designs (see Section 4.5). Too many uncertainties exist to allow such estimates to be made with any degree of confidence.

4. Except for the 125-B, all packages listed provide a single level of containment only. No allowance has been made for fuels with cladding whose integrity may have been compromised. Such fuels will probably require double containment transportation systems. In this case, either the 125-B must be used, if possible, or another level of containment (canister) must be incorporated in some other package. No allowance for such additional containment structure has been made in estimates of payload capacity.

5. Packages with limited capacity (GE-100 and NRBK-41) have not been included in the summary. Likewise, naval fuels and packages (M-130, M-140 and M-160) have not been included because sufficient information is not available to make payload capacity estimates for them with any degree of confidence. 


\subsection{Conclusions}

Key conclusions resulting from the study are summarized below.

- Much of DOE's spent nuclear fuel (approximately one-third of all SNF types) appears to be transportable in existing shipping packages. However, most of this fuel will require that extensive additional effort be made before shipments can commence. Significant amounts of time and resources will be required for most SNF shipping campaigns due to the following:

- Most campaigns utilizing existing packages will require changes to licensing documents, hardware, or facilities. In general, these changes are expected to be extensive in terms of time and resources.

- The majority of fuels require significantly enhanced or improved fuel characterization before compatibility with existing packages can be properly and completely assessed.

- Compatibility with existing packages does not appear to exist for many DOEowned fuels. Thus, completely new transportation systems will likely be required if all DOE fuel is to eventually be shipped in licensed packages.

- $\quad$ Although the total quantity of DOE-owned SNF is far less than the quantity of commercial SNF, total cost on a per-MTHM basis is expected to be significantly greater for transport of DOE fuels than commercial fuels (due to the wide variety of fuel types within the DOE system and the small quantities that exist at many individual storage facilities).

- Extensive public involvement during the entire process will be an absolute necessity in a successful completion of the transportation objectives.

- Coordination of the SNF characterization and transportation efforts should be accomplished by a centralized materials management and disposition program office with oversight authority in all pertinent DOE facilities.

- Planning should be founded on a global assessment of transportation economics. Greater initial effort and expenditure will likely result in long-term savings (fewer required total fuel shipments). Less initial effort will like result in a greater long-term expenditures. The balance between these two extremes must be carefully determined in order to produce the greatest programmatic efficiency. For example, completely new transportation system designs should be considered when fuel quantities are relatively large and use of existing packages is found to require a large number of inefficient shipments. While the initial outlay will be greater, resulting shipping efficiencies may provide an overall long-term economic advantage.

- DOE prioritization of SNF shipments and identification of desired and required schedules is needed early on to ensure optimized planning for all shipping campaigns as 
a whole. In those cases where current storage facilities are adequate, continued storage in such facilities or use of administratively controlled onsite transfers to such facilities (using nonlicensed packages) should be considered as potential alternatives to immediate shipment in licensed packages. Fuels in less-than-adequate storage facilities should be given the highest priority for transportation planing.

More detailed discussions of these conclusions follow.

The primary conclusion from this study is that a relatively large quantity, but not all, of DOE-owned spent nuclear fuel can likely be transported in existing, currently licensed, irradiated fuel shipping packages. It is estimated that 77 of the approximately 230 fuels listed in Reference 2-1 might potentially be accommodated by existing packages. These fuels represent approximately $34 \%$ of all the various fuel types, and about $87.5 \%$ of the total heavy metal in the DOE-owned SNF inventory. Therefore, of all the different fuel types in the DOE-owned SNF inventory, approximately two-thirds either do not have a packaging matchup, or there were not sufficient fuel characteristics data available to make a transportation assessment. Of the remaining one-third of the fuel types, $16 \%$ appear to have at least one compatible package as-is. Another $12 \%$ could potentially have at least one compatible package if minor Certificate of Compliance changes are made and a new basket assembly is designed and qualified, as necessary. The final $6 \%$ could potentially have at least one compatible package if extensive changes are made to the safety analysis report for packaging and the Certificate of Compliance, and a new basket assembly is designed and qualified, as necessary. In addition, it is possible that additional design changes to existing packages, other than new basket designs, could be undertaken to allow additional fuel types to be transported. However, due to the complexity and interrelated nature of the many factors involved in packaging design, no estimates have been made in this study for such a level of packaging-related modification. Additionally, such an undertaking would almost inevitably entail resource and schedule requirements similar to those which would be required to qualify and entirely new package design.

The above estimates are based on data engineering judgments that indicate, for many categories of fuels, geometries, weights, decay heat loads and shielding needs appear to fall within the capability of one or more of the various existing shipping packages (see Tables 4-1 through 4-6). Potential criticality concerns have not been specifically addressed by this study, since criticality assessments involve complex and detailed analyses, which themselves call for detailed fuel-related characteristics data that are largely not currently available. Instead, it has been assumed that criticality concerns for any specific fuel type can eventually be addressed by appropriately limiting the quantity of fuel per shipment, maintaining positive post-accident payload configuration control, or incorporating neutron poisoning materials. Ultimately, more detailed criticality assessments for such fuels will probably result in a need for a greater number of shipments than has been estimated in this report. In most cases, however, it is unlikely that complete elimination of an otherwise compatible fuel/package combination would result.

Although shipment in existing packages is considered possible for many spent fuel categories, timelines and costs associated with implementation of most shipments will be significant. With only a few exceptions (i.e., the specific fuel/package matchups that currently exist), changes to current licensing documents (SARPs and Certificates of Compliance) will be required at a minimum, prior to the start of actual shipping campaigns. For the existing fuel/package matchups, 
it is reasonable to expect that at least three months of planning, coordinating, and system acquisition activities will be required prior to initial shipments taking place.

For those fuels for which there is no specific transportation package matchup, a significant amount of resource expenditure is likely to be required in order to sufficiently characterize the SNF for purposes of adequately determining transportation package needs. In some cases, this effort may consist only of compiling existing records into an appropriately formatted, centralized data base. An example would be the Savannah River RBOF, for which comprehensive inventory data currently exist. At the other end of the spectrum, the Hanford K-Basin fuels will require a greater amount of detailed classification in terms of fuel age and condition before an efficient transportation campaign can be planned. It has been estimated that, given a reasonable amount of priority, a minimum of five years will be required to sufficiently characterize the entire inventory of noncommercial DOE-owned SNF. Obviously, transportation planning need not wait for the completion of the entire characterization effort. As characteristics for individual fuel types are determined, detailed transportation for those fuel types can commence.

Presently there appears to be little communication between the various fuel storage facilities regarding the definitions and eventual dispositions of their SNF inventories. For a reasonable transportation plan to be developed, greater facility coordination should be implemented. To this end, a materials management and disposition program should be initiated for purposes of detailed fuel characterization and transportation planning.

Once the SNF is adequately characterized and transportation planning is completed, detailed transport package modifications can commence, as required. If relatively simple and straightforward SARP and Certificate of Compliance modifications are required, initiation of shipments for a given fuel will realistically take a minimum of six months, even if the particular shipping campaign has high priority within the DOE system and with the DOE or NRC regulatory body having jurisdiction. In addition, depending on the actual characteristics of any specific fuel to be transported and the operational constraints imposed at the fuel's current storage location or ultimate receiving facility, much more extensive activities may be required prior to implementation of shipments. Potential activities range from development of new fuel baskets or repackaging of fuels to packaging or facility modifications to satisfaction of political and public concerns. Shipments requiring such hardware-related activities or those that are politically sensitive and publicly visible can be expected to require two or more years prior to initial shipment.

For certain fuel types or categories, it is expected that entirely new transportation package systems will actually be required if shipment is to occur in a licensed shipping package. It is also noted that even though use of existing packages is viable in many cases, such an approach may not always be the most cost-effective solution. If large quantities of fuel requiring multiple shipments are to be transported (e.g., N-Reactor fuel), and priorities and needs are such that upfront schedule delays are acceptable, it will likely be more cost effective to develop an entirely new, reasonably optimized transportation system. For these reasons, any detailed planning activities associated with management of DOE-owned SNF should include provisions for the development of at least one new transportation package system designed specifically for DOEowned SNF. Development of such a system should, however, be deferred until after all DOEowned SNF has been properly characterized, shipping priorities initially set, and detailed facility constraints appropriately factored in. At that time, a detailed specification identifying required and 
desired package system attributes can be developed, which will ensure a cost-effective approach to dealing with DOE-owned fuels. With a design specification in hand, a new transport system development effort will likely require three or more years prior to initial shipments of SNF.

In order to ensure an optimally designed new package transportation system (or optimal use of an existing system), fuel groups or fuel characteristics to be accommodated must be carefully selected. Attempting to accommodate too large of a group of fuels in a specific package will either lead to an over-constrained set of design requirements and hence, no transport capability, or result in an inefficient system for many shipping campaigns. Conversely, too few fuels in a category, although possibly being more efficient for a given shipping campaign, will likely require multiple packages to be developed. Consequently, once fuel characteristics are clearly understood and shipping priorities set, a proper balance will need to be made between a "one package does all" approach with its potential inefficiencies for a given fuel type and a "one package per fuel type" approach, which will require far more development and licensing effort. The HFIR cask development effort, which would have accommodated a subset of DOE fuels, serves as a good "lessons learned" example. The request for proposal for that development effort was issued in May of 1986. To date, the subsequent development effort has been unsuccessful and has apparently been abandoned. The problems associated with that program must be avoided if successful transportation of DOE-owned SNF in licensed packages is to become a reality.

Additionally, although the total quantity of DOE-owned SNF is small compared to the quantity of commercial SNF, it must be realized that costs per MTHM for shipping DOE-owned SNF can be expected to greatly exceed corresponding costs for shipping commercial fuel. This is due to the wide variety of fuel types within the DOE system and the small quantities that exist at many different facilities. The wide variety of fuels will lead to a need for numerous different shipping campaigns and the small quantities per facility will lead to a need for many shipments per campaign. This rather large mix of fuels and facilities again points to the need for thorough and complete fuel characterization efforts and carefully established shipping priorities as early on steps in preparation for transport of DOE-owned SNF.

Last, though by no means least, the importance of public involvement throughout the entire planning and operation of the SNF transportation program cannot be overemphasized. Public resistance to large-scale movements of highly radioactive material will be enormous. Accordingly, the resources expended to overcome this resistance must be equally great.

Based on the above discussions, it is apparent that transport of DOE-owned SNF will not be a simple or rapidly completed activity. A tremendous amount of time and resources will be required. Consequently, continued storage in current facilities or use of administratively controlled onsite transfers to alternate facilities using nonlicensed packages should be considered as potential near-term alternatives to shipment in licensed packages.

\subsection{Recommended Actions}

Based on the study results documented and discussed herein, the following recommendations are made. Ideally, these actions would be carried out in the order presented. However, if shipping priorities so dictate, many of the actions could be carried out in parallel, with an increased risk that certain shipping campaigns could become less efficient or delayed due to conflicts with the 
needs of the higher priority campaigns. At a minimum, an initial effort to address items 1 through 3 should be performed prior to completion of the remaining items.

1. In parallel with Items 2 and 3 below, DOE should prioritize shipping campaigns based on considerations such as the following. Note that significant funding and supporting studies may be required to adequately address these items.
a. Fuel condition
b. Fuel quantity at a given storage location
c. Storage facility condition
d. Political considerations.

2. Continue with the various fuels characterization efforts currently under way at many of the DOE sites, and complete the characterization of all DOE-owned spent nuclear fuel that must eventually be transported. This is a vital step, since packaging requirements directly relate to payload characteristics, and proper packagings can only be selected or developed once payloads are fully understood. In addition, past experiences indicate that successful licensing activities have always required well-understood and wellcharacterized payload definitions. Specific sub-steps include the following.

a. Correct inconsistencies between various reference documents, fill in the many unknowns currently identified in the data bases, identify special problems or restrictions associated with each fuel, etc. Note that prior characterization efforts were often based on voluntary, unfunded inputs. Significant funding may be required to adequately obtain all necessary data.

b. DOE should provide sufficient guidance to each site to ensure that all efforts are properly prioritized, coordinated, and consistently carried out. It appears that in many cases, fuels characterization activities being performed at one site are not visible to other sites.

3. The initial matrix of compatible fuels and packagings presented in this report should be revisited and corrected or expanded following, or in parallel with, completion of item 2. The potential use of new packages now under development, such as the multipurpose container (MPC) for commercial fuels (Request for Proposal issued June 1994, award expected in early 1995), the Office of Civilian Radioactive Waste Managementdeveloped (but not yet licensed) GA 4/9 package, etc., should also be more closely investigated for potential application to DOE-owned SNF.

4. Based on the first three items and available funding, a detailed implementation strategy and desired and required shipping schedule should be developed. As part of the strategy, consideration should be given to continued storage at current facilities or possible onsite transfers to appropriate facilities in other than licensed packages. Keeping in mind that an optimized solution for one fuel type may be rather inefficient 
for another type, this is also an appropriate time to revisit fuel categories or groups, the intent being to optimize transportation solutions for all fuels as a whole. A detailed assessment of the benefits of one or more entirely new package design development efforts geared specifically at DOE-owned SNF should also be included once governing characteristics for all fuels are fully understood. If a new system appears beneficial, it would be "speced out" at this time.

5. Select two or three specific fuels or fuel groups for detailed studies based on shipping priorities and the item 4 implementation strategy. Include the highest priority, the largest quantity, or one of the more difficult to address fuel groups. Include fuel at two or more sites, and shipping campaigns that involve both NRC and DOE certification activities, if possible.

6. Perform detailed paper studies for dealing with the selected fuel types, discuss details of intended design and licensing approaches in face-to-face meetings with the regulators, and document results for DOE consideration. These more detailed studies would include more rigorous structural, thermal, shielding or criticality analyses as appropriate to establish allowed fuel quantities per shipment, etc.

7. Fully implement study results for highest priority shipment including SARP and hardware modifications, certification activities, and actual transport.

8. Again considering shipping priorities, and applying lessons learned from the detailed studies of previously selected fuel types, refine or develop appropriate action plans for all remaining fuel groups.

9. Implement action plans for all remaining fuels.

As a final note, throughout this entire process, the need for public involvement must not be overlooked or underestimated. An overall public outreach strategy must be devised early on in the program. Large-scale efforts to inform and educate the public will be required continuously throughout the duration of the SNF shipping campaign. These educational programs should incorporate not only discussion but also demonstrations of actual program-related hardware, with an emphasis on the large degree of safety incorporated into its development and operation. Without such a public relations effort, no degree of technical effort will be sufficient to ensure a successful transportation program. 


\section{REFERENCES}

1. Idaho National Engineering Laboratory, Spent Fuel Background Report, Volumes I and II, EGGM-11249, March 1994.

2. U.S. Department of Energy, Characteristics of Potential Repository Wastes, Volumes 1-4, DOE/RW-0184-R1, July 1992.

3. K. H. Bergsman, Hanford Irradiated Fuel Inventory Baseline, WHC-SD-CP-TI-175, Rev. 1, January 1993.

4. U.S. Department of Energy, Spent Fuel Working Group Report, Volume I, November 1993.

5. Code of Federal Regulations, 10 CFR 71, "Packaging and Transportation of Radioactive Material," Office of the Federal Register, proposed rule, June 8, 1988.

6. U.S. Department of Energy, Radioactive Materials Packages Database (RAMPAC), maintained by Analysas Corporation for the DOE's Transportation and Packaging Safety Division.

7. U.S. Nuclear Regulatory Commission, Directory of Certificates of Compliance for Radioactive Materials Packages, NUREG-0383, Volume 2, Revision 16, NRC Office of Nuclear Material Safety and Safeguards, October 1993.

8. U.S. Department of Energy, Directory of Certificates of Compliance for Radioactive Materials Packaging, DOE Transportation and Packaging Safety Division, April 26, 1994.

9. M. J. Monthey, Engineering Study of the Transfer of Irradiated Fuels on the Hanford Site, WHC-SD-TP-ES-001, Rev 0, April 20, 1993.

10. U.S. Department of Energy, DOE Order 5480.3, "Safety Requirements for the Packaging and Transportation of Hazardous Materials, Hazardous Substances, and Hazardous Wastes."

11. Code of Federal Regulations, 49 CFR 173, "Shippers-General Requirements for Shipments and Packagings," Office of the Federal Register, October 1993.

12. T. L. Sanders et al., A Method for Determining the Spent-Fuel Contribution to Transport Cask Containment Requirements, SAND90-2406/TTC-1019/UC-820, November 1992.

13. American National Standards Institute for Radioactive Materials, ANSI N14.5-1987, "Leakage Tests on Packages for Shipment," 1987.

14. H. W. Reno et al., "Transporting Spent and Damaged Fuel in the United States: Recent Experience and Lessons Learned Related to the Evolving Transportation Policy of the U.S. Department of Energy," Proceedings of the 9th International Symposium on the Packaging and Transportation of Radioactive Materials (PATRAM), CONF-890631 (Vol 2), pp. 648-653, June 11-16, 1989. 
15. U.S. Department of Energy, History Summary of the Three-Mile Island Unit 2 Core Debris Transportation Campaign, DOE/ID-10400, March 1993. 


\section{Appendix A}

Fuel Data Sheets and Design Drawings/Sketches 
This appendix provides available drawings of U.S. Department of Energy spent nuclear fuel. The data are drawn from References 1 through 4. 


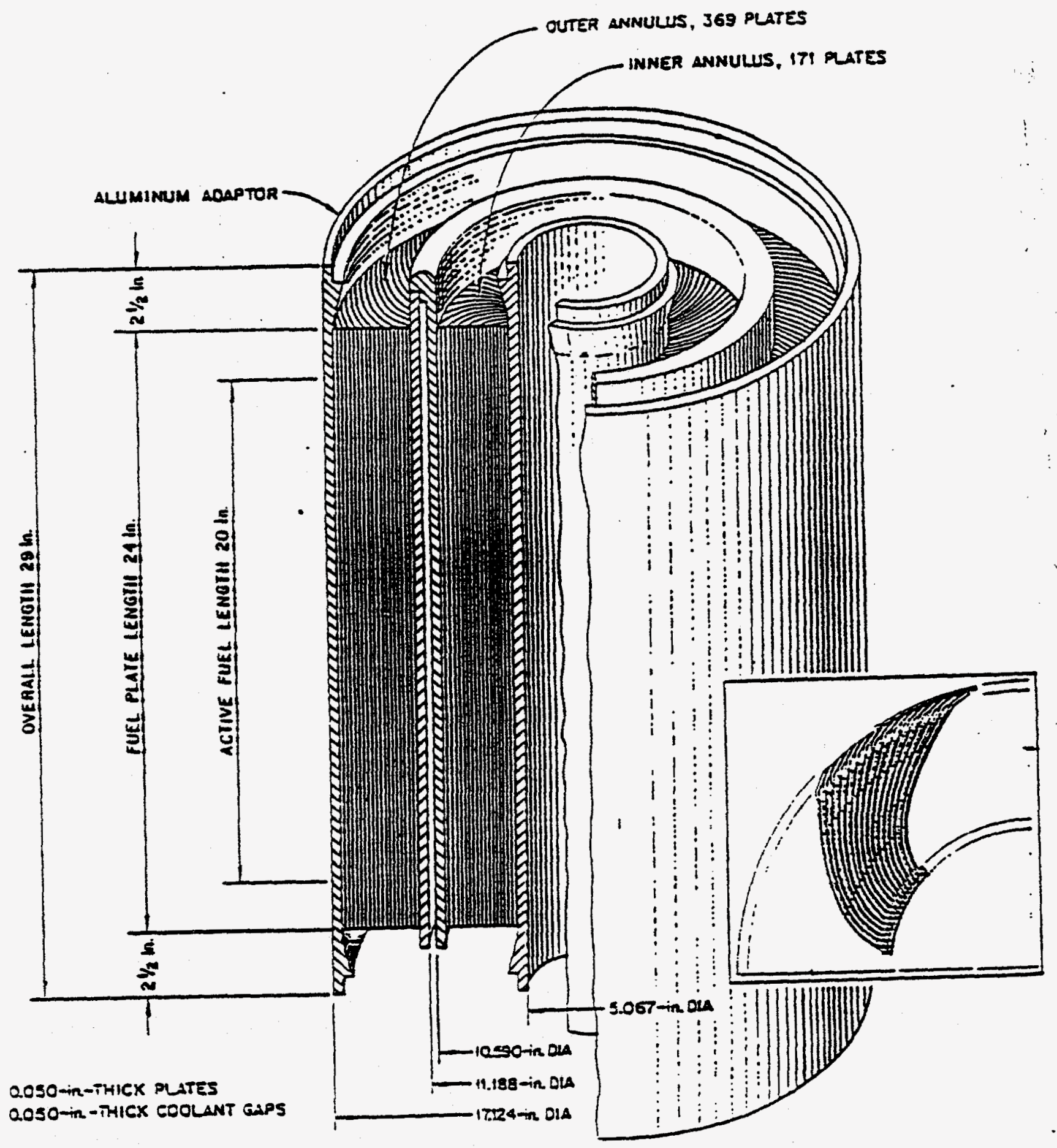

Dinmsienal Illuswertes of the Lstembiod HFIR Fuel Eloment. 


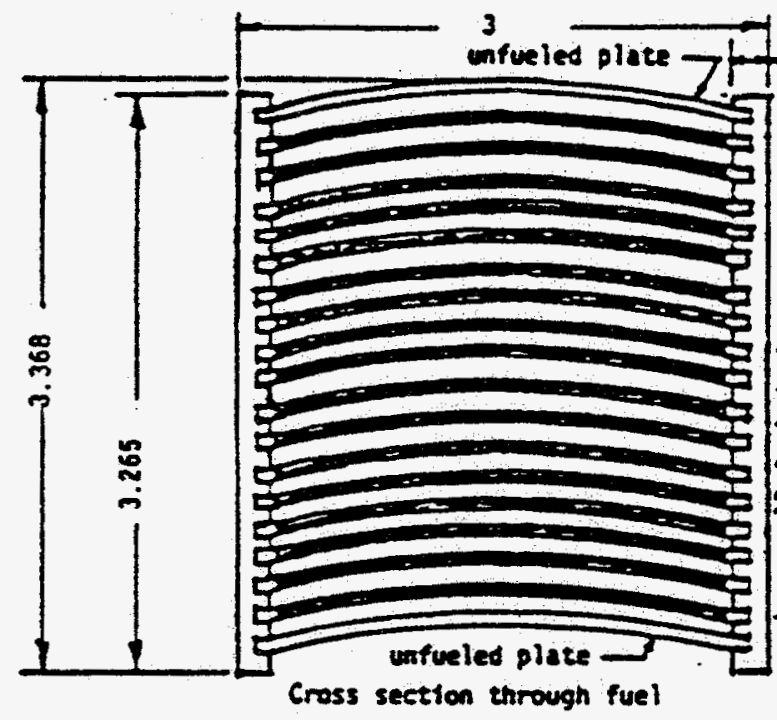

.188 erp.

sounting plate

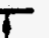

1

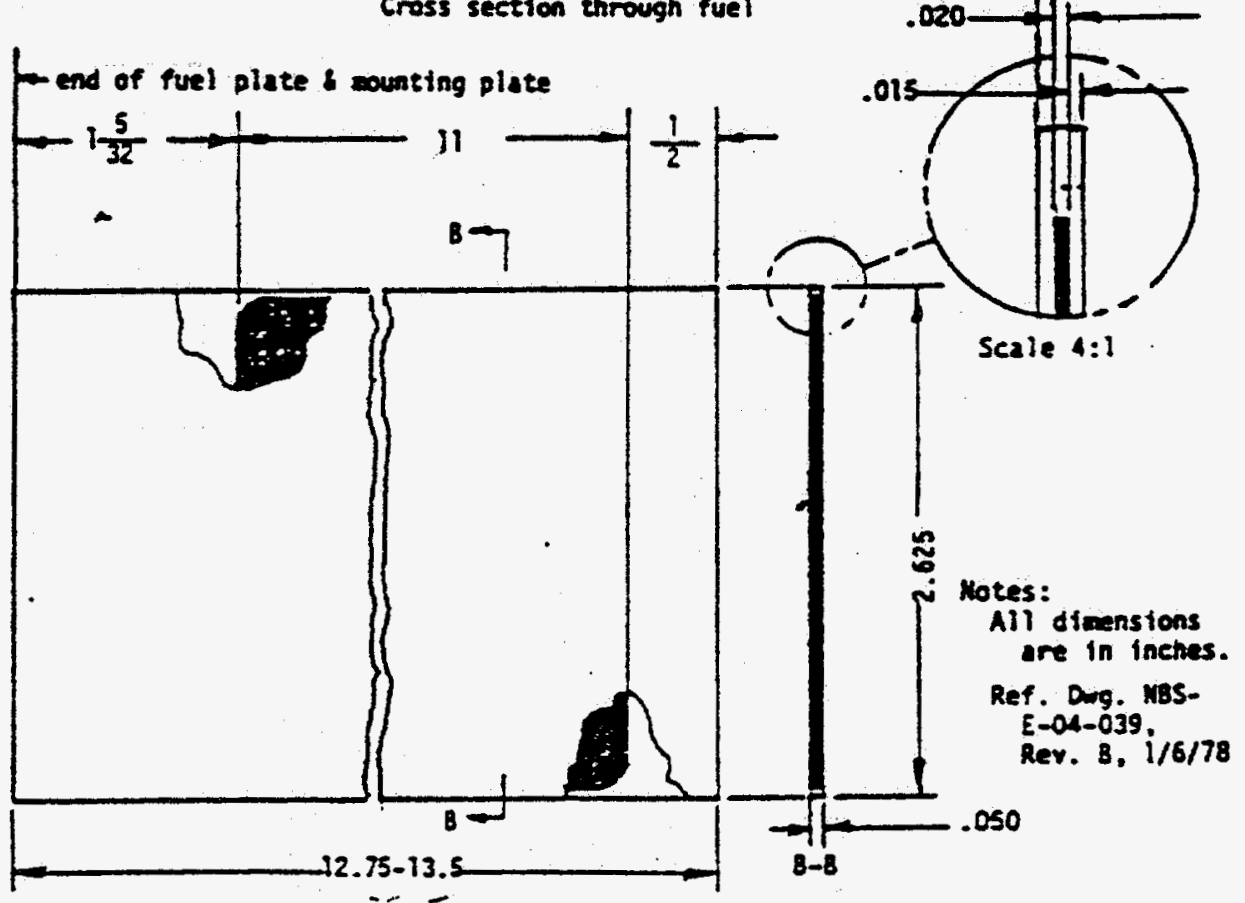


17 inner fuel plates $23.75 \times 0.050$ thick Fuel alloy core 22.75 long $\times 0.021$ thick Cladding 0.012 thick, both sides

2 outer fuel plates 25 long $\times 0.140$ thk. Fuel alloy core 22.75 long, 0.009 thick Cladding 0.015 thick, minimum
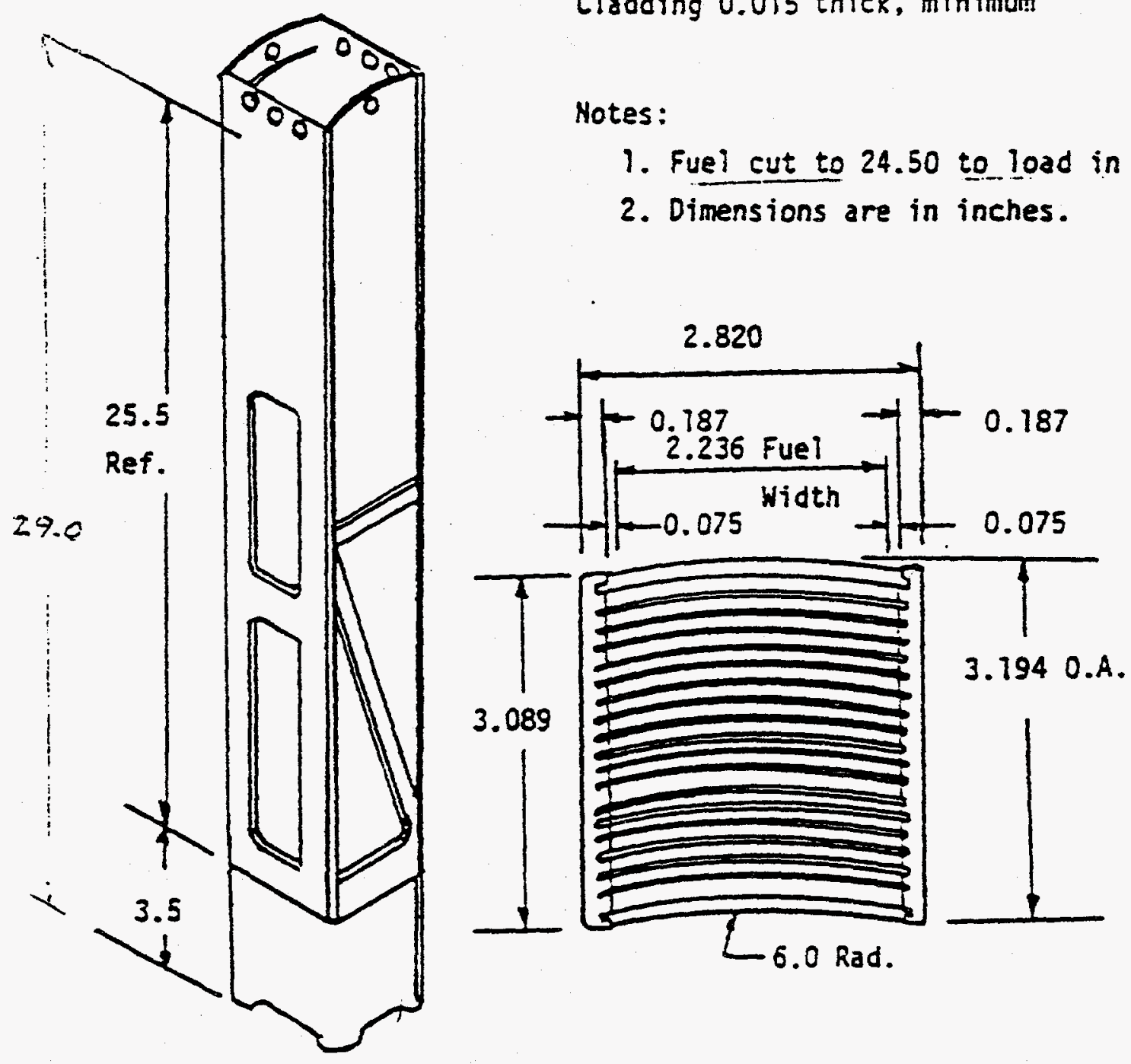

Notes:

1. Fuel cut to 24.50 to load in cask. 2. Dimensions are in inches. 
Revision 1

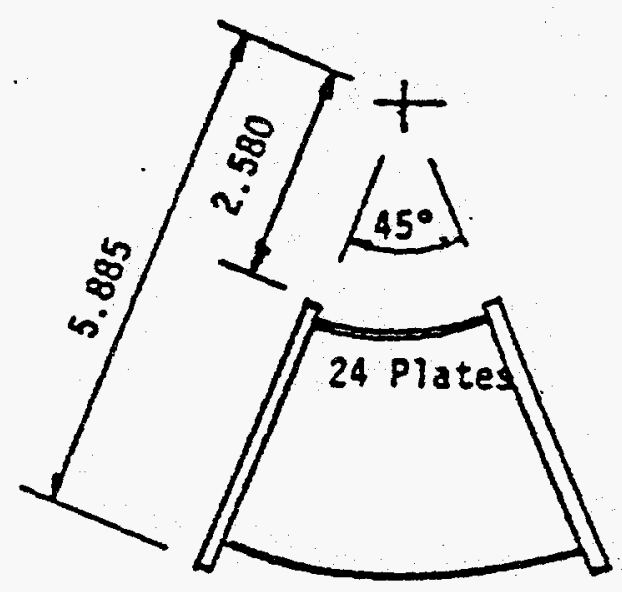

Dimensions are in inches.
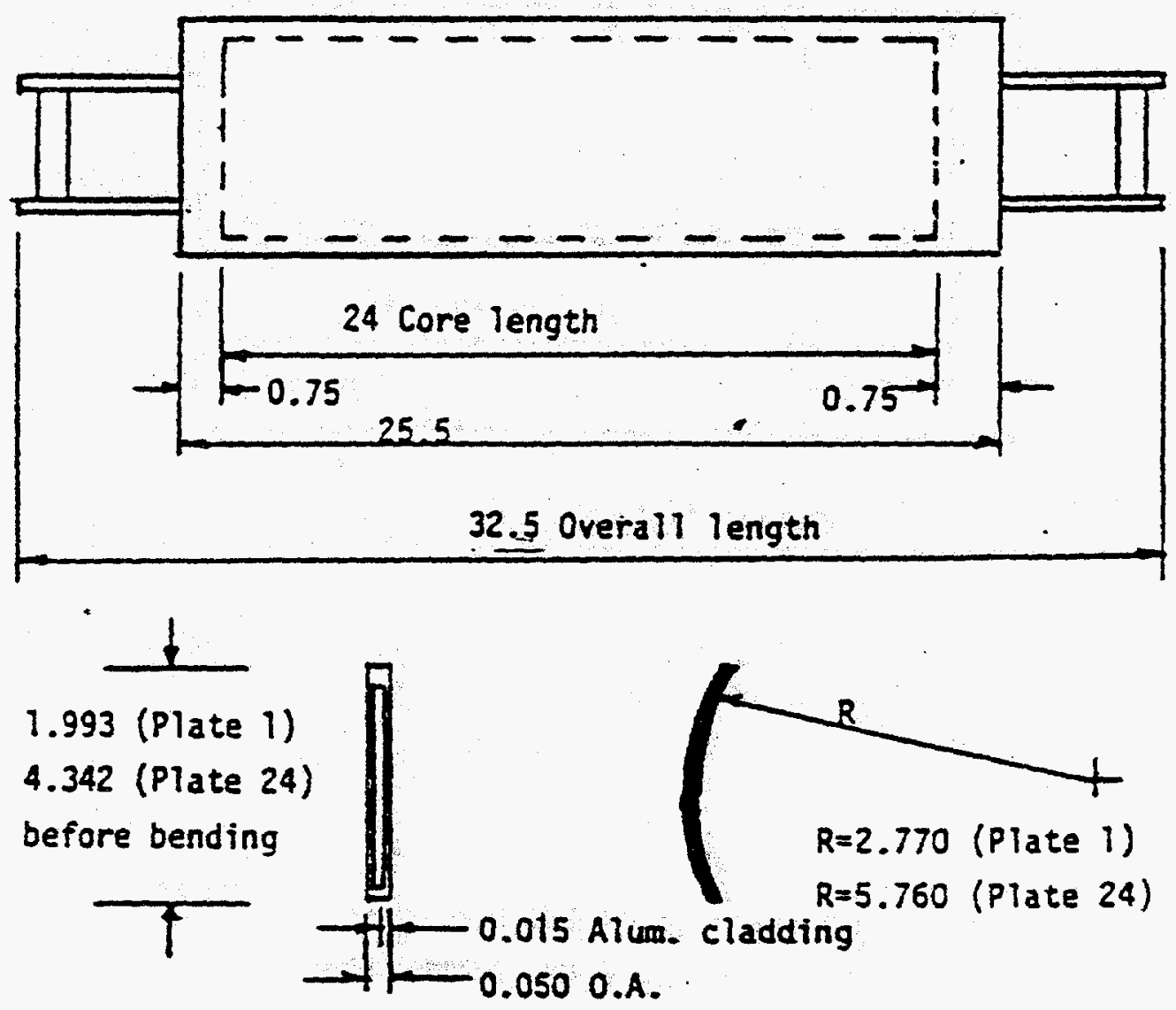

SCHEMATIC OF'MURR SPENT FUEL ASSEMBLY 


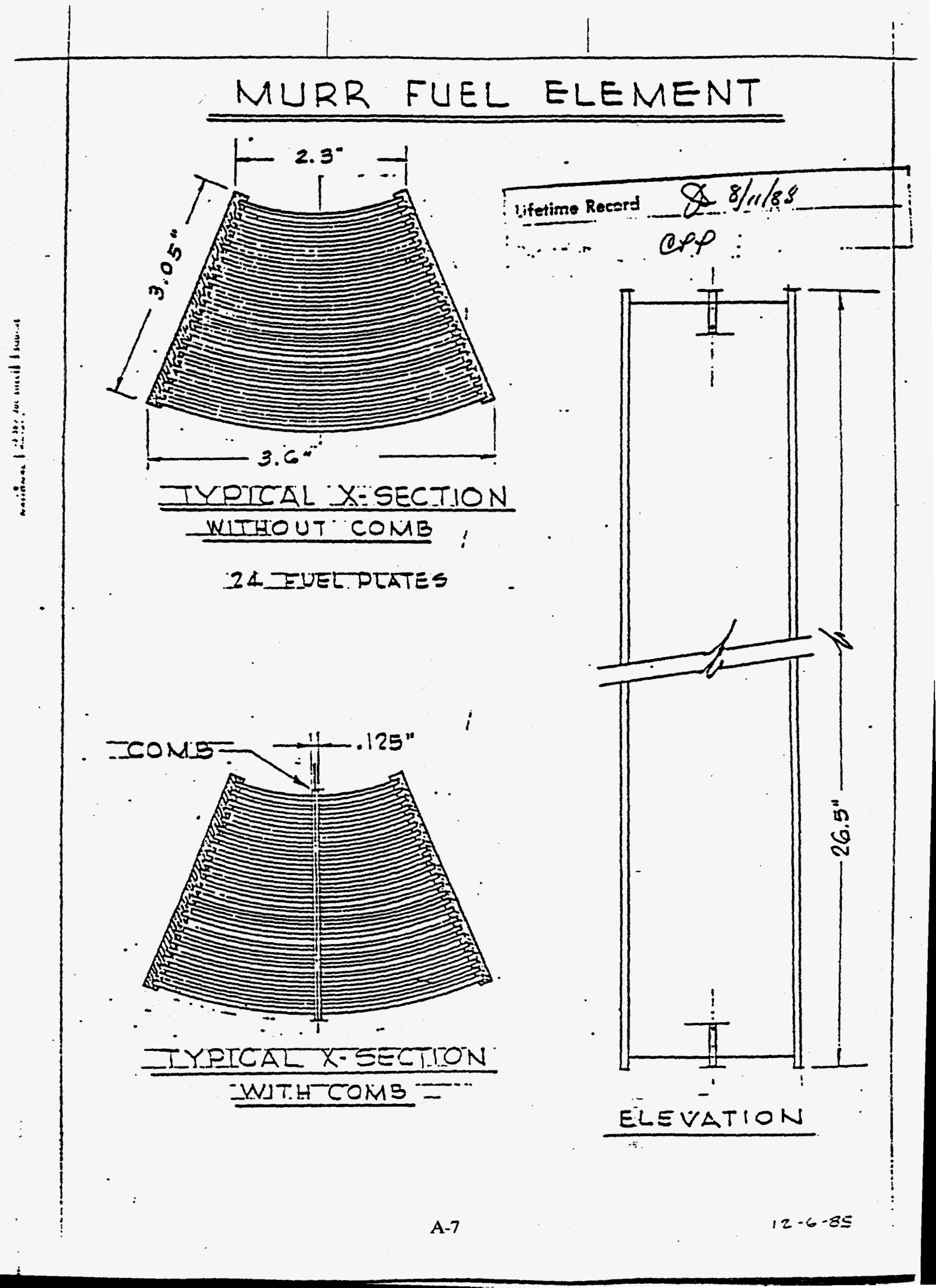




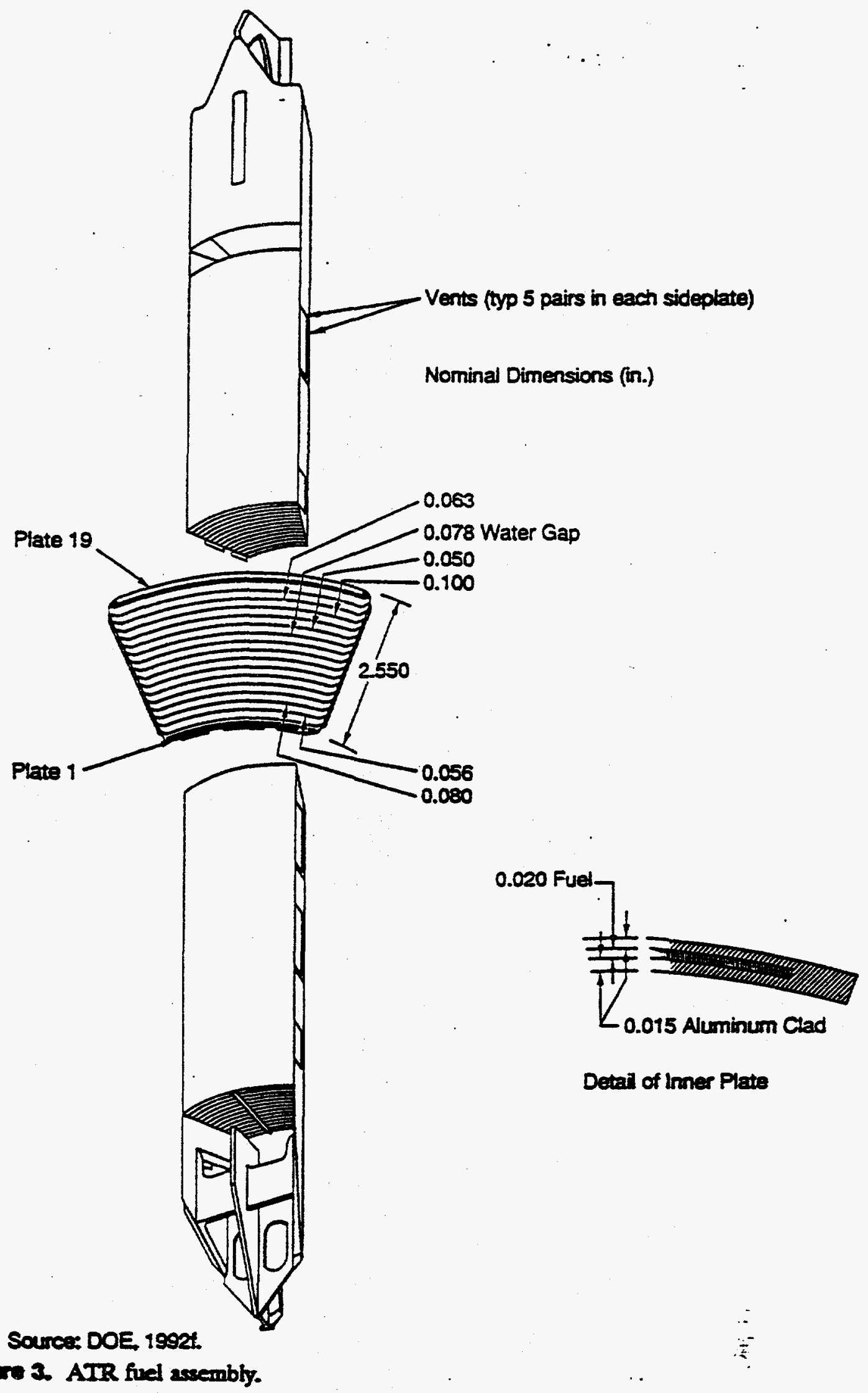


Inc- $50-00-17-175$ RY I

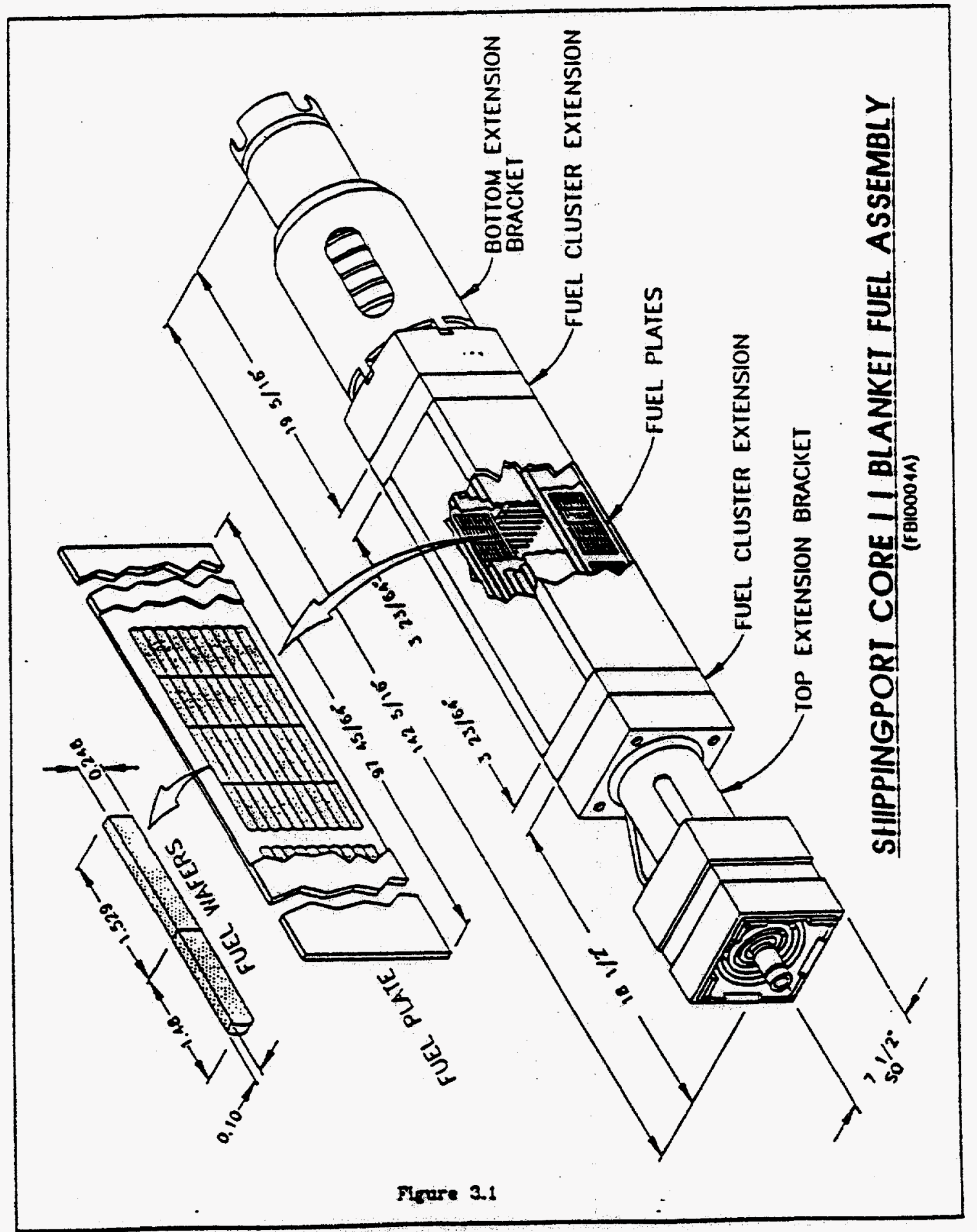




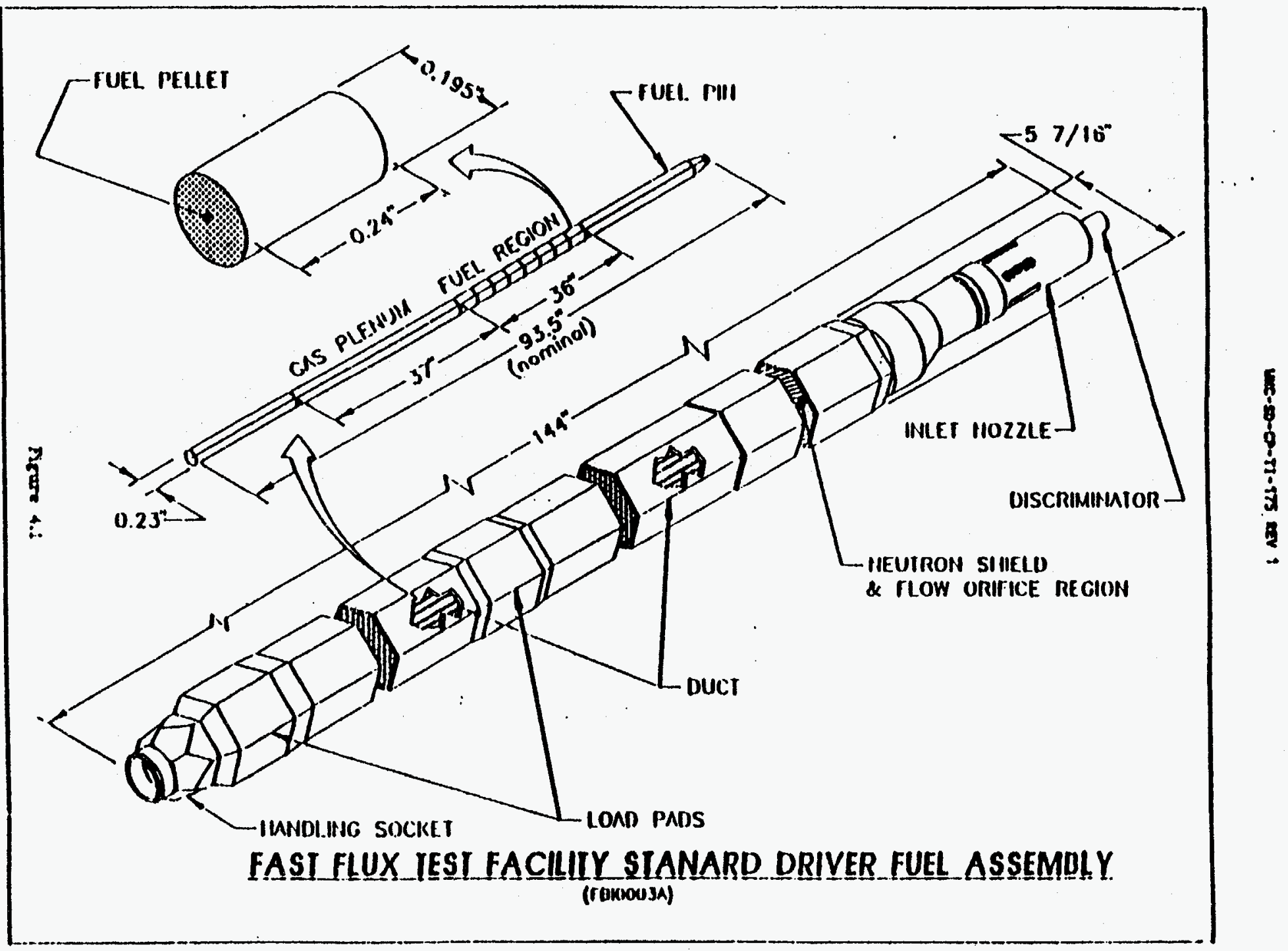


U15-sob-0-11-175 ReV :

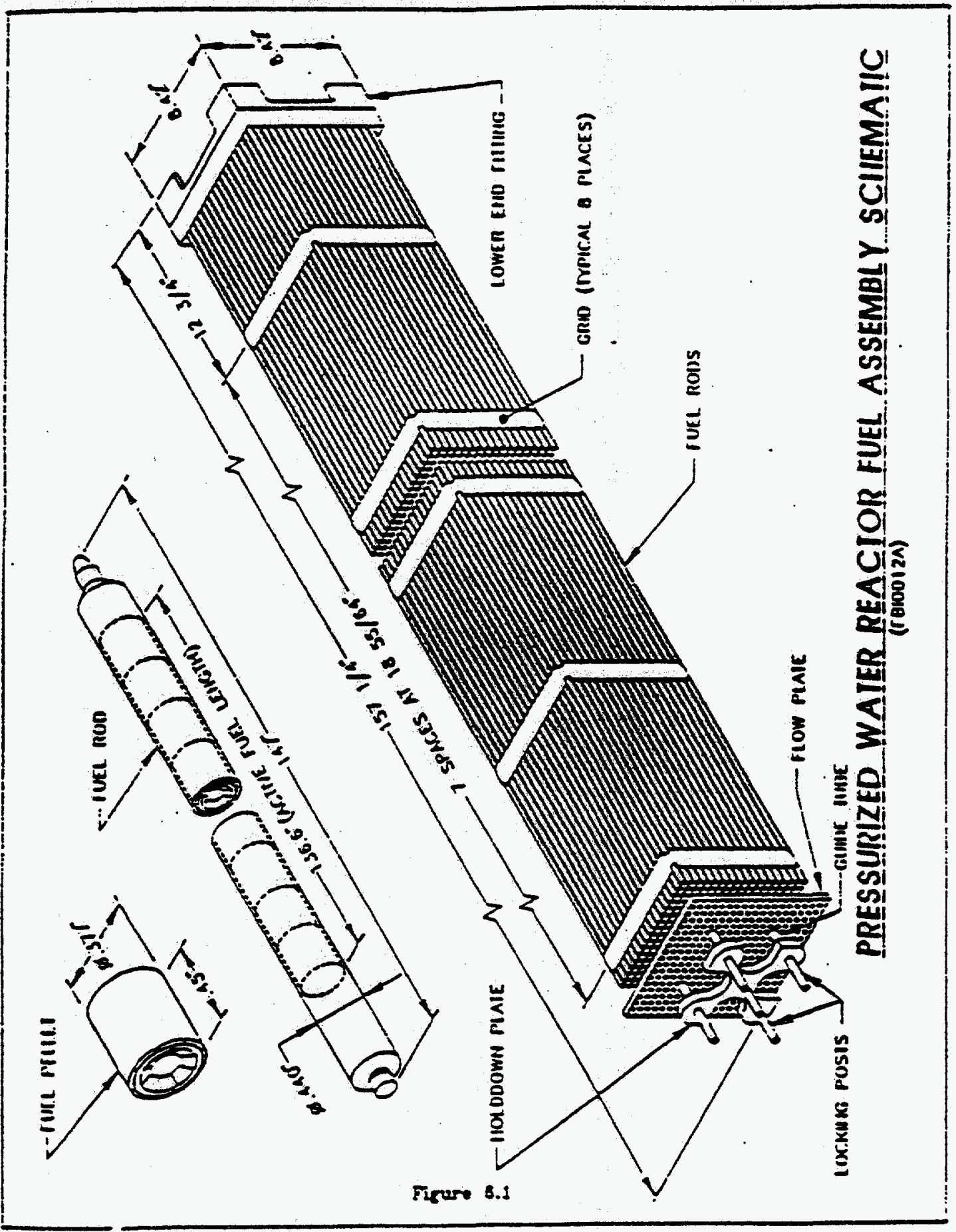




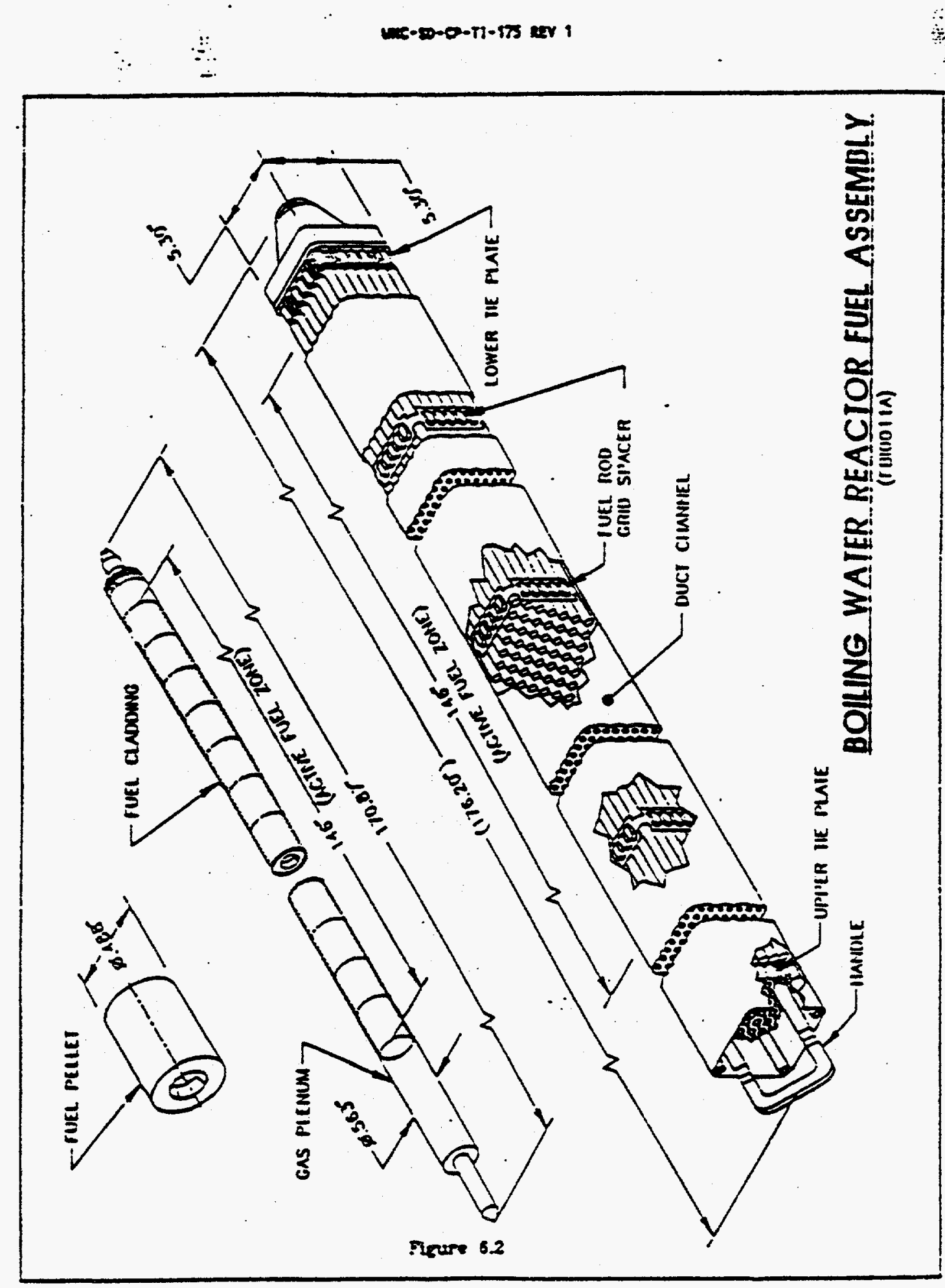


unce- $-00-0-11-175$ xed I

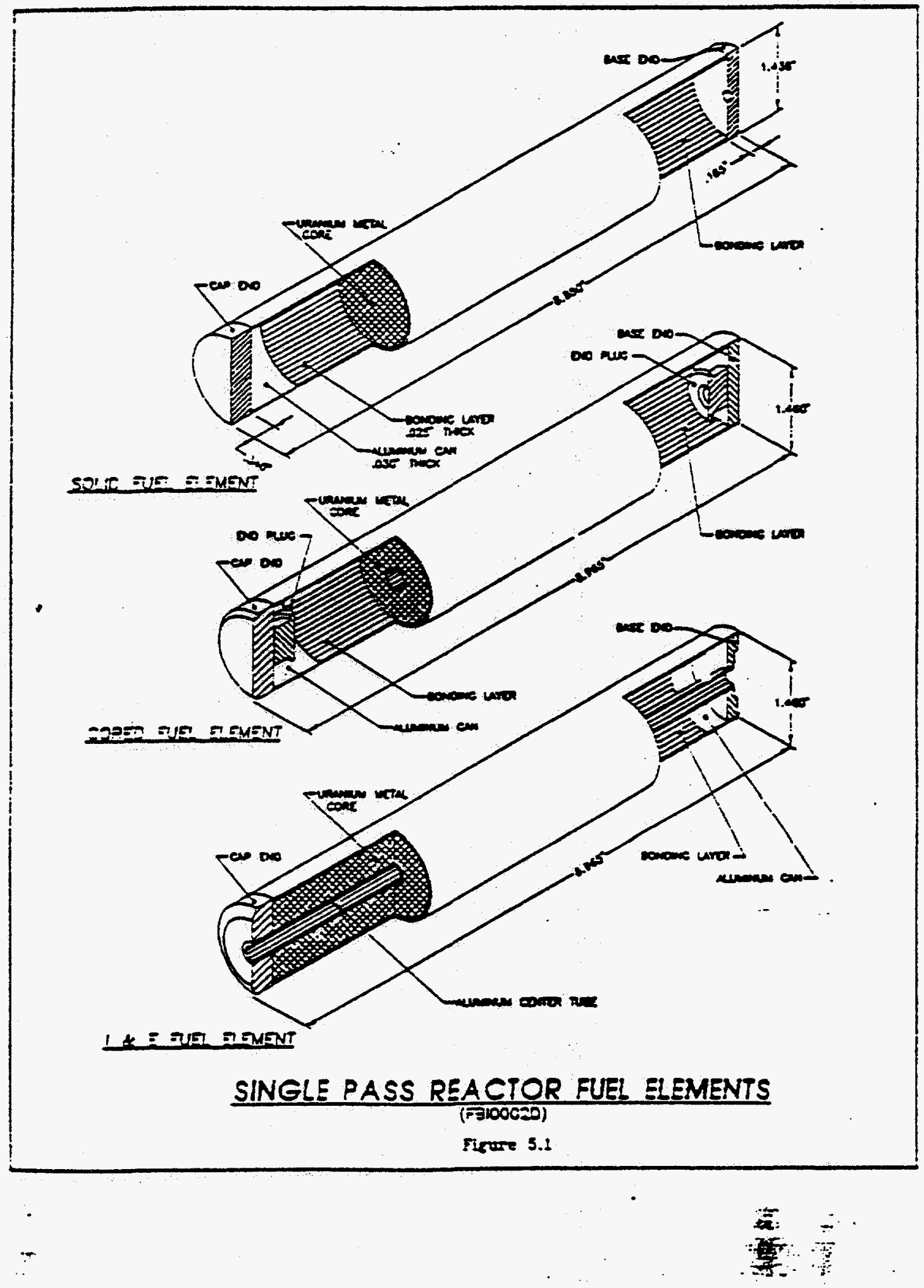




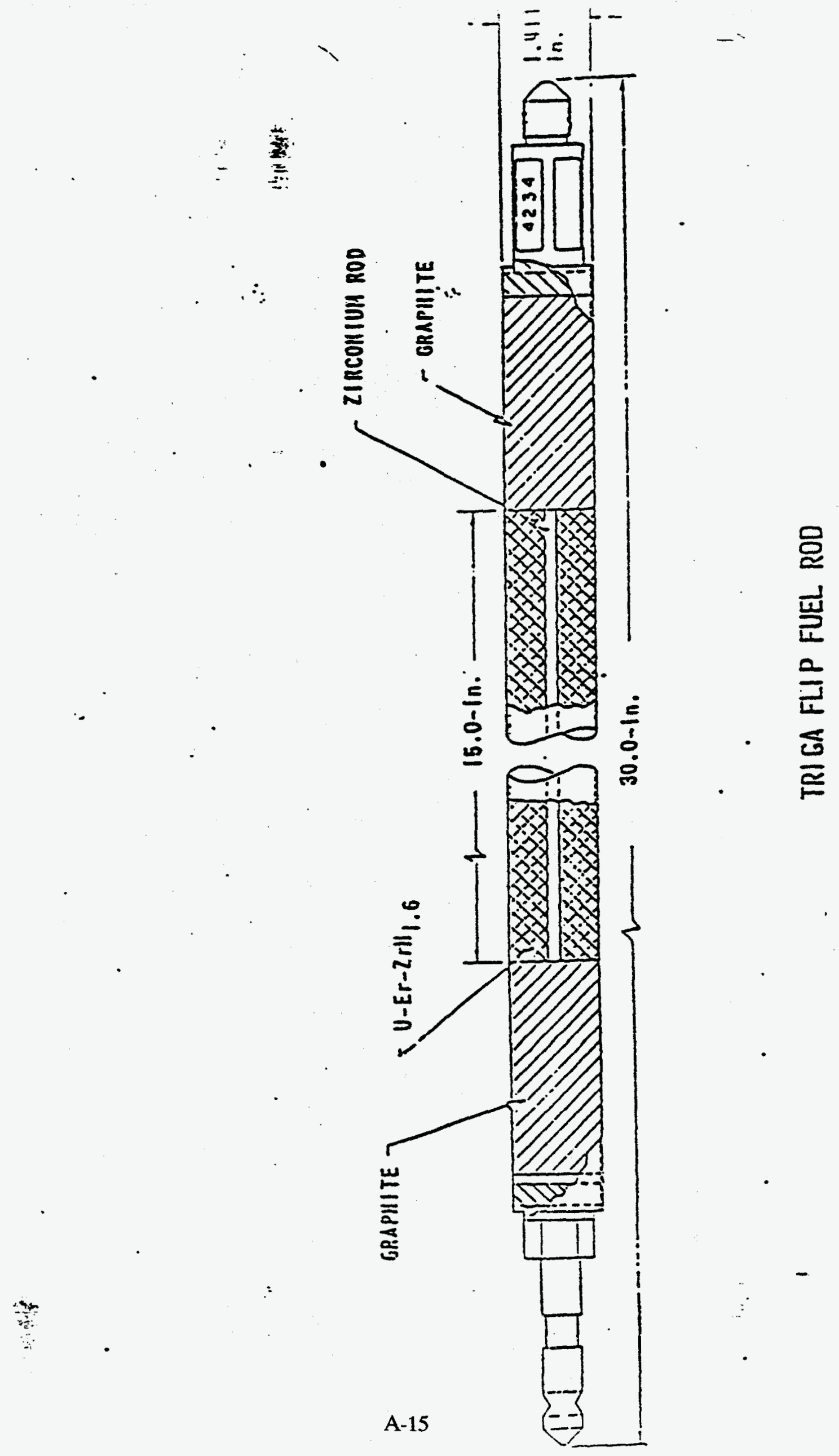




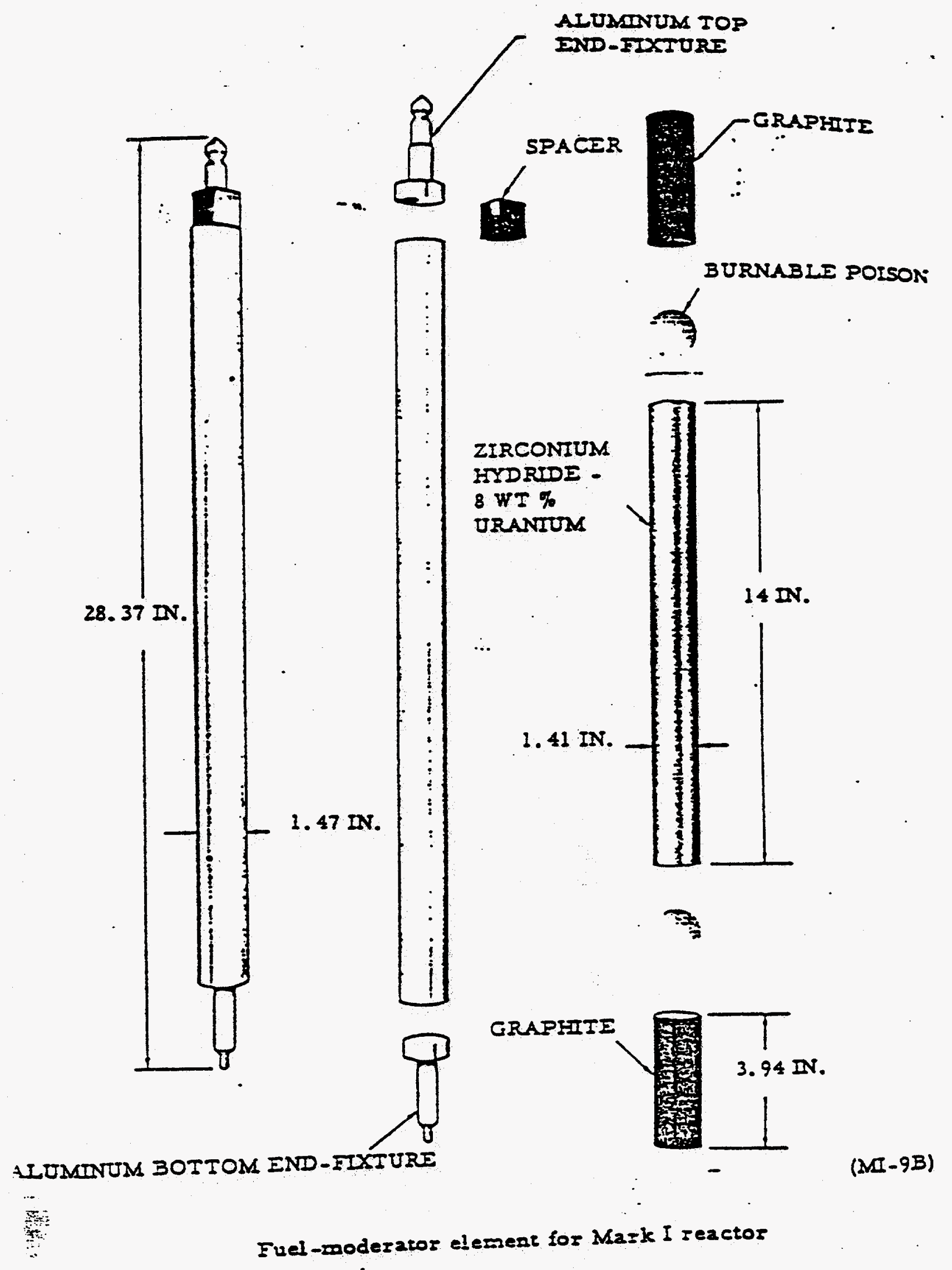




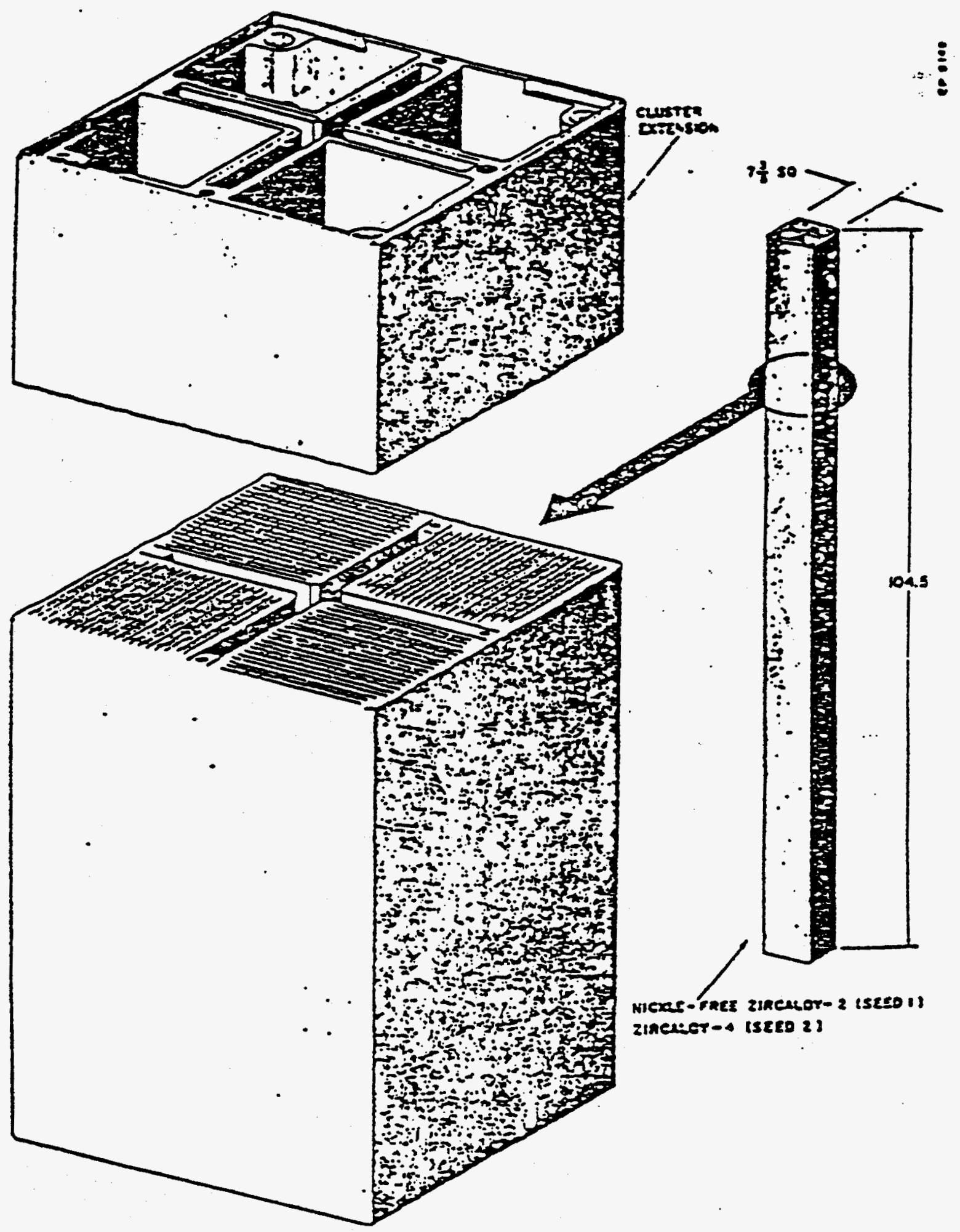

PIIR-2 Seed 1 and Seed 2 Fuel Cluster -

i-78 Original 


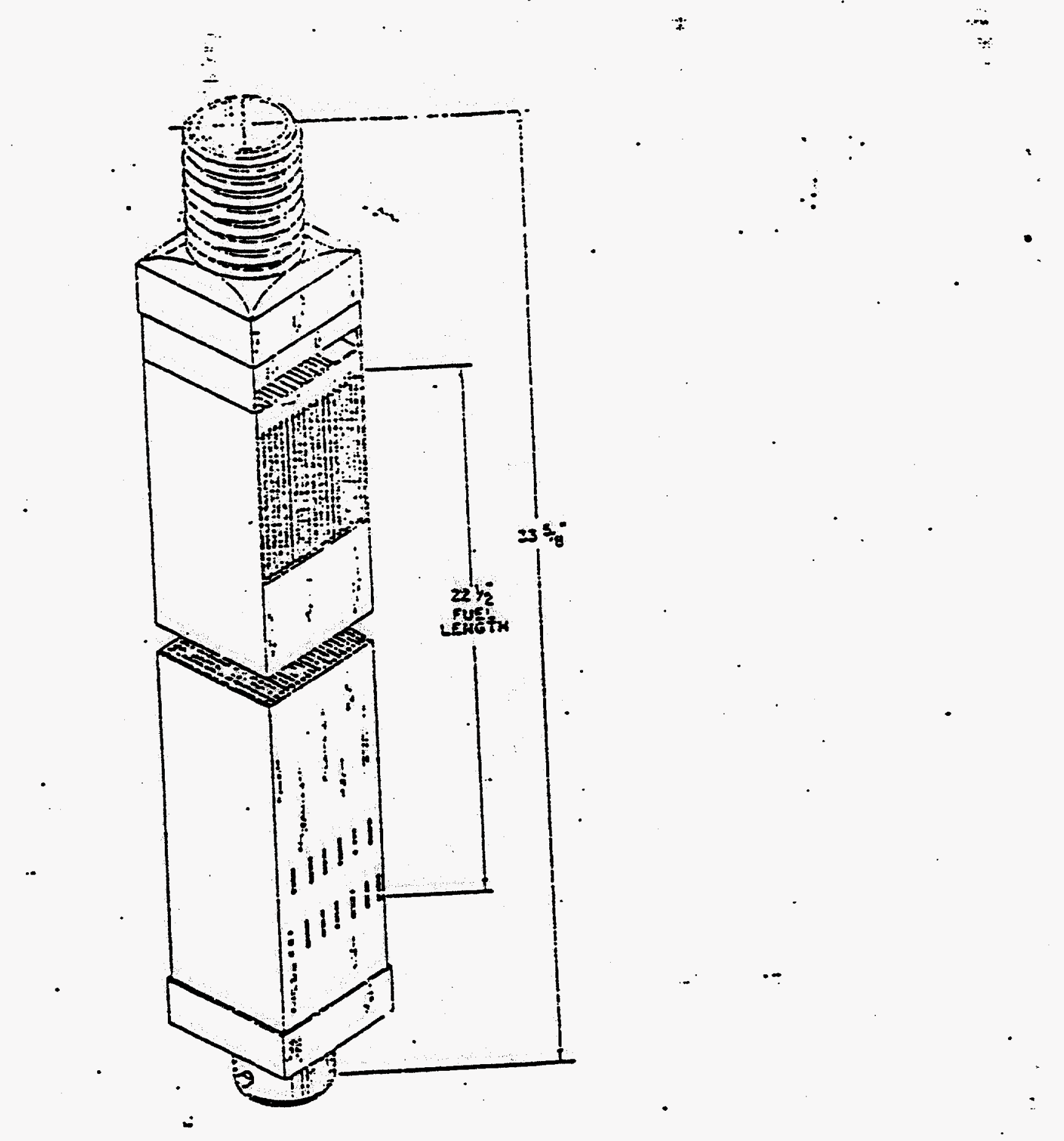




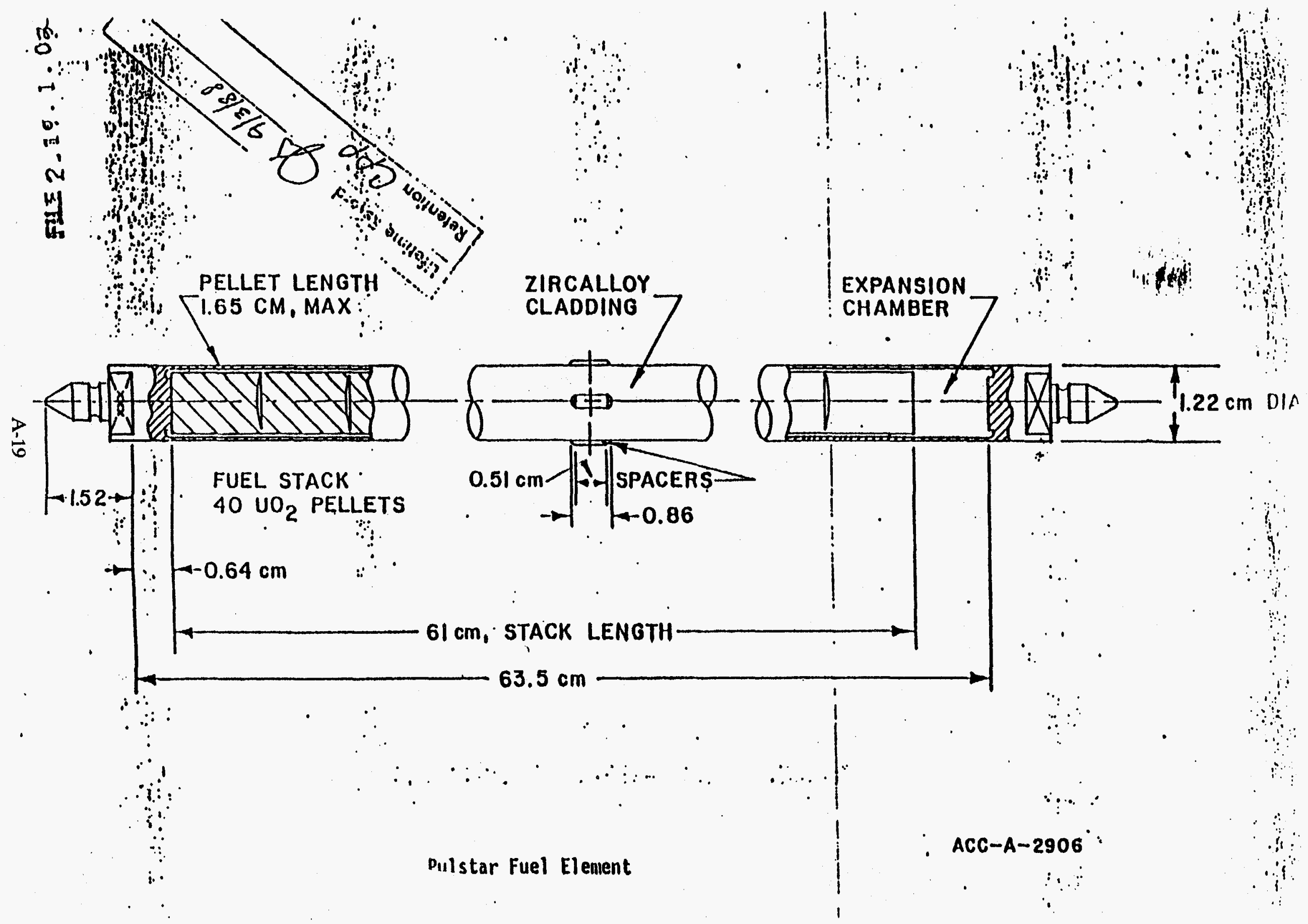




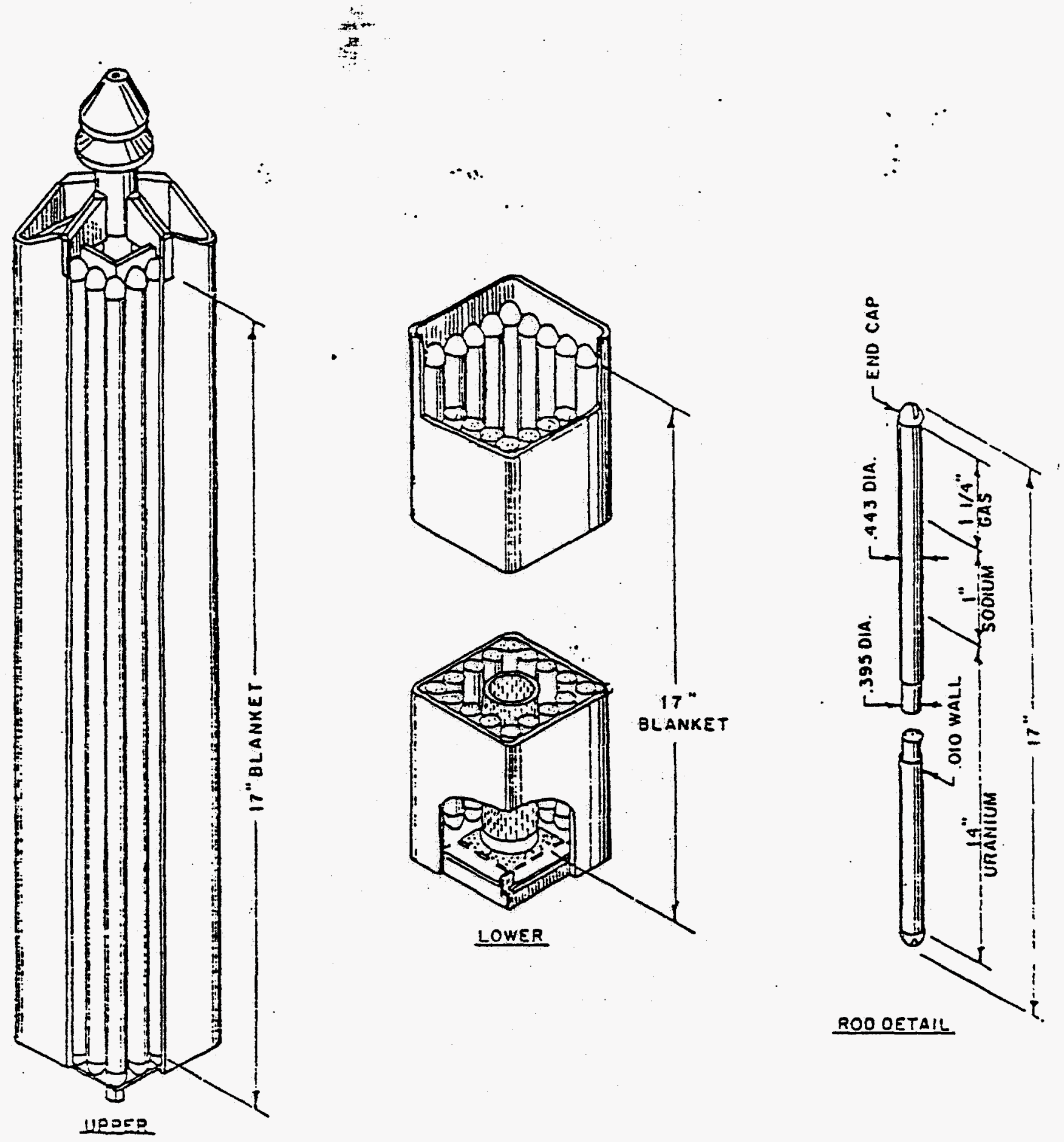

$$
\begin{gathered}
\text { Femi I ixial ollankec Subassemily } \\
\text { A-20 }
\end{gathered}
$$



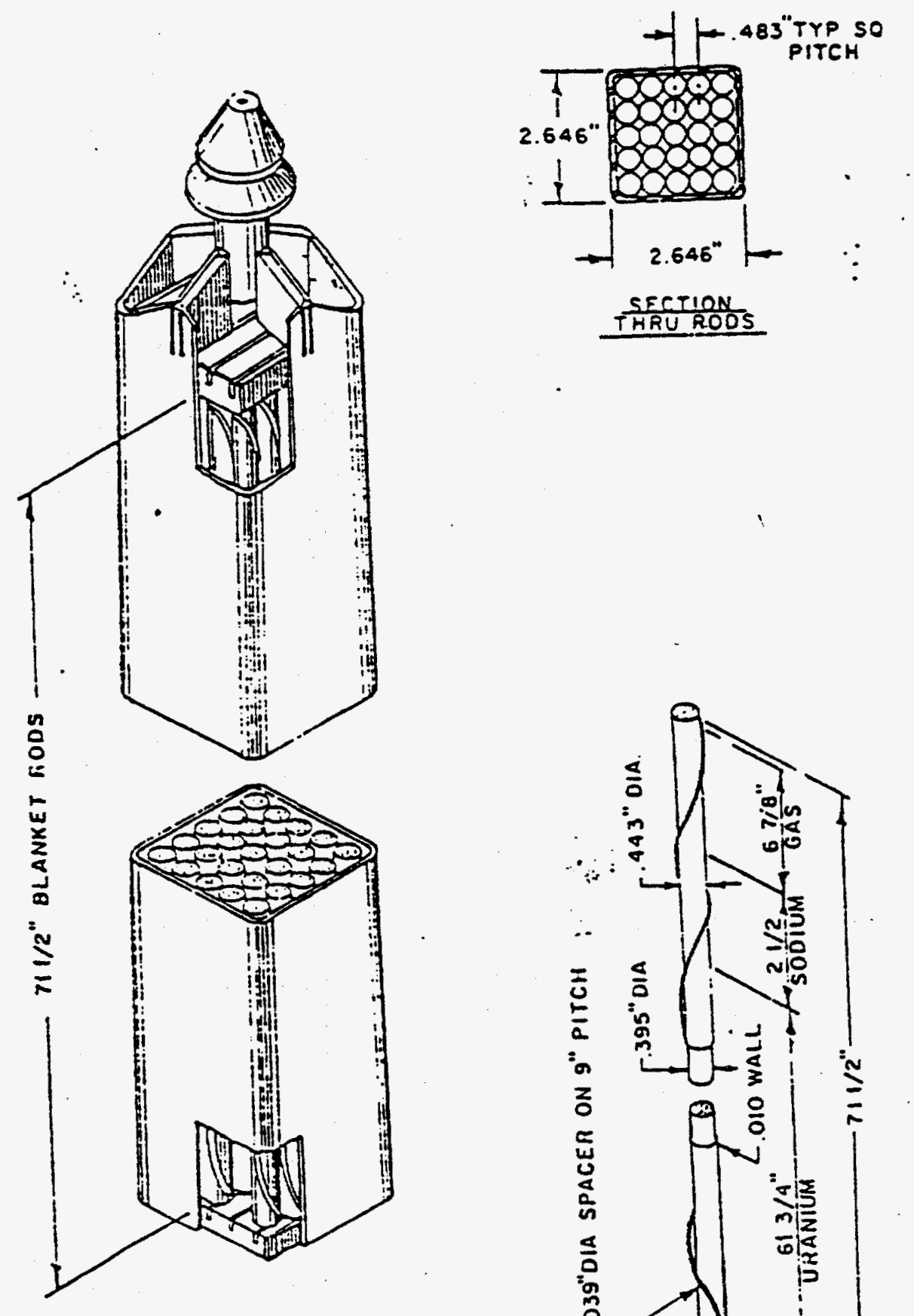

$\frac{\text { SECTION }}{\text { THRUROOS }}$

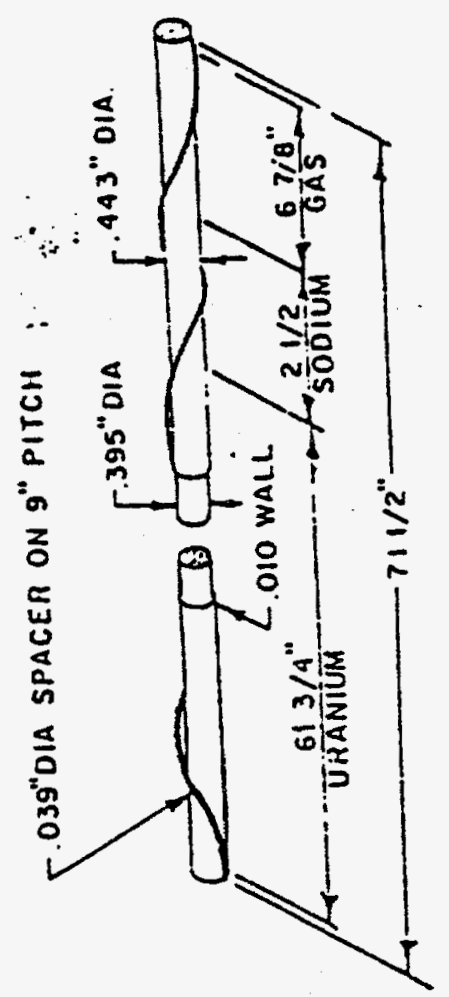

ROO OETAIL

Fermi I Radial Blanker Subassemily 


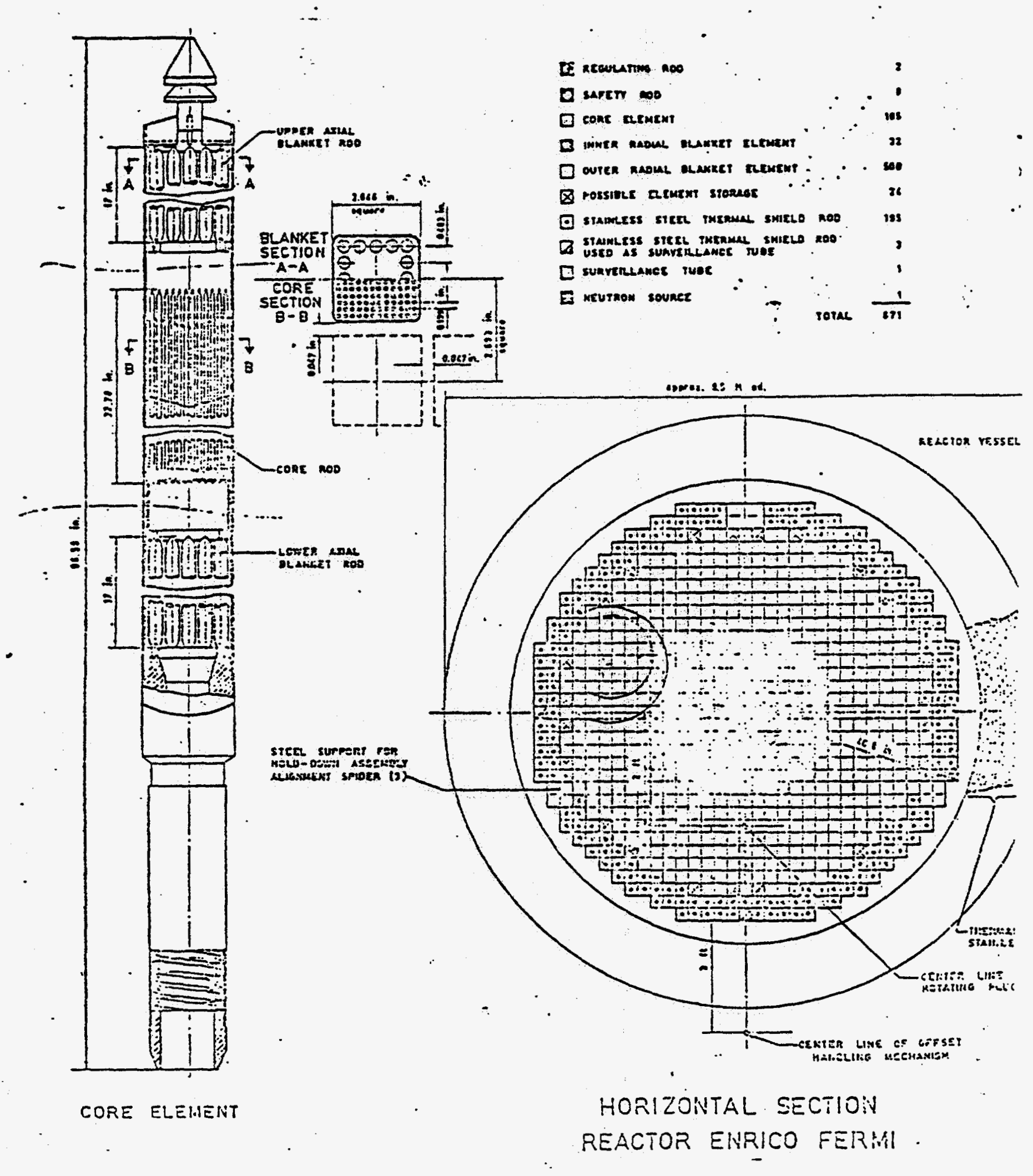


DATA SHEET I - ATTACHMENT I

MTR FUEL ELEMENT

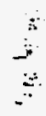

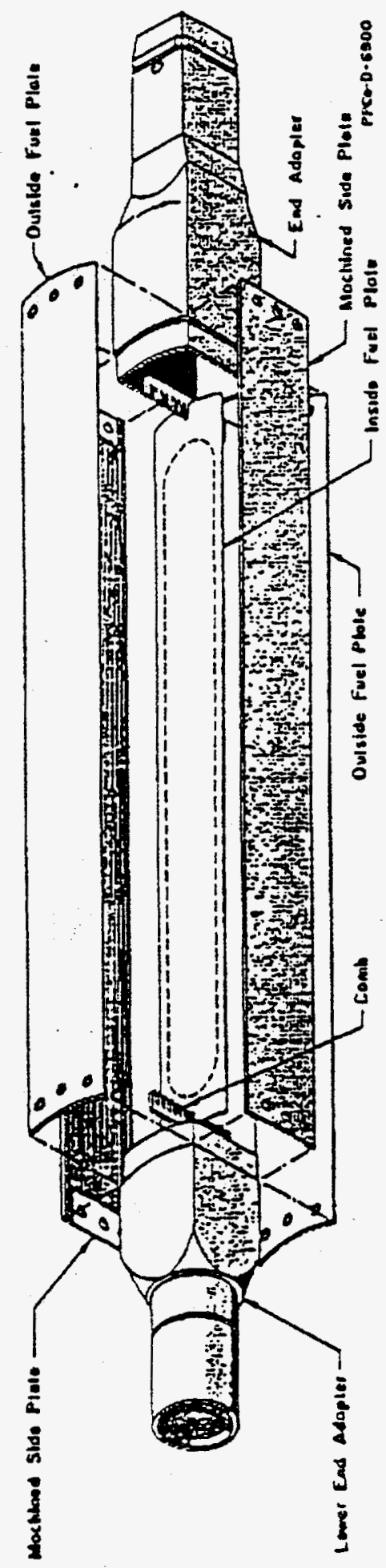




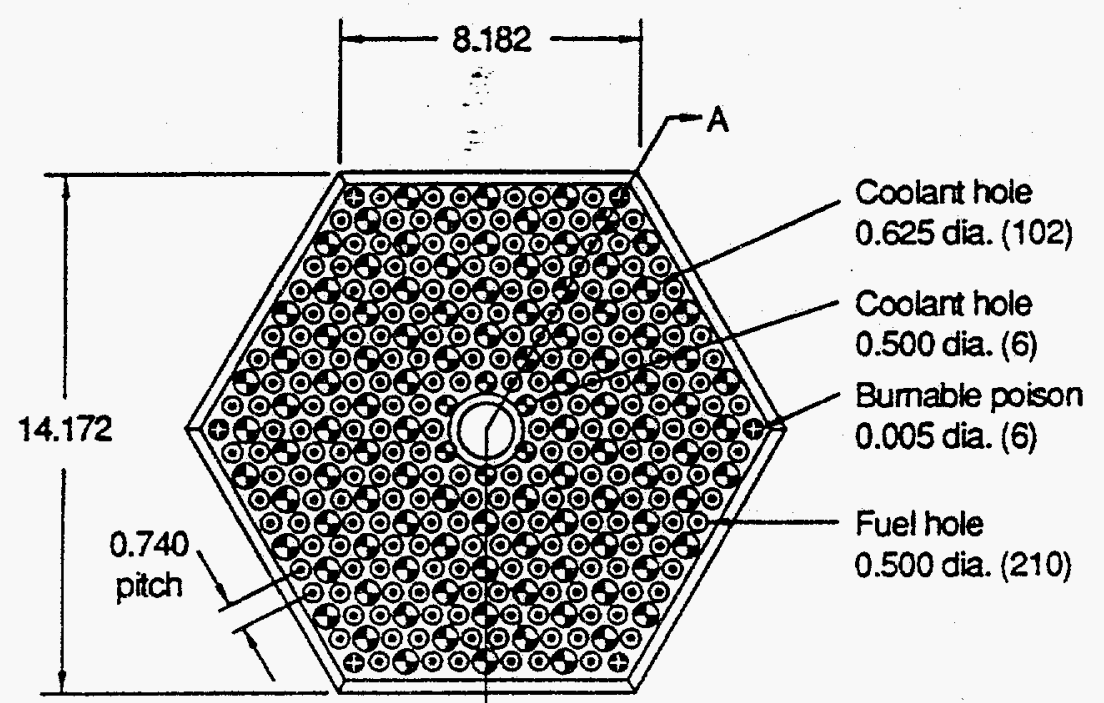

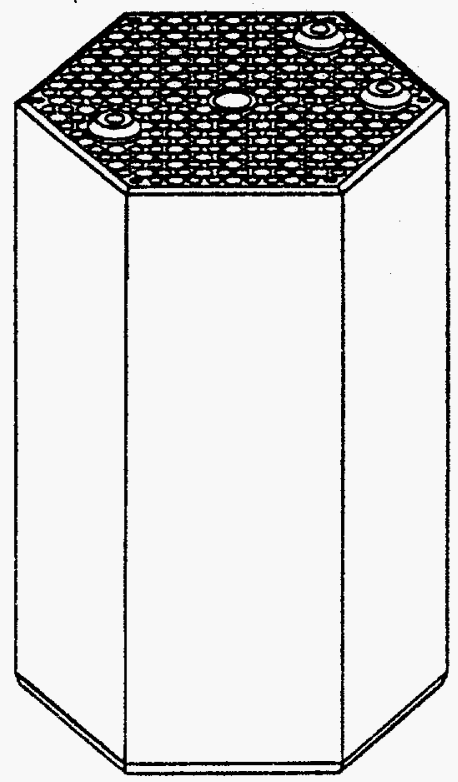

(B)

pictorial
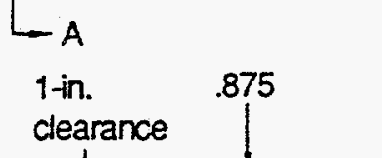

$$
1
$$
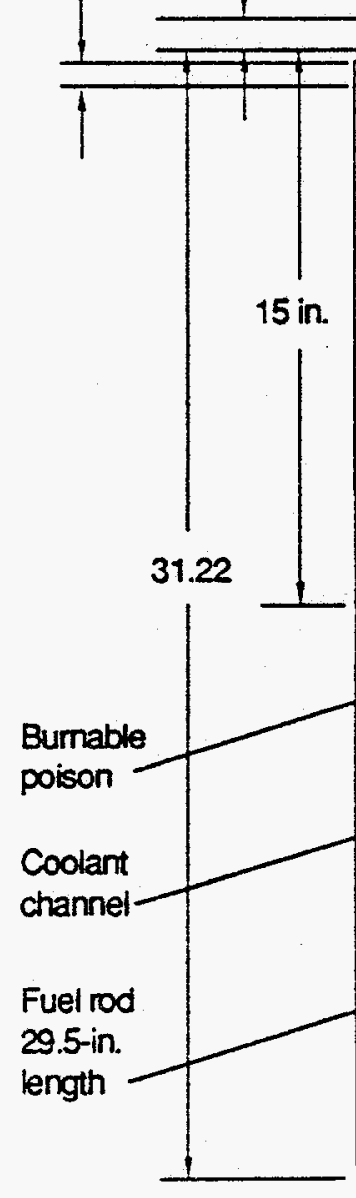


\section{EBR-II EUEL SUBASSEMBLY AND FUEL ELEMENT}
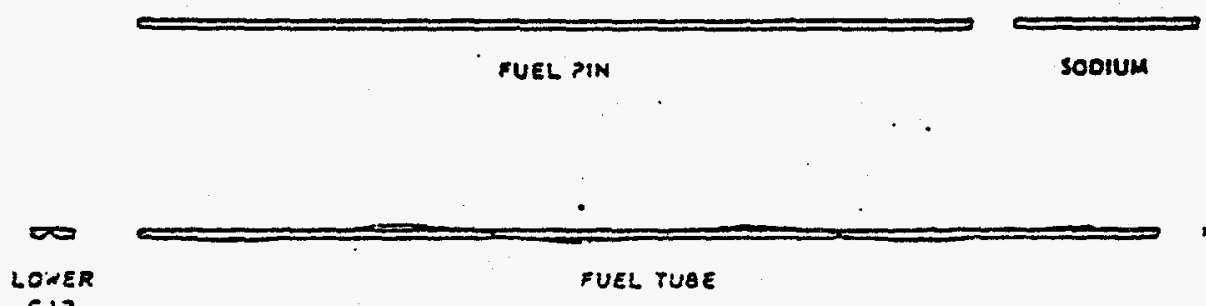

FUEL TUAE

UPPER

Cd?

12

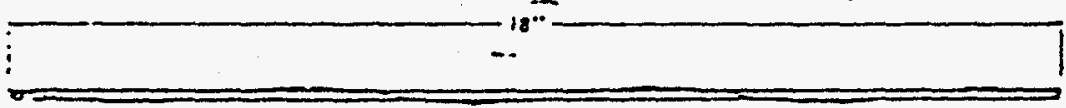

FUEL ELEMENT

$$
\therefore-\ldots+-2=" \because
$$

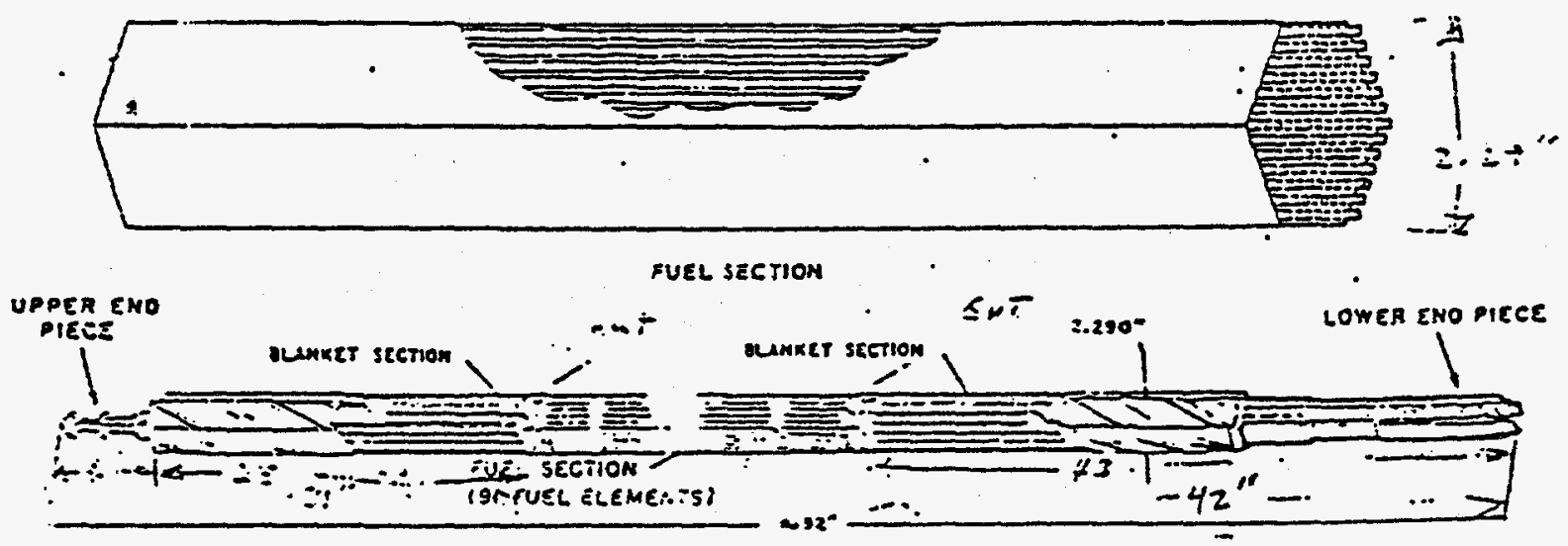




\section{Appendix B}

Packaging Data Sheets and Design Drawings/Sketches 
This appendix provides copies of packaging Certificates of Compliance and basic dimension data in the form of drawings and, in some cases, individual data summaries. 
GE T-2

B-1 


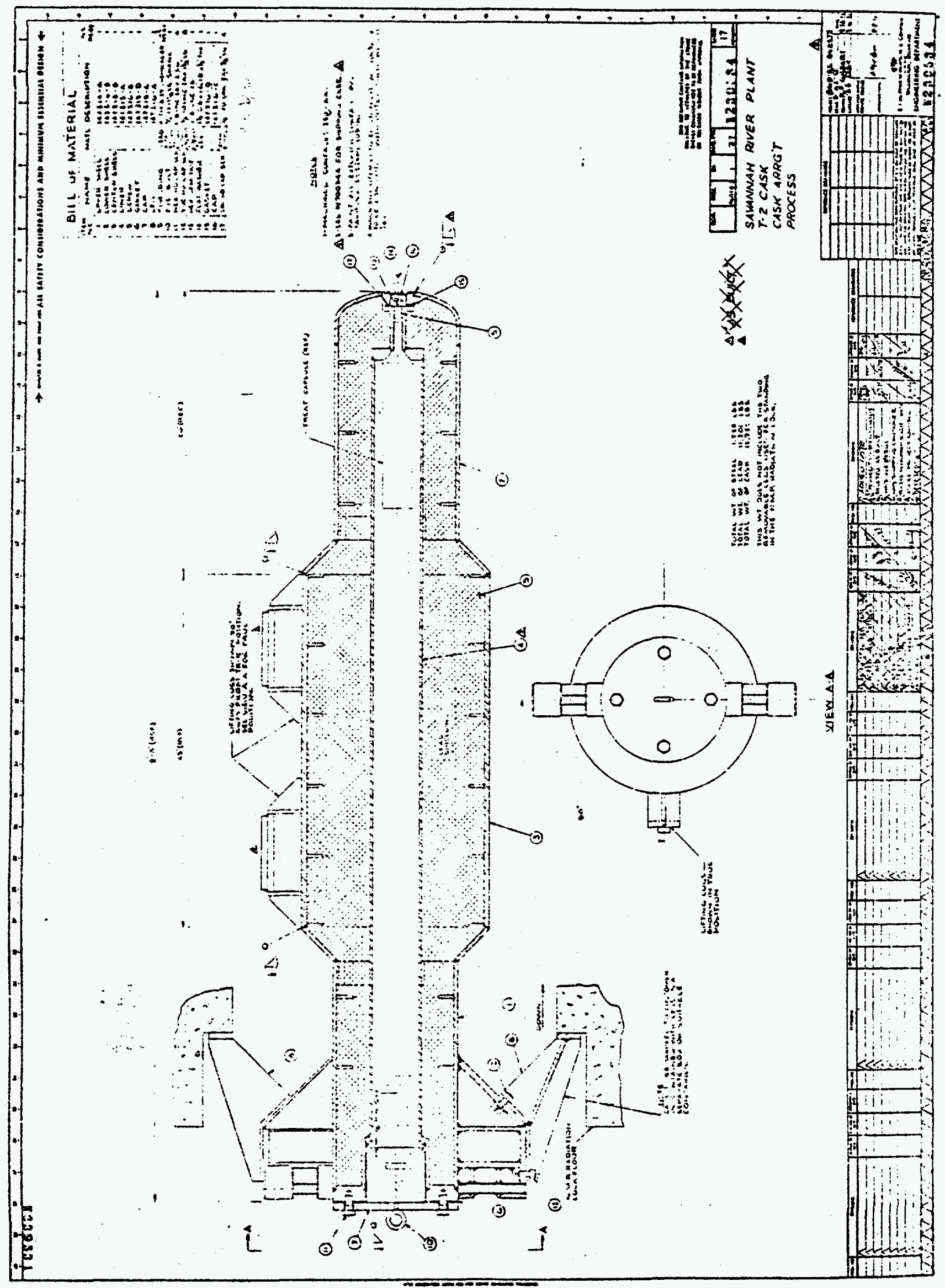




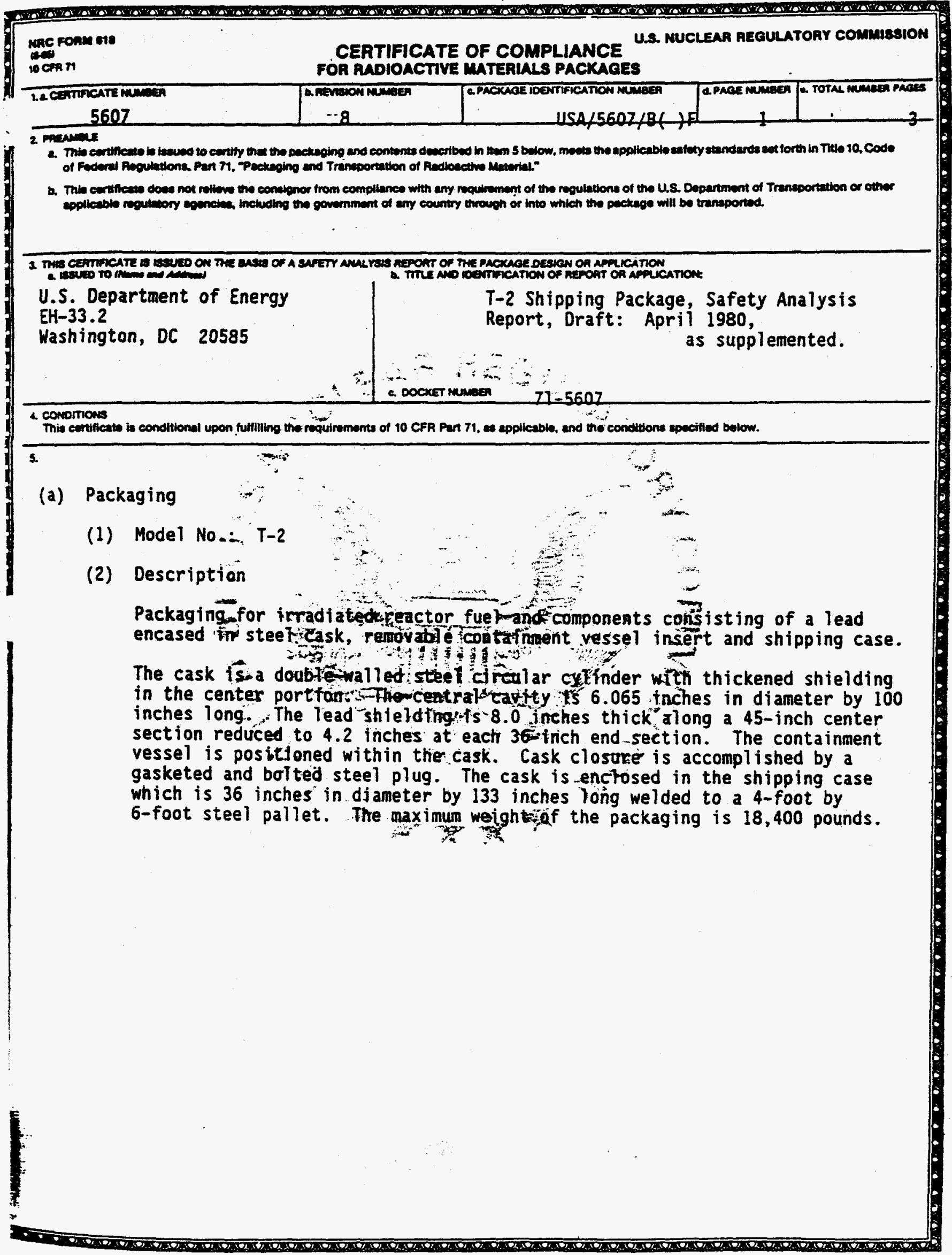


Page 2 - Certificate No. 5607 - Revision No. 8 - Docket No. 71-5607

5. (a) (3) Orawings

(i) The shipping case is constructed in accordance with DuPont Drawing Nos.: W716539, Rev. 0; 180191, Rev. 1; 180192, Rev. 0; 180193, Rev. 1; 180194, Rev. 0; 180197, Rev. 0; W716538, Rev. 0; 180195, Rev. 0; 180196, Rev. 0; and 180089, Rev. 0 .

(ii) The cask is constructed in accordance with General Electric Drawing Nos.: 9190755, Rev. 0; 135C5202, Rev.0; 153F966, Rev. 0; and 10603721, Rev. 0; or it is constructed in accordance with DuPont Drawing Nos.: W239534, Rev. 2*; 147214, Rev. 15; 147215, Rev. 2*; and 147216, Rev. 1.

(iii) The ANL insert is constructed in accordance with Argonne National Laboratory Drawing Nos.:- WOL47 $=0227-D D$, Rev 7; W0147-0228-DD, Rev. 6; W0147-0229-DC, Rev. 6;-KO1 47-0231-DD Rev. 3; W0147-0234-DC, Rev. 4; and W0147-0312-DE, Rey:-2.

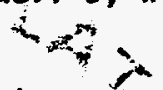

(iv) The TREAT Vessel insert is constructed in accordance with Westinghouse Hanford Company Drawing Nos.: H-3-39082, Sheets 1 through 4 and 6, Rev. 0 , and Sfieet 5, Rev. 1; H-3-36134, Sheet 1, Rev. 3 and Sheet 2, Rev. 2; and $\mathrm{H}-3 \mathrm{~g}=3623$, Sheet 1 , Rev. 1 and Sheets 2 through.4, Rev. 0.

(b)

(1)
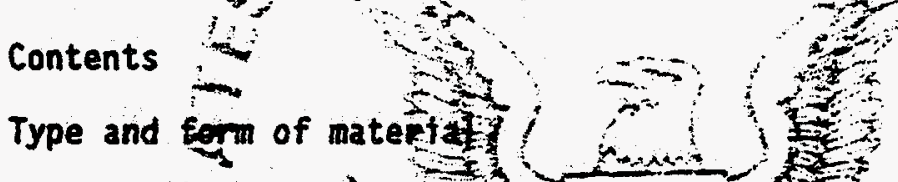

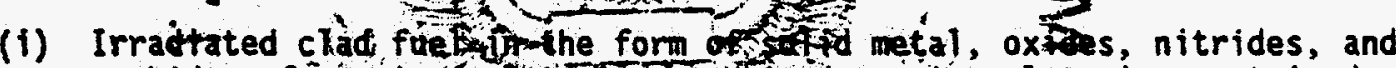

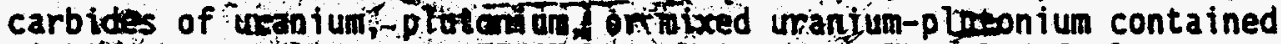

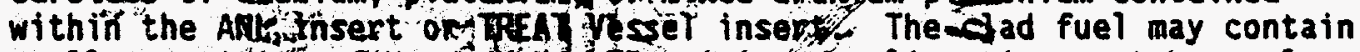

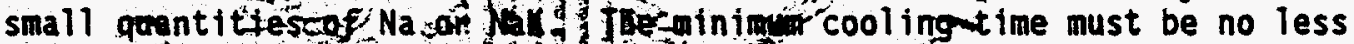
than $150^{\circ}$ days.

$$
\text { i- }
$$

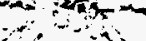

(ii) Irradiated clad fuel pins of uramium otoxide emajched to up to $3.0 \mathrm{w} / 0$ in U-235 contained within thei-ANL Insert or TREAT vessel insert. Average exposure of fuet not to exceed 18 megawatt:days per kilogram. The clad fuel may contain small quantities of $\mathrm{Na}$ or Mak. The minimum cooling time must be no less than:90 days... th

(iti) Irradiated reactor components held within the container shown in Drawing No. W0147-0234-DC, Rev. 4.

*As provided in the April 12, 1983, supplement. 
Page 3 - Certificate No. 5607 - Revision No. 8 - Docket No. 71-5607

5.(b) (2) Maximum quantity of material per package.

Internal decay heat not to exceed 208 watts, and:

(i) For the material described in $5(b)(1)(i)$, fissil material not to exceed $1.71 \mathrm{~kg}$.

(ii) For the material described in $5(b)(1)(i i)$, fissile material (U-235) not to exceed 300 grams.

(c) Fissile class for the material described in $5(b)(1)(i)$ and $5(b)(1)(i i)$, and limited in $5(b)(2)(i)$ and $5(b)(2)(i j)$

6. The contents must be shipped $d r y$. Whe töaded underwater, the package must be dried using Consumer Power Eompany"s procedure. : T-2 Cask Liner Assembly Drying Procedure," Proc. No. EEST-C12, Rev. 1, 11/12/81."

7. The ANL Insert and FREAT Vessel insert must be leak têsted prior to first use and annually thereafterin accordance with the procedures specified in Argonne National Laboratories Document No. W0195-0054-ES-00.

8. Prior to each shjpment, the package must be leakested in accordance with procedures specifled in Append ix A to-HEEF/N OML6202, Rev. 2, March 17, 1981.

9. In addition to the requirements of Sabpart $G$ o conditions of this

(a) The package)shalkibe openated 3ad pepared for shipment accordance with the

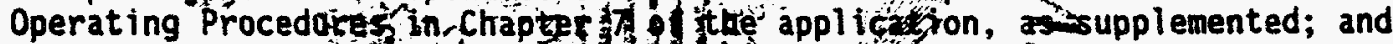

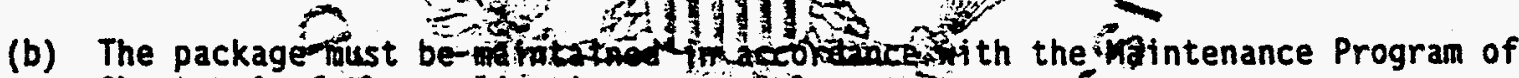
Chapter, 8 of the application, as soiplemente?

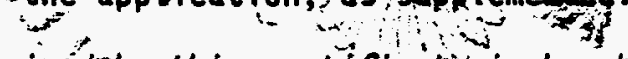

10. The package authorizedpby this certfficate is hereby appoved for use under the general license provisions of 10 CFR \$71.12.

11. Expiration date: May 31,1998

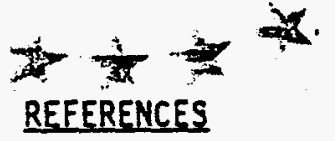

OuPont Safety Analysis Report, Draft Apri1 1980.

Department of Energy supplements dated: February 11, April 8 and 20, 1982; Apri1 12, 1983; February 26, 1992; and February 3, 1993.

FOR THE U.S. NUCLEAR REGULATORY COMMISSION

MAY 201998

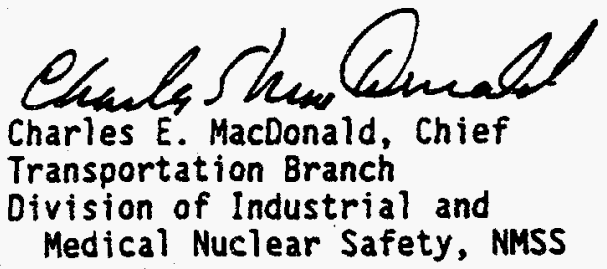


GE-100

B-6 


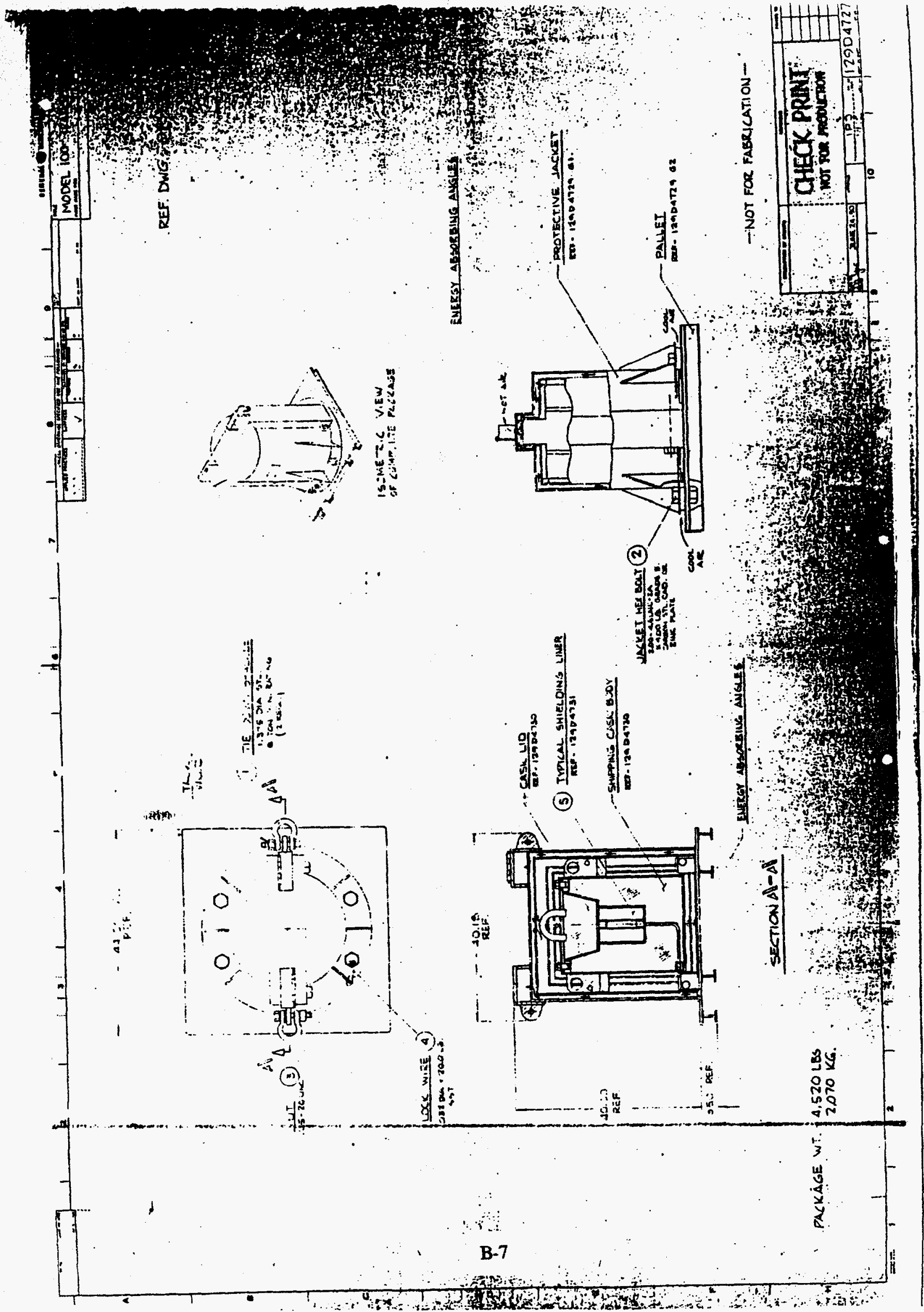




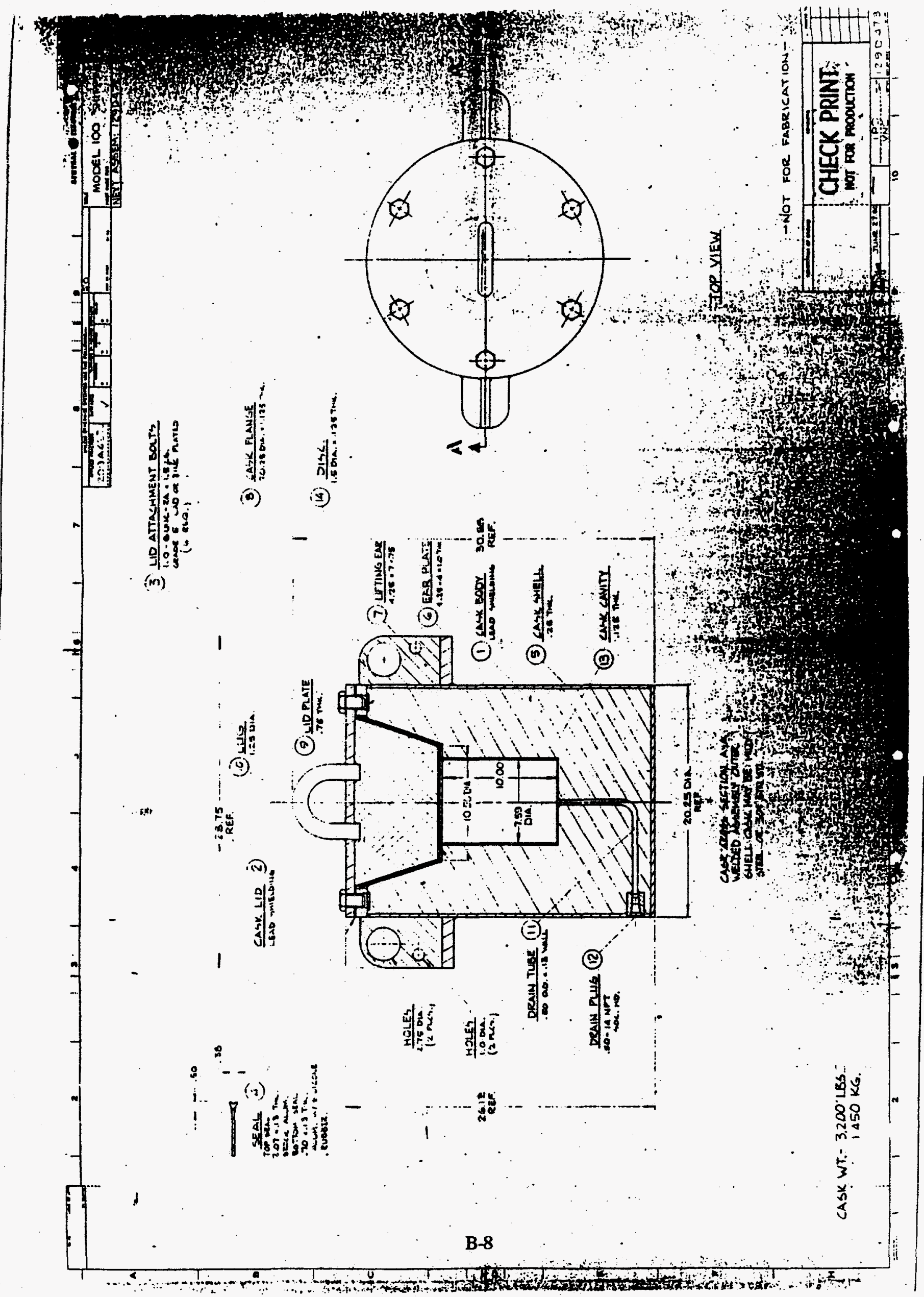


- Prisurer

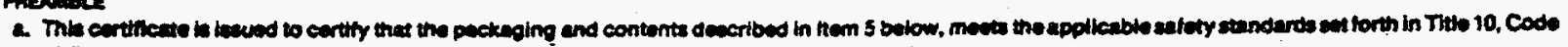
of Fudert Pegulationa, Part 71, "Pactaging and Tranaportition of Radiosetwe Matorial"

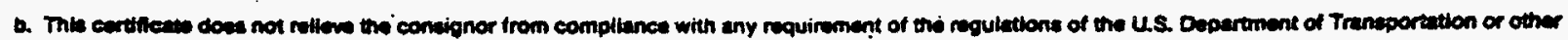

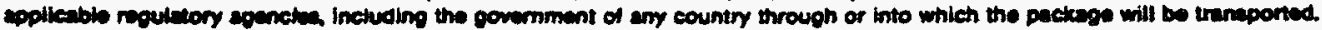

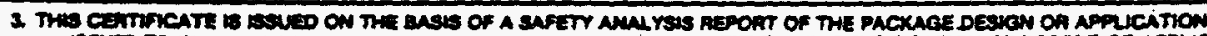

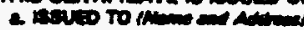

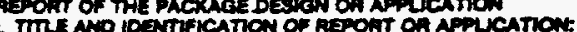

General Electric Company P.0. Box 460 , Vallecitos Road Pleasanton, CA 94566

General Electric Company application dated November 19, 1987, as supplemented.

DOCKET number $71-5925$

4. Condirnons

This centlicate is conditional upon tultiling the requirements of to CFR Part 71, as epplicable, and the conditions specified betow.

5.

(a) Packaging

(1) Model No.: GE-100

(2) Description

A steel encased lead shielded shipping cask. The cask is double-walled steel circular cy finder, 20-1/4-finch dfameter by $260 / 8$ inch high with a central cavtty approximately 7-5/8-inch diameter by 10 inches high. Approxtmately 5-7/8 inches of head surround the central cavity. The cask ts equipped with a cavity dicain line and lifting device. Closure is accomplished by.a gasketed and botted steel lead filled olug. For additional shielding lead, tungsten or uraniumpiners may be inserted in the cask cavity. The maximum weight af the packaging is 4,800 pounds.

(3) Drawings<smiles>C1CC[As]C1</smiles>

The packaging is constructed inaccordance with General Electric Company Drawing Nos. 12904727, Rev. 5; 12904729, Rev. 5; 12904730, Rev. 4; and 12904731, Rev. 1. 
Conomows (continued)

Page 2 - Certificate No. 5926 - Revision No. 15 - Docket No. 71-5926

j. (b) Contents

(1) Type and form of material

(i) Byproduct and irradiated special nuclear material in the form of fuel rods, or plates, fuel assemblies, or meeting the requirements of special form radioactive material; or

(ii) Solid nonfissile irradiated metal hardware and reactor control rods (blades).

(2) Maximum quantity of material per package

Radioactive decay heat nôt tó exceed 400 watts and 500 grams U-235

equivalent mass fissite material. "(U-235 equivalent mass equals U-235 mass plus 1.66 times $U-233$ mass plus 1.66 -times $P u$ mass).

Plutonium in excess of twenty (20) curies per package must be in the form of metal metal alloy or reactor elements,

(c) Fissile Class"

TI

Minimum transport index to be showm on labele :-

Contents $5 \cdot(b)(1)(i)$ :

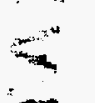

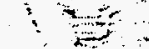

$5 \frac{\sqrt{6}}{2}$

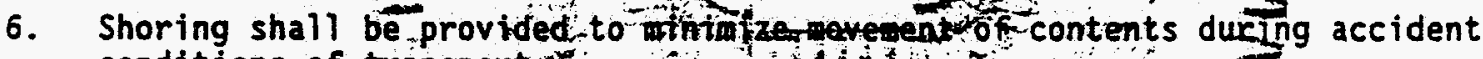
conditions of transporting

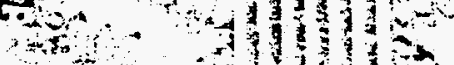

7. At the time of detivery of the loaded pachage to a carrier far transport, the package contents shall be dryand the frissi ematerial unmoderated ( $H$ to $X$ atomic ratio less than 2 ).

8. In addition to the requirements of Subpart $G$ of 10 CFR Part 71 :

(a) The package must be maintained in accordancerwith the maintenance procedures submitted with GE application dated Jangary T8, 1993.

(b) The package must be prepared for shipment and operated in accordance with the operating procedures submitted with GE application dated January 18, 1993.

9. The package authorized by this certificate is hereby approved for use under the general license provisions of 10 CFR $\$ 71.12$.

10. Expiration date: February $28,1998$. 
conortions (continuod)

Page 3 - Certificate No. 5926 - Revision No. 15 - Docket No. 71-5926

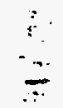

REFERENCES

General Electric Company application dated January 18, 1993.

Supplements dated: March 3, 1993.

FOR THE U.S. NUCLEAR REGULATORY COMMISSION

.

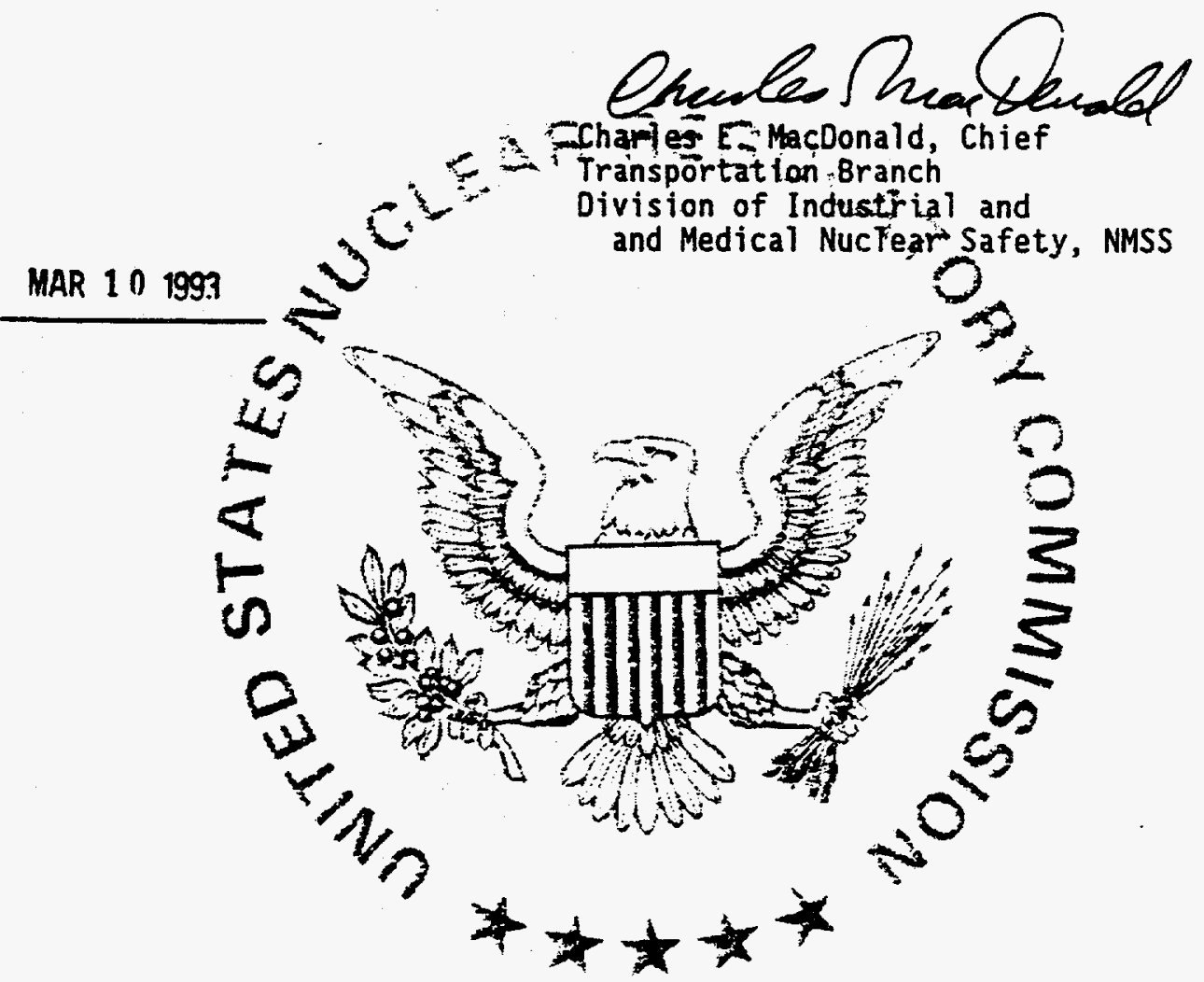


BMI-1

B-12 


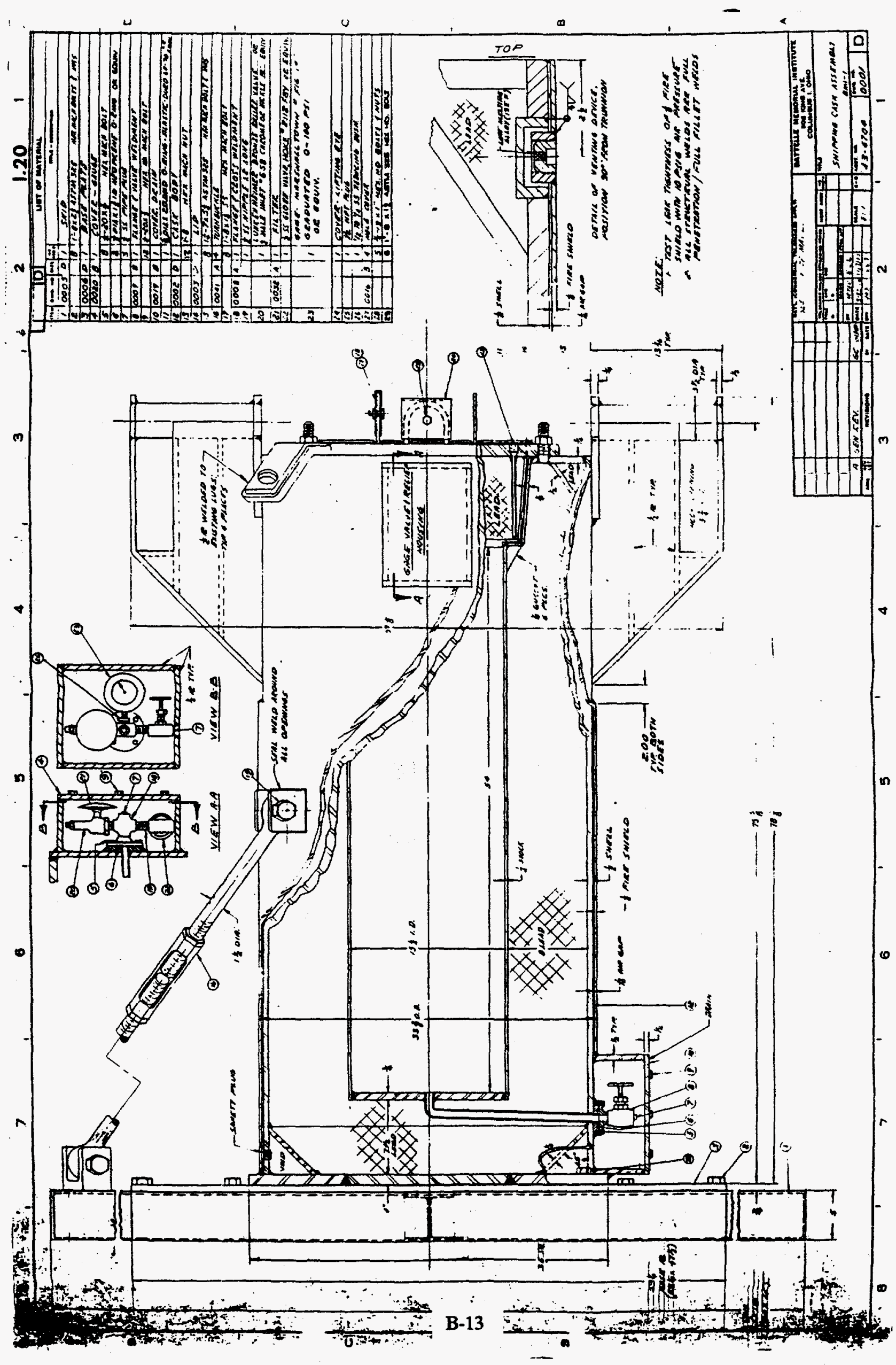




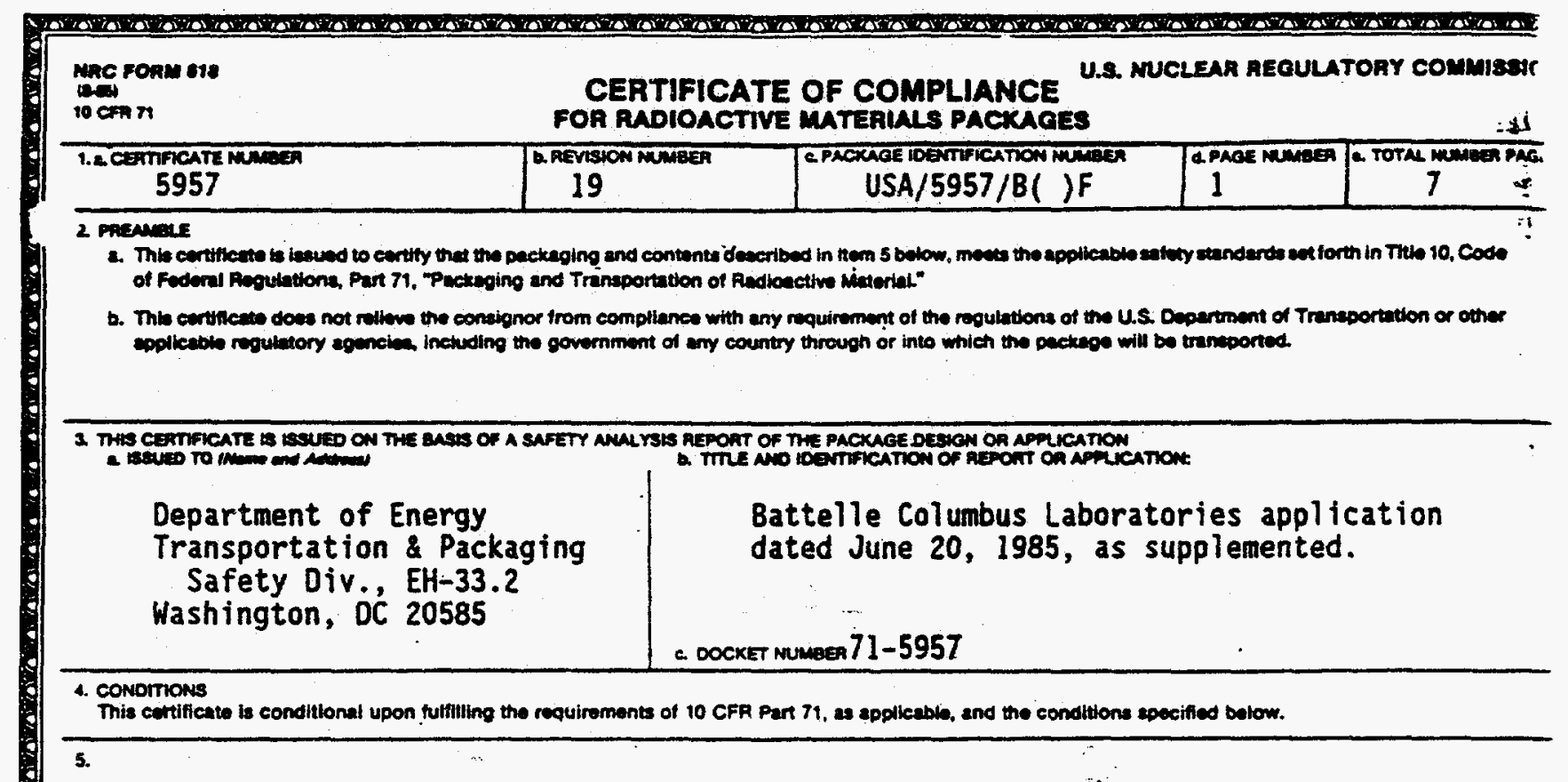

(a) Packaging

(1) Model No.: BMI-1

(2) Description

A steel-encased Tead sbielded shipping cask. The basic cask body is a cylinder 33.37 inches in- dianeter 73.37 inches high formed by two concentric stainless steel shel Ts. whose annular region is filled with lead:- The outer. 1/2-inct thick shell has:20.12-inch thick plate spot welded to it, providing 0 s06 inch thick air gap-insulator. The inner shell ts 15.5 inches inside: diameter by 54 inches inside length. The cask $l$ id is a stainless steel weldmeat having 7,75 inches of lead shielding. "The cask $l$ id is. secured to the cask by twelve steel studs which are welded to the cask body. The caski is provided with a drain line with needle valve and plug, pressure gauge, and a pressure relief valve. The total cask. weight, including maximum contents of 1,800 lbs, is 23,660 lbs.

(3) Drawings

The cask is constructed in accordance with the folluwing Battelle Memorial Institute (BMI) Drawing Nos.: 43-6704-0001, Rev. B; and 41-4409-0003, Rev. B. 


\section{CONDITIONS (continued)}

Page 2 - Certificate No. 5957 - Revision No. 19 - Docket No. 71-5957

5. (a) Packaging (continued)

(4) Product Containers

The various authorized product containers are constructed in accordance with the following Drawing Nos.:

(i) Inner can assembly as shown in BMI Drawing No. 00-000-421, Rev. C.

(ii) Basket Assembly as shown in BMI Drawing Nos. BCL-000-500, Rev. A; BCL-000-501, Rev. A; and 0048, Rev. A.

(iii) Fermi Fuel Element copper casting assembly as shown in BMI Drawing No. K5928-5 00490, Rev. to May 12, 1966.

(iv) Basket Assembly as shown in BMI Drawing-No. 1020, Rev. B (or with alternate spacer shown in CI Drawing No. 334D2193) or GA Drawing No. 9590001 , Rev. A. Failed fuel assemblies must be seal welded in aluminum or stainless steel tubes with wall and end cap thicknesses. of at least 0.015 inch. in. $-2$

(v) Basket Assembly defined by BMI Drawing No. BCL-000-500, Rev. A, $\ldots$ as modiffed by BMI Drawing Nos -00-000-236, Rev. C, and

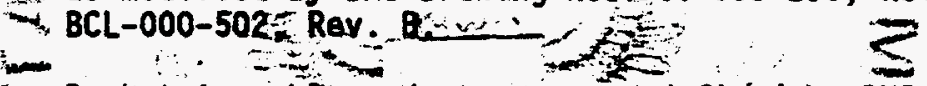

(vi). BasketiAssembly andrstoragefeap deftied by BuI Drawing No.

O 00-000-391, ReV -C, and Atome Intexpationa E, Drawing No. AIHL, S80R 0019-01, Rev. As respectively:-

(vii) Inner can assembly as shown in Union Carbide Corporation Drawing No. 101501, Rev. A.

$\because \rightarrow$

(viii) Basket Assembly as shown in University of Missouri Research Reactor (MURR) Orawing No. 2234, Sheets I through 5, Revision 0.

(b) Contents

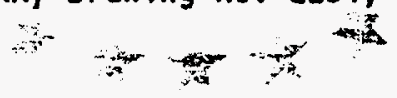

(1) Type and form of material

(i) Intact irradiated MTR- or BRR-type fuel assemblies containing not more than 200 grams U-235 per assembly prior to irradiation. Uranium may be enriched to a maximum $93.5 \mathrm{w} / 0$ in the U-235 isotope. Active fuel length shall be approximately 25 inches.

(ii) Intact irradiated Enrico Fermi Core. A fuel assembly containing not more than $4.77 \mathrm{kgs}$ U-235 prior to irradiation. Uranium may be enriched to $25.6 \mathrm{~W} / 0$ in the $U-235$ isotope. 
conotrions (continued)

Page 3 - Certificate No. 5957 - Revision No. 19 - Docket No. 71-5957

(iii) Greater than Type A quantity of radioactive material which may include uranium enriched in the $U-235$ isotope, $U-233$, plutonium, as metal, oxides, or compounds which are thermally stable up to $600^{\circ} \mathrm{F}$. Plutonium in excess of twenty $(20)$ curies per package must be in the form of metal, metal alloy, or reactor elements.

(iv) Greater than Type A quantity of byproduct material meeting the requirements of special form radioactive material.

(v) Greater than Type A quantity of byproduct material in normal form as metal, oxides, or compounds which are thermally stable up to $600^{\circ} \mathrm{F}$.

(vi) Irradiated Triga. Type füel assemblies described in Section 6.6 of the application (pp. 6-23 through 6-27).

$$
-\infty
$$

(vii) Irradiated S8DR fuel elements $0.56-i n c h$ oD by 18.7 inches long by 0.010 -inch wall thickness of Hastelloy=N. The fuel material is Wxt" fully enriched in U-235.<smiles>[CH]1C=C[CH+]C1</smiles>

(vili) Entact trradiate CP-5 fuel assemplies containing not more than - I76 gransi $U-235$ per assenbly, petop-to irradiation. Uranium may

- we enrichedito a maximum 93 w foy in the U-235-isotope. Active

- fuel lengthshall bei28.5 inches.

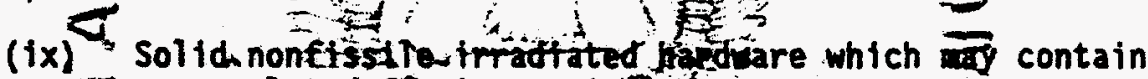

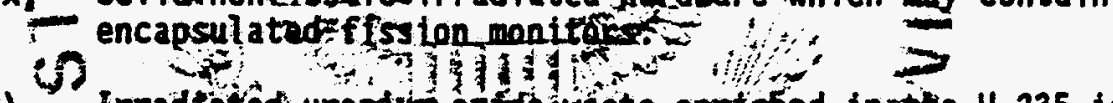

(x) Irradtated urantin oxforiwste emitefed in the U-235 isotope up to a nombial 93 w/osutinct fs then ally stabte up to $800^{\circ} \mathrm{F}$.

(xi) Fradiated uranium enriched inthe U-235-tsotope meeting the requirements of spectal form radioact ive-material.

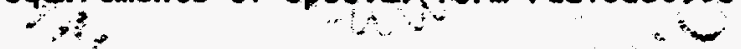

(xii) Intact, trradiated MURR fuel assembites containing not more than 775 grams of U-235 per assembix.prior to irradiation. Uranium may be enriched ta a maxim $93: 5 \mathrm{w} / 0$ in the U-235 isotope. Active fuel length shall be 24 inches.

(xiii) Intact irradiated MITR-II fuel assemblies containing not more than a nominal 510 grams of U-235 per assembly prior to irradiation. Uranium may be enriched to a maximum $93.5 \mathrm{w} / 0$ in the U-235 isotope. Active fuel length shall be approximately 24 inches.. 


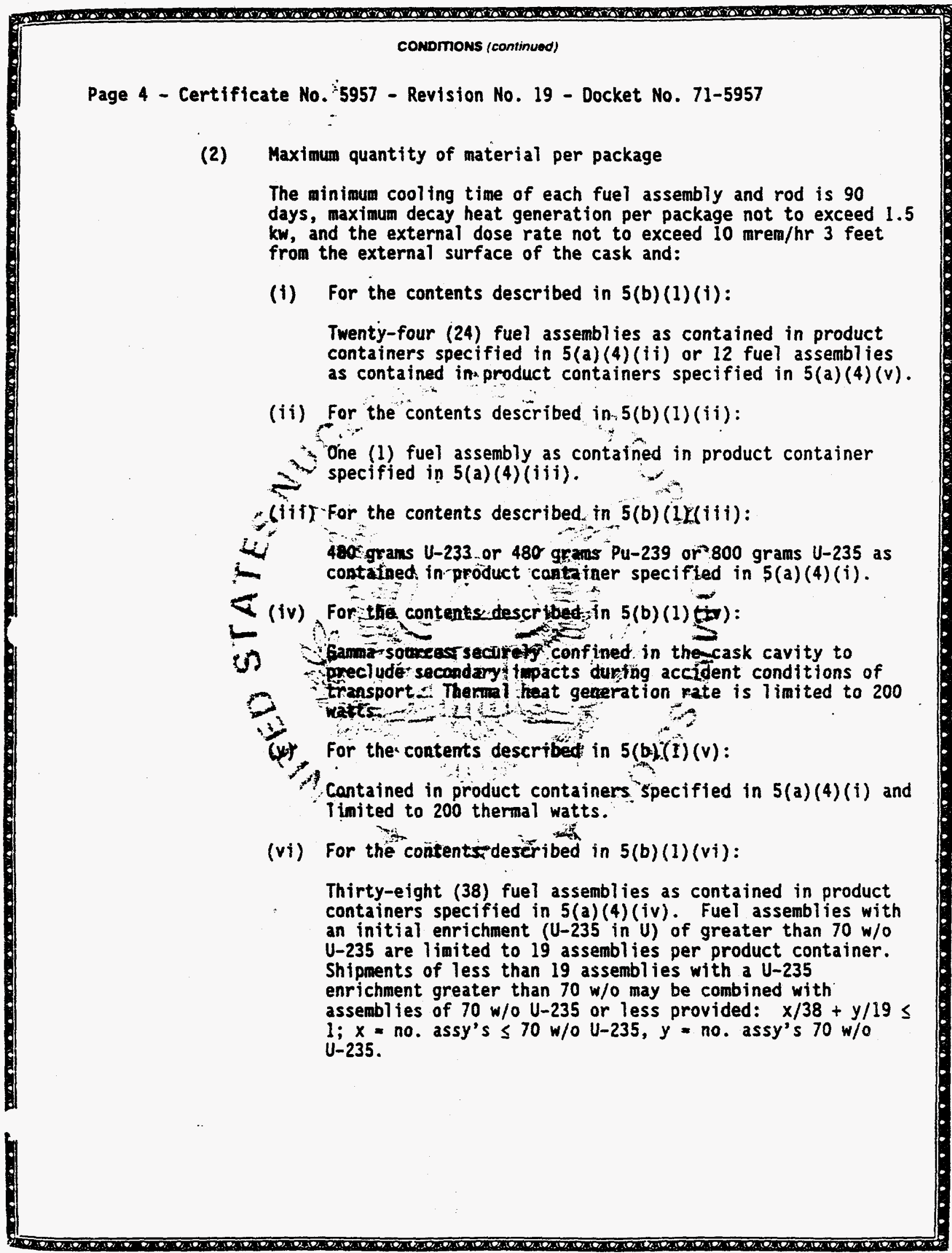


Page 5 - Certiftcate.No. 5957 - Revision No. 19 - Docket No. 71-5957

(vii) For the contents described in $5(b)(1)(v i i)$ :

Twenty-four (24) fuel elements per can and six sealed cans per basket as described in $5(a)(4)$ (vi). Each of the six cans may contain up to $818 \mathrm{~g} \mathrm{U}-235$ and $158 \mathrm{~g}$ hydrogen. The cask is 1 imited to $4.908 \mathrm{~kg} \mathrm{U-235}$.

(viii) For the contents described in 5(b)(1)(viii):

Twelve (12) fuel assemblies.

(ix) For the contents described in $5(b)(1)(i x)$ :

Thermal heat generation-rate is limited to 200 watts.

(x) For the contents described in $5(b)(1)(x)$ :

Twenty-four (24) containers each irited to 352 grams U-235 as contained in product containers specified in

$5(a)(4)$ ( $v i i)$. The decay heat per container is 1 imited to 20 watts. The containers-must be leak-tested in accordance with Union Carbfide Corporation letter dated November 17, 1980.

(xi) For the contents descrbed in. $5(b)(1)\left(x^{i}\right)$ :

Tuenty-four (24) capstes each timited to 100 grams $U-235$. 1.t.

(xii) Fongthe contents described fn $5(b)$ (n)(xii):

Eight (8) fuel assembliestas contained in the product container specified in $5(\mathrm{~d})(4)(v f i i)$. The maximum burnup -1s, 150 MND/Assembly and the minimum cooling time of each fuel assembiy is 150 days. The maximum radiation source term is 400,000 curies. ...ts

(xiii) For the contents described in $5(b)(1)(x i i j)$ :

Eight (8) fuel assemblies, contained in the product container specified in 5(a)(4)(viii). The maximum decay heat per package is 200 watts.

(c) Fissile Class

(1) Class I
$I$ and III

For the contents specified in 5 (b) (1) (iii) and limited in $5(b)(2)(i i i)$. 


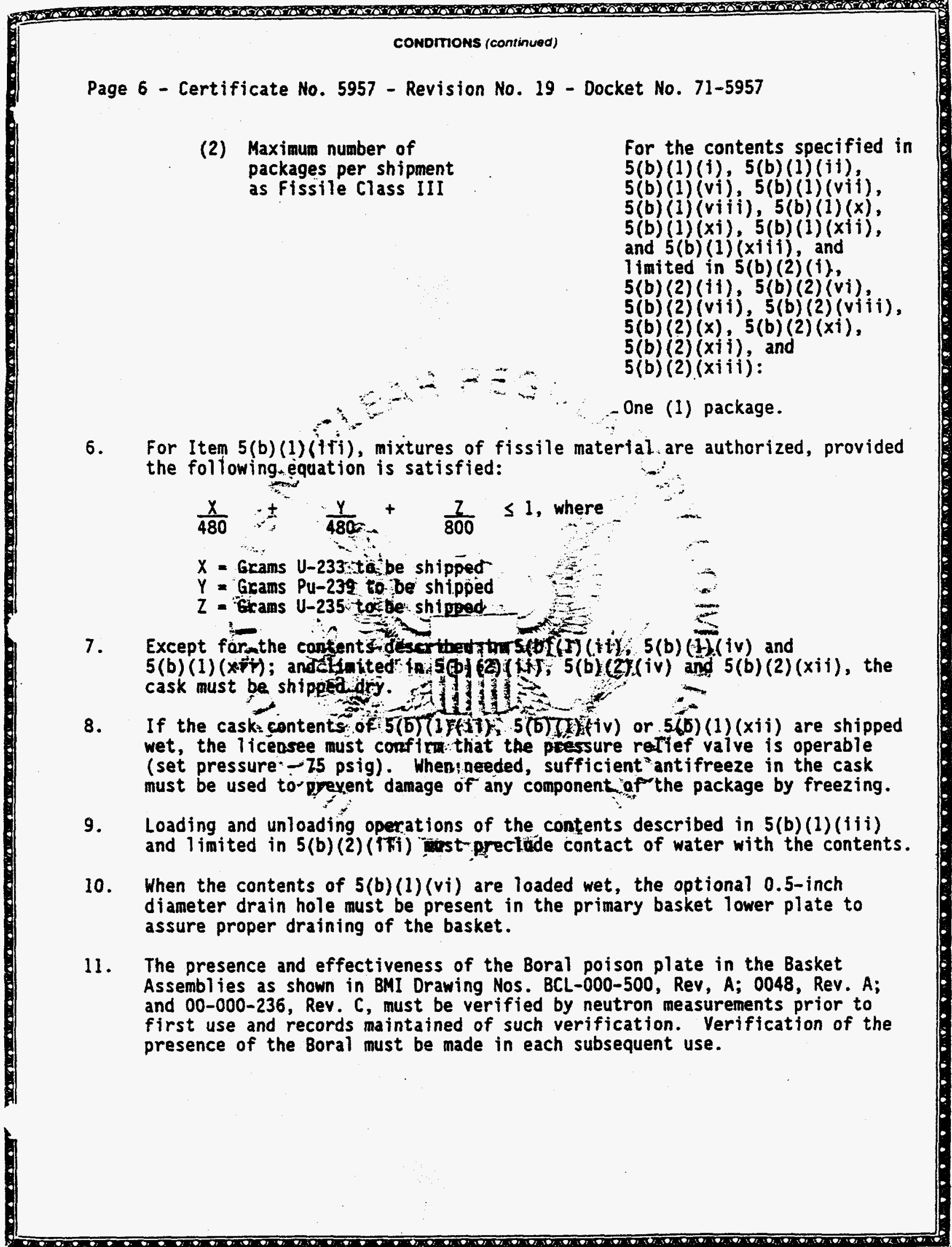




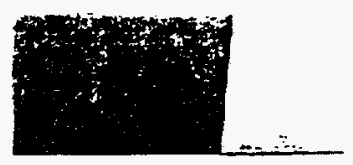

Page 7 - Certificate No. 5957 - Revision No. 19 - Docket No. 71-5957

12. Contents $5(b)(1)(i)$ and $5(b)(1)(x)$ may be mixed provided the sum of the product containers and fuel assembltes does not exceed 24.

13. For contents described in $5(b)(1)$ (xiii), removable spacers may be used in each section of the basket to limit axial movement of the assembilies.

14. Contents must be securely confined in the cask cavity to preclude secondary. impacts'during accident conditions of transport.

15. Prior to each use, adequacy of containment vessel must be demonstrated by performance of the leak test described in Section 7.1.1.1 of the application.

16. Gaskets and seals (cask and fuel canister) must be replaced at least every 12 . months or earlier if visibleodegiadation occurs.

an $=-3$ it

17. In addition to the regurrements of Subpart G af 10 CFR Part 71:

(a) The packages shall be prepared for shipment andoperated in accordance with the-0prerating Procedures of Chapter 7 of the application.

(b) The packaging must meet the Acceptance Jests and Maintenance Program of Chapter 8 of thesplication.

18. The packar authorized this cat fidate

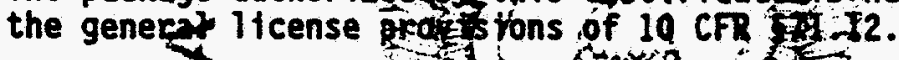

19.
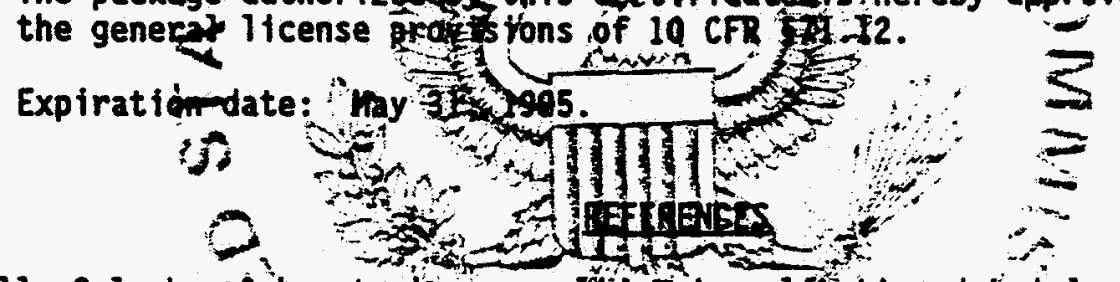

Battelle Columbus fiboratomes-consert trued applfeation dated June 20, 1985.

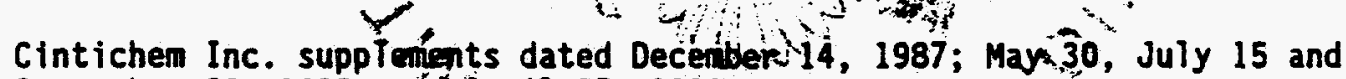
September 28, 1988; and April 17, 1990.

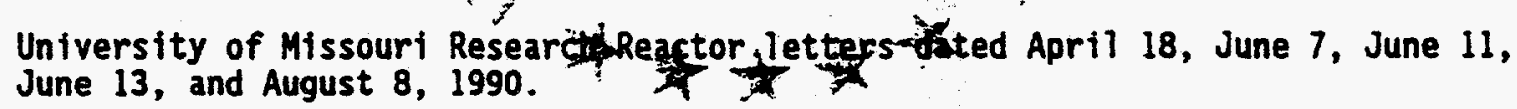

Massachusetts Institute of Technology Nuclear Reactor Laboratory letters dated October 19, 1992, and January 11, 1993.

FOR THE U.S. NUCLEAR REGULATORY COMMISSION

JAN 151993

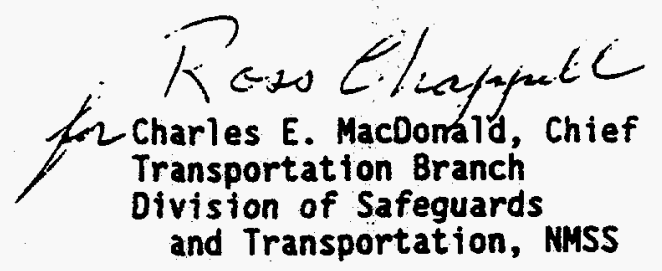

Date: 
FSV-1

B-21 


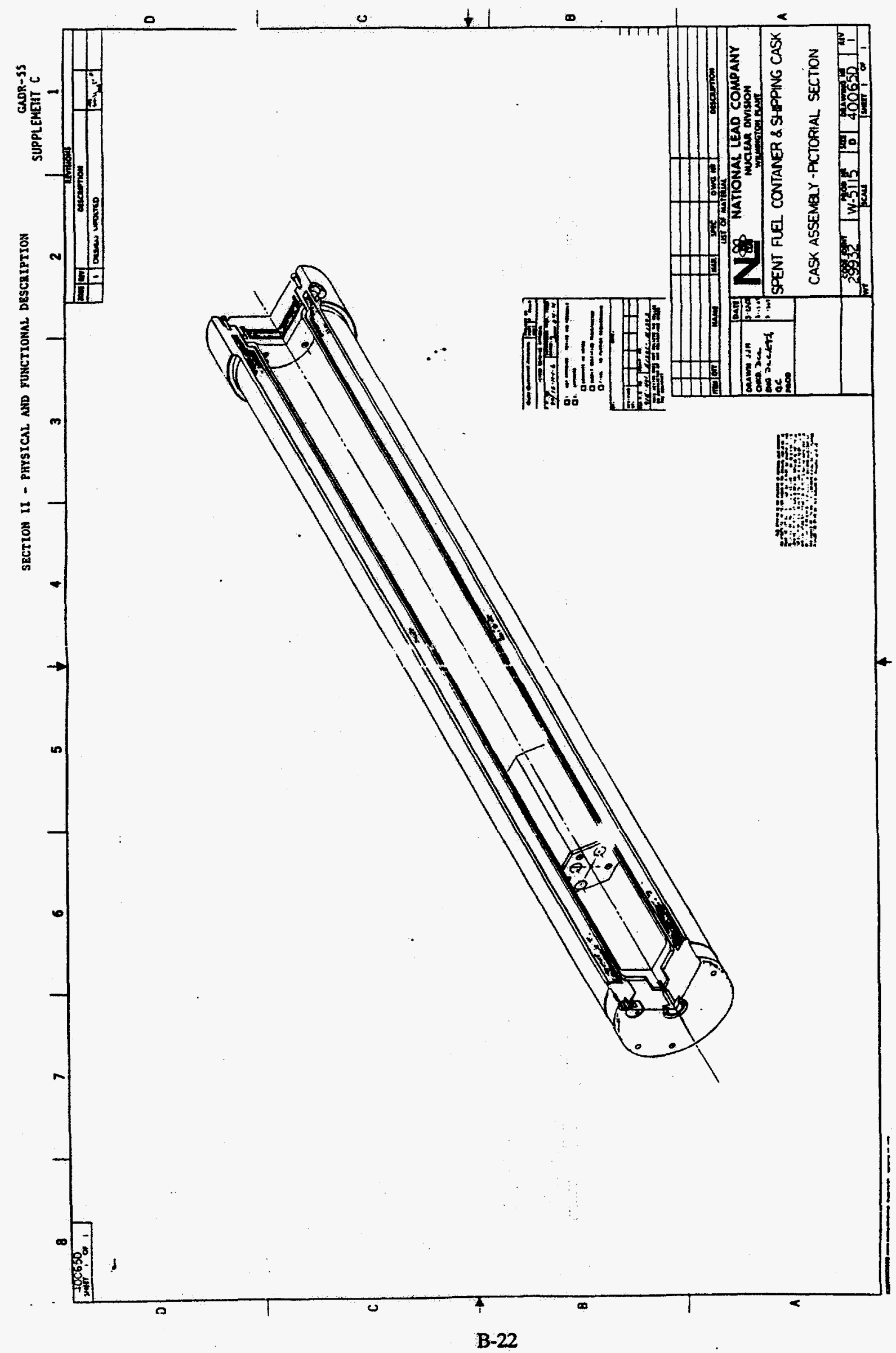




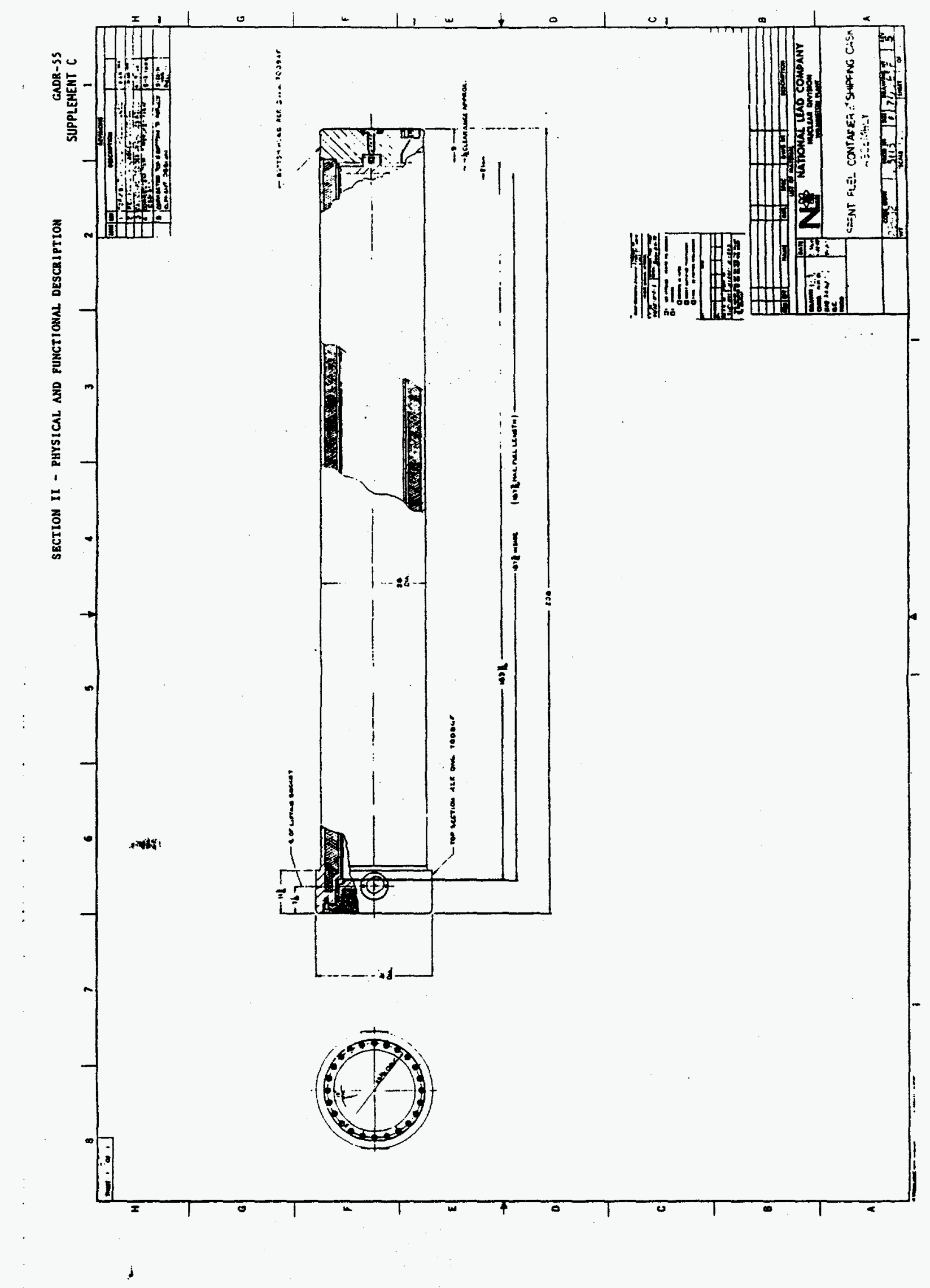

B-23 


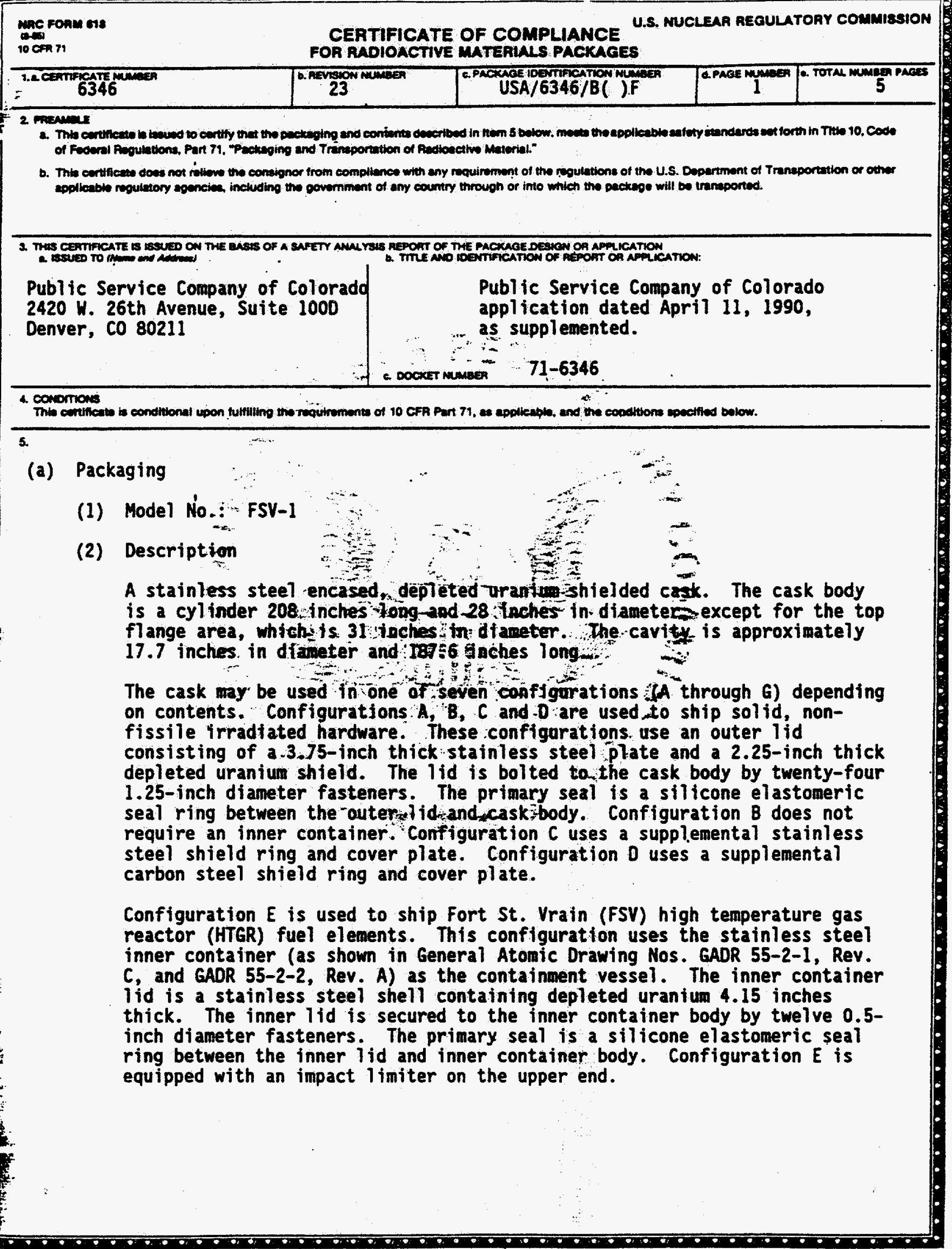




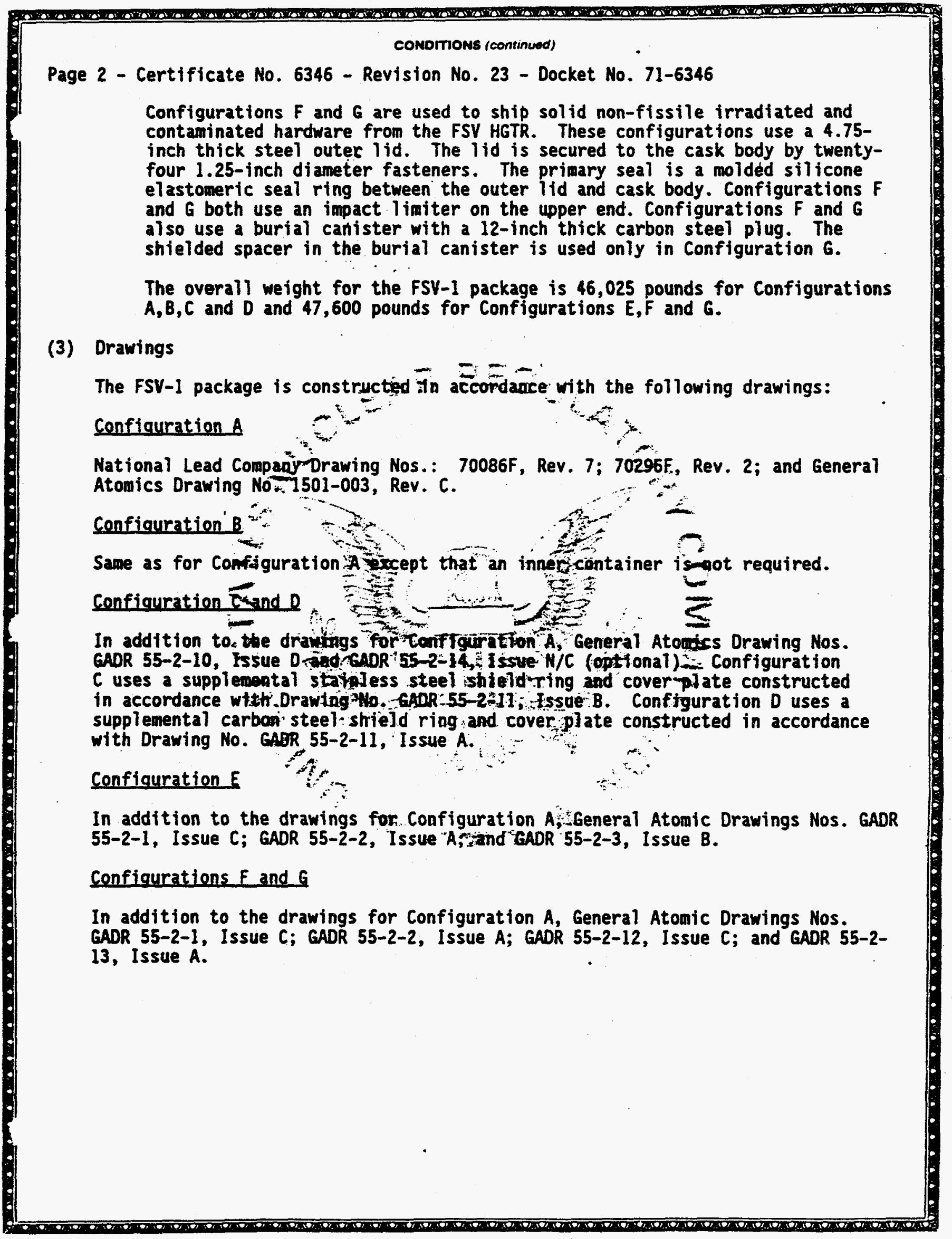


CONDITIONS (continued)

Page 3 - Certificate No. 6346 - Revision No. 23 - Docket No. 71-6346

5. (b) Contents

(1) Type and form of material

(i) Irradiated fuel elements consisting of graphite body, hexagonal in horizontal cross section, apploximately 31.2 inches high and 14.2 inches across the flats. Prior to irradiation, each fuel element contains thorium and uranium enriched to a maximum of 93.5 $w / 0$ in the $U-235$ isotope, or

(ii) Solid, irradiated and contaminated hardware, which may include fissile material, provided the quantity of fissile material does not exceed a Type A quantity and does not exceed the mass linits of 10 CFR $\$ 71.53$, and neutron source components, $\ldots$ r

(iii) Solid, nonfissile irradiated and contaminated hardware which has been removed from the Fort St. Vrain High+Jemperature Gas Cooled Reactor and the surface contamination does not exceed 51 millicuries per package. . "

$$
-\infty
$$

(2) Maximum quantity of material per package

Decay heatinot to exceed $4.1 \mathrm{kw}$ and:
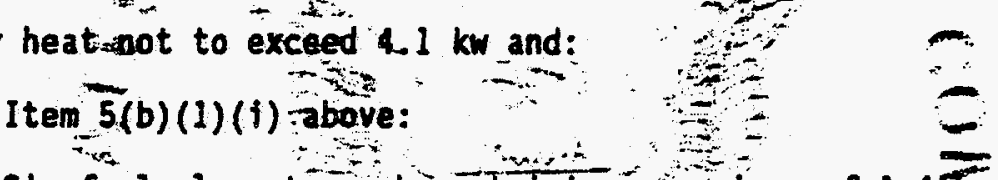

Six fuel elements ench containing of $1.4 \overline{\mathrm{kg}}$ of enriched uranium, having a thorium/uranimuratio greater than 8.1:1 and weighing approximately 300 lbs.. The uross weight of the cask cavity contents, inciading the componeatispacers; inner container and irradiated foel zelements shal 1 not exceed 4,430 pounds. Contents must be shipped in Configuration $E$.

(ii) Item $5(b)(1)(t i)$ above:

The gross weight of the cask cavity contents, including appropriate component spacers, liners, inner containers, shield rings and solid, nonfissile irradiated and contaminated hardware shall not exceed 3,720 Ibs. Contents must be shipped in Configuration $A, B, C$ or $\mathrm{D}$.

(iii) Item 5 (b)(1)(iii) above:

The gross weight of all of the cask cavity contents, including burial canister and spacers, with or without supplemental shielding shall not exceed 4,430 pounds. Contents must be shipped in Configuration $F$ or $G$. 
conomows (continued)

Page 4 - Certificate No. 6346 - Revision No. 23 - Docket No. 71-6346

5. (b) (2) Continued

(c) Fissile Class

III

Maximum number of packages per shipment

One (1)

6. As needed, appropriate component späcers must be used in the cask cavity when shipping the contents described in paragraph $5(b)$ to limit movement of contents during shipment.

7. For transport of the contents of Item (b)(1)(ii) in Conftguration $D$, the dose rate measured on the surface of the package must not exceed $200 \mathrm{mr} / \mathrm{hr}$. For the purpose of this requirement, the surface of any personnel barrier may not be considered the surface of the package.

8. The Model No. FSV-1 cask be wrapped with reinforced plastic when shipping the contents described in Item $5(b)(1)$ (ii) or (iii) provided the heat generation rate does not exceed 500"watts. The applicable requirements_of 10 CFR $\$ 71.87$ must be satisfied prior towrapping the cask.

9. Use of packaging Fabricated after August 31, 1986 is not authorized.

10. In addition to the requirewents of subpart 6 ofia CFR Parti 7 :

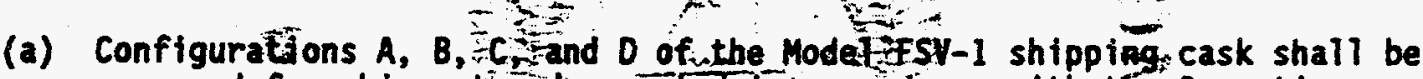
prepared for shipment andraperated inizecondance with the Operating

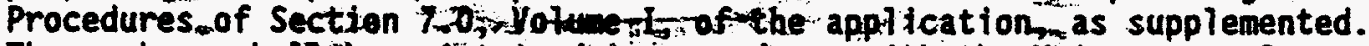
The package shall the amaintajned in accordance with the Waintenance Program in Section 8.0. Volume i, of the appitcation, as supplemented.

(b) Configurations $E, F$, and $G$ of the Hodel FSV $I$ shipping-cask shall be prepared for shipment and operated in accordance with the Operating Procedures of Section 7.0, Volume II, of the application, as supp] emented. The package shall be maintaibed in accordance with the Maintenance Program in Section 8.0, Volume II, of the application, as supplemented.

(c) The main flange seals must berreplaced-within (12) months prior to any use of the packaging and must be replaced if inspection shows any defect.

(d) The silicone O-ring on the inner container primary plug in Configuration $E$ must be replaced within the twelve (12) months prior to any use of the packaging and must be replaced if inspection shows any defect. 
Page 5 - Certificate No. 6346 - Revision No. 23 - Docket No. 71-6346

11. The package authorized by this certificate is hereby approved for use under the general license provistons of 10 CFR $\$ 71.12$.

12. Expiration date: April 30, 1996.

\section{REFERENCES}

Public Service Company of Colorado application dated April 11, 1990.

Supplements dated: June 28, and November 2, 1990; March 27, 1991; and March 30, 1992.

Date: JUN 231992
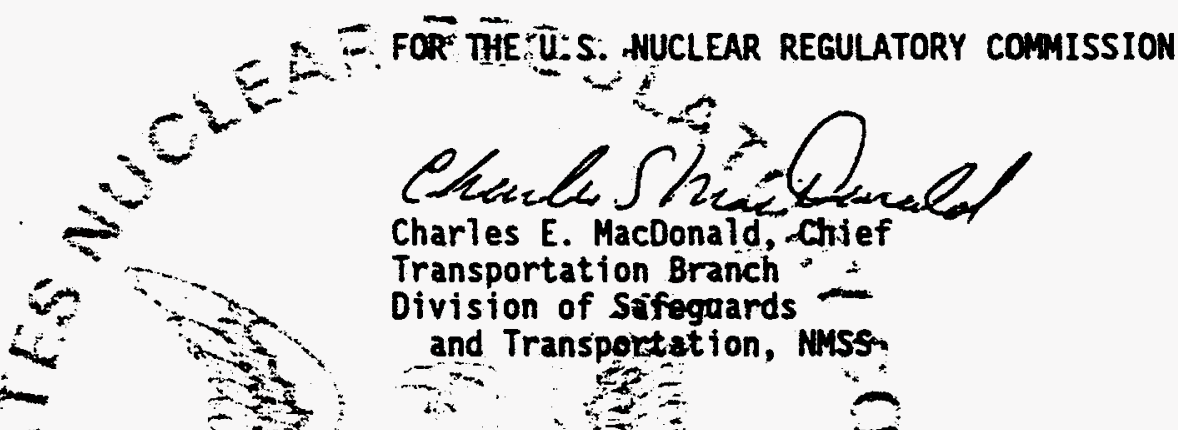

Charles E. MacDonald, Chjef

Transportation Branch "

Division of Safegaards

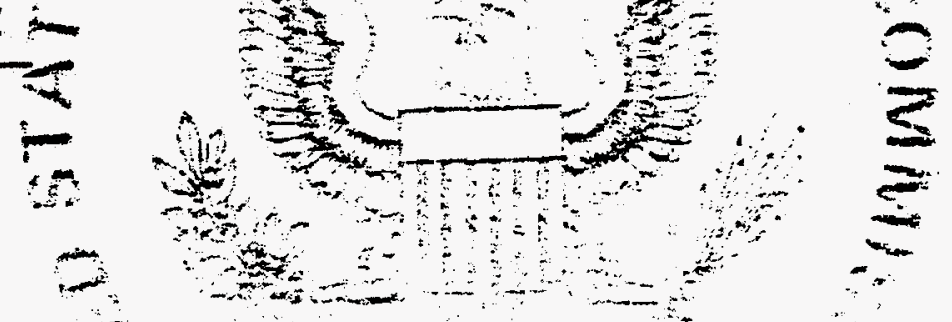

$-$

$+4$

in 
NLI-1/2

B-29 


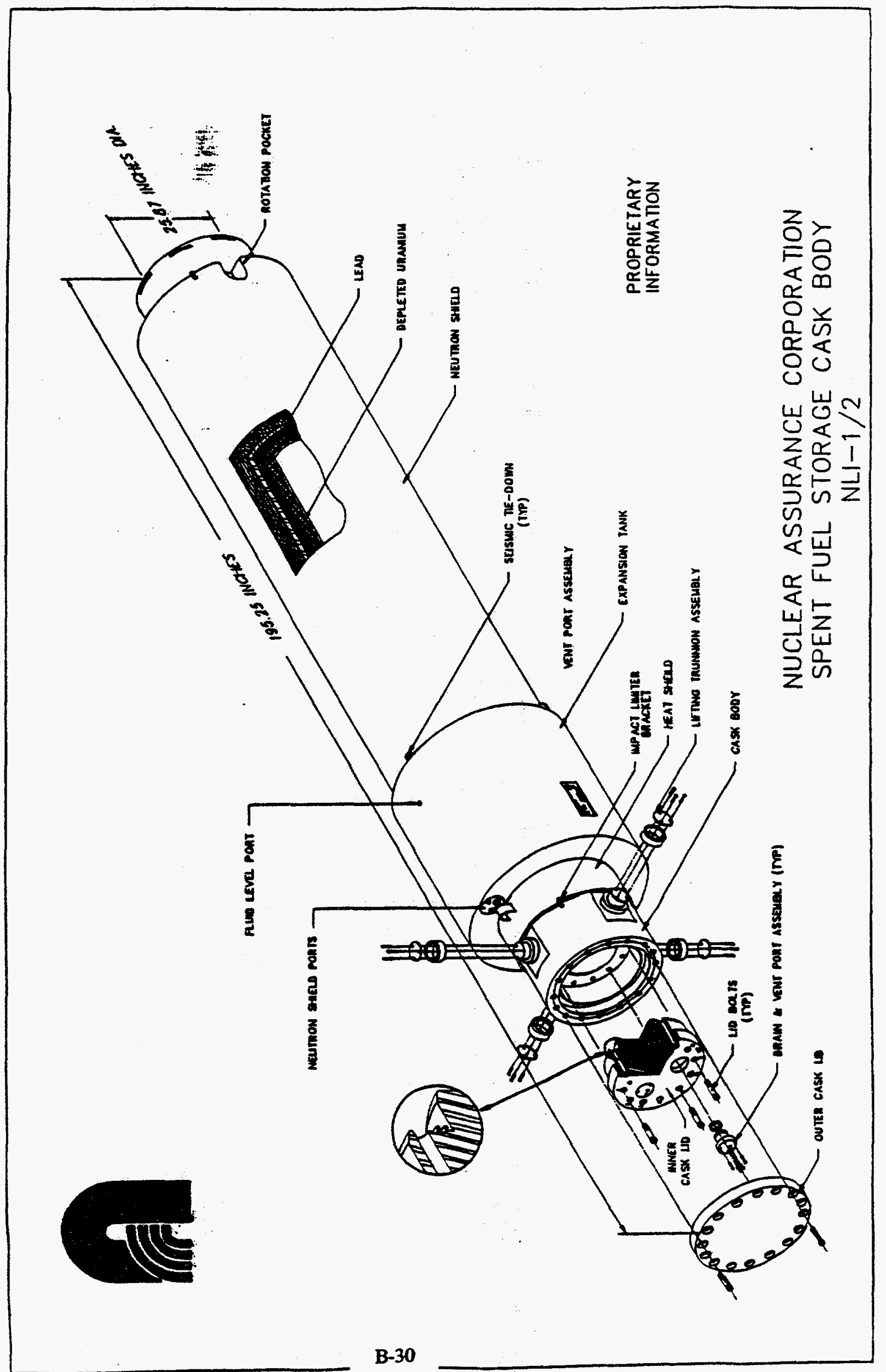


NLI-1/2

Owner

Type

Manufacturer/Vendor

Capacity

Intact Spent Fuel Assemblies

Cans Consolidated Fuel Rods

Other Configuration

Weight (tons)

Loaded

Empty

Design Heat Rejection (kW)

Shape

Dimensions

Overall Length (in)

Cavity Length (in)

Cavity Diameter (m)

Inner Shell Wall Thiciness (in)

Depleted U Wall Thickness (in)

Lead Shield Wall Thickness (in)

Outer Shell Wall Thickness (in)

Neutron Shicld Tank Thickness (in)

Inner Container Wall Thickness (im)

Materials of Construction

Cask Body

Neutron Shield

Sides

End

Basket

Cavity Atmosphere

Operating Temperature (F)

Operating Pressure (psig)

Outside Surface Dose (mrem/hr)
Nuclear Assurance Corporation

Legal Weight Truck Shipping Cask

NL Industries Inc.

1 PWR; 2 BWR

2 FWR; 4 BWR

Metallic Fuel

23.1

223

10.6

Cylindrical

19525

47.125

178

13375

0.5

275

2125

0.875

5.25

0.25

SSDepleted U/Lead/SS

Water \& Ethylene Glycol

None

SS and Alnminum

He, Air if heat is less than $25 \mathrm{~kW}$

1010 (maximum fuel cladding temperature)

117 (maximum)

53 (maximum)

Licensing Status - Initially licensed by the USNRC in 1974; can be used to ship either intact or consolidated LWR fuel.

Comments - Casks have separate baskets for PWR and BWR fuel. There are five casks curreatly in existence.

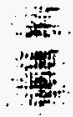




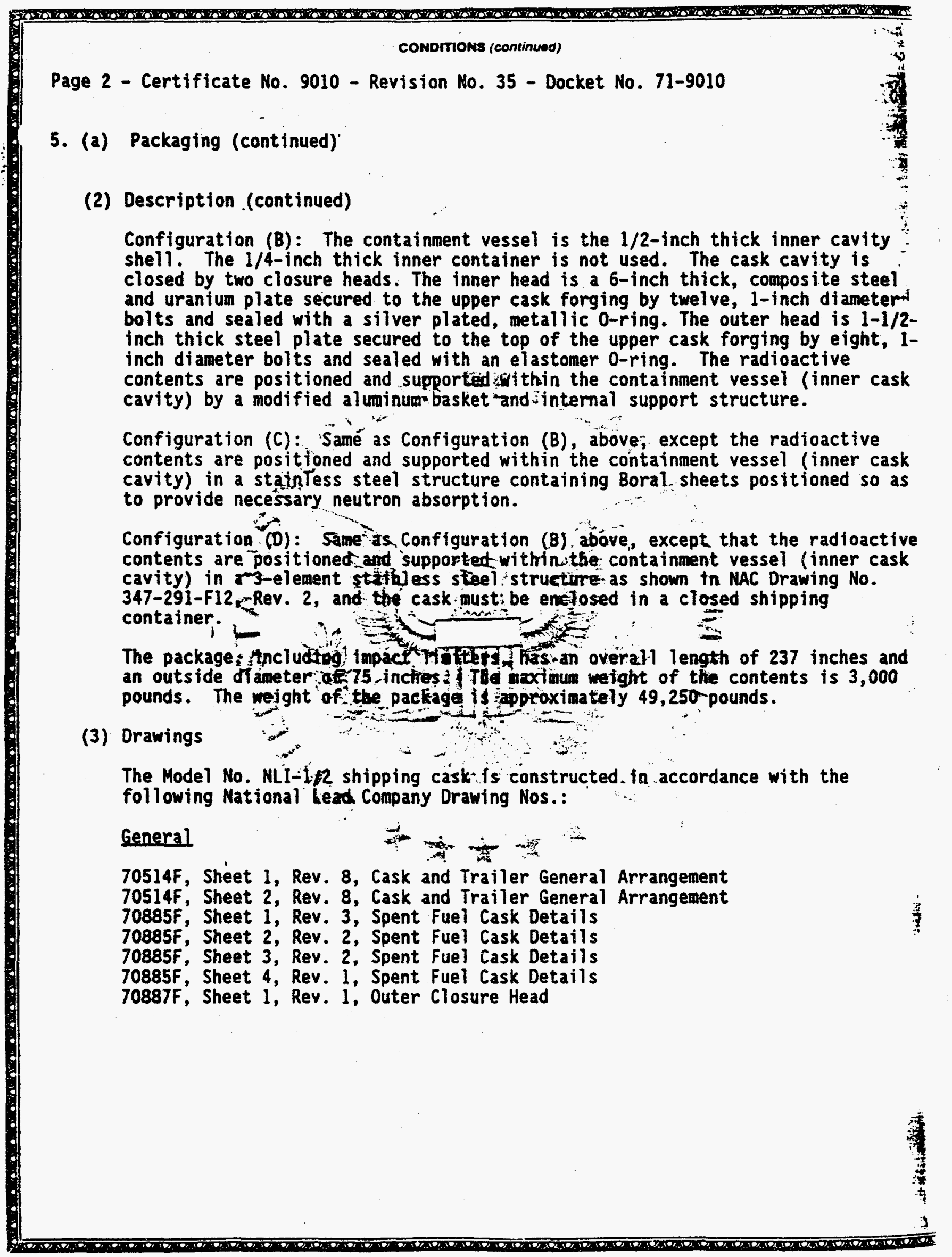




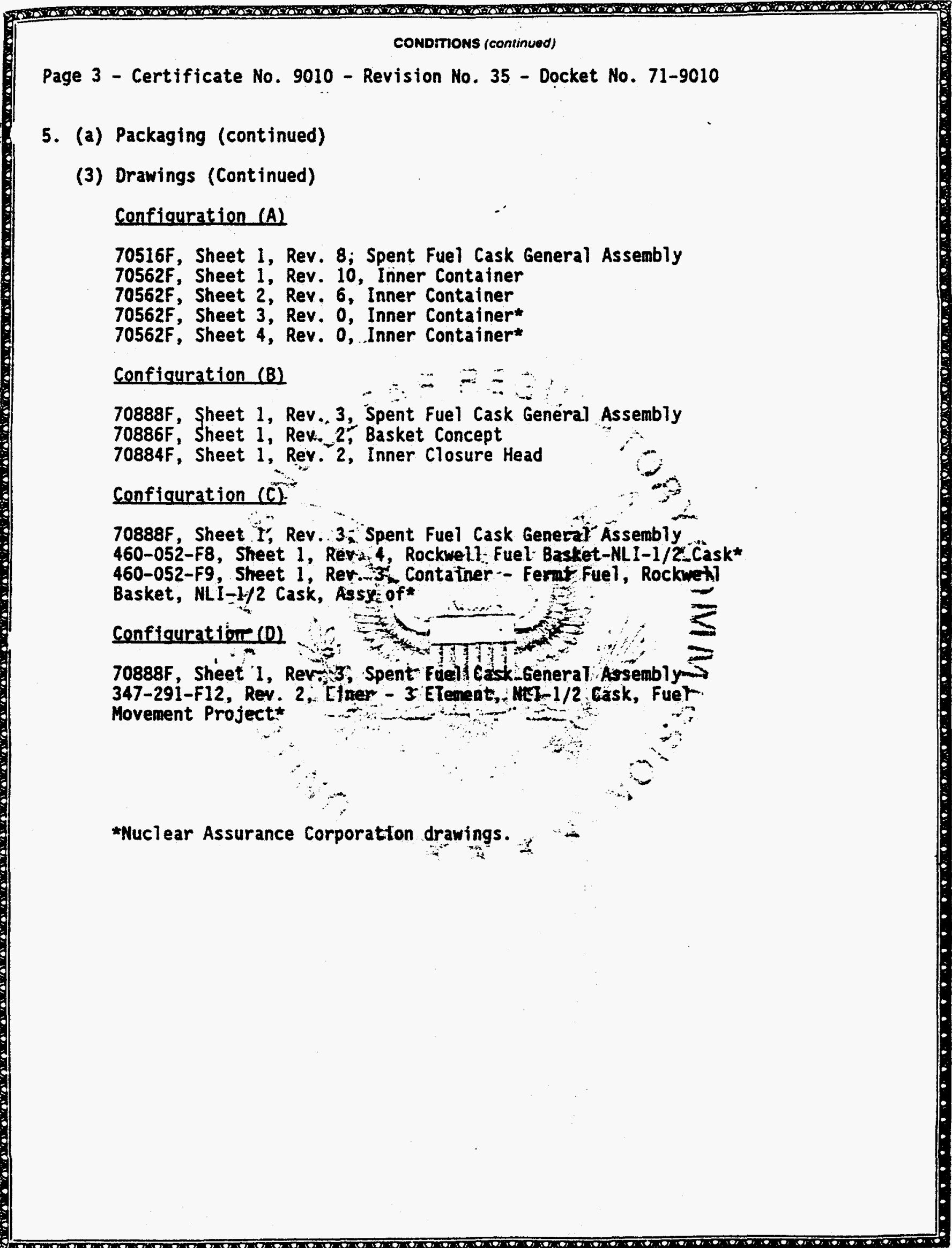


Page 4 - Certificate No. 9010 - Revision No. 35 - Docket No. 71-9010

j. (b) Contents

(1) Type and form of material

(i) Irradiated PWR or BWR uranium oxide fuel assemblies of the following specifications:

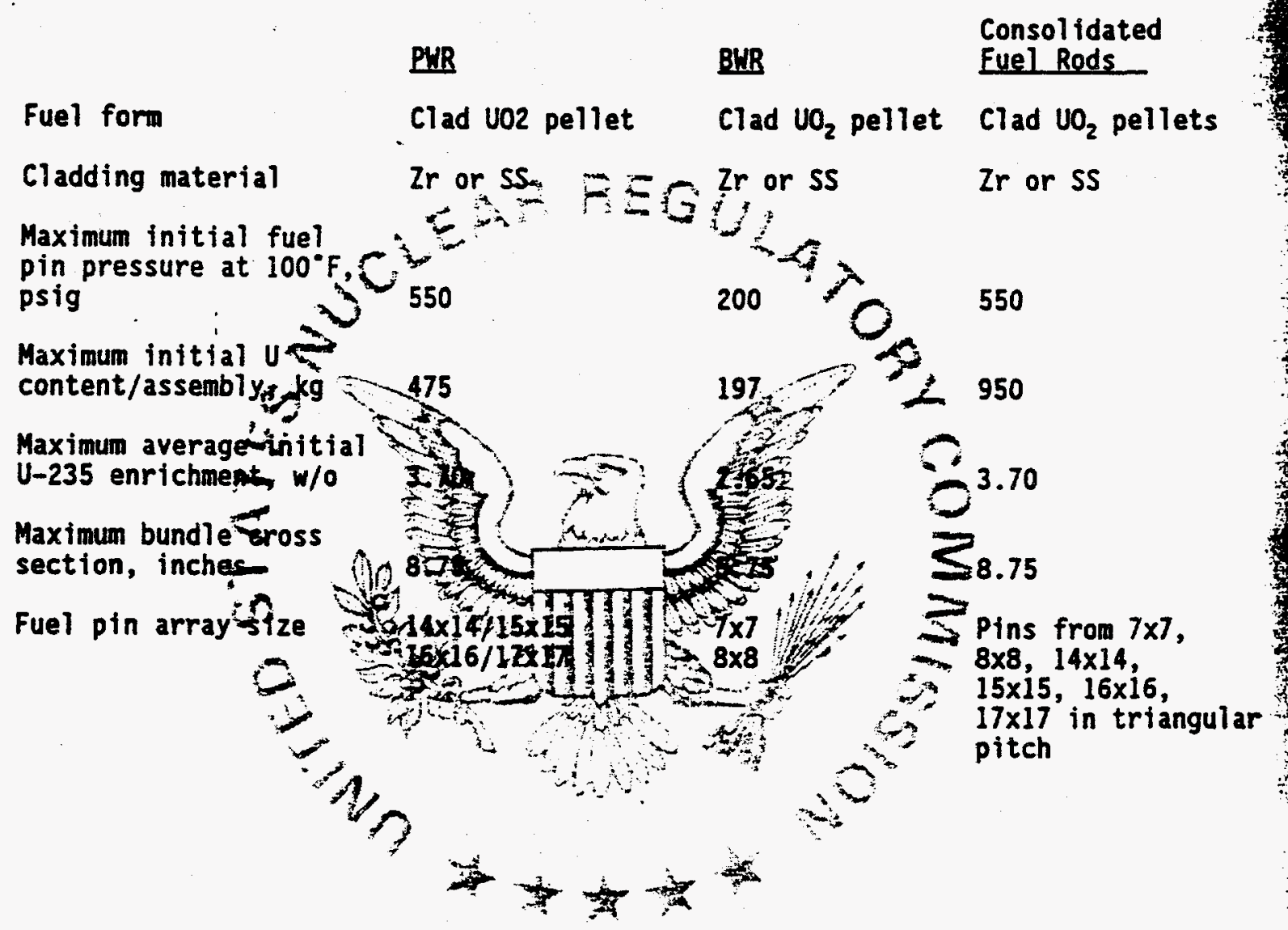


Page 5 - Certificate No. 9010 - Revision No. 35 - Docket No. 71-9010

(b) (1) (i) (continued)

Maximum active fuel

length, inches

PWR

BWR

Consol idated

144

145.25

Fuel Rods

Maxinum specific power, $\mathrm{kw} / \mathrm{kgU}$

40

27

144

Maximum average burnup,

MWD/HTU

$$
40,000^{* *}=34,000
$$

40,000

Maximum decay heat, $k$ 10.6 .

Minimum cooling

days

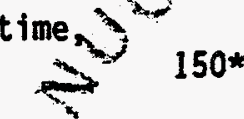

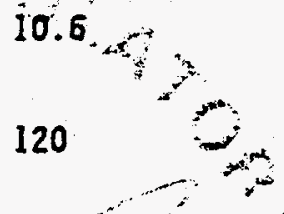

0.6

4,380

The PWR type assembly, may be shipiged either with or witfout burnabie poison rods or control rods.

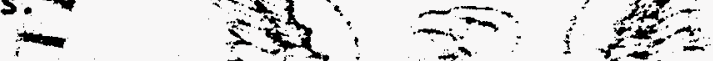

* Four (4) fuel rodsimay have a inititim cooling time of 120 days. -

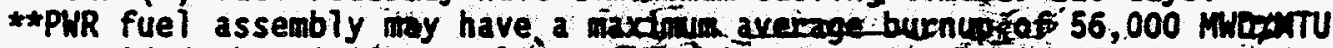

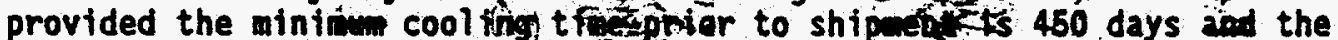

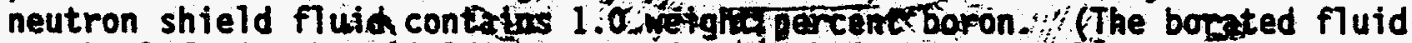
may be left in the shielding tanks danthg the shfpment of other contents.)

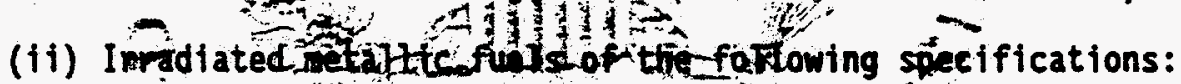

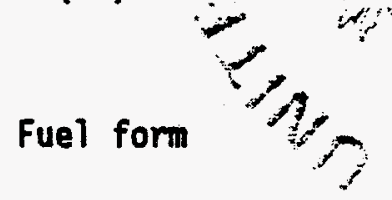
Cladding Material
Max. initial $u$ content/assembly, $\mathrm{kg}$ Fenmi-1 Uranium-Molybidenum
alloy pins $\frac{2}{2 r}-4$ - $18.7 /$ assy. $300 / 16$ assy. cask load

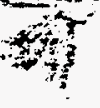
Max. avg. initial U-235 enrichment, $w / 0$
26.0

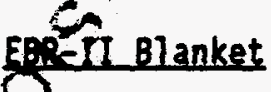<smiles>C1[C+]2C[As]1C2</smiles>
3 Oranium metal cylindrical slugs
Aluainum containers 292/container
$0.21(3.88 \mathrm{~kg}$
Pu/Canister) 


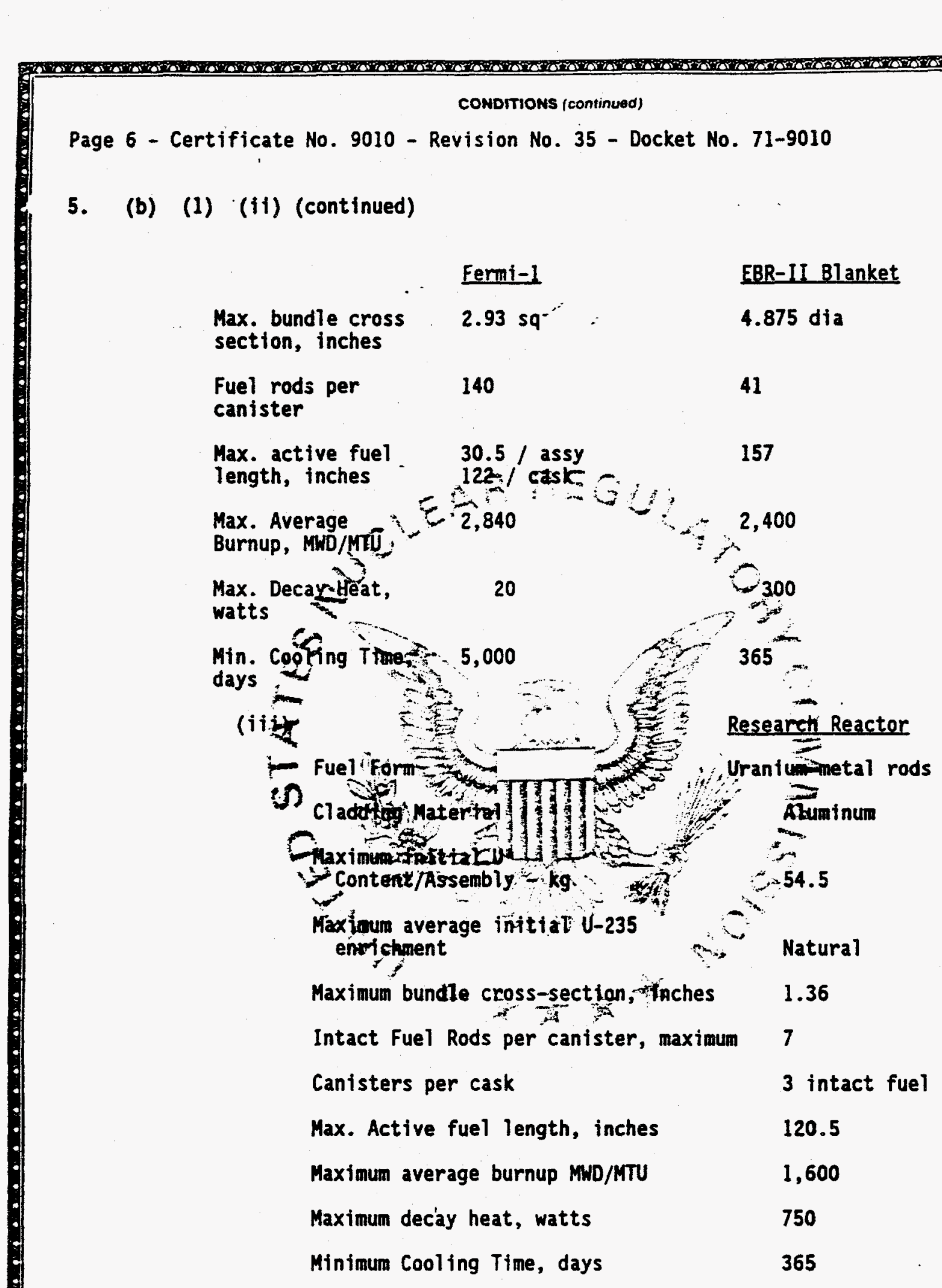




\section{CONDTrions (continued)}

Page 7 - Certificate No. 9010 - Revision No. 35 - Docket No. 71-9010

j. (b) (1) (Continued)

(iv) Irradiated PWR* or BWR uranium oxide fuel rods of the following specifications:

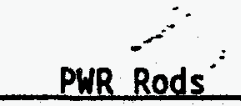

Fuel form

Cladding material
$\mathrm{Clad} \mathrm{UO}_{2}$ pellets

Zr or SS
BWR Rods

Clad $\mathrm{UO}_{2}$ pellets

Zr or SS

Maximum initial fuel pin

pressure at $100^{\circ} \mathrm{F}$, psig

Maximum initial $U$

content, $\mathrm{kg}$

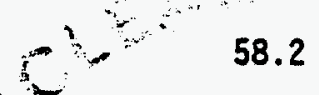

58.2

200

Maximum average initial

U-235 enrichment, wo

Maximum bundle cross

section, inches

Maximum active füel

Maximum specific power

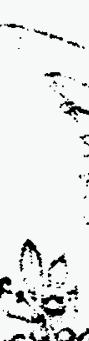

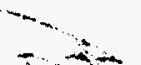

\subsection{5}

$=\infty$

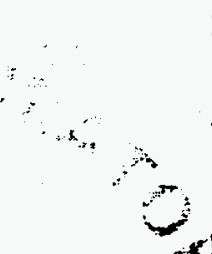

75

$\mathrm{kw} / \mathrm{kgU}$<smiles>[C]#[C]</smiles>

Maximum average bürnup, -

MND/MTU

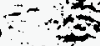

Maximum decay heat

Maximum decay heat, :

Minimum cooling time, days:
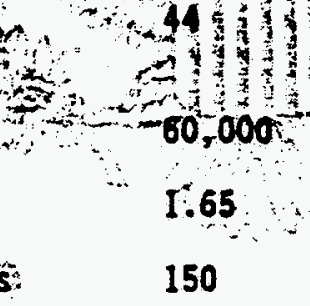

\section{I.65}

150

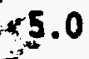

$\pm$

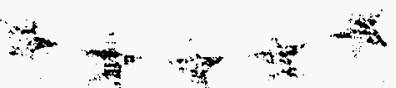

* For the shipments of irradiated PWR fuel rods, the neutron shield fluid must contain 1.0 weight percent boron (the borated fluid may be left in the shielding tanks during the shipment of other contents). . 


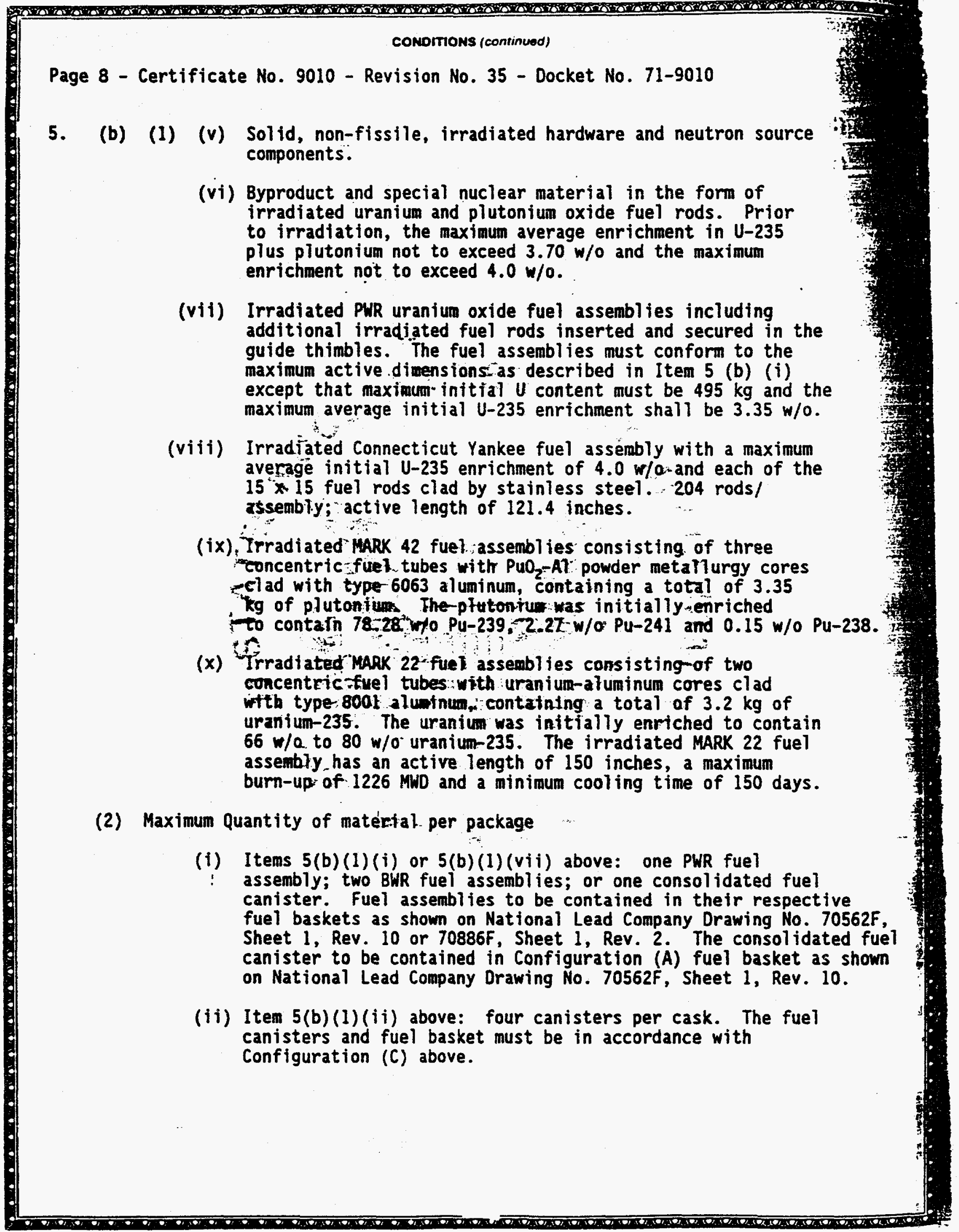


Page 12 - Certificate No. 9010 - Revision No. 35 - Docket No. 71-9010

20. The package authorized by this certificate is hereby approved for use under the general license provisions of 10 CFR $\$ 71.12$.

21. Expiration date: March 31, 1996.

\section{REFERENCES}

Nuclear Assurance Corporation application dated November 1, 1990.

Supplements dated: March 7 and 18, August 21, October 2 and 23, 1991; and April 14, 1992.

FOR THE.U.S. NUCLEAR REgULATORY COMMISSION

Date:

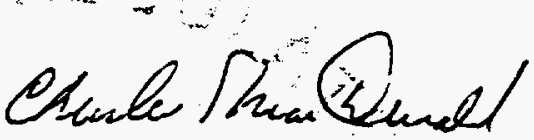

Charles E. MacDonald; Chief

Transportation Beanch $:$

Division of Safeguards

and Transportation, NMSS.

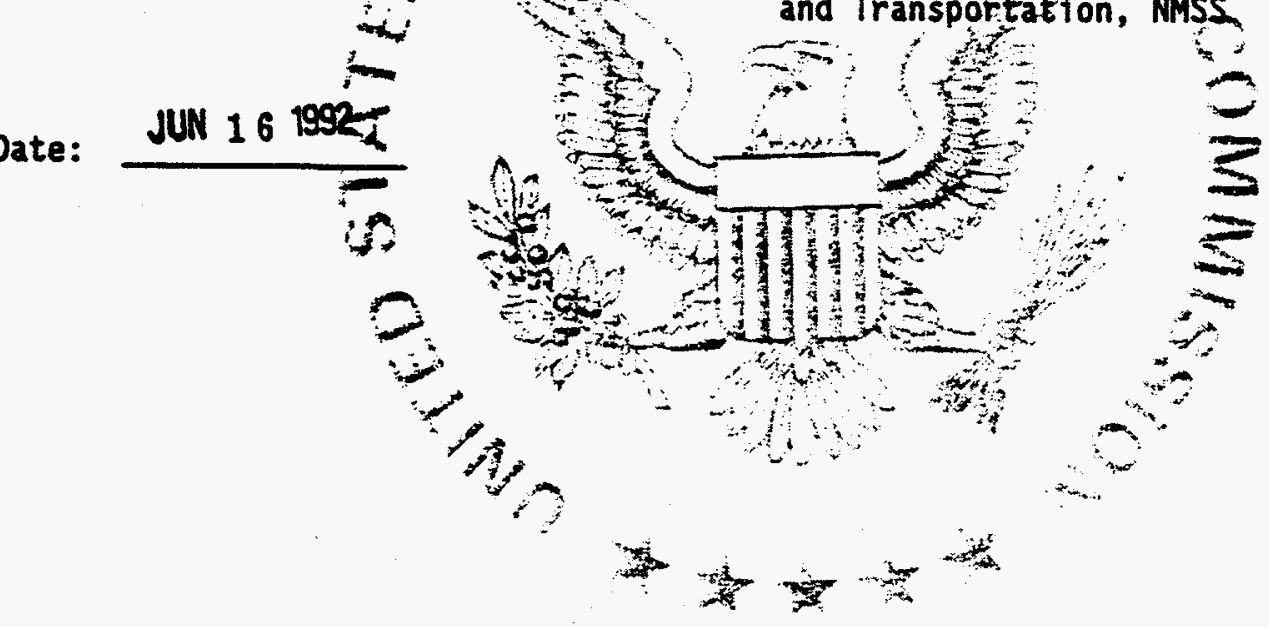


NLI 6502 
Drawing Not Available 


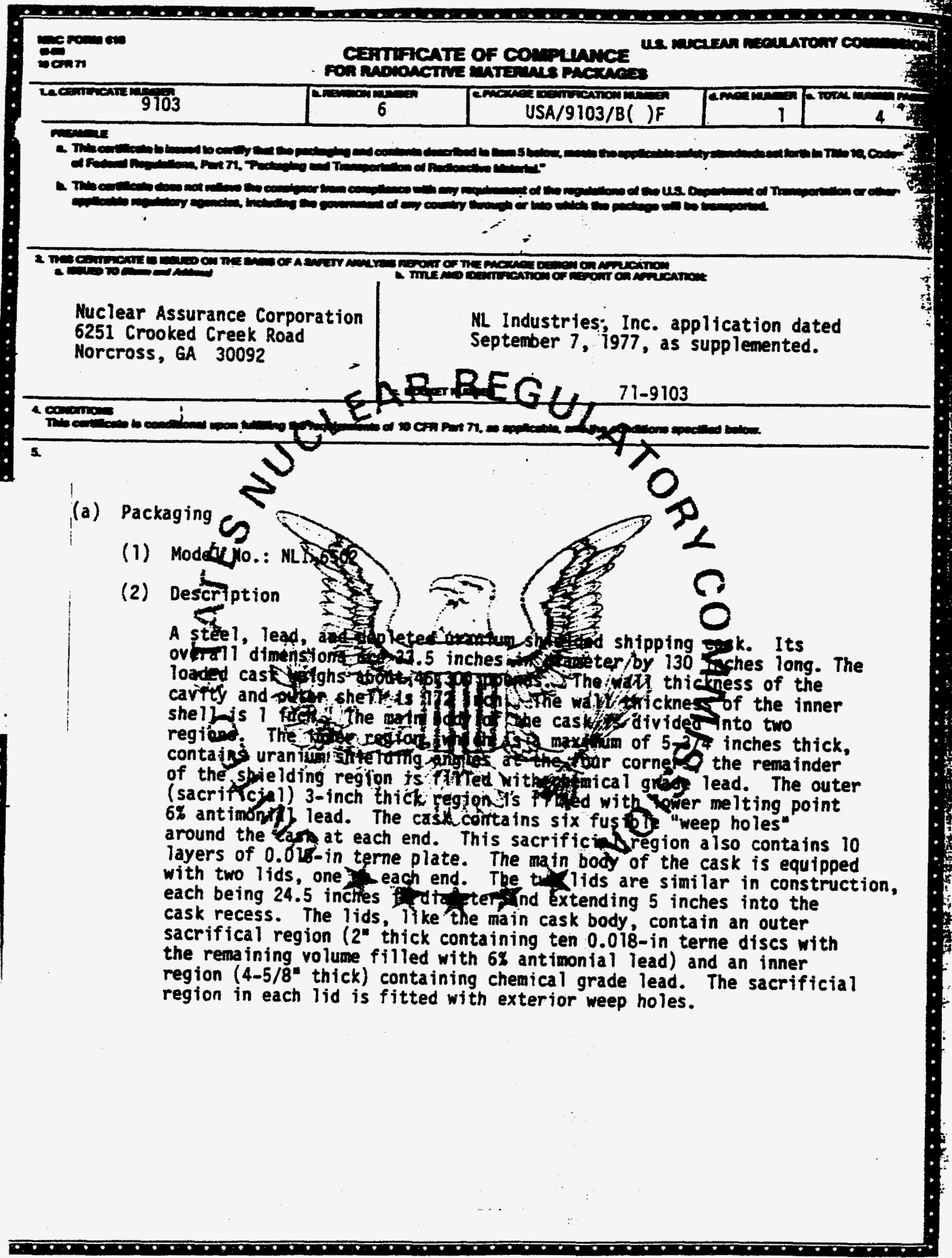

ML Industries; Inc. application dated 6251 Crooked Creek Road Norcross, GA 30092

September 7, 1977, as supplemented. 
Page 2 - Certificate No. 9103 - Revision No. 6 - Docket No. 71-9103

5. (a) (2) Description (Cont'd)

One lid contains a $1 / 2$-inch drain line and vaive. The other lid contains two, 1/2-inch lines for a pressure relfef valve and the other line is capped.

The fuel rods are contained within an approximate 11-1/2-inch square by 128-1/2-inch long opemwork basket constructed of stainless steel angle and strap. Theobstistras full-length lid along one side, for

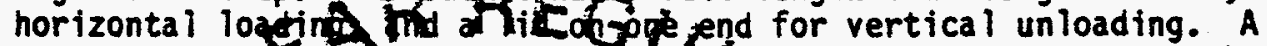
vertical, stailpess steel-clad Boral poison plate divides the basket into two $f y M-$-iength halves, and two pasial poison plates are incorgorgted into the sides of the basket?

A thith screen enclosure surrounds the package in transport to serve as a sumshade and prevent inadvertent use of 5. Wuctural parts of the palage, ethex than the tie-down devicfs for seculing the package to tof vehichioling transport.

L<smiles>C1=C[C@H]2C[C@H]1C2</smiles>

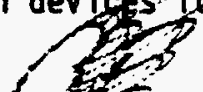

The cask rests

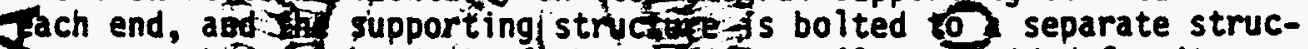
(3) ture

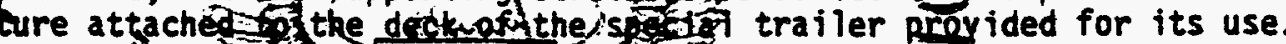

(3)

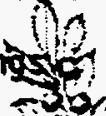
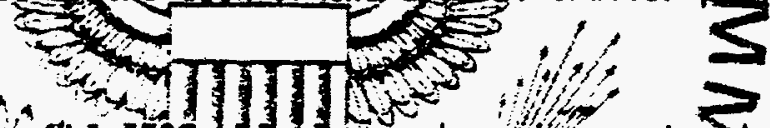

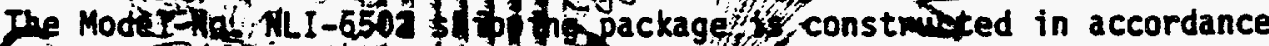

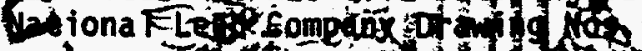

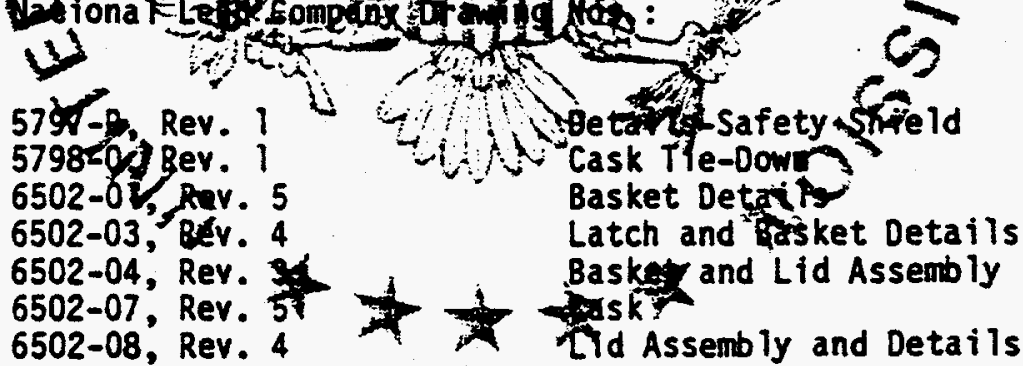

5. (b) Contents

(1) Type and form of material

Irradiated a luminum-uranium alloy fue 1 rods enriched in the U-235 isotope with the following specifications: 
Page 3 - Certificate No. 9103 - Revision No.: 6 - Docket No. 71-9103

5. (b) (1) (Cont'd)

$\underline{\text { MRU }}=-$ NRX and Miscellaneous* $^{*}$

Maximum U-235 content per rod $495 \quad 550$ prior to irradiation, $g$ (1.8 per $\mathrm{cm}) \quad(4.0$ per $\mathrm{cm})$

Minimum average burnup, * 45

Minimum decay $2=120$

* May be a nixture of NRX fuel rods, NRX füel. rod element sections in accon, bundles of SLOWPOKE fuel elements in a can and sections of fast neutron fuel tubes and plugs in a caing

(2) Maxjmumiquantity of material per package

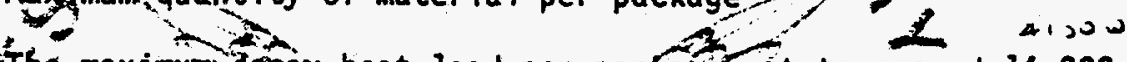
the maximun decax heat load per paekage not to exceed $14,000 \mathrm{Btu} / \mathrm{hr}$; fand $9.9 \mathrm{~kg} \mathrm{U-235}$ in the-form of URU fuei rods or $15.4 \mathrm{~kg} \mathrm{U-235}$ in the form of NRX fuef rods or misce I Taneous SLOWPOKE e Tements, fast neutron fubes and phogs.

(c) Fisstite class

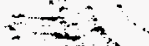
$+3$<smiles>C1#CC#CCCCCC#C1</smiles><smiles>CC#CC#CC#CCC#CC</smiles>

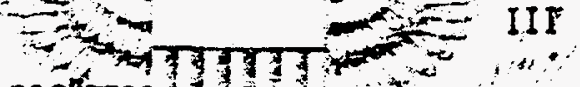
shim number of packages of 13 per spipment (n)

6. Poison inspection and loading of tuet rods mustbe subject to the following<smiles>C1CCC2(CC1)CCCC2</smiles>
one (1) $=$ conditions:

(a) Poison inspection and loading of fuel rods in accardance with Section VIIB.2.(B.)(3.Y (pp. VII-10 thru VII-II) of the app Heation, and

(b) The package must notbe loaded with a mass which exceeds $75 \%$ of critical, determined by extrapolatfor tockerosireciprocal count rate.

7. Dummy fuel rods consisting of empty aluminum pipes must be installed in every fuel rod position not occupied with fuel.

8. The cask cavity must be dry (no free water) when delivered to a carrier for transport. 
Page 4 - Certificate No. 9103 - Revision No. 6 - Docket No. 71-9103

9. The cavity pressure relief valve must be_tested at set pressure every twelve (12) months.

10. Additional contents in accordance with Nuclear Assurance Corporation application dated February 17, 1982. Fuel rods must be contained within a fuel basket to minimize damage to the rods during normal conditions of transport.

11. In addition to the requirements of Subpart $G$ of 10 CFR Part 71:

(a) Prior to each shipmentite Buha-libber ylid gasket(s) (if opened) must be inspected. Thesengaskets must be reptaced if inspection shows any defects or renlace both gaskets every twelve-(12) months, whichever occurs firsta tavity lines must be sealed with appropriate sealant applied ta threads of pipe caps or plugs.

$$
\text { tath }
$$

(b) The package must be maintained in accordance with sefation VIII of the appis cation.<smiles>[C]1[C]2CC3C1C23</smiles><smiles>C1CC2CCC1CC2</smiles>

(c) The package must be e prepared-for shipoent and operated-th accordance

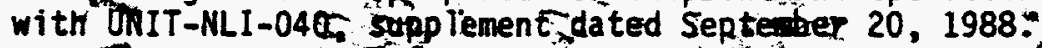

12. The cask contents must 5 s so. limitatinander nowaj conditions of transport that the doseftrte will not exteed $10 \mathrm{mrem} / \mathrm{hr}$ a of the cast.

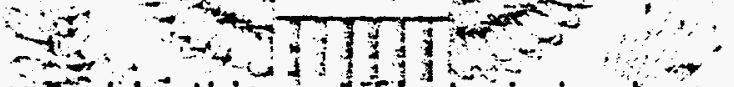

13. The packageauthorited by this: certif tate is hereby approd for use under the generaluticense prowisions af ta CFR \$21.12,

14. Expiration date: 0ctober 31,1993,

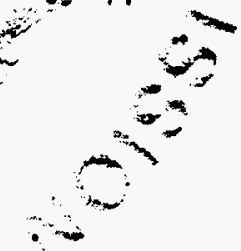

NL Industries, Inc. applicatigandated September 7977 .

Supplements dated: May 1, 1978; December 16, 1985; and May 11, July 5, and September 20, 1988.

FOR THE U.S. NUCLEAR REGULATORY COMMISSION

Date: OCT 131988

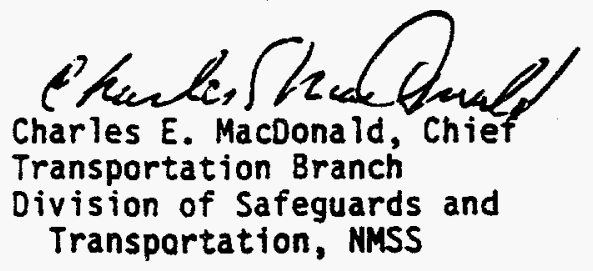


T-3

B-50 


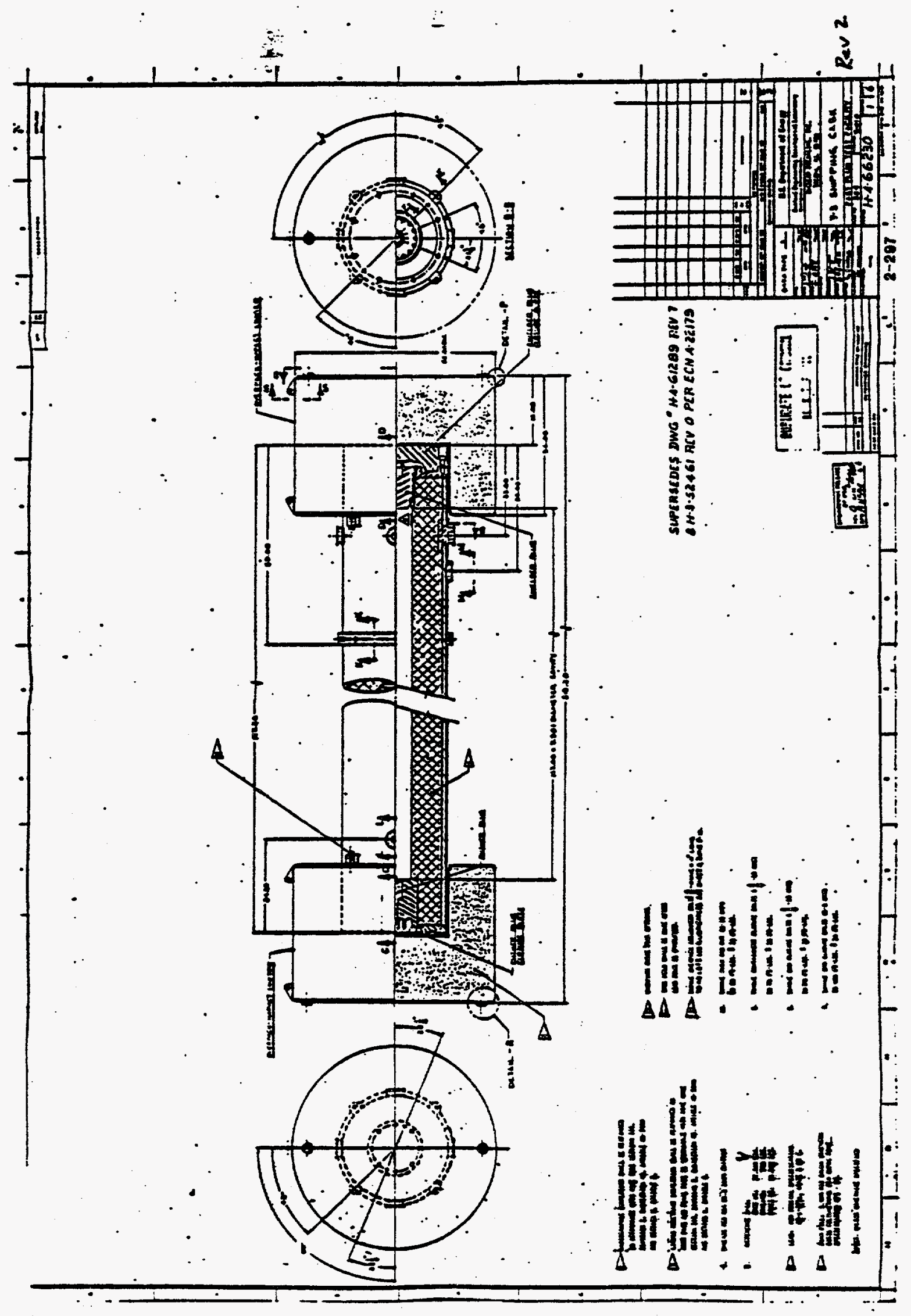

B-51 


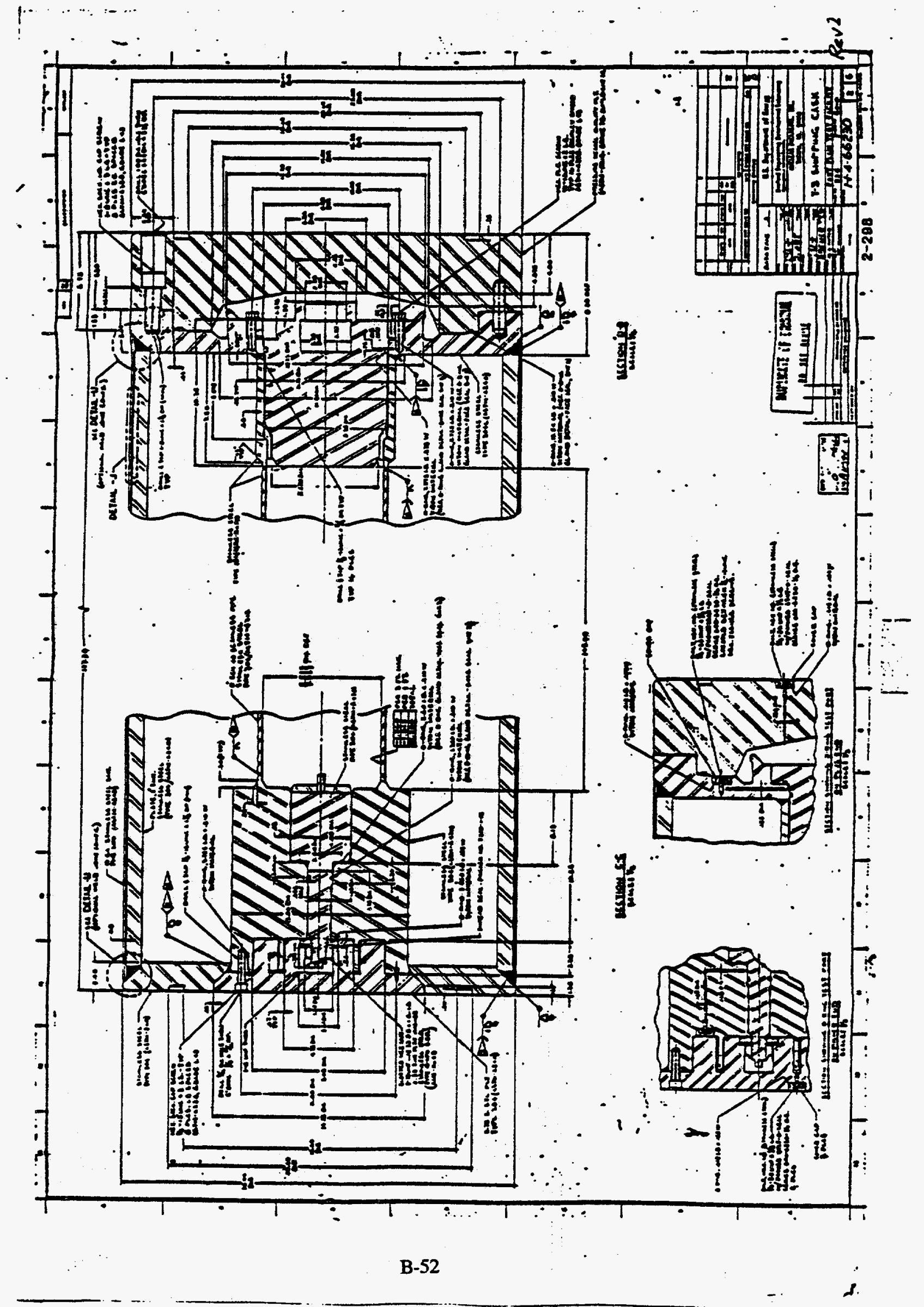




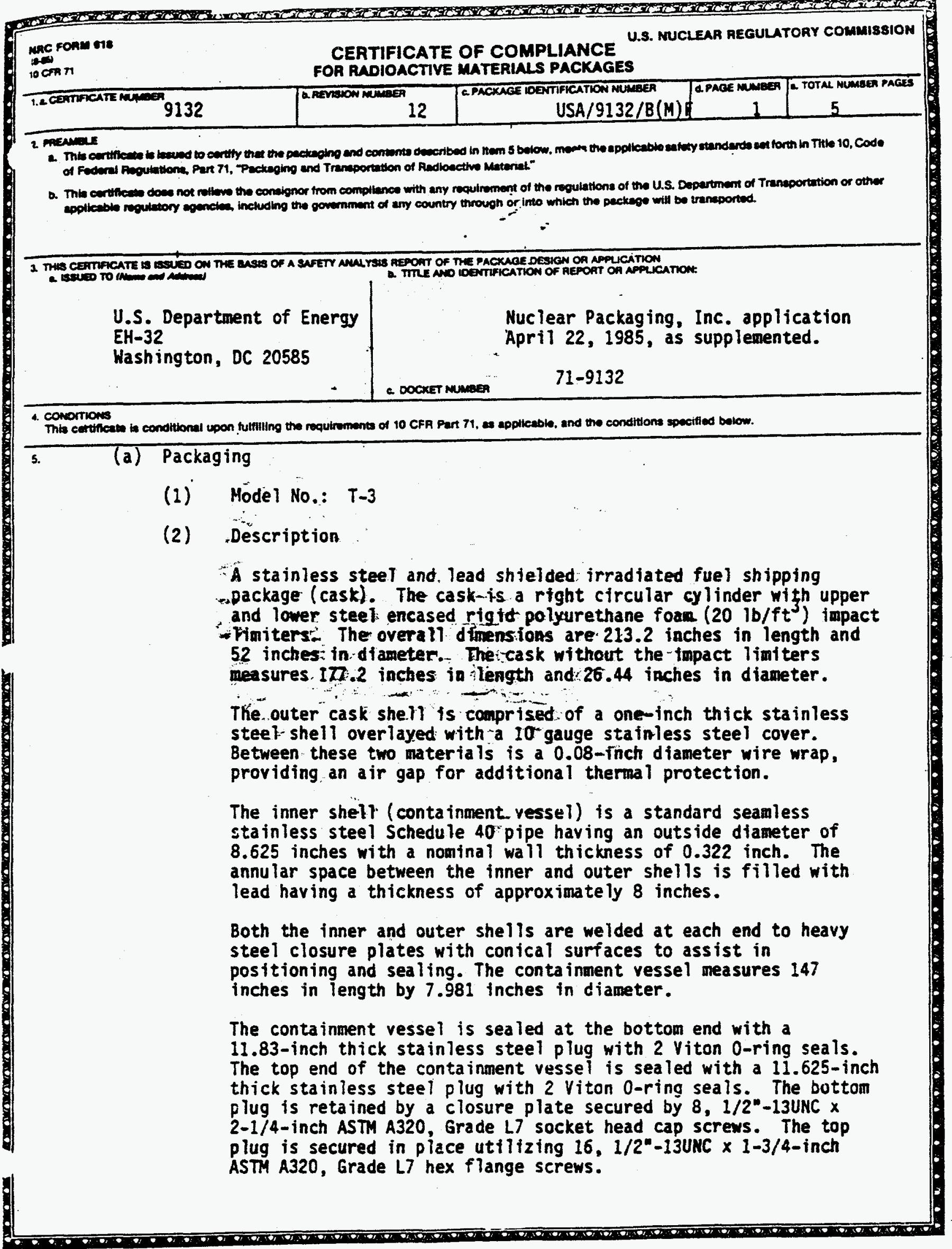




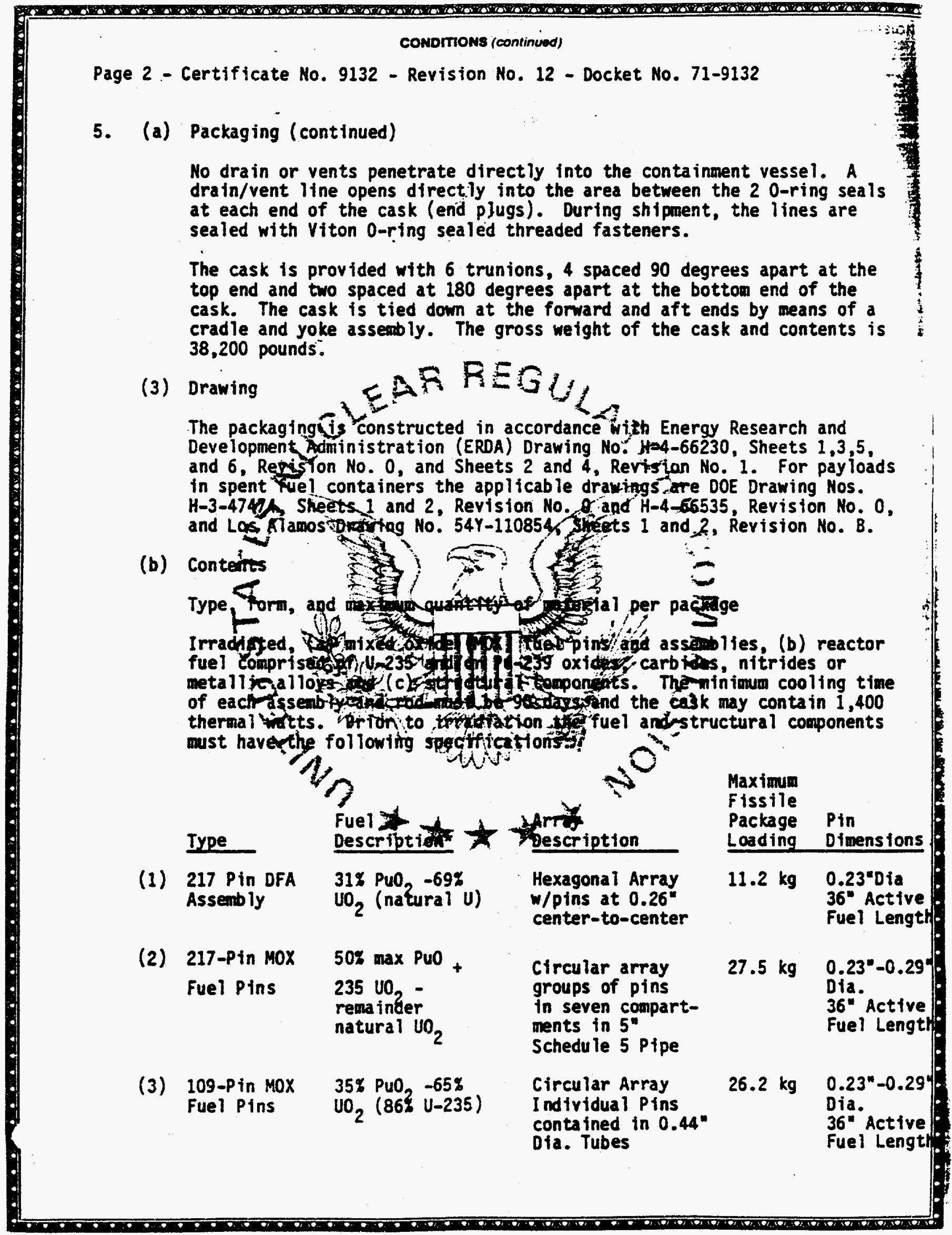


Page 3 - Certificate No. 9132 - Revision No. 12 - Docket No. 71-9132

5. (b) Contents (continued)

\begin{tabular}{|c|c|c|c|c|c|}
\hline & Type & $\begin{array}{l}\text { Fuel } \\
\text { Description* }\end{array}$ & $\begin{array}{l}\text { Arráy } \\
\text { Description }\end{array}$ & $\begin{array}{l}\text { Maximum } \\
\text { Fissile } \\
\text { Package } \\
\text { Loading } \\
\end{array}$ & $\begin{array}{l}\text { Pin } \\
\text { Dimensions } \\
\end{array}$ \\
\hline (4) & $\begin{array}{l}\text { 55-Pin Mox } \\
\text { Fuel Pins }\end{array}$ & $\begin{array}{l}35 \% \\
\mathrm{UO}_{2}\end{array}$ & $\begin{array}{l}\text { Circular Array } \\
\text { Individual Pins } \\
\text { contained in } 0.625^{m} \\
\text { Dia. Tubes }\end{array}$ & $13.2 \mathrm{~kg}$ & $\begin{array}{l}0.23^{\prime \prime}-0.29^{*} \\
\text { Dia. } \\
36^{*} \text { Active } \\
\text { Fuel Length }\end{array}$ \\
\hline (5) & $\begin{array}{l}37-P \text { in } \operatorname{MOX} \\
\text { Fuel Pins }\end{array}$ & $235)^{3}$ & 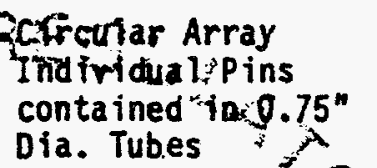 & $8.9 \mathrm{~kg}$ & $\begin{array}{l}0.23^{n}-0.29^{n} \\
\text { Dia. } \\
36^{\prime \prime} \text { Active } \\
\text { Fuel Length }\end{array}$ \\
\hline (6) & 42-P in MOX & 235 & $\begin{array}{l}\text { Circular Array } \\
\text { Individua } 1 \text { P ins } \\
\text { contained tn } 0.62 \\
\text { Dia. Tubes }\end{array}$ & & $\begin{array}{l}0.23^{\prime \prime}-0.29^{\prime \prime} \\
\text { Dia. } \\
36^{\prime \prime} \text { Active } \\
\text { Fuel Length }\end{array}$ \\
\hline (7) & $\begin{array}{l}\text { 40-P in } 10 x^{3} \\
\text { Fuel Pins }\end{array}$ & ? & 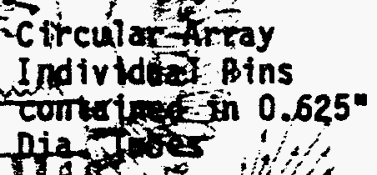 & $\sum^{9.6} \mathrm{~kg}$ & $\begin{array}{l}0.23^{\prime \prime}-0.29^{\prime \prime} \\
\text { Dia. } \\
36^{\prime \prime} \text { Active } \\
\text { Fuel Length }\end{array}$ \\
\hline (8) & $\begin{array}{l}\text { 19-Pin MOX } \\
\text { Fuel Pins }\end{array}$ & & 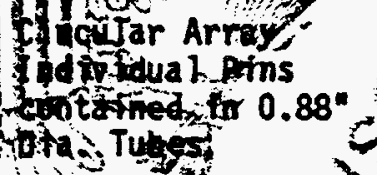 & & $\begin{array}{l}0.23^{n}-0.29^{n} \\
\text { Dia. } \\
36^{\prime \prime} \text { Active } \\
\text { Fuel Length }\end{array}$ \\
\hline (9) & $\begin{array}{l}\text { PU Compounds } \\
\text { Fuel Pins } \\
\text { (spent fue) } \\
\text { containers) }\end{array}$ & 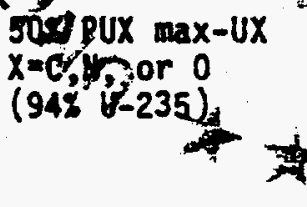 & $\begin{array}{l}\text { Unrestricted arkay } \\
\text { Individual P fas } \\
\text { contained in } 55 \\
5 \text {-ins sthedule } \\
\text { of pe }\end{array}$ & $8.0 \mathrm{~kg}$ & $\begin{array}{l}\text { Container } \\
\text { Cavity } \\
5.047^{" 1} \text { Dia. } \\
\text { by } 38.9^{\prime \prime} \\
\text { length }\end{array}$ \\
\hline (10) & $\begin{array}{l}\text { LAMPRE } \\
\text { Fuel Pins } \\
\text { (spent fuel } \\
\text { container) }\end{array}$ & $\begin{array}{l}97.5 \% \text { Pu max }-X \\
\text { alioy } \\
x=F e, \text { co or cs }\end{array}$ & $\begin{array}{l}\text { Circular Array } \\
\text { Individual Pins } \\
\text { contained in } 0.625^{\circ} \\
\text { or } 0.75^{\prime \prime} \text { Dia. } \\
\text { steel tubes }\end{array}$ & $8.0 \mathrm{~kg}$ & $\begin{array}{l}0.425^{\prime \prime} \text { Dia. } \\
38^{*} \text { Active } \\
\text { Fuel length }\end{array}$ \\
\hline (11) & $\begin{array}{l}\text { Structural } \\
\text { Components } \\
\text { (Incl. Control } \\
\text { assemblies) }\end{array}$ & Dosimetry Foils & 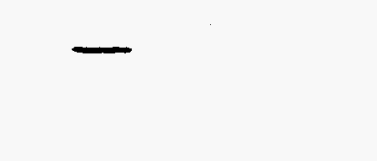 & $1.0 \mathrm{~kg}$ & - \\
\hline
\end{tabular}


Page 4 - Certificate No. 9132 - Revision No. 12 - Docket No. 71-9132

(12) 24 Max. Pins. U-Pu Carbide Fuel Pins

(13) 18 max. pins. Sodium bonded (fuelto-clad)

85-948(Pu-U)C -6 to Circular array; $15 \%\left(\mathrm{Pu}-\mathrm{U}_{2}\right) \mathrm{C}_{3}$. individual pins

Max 23\% $\mathrm{PQ}$, Oranium contained in is not enriched

10\% Zr-20\% Pu max. Rema inder U (U enriched to $40 \%$ max. $(v-235)$. 0.625-in. dia. - tubes within 5-in. Schedule 40 pipe

\section{Circular array;}

individual pins contained in 0.625 -

$3.0 \mathrm{~kg}$ $0.37 *$ outer of
$36 "$ Active Fue Length in. diam. tubes within

\#AT plutonium in the fuel Types (1) thru (8) contains at least 10\% Pu-240; fuel type (9) has no. Timit for PU-240; Type (10) contains at least $6 \%$ PU-240.

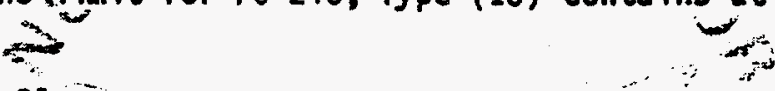
5. (c) Fissileclass
Maximum number of packages per shipment-
- One

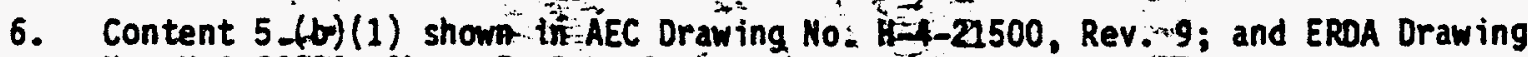
Mo. H-4-66230, Sheet 5 , Rer.

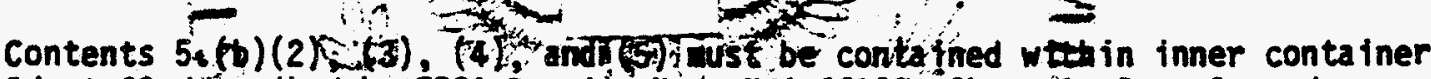
Ident 69 déscribed byr ERDA Drawiag Hos H-4-66230, Sheets 5-and;6, Rev.jos

$$
\text { Sto }
$$

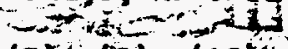

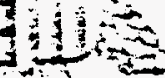

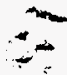

Contents $5 .(6)(6),(7),(8),(12)$ )and (13)- mitst be contained with in inner conta iner Ident 1578 descrtbed-by ERoA Orawing Nos. H-4-66160, Sheet 2, Rev. 0 ; and $\mathrm{H}-4-66230_{2}$, Sheets 5 and 6:2 Rev: 0 .

Contents 5.(b)(9) and (10) shown in DOE Drawing No. H-3-47474, Sheets 1 and 2 , Revision No. 0 and LOS A Tamos Drawing No, 54\%-110854, Sheets 1 and 2, Revision Mo. B must be contained within the deme 69 Liner shown in ERDA Drawing No. H-4-66230, Sheets 5 and 6, Revision No. O and DOE Drawing No. H-4-66535, Revision Mo. 0.

7. The cask must be shipped dry (no water coolant in cask cavity). Shipment of sodium wetted fuel rods (external) is authorized for up to $200 \mathrm{~g}$ of sodium provided the additional requirements of Section 7.4 of the application are adhered to. 


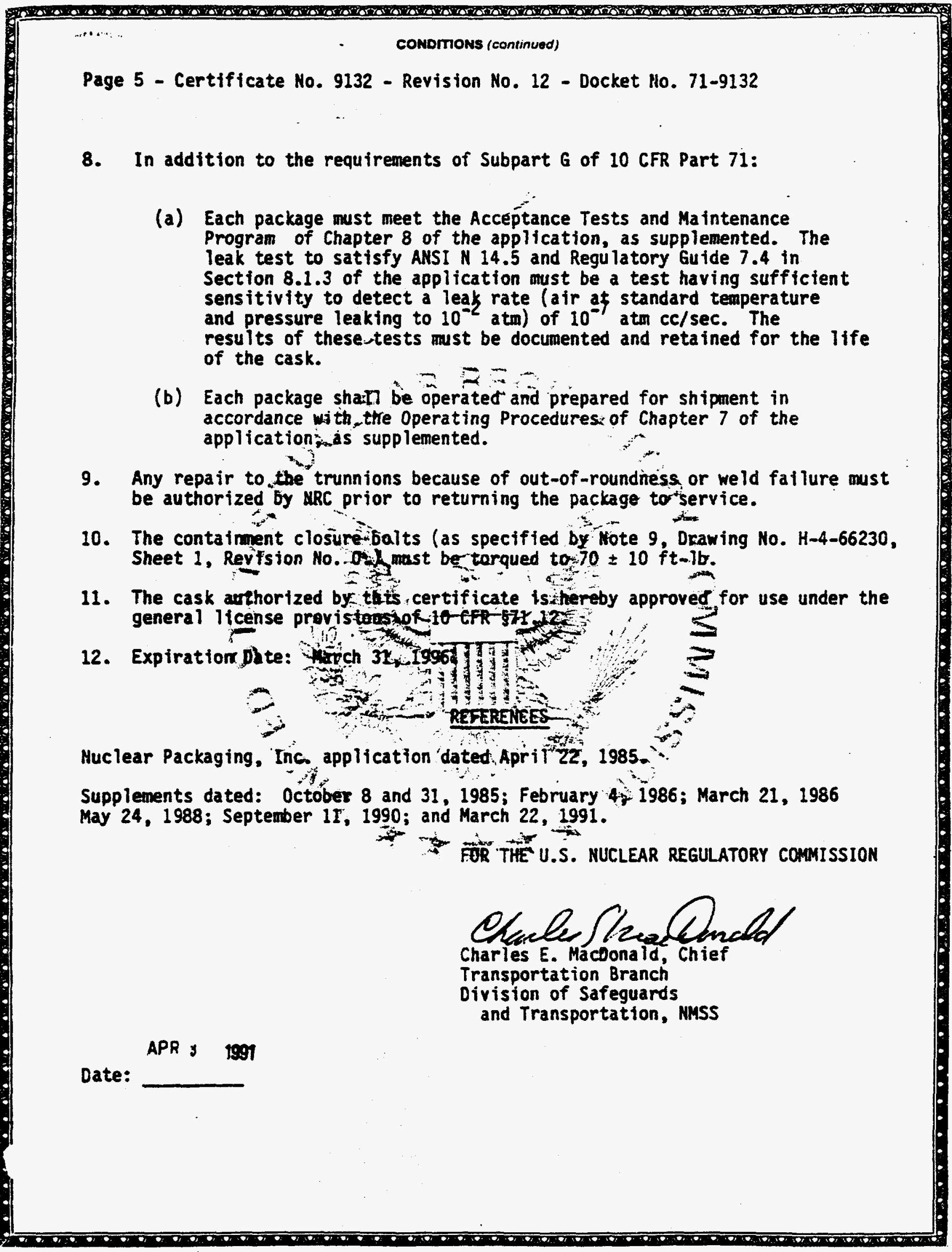


NAC-1

B-58 


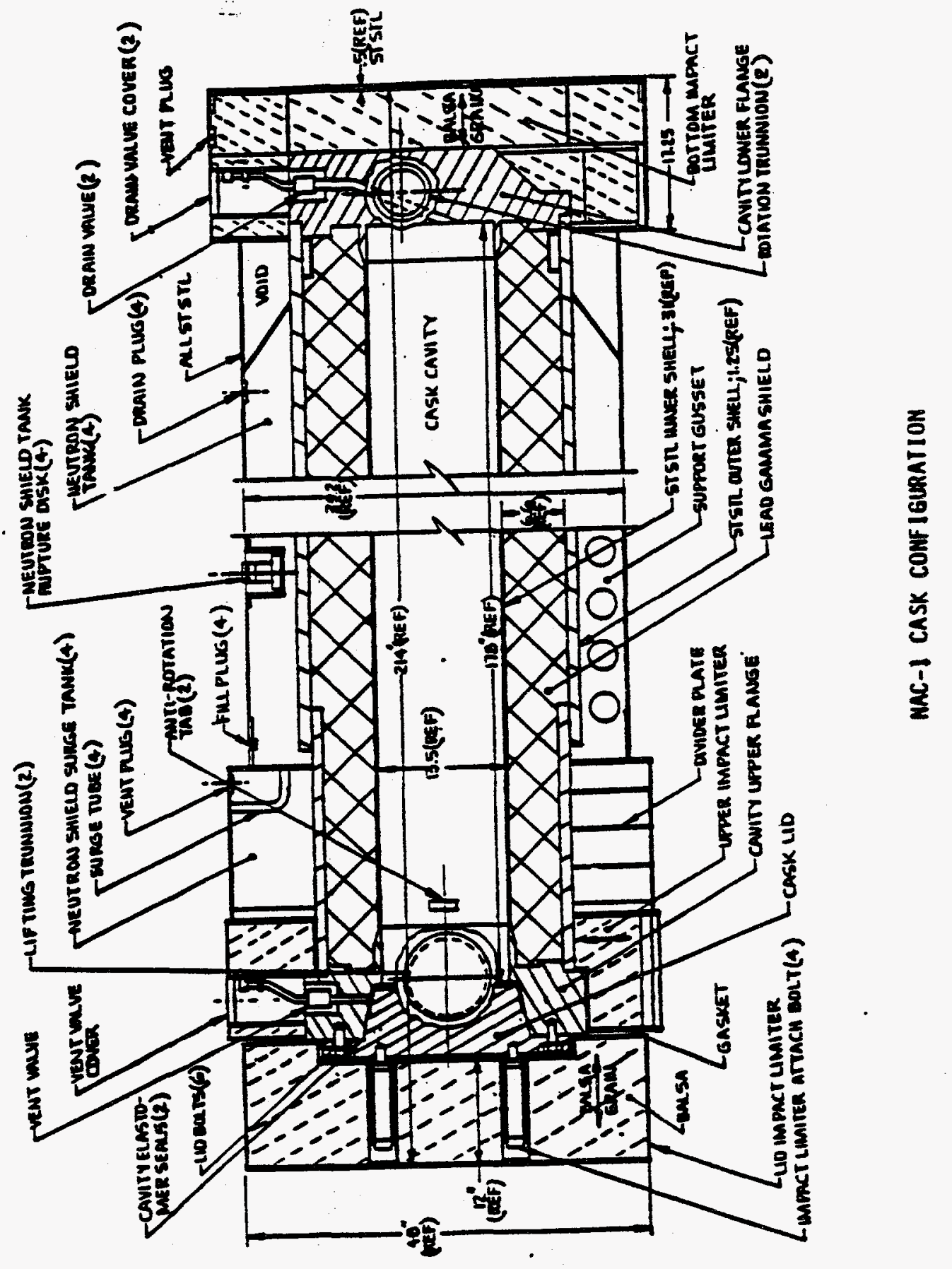




\section{NAC-1 (NFS-4)}

Designer

Type

Capacity

Intact Spent Fuel Assemblies

Otber Configarations

Weight (tous)

Loaded

Empty

Design Heat Rejection (kW)

Shape

Dimensions

Overall Length (in)

Overall Diameter (in)

Cavity Length (in)

Cavity Width (in)

Inner Shell Wall Thickness (in)

Lead Shield Wall Thicteress (in)

Outer Shell Wall Thickneas (in)

Neutron Shield Tank Thickness (ii)

Materials of Construction

Cask Body

Neutron Shieid

Sides

Ends

Basket

Cooling Fins

Operating Temperarure ( $\left.{ }^{\circ} \mathrm{F}\right)$

Operating Pressure (psig)

Outside Surface Dose (mrem/hr)

Cavity Atmosphere

\author{
Noclear Assurance Corporation \\ Legal Weight Truck Shipping Cask \\ 1 PWR; 2 BWR (on-site onty) \\ Metallic Fuel \\ 23 \\ 22
}

115

Cylindrical

214

38

178

13.5

0.3125

6.625

125

45

SShead/SS

Borated Water \& Ethylene Glycol

Nome

SS

None - Smooth Surface

345 (maximum)

5 (984 maximum)

200 (maximum)

Air

Licensing Stans - Originally licensed by AEC and USNRC. USNRC did not recertify the cask for off-site movement of LWR fuel due to some concerns about distortion in the inner cavity.

Comments - NAC obtained a license for the NFS-4 cask design for use by NAC. NAC later took possession of two NFS 4 casks and has rights to lease them. A total of seven casks are in existence, one of which is owned by Duke Power Company and the others are available for lease from NAC. Casks have interchangeable baskets for PWR and BWR fuel. 


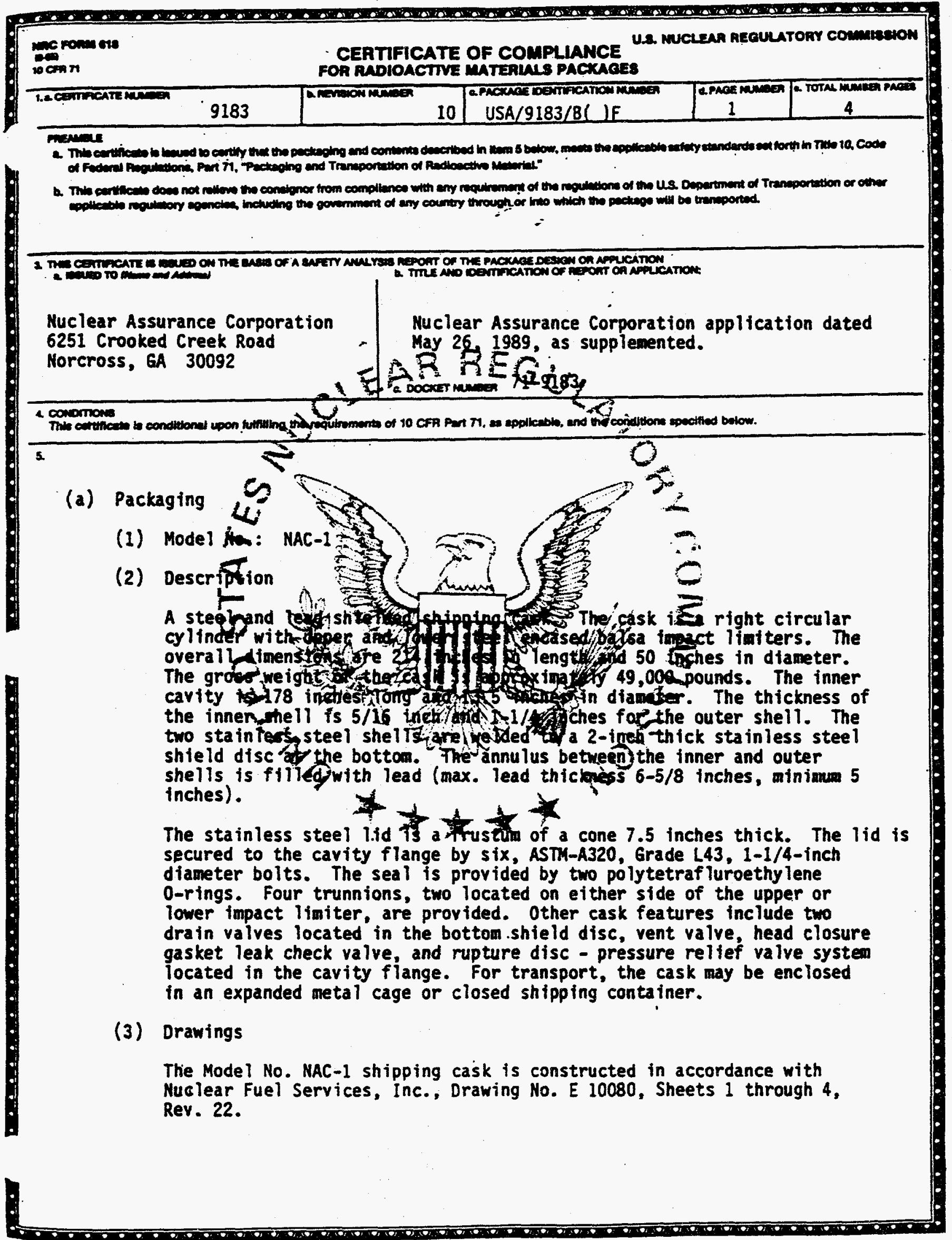


Page 2 - Certificate No. 9183 - Revision No. 10 - Docket No. 71-9183

\section{(b) Contents}

(1) Type and form of material

(i) Clad, irradiated metallic natural uranium fuel rods.

(ii) Solid nonfissile irradiated hardware.

(2) Maximum quantity of material per package

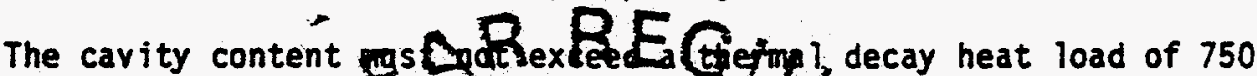
watts and a weight 3,700 ibs including light of component spacers (or fuel basket) used in the cask cavity to hill movement of contents during shipmizgt. Fuel rods are additionally ifmited as follows:

(i) 21 act rods or 6 encapsulated (defective) Roch defective rod $w i$ t be encapsulated in either a 2.75-inch 75 . failed fuel rod c.0 as showion Nuclear Assurance Cognoratton Drefuing No. 340-108-02,

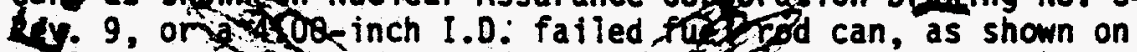
Nuclear Assumage Corporation Dravin ina. 340-108-03 Rev. 9.

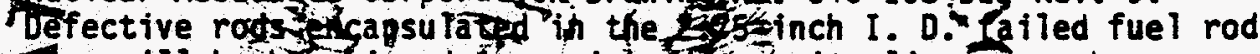
Gans will be beschipped in a isix refacity lineryas shown on

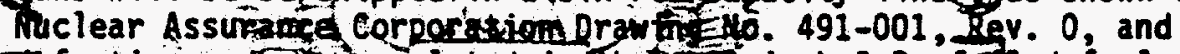

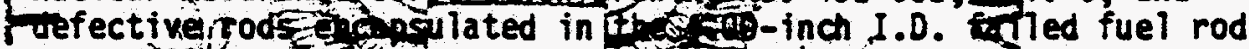

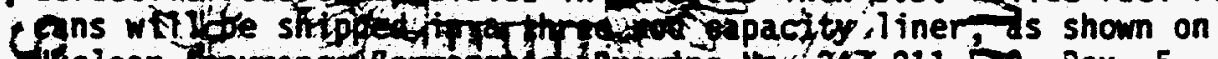

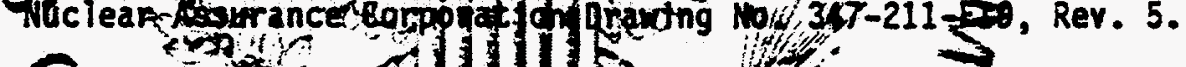

\section{(c) Fissile Class}
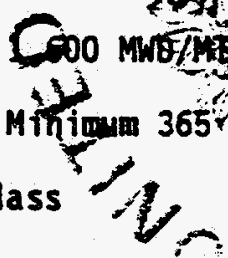

6. The cask cavity must be dry (no free water) when delivened to a carrier for transport.

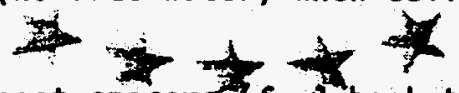

7. As needed, appropriate component spacers fuel basket and axial spacers for shipment of fuel rods) must be used in the cask cavity to limit movement of contents during accident conditions of transport.

8. The cask may' be shipped in a closed shipping container provided that the closed container, the cask tie-down and support system and the transport vehicle (trailer) meet the applicable requirements of the Department of Transportation. Tie-down devices which are a structural part of the package must comply with 10 CFR \$71.45.

9. When the cask is shipped in a closed shipping container, the center of gravity of the combined cask, closed shipping container and trailer must not exceed 75 inches.

10. When the cask is shipped in a closed shipping container, the internal heat load must not exceed 750 watts. 
Page 3 - Certificate No. 9183 - Revision No. 10 - Docket No. 71-9183

1.. In lieu of the requirements of 10 CFR $\$ 71.87(\mathrm{e})$, the licensee must perform periodic maintenance and testing of 0-rings, drain and vent ball valves, relief valves, and rupture discs of the cask as indicated in the table given below. During inactive periods, the maintenance and-testing frequency may be disregarded provided that the package is brought into full compliance prior to the next use of the package.

Cask Component Period $\quad$ Test/Action

Ball Valve

Ball Valve

0-rings

0-rings

Inner Containment Annually

Cavity Relief Vậto

Cavity Rupture:Disc

Each shipment Hydro test to 30 psig*

Neutron Shield fank

Rupture Disc

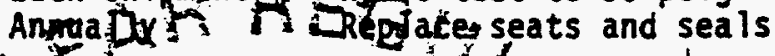

Impact Liaitersi;

Annually

Each shipment

Annually

Annmaris

Annuarly

$=2$

Anneàl ly

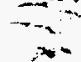

Antos

Sol Annatiay

Dor

*There must be nowisua f fpressure gauge 1 indications of pressure-drop for the component under test duringicu 10-minute test period atherwise, corrective action must be taken and the test repeated unt lisuchitime as the component meets the speciffed tests. "(Jest to pressures equal ta or greater than-those indicated.)

12. The package'shall be prepared for shipment and operated in accordance with the operating procedures in Chapter 7 of the application

13. Each package must be maintained, in accordance with the maintenance program in Chapter 8 of the application.

14. The package authorized by this certificate is hereby approved for use under the general license provisions of 10 CFR $\$ 71.12$.

15. Expiration date: September 30, 1994. 
Page 4 - Certificate No. 9183 - Revision No. 10 - Docket No. 71-9183

REFERENCES

Nuclear Assurance Corporation application dated May 26, 1989. Supplements dated January 29 and March $20,1990$.

FOR THE U.S. MUCLEAR REGULATORY COMMISSION

Date:

MAY O \& 1990
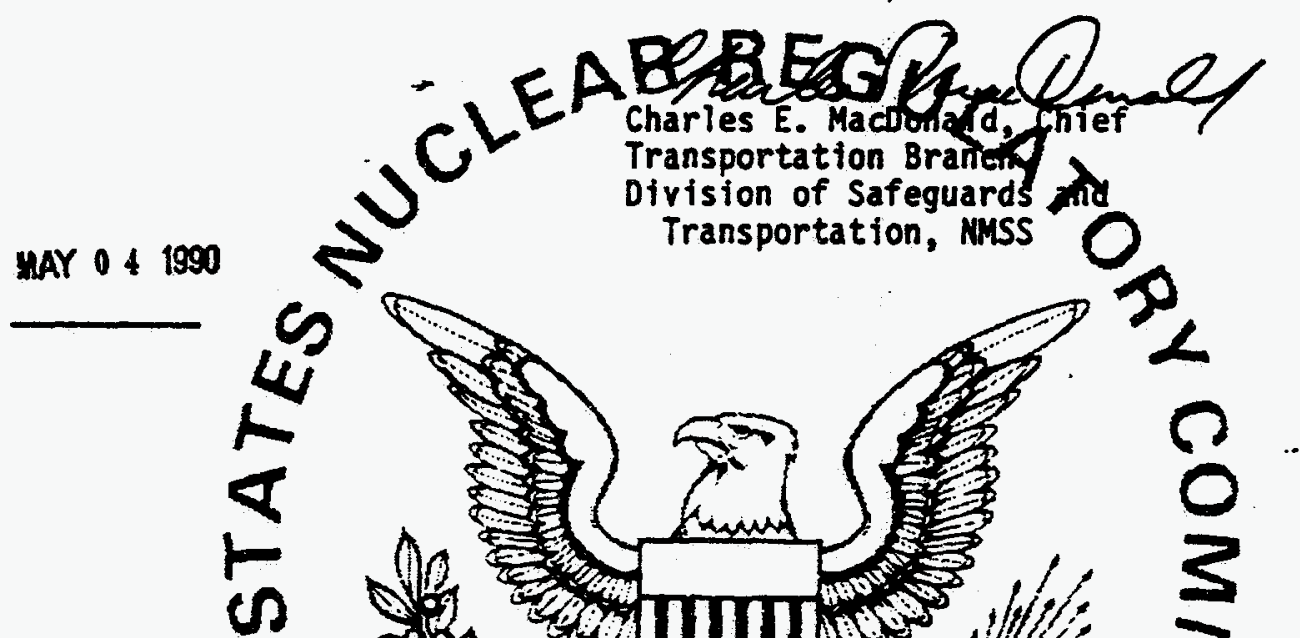

(1)

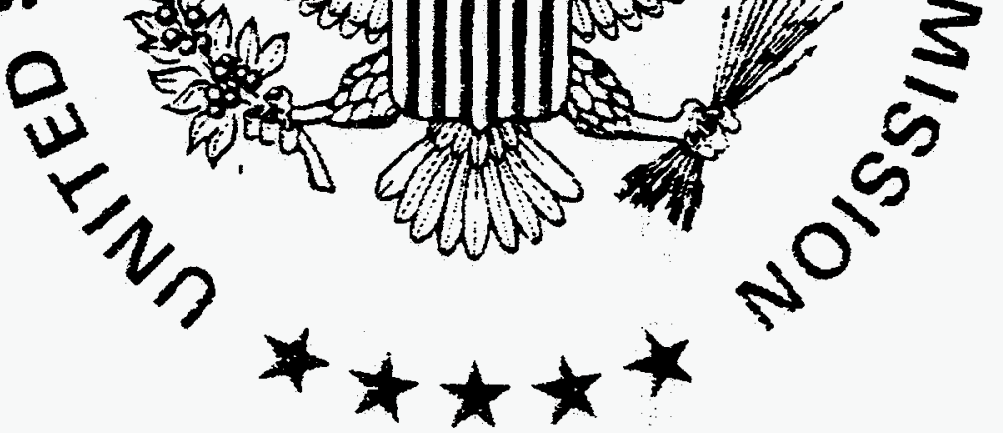

- 
CNS 1-13G 
80

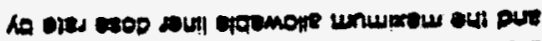

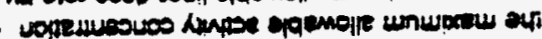

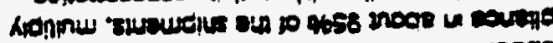

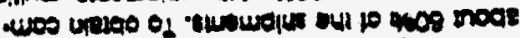

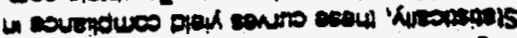

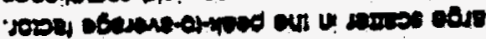

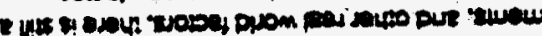
n

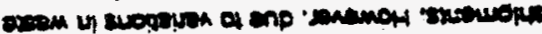

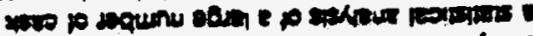

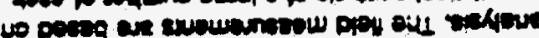

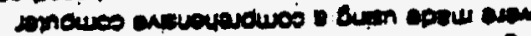

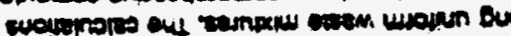

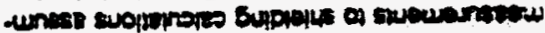

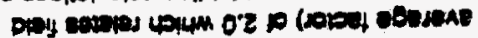

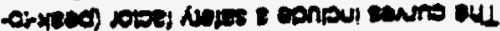
gLON AMmounvo

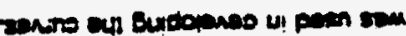

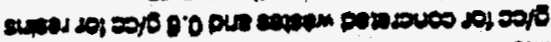

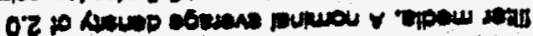

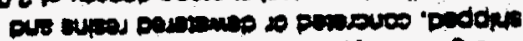

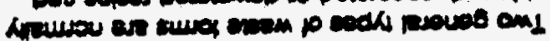

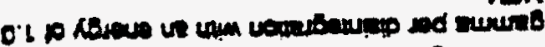

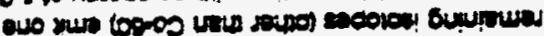

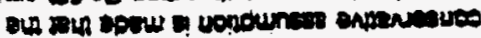

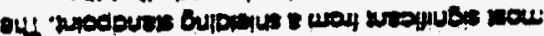

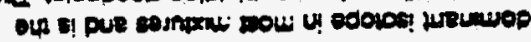

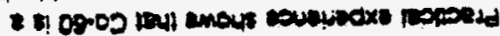

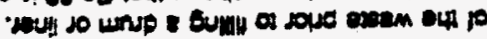

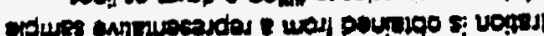

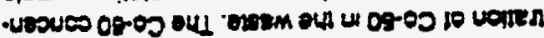

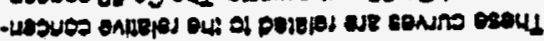

(DION krevounes

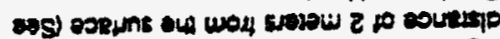

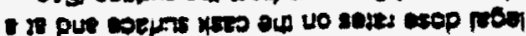

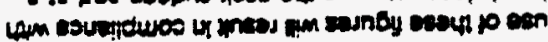

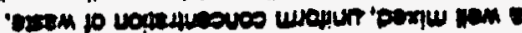

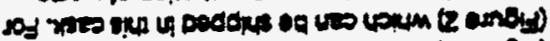

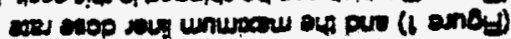

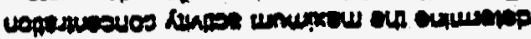

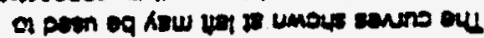

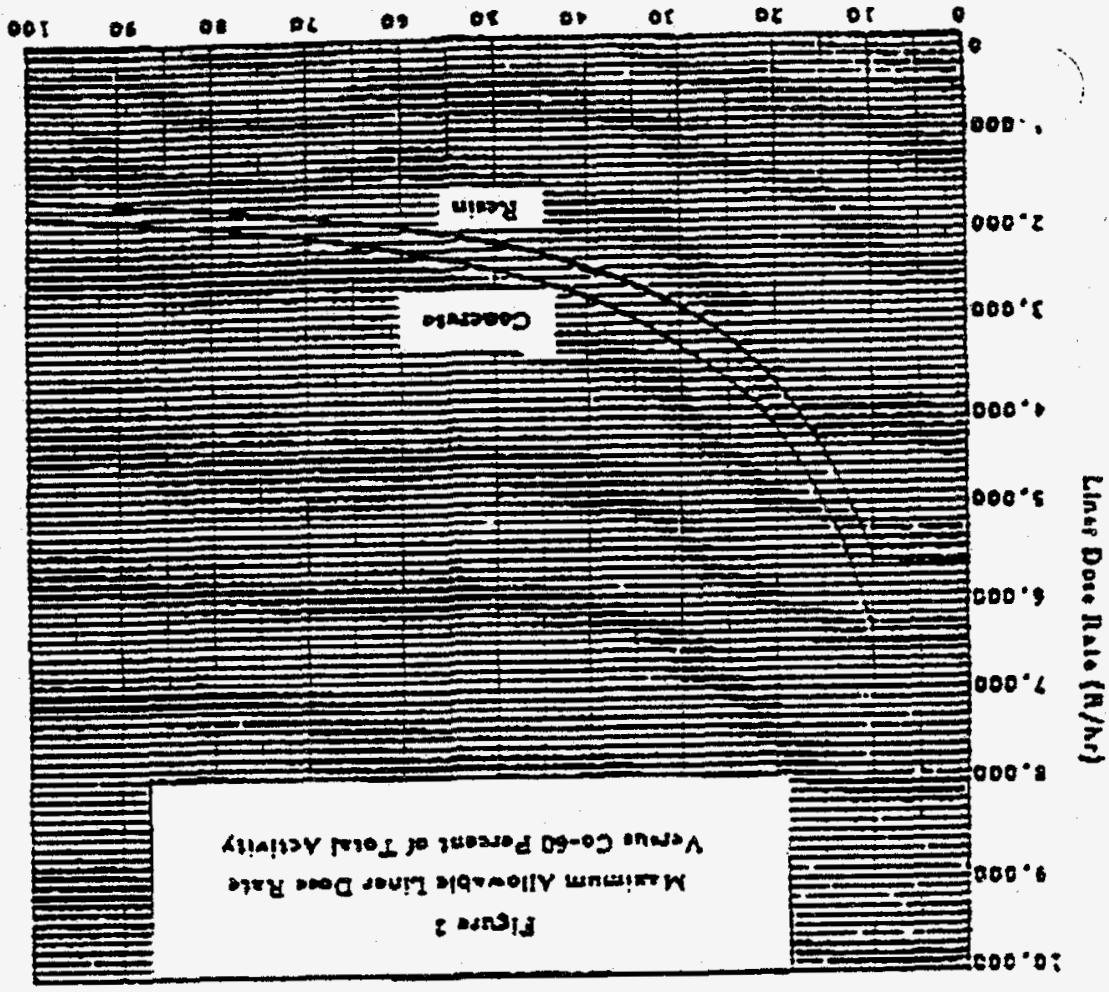

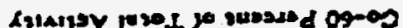

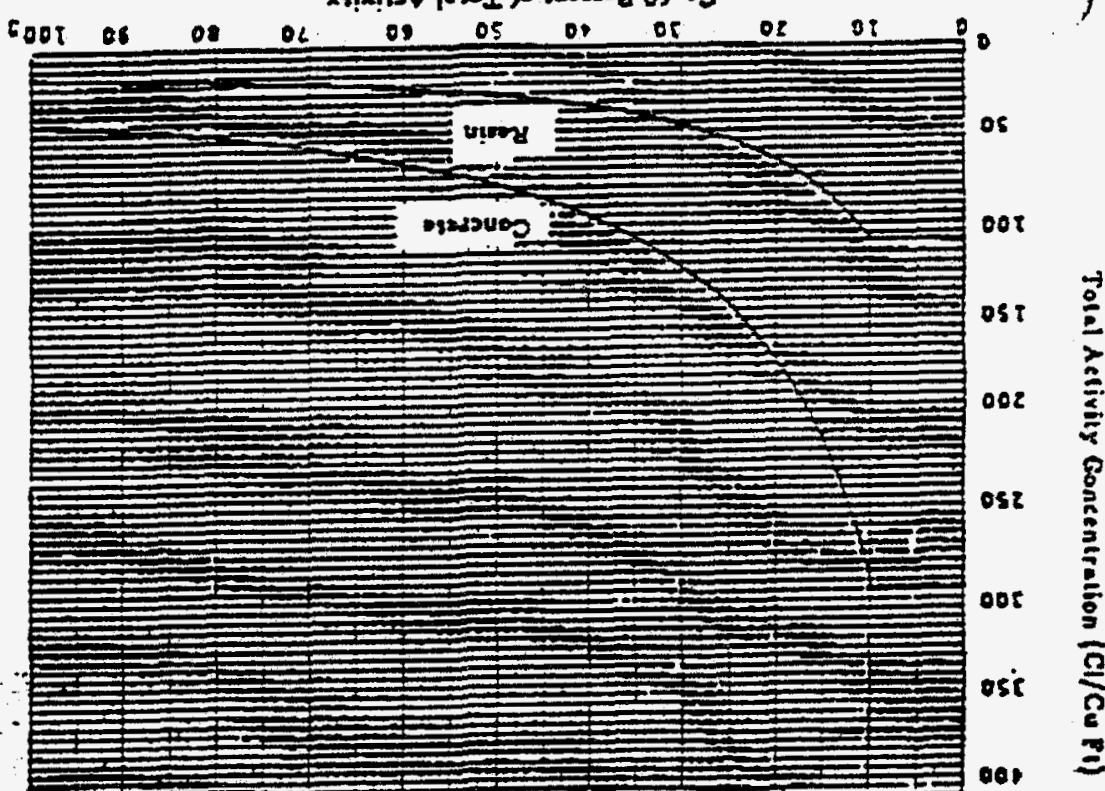

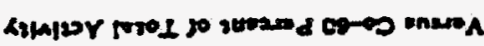

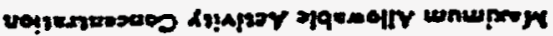

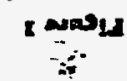

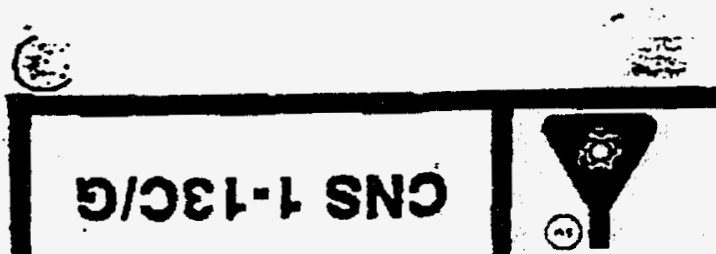




\section{CHEM-NUCLEAR SYSTEMS, INC.}

\section{s:}
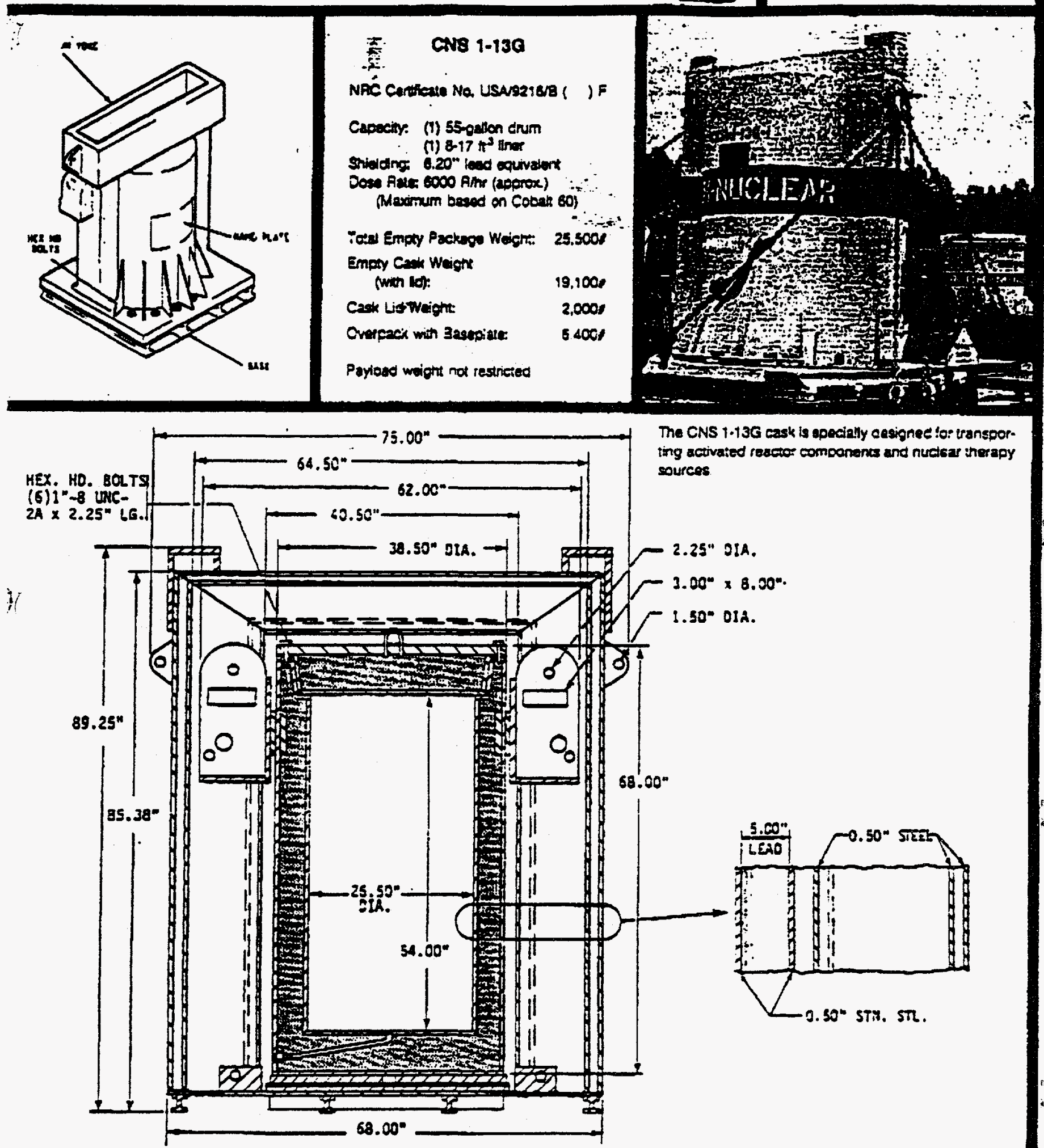

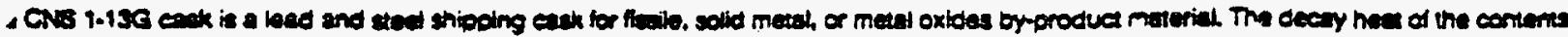

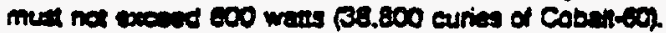

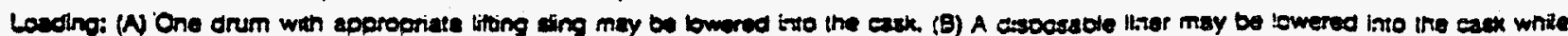

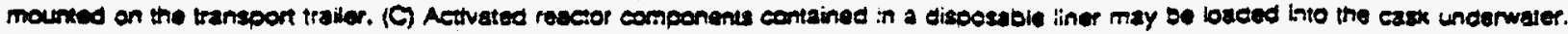




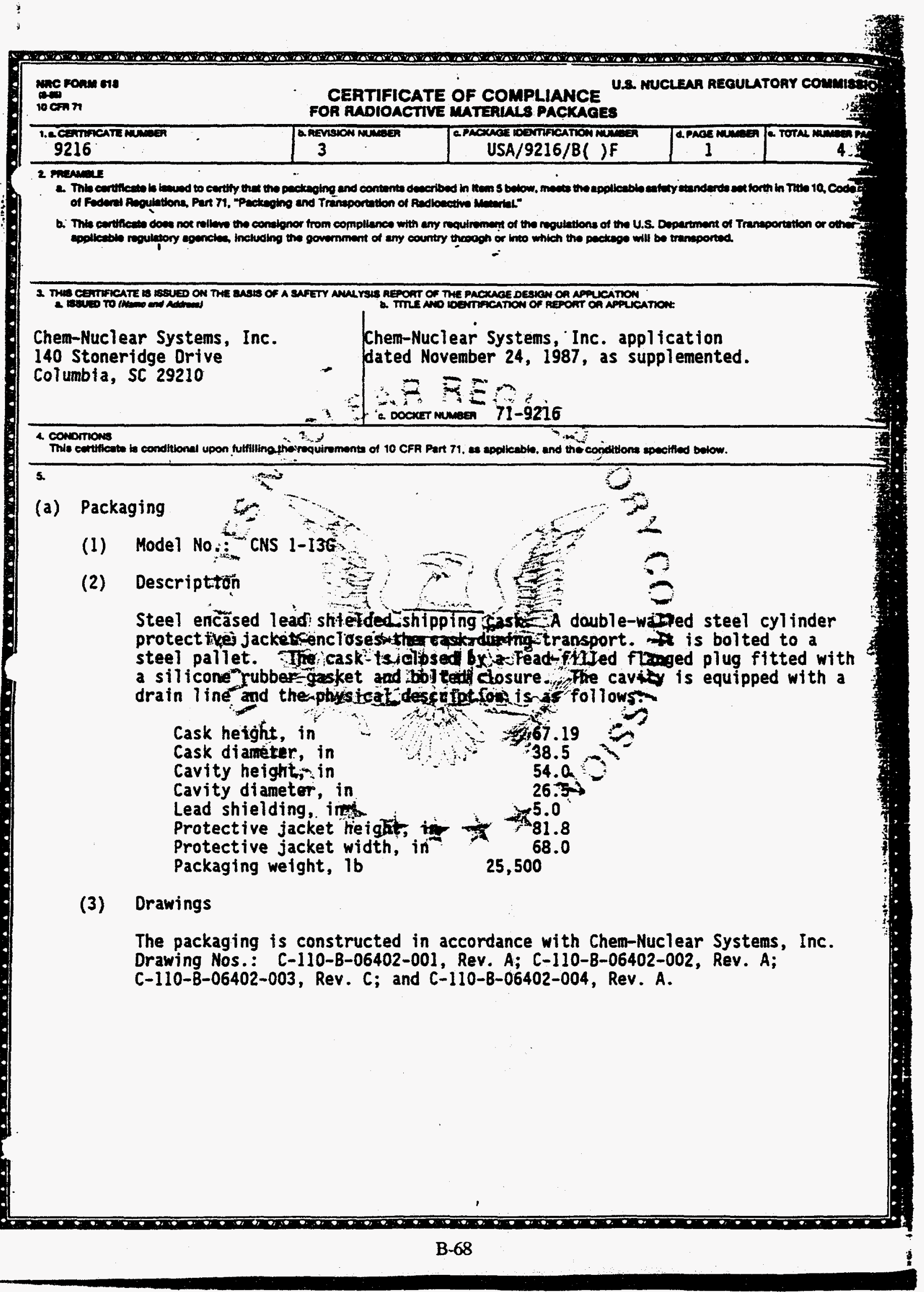




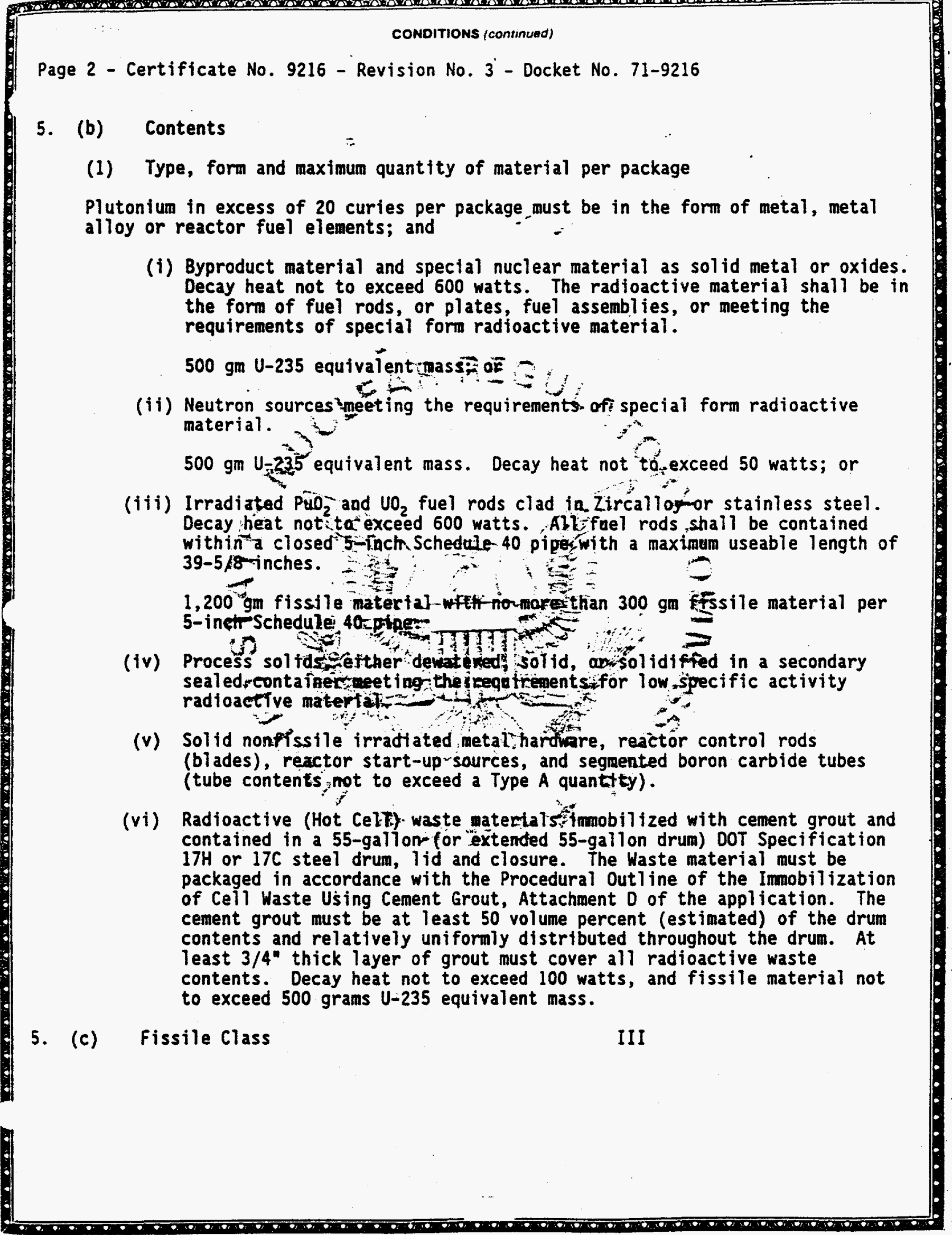


Page 3 - Certificate No. 9216 - Revision No. 3 - Docket No. 71-9216

Maximum number of packages per shipment
(1)

$$
\begin{aligned}
& \text { Contents } 5 . \text { (b) (1)(i), } \\
& 5 \text {. (b) (1) (ii), (b)(i)(iti), } \\
& \text { or } 5 \text {. (b)(1)(vi): } \\
& \text { Two (2) }
\end{aligned}
$$

6. The U-235 equivalent mass is determined by U-235 mass plus 1.66 times U-233 mass plus 1.66 times Pu mass.

7. (a) For any package containing water and/or organic substances which could radiolytically generáte combustible gases, determination must be made by tests and measurements or by anatysis of a representative package such that the following criteria ar of of a period of time that is twice the expected shipment times

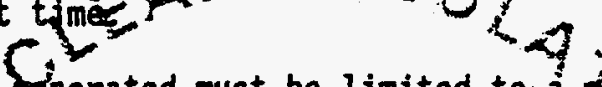

(i) The hydrogen generated must be limited to a molar quantity that would be no more than $5 \%$ by volume (or equivalent limi s for other inflamable gases) othe secondary container gas void if pregent at STP (i.e., no more than $0.063 \mathrm{~g}$-moles $/ \mathrm{ft}^{3}$ at $14.7 \mathrm{psia}$ and $70^{\circ} \mathrm{F}$ ) $\mathrm{z}$ or

(ii) The sudindary and and cask caviturst be inerted with a diluent to

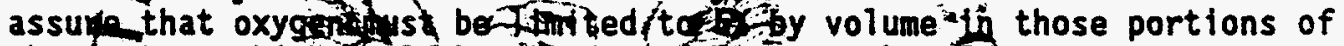
the package whicteond have hydroger 3 reater than 5 .

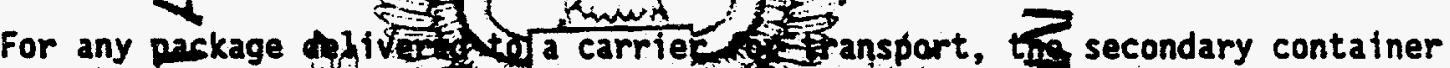

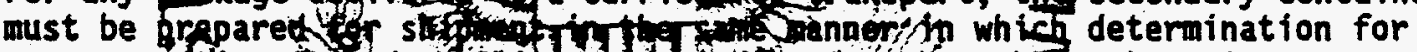

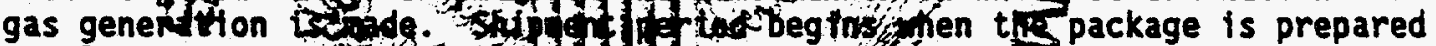

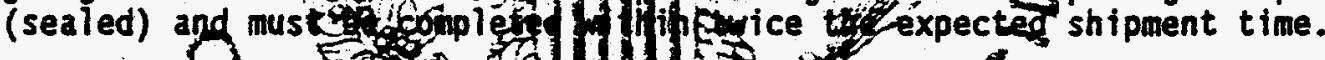

(b) For any packle conforing wate exceeding that for low spaciffichactiktty of preparation gr within 10 days lafter venting of drums or other secondary containers, the/ (termination if $(2)$ above need $n$ bot be made, and the time restriction in (a) 3 bove does not apply.

8. For packaging of neutron sourtis, with a melting temperature of $200^{\circ} \mathrm{F}$ and the cask cavity must be dry before delivery of the package to a carrier.

9. For packaging of other than neutron sources, the cask must be delivered to a carrier dry and the cavity drain line must be closed with a plug which will maintain its seal at temperatures up to at least $620^{\circ} \mathrm{F}$.

10. For the shipment of irradiated metal hardware, the use of the auxiliary shielded inner container and shoring plug shown in Chem-Nuclear Systems, Inc. Drawing Nos. 8651-E-02, Rev. A and 8651-C-01, Rev. B is authorized. The inner container must be provided with vent and drain lines. 
$\because \div \cdots$

conomons (continued)

Page 4 - Certificate No. 9216 - Revision No. 3 - Docket No. 71-9216

11. Shoring must be provided to minimize movement of contents during accident conditions of transport.

12. In addition to the requirements of Subpart, 6 of 10 CFR Part 71:

(a) The package shall be prepared for shipment and operated accordance with ChemNuclear Systems, Inc. Operating Procedures, Section T.0.

(b) Prior to each shipment the sillicone rubber lid gasket(s) must be inspected. This gasket(s) must be replaced if inspection shows any defects or every twelve (12) months, whichever occurs first. Cavity. drain line must be sealed with appropriate sealant applied to threads of pipe plug.

(c) Prior to each shipmentet $B$ aseptate to caslo shell weld must be visually inspected in accocdunct with Chem-Nuclear Systens, Inc. Operating Procedures, Section 7.0 .

1

(d) The packaging-gust meet Chem-Nuclear Systems, Inc. Acceptance tests and Maintenance gram, Section 8.0.

13. For packaging of neutromsaces, 50 times measube feutron dose rate at one meter

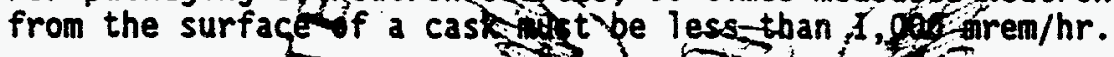

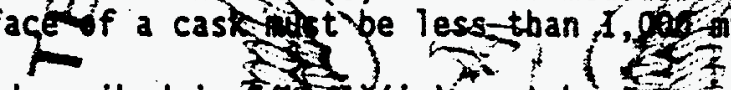

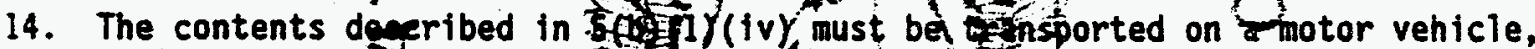

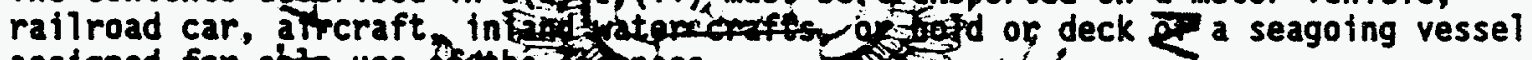
assigned for site use difthe
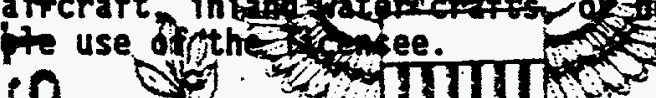

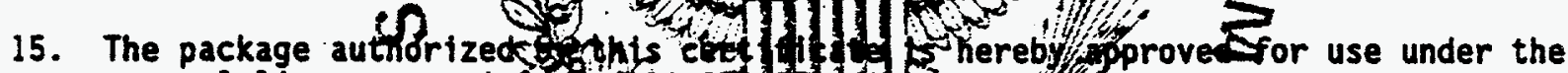
general license

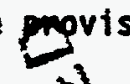

16. Expiration date:

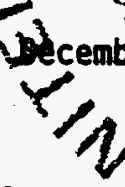

Chem-Nuclear Systems, Inc. app?
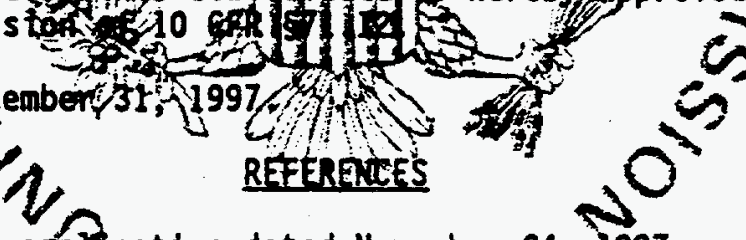

Pifion dated November 24, 1987.

Supplement dated: November 24, 1992. 가

FOR THE U.S. NUCLEAR REGULATORY COMMISSION

DEC 161992

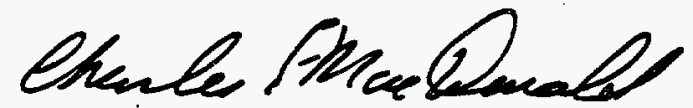

Charles E. MacDonald, Chief

Transportation Branch

Division of Safeguards and

Transportation, NMSS

Date: 
NRBK-41

B-72 
Drawing Not Available

B-73 


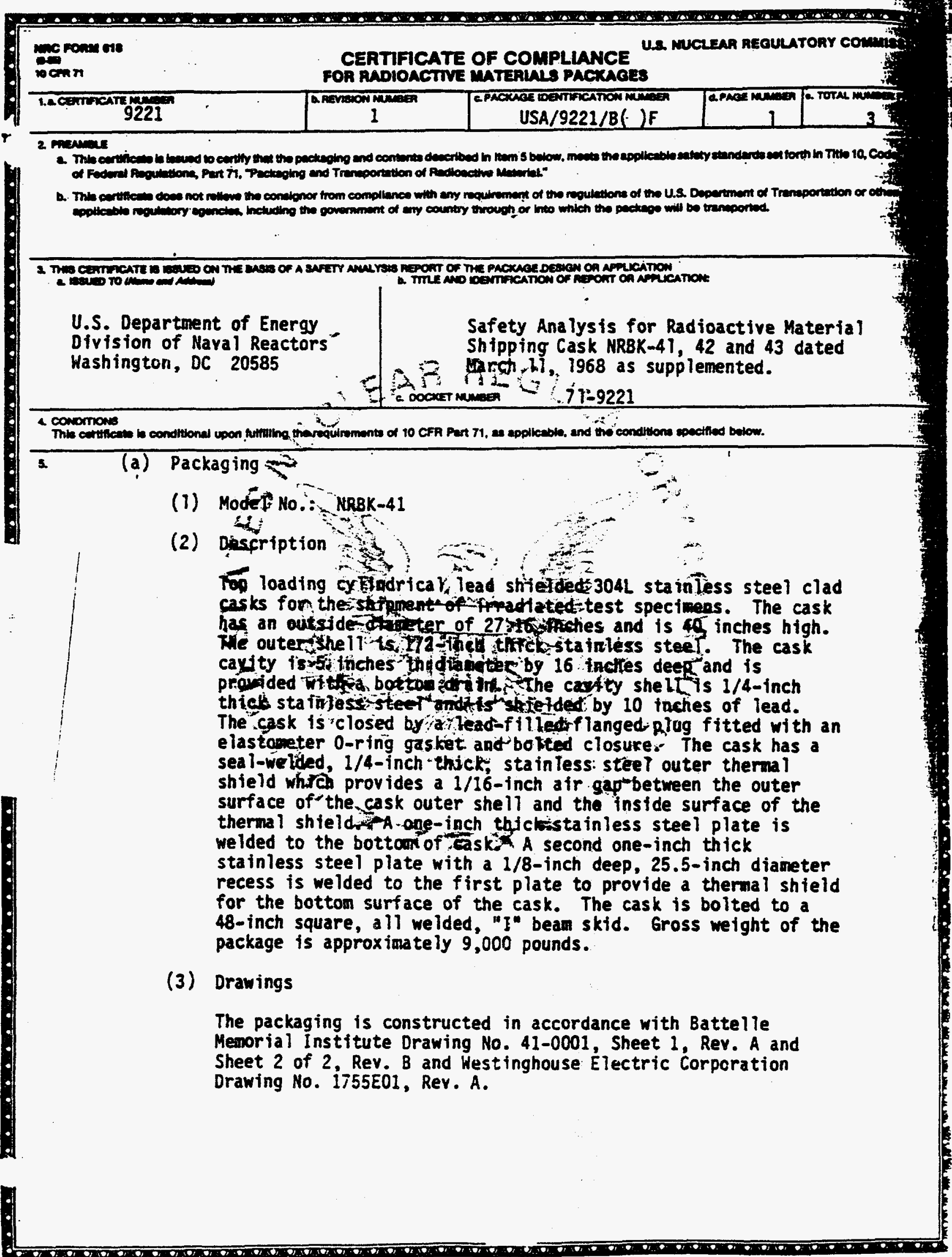




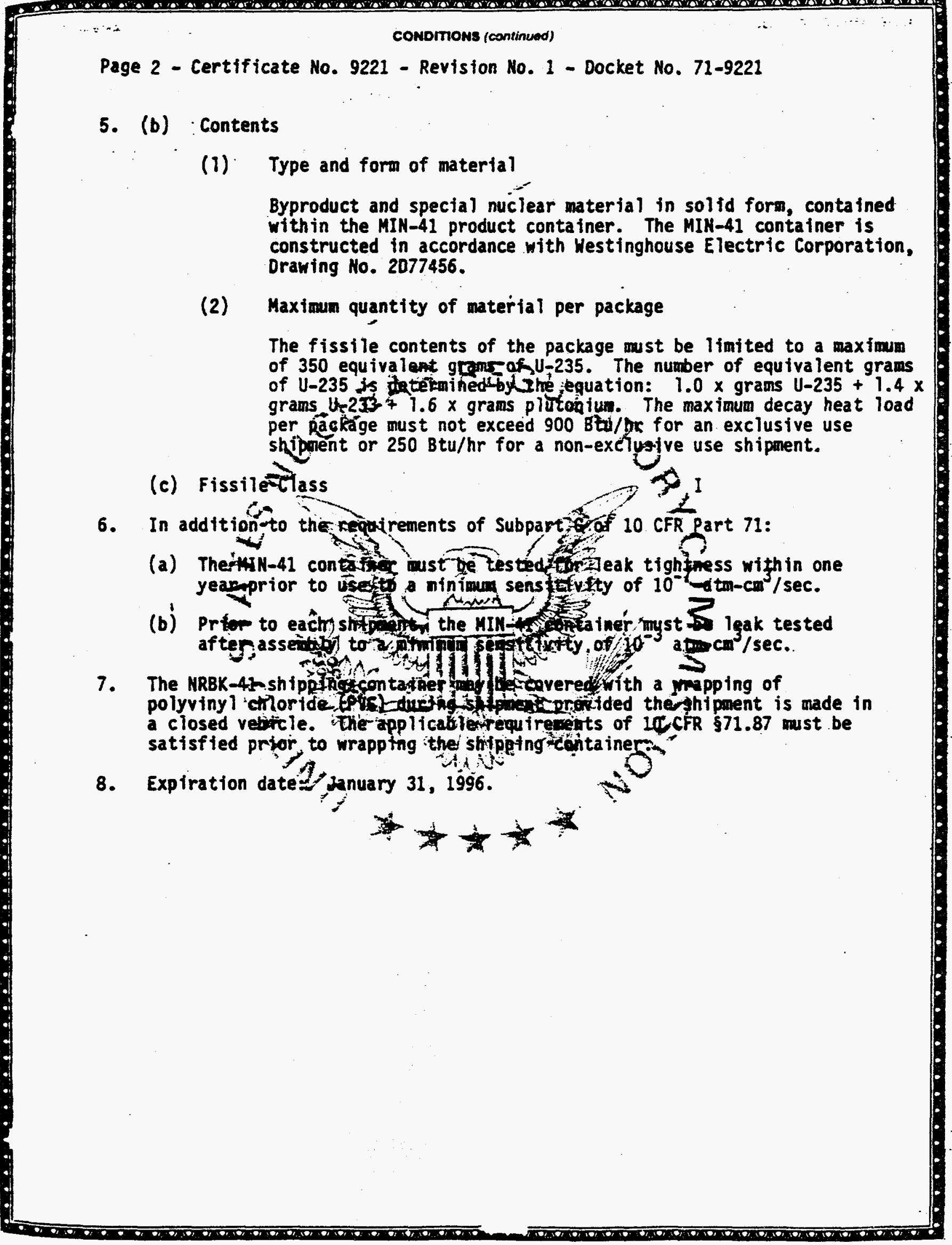




\section{conprnons (continued)}

Page 3 - Certificate No. 9221 - Revision No. 1 - Docket No. 71-9221

\section{REFERENCES*}

Safety Analysis for Radioactive Material Shipping Cask No. NRBK-41, 42 and 43 dated March 11, 1968.

Supplements: Division of Naval Reactors letters S\# 1458 dated June 19, 1968; Sf 1570 dated September 19, 1968; S\$ 1597 dated September 19, 1968; S\$ 1658 dated October 22, 1968; S* 1681 dated November 7,1968 ; S* 1690 dated November 22, 1968; S\& 1903 dated March 19, 1969; Sf 2000 dated June 2, 1969; S* 2509 dated June 10, 1970, and Bettis Atomic Power Laboratory letter WAPD-CL(IH)-733, dated October 10, 1968, and Division of Naval Reactors letters Zf 85-1605 dated Apri1 1,3 1985; S\# 86-3305 dated Fébruary 3, 1986; and S\$ 86-3332 dated June 16, 1986, and Bettis Atomic Power Laboratory letter KAPD"N(PAS)-526 dated June 20, 1986; and Division 'of Naval Reactors lettein S187-2738 däted September 18, 1987; and Naval Reactors letter S $\$ 90-12,039$, dated December $24,1990$.

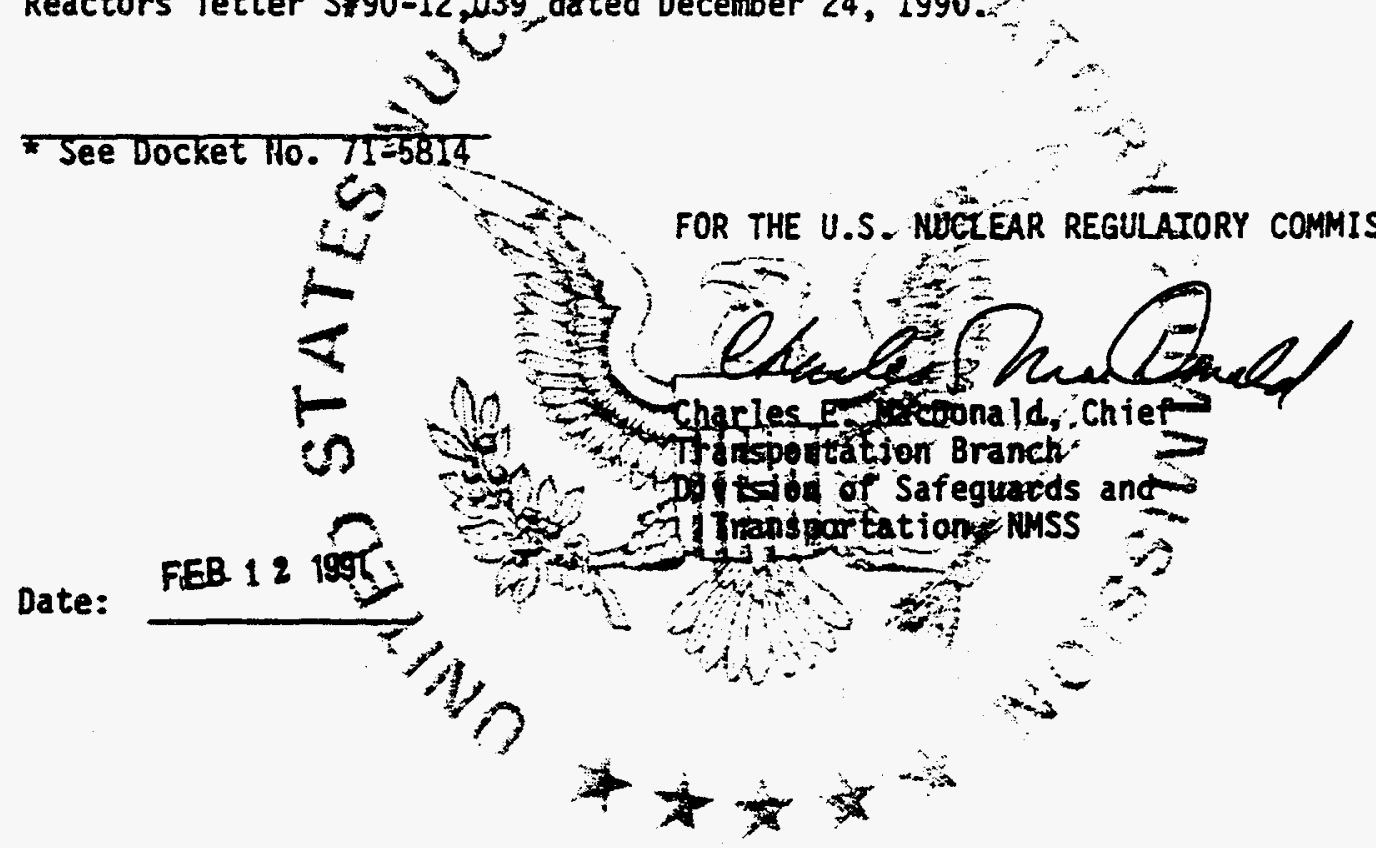


NAC-LWT

B-77 


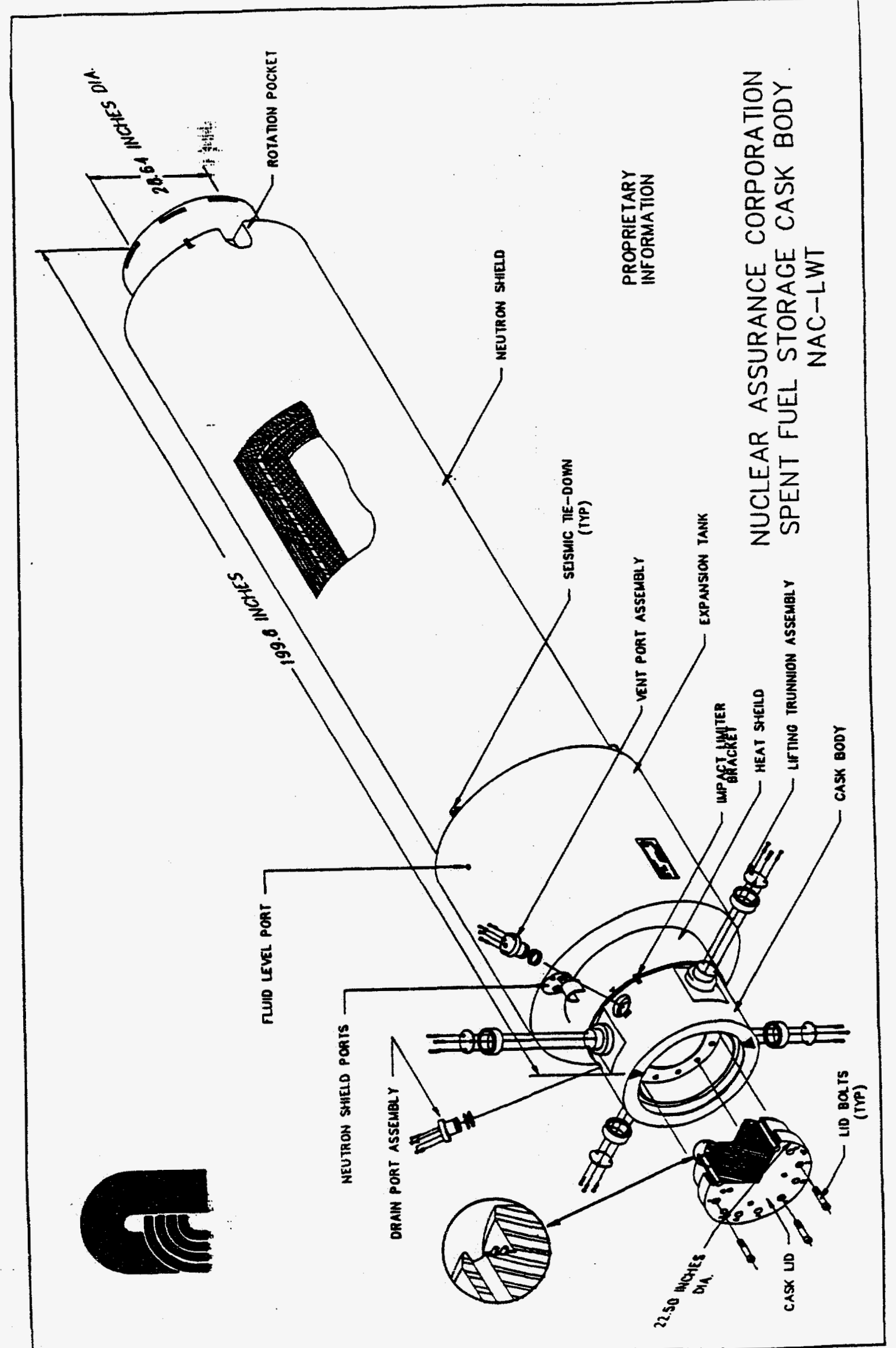




\section{NAC-LWT}

Designer

Type

Capaciry

Intact Spent Fuel Assemblies

Other Configurations

Weight (tons)

Loaded

Empty

Design Hear Rejection (kW)

Shape

Dimensions

Overall Leagth (in)

Overall Diameter (in)

Cavity Length (in)

Cavity Diameter (in)

Inner Shell Wall Thickness (in)

Lead Shield Wall Thickness (in)

Outer Shell Wall Thickness (in)

Neutron Shield Tank Thickness (ii)

Neutron Shield Wall Thickness (in)

Materials of Construction

Casik Body

Neutron Shield

Sides

End

Basket

Cooling Fins

Cavity Atmosphere

Operating Temperature ( $/ \mathrm{F}$ )

Operating Pressure (psig)

Outside Surface Dose (mrem/hr)
Nuclear Assurance Corporation

Legal Weight Truck Shipping Cask

1 PWR; 2 BWR (3.7 w/0; 4.0 w/o enrichment) 15 Metallic Fuel Rods

25.6

24.0

25

Cylindrical

199.80

442

180.90

13.375

0.75

5.75

1.20

5.00

0.25

SSReadSS

Water \& Ethylene Glycol (1.0 w/o Boron)

None

Aluminum

None

Air or Inert Gas

229 (cask radial surface; maximum)

0

100 (maximum)

Licensing Status - Safery Analysis Report submitted in March 1988 and licensed by the USNRC December 6, 1989.

Comments - Casks bave separate baskets for one PWR, two BWR or metallic fuel. Legal truck weight 80,000 pounds (maximum). 
Page 2 - Certificate No. 9225 - Revision No. 5 - Docket No. 71-9225

(2) Description (cont'd)

The cask is equipped with aluminum honeycomb impact limiters. The top impact limiter has an outside diameter of 65.25 inches and a maximum thickness of 27.8 inches. The bottom impact limiter has an outside diameter of 60.25 inches and maximum thickness of 28.3 inches. Both impact limiters extend 12 inches along the side of the cask body.

(3) Drawings

(i) The packaging is constructed in accordance with the following Nuclear Assurance Corporation Drawings:

LWT 315-40-01, ReV. 2

LWT 315-40-02, Rev. 4

LWT 315-40-03, Rev. 6

(Sheets 1 through 6 )

LWT 315-40-04, Rev. 5

LWT 315-40-05, Rev. 4

LWT $315-40-06$, ReV. 4

LHI 315-40-08, Rev. 4 (Sheets 1 and 2 )
Cask Assembly
Body Assembly
Transport Cask Body
Cask Lid Assembly Upper Impact Limiter
Lower Impact Limiter
Cask Parts Detail

(ii) Fhe fuel assembly baskets are constructed in accordance with the fol lowing Nuclear Assurance Corparation Drawings: LWT 315-40-09, Rev. 2 LWI 315-40-10, Rev. 2 ?

PWR Basket Späcer

LWT $315-40-11$ Rex: 1 PWR Basket LWT $315-40-12$, Rev. 3

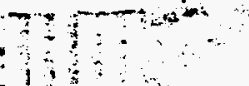

5. (b) Contents

(1) Type and form of material

Irradiated PWR or BWR uranium:oxide fuel assemblies or metallic fuel rods of the following specifications.

\begin{tabular}{|c|c|c|c|}
\hline & PWR & BWR & Metallic Rods \\
\hline Fuel Form & $\begin{array}{l}\mathrm{Cl} \text { ad } \mathrm{UO}_{2} \\
\text { pellets }\end{array}$ & $\begin{array}{l}\mathrm{Cl} \text { ad } \mathrm{UO}_{2} \\
\text { pellets }\end{array}$ & $\begin{array}{l}\text { Nat. Uranium } \\
\text { pellets }\end{array}$ \\
\hline $\begin{array}{l}\text { Nominal Pellet } \\
\text { Diameter, in. }\end{array}$ & 0.3659 & 0.487 & 1.36 \\
\hline Cladding material & Zircalloy -4 & Zircalloy-4 & Aluminum \\
\hline
\end{tabular}


Page 3 - Certificate No. 9225 - Revision No. 5 - Docket No. 71-9225

(b) (1) Continued

\begin{tabular}{|c|c|c|c|}
\hline & PHR & BWR & Metallic Rods \\
\hline $\begin{array}{l}\text { Nominal cladding } \\
\text { thickness, in. }\end{array}$ & 0.0243 & 0.032 & 0.080 \\
\hline $\begin{array}{l}\text { Maximum fuel rod } \\
\text { length, in. }\end{array}$ & 162 & 172 & 120.5 \\
\hline
\end{tabular}

Assembly array $\quad 15 \times 15 \quad 7 \times 7 \quad \mathrm{n} / \mathrm{a}$
Maximum Assembly
weight, lbs (15 rods)

Maximum initial fuel pin pressure at $70^{\circ} \mathrm{F}$, psig 565

Maxjouminitial is

Maximum initial of

content/assemb Iy, $k g$

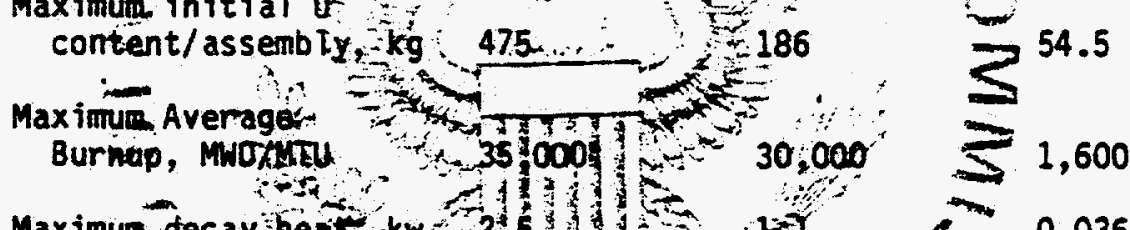

Maximundec ay hed -kw2 0.036

time, yr (2) Maximum quantity of material per package
Not to exceed 4,000 pounds inotuding contents and fuel assembly
basket.

(i) For contents described in Item 5.(b)(1): one PWR assembly, two BWR, or up to 15 intact metalific fuel rods positioned within the respective fuel assembly basket. Maximum decay heat not to exceed 2.5 Kilowatts.

(ii) For failed metallic fuel of the type described in Item 5.(b)(1):

(a) up to six canisters containing one defective metall ic fuel rod per canister. The canisters are 2.75-inch 1.0. failed fuel rod canisters as shown on Nuclear Assurance Corporation Drawing No. 340-108-02, Rev. 10, and are placed in a six-hole liner as shown on Nuclear Assurance Corporation Orawing No. 315-040-43, Rev. 1. The maximum decay heat load for a defective metallic fuel rod is limited to 5 watts; or. 
CONDITIONS (continued)

Page 4 - Certificate No. 9225 - Revision No. 5 - Docket No. 71-9225

s. (b) (2) Continued

(b) Up to three canisters containing either up to three defective metallic fuel rods per canister or up to 10 failed fuel filters per canister. The canisters are 4.00-inch 1.D. failed fuel rod canisters as shown on Nuclear Assurance Corporation Drawing No. 340-108-01, Rev. 10, and are placed in a three-hole basket as shown on Nuclear Assurance Corporation Drawing No. 315-40-12, Rev. 3. The weight of the filters is 1 imited to 125 pounds per canister. For canisters containing fuel rods, the maximum decay heat load is 15 watts per canister; and for canisters containing filters, the maximum decay heat load is 5 watts per canister. Plutonium content of the-fi]ters not to exceed 20 curies plutoniunde per package:

(c) Fissile Class

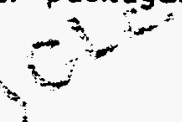

(i) For Metarlic Fuel Rods:

(ii) For BWR and PWR assemblies:

Maximum number of packages-in Fissine Class III shipment: One

failed fuet assemblies (rods) and fuel withriladding defects

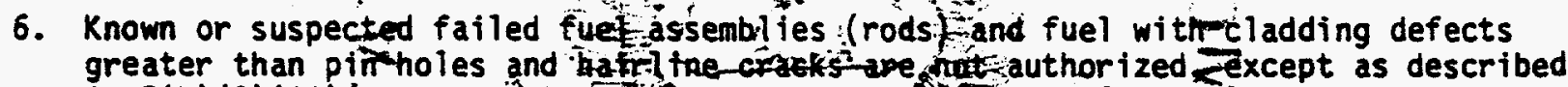
in 5(b)(2)(ii):

7. The cask must be dry (notree water)

8. Bolt torque:
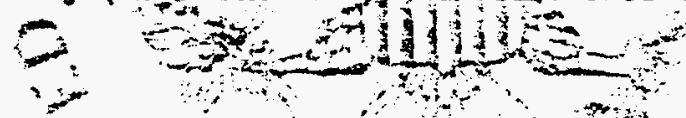

The cask lids bolts must be torqued-to 260 ft-Toss

it.

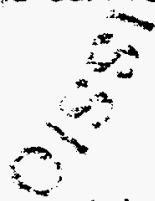

The bolts used to secure the vent and drain port covers must be torqued to 100 inch-ibs.

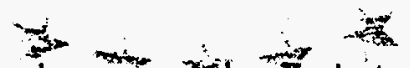

9. Prior to each shipment, the packagemus be Teak tested to $1 \times 10^{-3} \mathrm{std} \mathrm{cm}^{3} / \mathrm{sec}$.

Prior to first use, after third use, and at least once within the 12-month prior to each subsequent use, the package must be leak tested to $5.5 \times 10^{-7}$ std $\mathrm{cm}^{3} / \mathrm{sec}$.

10. In addition to the requirements of Subpart $G$ of 10 CFR Part 71:

(a) The metallic 0-ring seal must be replaced prior to each shipment; and

(b) Each package must meet the Acceptance Tests and Maintenance Program of Chapter 8 of the application; and

(c) The package shall be prepared for shipment and operated in accordance with the Operating Procedures of Chapter 7 of the application. If the cask is loaded under water or water is introduced into the cask cavity, the cask must be vacuum dried as described in Chapter 7 of the application. The cask cavity must be backfilled with $1.0 \mathrm{~atm}$ of helium when shipping PWR or BWR assemblies. 


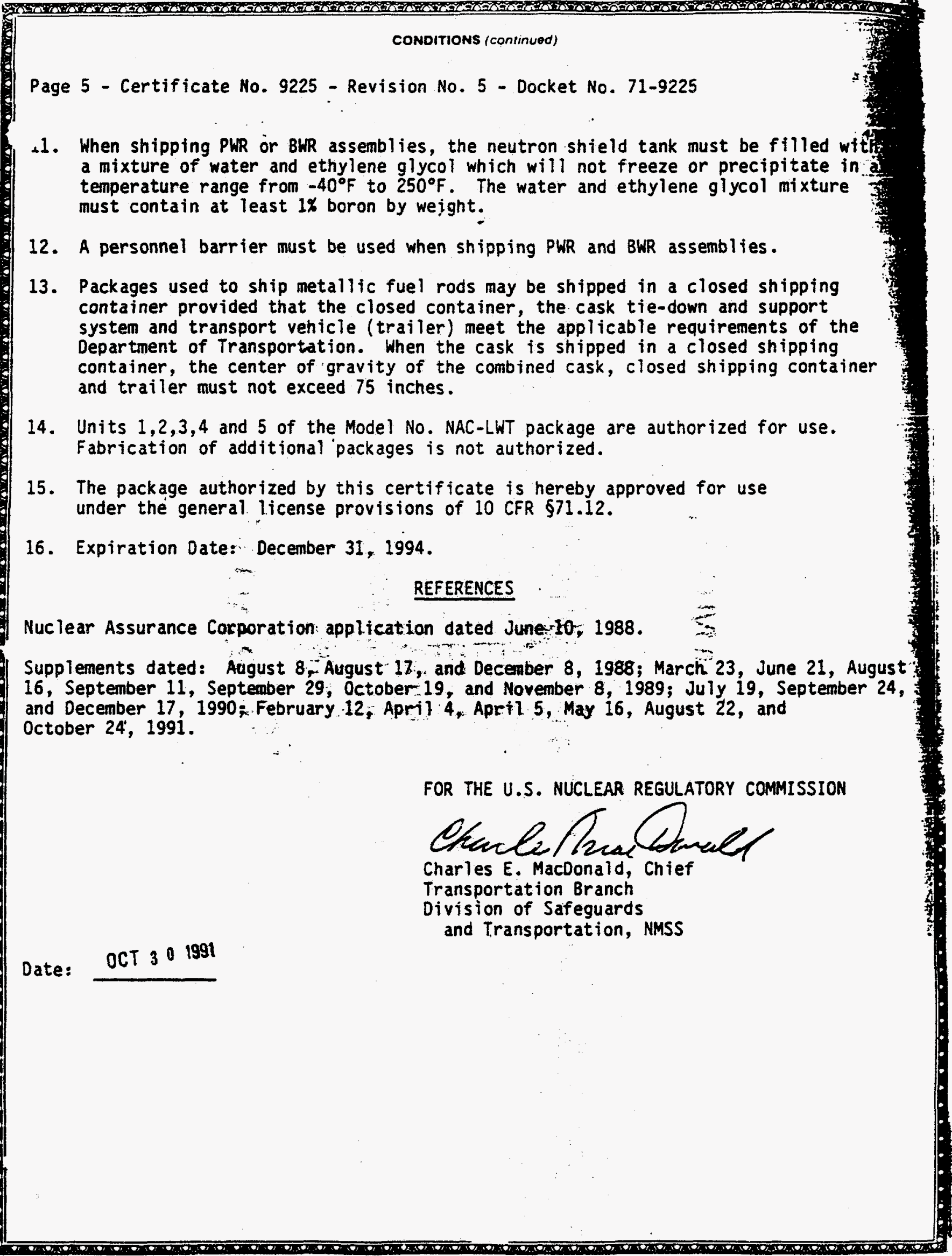


GE Model 2000 


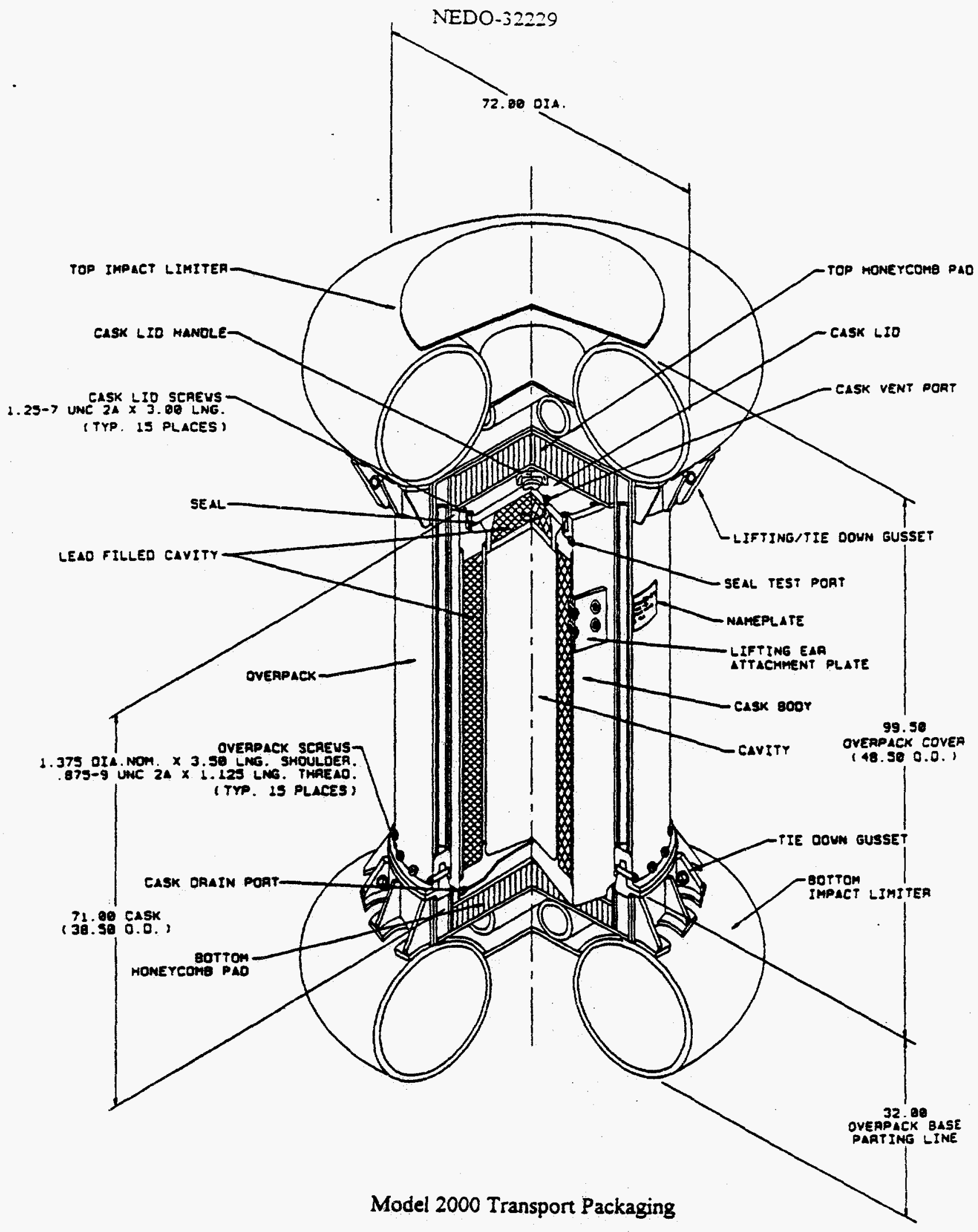


Page 2 - Certificate No. 9228 - Revision No. 4 - Docket No. 71-9228

Gussets on the top and bottom impact limiters provide tie-down points for the package. The cask body is equipped with attachment plates for lifting devices. The cask lifting devices are detached during transport. -

\section{(3) Drawings}

The packaging is constructed and assembled in accordance with General Electric Company Drawing Nos. 12904946, Rev. 8; 105E9520, Rev. 0; and 105E9521, Rev. 0.

Packaging Serial No. 2001 is constructed and assembled in accordance with General Electric Company Drawing Nos. 12904946, Rev. 8; 101 E8718, Rev. 11; and 101E8719, Rev. 9.

(b) Contents

(1) Type and form of material

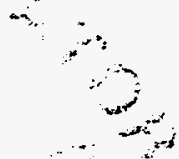

(i) Irradtated fuel rods, which may be cut or segmented.

(ii) Byproduct source, or-spectal nuctear material in solid form.

(2) Maximen quantity of material per package.

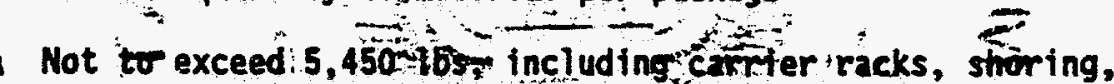
secondary containers and stiteld ing 1 toer; 600 , wat stedeay heat; and

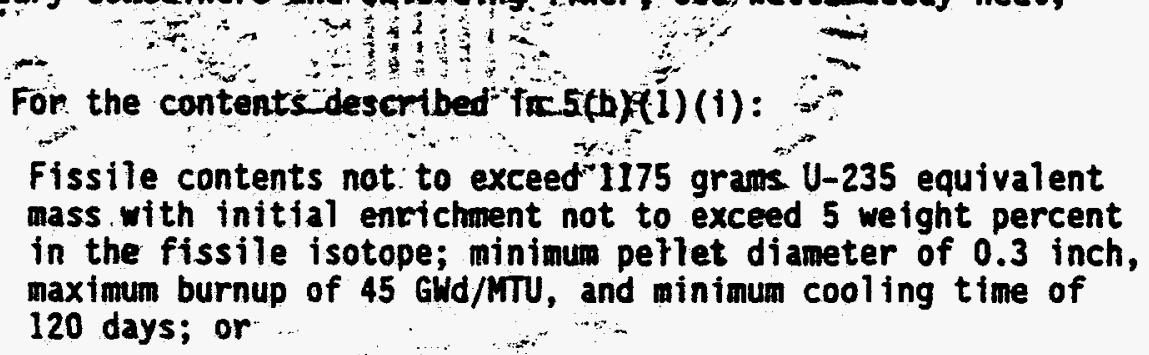

Fissile contents not to exceed 1750 grams $U-235$ equivalent mass with initial enrichment not to exceed 5 weight percent in the fissile isotope; minimum pellet diameter of 0.35 inch, maximum burnup of $38 \mathrm{GWd} / \mathrm{MTU}$, and minimum cooling time of 120 days. Fuel rods must be contained in closed, 5-inch schedule 40 pipe, with a maximum of 437.5 grams U-235 equivalent per pipe.

(ii) For the contents described in $5(b)(1)(i i)$ :

Fissile contents not to exceed 500 grams $U-235$ equivalent mass.

(c) Fisstle Class

Maximum number of packages per shipment One 


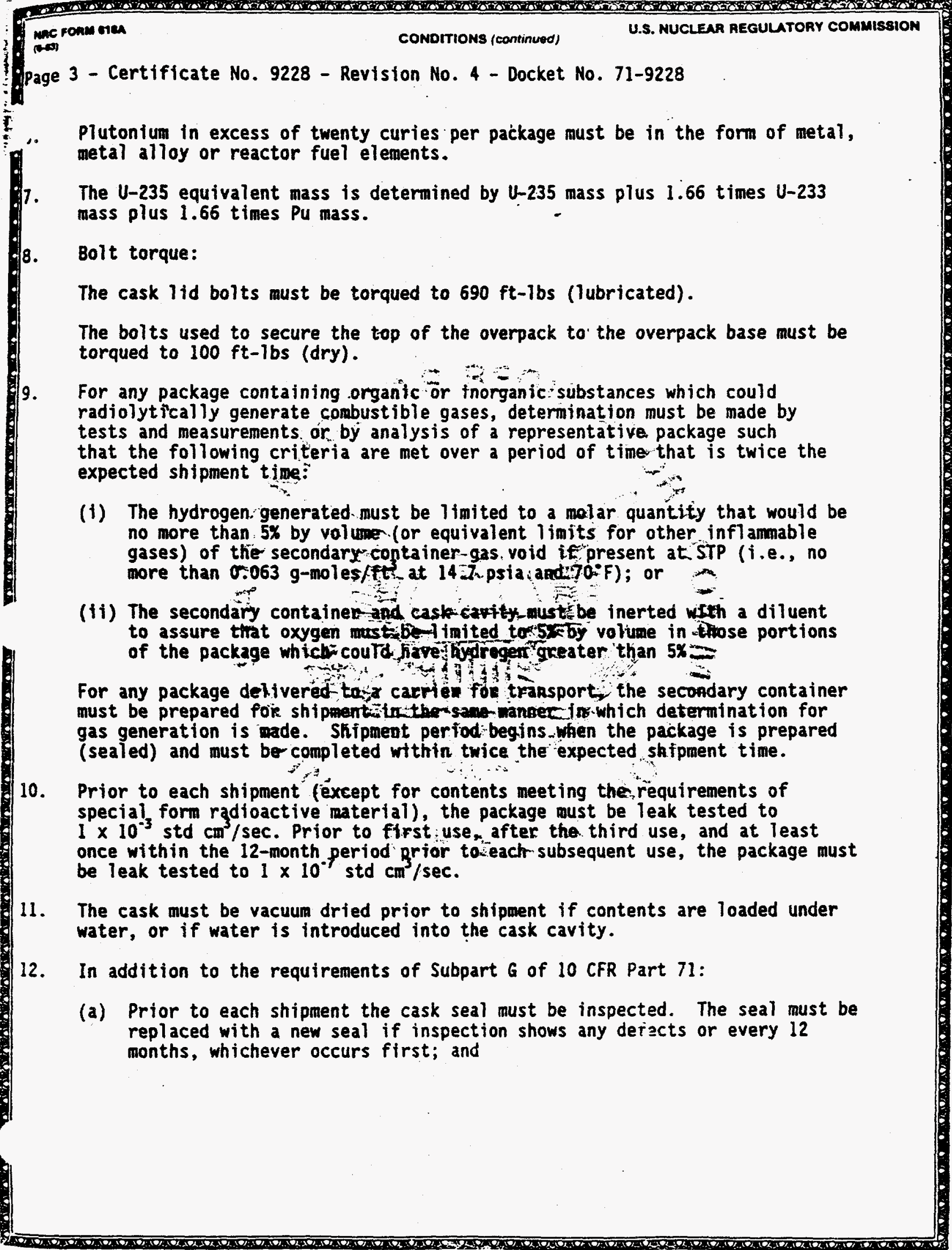


Page 4 - Certificate No. 9228 - Revision No. 4 - Docket No. 71-9228

(b) Each package must meet the Acceptance Tests and Maintenance Program of Chapter 8 of the application, except that inspections in Section 8.2 of the application must be performed at least once within the 12-month period prior to each use; and

(c) The package shall be prepared for shipment and operated in accordance with the Operating Procedures of Chapter 7 of the application.

13. Appropriate carrier racks or shoring must be provided to minimize movement of contents during accident conditions of transport. A lead liner, as shown in General Electric Company Orawing No. 12904922, Rev. -, which was included in the March 29, 1989 supplement, may be used inside the cask.

14. Each batch of ethylene propylene seals must be tested in accordance with Section 8.1.4.2 of the application.

15. The package authorized by this certificate is hereby approved for use under the general license provisions of 10 CFR $\$ 71.12$.

16. Expiration date: October 31, 1994.

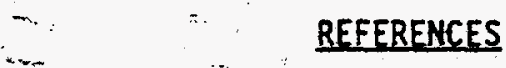

General Electric Company application-dated May 19, 1988.

pplements dated: March 29 and August 24, 1989; May 30, October 117-and

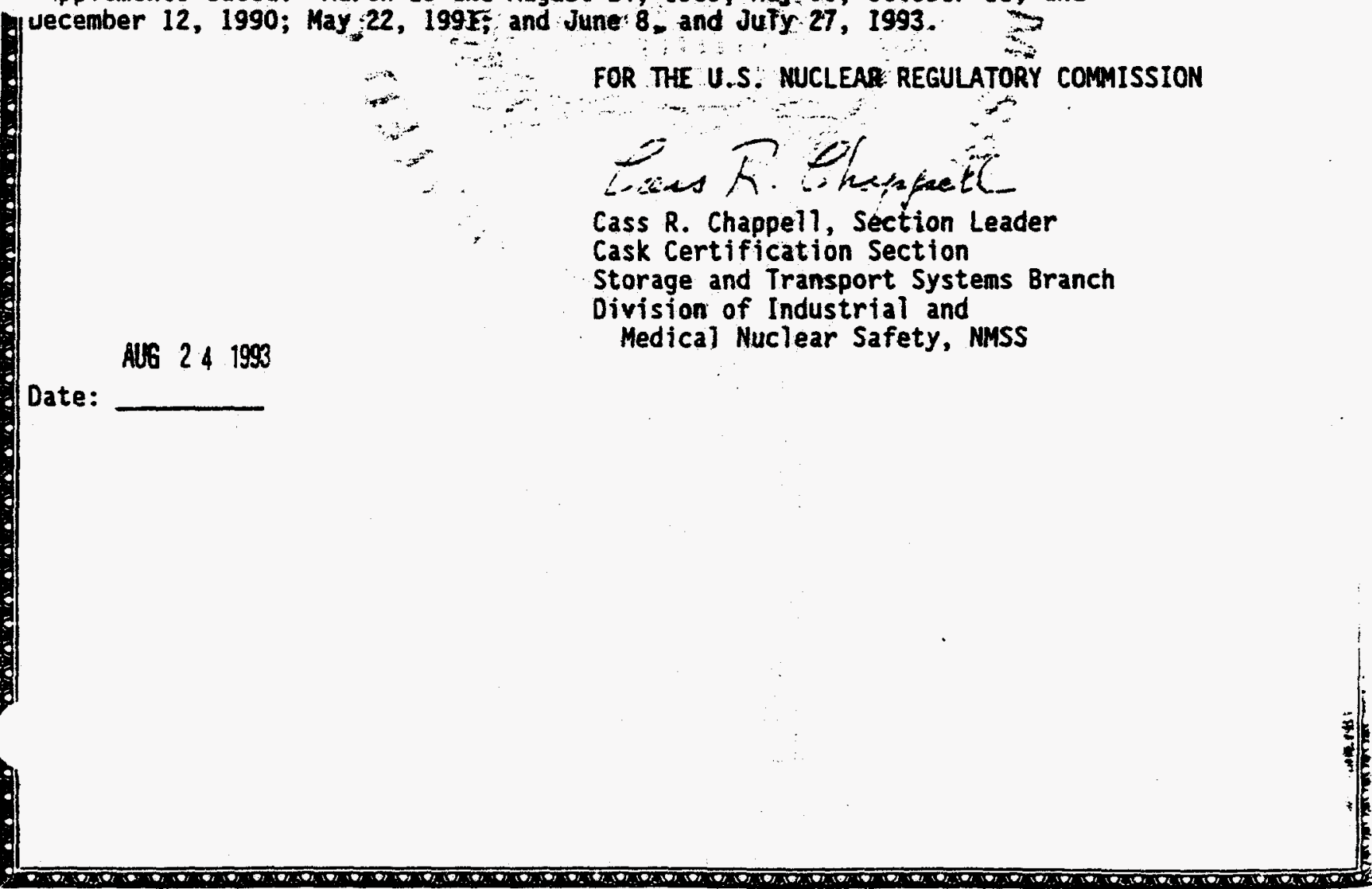


TN-FSV

B-91 


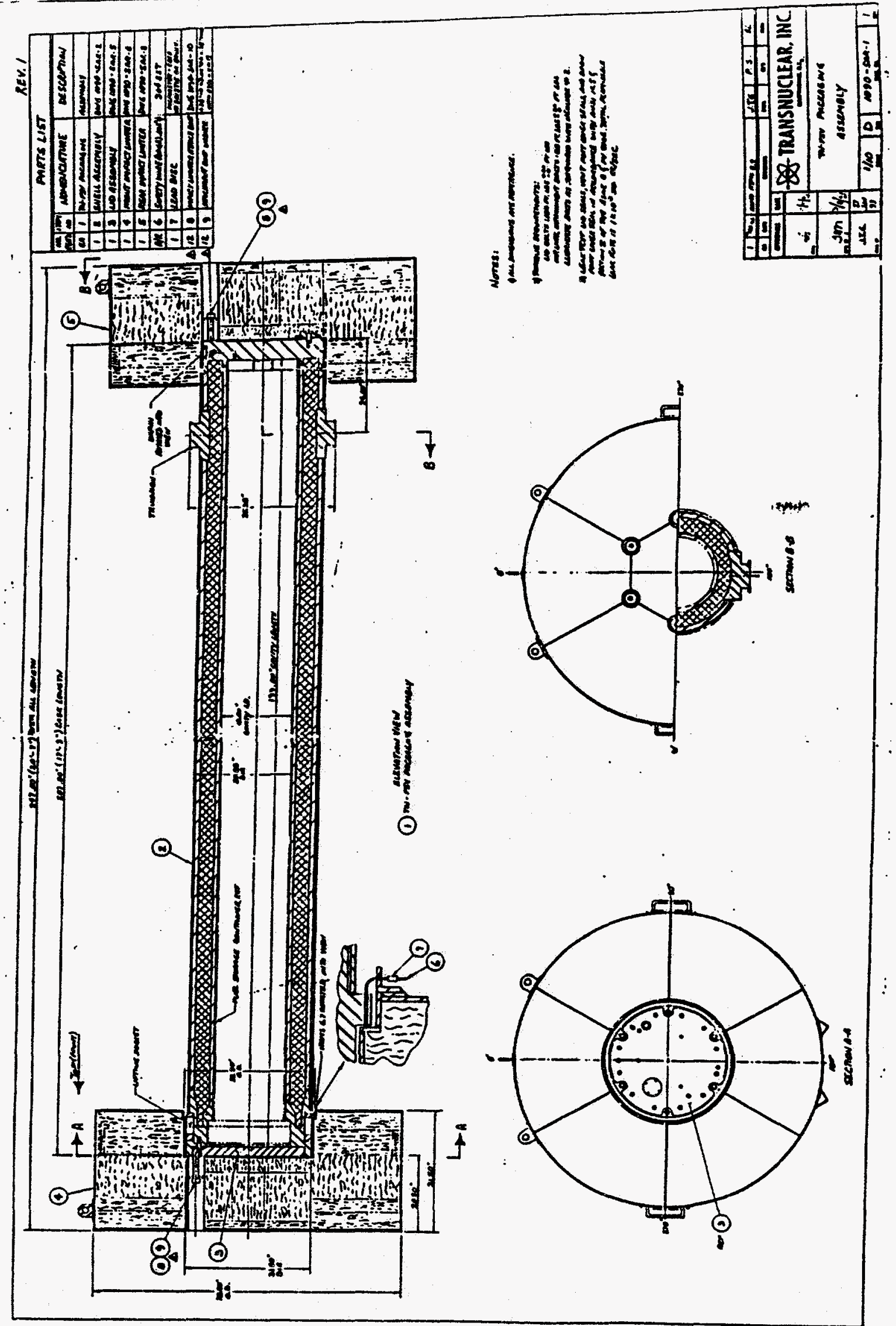




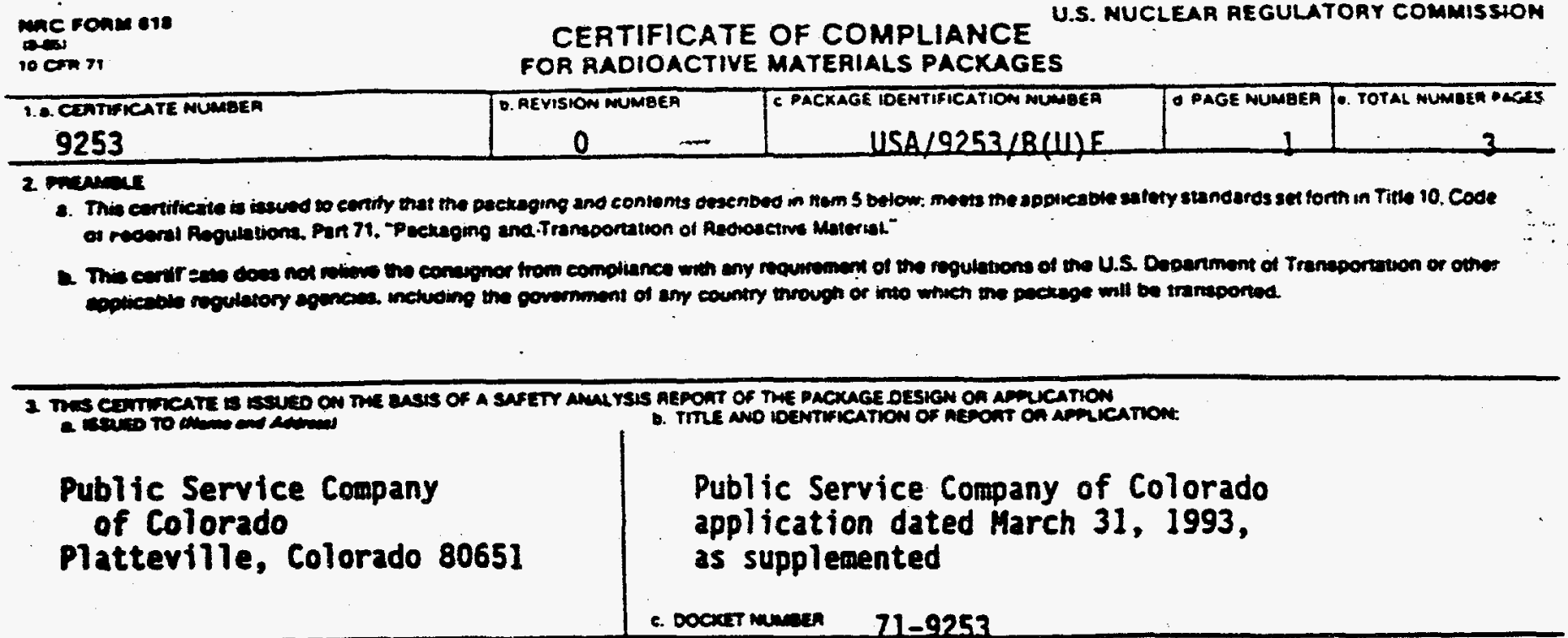

4 coromon:

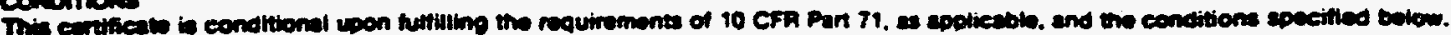

3.

(a) Packaging

(1) Model Ho.: TH-FSY

(2) Description

A steel and lead shielded shipping cask for irradiated high temperature gas cooled reactor (ITGR) fuel elements. The cask is a right circular cylinder, wth a baisa and redwood impact 1 imiter at each end. The package has approximate dimensions and weights as follows:

Cavity diameter
Cavity length
Cask body outer diameter
Lead shield thickness
Package overall outer diameter,
including impact limiters
Package overall length,
including impact limiters
Packaging weight
Gross package weight,
including contents

18 inches

199 inches

31 inches

3.44 inches

78 inches

247 inches

42,000 pounds

47,000 pounds

The cask body is made of two concentric shells of Type 304 stainless steel, welded to a bottom plate and a top closure fiange. The inner shell has an 10 of 18 inches and is 1.12 inches thick. The outer shell has an 00 of approximately 30 inches and is 1.5 inches thick. The annular space between the inner and outer shelis is filled with lead. The bottom plate is 5.5-inch thick Type 304 stainless steel. The closure 1id is 2.5-inch thick Type 304 stainless steel, and is fully recessed into the cask top fiange. The 1id is fastened to the cask body by 12, 1-inch diameter closure bolts. The lid is sealed with double-silicone 0 -rings, equipped. with a leak test port. A vent port and drain port are sealed with single silicone 0 -rings and cover plates. The cask body is covered with a stainless steel thermal shield composed of 0.25 -inch thick stainless steel plate over a wire wrap. The impact limiters are constructed of balsa and redwood encased in stainless steel shells. 
Page 2 - Certificate No. 9253 - Revision No. 0 - Docket No. 71-9253

5.(2)(2) Description (Continued)

The cask has two lifting sockets bolted to the cask top flange. Two rear trunnions are provided for cask tie-down.

The fuel elements are stacked in a carbon steel fuel storage container, which has an 00 of approximately 17.6 inches and an overali length of 195 inches. The fuel storage container has a 0.5 -inch thick shell, a 2.0-inch thick bottom plate, and a 1.5-inch thick lid. The lid accommodates a removable depleted uranium plug.

(3) Drawings

The packaging is constructed and assembled in accordance with the following Transnuclear, Inc. Orawing Nos.:

$$
\begin{aligned}
& \text { 1090-SAR-1, Rev. } 1 \\
& 1090-\text { SAR-2, Rev. 1 } \\
& 1090-\text { SAR-3, Rev. 1 } \\
& 1090-\text { SAR-4, Rev. 1 } \\
& \text { 1090-SAR-5, Rev. 1 }
\end{aligned}
$$

$$
\begin{aligned}
& \text { 1090-SAR-6, Rev. } 1 \\
& \text { 1090-SAR-7, Rev. 1 } \\
& 1090-S A R-8, \text { Rev. } 1 \\
& \text { 1090-SAR-9, Rev. 1 } \\
& \text { 1090-SAR-10, Rev. } 1
\end{aligned}
$$

\section{(b) Contents}

(1) Type and form of material

Irradiated HTGR fuel elements. Each fuel element consists of a graphite block containing fuel rods. The fuel is composed of thorium/uranium carbide and thorium carbide fuel particles within the fuel rods. The graphite block is hexagonal in cross section and is approximately 14.2 inches across the flats and 31.2 inches long. Each fuel element contains 2 maximum of $1.4 \mathrm{~kg}$ of uranium enriched to a maximum of 93.5 weight percent U-235 and approximately $11.3 \mathrm{~kg}$ of thorium. The maximum burnup is approximately 70,000 MND/MTIHM, and the minimum cool time is 1600 days.

(2) Maximum quantity of material per package

Six fuel elements, with decay heat not to exceed 60 watts per fuel element. The fuel elements are contained within a fuel storage container. Total weight of contents not to exceed 5,000 pounds, including fuel elements, fuel storage container, and depleted uranium shield plug.

(c) Fissite Class

III

Maxinum number of packages per shipment

One 
Page 3 - Certificate No. 9253 - Revision No. O - Docket No. 71-9253

6. The package must be leak tested as follows:

(a) Within the 12-month-period prior to shipment, and after seal replacement, the package must be \pm ested to show a leak rate no greater than $1 \times 10^{-3}$ std-cm/sec. The lesk-tést must have a sensitivity of at least $5: 10^{-6}$ std-cm/ $/ \mathrm{sec}$.

(b) Prior to each shipment, the package seals (main seal and vent seal) must be leak tested in accordance with Section 7.1.2 of the application, The acceptance criterion is a leak rate no greater than $1 \times 10^{-3} \mathrm{std}-\mathrm{cm}^{3} / \mathrm{sec}$. The test must have a sensitivity of at least $1 \times 10^{-3} \mathrm{std}-\mathrm{cm}^{3} / \mathrm{sec}$. The drain seal must also be tested if the drain port cover has been removed since the seal was last leak tested.

7. In addition to the requirements of Subpart 6 of 10 CFR Part 71:

(a) The package must be prepared for shipment and operated in accordance with the Operating Procedures of Chapter 7 of the application.

(b) Each packaging must meet the acceptance tests and must be maintained in accordance with the Acceptance Tests and Maintenance Program of Chapter 8 of the application.

(c) Prior to each shipment, the cask main closure seal and vent seal wust be inspected. The drain seal must be inspected if the drain port cover has been removed during preparation for shipment. All seals must be replaced within the 12-wonth period prior to shipment, or earlier if inspection shows any defect.

8. The package authorized by this certificate is hereby approved for use under the general license provisions of 10 CFR $\$ 71.12$.

9. Expiration date: May 31, 1999.

\section{REFERENCES}

Public Service Company of Colorado application dated Harch 31, 1993. Supplements dated: February 24, June 2, and June 14, 1994.

FOR THE U.S. RUCLEAR REGULATORY CONAISSION

ant $! 5194$.

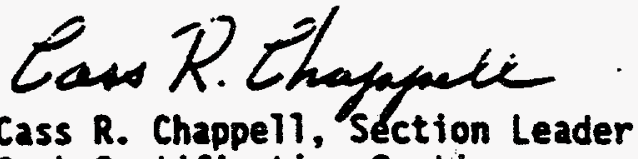

Cask Certification Section

Storage and Transport Systems Branch

Division of Industrial and Medical Nuclear Safety, NuSS

Date 
TN-8/8L

B-96 


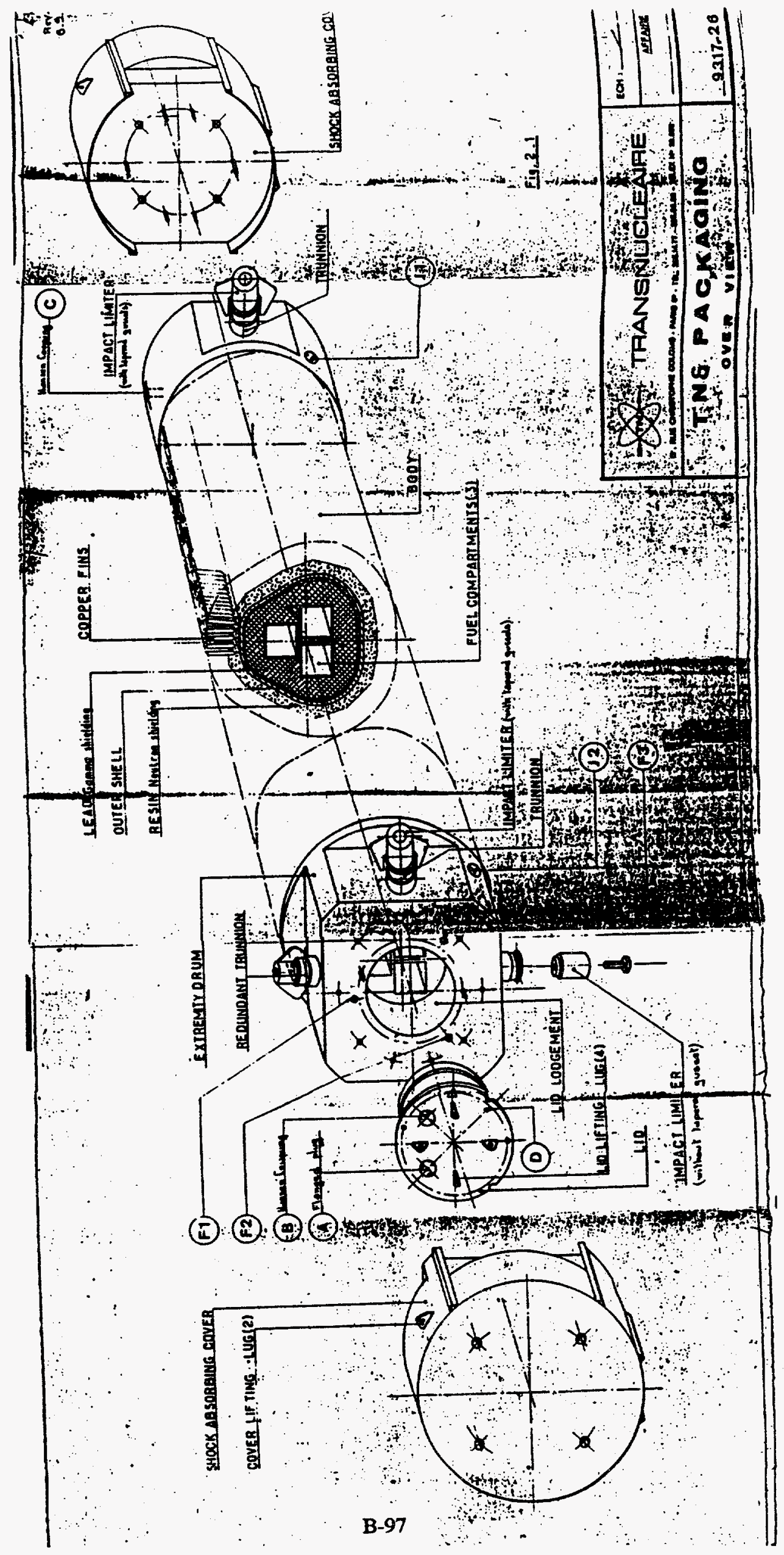




\begin{tabular}{|c|c|}
\hline a.PACE MUASA & - Total mumere bate \\
\hline 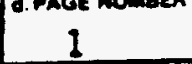 & 5 \\
\hline
\end{tabular}

2 menter:

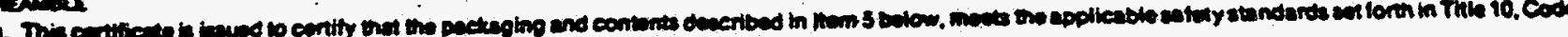

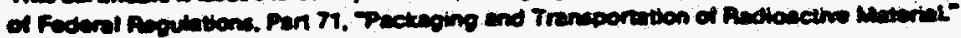

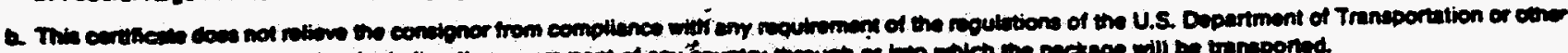

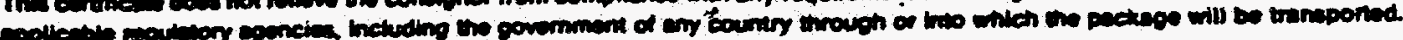

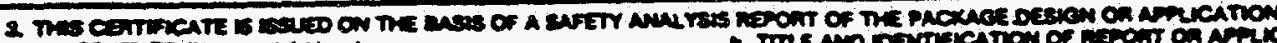

its

Transnuclear, Inc. Two Skyline Drive

Hawthorne, IY 10532-2120

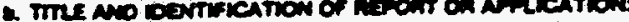

Transnuclear, Inc. application dated

March 25, 1991, as supplenented.

a Dooct 71-9015

4. coromous

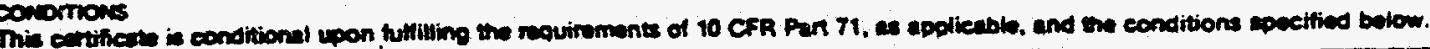

5

(a) Packaging

(1) Model Mos.: TN-8 and TK-8L

(2) Description

Lead, steel and resin shielded irradiated fuel shipping casks. The casks approximates a right circular cylinder 1,718 m in diameter and $5,516 \mathrm{~mm}$ long." The cavity consists of three sta inless steel square pressure vessels velded to an end plate and a circular stepped top flange, separated by a T-shaped copper plate and surrounded with $B 4 C+C u$ plates. Each cavity is $230 \times 230 \mathrm{~mm}$ and 4,280 an long. The ma in shielding consists of $135 \mathrm{~mm}$ of $7 \mathrm{ead}, 26 \mathrm{~mm}$ of stee 1 and $150 \mathrm{um}$ of resin. A wet cement layer is located between the lead and the outer shell. Radial copper fins are welded to the outer shell and cover the surface of the cask between each end drum. The Model No. TH-8 has 150 rows of fins and the Model Ho. TN-8L has 104 rows of $f$ ins.

The lid is a welded stainiess steel shell containing lead and resin shields. The pressure vessels are closed and sealed by sixteen, 1-1/4-inch diameter bolts and two stlicone rubber or Viton 0-rings located within recessed grooves on the top flange. Each extremity of the cask is surrounded by circular stainless steel drums reinforced by radial gusset plates and filled with balsa wood. A disk shaped impact limiter, constructed of carbon steel and balsa wood is fastened to each drum with four, 1-1/4-inch bolts. The vent and drain lines which penetrate the inner cavity are equipped with positive closures. In addition, all access ports are protected by the impact liniters.

The lid of the cask may be replaced with a modified lid which increases the cavity length to $4,362 \mathrm{~mm}$ or to $4,394 \mathrm{~mm}$ with the lif plate removed. This arrangement will be referred to as "Configuration $x$."

Trunnions are used for lifting and tte-down of the package. The package weighs approximately $36,000 \mathrm{~kg}$. 
CONDmONE (continued)

Page 3. - Certificate No. 9015 - Revision No. 17 - Docket No. 71-9015

(iif) Intact BHR and PUR fuel rods. The rods shall be constrained by a basket or grid structure; initial U-235 content shall be less than $15.0 \mathrm{~kg}$ per rod bundle; cross sectional area of the rods, tubes, and full length: structural material shall not be less than 29.6 square inches; and the bundle cross section shall not be greater than 8.5 inches. Maxinum weight per bundle shall not exceed $733 \mathrm{~kg}$. The Group I and Group II burnup Iinits of paragraph 5.(b)(1)(i) apply.

(2) Maximum quantity of material per package

(i) For the contents described Yn Item $5 .(b)(1)(i)$, Group I fuel assemblies:

Three PWR assemblies. The maximum decay heat load is not to exceed 35.5 kilowatts per package and 12 kilowatts per assembly for the Model Mo. TN-8 packaging and 23.7

kilowatts per package and 7.9 kilowatts per assembly for the Model Ko. TH-8L packaging.

(ii) For the contents described in Item 5.(b)(1)(i). Group II fuel assemblies: :

Three PUR asseabltes. - The maximan decay heat foad and the naximum free gas volune are not to exceed the linits listed in the table below:

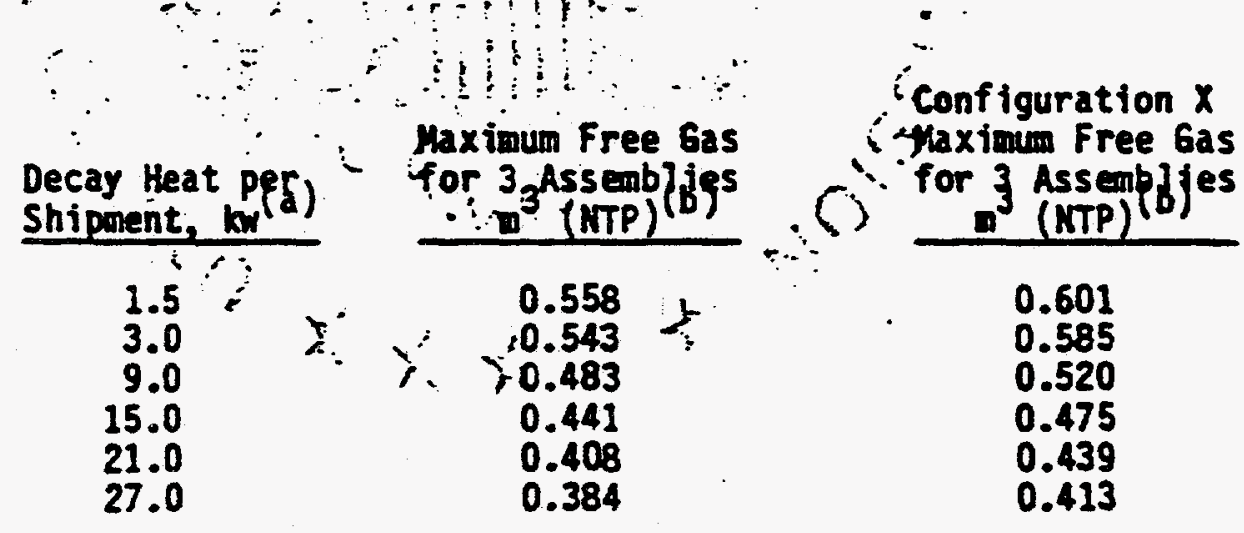

(a) Decay heat load per assenbly must not exceed 7.9 kilowatts for Model No. TH-8L packaging.

(b) NTP conditions are $25^{\circ} \mathrm{C}$ and one (1) bar. 
conomons (continuod)

Page 4 - Certificate No. 9015 - Revision No. 17 - Docket No. 71-9015

5. (b)(2) (Continued)

(iii) For the contents described in Item 5.(b)(1)(iii):

Three rod bundles. The maximum decay heat load and maximum free gas volume are not to exceed the linits listed in Paragraph 5.(b)(2)(ii).

(c) Fissile Class

III

Maximum number of packages per shipment One (1)

6. Group $I$ and Group II fuel assenblfes, efther Ir or SS clad, and bundles of PWR and/or BWR fuel rods that indivjdualiy meet 211 the appropriate specifications of $5 .(b)(1)(i), 5 .(b)(2)(i), 5 .(b)(1)(1 i i) ;$ and $5 .(b)(2)$ (iii) above may be packaged in any combination.<smiles>[Y]C1CCCC1</smiles>

7. PWR assemblies may be shipped either with or without burnable poison rod, thimble plug, or control rod assemblies.

8. As needed, appropriate component spacers may be used in the cask cavity to properly position the fue? assemblies.

9. The maximum weight of the contents (fuei assemblies, component spacers, inserts, irradiated hardirare, etc.) Tust pot exceed $2,200 \mathrm{~kg}$.

10. The cask cavity must he' Jry trio free pater) When ielivered to zarrier for transport. Restoual nolsture uist be pronptly renoved from the cask cavity by the methods described in Afriex I' to chapter yIII of the Application. For contents 5.(b)(1)(i) and $5 .(b)(1)($ ilfi); the carty ast be promptly backfilled with 1.0 atm of heliun, nitrogen, or argongas, gu

11. Known or suspected fájled fuel assemblies (rodś) and fuel cladding defects greater than pin holes and hajpine cracks aré not authorized.

12. For contents $5 .(b)(1)$ (iif the dryness verification test is required but leakage tests for containment asseably verification are not required.

13. The package contents wust be so linited that under normal conditions of transport, the total dose rates must not exceed $17 \mathrm{aren} / \mathrm{hr}$ at one neter from the surface of the package.

14. Any system used for cooling down the package must be provided with a pressure relief device set so that the maximum pressure in the containnent vessel cannot exceed 7 atmospheres during the cool-down process.

15. The systems and components of each packaging must meet the periodic tests and criteria specified in Chapter VIII of the Application. The $K$ fe verification and shielding efficiency verification tests in Chapter VIII of the Application must be performed on each packaging within the two year period preceding any shipment of contents $l$ isted in $5(b)(i)(i)$ and $5(b)(1)(i i i)$. The $K$ verification and shielding efficiency verification tests need not be pefformed on packaging during perfods (which may exceed two years) when only irradiated hardware as spectfied in $5(b)(1)(i i)$ is shipped. 
CONDmons (continued)

Page 5 - Certfficate No. 9015 - Revision No. 17 - Docket No. 71-9015

16. In addition to the requirements of 10 CFR Part 71:

(a) The package must be prepared for shipment and operated in accordance with the Operating Procedures in the application dated March 25, 1991 :

(b) Each package must be tested, repaired and maintained in accordance with the Acceptance Tests and Maintenance Procedures in the application dated March 25, 1991.

17. All valves, fittings, seais and rellef devices must be of the type, size, wodel and manufacture as indicated on the design drawings. The resin material must be of the specifications stated in Annex $A$ to Chapter II of the Application.

18. In accordance with Annex $L$ to Chapter $V I 1$, Gi periodic intervals not to exceed two years, the thermal performance of the cask nust be analyzed to verify that the cask operation has not degraded below that which is licensed*. Following the initial acceptance tests, the heat source may be that provided by the decay heat from the loading of -the package, provided that the heat source is equal to at least $25 \%$ of the design heat load for the package. Each cask that fails to meet the thermal acceptance criteria given in Annex $L$ of the Application must be withdrawn from service until corrective action can be completed or the license amended to limit the package zo lower heat joad. s..

ॠ The thermai.perforpance test is inot requifed at periodic-intervals when the baximum decay int Hoad per pactiage Hoes not exceed $25 \%$ of the design heat Joad.

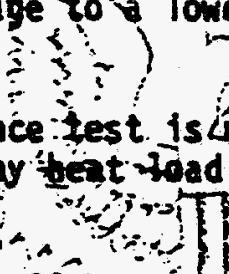
$\therefore$

19. The Configuration $X$ lid salf be operted ind ma intained in accordance with Annex N to Chapter WII; in the Fppitication dated March 25, 1991.

20. The package authoriat by this bertificate. is hereby approved for use under the general license provisjpons of 10 CFR 1712.12.

21. Expiration date: May 31,2996.

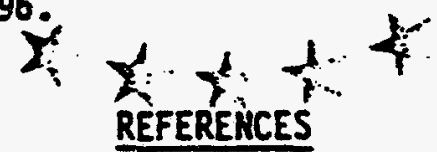

Transnuclear, Inc. application dated March 25, 1991

Supplements dated: Aprit 22, 1991.

FOR THE U.S. NUCLEAR REGULATORY COMISSION

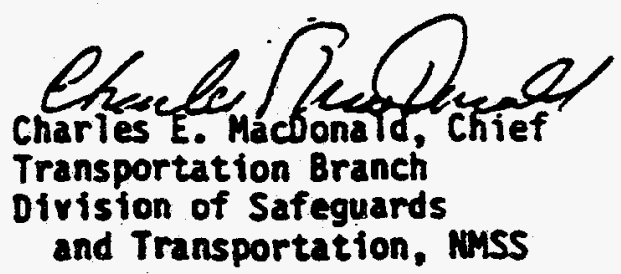

Date: MAY 111991 
TN-9

B-103 


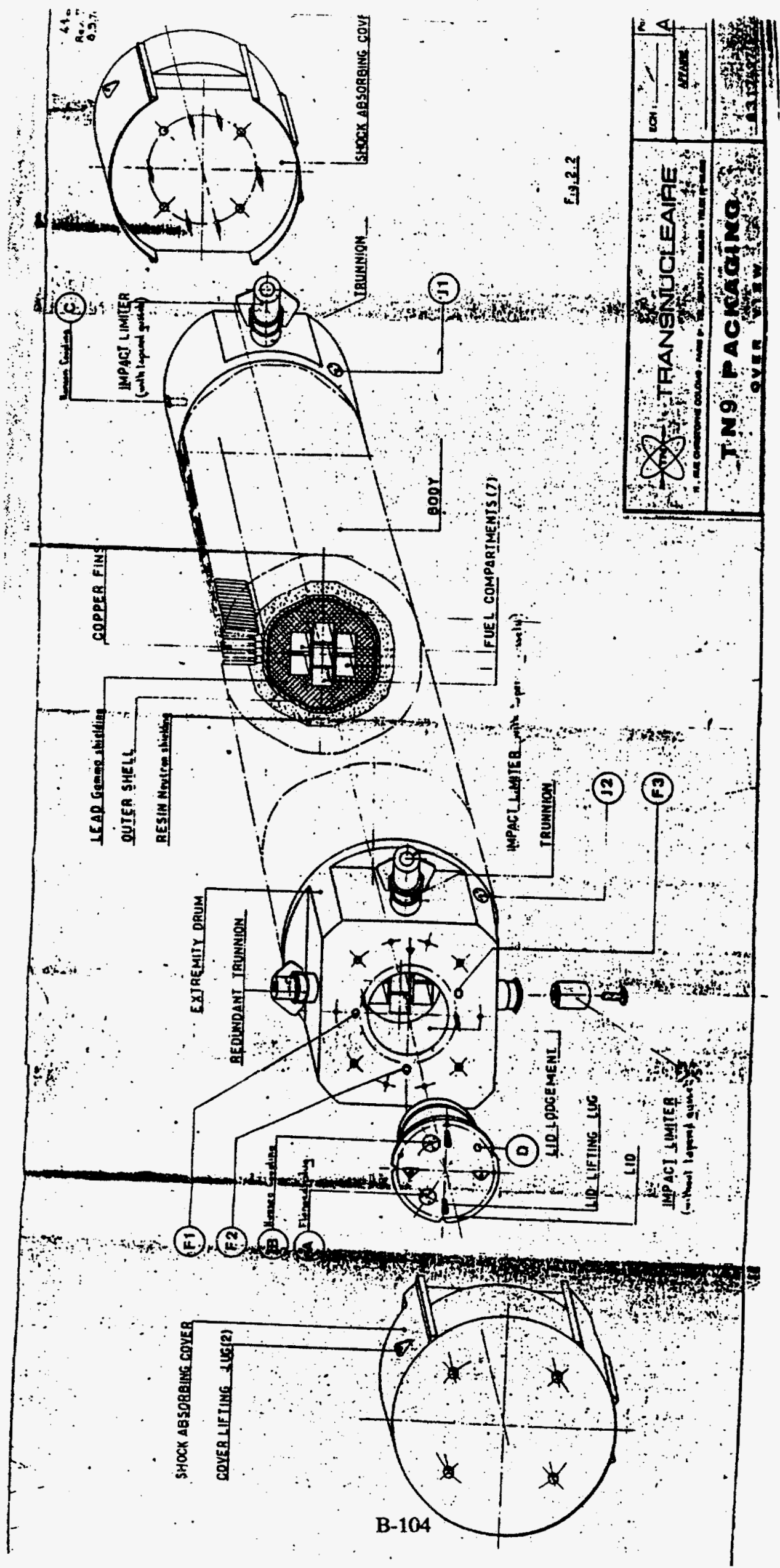


CEATIFICATE OF COMPLIANCE

\begin{tabular}{|c|c|c|c|c|}
\hline 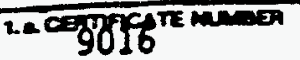 & $\begin{array}{l}\text { a pronson MUMBef } \\
9\end{array}$ & $\begin{array}{l}\text { C.PACKUGE IOENTIFICATION MUMAEA } \\
\text { USA/9016/B( )F }\end{array}$ & $\begin{array}{l}\text { A. PAGE MUMEER } \\
1\end{array}$ & $\begin{array}{c}\text { - TOTAL NUMAg paess } \\
4\end{array}$ \\
\hline
\end{tabular}

2 menimer

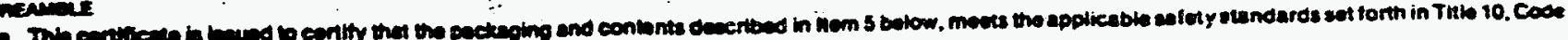

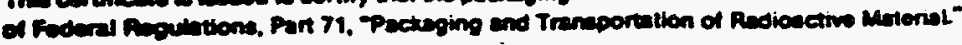

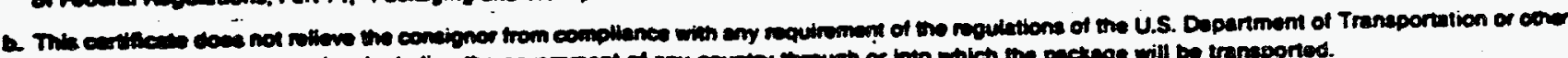

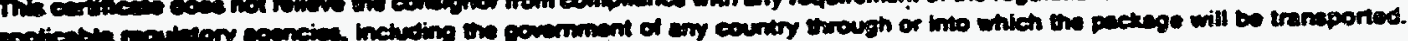

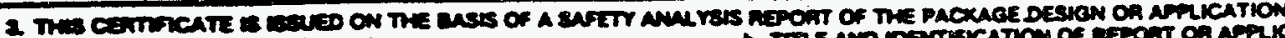
a

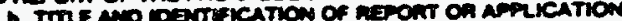

Transnuclear, Inc. Two Skyline Drive

Hawthorne, WY 10532-2120

Transnuclear, Inc. application dated

March 25, 1991, as supplemented.

e dockrt mangs $71-9016$

conomons

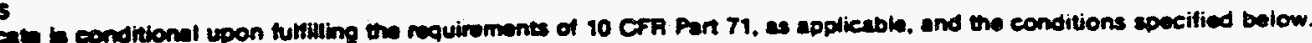

s.

(a) Packaging

(1) Model Ho.: TH-9

(2) Description

A lead, steel and resin shielded irradiated fuel shipping casks. The casks approximates a right circular cylinder 1,718 in in diameter and 5,756 mul long. The cavity consists of three rectangular, stainless steel pressure vessels welded to end plates and a circular stepped top flange, separated by thin cooper plates. The bays are divided into a total of seven square compartnents, $150 \times 150 \mathrm{~mm}$ and $4,520 \mathrm{~mm}$ long. The main shielding consists of $128 \mathrm{~mm}$ of lead, $26 \mathrm{~mm}$ of steel and $150 \mathrm{~mm}$ of resin. A wet cement layer is located between the lead and the outer shell. Radial copper fins are welded to the outer shell and cover the surface of the cask between each end drum.

The lid is a welded stainies's stee! stell containing lead and resin shields. The pressure vessels are closed and sealed by sixteen, 1-1/4-inch diameter bolts and two silicone rubber or Viton 0-rings located with in recessed grooves on the top flange. Each extremity of the cask is surrounded by circular stainless steel drums reinforced by radial gusset plates and filled with balsa wood. A disk shaped impact limiter, constructed of carbon steel and balsa wood is fastened to each drum with four, 1-1/4-inch bolts. The vent and dra in lines which penetrate the inner cavity are equipped with positive closures. In addition, all access ports are protected by the impact limiters. Trunnions are used for lifting and tie-down of the package. The weight of the package is approximately $36,000 \mathrm{~kg}$.

\section{(3) Drawings}

The package is constructed in accordance with Transnuclear Drawing No. 9317.03, Rev. J. The materials of construction and welds must be in accordance with Annex $A, B$, and C to Chapter $I I$ of the Application.

$$
\text { B-105 }
$$


conomons (coninued)

Page 2 - Certificate No. 9016 - Revision No. 9 - Docket No. 71-9016

5. (b) Contents

(1) Type and form of material

(i) Irradiated BWR uraniun oxide fuel assemblies of the following specifications:

Fuel form

Cladding material

Initial fuel pin pressure at $100^{\circ} \mathrm{F}$, psig

Maximum initial $U$ content/. assembly, $\mathrm{kg}$ :

Maxinum average initfal U-235

enrichment, $w / 0$

Clad $\mathrm{UO}_{2}$ Pellets

Zr or $S S$

Maximum bundle cross section, in

Maximum active fuel length, in

Average burnup, MWD/MTO

Minimum cooling time, day

Maxinum weight/fuel assembly, kg

200

201

2.65

5.52

144

36,500

150

300

(ii) Solid non-fisslle irradlated hardware. As needed, appropriate component spacers iust be used when loading Arradiated hardware into the cask cavity to limit movement of the contents during accident conditions of transport.

(2) Maximin quantity of material per package

(i) Seven BWR zssenbities. The maximum decay heat joad per package is not to exceed 24.4 kilowratts and $3.5 \mathrm{kllowatts}$ per assembly. As needed, appropriate component spacers may be used in the cask cavity to properly position the fuel assemblies.

(ii) The maximim weight of the contents (fuel assemblies, component spacers, inserts, irradiated hardure, etc.) wust not exceed $2,110 \mathrm{~kg}$.

(c) Fissile Class

III

Maximum number of packages per shipment

One (1)

6. The cask cavity must be dry (no free water) when delivered to a carrier for transport. Residual moisture must be promptly renoved from the cask cavity by the methods described in Annex I to Chapter VIII of the application. For contents 5.(b)(I)(i), the cavity must be promptly backfilled with 1.0 atm of helfum, nitrogen, or argon gas. 
conomons (continued)

Page 3 - Certificate No. 9016 - Revision No. 9 - Docket No. 71-9016

7. In addition to the requirements of Subpart 6 of 10 CFR Part 71:

(a) The package must be prepared for shipment and operated in accordance with the Operating Procedures in Chapter VIII of the Application.

(b) Each package must be tested and maintained in accordance with the Acceptance Test and Maintenance Procedures in Chapter VIII of the Application.

8. Known or suspected failed fuel assemblies (rods) and fuel with cladding defects greater than pin holes and hairline cracks are not authorized.

9. For cortents $5 .(b)(1)(i f)$, the dryness jerification test is required but leakage tests for assembly verification are not required.

10. The package contents must be so limited that under normal conditions of transport, the total dose rates must not exceed $14 \mathrm{mrem} / \mathrm{hr}$ at one eter from the surface of the package.

11. Any system used for cooling down the package must be provided with a pressure relief device set so that the maximum pressure in the containment vessel cannot exceed 7 atmospheres during the cool down process. :

12. The systems and components of each packaging wist meet the perfodic tests and criteria specified in Chapter. YIII of the Application. . Each packaging that fails to meet these iriteria wist be withdrawn from service unitil corrective action has been completed. $?$ seats and

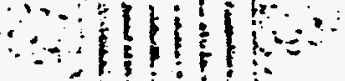

13. All valves, fittings, seals and felief devlces must be of the type, size, nodel and manufacture as indicated on the design' drawings. The resin material must be of the specifications stated in Annex $A$ to Chapter 11 of the Application.

14. In accordance with Annéx $L$ to Chapter $v y$ ily, at periodic intervals not to exceed two years, the thermal performance of the cask must be analyzed to verify that the cask operation has not degraded below that which is licensedt. Following the initial acceptance tests, the dreat source nay be that provided by the decay heat from the loading of the package, provided that the heat source is equal to at least $25 \%$ of the design heat load for the package. Each cask that fails to weet the thermal acceptance criteria given in Annex $L$ of the Application must be withdrawn from service until corrective action can be completed or the license amended to linit the package to lower heat load.

TTe thermal performance test is not required at periodic intervals when the maxinum decay heat load per package does not exceed 258 of the design heat load. 
commons (continued)

age 4 -Certificate No. 9016 - Revision No. 9 - Docket No. 71-9016

15. The package authorized by-this certificate is hereby approved for use under the general license provisions of 10 CF $\$ 71.12$.

16. Expiration date: Mar 31, 1996.

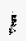

REFERENCES

Transnuclear. Inc. application dated March 25, 1991

Supplements dated: April 22, 1991.

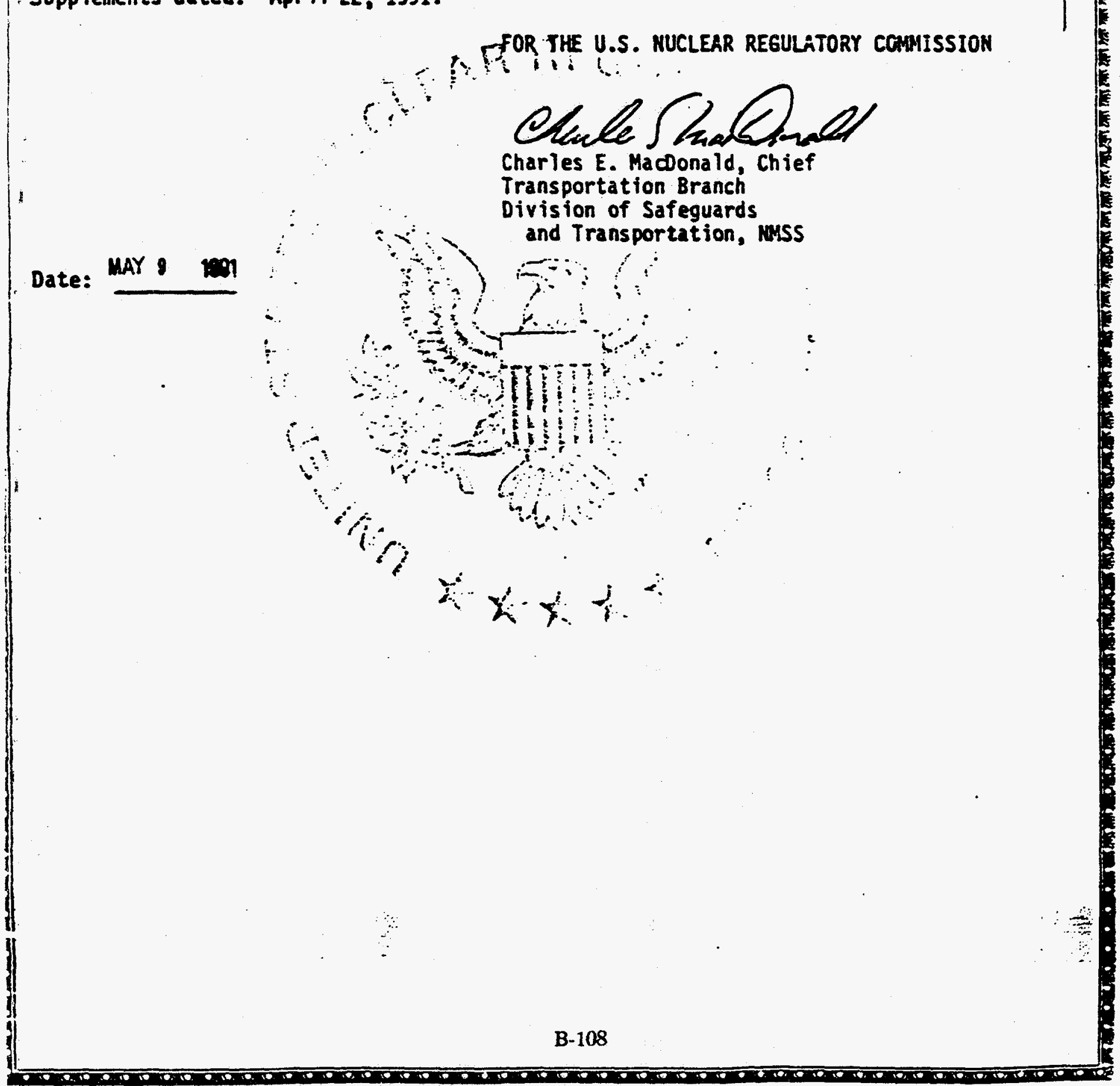


M-130

B-109 
Drawing Not Available 


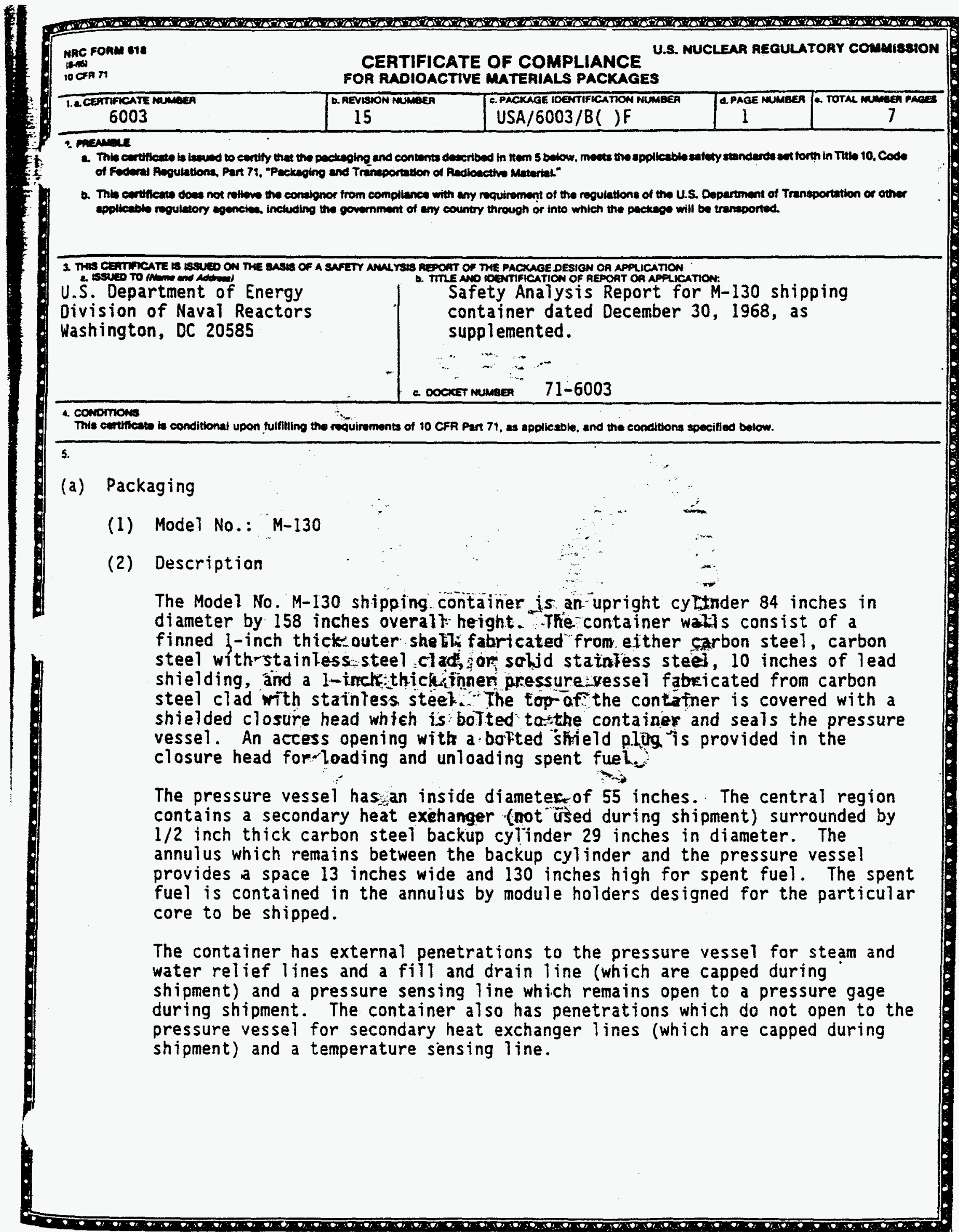


CONOITIONS (continued)

Page 2 - Certificate No. 6003 - Revision No. 15 - Docket No. 71-6003

i. (a) Packaging (cont'd)

(2) Description (cont'd)

For LWBR spent fuel shipments, the heat exchanger and associated structures have been removed, external penetrations plugged and seal welded, and an external shield and energy absorber added during modifications.

The container is supported on its transport vehicle by an " $A$ " frame structurf Gross weight of the loaded container without its support structure is approximately 228,000 pounds.

(3) Drawings

$$
-\infty
$$

The packaging is constructed in accordance with General Electric Drawing Nos. 247E209; Sheet 1, Rev. R; Sheet:2,-Rev. K; Sheet 3, Rev. T; Sheet 4, Rev. U: Sheet 5 of 5 , Rev. F and 247E228, Rev. F.

For LWBR spent fivel shipments, the container has been modified in accordance with Westinghouse Electric Orawing 1176J48, Sheet 1 Rev. G, Sheet 2 Rev. E an an external energx absorber added in accordance with Westinghouse Electric Drawing 1525E32, Rev. A-.

(b) Contents

(1) Type and
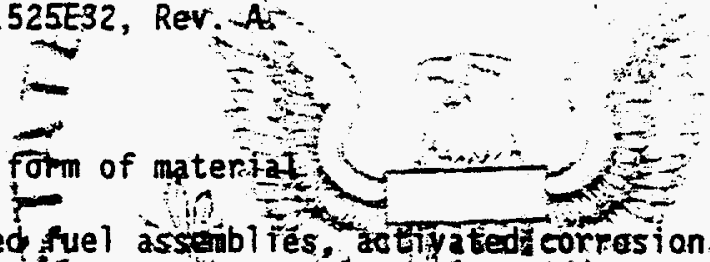

Irradiated containing

(viii) 53G-3/3A fuel module with or without control rods.

(ix) SAD cell.

S3Nis4W fuel subassembities of conetype 2.

S5W fure modules of cone types 2 or 3 .

S5W corner fuel modules of core types 2 or 3.

DIG fuel modules of rerestypes 1 or 2 .

DIG removable fuel assemblies of core types 1 or 2 .

SIC/S2C fuel modules with control rods.

SIC/S2C peripheral fuel modules.

(ix) SAD cell. 


\section{CONDTrions (continued)}

Page 3 - Certificate No. 6003 - Revision No. 15 - Qocket No. 71-6003

i. (b) Contents (cont'd)

(1) Type and form of material (cont'd)

(x) S3G-3/3A irradiated thermocouples and thermocouple cases.

(xi) S8G'full size fuel cell with or without control rod.

(xii) S8G partial size fuel cell with or without control rod.

(xiii) S5W-4A recoverable irradiated fuel modules with control rod.

(xiv) S7G recoverable irxadiated fữelicezlls.

(xv) D2W fuel cells with control rods.

(xvi) NR-1 fuel-midules with or without control rods

(xvii) ATC fuel moduies with or without control rods.

(xviii) AlW-3 iccoverabifiradiated fuel modules. fuel modules that use contral rods sharfye control rods fiserted.

(2) Maximum quowtity of matetat per-paclage:

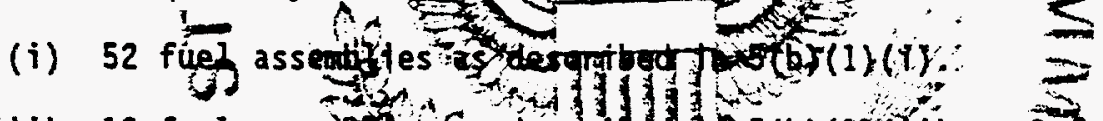

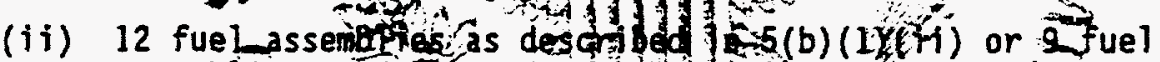

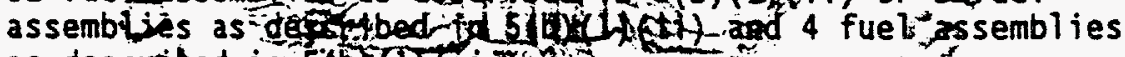

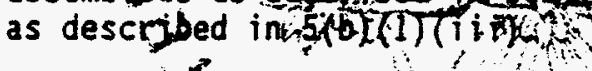

(iii) 6 fuel assemblies as described in 5 (b) (1) (iv) $x$ and

4 fuel assemity as described in 5(b)(1)(k):

(iv) 9 fuel assemblies asyescribed in 5(b)(ta) (vi) and 8 fuel assemblies as desilpiber $(b)(1)(v i i)$.

(v) 10 fuel assemblies as described in $5(b)(1)$ (viii).

(vi) 9 fuel assemblies as described in $5(b)(1)$ (viii) and one fuel assembly as described in 5 (b) (1)(ix).

(vii) 9 fuel assemblies as described in $5(b)(1)(v i i i)$ and one structure as described in $5(b)(1)(x)$. 
Page 4 -Certificate No. 6003 - Revision No. 15 - Docket No. 71-6003

i. (b) (2) Contents (cont'd)

(viii) 4 fuel cells as described in $5(b)(1)(x i)$; or

2 fuel cells as described in $5(b)(1)(x i)$ and

2 fuel cells as described in $5(b)(1)(x i i)$.

(ix) 6 fuel assemblies as described in $5(b)(1)(x i i i)$.

(x) 8 fuel cells as described in $5(b)(1)(x i v)$.

(xi) 4 fuel cells as described in $5(b)(1)(x v)$ plus

2 corner fuel cells or I RFA fuel cell.

(xii) 4 fuel modules as described in $5(b)(1)(x v i)$.

(xiij) 10 fuel modules as described in $5(b)(1)(x$ ii $i)$.

(xiv) For contents described in $5(b)(1)$ (xviii), 6 fuel modules, or 8 fuel medoles, as described in supplement dated March 30, 1992.

(3) Shipments shaj be further limited by thermal requirements as follows:

(i) Shipment of contents specified in $5(b)(1)(i v)$ and $5(b)(1)(v)$ and limited in $5(b)$ (2) (iii) shal be made no earloter than 75 days after-shutdown and sfiall havena-decay feat load not =to exceed $33,50 \mathrm{R} B \mathrm{Btu} / \mathrm{hr}$ per stitpaept.

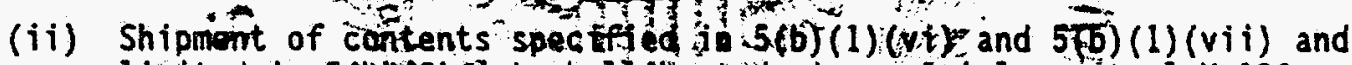
limited in $5(b)(2)(j v)$ shalj $b$ ade in austainless steel $M-130$ containe and shatk haye ad dgcaytheal load not ta-exceed 18,960 Btu/hr per shipment.

(iii) Shipment of contents speciffed ia $5(b)(1)(v i$ in $5(b)(1)(i x)$ and $5(b)(1)(x)$ and limited in $5(b)(2)(v), 5(b)(2)(v i)$ and $5(b)(2)$ (vii) shall be made at a time after shutdown as determined from Bettis Atomic Power Laboratory report WAPD-OP(PP) S-4401 dated June 29,1979 and shall have a decay heat load not to exceed $28,620 \mathrm{Btu} / \mathrm{hr}$ for the shipboard core and $30,000 \mathrm{Btu} / \mathrm{hr}$ for the prototype core.

(iv) Shipment of contents specified in $5(b)(1)(i), 5(b)(1)$ (ii) shall be made no earlier than 72 days after shutdown and shall have a decay heat load not to exceed $33,500 \mathrm{Btu} / \mathrm{hr}$ per shipment.

(v) Shipment of contents specified in $5(b)(1)(x i)$ or $5(b)(1)(x i i)$ as limited by $5(b)(2)$ (vii) shall have a fully loaded container heat load not to exceed $15,400 \mathrm{Btu} / \mathrm{hr}$ per shipment.

(vi) Shipment of contents specified in $5(b)(1)(x i i i)$ and limited in 5 (b) (2) (ix) shall have a heat load not to exceed 23,800 Btu/hr and shall be made no earlier than 92 days after shutdown. 
5 - Certificate No. 6003 - Revision No. 15 - Docket No. 71-6003

T5. (b) (3) Contents (cont'd)

-

(n)

(vii) Shipment of contents specified in $5(b)(1)(x i v)$ and 1 imited in $5(b)(2)(x)$ shall have a heat load not to exceed $22,400 \mathrm{Btu} / \mathrm{hr}$ and shall be made no earlier than 122 days after shutdown.

(viii) Shipment of contents specified in $5(b)(1)(x v)$ and limited in $5(b)(2)(x i)$ shall have a heat load not to exceed $19,100 \mathrm{Btu} / \mathrm{hr}$ and shall be made no earlier than 420 days after shutdown.

(ix) Shipment of contents specified in $5(b)(1)(x v i)$ and limited in 5 (b) (2) (xii) shall have a heat load not to exceed $6,000 \mathrm{Btu} / \mathrm{hr}$ and shall be made no earlier.than 50 days after shutdown.

(x) Shipment of contents specified in $5(b)(I)(x v i i)$ and limited in 5 (b)(2) (xiii) shalt have a heat load not to exceed $27,400 \mathrm{Btu} / \mathrm{hr}$ and shall be made no earlier than 195 days after shutdown.

(xi) Shipment of contents specified in 5(b)(1)(xvilif)rand limited in 5 (b) (2) (xiv) shall have a heat load not to-exceed 43,800 BTU/hr and shall be, imade no earlier than 400 days after shutdown.

(c) Fissile Class

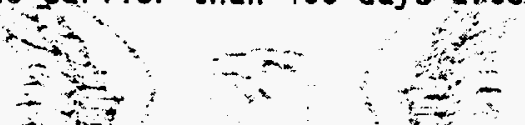

Maximum number packages perfishipment,

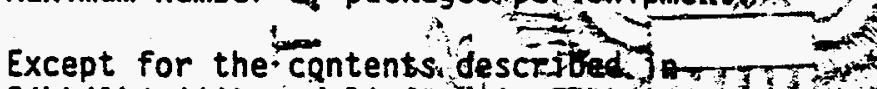
Except for the contents described
$5(b)(1)$ (viii) and limited in $5($ b) (2)

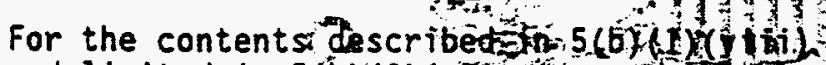
and limited in $5(b)(2)(v)$,

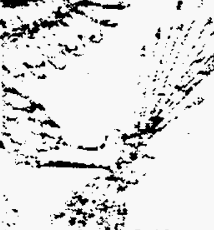

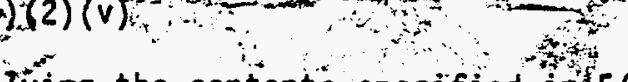

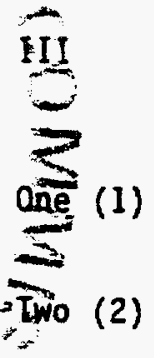

6. For shipments invorving the contents specified inf $5(b)(f)$ (ii) or $5(b)(1)$ (iii) the Model No. M-130 package shall be inspected to verify-that boron poison plates are in the moduTe holders.

7. For shipments involving the contents specified in $5(b)(1)(v i i i), 5(b)(1)$ (ix) or $5(b)(1)(x)$ the thermocouples and thermocouple cases if included or the vacant module holder shall be located in the mid-position of either cage and module holder assembly. 
Page 6 - Certificate No. 6003 - Revision No. 15 - Docket No. 71-6003

3. Shipments shall be made in the dry condition, except for residual water as limited in $5(b)(1), 5(b)(3)(v)$, and $5(b)(3)(v i i)$.

9. Container number three $(M-130-3)$ has been modified by adding two 4-inch thick by 8 -inch wide steel plates welded between fins 25 and 50 and between fins 110 and 135 at approximately 14.75 inches from the bottom of the container. The cooling fins in this localized area are removed to permit attachment of the plate directly to the outer shell of the container.

10. Container number four $(M-130-4)$ has been modified by adding a 2 -inch thick by 4-inch wide steel plate welded between fins 32 and 49 at approximately 18.4 inches from the bottom of the container. The cooling fins in this localized area removed to permit attachment of the plate directly to the outer shell of the container.

11. Containers $M-130-3, M-130-4, M-130-6$, and $M-130-7$ may be used for the contents specified in $5(b)(1)(v i i i)$ and $5(b)(1)(x)$ only. Containers $M-130-10$ and $M-130-15$ may be used for the-contents specified in $5(b)(1)(v i i j), 5(b)(1)(x)$ and $5(b)(1)(x v i i i)$ only:

12. Container M-130 1 is may be used for NR-1 shipments onjy.

13. For shipments inyolving the coutents specified 10 (b)(1)(xvi) which do not contain a full-complement of füel modules: (i.e one position is. occupied by either two flux thimbles or asvacant fuet module holder with jocket shield plug), that position shall be Focated-ia-thexmfde madule halder of either half of the cage fassembin.

14. Expiration date Decem5er 31,1997

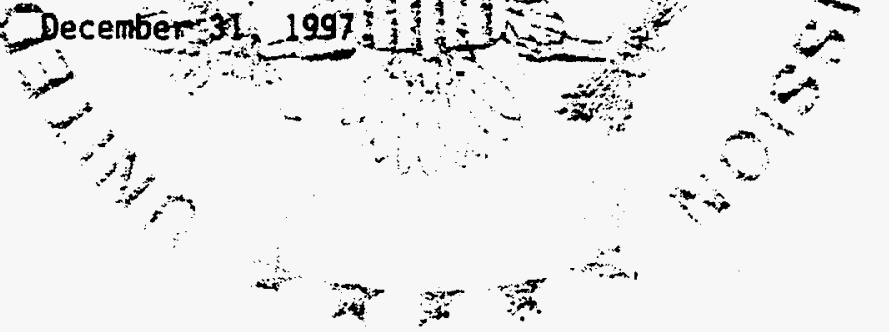




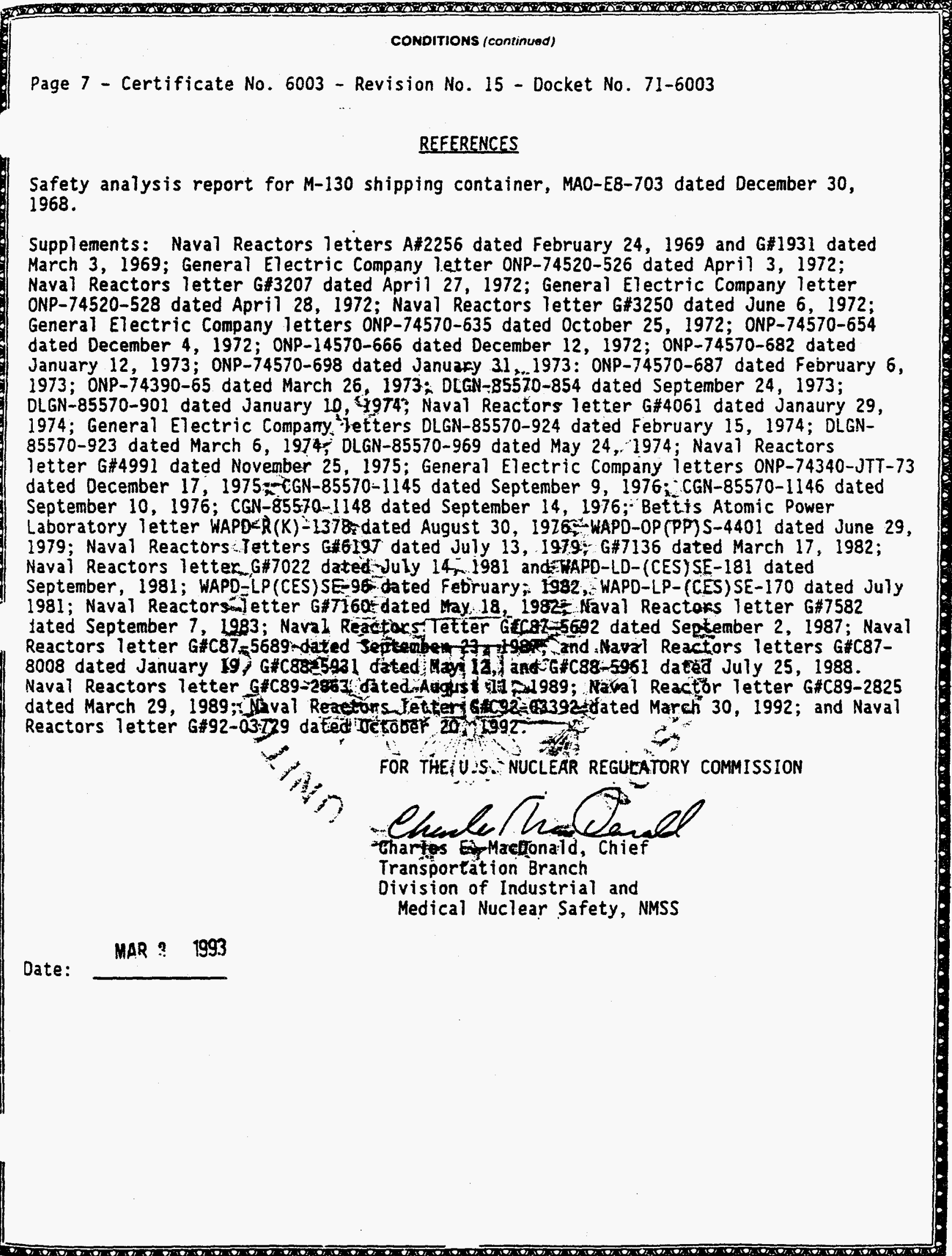


IF-300

B-118 


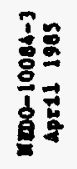

苟点

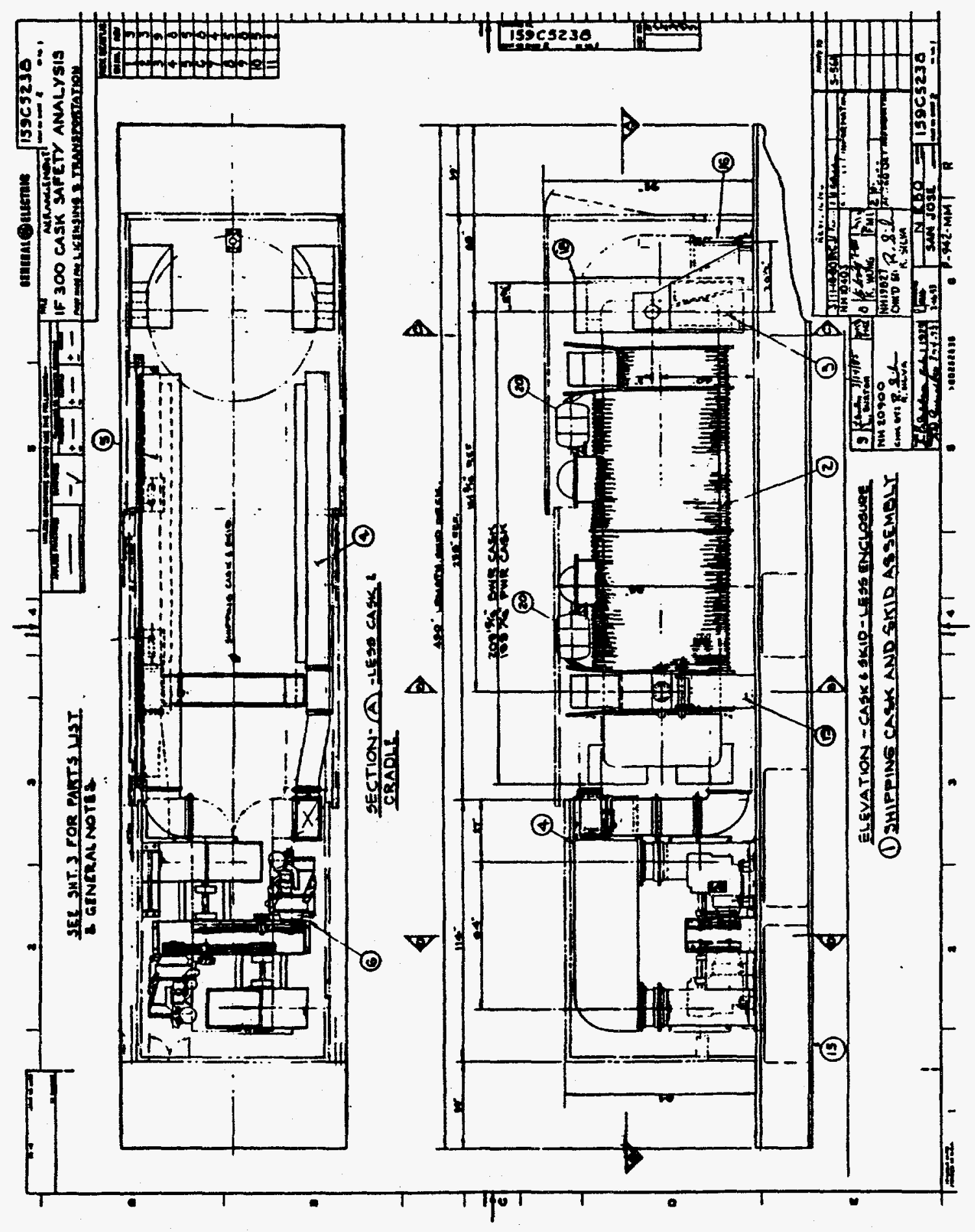

B-119 


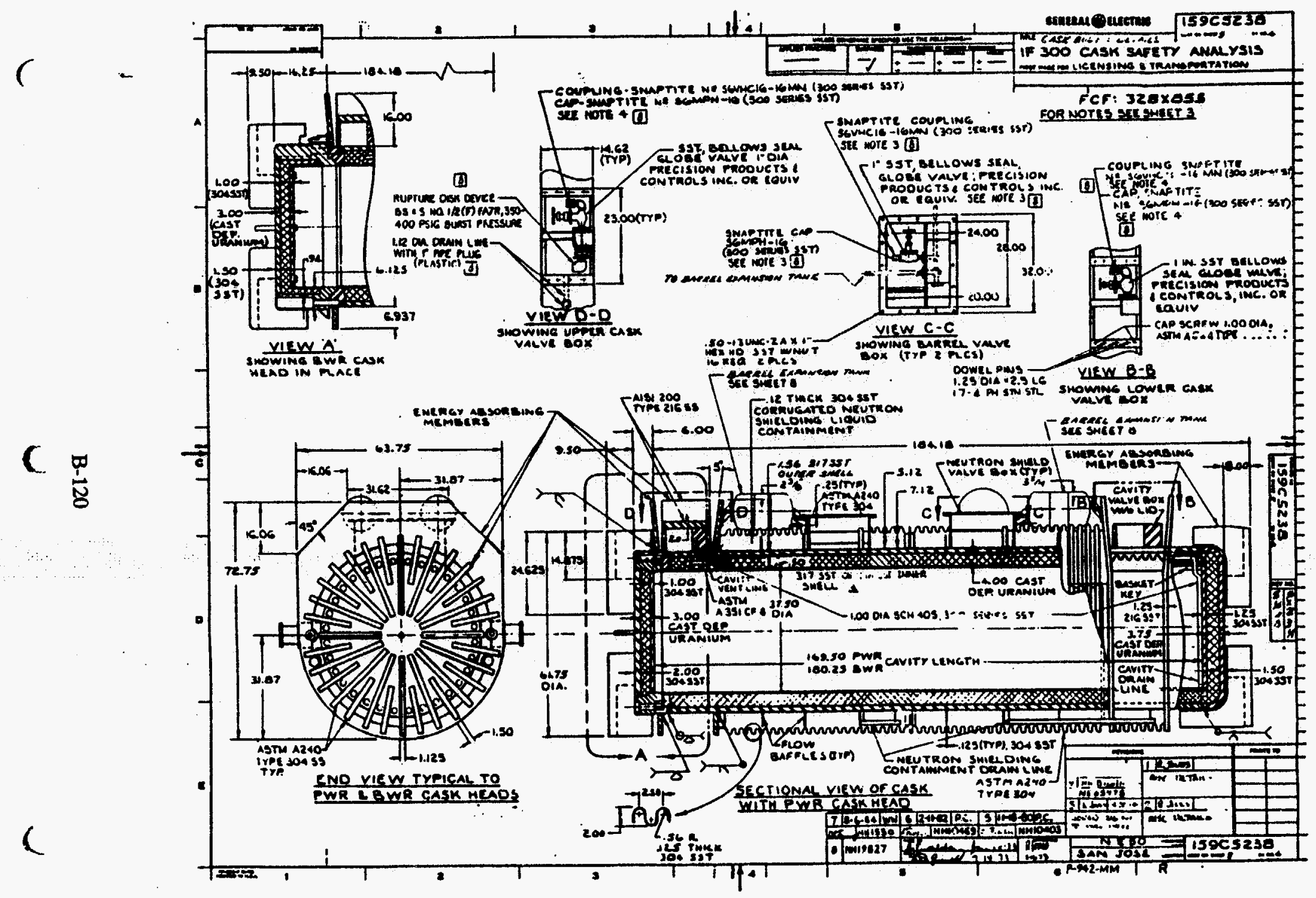




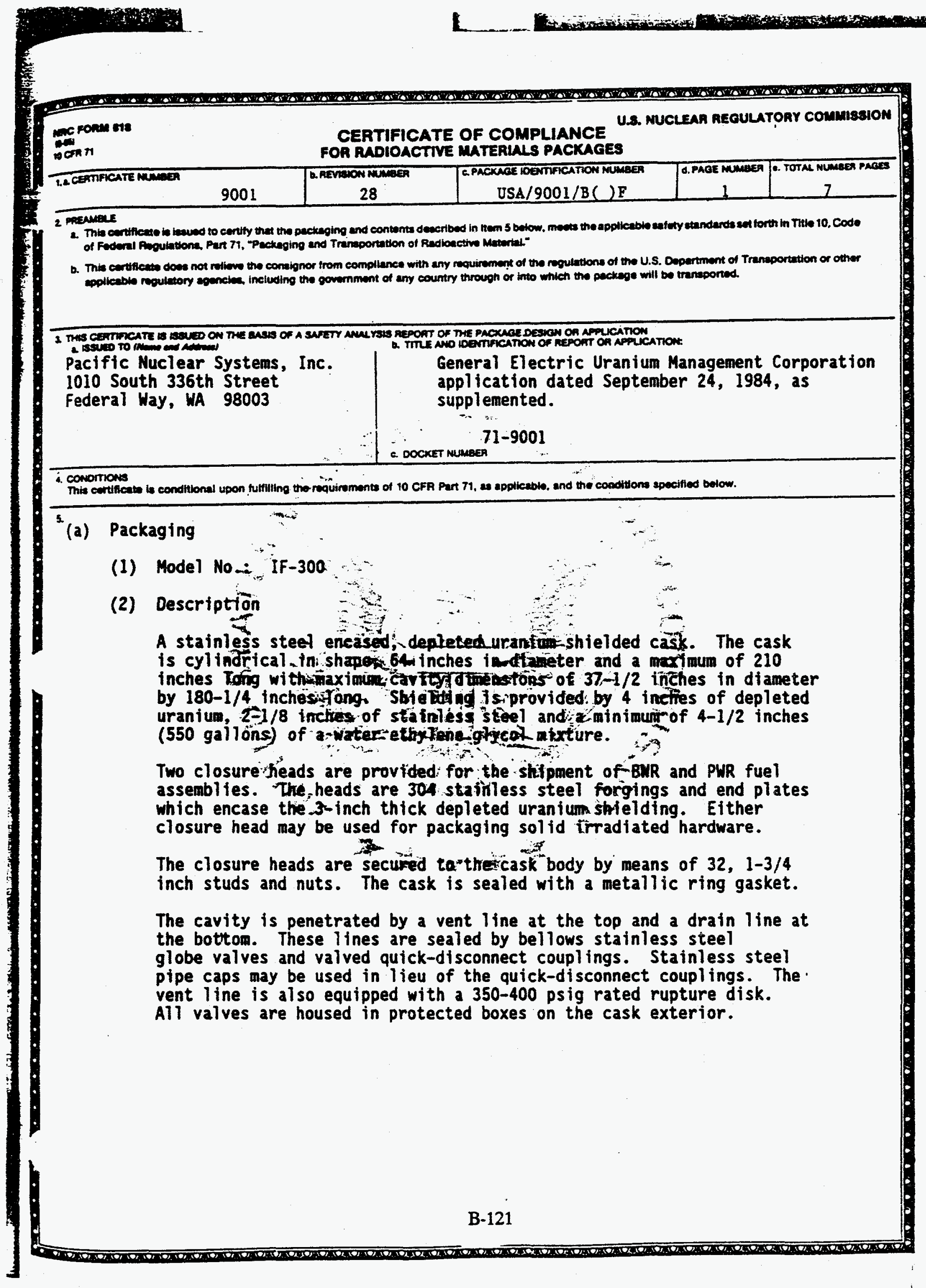




\begin{tabular}{|c|c|c|}
\hline $\operatorname{Mac}_{i \rightarrow \infty}$ Fonw eren & \multicolumn{2}{|c|}{ U.S. MUCLEAR AEGULATOAY COMMIS } \\
\hline Page 2 - Certificate No. 9001 - R & ision No. 28 - Docket No. 71-9001 & $\because$ \\
\hline$-. \quad-$ & & 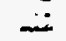 \\
\hline 5. (a) Packaging (continued) & & $\therefore$ \\
\hline (2) Description (continued) & , & - \\
\hline
\end{tabular}

Neutron shielding is provided by a liquid-filled, thin-walled, corrugated containment on the cask exterior. This cylindrical structure is separated two longitudinal compartments, each equipped with two expansion tanks, fill relief valves. The fill line from each compartment is terminated by a stai less steel globe valve in a protected box (separate from cavity boxes) on $t$ cask exterior. The stainless steel globe valves may be replaced by stainle steel blind flanges. The vent line from each compartment goes to an expans tank which is provided with a: pressure relief valve set at 200 psig.

The cask has four types of fuel baskets which can be interchanged to accommodate various fuels. The PWR basket holds seven assemblies, the unchannel BWR basket holds eighteen assemblies, the channelled, BWR basket holds seven assemblies, and the Shoreham BWR fuel basket holds'-seventeen Shoreham BWR f: assemblies. The channelled and unchannelled BWR fueT-baskets may be provide with supplementary shielding (depleted uranium), near thio cask closure.

The cask is-shipped horizontally with the battom supported in a tipping crac between two:pedestals-and, the upper end resting in a sent-circular saddle; : upper end is pinned to the saddle. The cask supports are welded to the fran of a 37-1/2-foot long by 8-foot wide-structural steel ska. The skid may al have instatled on it an auxiliary cooflhgisystems consisting of two diesel engines drfving two blowers which dischange cooling air-to the corrugated surface of the caskevia. comond ductiag. Neithen instartation nor operation all or partwof this auxiliary coatjig system is a requtrement of this packag approval.
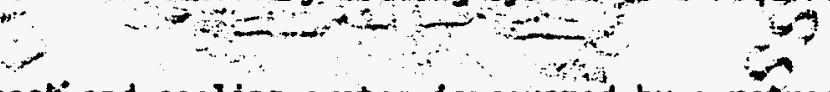

The entire cask and cooling system is covered by a retractable aluminum enclosure. Access to the enclosure is via locked panels in the side and a locked door in one ead. Although the Model Mo-1f-300 cask can be transport for short distances on the highway, its principal mode of transportation is. railiroad.

The gross weight of the cask is approximately 140,000 pounds. The skid and: other external components weigh approximately 45,000 pounds.

(3) Drawings,

The Model No. IF-300 shipping cask is described by the following General Electric Company Orawing Mos.: 159 C5238 - Sheet 1, Rev. 9; Sheet 2, Rev. 3; Sheet 3, Rev. 9; Sheet 4; Rev. 8; Sheet 5, Rev. 5; Sheet 6, Rev. 8; Sheet 7, Rev. 4; Sheet 8, Rev. 5; Sheet 9, Rev. 8; Sheet 10, Rev. 5; and Sheet 11, Rev 2, and Pacific Nuclear Systems, Inc. Orawing Nos.: 420-11-3000, Sheets 1 , through 9, Rev. 0; 420-11-3001, Sheet 1, Rev. 0; 420-11-3002, Sheets 1 and 2 Rev. 0; 420-11-3003, Sheets 1 and 2, Rev. 0; 420-11-3004, Sheets 1 and 2, Rev $0 ; 420-11-3005$, Sheets 1 and 2, Rev. 0; 420-11-3006, Sheet 1, Rev 0; 2045.300 Sheets 1 to 4, Rev. 1; 2045.3001, Sheets 1 and 2, Rev. 1; 2045.3002, Sheet: Rev. 1; and 2045.3003, Sheet 1, Rev. 0. 
Page 3 - Certificate No. 9001 - Revision No. 28 - Docket No. 71-9001

\section{5. (a) (4) Basic Components}

The basic components of the Model No. IF-300 shipping cask that are important to nuclear safety are listed in Section IX, Table IX-1.

(b) Contents

\section{(1) Type and form of material}

(i) Irradiated PWR and BUR uranium oxide fuel assemblies. PWR assemblies may be shipped with or without control rods. Partial fuel assemblies, that is, assembltes, from which fuel pins are missing, must not be shipped unless dumy fuel pins are used to displace an amount of water equal to thiat displaced by the original pins. The specific power of each fuel assembly must not exceed $40 \mathrm{kw} / \mathrm{kgU}$ and the burnup of each fuel assembly must not exceed 35,000 MND/MTU. The minimum cooling time of each assembly must be no less than 120 days. Prior to irradiation, the BUR and PtiR fuel assemblies must have the following dimensions and specifications:

$$
\text { Grout }
$$
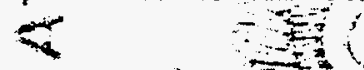

Pac pitR
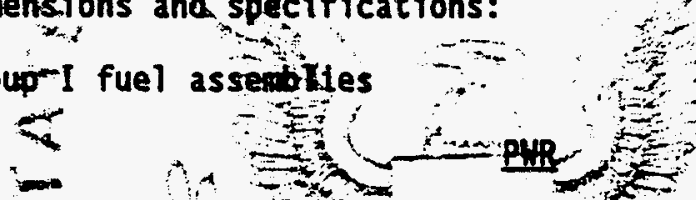

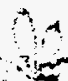<smiles>C1#CCCC#C1</smiles><smiles>[C]1C#C1</smiles>

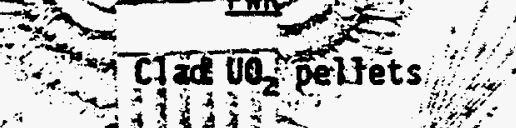

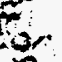

Cladding matorfat

Maximunititiar $\mathrm{U}^{-}$ content/assembly, $\mathrm{kg}$

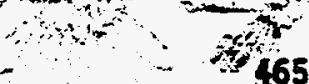
st

Maximum inițał U-235 enrichment, $w / 0$<smiles>C1=[Ge]C[Si]1</smiles>

Maximum bundle cross section, in sक

Fuel pin array $14 \times 14 / 15 \times 15$ 4.0

Fuel diameter, in

$0.380-0.460$

$0.500-0.600$

Fuel pin pitch range, in

$0.502-0.582$

$0.647-0.809$

Maximum active fuel length, in 
Page 4 - Certifićate No. 9001 - Revision No. 28 - Docket No. 71-9001

5. (b) Contents (continued)

Group II fuel assemblies .-

PWR

BWR

Fuel form

Clad $\mathrm{UO}_{2}$ pellets

Clad $\mathrm{UO}_{2}$ pellets

Cladding material

Zr or SS

Zr or SS

Maximum initial $U$ content/assembiy, $\hat{\mathrm{kg}}$

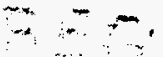

Maximum inittâ $u-235$

enrichment, $w / 0$ .

Maximum bundle cross section, in

Fuel pin array :

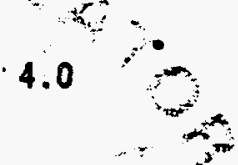

8.75

5.75

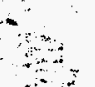

Fuel diameter, in

$16 \times 16 / 17 \times 17$

$$
20
$$

$0.376-0.400$

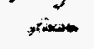

$8 \times 8$

fue pin pitch: range in $=4 \quad:-1$

Maximum actives fuel length, in

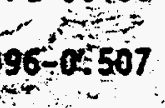

$$
=
$$

$0.475-0.505$

$0.630-0.645$

(ii) Irradiated Shoreham BUR fuel assemblies composed of $\mathrm{UO}_{2}$ fuel rods in an $8 \times 8$ square array. Each fue T assembly has a maximum average enrichment of $2.19 \mathrm{w} / 0 \quad U-235$. The fuel rods have the following-nominal dimensions: 0.410 -inch fuel pellet diameter, 0.032 -inch thick zircalloy cladding, 0.64-inch rod pitch, and 150-inch activeafuel length. The maximum assembly burnup is 87.0 MWD/MTU, and the minimum cool time is three years. Fuel assemblies may be shipped with protective packaging as described in Section 1.2.3.1 of application dated February 25, 1993. Fuel assemblies must be positioned within stainless steel channels which have a nominal thickness of 0.0595 inch and a nominal length of 167 inches. For natural uranium fuel assemblites, zircalloy channels, having a nominal thickness of 0.1 inch and a length of approximately 167 inches, may be used in lieu of stainless steel channels. 
Page 5 - Certifiçate No. 9001 - Revision No. 28 - Docket No. 71-9001

\section{5. (b) Contents (continued)}

(iti) Solid irradiated hardware, which may include fissile material, provided the quantity of fissile material does not exceed a Type A quantity and does not-exceed the mass linits of 10 CFR 571.53. As needed, appropriate component spacers must be used when loading irradiated hardware into the cask cavity to 11 mit movement of the contents during accident conditions of tranpsort. Use of a steel liner is authorized provided: its outside dimensions are approximately those of the cask cavity inside dimensions, (2) constructed of single thickness of steel plate with full penetration welds, (3) thickness of steel plate does not exceed one inch, and (4) the liner is provided with a drain and vent to insure water removal.

(2) Maximum quantity of material per package

(i) Maximun decay heat per package not to exceed $40,000 \mathrm{Btu} / \mathrm{hr}$. Maxtmuin 5,725 Btu/hr/PWR assembly. Maximum 2,225 Btu/hr/BWR assembly.

(ii) Seven PWR fuel assemblies, seventeen-channelled BWR assemblies, éighteen unchannelled BUR fuer assemblies, or seventeen shoreham - BUR fuel assembilies.

(iii) Above fuel assenblias-tobe-contatined in theichespective fuel

-baskets as shown th GE Drawtng $150-159 C 5238$ - - Sheet 6,

Rev. 8, PNSI Draiting $\mathrm{NO}_{2}$ 420=11L-3000; Sheet through

9, Rev. Q or PISI Drawing Mo. 2045.3002, Sheet 1, Rev. 1.

(c) Unloaded package - contents and maxtmuantityo material

Greater than a Type A quantity of residual radfoactive material consisting of mixed-fission and activation products adhering to interior cavity and fuel basket surfaces. ...

(d) Fissile Class

6. The end of life total calculated residual gas that could become available from the fuel pins must not exceed 0.50 ib moles for content 5 .(b).

7. The maximum gross weight of the cavity contents must not exceed 21,000 pounds.

8. For the shipment of irradiated fuel assemblies, the cask cavity (containment vesse1) must be promptly inerted following removal of the water from the cavity. The cask cavity must be purged at least three times with argon, nitrogen, or helium. Each purge volume must be equivalent to or greater than the cask cavity volume. After the final purge, the cavity must be promptly filled with argon, nitrogen, or helium at 1.0. atm pressure.

9. Known or suspected falled fuel assemblies (rods) and fuel with cladding defects greater than pin holes and hairline cracks are not authorized. 
Page 6 - Certificate No. 9001 - Revision No. 28 - Docket No. 71-9001

10. Prior to each shipment, the licensee must confirm that the cask contains no more than 1 cubtc, foot of water in the cavity and the licensee must prepare the cask for shipment, in accordance with Subsection 10.1 of the application.

11. The cask contents shall be so limited that under normal conditions prior to transport, 62 times the neutron dose rate plus 6.3 times the gamma dose rate will not exceed $560 \mathrm{mrem} / \mathrm{hr}$ at a distance of six feet from the side of the cask (ten feet from the cask center-line).

12. The neutron shielding tanks must be filled with approximately a 50/50 volume percent mixture of ethylene glycol and water during the months of October through May.

13. Replacement globe valves other than the valve specified on Drawing No. 159C5238-Sheet 4, Rev: 8. must be tested as stated in Subsection 6.6.3.2 of the application.

$$
+2
$$<smiles>C1CCCC1</smiles>

14. The packaging must be maintained in accordance with the requirements of Subsection 10.2 of the application. During inactive periods, the maintenance and testing frequency may be disregarded provided that the package is brought finta full compliance with these requirements prior to the next use of the package.

15. The cask cavity must be equipped wfth-a expture disk dektee with a burst pressure with in the range of $350-400$ pstg $\left(943^{\circ} \mathrm{F}\right.$ ). Includifing all tolerances:

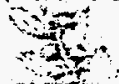

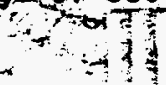
$\rightarrow 1]$

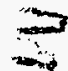

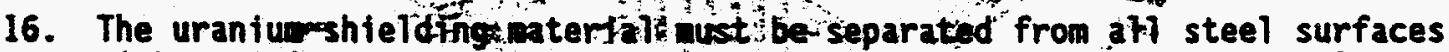
with a mintmum coppertificknessiof 4 -indis, except that the stud bolts attaching the-shield assenblies to top of the unchannel led BWR basket must be coated with a minimum of 1/2-mit of copper.

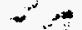

17. A shutoff valve mist not be installed between each neutron shield tank and its respective thermal expansion tank.

18. The cask may be wrapped with reinforced plastic during shipment, provided that the decay heat of the contents does not exceed $1.5 \mathrm{Kw}$. The reinforced plastic used to wrap the cask must not be greater than 0.015 inches thick or have a thermal conductivity less than $0.0242 \mathrm{Btu} / \mathrm{hr}-\mathrm{ft}-{ }^{\circ} \mathrm{F}$. The reinforced plastic wrapping cannot be used as the cask surface for purposes of complying with 10 CFR $\$ 71.87$.

19. For transport of Shoreham BWR fuel asemblies, the package may be covered with a tarpaulin. The tarpaul in may not be used as the package surface for purposes of complying with 10 CFR $\$ 71.87$.

20. The package authorized by the certificate is hereby approved for use under the general license provisions of 10 CFR $\$ 71.12$.

21. Expiration date: May 31, 1995. 
Page 7 - Certificate No. 9001 - Revision No. 28 - Docket No. 71-9001

\section{REFERENCES}

General Electric Uranium Management Corporation consolidated application dated September 24, 1984.

General Electric supplements dated: February 8, April 4, and May 10, 1985, and March 12, 1990.

Pacific Nuclear Systems, Inc. supplements dated: July 26, 1990; March 28, April 12, July 19, and August 30, 1991; January 3, 1992; and February 25, Aprit 9, July 29, and August 10, 1993.

$$
\begin{aligned}
& \text { FOR THE U.S. NOCL } \\
& \text { FOR }
\end{aligned}
$$

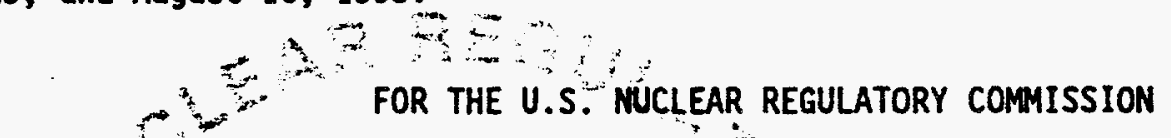

1060

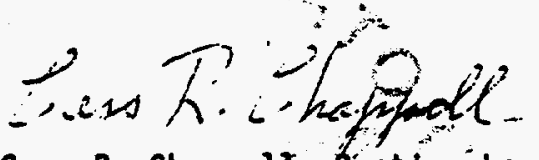

Cass R. Chappelt, Section-Leader

Cask Certification Section.

Storage and Transport Systems Branch

$\rightarrow \quad 2+$

Dfxision of Industrial andwedical

$1066 \overline{1}_{1903}$

Nuclear Saffety, MMSS

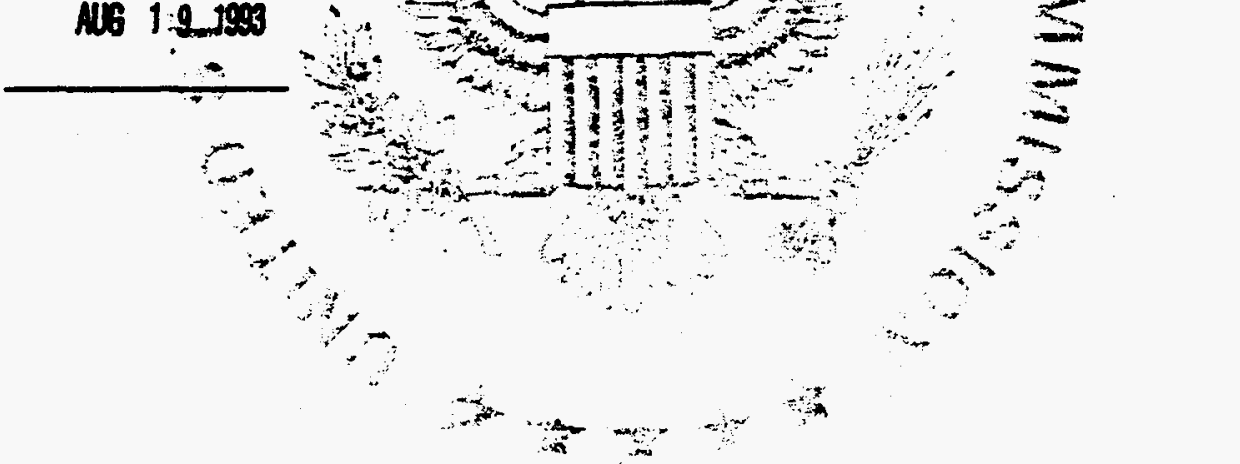

8 
NLI-10/24

B-128 

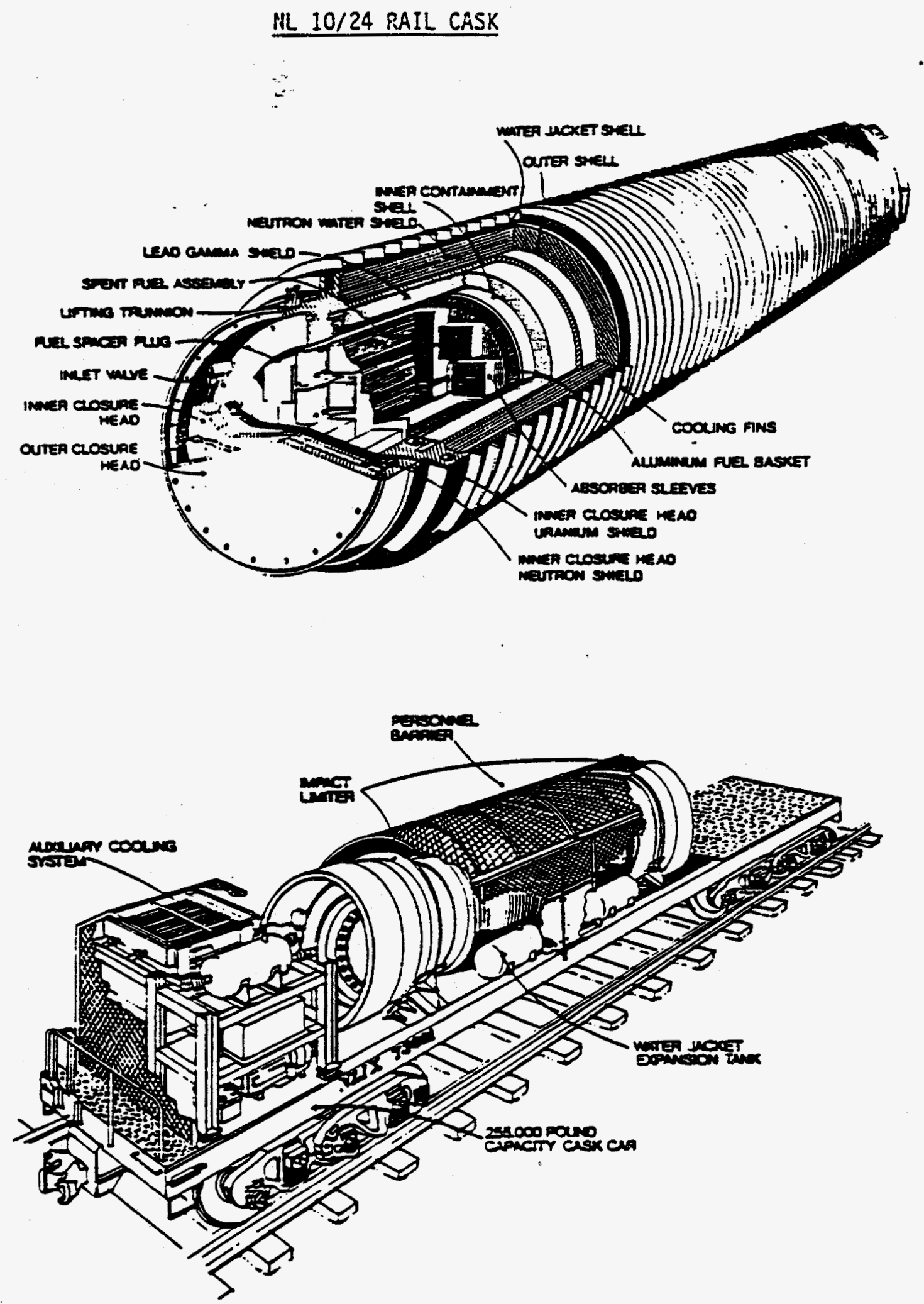


\section{NLI-10/24}

Owner

Type

Manufacturer/Vendor

Capacity

Intact Spent Fuel Assemblies

Weight (tons)

Loaded

Empty

Design Heat Rejection (kW)

Shape

Dimensions

Overail Length (in)

Overall Diameter (in)

Cavity Length (in)

Cavity Diameter (in)

Inner Shell Thickness (in)

Outer Shell Thickness (in)

Lead Shield Wall Thickness (in)

Neutron Shield Tank Thickness (in)

Outer Closure Head Thickness (in)

Materials of Construction

Cask Body

Nentron Shield

Sides

Ends \& Strategic Wall Locations

Basket

Cooling Fins

Dry Shipment

Atmosphere

Cavity Pressure (psig)
Nuclear Assurance Corporation

Rail Shipping Cask

NL Industries Inc.

10 PWR; 24 BWR

965

89

70

Cylindrical

2045

88

179.5

45

0.75

2

6

9.75

25

SSTeadss

Water

Ricorad

Aluminum-lined with Ag-In-Cd plates in SS

Concentric (forced water circulation cooling from separate cooling circuit)

He

23.1 (norral); 500 (maximum.)

Normal Operating Temperatures

(with only one of two cooling systems operative)

$\begin{array}{ll}\text { Outer Surface ("F) } & 227 \\ \text { loner Seal ( } \mathrm{F}) & 268 \\ \text { Basiket }\left({ }^{\circ} \mathrm{F}\right) & 451 \text { (maximum) } \\ \text { Fuel Assembly ( } \mathrm{O} \text { ) } & 690 \text { (average) } \\ \text { tside Surface Dose (mrem/hr) } & 200 \text { (maximum) }\end{array}$

Licensing Stanus - Licensed by the USNRC for shipment using Ag-In-Cd basket.

Comments - Cask has two separate baskets-one for PWR and one for BWR. Due to no demand for the use of this cask, previous owner recovered silver from the baskets in the late 1970 ; thus, no baskets exist for the cast at present. There are two casks currently in existence. 


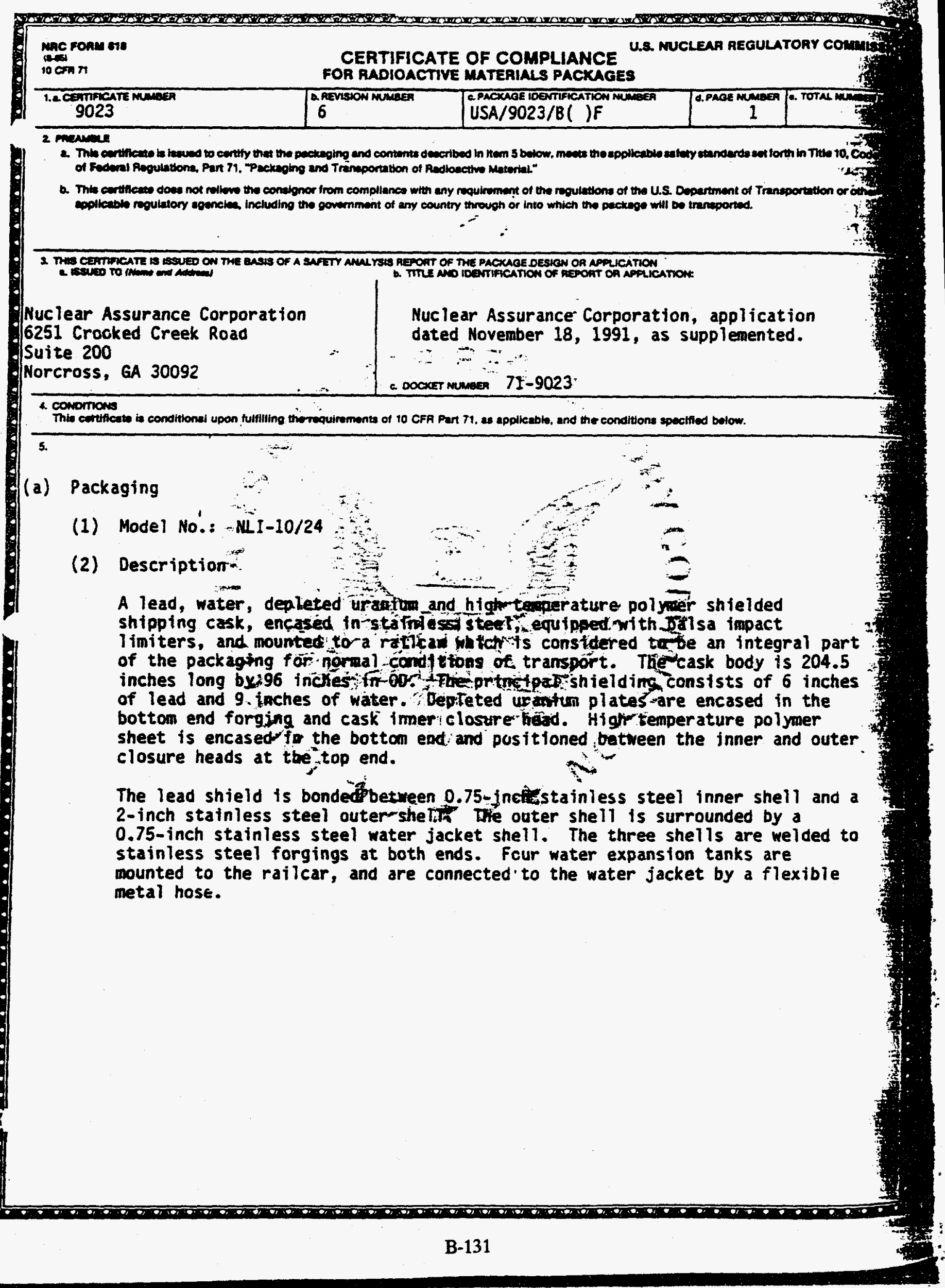




\section{CONDmONS (continued)}

Page 2 - Certificate No. 9023 - Revision No. 6 - Docket No. 71-9023

5. (a) Packaging (continued)

(2) Description (continued)

The primary containment vessel is comprised of the 0.75 -inch inner shell and the inner closure head. It is 179.5 inches long and has a $45-i n c h$ inside diameter. The inner closure head is held in place by sixteen bolts, and is sealed with a metallic 0-ring. Secondary containment is provided by the outer closure head which is bolted, and has a Viton or silicone 0-ring seal. There is no direct penetration between the contalnment cavity and the ambient. The two penetrations into the containment cavity are from the space between the inner and outer closure heads, which has a single penetration through the cask body connecting ft id th the ambient. The two lid penetrations are sealed with 1.5-inch qüick-disconnect valves and metal 0-ring seals each iff arvalue box arrangement."

The radioactive contents are positioned with in the containment cavity using neutron poisoned aluminum baskets and internal supportstructures. The PWR and BWR fuel basket-cavities are lined with neutron absorber sleeves composed of a silver-indiun-cadirium $(80-15-5 \mathrm{w} / \mathrm{0})$ aljor

$$
\text { a }
$$

An auxillacy cooling system, mounted to the tatlcar, is used to maintain the cask and:fuel temperaturesiso as to factilitate handing and cooldown.

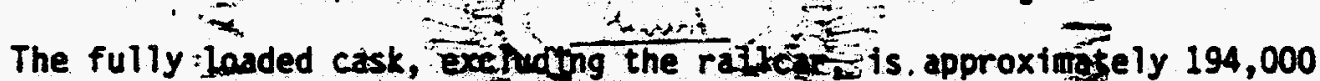

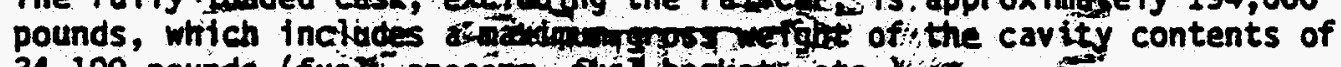
34,100 pounds (fueficspacersy fue 1 pastet etc.)

(3) Drawings
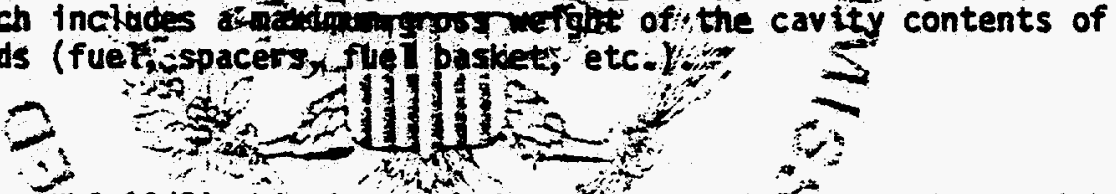

The Model No. NL1-10/24 shipping cask is coistructed fir accordance with the ML Industries, Inc and National Lead Company Drawiting Nos. as specified on page XVIII-1, ReV , and page XVIII-2, Rev. 8, injection XVIII of the application.

5. (b) Contents

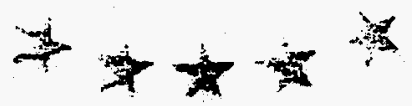

(1) Type and form of material

Irradiated PliR and BWR uranium oxide fuel assemblies of the following specifications: 
Page 3 - Certificate No. 9023 - Revision No. 6 - Docket No. 71-9023

5. (b) Contents (1) (continued)

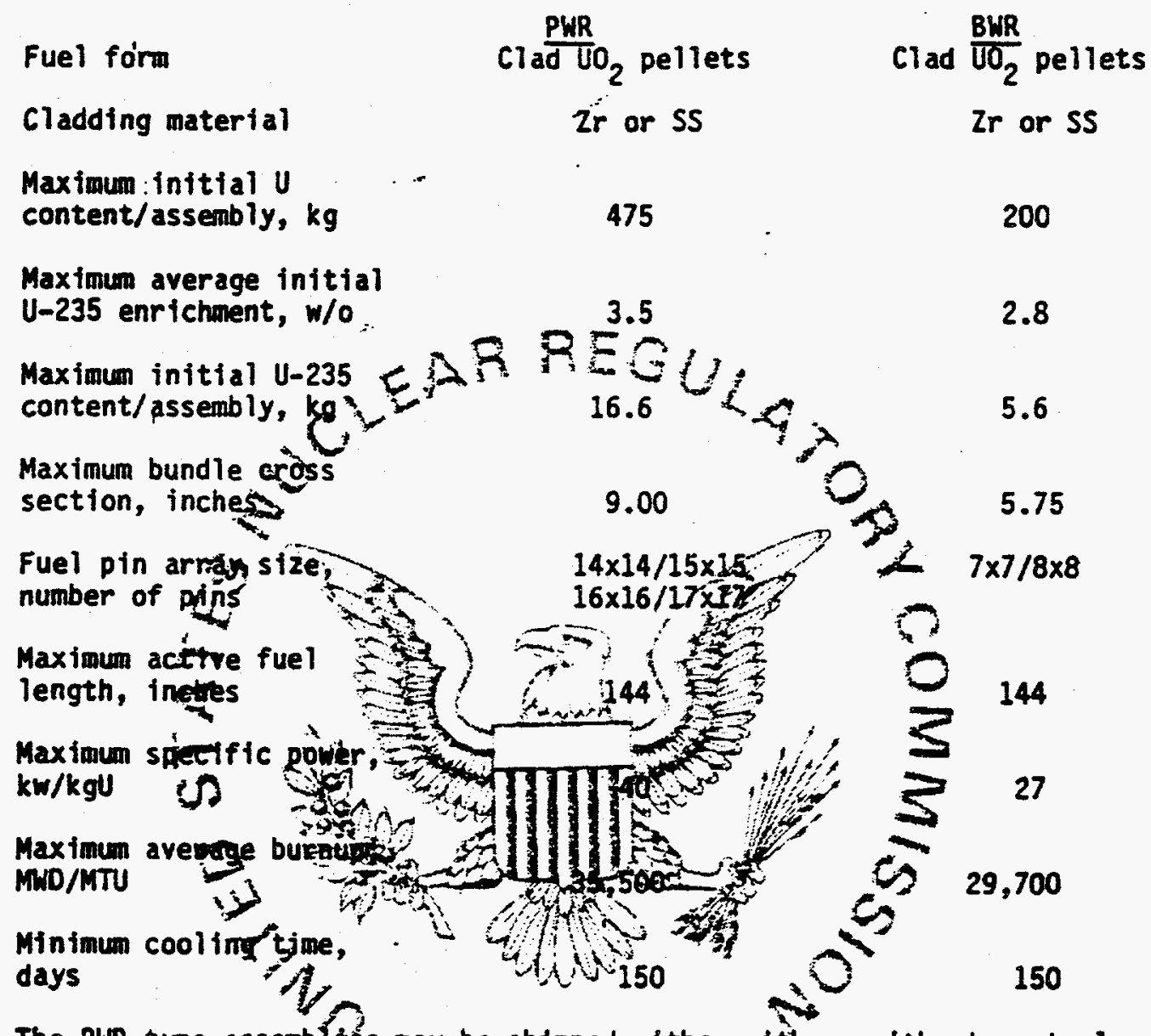

The PWR type assemb fies may be shipped eithey with or without control rods.

(2) Maximum quantity of material pur parkage

The maximum decay heat load per package not to exceed 70 kilowatts, and:

Ten PWR fuel assemblies or twenty-four BWR fuel assemblies.

Above assemblies must be contained in their respective fuel baskets as shown on $\mathrm{Ml}$ Industries, Inc. and National Lead Company Orawing Nos.:

70652F, Sheet 1, Rev. 7 PWR Fuel Basket, Sheet 2, Rev. 5 10/24 Rail Cask

70653F, Sheet 1, Rev. 7 BWR Fuel Basket, Sheet 2, Rev. 5 10/24 Rail Cask 


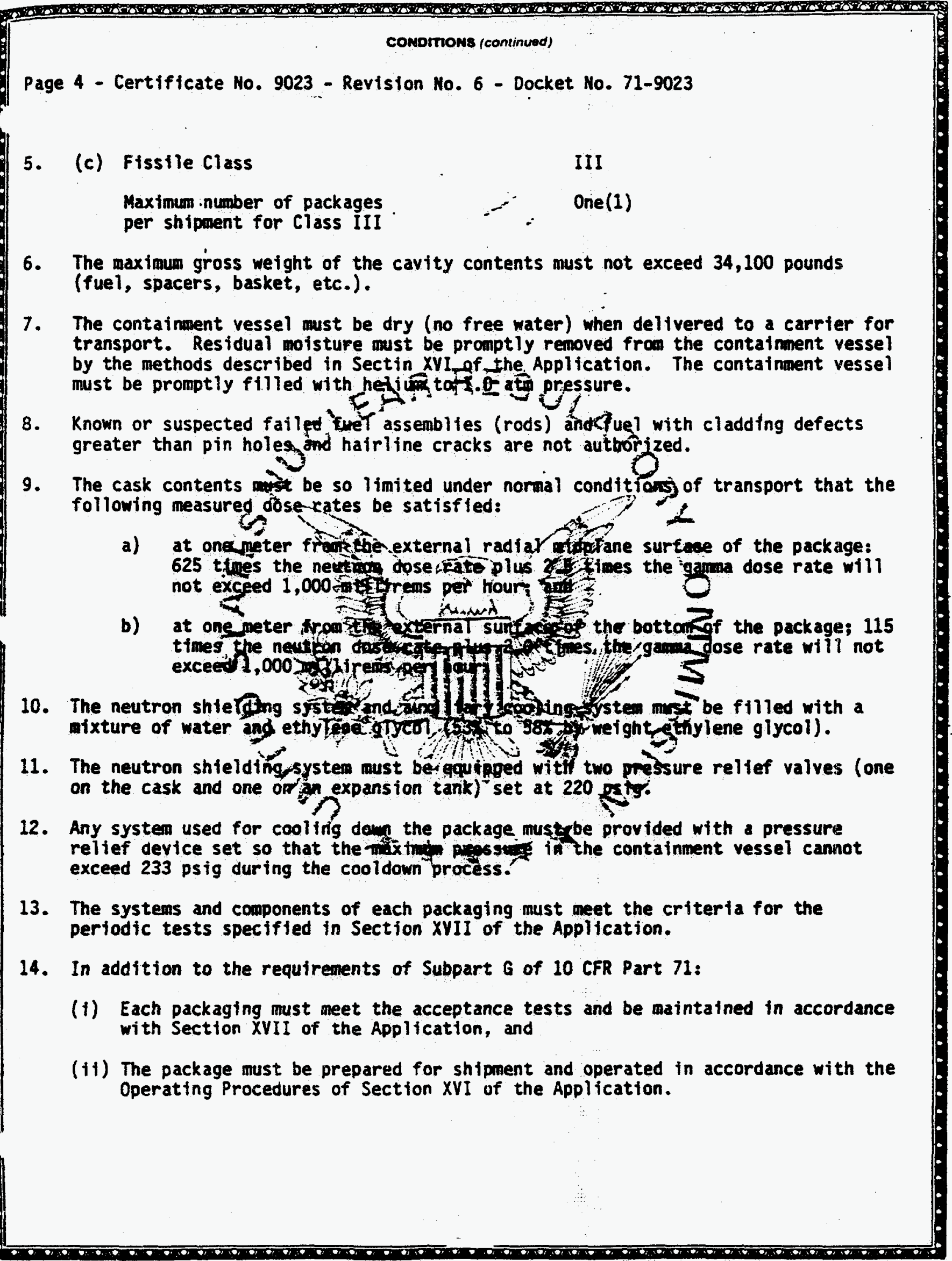




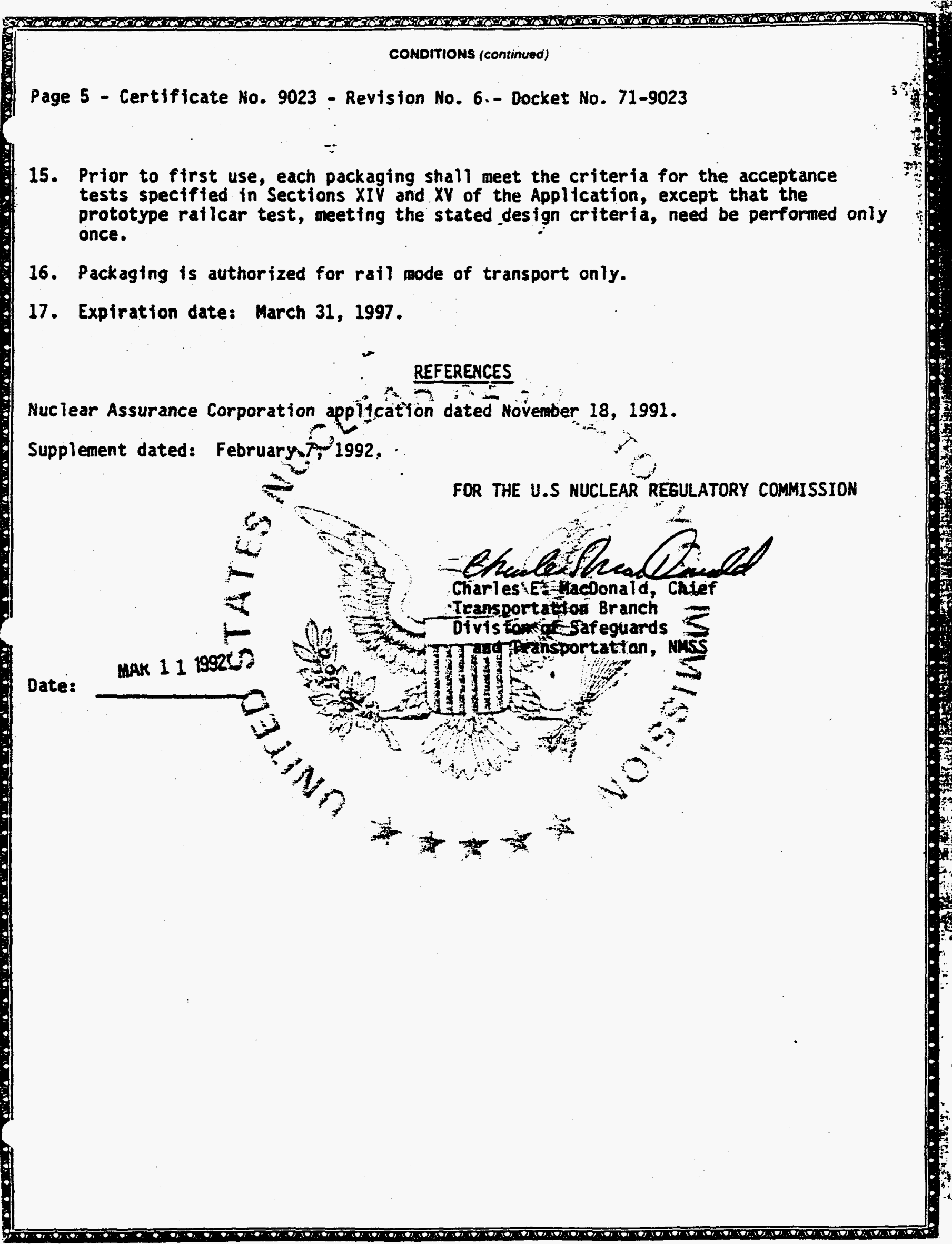


125-B

B-136 


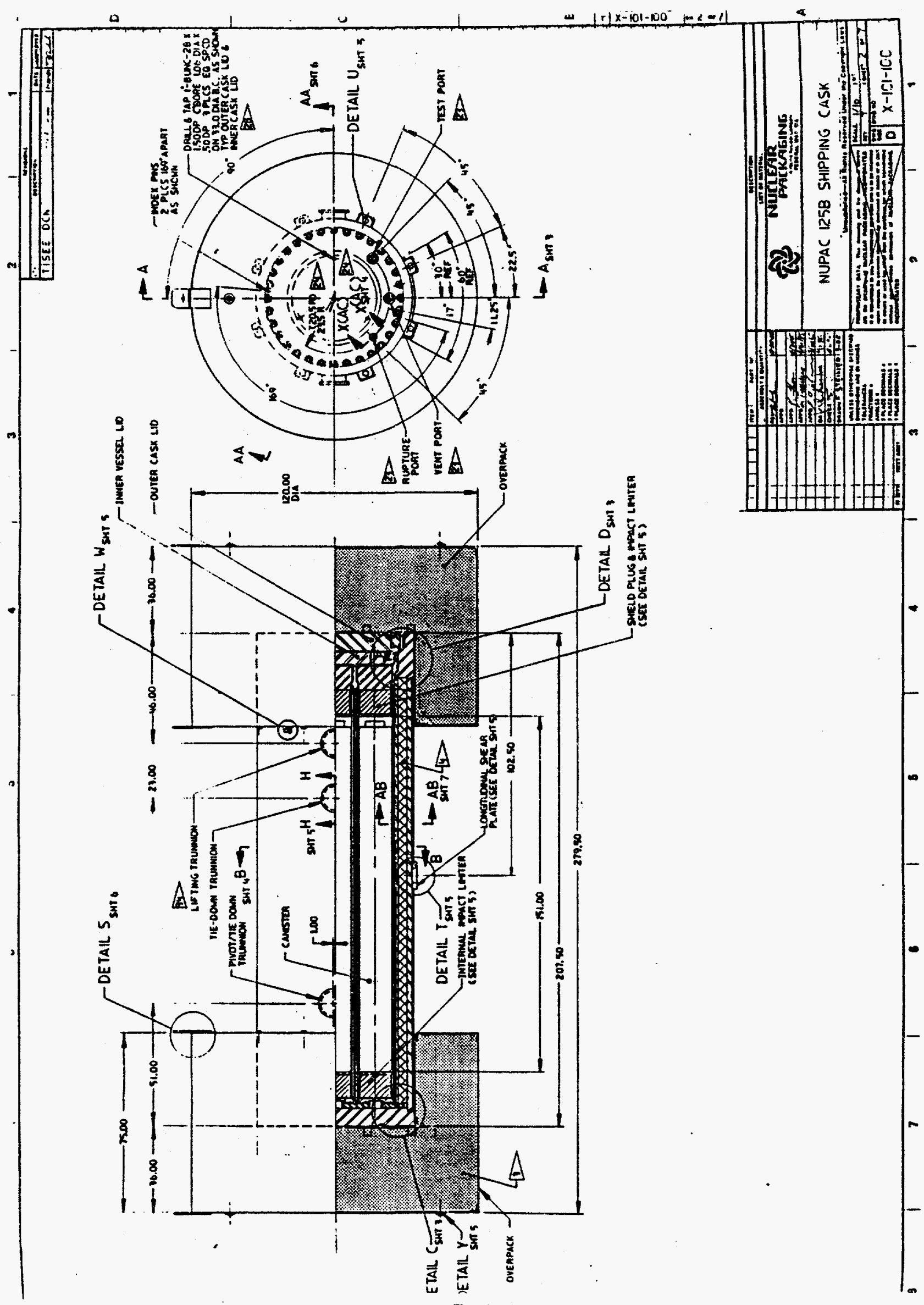




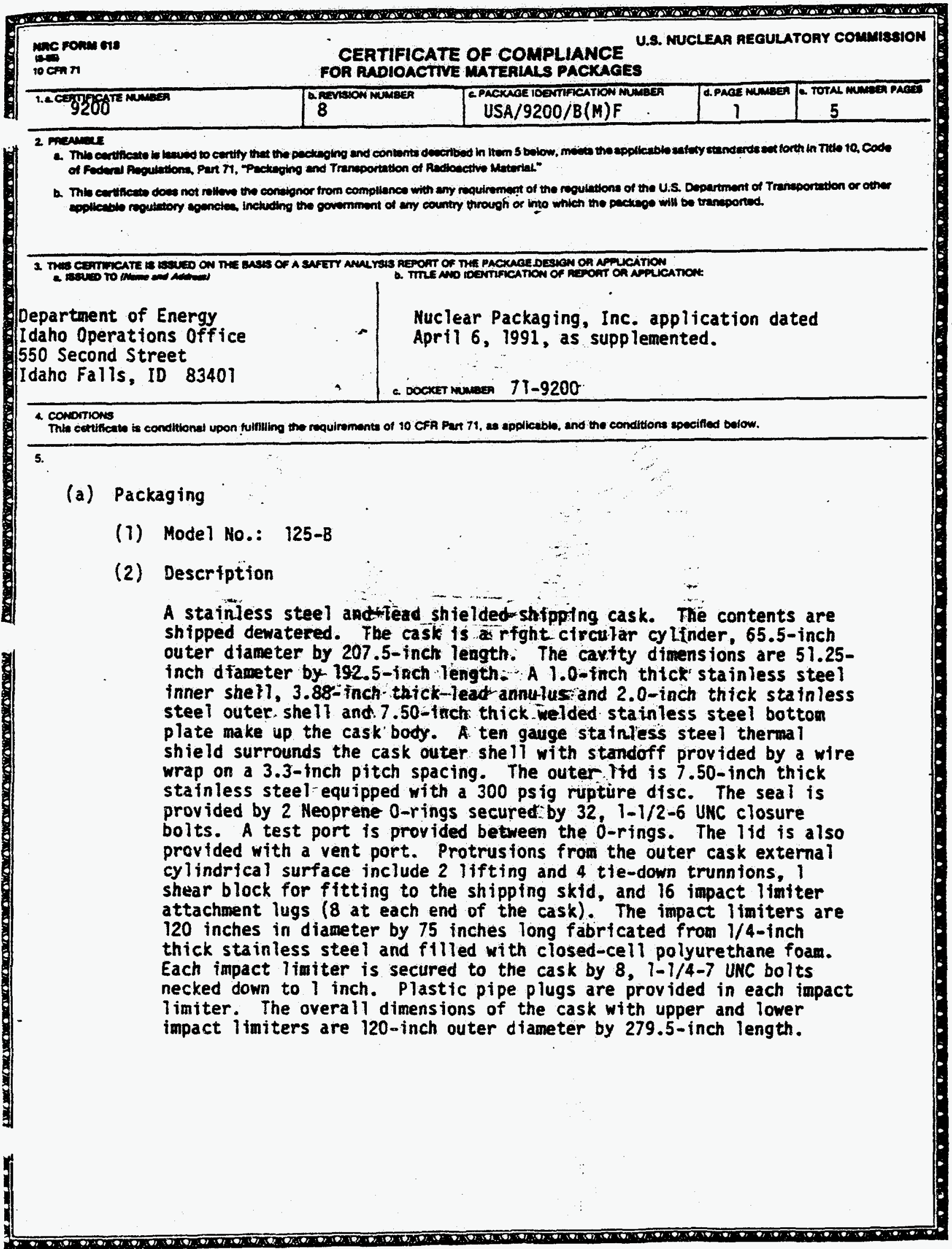

B-138 


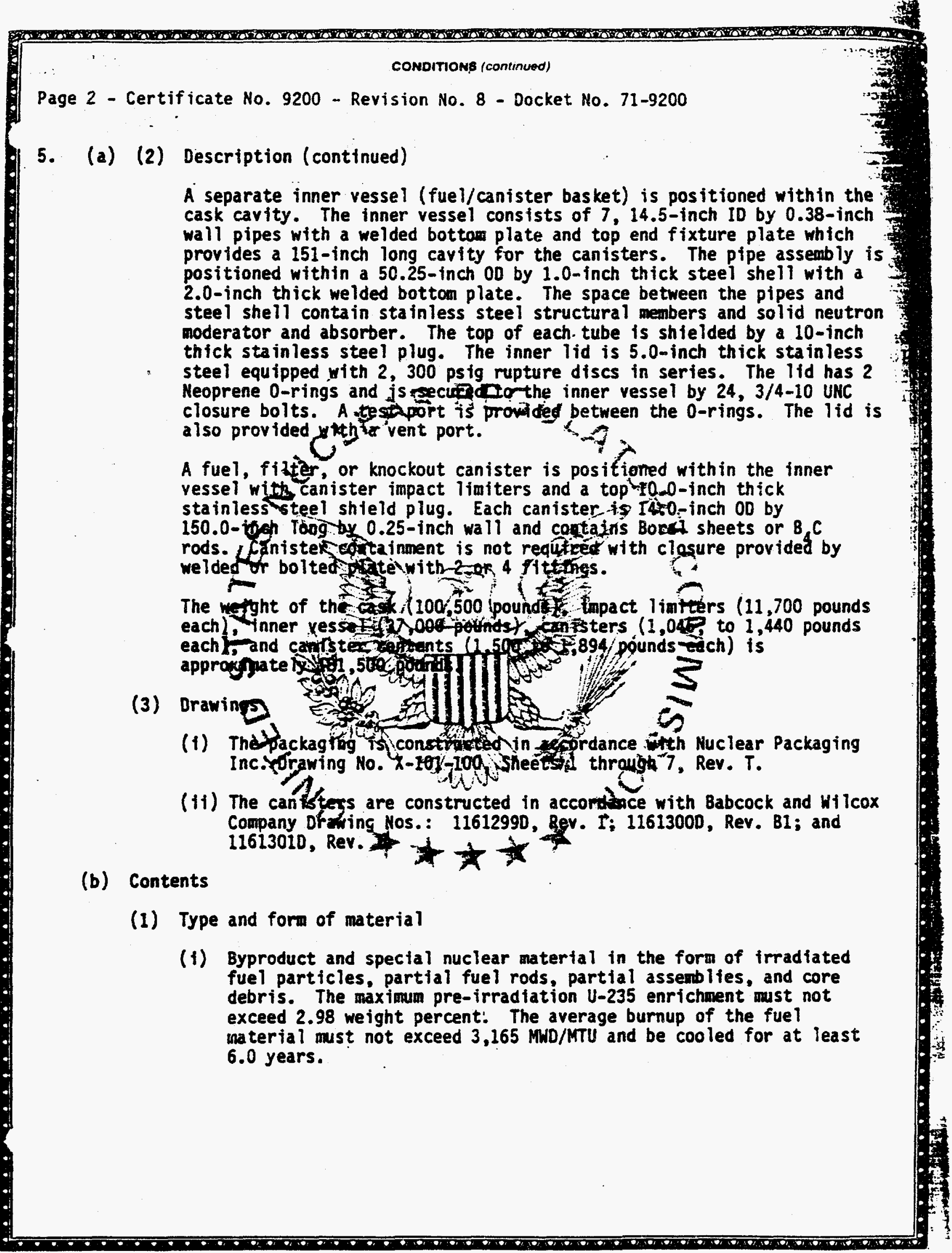


Page 3 - Certificate No. 9200 - Revision No. 8 - Docket No. 71-9200

5. (b) (1) (Continued)

(ti) Irradiated core structural components, contaminated defueling equipment, and filter-aid materiais.

Except for close fitting contents, dunnage must be provided in the shipping cask cavity sufficient to prevent significant movement of the contents and secondary containers relative to the outer packaging under accident conditions.

(iii) Byproduct and special nuclear material in the form of internal contamination inside the inner vessel. Internal contamination shall not exceed the ling to for low specific activity material as

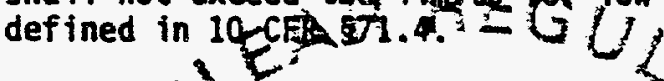

(2) Maximum quantety of material per package 7 Seven fuet knockout, or filter canisters or amy combination thereof within the inner vessel. The radioactive decay teid load must not exceed dap witcs in each canister. The gross weightof each canister must not exceet 2740 pounds.

(c)<smiles>C1CCCCC1</smiles>

Fissile chas

Maximum number of packig.
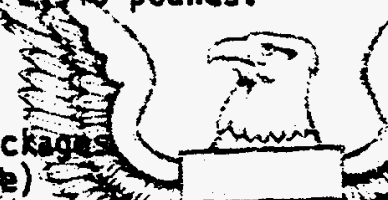

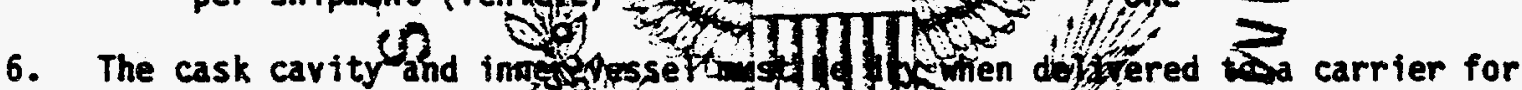<smiles>[Ge]</smiles><smiles>C1CC[Al]C1</smiles>

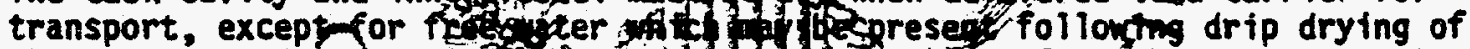

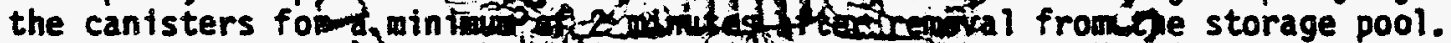

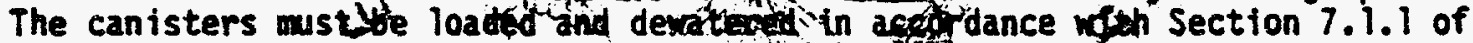

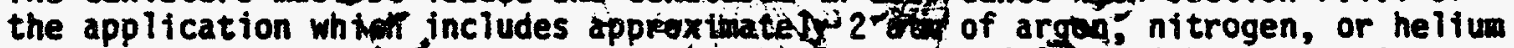
cover gas. The cask byity and inneviyessel must be filled with argon, nitrogen, or helium at $1.0 \mathrm{~atm}$ pilfyeyre.

7. In addition to the requirementof Subpart Gof $\$$ CFR Part 71:

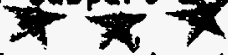

(a) Prior to each shipment, the inner and outer lid seals must be inspected. The seals must be replaced with new sea is if inspection shows any defects or every 12 months, wichever occurs first; and

(b) Each package must meet the Acceptance Tests and Ma intenance Program of Section 8.0 of the appitication.

(c) The package must be prepared for shipment and operated in accordance with Section 7.0 of the application.

8. For any canister containing water and/or organic substances which could radiolytically generate combustible gases, a determination must be made by tests and measurements or by analysis of a representative canister that the following criteria are met over a period of time that is twice the expected shipment time: 
Page 5 - Certificate No. 9200 -iRevision No. 8 - Docket No. 71-9200 $\therefore$

\section{REFERENCES}

Nuclear Packaging, Inc. application dated April 6, 1991. Supplement dated: Apr11 9 and 15, 1991.

FOR THE U.S. NUCLEAR REGULATORY COMMISSION

Date:
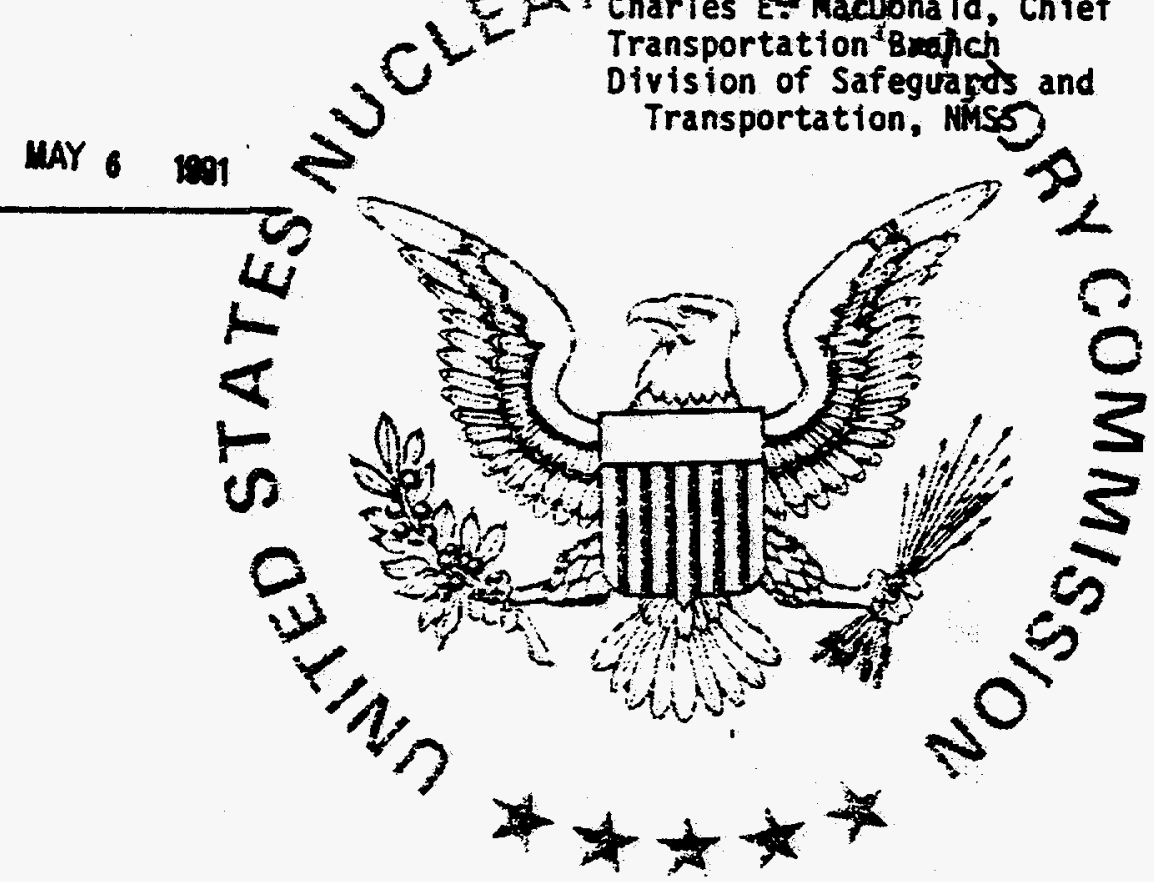
TN-BRP

B-143 


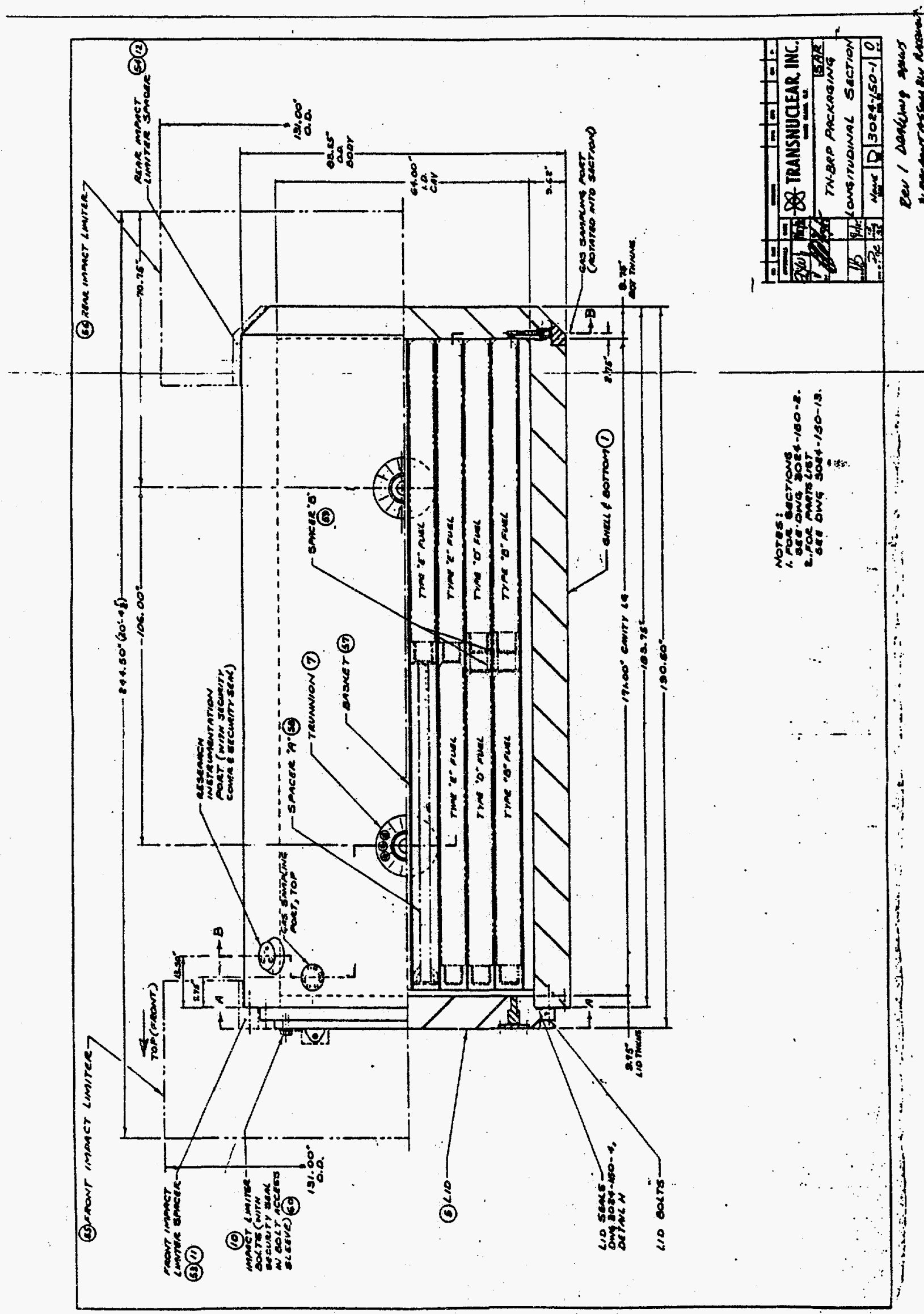


CONDITIONS (continued)

Page 2 - Certificate No. 9202 - Revision No. 2 - Docket No. 71-9202

\section{5. (a) Packaging (continued)}

\section{(2) Description (continued)}

The cask is provided with steel encased balsa-red wood impact limiters. The 1 imiters have an outer diameter of 131 inches, an inner diameter of 91 inches, and a thickness of 20 to 26 inches. Each impact limiter is attached to the cask by four equally spaced 2.25 -inch diameter bolts. The impact limiters are also connected to each other with fourteen 1.50-inch dianeter tie rods.

The cask has four iffting lugs welded to the lid, and four lifting/ tledown trunnions bolted to the is ask body.

(3) Drawings

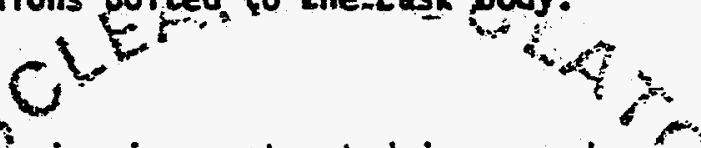

(i) The patkaging is constructed in accordance with the following Tranisnuclear, Inc. Drawings:

3024-150-1, Rev. 1 $3024-150-2$. Rev. 4 $3024-150-3$, Rey. 2 $3024-150-4, \operatorname{Rer}=2$ $3024-150-5$, Bet 3 B024-150-6; Rev, 2 3024-150-7, Rev-2

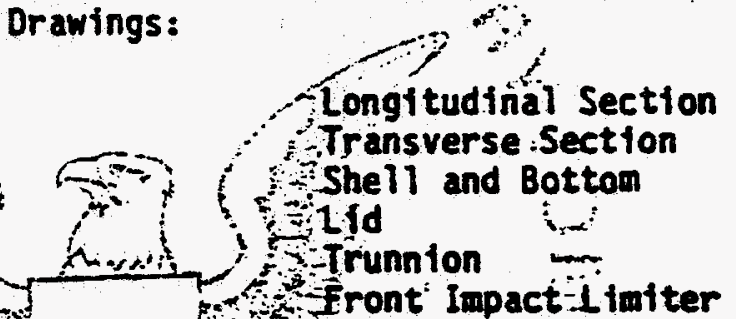
3024-150-11, Revi 1 $3024-150-18$ Rer. 21 $3024-150-13$, Rev -2 3024-150-14, Rey: 2 3024-150-16, Rev:.

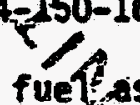

(ii) The fuefassembly basket is constructed In accordance with the followingt Transnuclear, Inc. Drawings: :-
3024-150-8, Revis 3024-150-9, Rev. 0 3024-150-10, Rev. 1 3024-150-15, Rev. 0 3024-150-17, Rer. 1 3042-150-18, Rev. 1 Basket Plane View Type A and B Spacers Basket Peripheral Inserts Fuel Replacenent Inserts 
CONOTIONS (continued)

Page 3-Certificate No. 9202 - Revision No. 2 - Docket No. 71-9202

5. (b) Contents

(1) Type and form of quaterial

(1) Irradiated Bip uranium oxide fuel assemblies as described in the application and including the following specifications:

\begin{tabular}{|c|c|c|c|c|c|c|}
\hline $\begin{array}{l}\text { Assembly } \\
\text { Type }\end{array}$ & Array & $\begin{array}{l}\text { Pellet } \\
\text { dia. (in.) }\end{array}$ & $\begin{array}{l}\text { Clad } \\
\text { Thickness }\end{array}$ & $\begin{array}{l}\text { Rod } \\
\text { OD (in.) }\end{array}$ & $\begin{array}{l}\text { Pitch } \\
\text { (in) }\end{array}$ & $\begin{array}{l}\text { Mass } \\
\text { (U) } \mathrm{Kg}\end{array}$ \\
\hline $\begin{array}{l}B \\
C \\
D \\
D \\
E \\
F \\
D(E G) \\
E P\end{array}$ & $\begin{array}{l}11 \times 11 \\
11 \times 11 \\
7 \times 7 \\
8 \times 8 \\
9 \times 9 \\
9 \times 9 \\
9 \times 9 \\
9 \times 9\end{array}$ & $\begin{array}{l}0.275 / 0.373 \\
0.275 / 0.373 \\
0.620 \\
0.500 \\
0.471 \\
0.471 \\
0.471 \\
0.471\end{array}$ & $\begin{array}{l}0.034 \\
0.034 \\
0.010 \\
0.035 \\
0.040 \\
0.040 \\
0.040 \\
0.040\end{array}$ & $\begin{array}{l}0.344 / 0.449 \\
0.344 / 0.449 \\
0.700 \\
0.570 \\
0.562 \\
0.562 \\
0.562 \\
0.562\end{array}$ & $\begin{array}{l}0.577 \\
0.577 \\
0.921 \\
0.807 \\
0.707 \\
0.707 \\
0.707 \\
0.707\end{array}$ & $\begin{array}{l}132 \\
121 \\
133 \\
113 \\
141 \\
137 \\
136 \\
118\end{array}$ \\
\hline
\end{tabular}

The BWR fuel assemblies have a maximum burnup of 25,000 MWD/MTU.

The minimum coolingtime for any assembiy is fourteen years.

(2) Maximim quantity

(1) Forty-foir Bin-assembities.

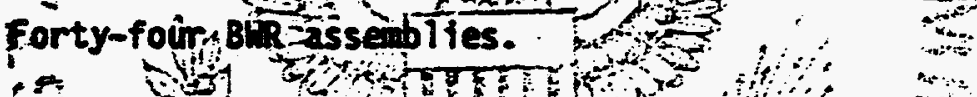

(i1) Maximu decay heat per package not to exceed 3.1 kilowatts. Maximu 103 watts per BWR issenbly. L.

(iii) Above fuel fissembires to be positioned in the fuel baskets as shown. In the draitings referenced $\$$ in $5(a)(3)$ (ii).

(c) Fisstie Class

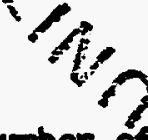
Q

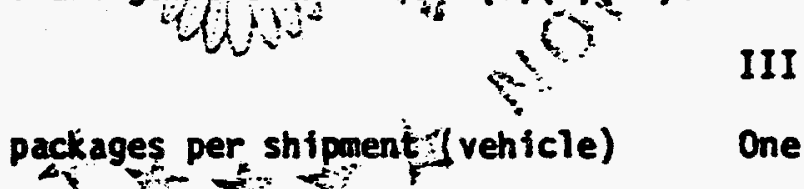

Maximu naber of packages per shipment (vehicle)

One

6. Shipaents must be completed between Aprifi 1 and October 31.

7. In addition to the requirements of Subpart 6 of 10 CFR Part 71:

a. The packaging must be prepared for shipment and operated in accordance with the Operating Procedures of Chapter 7 of the applicatiog, except that the required assembly verification leak test is $1 \times 10^{-3}$ atm-cm $/ \mathrm{sec}$.

b. The packaging must meet the Acceptance Tests and Maintenance Program of Chapter 8 of the application.

C. The packaging must be loaded in accordance with Section 7.1.2.19 and Chapter 1 of the application. 
Page 4 - Certificate No: 9202 - Revision No. 2 - Docket No. 71-9202

8. The package authorized by this certificate is hereby approved for use under the general license provisions: of 10 CFR $\$ 71.12$.

9. Expiration Date: June 30, 1994.

\section{REFERENCES}

Transnuclear Inc. application dated January 19, 1989.

Supplement dated March 22, 1989, December 19, 1990, March 4, and October 3, 1991.

FOR THE U.S. NUCLEAR REGULATORY COMNISSION

NOV 271991

Date:
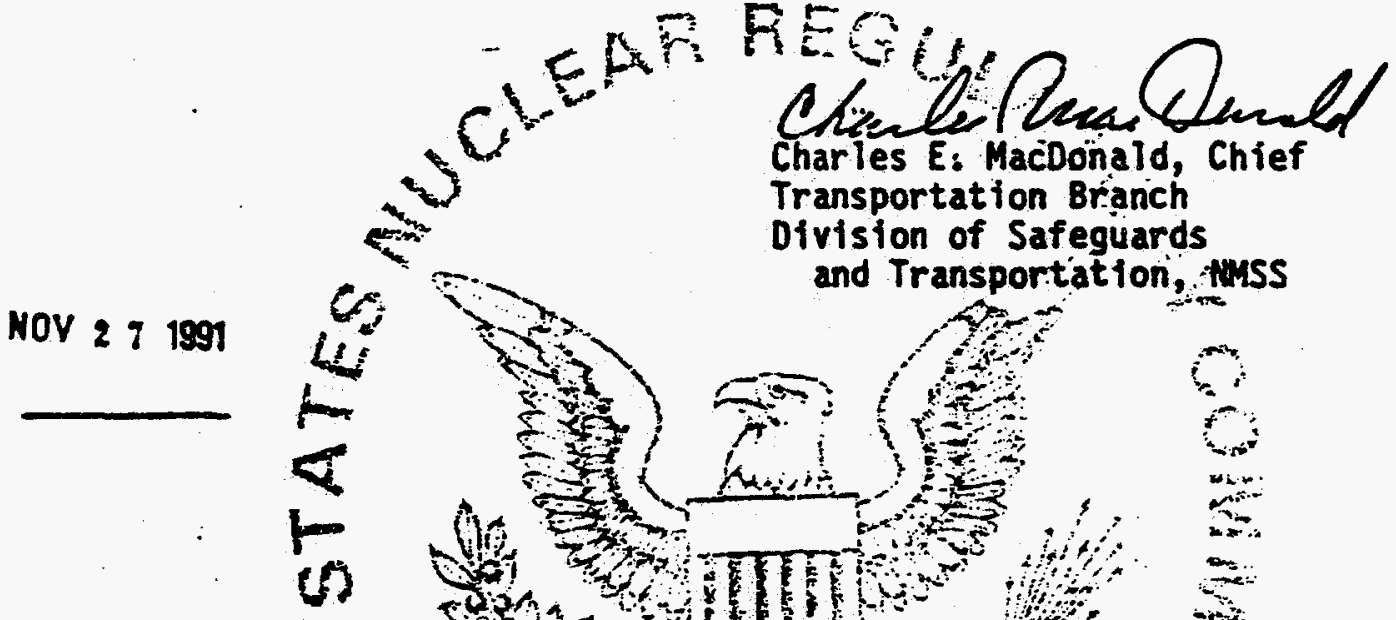

Charles E: MacDoñald, Chief

Transportation Branch

Division of Safeguards and Transportation, twSS

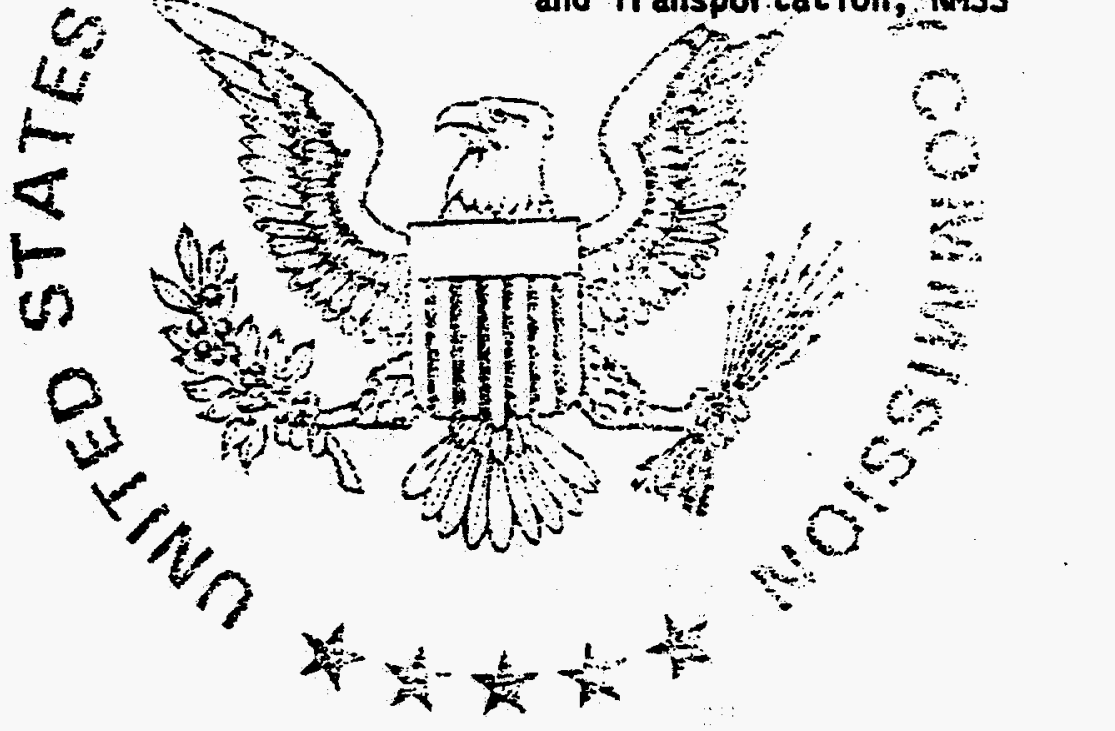


TN-REG

B-149 


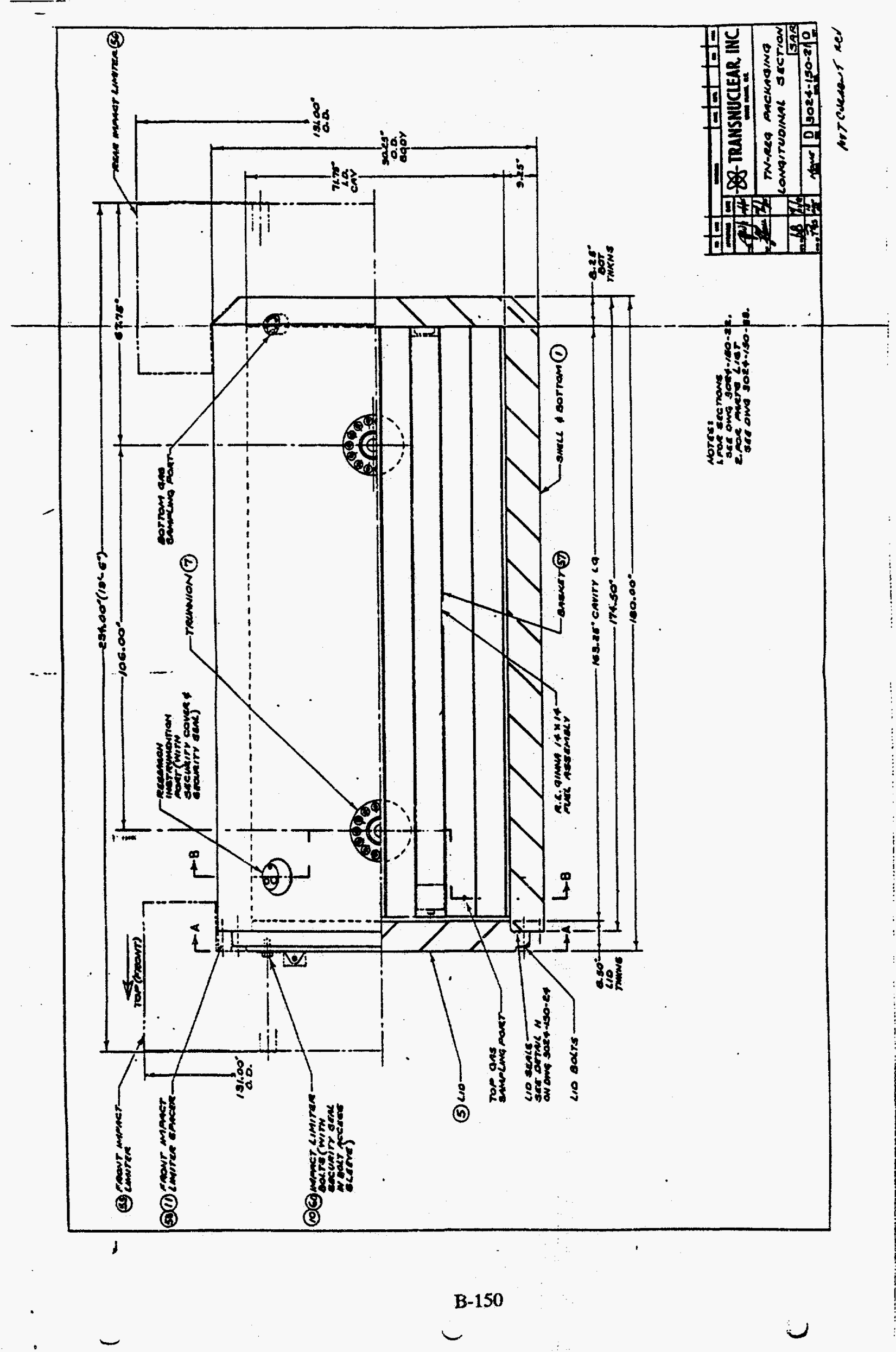




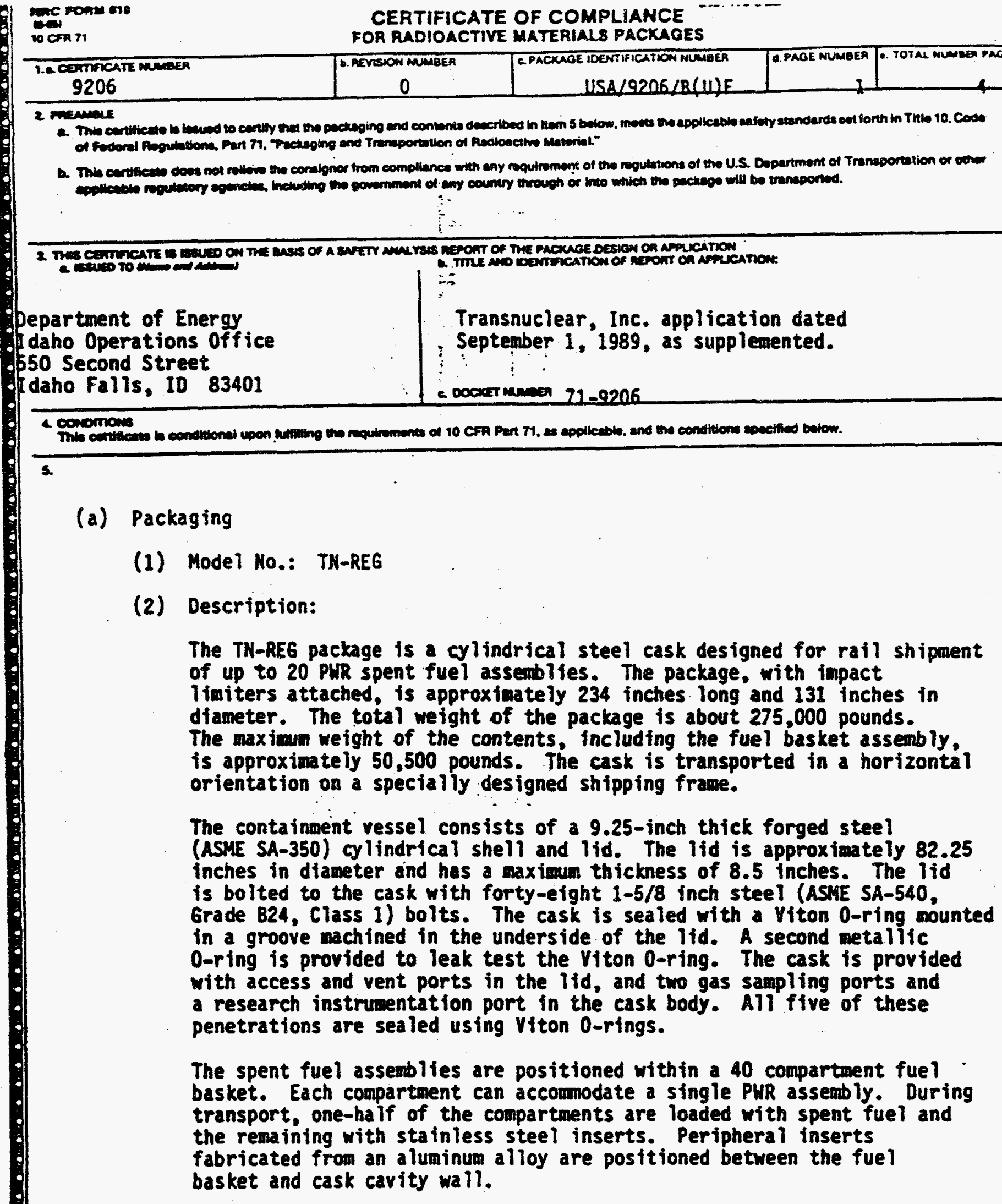


Page 2 - Certificate No. 9206 - Revision No. 0 - Docket No. 71-9206

5. (a) (2) Continued

The cask is equipped with impact 1 imiters made of balsa and redwood encased in carbon steel shells. The inpact limiters have an outer dianeter of 131 inches, an inner diameter of 91 inches, and a thickness ranging from 20 to 26 inches. Each impact limiter is attached to the cask by four 2.25-inch diameter bolts. The Impact limiters are also connected to each other with fourteen 1.5-inch dianeter tie rods.

The cask has four lifting lugs foreled to the lid, and four lifting/tfedown trunnions bolted tophs cask body:- $\mathrm{OCO}$

(3) Drawings<smiles>c1ccccc1</smiles><smiles>CCCCCCCCCCCCCC</smiles>

(i) The packaging is constructed in accordance with the following Transnuclear, Inc. Drawings:

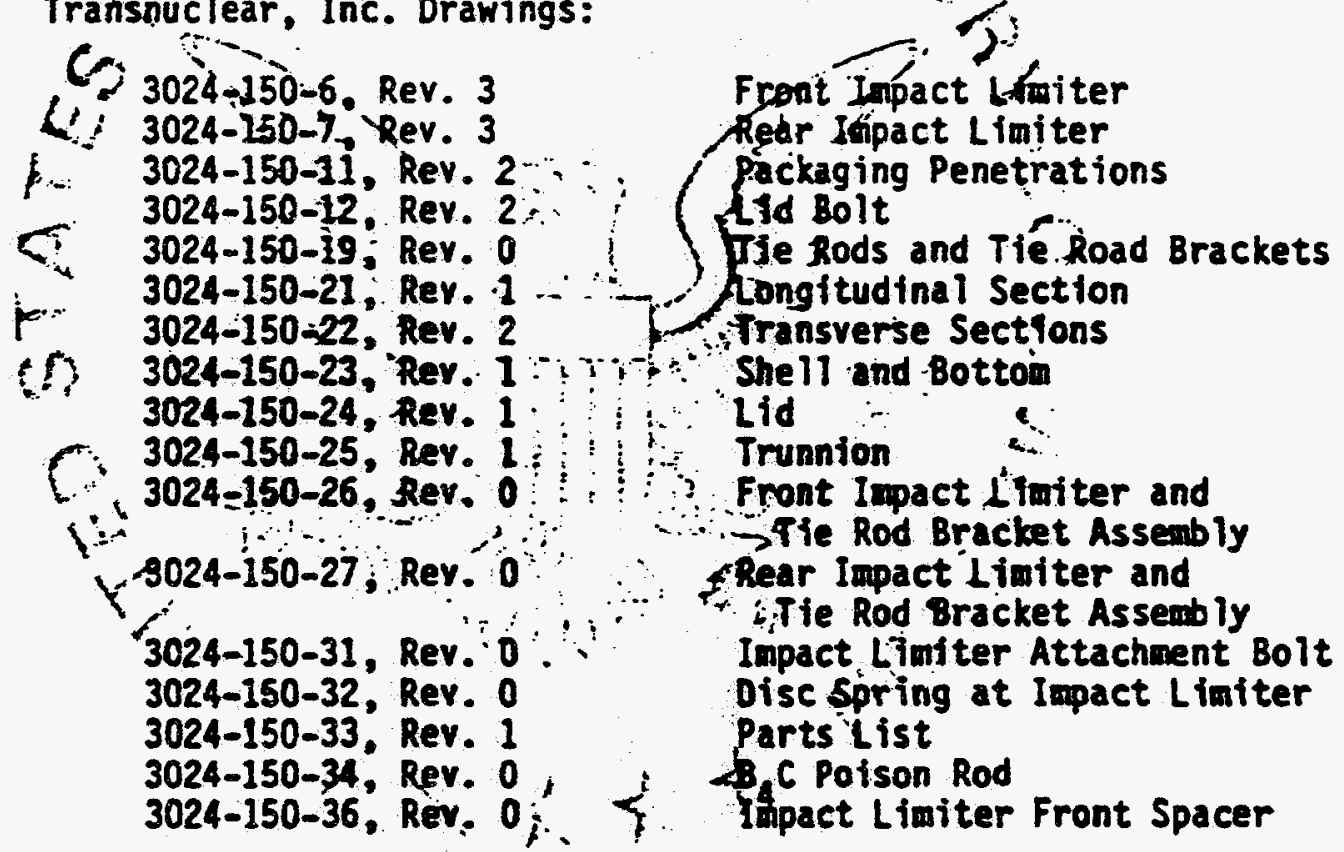

(ii) The fuel basket assembly is constructed in accordance with the following Transnuclear Drawings:

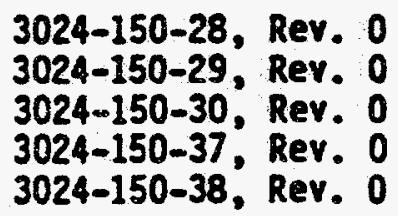

Basket-6eneral Arrangement

Basket-Cross Section

Basket-Plan View

Peripheral Insert

Fuel Replacement Insert 
Page 3 - Certificate No. 9206 - Regision No. 0 - Docket No. 71-9206

\section{5. (b) Contents}

(1) Type and form of miterial

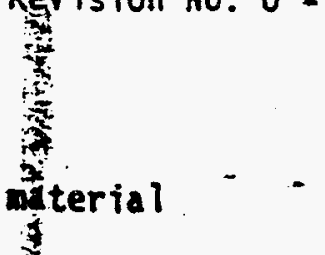

(i) Irradiated PH uranium oxide fuel assemblies as described in the application including the following specifications:

Fuel fornt

Mominal pellet diameter

Cladding materits 2 Z Zircalloy

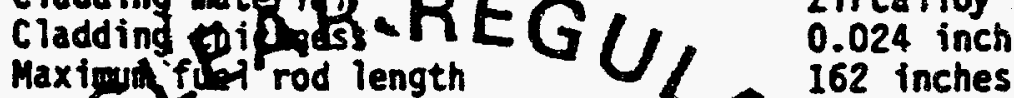

Maximut fun rod length

Max wernactive fuel rod length

Assembily array aximum initial fuel pin

pressure at $70^{\circ} \mathrm{F}$ Initiai xaranium loading

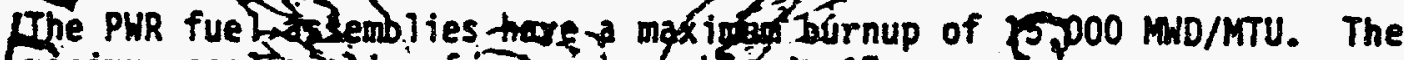

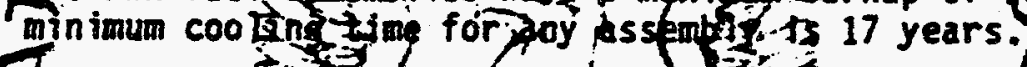

(2)

\section{Maxim}

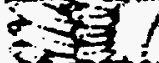

0.024 inch
162 inches

$\mathrm{UO}_{2}$ pellets

0.367 inch

144 inches

$14 \times 14$

2

enrichment<smiles>[Te]</smiles>

3.5. $1 / 0$

383 \&

(ix)

(i) paximen of

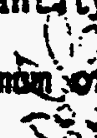

twedtatar

Sin 4 in $y$,

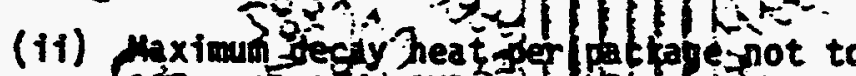

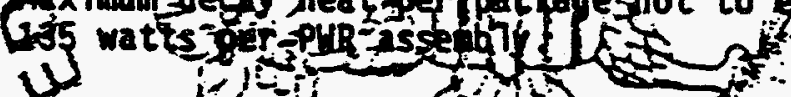

(iii) hove fuel assemblifs to be pasjyoued in thepyel baskets as shown in the drawings refetenced its (a)f $(i i)$.

(3) Fissile class?

Max imm number of $P$ kages per shippent

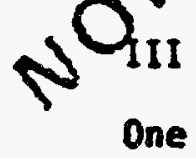

6. Shipments must be completed between Aprfi 1 and October 31. 
Page 4 - Certificate No. 9206 - Revision No. 0 - Docket No. 71-9206

7. Bolt torques:

(a) The cask lid bolts must be torqued to 1120 -ft-lbs."

(b) The bolts used to secure the vent and drain port covers must be torqued to $50 \mathrm{ft}$-lbs.

(c) The bolts used to secure the upper gas sampling port transport plug must be torqued to $30 \mathrm{ft}$-lbs.

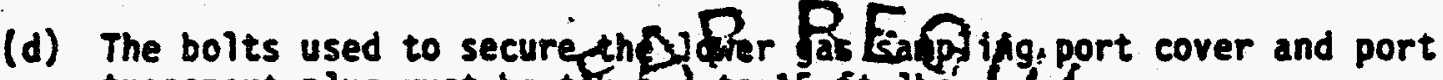
transport plug must be thequed to $15 \mathrm{ft}$-lbs.

Cin

8. Known or suspected farthol assemblies (rods) and fue $y^{\text {ath }}$ cladding defects greater than hairline tacks are not authorized.<smiles>C1=C[Ge]=C1</smiles>

9. In addition to the requicements of Subpart $G$ of 10 CFR Part-71:j:

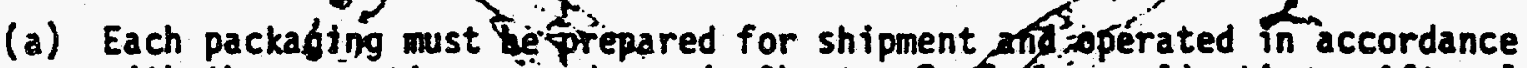 with the gperating procedures in Chapter 7 of the epplicatipn: After loading, the cask hust be vacuuin aried and backfill ed filth, nitrogen at one atmosphere as described in Chapter jo the applicationt

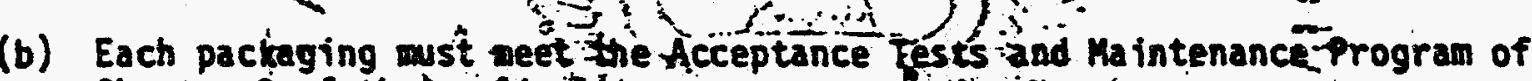 Chapter 8 of the application
(c) The packaging must josed in focordance with section 7.12 .19 of the application.

10. The package authorized by this certificate is hereby approved for use under the general license provisions of 10, CFR $71,12 . \cdots i_{i}$

11. Expiration Date: May bi, 1995.

\section{$Y$ REFERENCES}

Transnuclear, Inc. application dated September-1, 1989.

Supplement dated March 7, 1990.

MAY 151990

FOR THE U.S. NUCLEAR REGULATORY COMMISSION

Date:

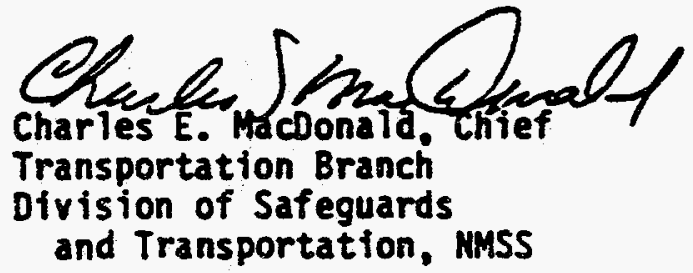


M-160

B-155 
Drawing Not Available 


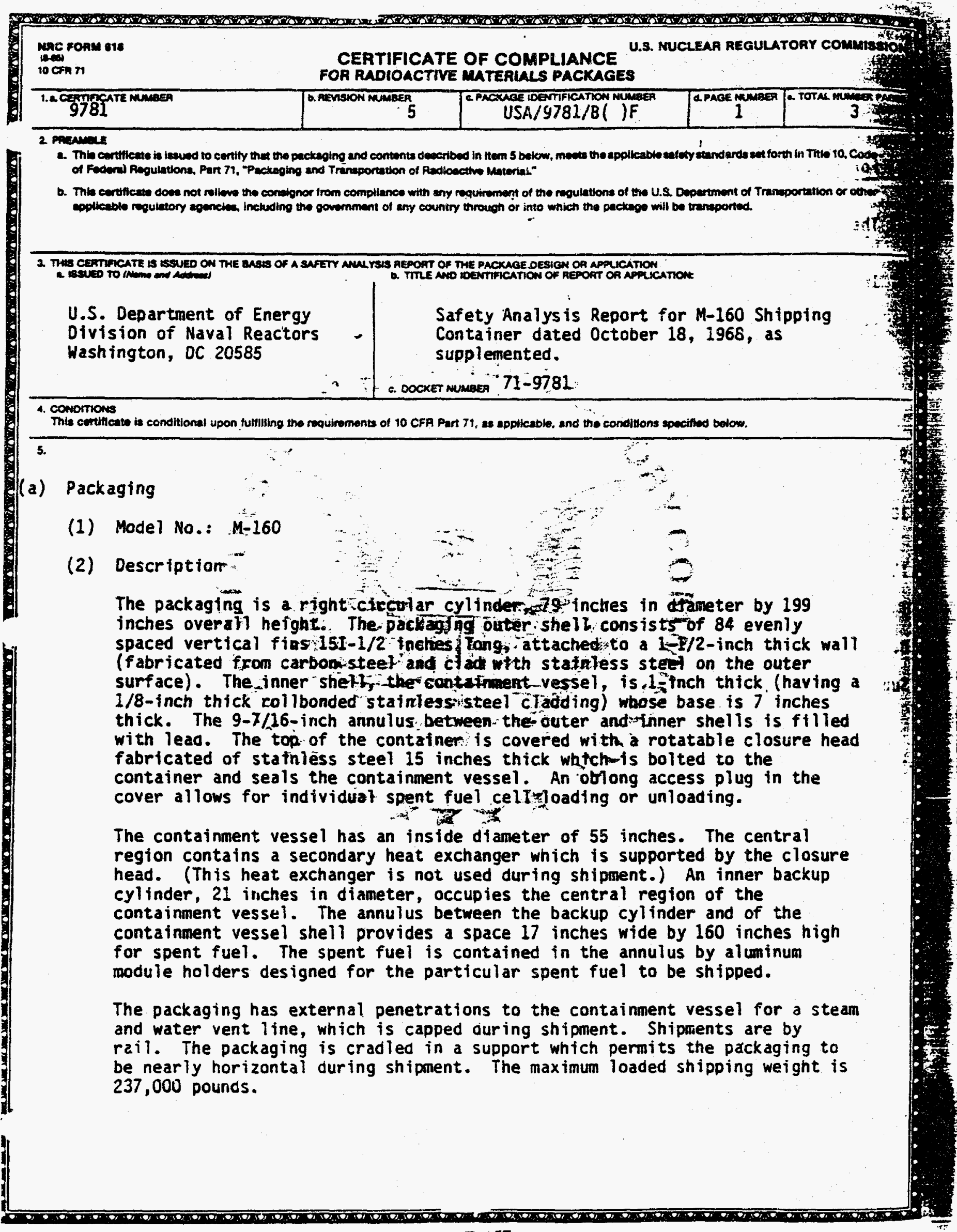


Page 2 - Certificate No. 9781 - Revision No. 5 - Docket. No. 71-9781

\section{5. (a) Packaging (continued)}

\section{(3). Drawings}

The packaging is constructed in accordance with the description and Drawing Nos. contained in the Bettis Atomic Power Laboratory Safety Analys is Reports (WAPD-OP (R)C-243, WAPD-OP(R)C-558 and WAPD-OP(R)C-621 dated May 1973, October 1, 1976 and March 1977.

(b) Contents

(1) Type and form of material.

Irradiated fuel assemblies and blanket modules of the following type:

(i) PWR Core 2 Seed 1 Fuel Assembly.

(ii) PWR Corre 2 Seed 2 fuel Assembly.

(iii) PWR Core 2 Blanket Fuel Assenbiy.

(iv) S5G Fuet Module, rodded or unrodded.

(v) S5E.Center-Cell.

$$
\text { shom? }
$$

All shipments shat the trade $\overline{d r y}$ and shati use one hädown device per PWR module- Each PhfC Care 2 Seed 1 or Seedzfoul assembiy shall conta in a

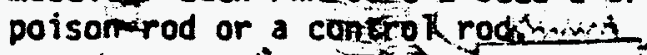

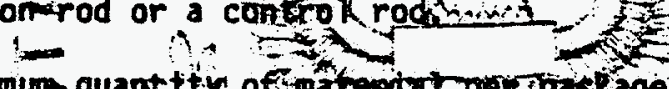

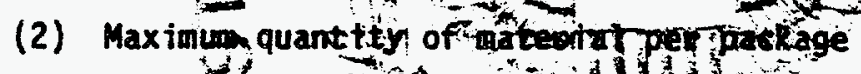

(1) 12fuel assemblies as descutber in $5(b)(1)$ (i) or 11 fuel assemblies and-one spectific btankete fulek assembty, Serial-No. 62A-W01-67. Shipwent shaly nat be- oudenprior to:1,614 days" after last power operation of the fuel and shall nat exceed-12;846 Btu/hr of decay heat peri:shipment.

$$
\text { assenb }
$$$$
+2
$$

(ii) 12 fuel assemblies as described in $5(b)(i)(i i)$ which shall not exceed 1,100 Btu/hr per fuel assentily of decay heat or 13,200 Btu/hr per shipment

(iii) 12 blanket fuel assemblies as described in $5(b)(1)$ (iii) which shall not exceed $21,300 \mathrm{Btu} / \mathrm{hr}$ of decay heat per shipment. Shipment shall not be made prior to 1,123 days after last power operation of the fuel. 
conomions (continued)

Page 3 - Certificate No. 9781 - Revision No. 5 - Docket No. 71-9781

(b) Contents (continued)

(2) Maximum quantity of material per package (continued)

(iv) 8 fuel assemblies as described in $5(b)(1)(i i)$ and 4 spectific blanket fuel assemblies, Serial Numbers G2A-F01-26B, G2A-F01-02, 62A-F01-10 and 62A-W01-73, which shall not exceed 12,016 Btu/hr of decay heat per shipment. Shipment shall not be made prior to 1,487 days after last power operation of the fuel, with the four blanket fuel assemblites located adjacent to each other in a clockwise or counter-clockwise direction as specified by the serfal numbers previously stated.

\section{Q R R B}

(v) 4 fuel assemb fles as described in $(b)(1)$ (iv) or 3 fuel assemblies and one (eater cell as described in $5(6), 4)(v)$. Shipment shall not be madepprior to 168 days after last powec operation of the fuel rod aga-shall not exceed $12,800 \mathrm{Btu} / \mathrm{hr}$ ofudecay heat per shipment.

(c) Fisstle Clas Maximum number of padkoses per shipment

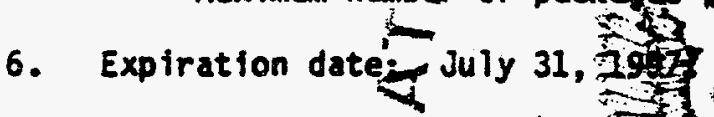

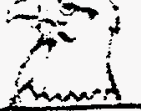<smiles>[C]1[CH]CC1</smiles><smiles>C1CCCCC1</smiles>

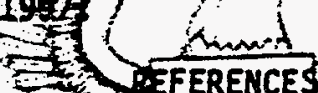

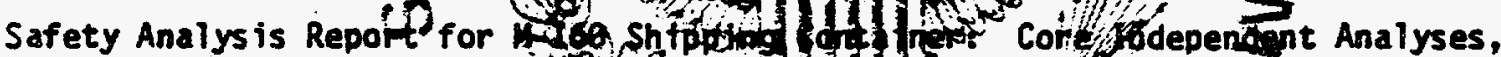

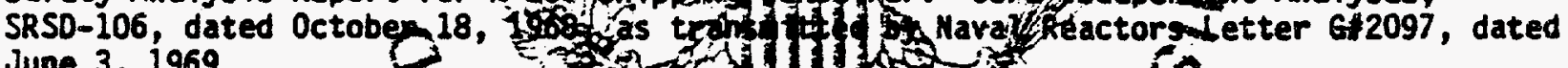
June 3, 1969.

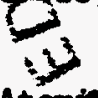

Supplements: Knolls Atcuit Power Laboratory Tetter 747520-4If, dated Novenber 26, 1969; Naval Reactors 1etter 6\$3742, dated Mayn15, 1973; Bett. (S) Átonic Power Laboratory letters WAPD-OP(R)C-284, datedugust 23, 1973 and WAPD-0RAN 297 , dated October 8, 1973; Naval Reactor letters GF582, dated Decenber 17, 1976 ; G\$567i, dated April 15, 1977; G\$5702, dated May 23, 1977; G:5792, dated Septe 'yer 22, 1977; G\$5793, dated Septenber 29, 1977; G\$5872, dated Detem 21 19, ; G\$5897, dated January 11, 1978; 6\$6658, dated Aprl1 14, 1980, and 6\$92-03424, Dated March 20, 1992.

FOR THE U.S. NUCLEAR REGULATORY COMMISSIOH

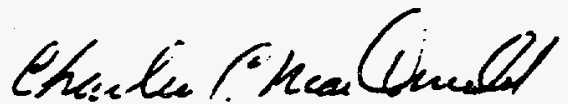

Charles E. MacDonald, Chief

Transportation Branch

Division of Safeguards and Transportation, NisS

Date:

APR : $: 199 ?$ 
M-140

B-160 
Drawing Not Available

B-161 


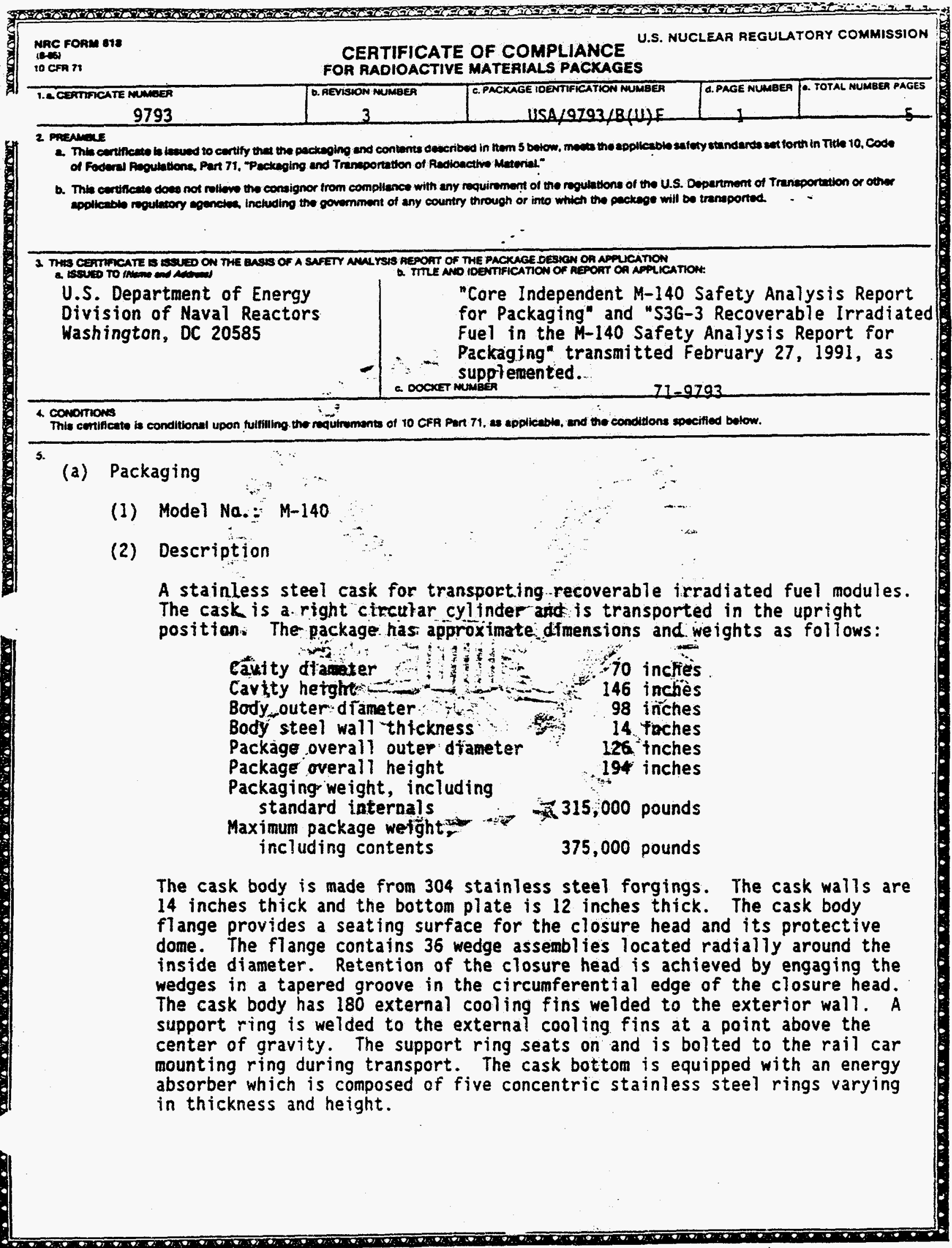


Page 2 - Certificate No. 9793 - Revision No. 3 - Docket No. 71-9793

\section{(2) Description (con't)}

The closure head is made from forged 304 stainless steel, and is approximately 13 inches thick and 81.7 inches in diameter. The closure head is equipped with an access port, which is approximately 24 inches in diameter, and is offset from the center of the closure head. The access port plug is a stepped design with a maximum diameter of approximately 31 inches and is attached to the closure head by 24 bolts. The closure head and access port are sealed with double ethylene propylene 0-ring seals. Seal test ports are provided for the closure head and access port seals. stainless steel protective dome is positioned over the closure head and is secured to the cask body frange-by: 12 ; 1.38-inch diameter, 38.5-inch long studs installed in a-vertical direction and: 6, 2.5-inch diameter, 9-inch long shear bolts jinstatled in the radial direction.

The containment system is composed of the cask body, the closure head and the closure head access port plug. There are five penetrations in the containment system: a drain port and vent port in the closure head, and a thermocouple penetration, a water inlet pepetration, and a water outlet penetration in the cask-body. Each penetration is sealed with a plug and a double ethylene propylene 0-ring-seal, and is equipped with a leak test port.

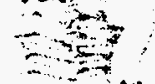
$\rightarrow$

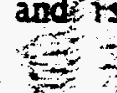

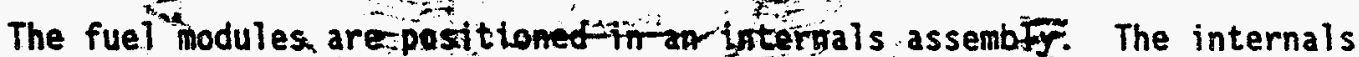
assemblints composed of stacked interna 7 spacer pl ates wich have openings for the thel modares. "The fritefualis assemblyithas a topoplate or top plate subassembiy whrobs sipreloaded bj springs agaiust a retining ring fitted in

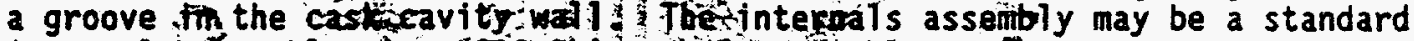

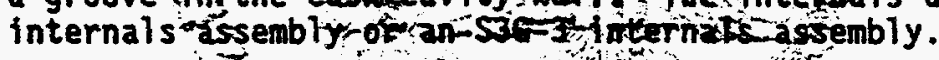

(3) Drawings

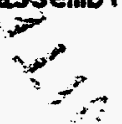

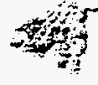

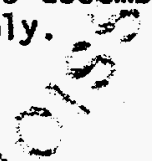

The packaging is constructed and assembled inacordance with the Westinghouse Electric Corporation Drawings in Appendix 1.3.2 of the application.

\section{(b) Contents}

\section{(1) Type and form of material}

Recoverable irradiated fuel modules, limited to the following types, including associated activated corrosion products: S3G-3 recoverable irradiated fuel modules.

(ii) 586 recoverable irradiated fuel modules.

(iii) D1G Core 2 recoverable irradiated fuel modules. 
Page 3 - Certificate No. 9793 - Revision No. 3 - Docket No. 71-9793,

(2) Maximum quantity of material per package

Total package weight, including fuel modules and internals assembly, not to exceed 375,000 pounds; and

(i) For contents described in $5(b)(1)(i)$ :

\$3G-3 recoverable irradiated fuel modules, not to exceed 62,300

Btu/hr decay heat per package.

For contents described in $5(\mathrm{~b})(1)(\mathrm{i} i)$ :

S8G recoverable irradiated fuef modules, not to exceed $51,609 \mathrm{Btu} / \mathrm{hr}$ decay heat.per package (prototype modules), or $45,713 \mathrm{Btu} / \mathrm{hr}$ decay heat per package (shipboard modules).

(iii) For cantents described in $5(b)(1)(i i i)$ :

DIE,Core 2 recoverable irradiated fuel-modulesi-not to exceed 37,750 Btw/hr decar theat per package.

(c) Fissile Classis
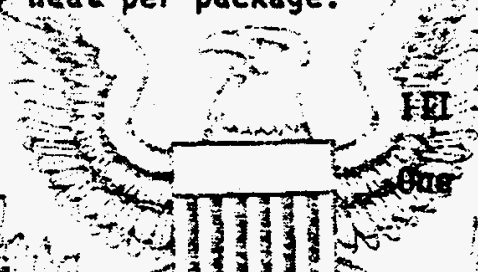

Maximum number of packages per shipmenty

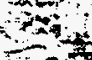

\section{$-1$}
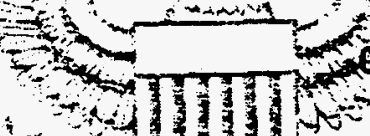

(a) Only a full road is authorized. A minimuthof twe pre fuel modules must have either contror rods or poison-shipping rods. Al1 rodded and unrodded modules must be positioned as specified on pago-36-11 (Rev. 1) of "S3G-3 Recoverable Irradiated Fuel in the M-140 Safety Analysis Report for Packaging;"

$$
\text { is }
$$

(b) Minimum fuel cooling time is 130 , days after shutdown;

(c) Core age must be at least 4,000 logging corrected full power hours;

(d) Control rod hold-down devices must be installed on cells which have control rods;

(e) All cells must have top and bottom energy absorbers;

(f) The weight of the fuel modules must be limited as specified on page 1-23 (Rev. 2) of "S3G-3 Recoverable Irradiated Fuel in the M-140 Safety Analys is Report for Packaging;" and

(g) S3G-3 internals assembiy must be used for shipment of \$3G-3 fuel modules. 
Page 4 - Certificate No. 9793 - Revision No. 3 - Docket No. 71-9793

7. For $\$ 8 G$ recoverable irradiated fuel shipments:

(a) Only a full load is authorized. Full and partial fuel modules may be shipped in any combination. All full and partial fuel modules must have control rods;

(b) Minimum fuel cooling time is 248 days after shutdown for prototype modules, and 157 days after shutdown for. shipboard modules;

(c) All fuel modules must have lower supports and grapple adapters;

(d) Standard internals assembly must:benused for shipment of S8G fuel moduies. Full fuel modules must have two fult (side) spacers, partial fuel modules must have two full. (side) spacers and one partial (inside) spacer; and

(e) The weight of the fuel modules must be limited as specified on page 1.23 (Rev. 4) of "S8G" Recoverable Irradiated Fuel in the-Mr140 Safety Analys is Report For Packaging."

8. For 016 Core 2 recoverable irradiated fuel shipments:

(a) Up to eight fuel modules may be shipped per package. Fuel modules of different types may be shipped in any combination.

(b) Minimum foel cooling ttmos rs 181 days after shutdown.

(c) All normalty rodded fuel modeles nust have control rodsin control rod holddown devices must beripstalled on rodded modules.

(d) Rodded modules must have top and bottom energy absorbers. Unrodded modules must have top energy absorbers.

(e) Standard internals assembly must be used for shipment of D1G Core 2 fuel modules. Fuel moduTe cavity spacers must be used for all fuel modules.

9. The package must contain no more than 6 gatlons of residual water.

10. Failed fuel, or fuel with defective cladding is not authorized for shipment.

11. Each packaging must meet the Acceptance Tests and Maintenance Program of Chapter 8 of the application, except:

All containment seals, including the main closure head seal, must be replaced with new seals within the 12 -month period prior to each shipment, or earlier if inspection shows any defect.

12. The package must be prepared for transport and operated in accordance with Chapter 7 of the application, except:

The containment seals, excluding the main closure head seal, must pass a leak test after final closure prior to each shipment. The leak test must have a sensitivity of at least $1 \times 10^{-3} \mathrm{std}-\mathrm{cm}^{3} / \mathrm{sec}$. 
Page 5 - Certificate No. 9793 - Revision No. 3 - Docket No. 71-9793

13. Prior to first use, and within the 12-month period prior to each shipment, all containment seals, including the main closure head seal, must be leak tested to show a leak rate no greater than $1 \times 10^{-4} \mathrm{std}-\mathrm{cm}^{3} / \mathrm{sec}$. The leak test must have a sensitivity of at least $5 \times 10^{-5} \mathrm{std}-\mathrm{cm}^{3} / \mathrm{sec}$.

14. Expiration date: October 31, 1996.

\section{REFERENCES}

"Core Independent M-140 Safety Analysis Report For Packaging," and "S3G-3 Recoverable Irradiated Fuel in the M-140 Safety Analysis Report For Packaging," transmitted February 27, 1991.

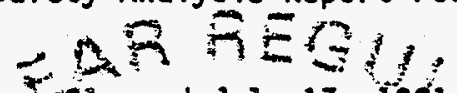

Supplements dated: May 23,June-21, and July 17, 1991, and February 4 and 7 , August 17, and December $2,1992$.

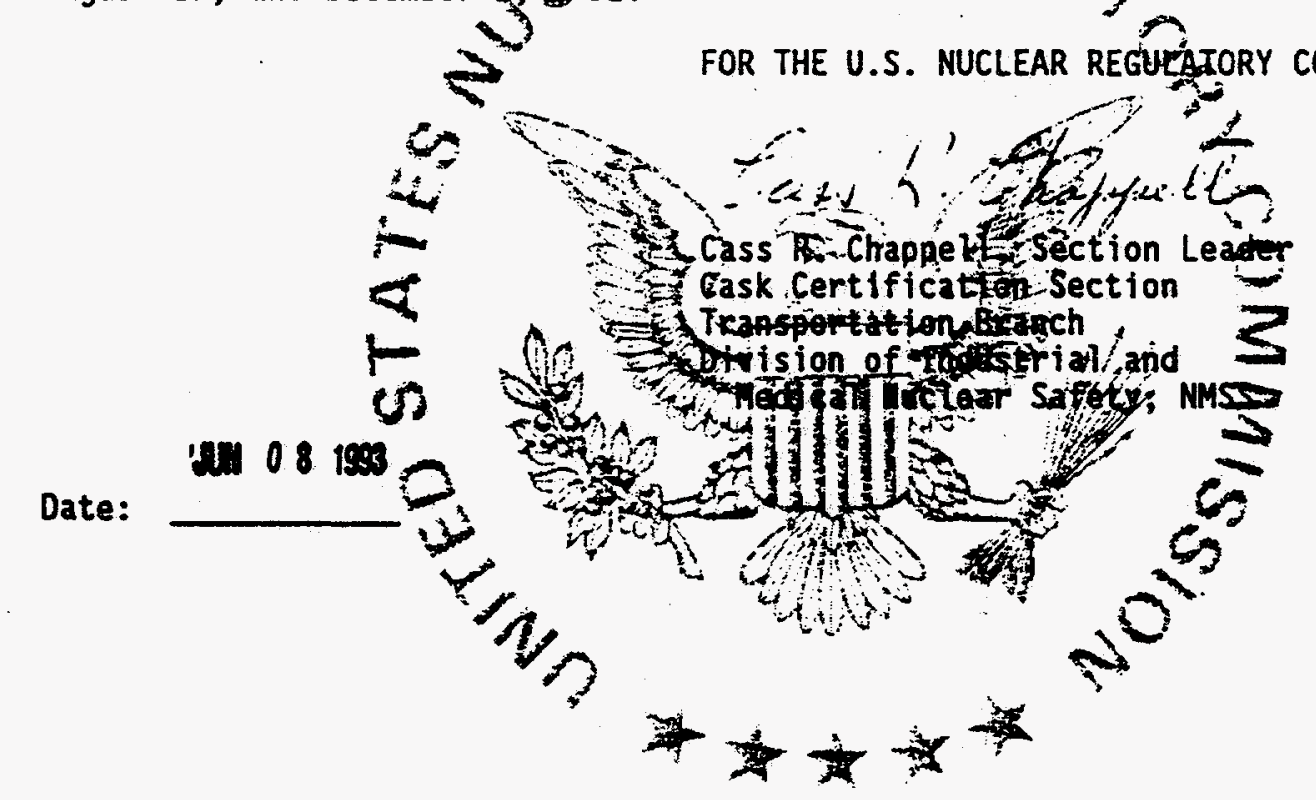

